\title{
Studies of Some Strained Organic Molecules
}

\author{
by
}

Carissa Susan Jones

\begin{abstract}
A thesis
Submitted to the Victoria University of Wellington

in fulfilment of the

requirements for the degree of

Doctor of Philosophy

in Chemistry
\end{abstract}

Victoria University of Wellington,

2001 


\section{Abstract}

The characterisation of rare examples of $\mathrm{C} 1$-substituted cyclopropanaphthalenes has been achieved with silanes (104) and (112) by employing the $\mathrm{C} 1$ anion (106). With toluene, $N, N$-dimethylacetamide, and cyclopropanaphthalene (58) this same anion gives the novel 6-methyl-7H-dibenzo[b,g]fluorene (179), a formal dimer of cycloproparene (58). Hydrocarbon (179) is the sole dibenzo[ $b, g]$ hydrocarbon characterised and this has required extensive spectroscopic study with confirmation from X-ray analysis.

A possible new route to alkylidenecyclopropanaphthalenes (114) employing lithiate (170) and either cycloproparene (58) or its disilyl analogue (105) was found to offer no advantage over known procedures. Application of the protocols embodied in this procedure to brominated synthons (114o) and (114p) has afforded novel $\pi$-extended methylidene compounds (197a) and (199) in low yield. Cyclopentadienylidene (197a) has also been prepared in better yield from benzophenone-containing methylidenecycloproparene (200). Initial attempts to obtain (200) from anion (193) and $N, N$-dimethylbenzamide were unsuccessful and gave instead the new phenol (114q).

The first acylcycloproparenes (189) and (202) have been obtained in modest yield from anion (103) and $N, N$-dimethyl-acetamide, and -benzamide. With $N, N$-dimethylcarbamoyl chloride anion (103) gives the bis-amide (205). With hydrochloric acid these acylcycloproparenes give rise to 2,3-disubstituted naphthalenes rather than 2-substituted naphthalenes that typically arise from protonation at the aromatic ring. Thermolysis leads to ring expansion and naphthofuran formation. Enolate formation from the 1-acyl-cyclopropanaphthalenes (189) and (202) and anion capture at oxygen affords the first cyclopropanaphthalenylidene enol ethers (219) and (220).

$1 H$-Cyclopropa $[b]$ naphthalene-3,6-dione (154) adds buta-1,3-diene across the enedione $\pi$-bond to give the tetrahydrocyclopropanthraquinone (160). Enolisation of (160) provides phenolate (234) that can be diverted to ether (229) or oxidised to the dihydroanthraquinone (230). Dehydrogenation of (229) is readily achieved and gives the first anthraquinone of the cycloproparene series $1 H$-cyclopropa $[b]$ anthracene-3,8-dione (162); quinone (162) is only the second cyclopropaquinone to have been characterised. Alternative routes to quinone (162) and its 3,8-dimethoxy analogue (163) have been examined with a view to providing the first alkylidenecyclopropanthracenes.

The first examples of cross-conjugated dithiole-containing cycloproparenes, (169) and (267), have been prepared from cyclopropanthraquinone (162) but they are unstable solids. The $\pi$-extended dithiole-containing methylidene compound (273) has been 
prepared in good yield from Wittig-Horner olefination of the benzoylmethylidene compound (200). Evidence was obtained to support the formation of a charge-transfer complex from it. Ketones already carrying a conjugated dithiole moiety participate in the Peterson olefination with the $\alpha$-silyl anion (106) and give the new $\pi$-extended methylidenecyclopropanaphthalenes (274) and (277) of limited stability. 


\section{Acknowledgments}

Of the many individuals that have contributed to this project it is my supervisor Professor Brian Halton who deserves, without a doubt, my most heart-felt thanks. I would like to express my sincere gratitude to Professor Halton for his unwavering help, support, and patience over the last three-and-a-half years; he has been an inspiration to me. As a result of his guidance I feel that I have evolved both as an individual and in a professional sense and these words alone can not describe the positive impact he has had on me.

I would like to thank Dr. Peter Northcote for his support, hospitality and for all the help and advice he has given me over the last few years. Thank you to Dr. John Hoberg for his useful tips on laboratory techniques and to Dr. Jim Pearce for assisting me with the preparation of silver/silver chloride electrodes and measuring cyclic voltammograms.

The graduate students and technical and general staff members in the School of Chemical and Physical Sciences have been invaluable. However, in particular, I would like to thank Dave Gilmour, Alan Rennie, Rhyl Singleton, Grant Franklin, Bill Leck, Peter Bickers, Matt Freeman, Bridget Stocker, Irina Chamritskaia, and Sanja Sretenovic for their support, friendship and for being there for me.

Parts of this dissertation were proof read by Dr. Barry Dent and Dr. Dennis Page and their assistance is appreciated.

I would like to thank the following sources for financial support: the CurtisGordon Research Fund, the Victoria University of Wellington Alumni Association, the Claude McCarthy Fellowship Trust, the Royal Society of New Zealand, the New Zealand Institute of Chemistry, and the Victoria University of Wellington Internal Grants Committee.

Finally, I would like to thank my partner and best friend Dr. Lyndon West for his unconditional love, patience, and help he has given me over the last three-and-a-half years. 


\section{Table of Contents}

Abstract...............................................................................

Acknowledgments....................................................................

Table of Contents.........................................................................

List of Tables......................................................................vi

Chapter One: $\quad$ Introduction......................................................

Chapter Two: $\quad$ Chemistry of the Monosilylcycloproparenyl anion..............29

Chapter Three: $\quad$ Approaches to Methylidenecyclopropanaphthalenes.............44

Chapter Four: $\quad$ 1-Acyl-1H-cyclopropa $[b]$ naphthalenes and the

Preparation of Cyclopropa $[b]$ naphthalenylidene

Enol Ethers....................................................64

Chapter Five: $\quad$ Approaches to Oxygenated Cyclopropanthracenes...............80

Chapter Six: $\quad$ Approaches to Dithiole-Containing Cycloproparenes............103

Chapter Seven: $\quad$ Experimental................................................116

Appendix A Re-prints of Published Papers.................................164

References......................................................................187 


\section{List of Figures}

Fig. 1. ${ }^{1} \mathrm{H}$ n.m.r. spectrum of the dimer in $\mathrm{CDCl}_{3}$ solution at $300 \mathrm{MHz}$

Fig. 2. Expansion of the aromatic region of the ${ }^{1} \mathrm{H}$ n.m.r. spectrum of the dimer in $\mathrm{CDCl}_{3}$ solution at $300 \mathrm{MHz}$

Fig. 3. HOMO 2DJ spectrum for a $\mathrm{CDCl}_{3}$ solution of the dimer $(300 \mathrm{MHz})$....

Fig. 4. Substructures of the dimer with selected ${ }^{1} \mathrm{H}$ n.m.r. chemical shift data (ppm)...38

Fig. 5. $\quad{ }^{1} \mathrm{H}$ n.m.r. chemical shifts (ppm) and nuclear Overhauser enhancements (arrows) recorded for 6-methyl-7H-dibenzo[b,g]fluorene (179) in $\mathrm{CDCl}_{3}$ at $300 \mathrm{MHz}$.

Fig. 6. ORTEP plot of 6-methyl-7H-dibenzo[b,g]fluorene (179) with crystallographic numbering at $50 \%$ probability

Fig. 7. Selected bond lengths $(\AA)$ of dibenzo[b,g]fluorene (179) and fluorene $(180) \ldots 41$

Fig. 8. $\quad{ }^{1} \mathrm{H}$ n.m.r. spectrum of the compound proposed as alkylidene derivative (114n) $\left(\mathrm{CDCl}_{3}, 300 \mathrm{MHz}\right)$

Fig. 9. $\quad{ }^{1}$ H n.m.r. spectrum of alkylidenecyclopropanaphthalene (197a) $\left(\mathrm{CDCl}_{3}, 300 \mathrm{MHz}\right)$.

Fig. 10. ${ }^{1} \mathrm{H}$ n.m.r. spectrum of dicarboxamide (205) $\left(\mathrm{CDCl}_{3}, 300 \mathrm{MHz}\right)$ .66

Fig. 11. ${ }^{1} \mathrm{H}$ n.m.r. spectrum of siloxyalkene (220c) $\left(\mathrm{CDCl}_{3}, 300 \mathrm{MHz}\right)$. .75

Fig. 12. Two views of the favoured conformer of silyl enol ether (220c) showing the shielding of $\mathrm{H} 2$ by a $\mathrm{Si}-\mathrm{Ph}$ ring; the distance of $\mathrm{H} 2$ to the centre of the pendant phenyl is $411 \mathrm{pm}$ .76

Fig. 13. Calculated $\left(6-31 G^{*}\right)$ conformation of $(160)(a)$ without geometrical constraints and $(\boldsymbol{b})-(\boldsymbol{e})$ with the $\mathrm{H} 3 \mathrm{a}-\mathrm{C} 3 \mathrm{a}-\mathrm{C} 7 \mathrm{a}-\mathrm{H} 7 \mathrm{a}$ dihedral angle constrained to $0^{\circ} \mathrm{C}$. Data taken from reference 119 .... 85

Fig. 14. Calculated (6-31G $\left.{ }^{*}\right)$ geometry of butadiene adduct (160) and X-ray geometry of quinone (154). Angles are shown in degrees $\left({ }^{\circ}\right)$ and the bond lengths in $\AA$. Data for (160) and (154) are taken from references 119 and 116 , respectively.

Fig. 15. ${ }^{1} \mathrm{H}$ n.m.r. spectra for compounds (a) (160), (b) (234) and (c) (230). Spectra were recorded in $d_{6}$-acetone at $300 \mathrm{MHz}$.

Fig. 16. ${ }^{13} \mathrm{C}$ n.m.r. spectra for compounds (a) (160), (b) (234) and (c) (230). Spectra were recorded in $d_{6}$-acetone at $300 \mathrm{MHz}$.

Fig. 17. ${ }^{1} \mathrm{H}$ and ${ }^{13} \mathrm{C}$ n.m.r. (bold) chemical shifts (ppm) for cyclopropanthraquinone (162), cyclopropanaphthalene (154), and anthraquinone. The ${ }^{1}$ H n.m.r. data were taken from reference 116 . 
Fig. 18. (a) ${ }^{1} \mathrm{H}$ n.m.r. and (b) ${ }^{13}$ C n.m.r. spectra for cyclopropanthraquinone (162). Spectra were recorded in $\mathrm{CDCl}_{3}$ at $300 \mathrm{MHz} \ldots \ldots \ldots \ldots \ldots \ldots \ldots \ldots \ldots \ldots . \ldots . \ldots \ldots$

Fig. 19. Projected minimum energy conformers of dithiole cycloproparene (169)....108

Fig. 20. Proton spectrum of the dithiole-containing cyclopropanthracene (267)

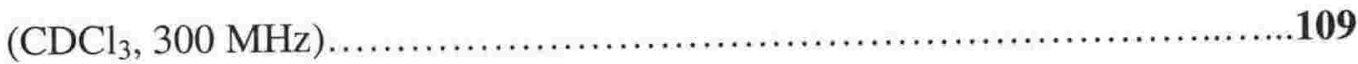

Fig. 21. Proposed minimum energy of conformers of dithiole (267) ..............109

Fig. 22. Alternate views of the sheet-like packing array of (275) in its crystalline form $(a)$ view of the array in which compound (275) packs and $(b)$ an alternative view of the packing of (275) ( $\mathrm{S}$ * S contacts shown as broken lines) 


\section{Chapter 1}

\section{Introduction}

Strained organic molecules have attracted considerable attention for more than a century. ${ }^{1}$ Because of the high energy that is associated with strain, such molecules often possess unusual chemical, physical, and spectral properties. Moreover, the limits to which stress and strain may be imposed upon a hydrocarbon framework continues ${ }^{2}$ to fascinate and challenge the chemist, theoretician and experimentalist alike.

Angle strain results from the distortion of bond angles from their customary values and is an inherent property of small-ring compounds. The smallest cyclic compound, cyclopropane, has a measured ${ }^{3}$ strain energy of $115 \mathrm{~kJ} \mathrm{~mol}^{-1}$ as a result of internal bond angles of $60^{\circ}$; it is somewhat less stable and more reactive than its linear analogue. The more highly strained cyclopropene has a measured ${ }^{4}$ energy of $220 \mathrm{~kJ} \mathrm{~mol}^{-1}$ and, although stable at liquid nitrogen temperature, it readily polymerises above $-80^{\circ} \mathrm{C} .^{5}$

One way to stabilise a strained system is to incorporate bulky substituents around the reactive centre. For example, whilst tetrahedrane is too reactive to be isolated, its sterically congested tetra-t-butyl derivative (1) is a stable crystalline solid that melts at $135^{\circ} \mathrm{C}$. The strain energy of (1), measured as $541 \mathrm{~kJ} \mathrm{~mol}^{-1}$, gives a strain enthalpy of $\sim 90$ $\mathrm{kJ} \mathrm{mol}^{-1}$ per ring bond; this is the highest value found for any compound. ${ }^{6}$

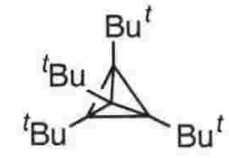

(1)

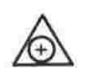

(2)

An alternative means of stabilising a reactive system is through aromaticity. The cyclopropenyl cation (2) is the simplest example in which strain and aromaticity work in juxtaposition. Early calculations ${ }^{7}$ suggested that converting cyclopropene to cation (2) would increase the strain energy in the monocyclic framework by $84 \mathrm{~kJ} \mathrm{~mol}^{-1}$. However, $\sim 167 \mathrm{~kJ} \mathrm{~mol}^{-1}$ of aromatic stabilisation energy was expected to be gained in the process. ${ }^{7}$ Consequently, cation (2) was predicted to be $83 \mathrm{~kJ} \mathrm{~mol}^{-1}$ more stable than cyclopropene and it has been detected in the tail of Halley's comet. ${ }^{8}$ In fact, the stability of (2) is such that its salts are easily isolated. ${ }^{9}$ Glukhovtsev and coworkers have now recalculated ${ }^{10}$ the stabilisation energy associated with (2) as $247 \mathrm{~kJ} \mathrm{~mol}^{-1}$. This value is nearly $100 \mathrm{~kJ} \mathrm{~mol}^{-1}$ higher than that of benzene. ${ }^{11}$ Due to this unusually high stabilisation energy the cyclopropenyl cation may be regarded as "superaromatic".

ortho-Annelation of the benzenoid framework should provide strained entities such as (3)-(5) which are expected to be stabilised, in part, through aromaticity. Such 
compounds serve as useful models to test the boundaries of stress and strain imposition on an aromatic moiety and, therefore, are of great importance. Didehydrobenzene ${ }^{12}$ (benzyne, 3) may be regarded as the parent member in this series of ortho-bridged aromatics. It is capable only of transient existence at ambient temperature. It has, however, been intercepted in Diels-Alder cycloadditions and was directly observed ${ }^{13}$ in an argon matrix at $8 \mathrm{~K}$ as early as 1973 . In contrast, cyclobutabenzene ${ }^{14}(5)$ is a stable, isolable, crystalline solid and was first prepared in 1958.<smiles>c1ccccc#1</smiles>

(3)

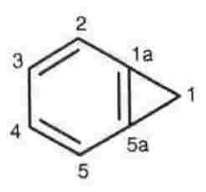

(4)

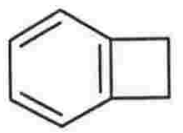

(5)

In 1963 Ullman and Buncel predicted ${ }^{15}$ that bicyclo[4.1.0]hepta-1,3,5-triene (4) (cyclopropabenzene) should be capable of transient existence with a stability lying between that of the known compounds benzyne and cyclobutabenzene; its strain energy was expected ${ }^{15}$ to be at least $190 \mathrm{~kJ} \mathrm{~mol}^{-1}$. Two years after this prediction, Vogel et al. reported $^{16}$ the synthesis of cyclopropabenzene which was obtained as an isolable, stable, liquid, albeit highly malodorous. It is towards this last entity and its derivatives, a class of compounds known as the cycloproparenes and characterised by the ortho-fusion of a single carbon atom across an aromatic system, that this dissertation is directed.

The cycloproparenes are the simplest ortho-bridged aromatic compounds capable of isolation and serve as useful models for assessing the boundaries that stress and strain may impose on the benzenoid framework. Moreover, their role in the debate over the concept of bond localisation in the aromatic ring is crucial. As a consequence of this so-called Mills-Nixon ${ }^{17}$ effect, cyclopropabenzene is expected to favour one of the two canonical forms in the direction of either (4a) or (4b).

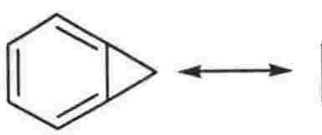

(4a)

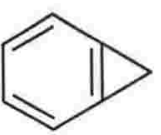

(4b)

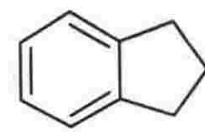

(5a)

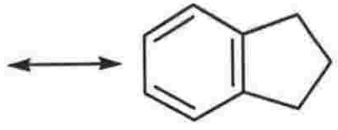

(5b)

The concept of aromatic bond localisation was first proposed by Mills and Nixon in 1930 in an attempt to explain the differences in the chemical reactivities of indane and tetralin. The authors predicted ${ }^{17}$ that indane would favour the Kekule structure with the least amount of $\pi$-character at the bridge bond in order to minimise strain, i.e. (5a) vs (5b). Although the experimental evidence that Mills and Nixon based their original hypothesis upon proved to be incorrect, early calculations ${ }^{18}$ suggested that aromatic bond localisation should become more pronounced as the size of the fused ring decreases. Cyclopropa- 
benzene which is the simplest isolable member of the ortho-annelated aromatics may be expected, therefore, to favour the conical structure (4a) over (4b).

In 1986 Apeloig $^{19}$ and his group calculated that, for cyclopropabenzene, the $\pi$ distortion in the aromatic framework would be minimal but would lie in the direction of (4a). Furthemore, a significant distortion to the $\sigma$-framework of (4) was expected. Six years later Baldridge and Siegel $^{20}$ reported that neither the cycloproparenes nor cyclobutarenes would have measurable bond localisation; calculations by other groups ${ }^{21}$ suggested otherwise.

An X-ray crystal structure for cyclopropabenzene has now been obtained ${ }^{22}$ after crystal growth at low temperature; the interbond distances are shown in Table 1. Clearly evident from these data is that the shortest bonds ( $a$ and $b$ ) are those which are closest to the sites of ring fusion. Furthermore, these are short in comparison to the benzene bond length $(1.395 \AA)$. The bonds ( $c$ and $d$ ) remote from the fusion site compare more favourably in length to the benzene bond. Hence, it can be concluded that the bonding in cyclopropabenzene does not compare favourably with either model (4a) or (4b). Instead, compound (4) suffers from bond deformation and a reduction in symmetry as a result of small-ring fusion. In addition to the X-ray crystal structure of cyclopropabenzene X-ray crystallographic data has been obtained ${ }^{22-28}$ for other members of the cycloproparene family; no evidence for aromatic bond localisation was obtained.

Table 1. Bond lengths of cyclopropabenzene from X-ray crystallography. ${ }^{a}$

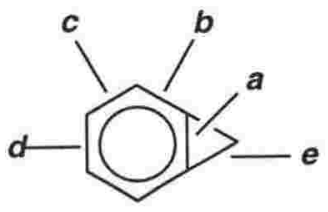

\begin{tabular}{ll}
\hline Bond & Interbond distance ${ }^{b}$ \\
\hline $\boldsymbol{a}$ & $1.334(4)$ \\
$\boldsymbol{b}$ & $1.363(3)$ \\
$\boldsymbol{c}$ & $1.387(4)$ \\
$\boldsymbol{d}$ & $1.390(5)$ \\
$\boldsymbol{e}$ & $1.498(3)$ \\
\hline
\end{tabular}

\footnotetext{
${ }^{a}$ Values taken from reference 22 .

${ }^{b}$ Bond lengths in Angstrom units $(\AA)$.
}

The existence of alternating double and single bonds within the aromatic framework as required by the Mills-Nixon effect has now been observed in the tris-substituted 
benzene derivatives $(6)^{29}$ and $(7)^{30}$. Seigel et al. suggest ${ }^{30}$ that bicyclic ring fusion at least is required in order to effect aromatic bond localisation.

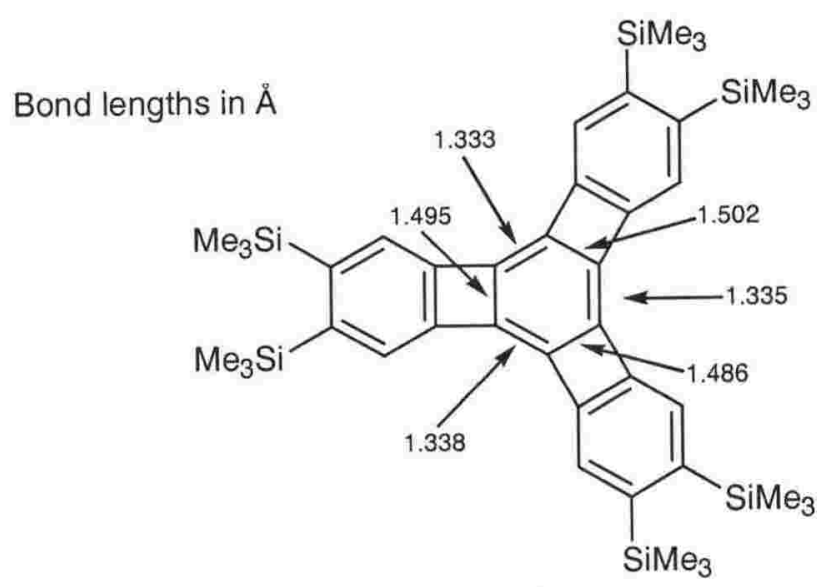

(6)

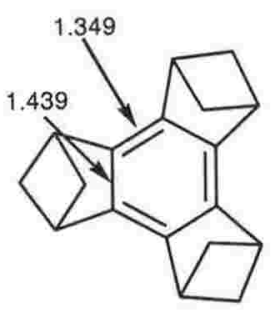

(7)

The earliest claim to the preparation of a cycloproparene was made in 1930 by De and Dutt ${ }^{31}$ who reported that a series of aryliminosemicarbazones (8) decomposed to afford iminocyclopropa[l]phenanthracenes (9) (Scheme 1). Unfortunately, all attempts to reproduce these findings in our laboratories proved unsuccessful and the 4-arylsemicarbazones (10) were the only isolable products of reaction. ${ }^{32}$<smiles>CC(C)(C)N=c1c2c3ccccc3c3ccccc3c12</smiles>

(9)

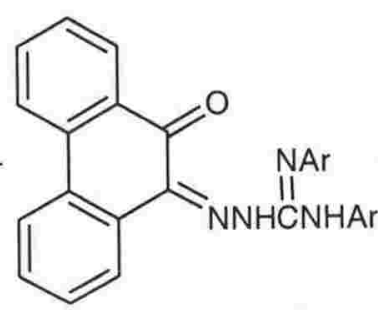

(8)

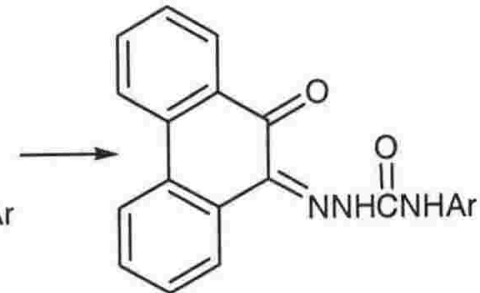

(10)

Scheme 1

In 1964, Anet and $\mathrm{Anet}^{33}$ laid claim to the first authenticated synthesis of a cycloproparene derivative. The authors reported that the photolysis of the $3 H$-indazole (11) afforded the cyclopropabenzene derivative (12), along with the styrene (13) and nitrogen (Scheme 2). However, this route for preparing cycloproparenes is seldom used because the synthesis of the indazole precursors is difficult. Furthermore, C1-monosubstituted cycloproparenes cannot be obtained using this methodology since the requisite indazole substrates (14), which exist as the more stable tautomer (15), and do not lose dinitrogen.<smiles>[R]c1ccc2c(c1)C(C)(C)N=N2</smiles>

(11)

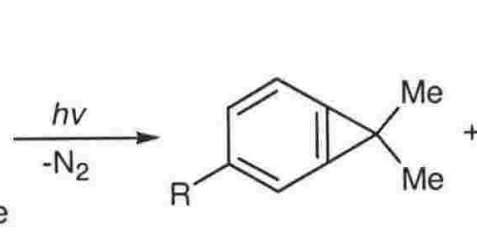

(12)<smiles>[R]c1ccc(C(=C)C)cc1</smiles>

(13) 
<smiles>[R]c1n[nH]c2ccc(C)cc12</smiles>

(14)

(15)

The ability of spiro- $3 H$-pyrazoles to ring-expand and provide cycloproparenes was discovered by Dürr ${ }^{34}$ and his group in the late 1960s. The authors found that upon photolysis the spiro-3H-pyrazole (16) undergoes two [1,5] carbon shifts to provide the indazole (17) via (18) (Scheme 3). Compound (17) then ejects nitrogen and affords the cyclopropabenzene derivatives $(19 \mathrm{a}-\mathrm{c})$; yields are often as high as $80 \%$. This route also affords the only known ${ }^{35,36}$ spirocyclopropabenzenes $^{37}$ (20a-c).<smiles>[R]C1=C([R7])[C@@]2(N=N1)C([R])=C([R])C([R])=C2[P]</smiles>

(16)

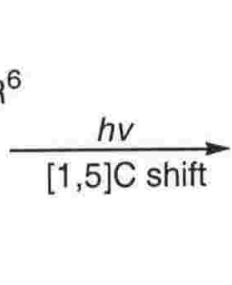<smiles>[R]C1=C([R])C([R7])C2([10B])C([P])=NN=C2C1[R]</smiles>

(18)

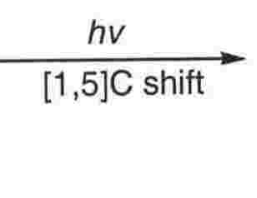<smiles></smiles><smiles>[R7]c1c([R])c([R7])c2c(c1[R])N=NC2(P)P</smiles>

(17)

(a) $R^{1}=R^{2}=R^{3}=R^{4}=P h$

(b) $\mathrm{R}^{1}=\mathrm{R}^{4}=\mathrm{Ph} ; \mathrm{R}^{2}=\mathrm{R}^{3}=\mathrm{H}$

(c) $R^{1} R^{2}=$ benzo fusion; $R^{3}=R^{4}=H$

(19)

cheme 3

Some two years after Ullman and Buncel predicted ${ }^{15}$ that cyclopropabenzene should be capable of existence this sought after molecule was prepared by Vogel et al. ${ }^{16}$ as noted above. The authors found that the flash vacuum pyrolysis (fvp) of compound (21) afforded cyclopropabenzene (4) in approximately $45 \%$ yield by way of a thermal $\left[\pi 2_{s}+{ }_{\sigma} 2_{s}+\sigma_{s} 2_{s}\right]$ cycloreversion; the ester (22) was also isolated. The synthon (21) was, in turn, easily prepared from the methano[10]annulene (23) and dienophile (24) by Diels-Alder methodology (Scheme 4). 


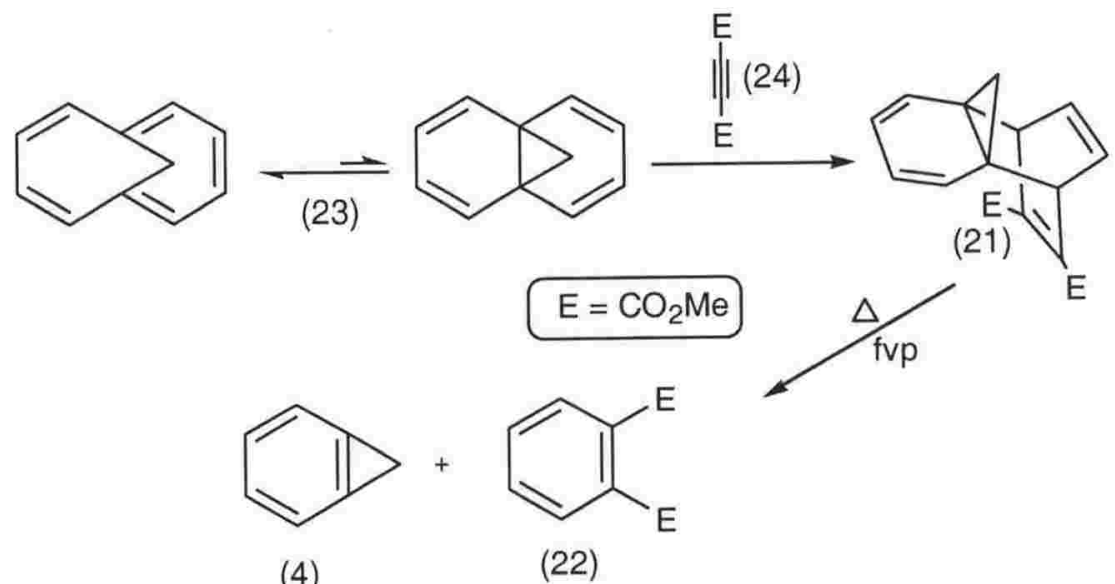

Scheme 4

In 1973, some eight years after cyclopropabenzene was initially prepared, the strain energy of this molecule was established as $\sim 285 \mathrm{~kJ} \mathrm{~mol}^{-1}$ by Billups et al. ${ }^{23}$ To determine the amount of strain energy present in the cyclopropabenzene framework, the authors measured the enthalpy change for the $\operatorname{Ag}(\mathrm{I})$ catalysed methanolysis of (4) which quantitatively affords benzyl methyl ether. The difference between $\Delta H_{\mathrm{f}}$ for this ether and $\Delta H_{\mathrm{rxn}}$ for the methanolysis of cyclopropabenzene gives the strain energy of (4). The results show that (4) is $\sim 75 \mathrm{~kJ} \mathrm{~mol}^{-1}$ more highly strained than cyclopropene ${ }^{4}\left(220 \mathrm{~kJ} \mathrm{~mol}^{-}\right.$ ${ }^{1}$ ) and $\sim 100 \mathrm{~kJ} \mathrm{~mol}^{-1}$ more strained than Ullman and Buncel predicted. ${ }^{15}$ This presumably results from distortions to the aromatic framework caused by ring fusion.

It should not be of any surprise that the chemical reactivity of cyclopropabenzene is directly influenced by its high energy. It has been established ${ }^{38}$ by experiment as well as predicted $^{19}$ by theory that the HOMO of (4) is located at the bridge (C1a-C5a) and C3-C4 bonds. Consequently, (4) should react with electrophiles at the bridge bond to relieve ring strain. That it does is evidenced from the isolation of a 1,2-disubstituted benzene (25), typically in high yield, when (4) is treated with acid ${ }^{39}$ or halogen. ${ }^{16,40-42}$ The mode of ring opening is thought ${ }^{41}$ to proceed as shown by path $a$ in Scheme 5 . Thus, $\pi$-capture of electrophile $\left(\mathrm{E}^{+}\right)$at the bridge bond generates cation (26) which can then rearrange to provide the more stable benzylic intermediate (27). Ion (27) is then intercepted by nucleophile $\left(\mathrm{Nu}^{-}\right)$to give the observed product (25). However, it should be noted that when (4) is treated with iodine a small amount $(\sim 7 \%)$ of the cycloheptatriene $(28)$ is formed. ${ }^{16,40}$ This suggests that cation (26) can capture halide ion before ring opening. The resultant norcaradiene (29) then gives rise to the observed product (28) after electrocyclic ring-opening (path $b$, Scheme 5). 


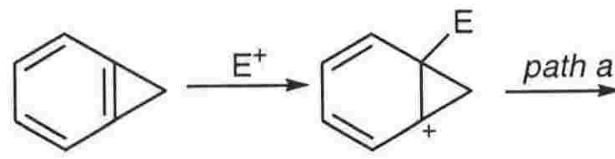

(4)

(26) path b

(29)<smiles>[CH2]c1ccccc1F</smiles>

(27)

(25)

\section{Scheme 5}

Evidence to support the mode of electrophilic ring-opening of the cycloproparenes, as depicted by path a Scheme 5 comes from examining ${ }^{42}$ the regioselectivity of products obtained when the 2- and 3-methylcyclopropabenzenes (30) and (31) are treated with halogen or hydrochloric acid (Scheme 6). It was found ${ }^{42}$ that the 2 -substituted cycloproparene (30) affords (32) as the major and (33) as the minor product of reaction. Conversely, the cyclopropabenzene (31) affords (34) as the major product of reaction together with a small amount of (35). The nature and respective yields of products formed from the ringopening of the unsymmetrical cycloproparenes is consistent with the $\pi$-bridge bond of (30) and $(31)$ capturing the electrophile, $\mathrm{E}^{+}$, at the centre which provides the most stabilised cations, (36) and (37), respectively.<smiles>[C+]C1c2cccc(C)c21</smiles>

(30)<smiles>CC1=CC=C[C+]2CC12F</smiles>

(36)<smiles>C#[N+]C1=C2C=CC=C(C)C21F</smiles>

(1)<smiles>Cc1cccc(CN)c1F</smiles>

(32) major<smiles>Cc1cccc(F)c1CN</smiles>

(33)

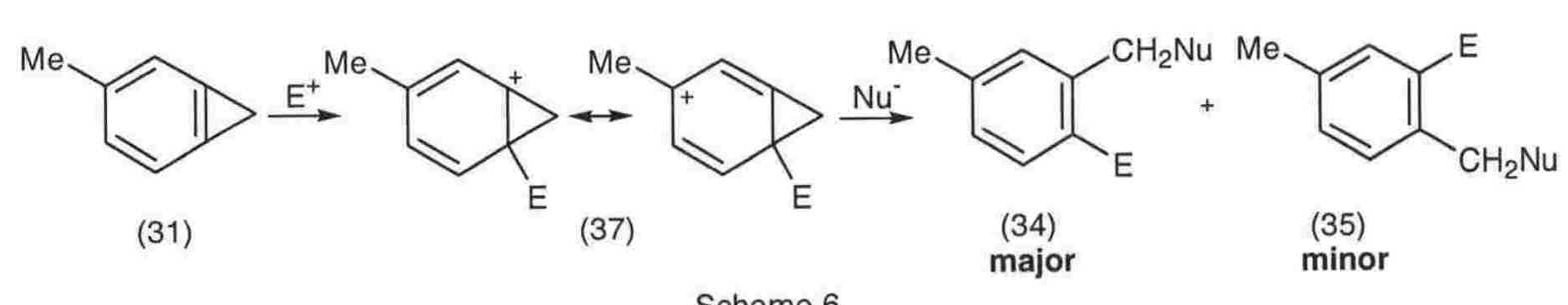

Scheme 6

When the methylcycloproparenes (30) and (31) are treated with acid or halogen in the presence of $\operatorname{Ag}(\mathrm{I})$ the regioselectivity of the isolated products is reversed from that shown in Scheme 6, i.e., (30) affords (33), and (31) gives (35), as the major product of reaction, respectively. ${ }^{42}$ The $\mathrm{Ag}(\mathrm{I})$ catalysed ring-opening of the cycloproparenes must proceed via a different mechanism to that shown in path a Scheme 5. The reaction is thought ${ }^{36}$ to proceed by silver ion attacking the $\sigma$-bond of the cyclopropyl ring with generation of the bridged cation (38) and strain relief. The subsequent interaction of ion 
(39) with acid, halogen, alcohol, thiol, or amine then affords the appropriate orthodisubstituted benzene (25) (Scheme 7). ${ }^{43,44}$

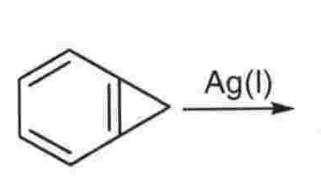

(4)

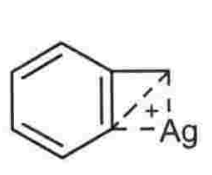

(38)<smiles>[CH2]c1ccc(I)cc1[GeH3]</smiles>

(39)<smiles>N#Cc1ccc(CN)c(F)c1</smiles>

(25)

\section{Scheme 7}

As already discussed, the HOMO of (4) is located at the C1a-C5a bridge and C3-C4 bonds. ${ }^{38,19}$ Consequently, cyclopropabenzene should participate as an electron-rich olefin in Diels-Alder cycloaddition reactions. That it does was ably demonstrated by Korte who isolated the cycloadducts (23) and (40) from the $\left[{ }_{\pi} 2_{\mathrm{s}}+{ }_{\pi} 4_{\mathrm{s}}\right]$ addition of $\alpha$-pyrone and buta1,3-diene, respectively, across the bridge bond of cyclopropabenzene (Scheme 8). ${ }^{39}$ However, while the addition of $\alpha$-pyrone to (4) (followed by loss of $\mathrm{CO}_{2}$ ) affords the methano[10]annulene (23) as the major reaction product, the analogous reaction with butadiene predominantly affords the 1-vinylindane (41) along with small amounts of (40) and (42). ${ }^{39}$ Compounds (41) and (42) most likely arise from the opening of the threemembered ring to generate a diradical intermediate which is then intercepted by diene.

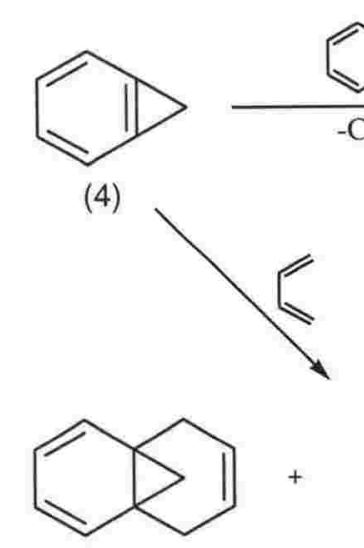

(40)

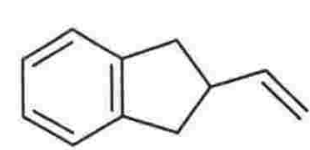

(41)<smiles>C1=CC23C=CC=CC2(C=C1)C3</smiles>

(23)

\section{Scheme 8}

In addition to the Diels-Alder adducts isolated above, the cycloadducts (43)-(46) were obtained from the reaction of (4) with diphenylisobenzofuran and the eneophiles (47)-(49), respectively (Scheme 9). ${ }^{47-48}$ Compound (43) results from the addition of diphenylisobenzofuran across the bridge of (4) in an exo sense ${ }^{45}$ which implies the cycloaddition occurs by a symmetry allowed $\left[{ }_{\pi} 6_{s}+{ }_{\pi} 4_{s}\right]$ process. However, adduct (44) arises from the endo-addition of diene across the bridge of (4) in a $\left[\pi 2_{s}+{ }_{\pi} 4 s\right]$ sense. ${ }^{46}$ 


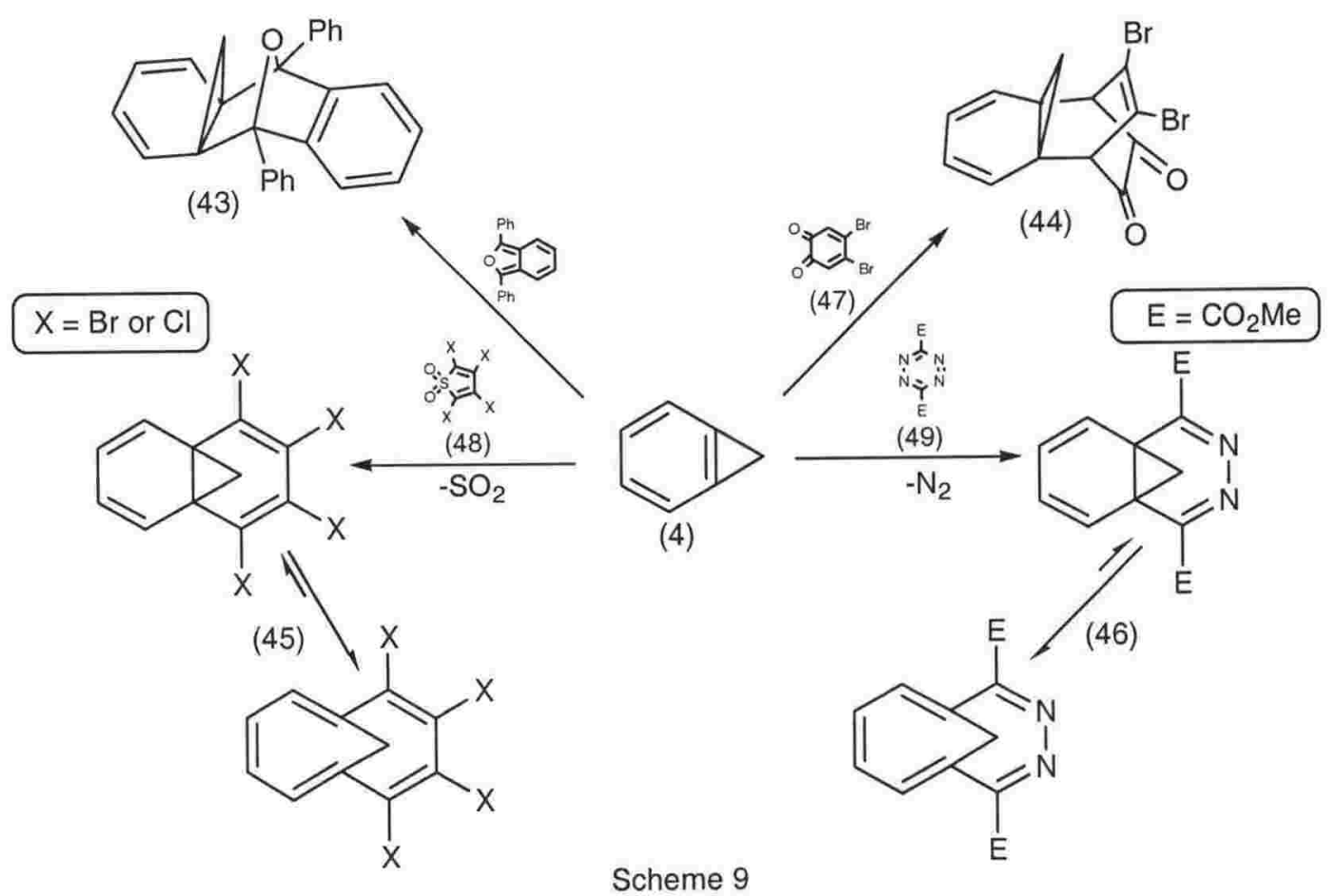

As anticipated, cyclopropabenzene reacts with both dichloro- and dibromocarbene by way of cheleotropic addition of carbene across the C1a-C5a bridge of (4). ${ }^{49}$ The products of reaction, the ring-expanded cyclobutarenes (50) (Scheme 10), are obtained almost quantitatively. The reaction probably proceeds via the norcaradiene intermediate (51) which can rearrange to afford the appropriate cyclobutarene.

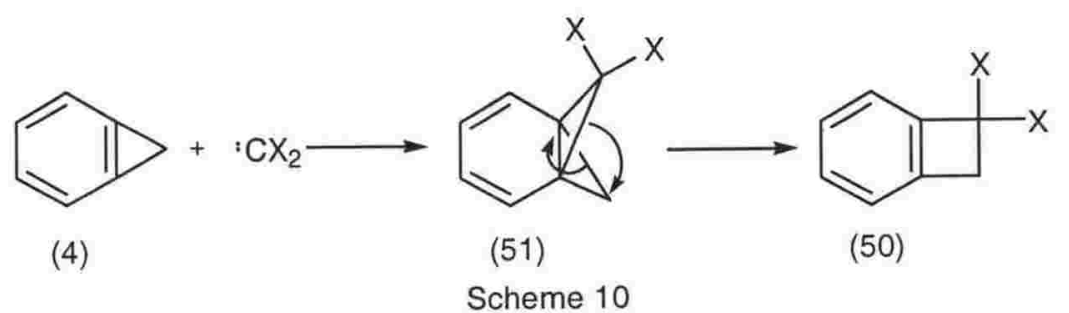

The vapour-phase thermolysis of (4) has been studied at $80^{\circ} \mathrm{C} .{ }^{39}$ The major product of reaction, 9,10-dihydrophenanthrene, most likely arises from the dimerisation of diradical (52) in a head-to-head fashion. Under flash vacuum pyrolytic conditions ${ }^{50}$ $\left(>500^{\circ} \mathrm{C}\right)$ the allene $(53)$ is the major product of reaction and is thought to arise from a Wolff-type rearrangement of carbene (52) (Scheme 11).

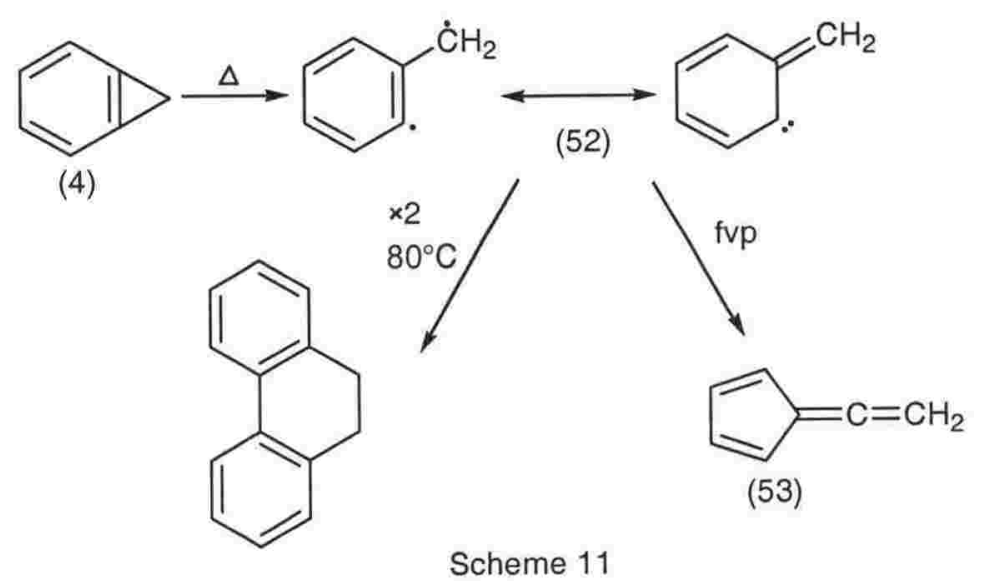


Upon photolysis, the C1-diester of cyclopropabenzene (54) affords allene (55) and benzofuran (56) as the major and minor products of reaction (Scheme 12). ${ }^{51,52}$ An intermediate of the type (57) is most likely involved which can ring-close to (55) and (56).

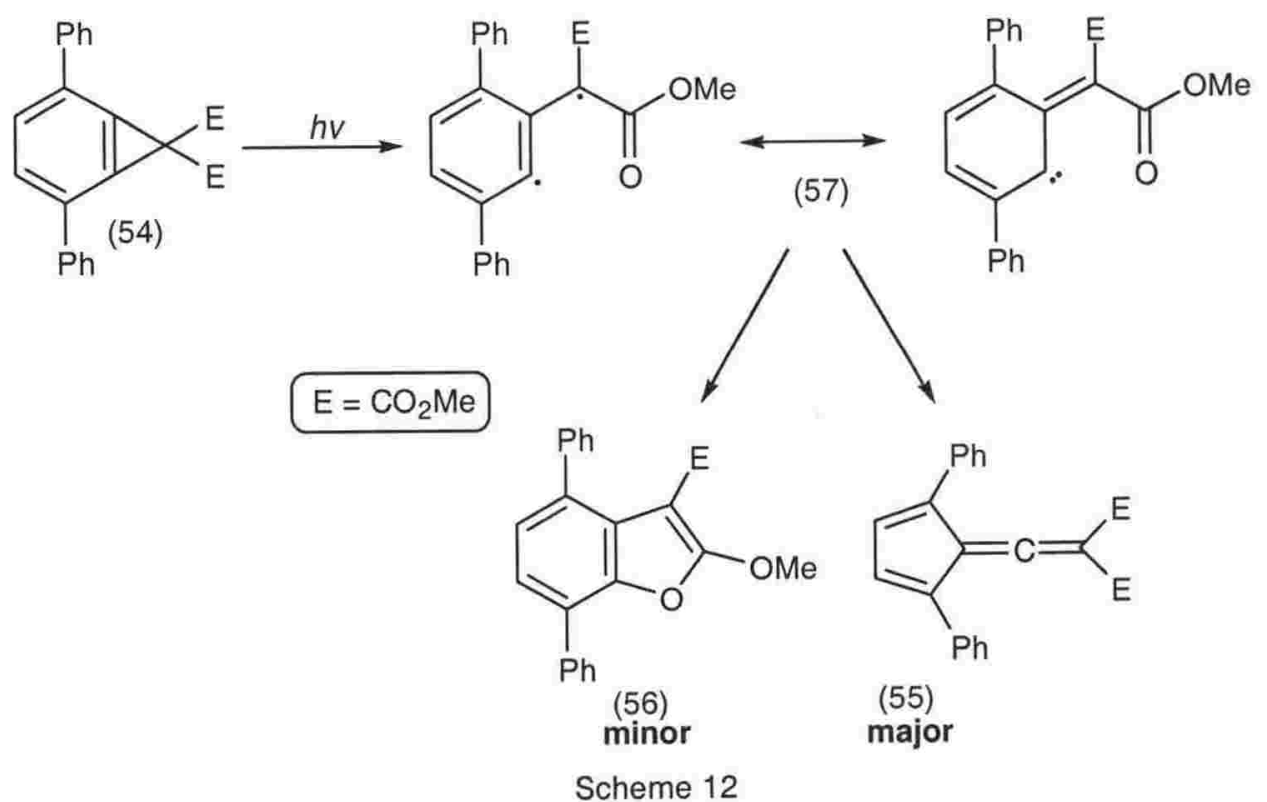

Since the initial preparation of cyclopropabenzene in 1965 , the fusion of a threemembered ring to other aromatic systems has been accomplished. Thus, linear ${ }^{53}$ and $\operatorname{angular}^{54}$ fusion of a three-membered ring to naphthalene affords the stable ${ }^{53}$ cyclopropa$[b]$ naphthalene (58) and its somewhat less stable ${ }^{54}$ angular isomer (59). The strain energy of (58) $\left(284 \mathrm{~kJ} \mathrm{~mol}^{-1}\right)^{23}$ is comparable to that of cyclopropabenzene, whilst that of (59) is higher and calculated ${ }^{55}$ as $297 \mathrm{~kJ} \mathrm{~mol}^{-1}$. More recently, the fusion of a three-membered ring to anthracene, ${ }^{56}$ and the separate rings of phenanthrene ${ }^{57,58}$ has been accomplished to give the corresponding cycloproparene derivatives (60)-(62), respectively.<smiles>c1ccc2cc3c(cc2c1)C3</smiles>

(58)<smiles>c1ccc2c3c(ccc2c1)C3</smiles>

(59)<smiles>c1ccc2cc3cc4c(cc3cc2c1)C4</smiles>

(60)<smiles>c1ccc2c(c1)ccc1cc3c(cc12)C3</smiles>

(61)<smiles>c1ccc2c(c1)CC1=C2c2ccccc21</smiles>

(62)

Although the parent member in the cycloproparene series is readily prepared in moderate yield (45\%) from the fvp of the Diels-Alder adduct (21) (Scheme 4, p. 6), ${ }^{16}$ the preferred method for the synthesis of (4) and its derivatives involves the construction of an appropriate bicyclo[4.1.0]heptene framework, followed by aromatisation. In this regard the 7,7-dihalobicyclo[4.1.0]heptenes, e.g. (63), have proved useful as synthons for cycloproparene preparation. Cyclopropabenzene (4) is easily prepared (40\%) from the 
base promoted didehydrochlorination of bicycloheptene (63) (Scheme 13). ${ }^{59,60}$ The reaction is believed to proceed via the high-energy ortho-fused intermediates (64)-(66) (Scheme 13). Evidence to support the involvement of these intermediates comes from a study conducted by Prestein and Günther ${ }^{61}$ who prepared (63) with ${ }^{12} \mathrm{C}$ label at C7. The didehydrohalogenation reaction proceeds smoothly and the isolated cyclopropabenzene contains label exclusively at the cyclopropyl carbon. This infers that the 7,7-dichlorobicyclo[4.1.0]heptene framework is preserved throughout the reaction sequence. Furthermore, the intermediate (64) has been intercepted with 1,3-diphenylisobenzofuran (dpibf) and the Diels-Alder cycloadduct isolated. ${ }^{62}$

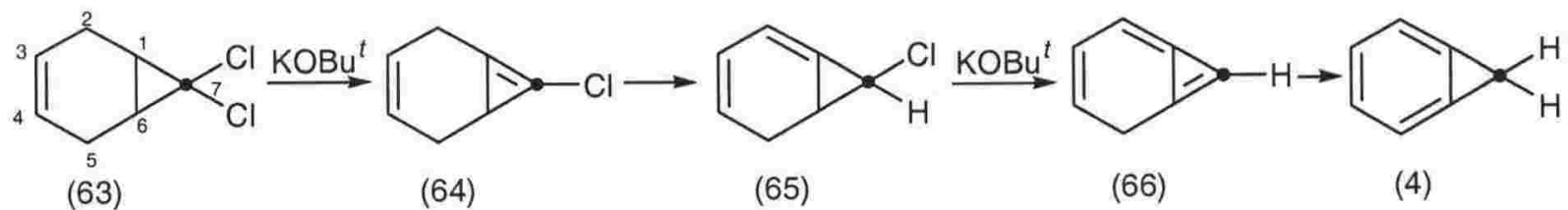

Scheme 13

By extending the methodology described above to the benzo analogue (67), cyclopropanaphthalene (58) becomes available (Scheme 14). ${ }^{53}$ However, in addition to (58), the ether (68) and the 6-substituted cycloheptatriene (69) have also been isolated from the reaction. ${ }^{53,63}$ The relative yields of compounds (58), (68), and (69), are found to correlate with the amount of base employed. Thus, cyclopropanaphthalene is formed in $38 \%$ yield along with a small amount $(7 \%)$ of solvolysis product (68) when (67) is treated with sixteen molar equivalents of potassium $t$-butoxide; none of (69) was isolated. However, when (67) is treated with four molar equivalents of base the major product of reaction is the 6-substitituted cycloheptatriene (69) (27\%); compounds (58) and (68) are formed in yields of 11 and $17 \%$, respectively.

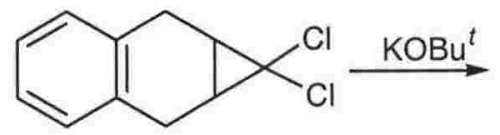

(67)

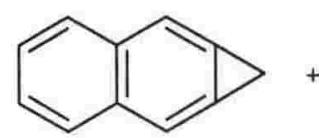

(58)<smiles>CCOCc1ccc2ccccc2c1</smiles>

(68)

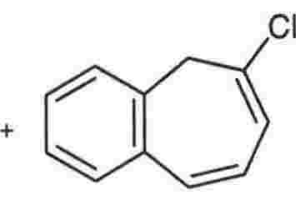

(69)

Scheme 14

The methodology described above has been extended to provide the dimethoxycyclopropanaphthalene $^{64}(70)$ and the shock sensitive biscyclopropanaphthalene ${ }^{65}$ from didehydrohalogenation of the appropriate bicyclo[4.1.0]heptene frameworks (72) and (73), respectively. Diether (70) is one of the few stable oxygenated cycloproparenes known, whilst (71) is the simplest bis-ortho-annelated aromatic system capable of isolation and has a measured ${ }^{65}$ strain energy of $\sim 694 \mathrm{~kJ} \mathrm{~mol}^{-1}$. 
<smiles>COc1ccc(OC)c2c1CC1C(C2)C1(Cl)Cl</smiles>

(72)<smiles>ClC1(Cl)C2CC3=C(CC21)CC1C(C3)C1(Cl)Cl</smiles>

(73)<smiles>COc1ccc(OC)c2cc3c(cc12)C3</smiles>

(70)
$\stackrel{\mathrm{KOBu}^{t}}{\longrightarrow}$<smiles>c1c2c(cc3cc4c(cc13)C4)C2</smiles>

(71)

Attempts to prepare cyclopropanthracene (60) from the didehydrochlorination of the appropriate 7,7-dihalobicycloheptene manifold (74) has proved unsuccessful and only the alkyl halide (75) and ether (76) were isolated (Scheme 15). ${ }^{66}$ In similar vein, the attempted didehydrochlorination of the compounds (77) and (78) provides products that correspond to opening of the three-membered ring and none of the desired cycloproparenes (61) and (62), respectively, were isolated. ${ }^{67,68}$<smiles>ClC1(Cl)C2Cc3ccc4ccccc4c3C21</smiles>

(77)<smiles>ClC1(Cl)C2c3ccccc3-c3ccccc3C21</smiles>

(78)

The formation of (75) is illustrated in Scheme 15. Thus, the base promoted dehydrochlorination of (74) followed by the rearrangement of the ortho-fused intermediate so produced, gives rise to (79) (Scheme 13, p. 11). The abstraction of an allylic proton from (79) followed by ring cleavage then generates anion (80) which, affords the observed product (75) upon proton capture. Solvolysis of (75) by $t$-butanol accounts for the formation of (76).

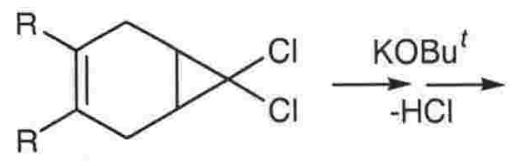

(74)

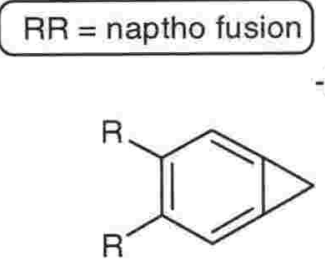

(60)

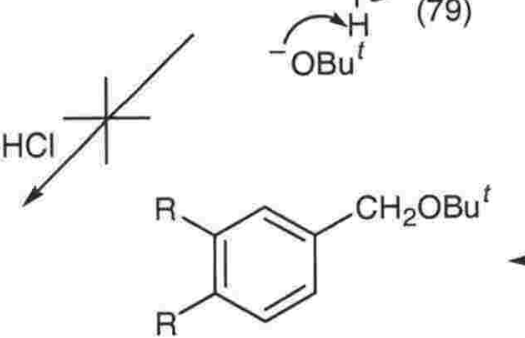

(76)

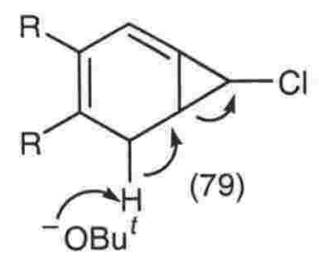<smiles></smiles> 
The use of a 1,6-dihalobicyclo[4.1.0]hept-3-ene, e.g., (81) and (82), for preparing the cycloproparenes, e.g., $(60)^{56}$ and $(61),{ }^{57}$ has proved successful; the compounds were prepared in yield of 41 and $89 \%$, respectively. Furthermore, a range of cycloproparenes which includes the bis and triscycloproparenes $(83)^{69}$ and $(84),{ }^{70}$ respectively, the C1dihalogenated cycloproparenes, e.g. $(85 \mathrm{a}-\mathrm{d}),{ }^{71-75}$ the nitrogen containing (86), ${ }^{76}$ and the dicyclobutabenzene (87), ${ }^{77}$ have all been prepared from dehydrohalogenation of an appropriate synthon containing the 1,6-dihalobicyclo[4.1.0]heptene framework(s).<smiles>ClC12Cc3cc4ccccc4cc3CC1(Br)C2</smiles>

(81)<smiles></smiles>

(84)<smiles>BrC(Br)(Br)C1(Br)Cc2c(ccc3ccccc23)CC12CC2(Br)Br</smiles>

(82)<smiles></smiles>

(83)<smiles>[R]c1c([R])c(-c2ccccc2)c2c(c1-c1ccccc1)C2([X])[X]</smiles>

(85a) $\mathrm{R}=\mathrm{H} ; \mathrm{X}=\mathrm{Y}=\mathrm{Cl}$

(85b) RR = benzo fusion; $\mathrm{X}=\mathrm{Y}=\mathrm{Cl}$

(85c) $\mathrm{R}=\mathrm{H} ; \mathrm{X}=\mathrm{Y}=\mathrm{F}$

(85d) $\mathrm{R}=\mathrm{H} ; \mathrm{X}=\mathrm{Cl} ; \mathrm{Y}=\mathrm{F}$<smiles>c1cnc2cc3c(cc2c1)C3</smiles>

(86)

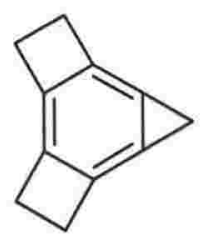

(87)

The syn-elimination from 1,6-disubstituted bicyclo[4.1.0]heptenes that contain substituents other than halide at the 1- and 6- positions has been studied. ${ }^{78-81}$ The preparation of the cycloproparene (62), along with its isomer (89), was effected from the ejection of dimethyl selenide ${ }^{58,78}$ from ylide (88a) or by loss of dimethyl sulfide ${ }^{79}$ from ylide (88b) (Scheme 16). Compound (62), which is stable only in the solid state for only a few days at $-78^{\circ} \mathrm{C}$, has been intercepted by furan as the corresponding Diels-Alder $\left[{ }_{\pi} 6_{\mathrm{s}}+\right.$ ${ }_{\pi} 4_{\text {s }}$ ] cycloadduct. ${ }^{58}$ Furthermore, the elimination of benzeneselenenic acid ( $\left.\mathrm{PhSeOH}\right)$ from (90) results in the isolation of ester (91) via the highly reactive (92). ${ }^{80,81}$ Although dichlorocycloproparene (92) has eluded isolation, it has been implicated in the generation of (91) as gem-dihalocycloproparenes are known to be unstable in the presence of protic media and opening of the three-membered ring is particularly facile under these conditions. ${ }^{35,84,85}$ 


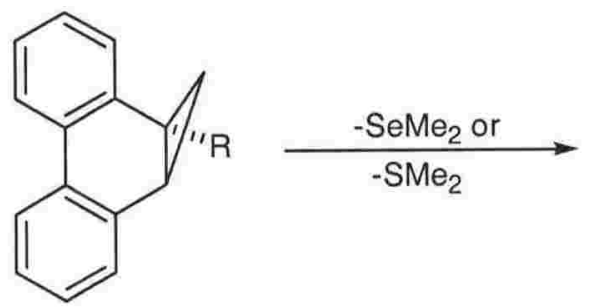

(88a) $\mathrm{R}=\mathrm{Se}^{+} \mathrm{MeCH}_{2}^{-}$

(88b) $\mathrm{R}=\mathrm{S}^{+} \mathrm{MeCH}_{2}^{-}$

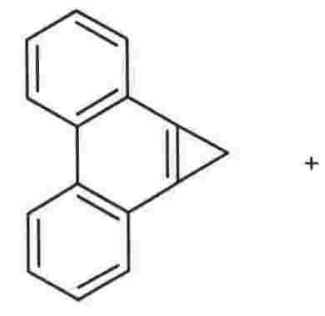

(62)<smiles></smiles>

(89)<smiles>O=[SnH]c1ccccc1C(=O)O</smiles>

(90)<smiles>ClC1(Cl)C2=C(c3ccccc32)c2ccccc21</smiles>

(92)<smiles>CC(=O)c1cc2ccccc2c2ccccc12</smiles>

(91)

Scheme 16

The possibility that ${ }^{84}$ a $\mathrm{C} 1$ cycloproparenyl cation might exist originally received theoretical consideration ${ }^{85}$ in 1952 . However, definitive evidence for the generation of such an ion was not obtained until the mid-1970s. ${ }^{82,83}$ It was found that treating dichlorocyclopropabenzene (85a) with antimony pentachloride gave rise to the salt (93) which, although extremely temperature and moisture sensitive, was capable of isolation. Furthermore, the chloro substituents of (85a) are readily exchanged with hydride, ${ }^{85}$ fluoride, ${ }^{86}$ and carbanion ${ }^{74,87}$ to give products in which the structural integrity of the cycloproparenyl framework remains intact. To date, the parent cycloproparenyl cations $(94 a, b)$ have eluded detection. However, they have been implicated in the generation of the aryl aldehydes $(95 \mathrm{a}, \mathrm{b})$ via the alcohols $(96 \mathrm{a}, \mathrm{b})$ which were obtained from treating the respective cycloproparenes with trityl tetrafluoroborate. 88,89<smiles>ClC1(Cl)c2c(-c3ccccc3)ccc(-c3ccccc3)c21</smiles>

(85a)

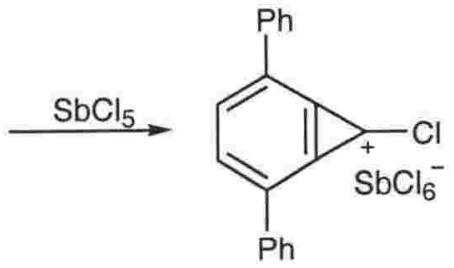

(93)<smiles>[R]c1cc2c(cc1[R])C2[18OH]</smiles>

(4) $\mathrm{R}=\mathrm{H}$

(58) $R R=$ benzo fusion
(94)<smiles></smiles>

(96)<smiles>[R]c1ccc(C=O)cc1P</smiles>

(95)

(a) $\mathrm{R}=\mathrm{H}$

(b) RR = benzo fusion

Scheme 17 
Due to the benzylic nature of the methylene protons of a cycloproparene, their abstraction should be facilitated easily with a strong base to generate a $\mathrm{C} 1$ cycloproparenyl anion. That this is so was ably demonstrated by Eaborn and coworkers who found that the treatment of (4) with butyllithium at low temperature provides the lithiate (97) (Scheme 18) which subsequently can be quenched with trimethylsilyl chloride to provide the silane (98). ${ }^{90}$ Furthermore, cleavage of the C-Si bond of (98) with aqueous sodium hydroxide regenerates (4) via (97) some 64 times faster than the analogous desilylation reaction of benzyltrimethylsilane. From this datum, a $K_{\mathrm{a}}$ value for (4) has been calculated as $\sim 36$, implying that cyclopropabenzene is more acidic than toluene $\left(\mathrm{p} K_{\mathrm{a}} 41\right) .^{90}$ More recently, the gas phase acidity of (4) was measured by Moore et al. who found it to be $17 \mathrm{~kJ} \mathrm{~mol}^{-1}$ less acidic than toluene when measured under identical conditions. ${ }^{91}$ This reversal in the relative acidities in going from liquid to gas phase presumably results from a diminished importance in charge delocalisation in (97) in solution due to solvation effects, and the greater influence exerted by delocalisation. ${ }^{91}$<smiles>c1ccc2c(c1)C2</smiles>

(4)

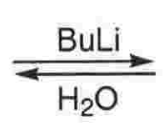<smiles>ClC1c2ccccc21</smiles>

(97)

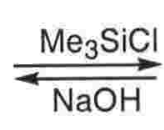<smiles>CCC1c2ccccc21</smiles>

(98)

Scheme 18

Further evidence for the existence of anion (97) has come from a study by Szeimies and Wimmer ${ }^{92}$ who were able to intercept (97) with simple electrophilic reagents including benzyl chloride, methyl fluorosulfate, various aldehydes, and carbon dioxide. The products of reaction, (99)-(102), demonstrate that C1-substituted cycloproparenes may be prepared without destroying the structural integrity of the cycloproparenyl framework.

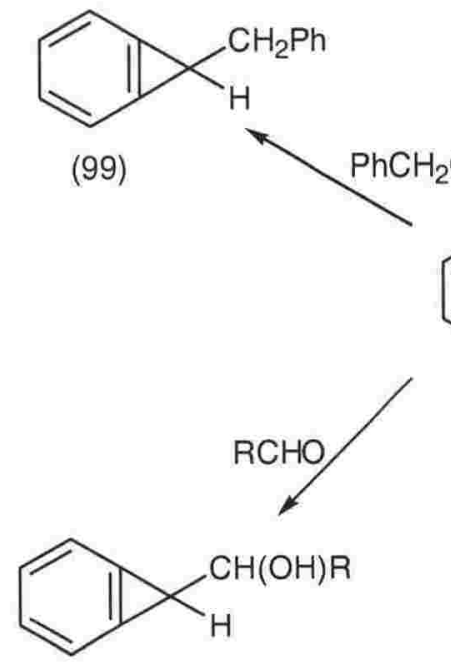

(101)

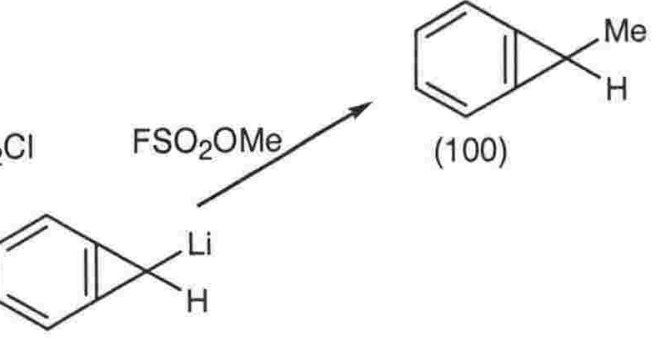

(97)
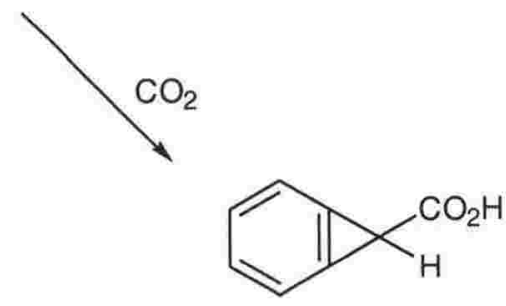

(102)

Scheme 19

The generation of the lithiated cyclopropanapthalenyl anion (103), from metalation of (58) at low temperature, followed by its interception with trimethylsilyl chloride does 
not provide the monosilylated derivative (104) as anticipated (Scheme 20). ${ }^{24}$ Instead, the disilane (105) and regenerated cyclopropanaphthalene are produced in almost equivalent amounts. $^{24}$ It has been proposed ${ }^{24,93}$ that the ability silicon has to stabilise an $\alpha$-anion ${ }^{94}$ will result in the anion (106) being more stable than anion (103). Thus upon formation of silane (104), abstraction of the benzylic proton by unreacted anion (103) is facile thereby regenerating cyclopropanaphthalene (58) and giving the more stable $\alpha$-silyl anion (106). Anion (106) is then silylated to furnish the observed disilane (105).<smiles>CC(C)(C)C1c2cc3ccccc3cc21</smiles>

(58)<smiles>ClC1c2cc3ccccc3cc21</smiles>

(103)

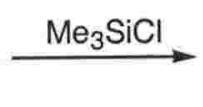

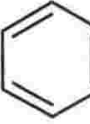

(104)<smiles>CCC1([SiH3])c2cc3ccccc3cc21</smiles>

(105)

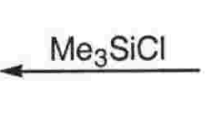

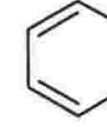

$(106$

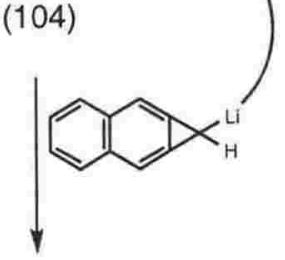

Scheme 20

A recent study by Halton and Cutler $^{93}$ into the nucleophilic behaviour of the $\mathrm{C} 1$ cycloproparenyl anion (103) with reagents other than trimethylsilyl chloride was recently undertaken. The authors found that the capture of anion (103) with simple electrophiles such as benzoyl chloride, methyl benzoate, and benzyl bromide was unsuccessful. However, quenching the anion with methyl iodide, acetone, or benzophenone did provide the products of monosubstitution, namely (107)-(109) (Scheme 21), although in low yield. When lithiate (103) was treated with toluoyl chloride it was the alcohol (110) that was isolated and no evidence was obtained to support the formation of the cycloproparenyl tolyl ketone (111). The authors concluded, that "the ease by which anion (103) deprotonates (104) suggested that its capture by other electrophiles could be difficult. This has been borne out and few simple substitution products have been isolated. Proton abstraction appears to be particularly facile thus rendering many reactions with electrophilic reagents ineffective". 93 
<smiles>CC1c2cc3ccccc3cc21</smiles>

(107)

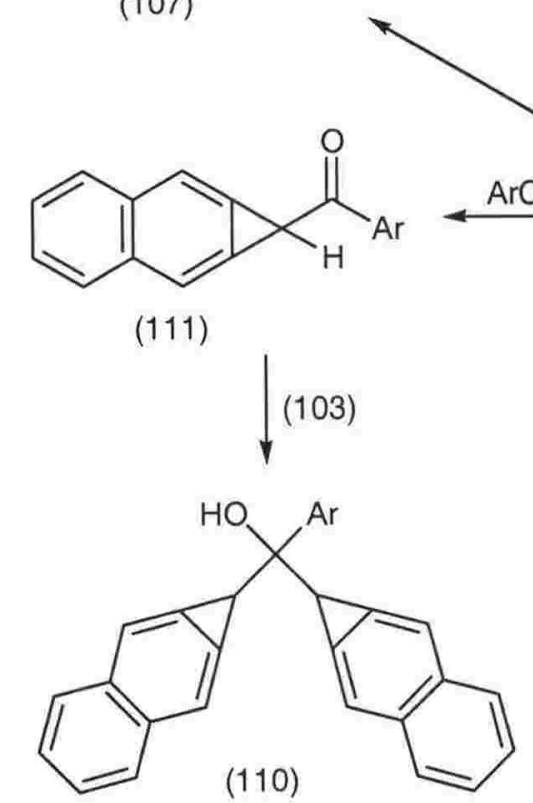<smiles>CC(C)(O)C1Cc2cc3ccccc3cc21</smiles>

(108)

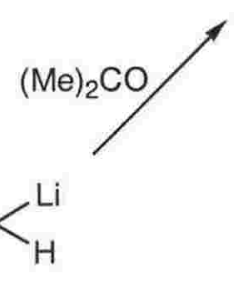

(103)

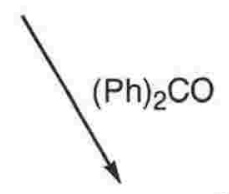

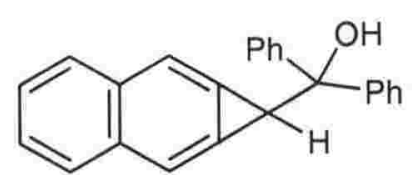

(109)

Scheme 21

In contrast to the reactions discussed above, the $\alpha$-silyl anion (106) (best prepared from treating disilane (105) with potassium $t$-butoxide) is readily intercepted by a range of acid cyanides or acid halides to provide the $\mathrm{C} 1$ disubstituted cycloproparenes, e.g. (112), in good yield. ${ }^{95}$ Furthermore, Halton and Jones ${ }^{96,97}$ found that quenching anion (106) with water and methyl iodide gave product data that were compatible with the silanes (104) and (113), respectively, (Scheme 22). However, the authors were unable to characterise the compounds. It should be noted, that quenching anion (106) with water not only produces silane (104) but also potassium hydroxide. This last reagent induces the cleavage of the CSi bond of (104) and generates anion (103) which, after being intercepted by water, affords cyclopropanaphthalene (58). ${ }^{97}$ Re-examination of the behaviour of anion (106) with simple electrophilic reagents along with efforts to characterise silanes (104) and (113) is one of the objectives of this project.

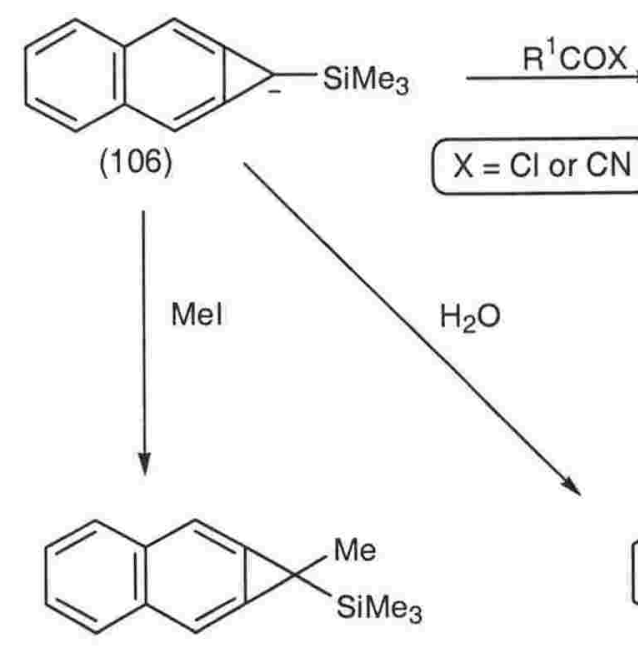

(113)<smiles>[R]C(=O)C1([SiH3])c2cc3ccccc3cc21</smiles>

(112a) $R^{1}=M e$

(112b) $R^{1}=M e$

(112c) $\mathrm{R}^{1}=\mathrm{Et}$ (112d) $R^{1}=$ OMe (112e) $R^{1}=\mathrm{NMe}_{2}$ (112f) $R^{1}=C N$ (112g) $\mathrm{R}^{1}=\mathrm{NPh}_{2}$<smiles>CCC1Cc2cc3ccccc3cc21</smiles>

(104) 
By treating the $\alpha$-silyl anion (106) with an appropriate carbonyl-containing compound, alkylidenecycloproparenes (114) are produced via Peterson olefination. This procedure, which converts the $\mathrm{sp}^{3}$-hybridised $\mathrm{C} 1$ centre of the cycloproparene precursor to that of an $\mathrm{sp}^{2}$-hybridised one has yielded over sixty alkylidenecycloproparenes to date. $^{24,27,28,98-104}$ The $\alpha$-silyl cyclopropabenzenyl anion (115), generated by metalating silane (98) at low temperature, also affords alkylidenecycloproparenes (116); nine such compounds have been prepared. ${ }^{24,101,103,105}$ Unfortunately, all attempts to prepare the parent alkenes (117) and (118) have been unsuccessful. ${ }^{100}$ Moreover, because of the strongly basic conditions required to generate anions (106) and (115), the olefination procedure often fails when enolisable aldehydes and ketones are used, presumably, due to competing reaction pathways. ${ }^{100}$

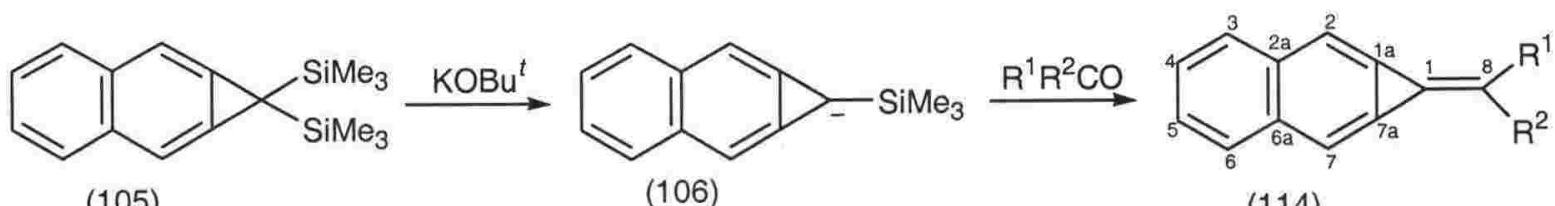

(106)<smiles>CC(C)(C)C1Cc2ccccc21</smiles>

(4)<smiles>C[SiH3]C1c2ccccc21</smiles>

(98)

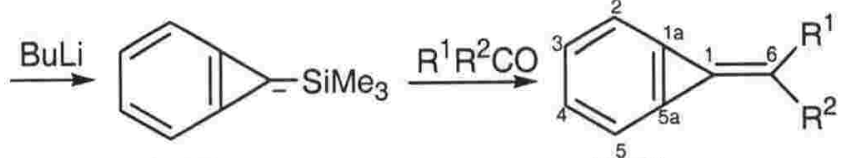

(115)

Scheme 23

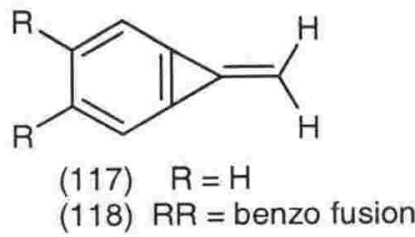

A second method for preparing methylidenecycloproparenes was developed by Stang et al. in 1994 and utilises the C1 acyl-substituted silanes (112). ${ }^{95}$ These cyclopropanaphthalene derivatives which are readily intercepted by nucleophiles, usually as their lithium salts, afford the appropriate alkylidenecycloproparenes (114) via Peterson olefination. To date, this procedure has been applied only to the naphthalene series, however, this methodology has yielded nine new and novel alkylidenecyclopropanaphthalenes (114).

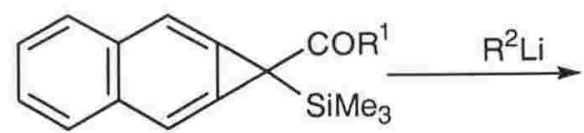

(112)

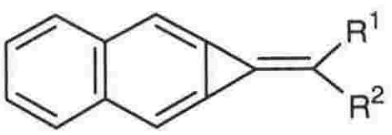

(114)

\begin{tabular}{|cccccccccc|}
\hline & (a) & (b) & (c) & (d) & (e) & (f) & (g) & (h) & (i) \\
\hline$R^{1}$ & $\mathrm{Me}$ & $\mathrm{Me}$ & $\mathrm{Et}$ & $\mathrm{OMe}$ & $\mathrm{NMe}_{2}$ & $\mathrm{CN}$ & $\mathrm{NMe}_{2}$ & $\mathrm{CN}$ & $\mathrm{NPh}_{2}$ \\
$\mathrm{R}^{2}$ & $\mathrm{H}$ & $\mathrm{Me}$ & $\mathrm{Et}$ & $\mathrm{OMe}$ & $\mathrm{OMe}$ & $\mathrm{OMe}$ & $\mathrm{NMe}_{2}$ & $\mathrm{NMe}_{2}$ & $\mathrm{~N} \mathrm{Me}_{2}$ \\
\hline
\end{tabular}


The alkylidenecycloproparenes which are prepared in yields of $8-95 \%$, are, in most cases, brightly coloured, shelf stable, non-odoriferous compounds. ${ }^{35,100}$ The valence isomers of the parent compounds (117) and (118) show they may exist as simple methylidene compounds (a), benzannelated methylidenecyclopropenes (b) which can exhibit triafulvene-like charge separation (c), or cross-conjugated radialenes (d) that can be polarised in two directions, viz. (e) or (f) (Scheme 24).

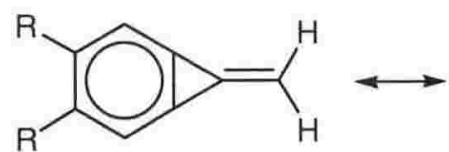

(a)

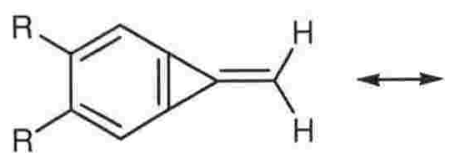

(b)<smiles></smiles>

(c)

(117) $\mathrm{R}=\mathrm{H}$

(118) $\mathrm{RR}=$ benzo fusion

$\uparrow$<smiles></smiles>

(e)

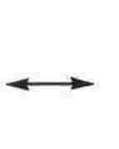

$R^{\prime}$ (d)

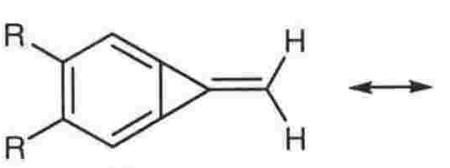

(d)<smiles></smiles>

(f)

Scheme 24

It has been predicted by theory that the alkylidenecycloproparenes should have measurable polarities. ${ }^{22,23,106}$ The calculated magnitude and direction of the dipole moments for compounds (117) and (119)-(121) are shown below. Although attempts to prepare these molecules have proved unsuccessful, the derivatives (122) and (123) of (120) and (121), respectively, have been prepared and their dipole moments measured; ${ }^{103,105}$ the experimentally determined values compare well with those calculated. It should be noted however, that while the magnitude of a dipole moment can be measured its direction cannot. Instead, direction comes from intuition or by calculation. The dipole moments for a range of other alkylidenecycloproparenes have been measured; their values of 0.4-9.1 D are consistent with the polar nature of the compounds. ${ }^{24,98,101,103,104}$<smiles>C=C1c2ccccc21</smiles>

$\underset{\mu=1.2 \mathrm{D}}{\stackrel{(117)}{\longrightarrow}}$

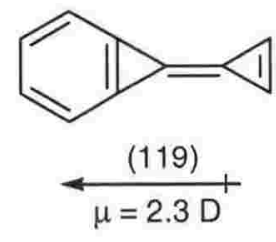

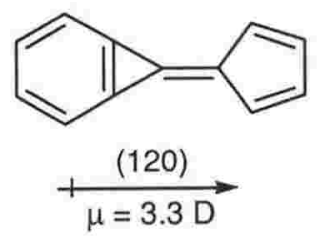

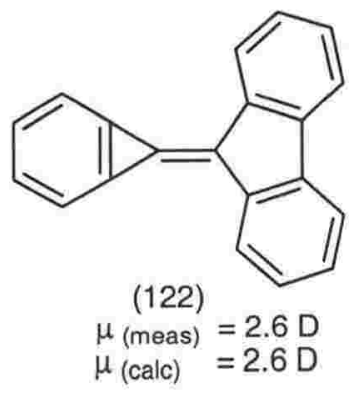

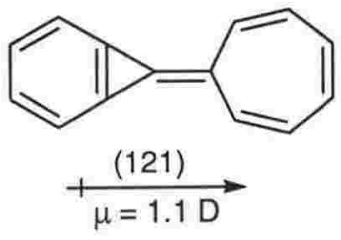

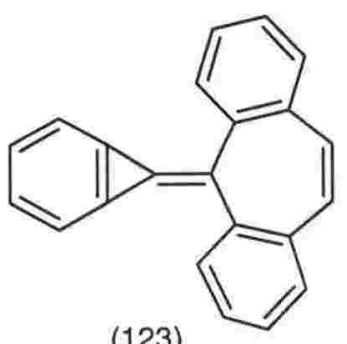

(123)

$\mu_{\text {(meas) }}=1.2 \mathrm{D}$

$\mu_{\text {(calc) }}=1.3 \mathrm{D}$ 
Apeloig has predicted ${ }^{107}$ that the unknown parent methylidene (117) will be $\sim 8.4 \mathrm{~kJ}$ $\mathrm{mol}^{-1}$ less strained than cyclopropabenzene (4) as a result of stabilisation by charge separation; the calculated bond lengths and bond angles are shown in Table 2. Although it has not been possible to generate (117) or its naphtho analogue (118) the X-ray crystal structures of other members in the alkylidenecycloproparene family have been obtained. ${ }^{24,26,28,102,103}$ These results show that as a consequence of converting the $\mathrm{sp}^{3}$ hybridised $\mathrm{C} 1$ centre to an $\mathrm{sp}^{2}$ one, angle $\alpha$ is widened by $\sim 4^{\circ}$. Moreover, the bridge bond $a$ is lengthened with respect to the parent cycloproparenes (4) and (58) while that of the lateral strained ring bond $e$ is shorter than those measured for (4) and (58). The exocyclic double bond $f$ ranges in length from 1.329 to $1.362 \AA$.

Table 2. Bond lengths and selected bond angles calculated for compound (117). ${ }^{a}$

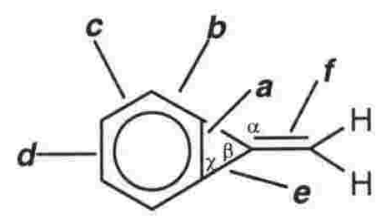

\begin{tabular}{|c|c|c|}
\hline Bond & Interbond distance $^{b}$ & Angle $^{c}$ \\
\hline$a$ & 1.347 & $\alpha 152.2$ \\
\hline$b$ & 1.382 & $\beta \quad 56.0$ \\
\hline$c$ & 1.388 & $\chi \quad 62.0$ \\
\hline$d$ & 1.407 & \\
\hline$e$ & 1.435 & \\
\hline$f$ & 1.318 & \\
\hline
\end{tabular}

${ }^{a}$ Data taken from references 106 and 107 .

${ }^{b}$ Bond lengths in angstrom units $(\AA)$.

${ }^{c}$ Bond angles in degrees $\left({ }^{\circ}\right)$.

The electronic absorption (UV) spectra of the alkylidenecycloproparenes show long wavelength maxima that are consistent with the extended conjugation in these molecules. Furthermore, the positions of the absorbance maxima are solvent dependent and, when the solvent polarity is increased (from hexane or cyclohexane to acetonitrile) hypsochromic shifts of typically 5-7 $\mathrm{nm}$ are observed. ${ }^{100}$

The brightly coloured anilinomethylidene $(114 \mathrm{j})$ has been found to fluoresce in dilute solution. ${ }^{108}$ The absolute quantum yield of this is 0.96 , which is comparable to that of fluorescein. Consequently, $(114 \mathrm{j})$ has the potential ${ }^{100,102,108}$ for use as a laser dye or in the field of non-linear optics. 


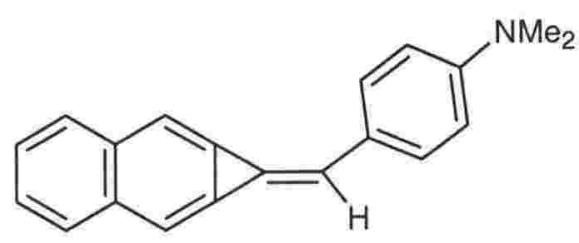

(114j)

The ${ }^{1} \mathrm{H}$ n.m.r. chemical shifts of the aromatic protons of the methylidenecycloproparenes lie in the usual range. ${ }^{100}$ However, the aromatic ${ }^{13} \mathrm{C}$ n.m.r. resonances of the alkylidenecycloproparenes generally appear in the range 94 to $136 \mathrm{ppm}$. The methine carbons adjacent to the sites of three-membered ring fusion are shielded and typically resonate between 96-115 ppm, while those of the exocyclic double bond are observed between 100-120 ppm. Moreover, when a para substituted aryl group is attached to the exocyclic alkene, e.g. (114j), the carbon chemical shifts of the exocyclic olefin, the threemembered ring and $\mathrm{Cla}-\mathrm{C} 7 \mathrm{a}$ are affected by the nature of the para substituent

The chemistry of the alkylidenecycloproparenes is dominated by reactions that involve opening of the three-membered ring by nucleophiles ${ }^{109}$ and electrophiles; ${ }^{110}$ the compounds are more sensitive to the last reagent. ${ }^{100,110}$ With (114k) and aqueous acid, the product of reaction is ketone (124) (Scheme 25). ${ }^{111}$ The reaction most likely proceeds by (114k) capturing proton at C-8 to generate the benzylic cation (125). Quenching ion (125) with water then generates alcohol (126) which can then ring open to product (124).

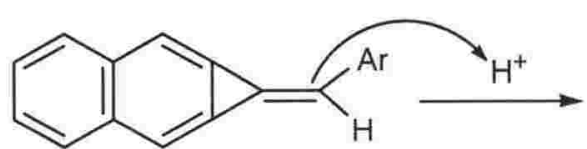

(114k)

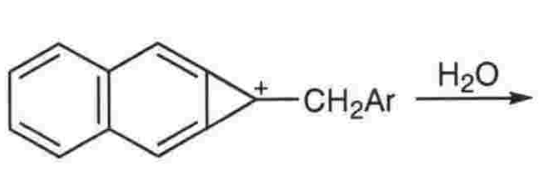

(125)

$$
\mathrm{Ar}=p-\mathrm{MeOC}_{6} \mathrm{H}_{4}
$$

Scheme 25<smiles>[3H]CC1(C)c2cc3ccccc3cc2C1(O)CO</smiles>

(126)<smiles>CCCC(=O)c1ccc2ccccc2c1</smiles>

(124)

Upon treating the methylidenecycloproparenes with $\mathrm{Ag}(\mathrm{I})$ the three-membered ring is cleaved analogously to cyclopropabenzene (4). Thus, when compound (1141) or (116a) is treated with $\operatorname{Ag}(\mathrm{I})$ in the presence of alcohol the product formed is the enol ether (127) (Scheme 26). ${ }^{110}$ However, if one of the exocyclic olefin substituents is proton, e.g., as in compounds (114m) and (116b), ethynes (128) are formed. ${ }^{110}$ The cations (129) are most likely involved. 
<smiles>[R]c1cc2c(cc1[R])C2=C(c1ccccc1)c1ccccc1</smiles>
$\stackrel{\mathrm{Ag}^{+}, \mathrm{R}^{1} \mathrm{OH}}{\longrightarrow}$

(114l) $R R=$ benzo fusion (116a) $\mathrm{R}=\mathrm{H}$<smiles>[R]OC(=C(c1ccccc1)c1ccccc1)c1ccc([R])c([R])c1</smiles>

(127a) $R R=$ benzo fusion (127b) $\mathrm{R}=\mathrm{H}$<smiles></smiles>

(114m) RR = benzo fusion (116b) $\mathrm{R}=\mathrm{H}$<smiles>[R]c1cc2c(cc1[R])[Al]2[Al]=C(CC)c1ccccc1</smiles>

(129a) RR = benzo fusion (129b) $\mathrm{R}=\mathrm{H}$

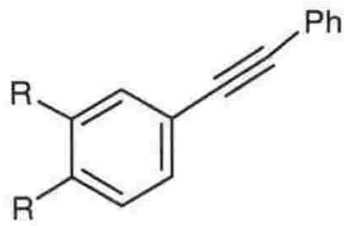

(128a) $R R=$ benzo fusion (128b) $\mathrm{R}=\mathrm{H}$

\section{Scheme 26}

The addition of bromine in water to (116a) gives rise to the heptafulvene (130). ${ }^{110}$ The reaction probably proceeds by bromine adding across the strained bridge bond to generate (131) which affords (130) on electrocyclic ring-opening (Scheme 27). However, when the naphtho derivative (114l) is treated analogously it is cyclobutarene (132) that is isolated. ${ }^{110}$ The reaction probably occurs via addition of bromine across the exocyclic olefin which generates (133) and thus avoids the high energy orthoquinodimethane intermediate (134) that would result from addition across the bridge bond. The subsequent loss of bromide then affords cation (135) which can then furnishes the observed product (132) after ring-expansion.
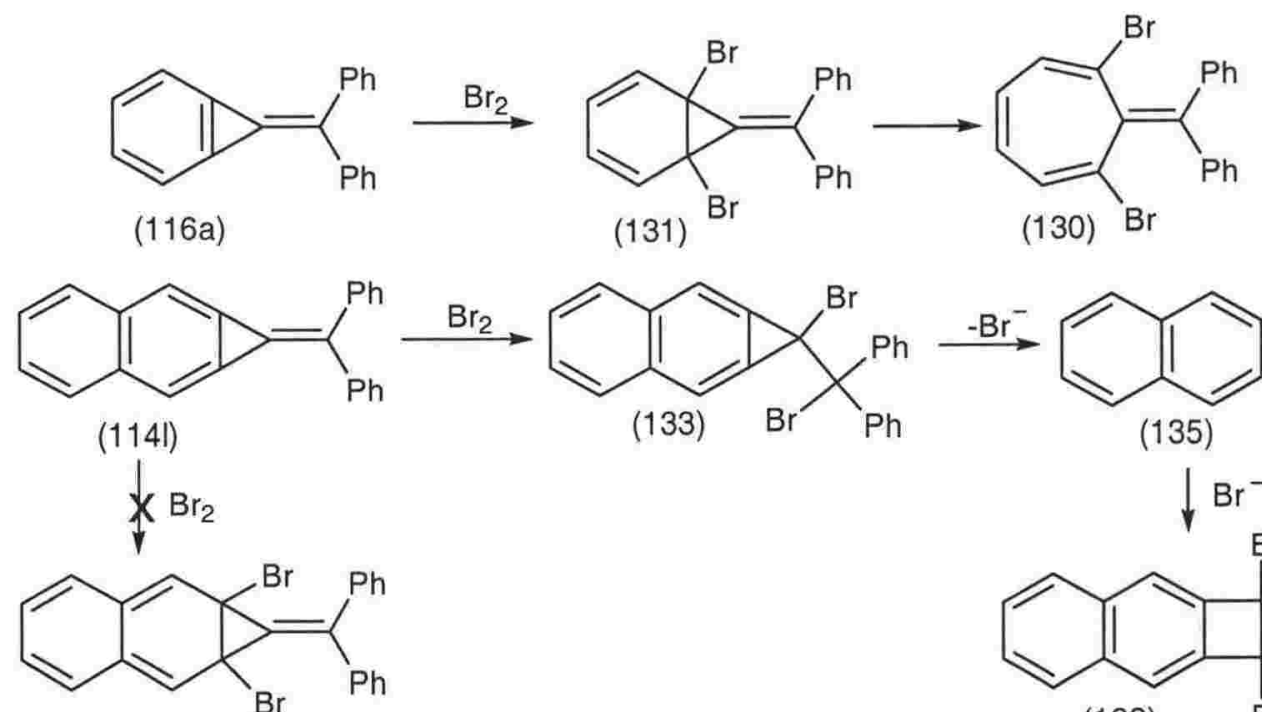

(134)
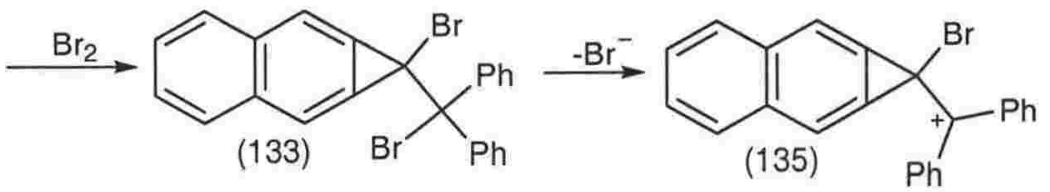

(135)<smiles>BrC(Br)(Br)c1cc2ccccc2cc1C1(Br)c2ccccc2C1(Br)c1ccccc1</smiles>

Scheme 27

The alkylidene compounds are sensitive to electrophiles but they react only slowly with nucleophilic reagents. ${ }^{109}$ With potassium $t$-butoxide in thf (tetrahydrofuran), $(1141, \mathrm{~m})$ and $(116 \mathrm{a}, \mathrm{b})$ require several days of reflux before conversion into product heptafulvenes (136) and (137) is complete (Scheme 28). ${ }^{109}$ The $t$-butoxide ion probably adds to the strained bridge bond to give anions (138) and (139) which, on protonation, give 
norcaradienes (140) and (141); electrocyclic ring-opening then accounts for the observed products.<smiles>[R]C(=C1c2cc([R])c([2H])cc21)c1ccccc1</smiles>

(114l) $\quad R R=$ benzo fusion; $R^{1}=P h$

$(114 \mathrm{~m})$ RR = benzo fusion; $\mathrm{R}^{1}=\mathrm{H}$<smiles>[R]C1=CC2=C(c3ccccc3)C2(OCCC)C=C1[R]</smiles><smiles>[R]C1=CC2C(=C([R])c3ccccc3)C2(OCCC)C=C1[R]</smiles>

(138) $R R=$ benzo fusion

(139) $\mathrm{R}=\mathrm{H}$

(140) $R R=$ benzo fusion

(116a) $\mathrm{R}=\mathrm{H} ; \mathrm{R}^{1}=\mathrm{Ph}$

(116b) $\quad \mathrm{R}=\mathrm{H} ; \mathrm{R}^{1}=\mathrm{H}$<smiles>[R]C1=C([R])C=C(OCC)C(=C([Tl])c2ccccc2)C=C1</smiles>

(136) $R R=$ benzo fusion

(137) $\mathrm{R}=\mathrm{H}$

Scheme 28

The alkylidenecycloproparenes can react with dienes either across the strained bridge bond or the exocylic olefin. When the cyclopropanaphthalene derivative (1141) is treated with diphenylisobenzofuran either in refluxing toluene or in ethylene glycol the cyclobutarene (142) is isolated via rearrangement of the strained spirocycloproparene (143) (Scheme 29). ${ }^{112}$ With $\alpha$-pyrone, the product of reaction is (144) and it is most likely formed via the highly strained intermediate $(145){ }^{112}$ To date, no evidence to support cycloaddition across the bridge bond of (1141) has been obtained.<smiles></smiles>

(114l)

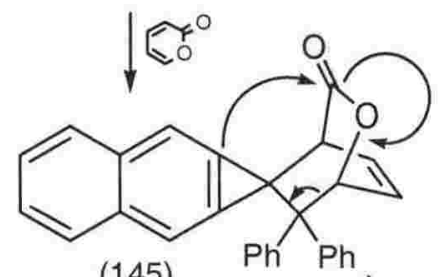

(145)

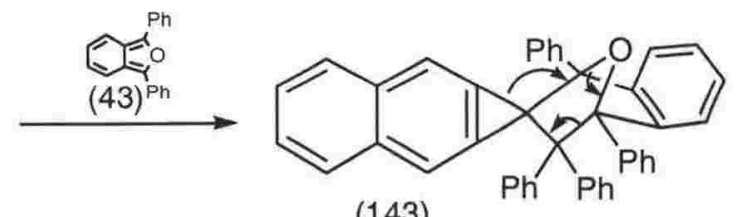

(143)<smiles>O=C(c1ccccc1)c1cccc(C2(c3ccccc3)C(=Cc3ccccc3)c3cc4ccccc4cc32)c1</smiles><smiles></smiles>

Scheme 29

In contrast to the Diels-Alder reactions discussed above, alkylidenecyclopropabenzenes (116a) and (116c) do not form cycloadducts with $\alpha$-pyrone. ${ }^{113}$ However, the diene component of diphenylisobenzofuran adds across the bridge bond of (116a) and 
(116c) in an endo-sense to give adducts (146) and (147), respectively (Scheme 30), as shown by X-ray crystallography. ${ }^{113}$

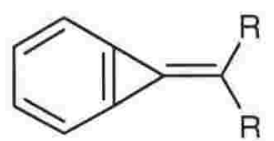

(116a) $\mathrm{R}=\mathrm{Ph}$

(116c) $\mathrm{R}=p-\mathrm{MeOC}_{6} \mathrm{H}_{4}$
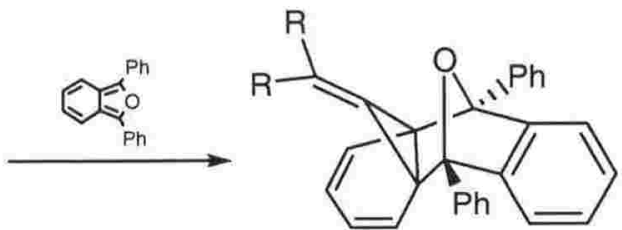

(146) $\mathrm{R}=\mathrm{Ph}$

(147) $\mathrm{R}=p-\mathrm{MeOC}_{6} \mathrm{H}_{4}$

Scheme 30

When alkylidenecycloproparenes (114l) and (116a) are treated with dimethyldioxirane $\left(\mathrm{Me}_{2} \mathrm{CO}_{2}\right)$ the corresponding oxaspiropentene $(148)^{114,115}$ is generated from epoxidation of the exocyclic olefin (Scheme 31). These strained molecules have eluded isolation under normal conditions; however by performing the epoxidations in n.m.r. tubes at low temperature, carbon and proton n.m.r. data fully compatible with these compounds are obtained. At $0^{\circ} \mathrm{C}$ the oxaspiropentene (148a) rearranges to afford cyclobutarene (149a); the conversion is $\sim 50 \%$ complete after $8 \mathrm{~h}^{114}$ Evidence for the analogous rearrangement of $(148 \mathrm{~b})$ into $(149 \mathrm{~b})$ is obtained after $1 \mathrm{~h}$ at $20^{\circ} \mathrm{C}$. However, only after heating (148b) at $35^{\circ} \mathrm{C}$ for $5 \mathrm{~min}$ and then $50^{\circ} \mathrm{C}$ for $30 \mathrm{~min}$ is convincing evidence obtained for cyclobutenone $(149 \mathrm{~b}){ }^{115}$ The conversion is complete after heating the sample for a further $1 \mathrm{~h}$ at this temperature.<smiles>[R]c1cc2c(cc1[R])C2=C(C)c1ccccc1</smiles>

(114k) RR = benzo fusion (116a) $\mathrm{R}=\mathrm{H}$

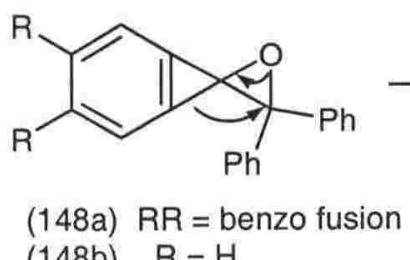
(148b) $\quad \mathrm{R}=\mathrm{H}$

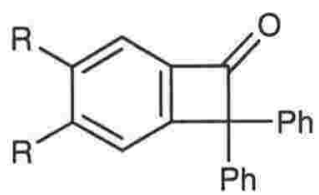

(149a) $R R=$ benzo fusion (149b) $\quad \mathrm{R}=\mathrm{H}$

Scheme 31

Halton et al. have recently demonstrated that bis-ether (70) is a synthon for preparing dimethoxyalkylidenecyclopropanaphthalenes (150). ${ }^{116}$ His group found that the metalation and silylation of (70) gives the disilane (151) (Scheme 32) in direct analogy to the silylation of cyclopropanapthalene (58) (Scheme 20, p. 16). The desilylation of (151) and interception of anion (152) with an appropriate aldehyde or ketone affords the corresponding methylidene compound (150) (Scheme 32); approximately 20 examples are known. ${ }^{104,116,117}$ Alkylidenecyclopropanaphthalenes (150) are oxygen-sensitive and slowly darken on storage in air. ${ }^{116}$ 


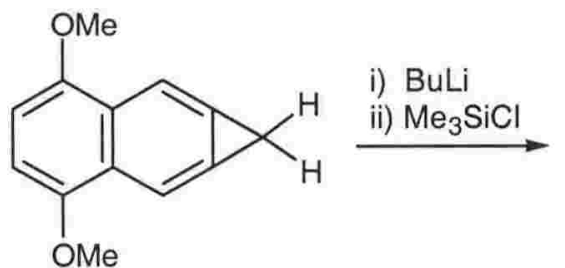

(70)

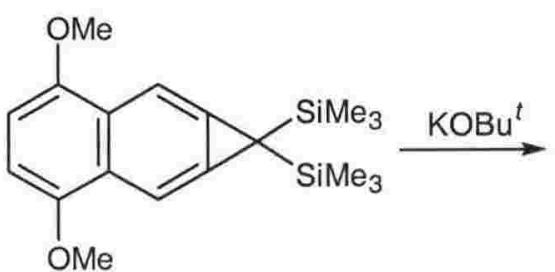

(151)<smiles>COc1ccc(OC)c2cc3c(cc12)C3C</smiles>

(152)

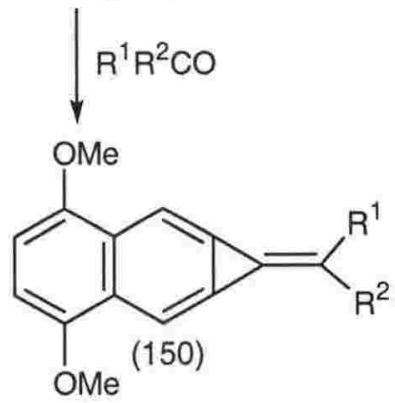

Scheme 32

Dimethoxymethylidenes (150) can couple the electron donating ability of the C3 and C6 ether functions with either electron-donating or electron-withdrawing groups on the exocyclic olefin to produce compounds with interesting electronic features; as expected these compounds have measurable ${ }^{104}$ dipole moments. In general, the $\mathrm{C} 8$ resonance of (150) is $\sim 2$ ppm more shielded than for their unsubstituted alkylidenecyclopropanaphthalene analogues (114). ${ }^{116}$ The chemical shifts for $\mathrm{C} 2 / 7$ are shielded as expected. ${ }^{116}$

Recent attempts to synthesise methylidenecyclopropaquinones (153) in which the quinone moiety can act as electron acceptor, and therefore polarise the alkylidene compounds in an opposite sense to that of the ether analogues (150), have proved unsuccessful. ${ }^{116}$ It was found that treating ether (70) with cerium ammonium nitrate (CAN) affords cyclopropaquinone $(154)^{116,118}$ in excellent yield as the first example of a stable cyclopropaquinone (Scheme 33). However, attempts to prepare the requisite silane (155) for Peterson olefination proved unsuccessful. ${ }^{116}$ An alternative route to silane (155) involves reducing the disilyldialkoxyether (151) with CAN. However, only on one occasion was evidence to support the formation of (155) obtained; this result proved nonreproducible. An alternative route to the alkylidenecyclopropaquinones would involve converting the methylidene ethers (150) into compounds (153) with CAN. Unfortunately, these reactions resulted in significant decomposition of starting material and the reaction products eluded isolation. ${ }^{116}$ 


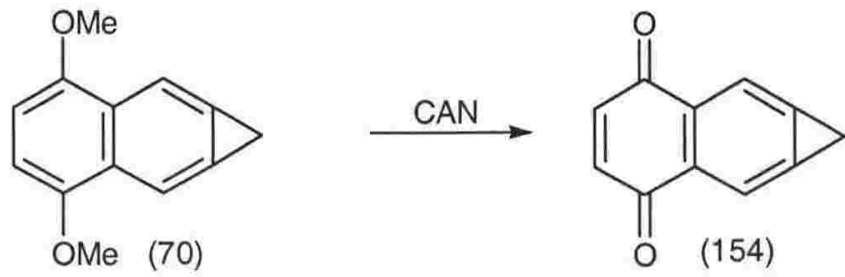

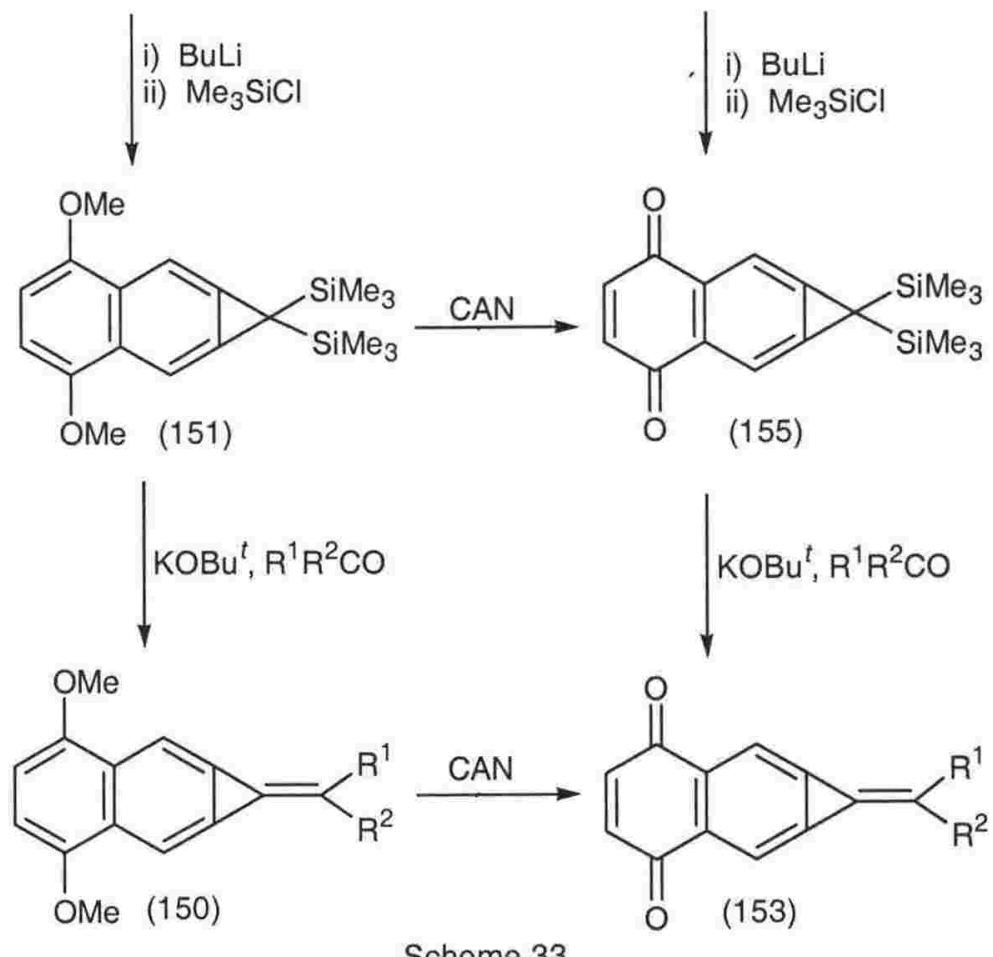

Scheme 33

Quinone (154) adds bromine across the C4-C5 $\pi$-bond to afford the halogenated cycloproparene (156) and with cyclopentadiene the Diels-Alder adduct (157) is isolated from addition of diene across the same bond; ${ }^{19}$ no evidence to support the formation of the cycloadduct from the addition of diene across the strained bridge was obtained. ${ }^{119}$<smiles>CC1(C(=O)Br)c2cc3c(cc21)C(=O)C(Br)C3=O</smiles>

(156)<smiles>O=C1C=CC(=O)c2cc3c(cc21)C3</smiles>

(154)
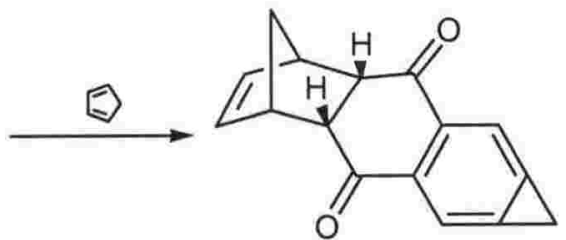

(157)

A recent study by Halton et al. ${ }^{120}$ has shown that while furan does not add across the enedione $\pi$-bond of cyclopropaquinone (154) (even when the reaction is performed at $45^{\circ} \mathrm{C}$ under $14 \mathrm{kbar}$ of pressure) the air sensitive Diels-Alder adduct (158) is obtained from isobenzofuran at ambient temperature (Scheme 34). Attempts to dehydrate the epoxytetracene (158) to (159) proved unsuccessful. ${ }^{120}$ The addition of butadiene to quinone (154) can be effected at $40-50^{\circ} \mathrm{C}$ and an inseparable ${ }^{121}$ mixture of adducts (160) and (161) along with unchanged (154) is formed. Compound (161), which results from the addition of butadiene to the strained $\sigma$-bond of (160), is the major product of reaction at $100^{\circ} \mathrm{C}$ and it has been characterised. ${ }^{119,121}$ 


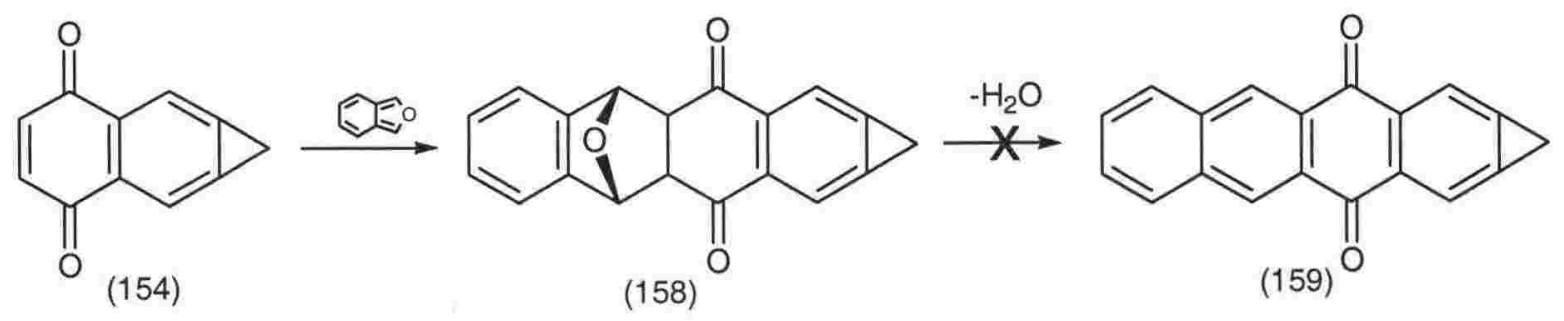

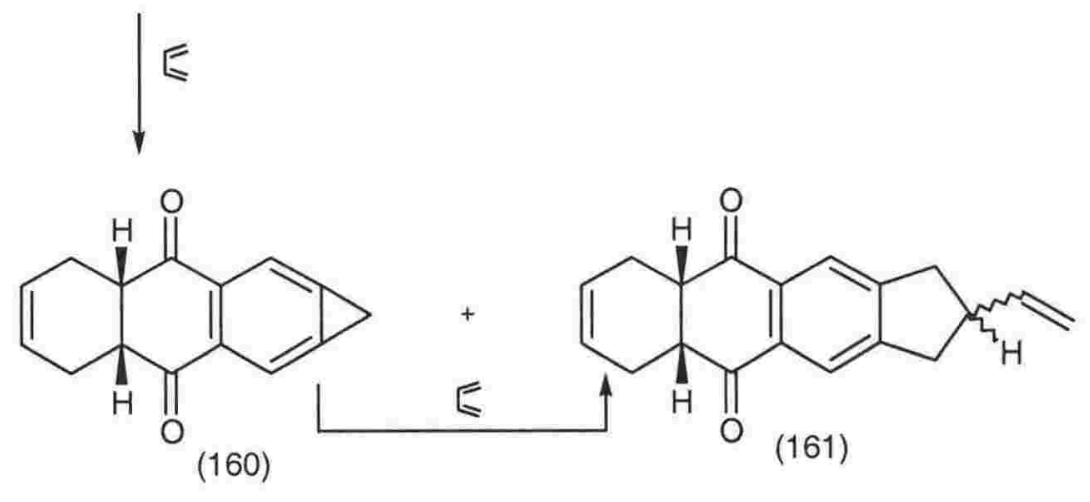

Scheme 34

One objective of this project is to extend the cyclopropaquinone family by preparing the unknown cyclopropanthraquinone (162) and its diether analogue (163) from synthon (160). Attempts to further conjugate these compounds by preparing their alkylidene derivatives (164) and (165), respectively, will be discussed.<smiles>O=C1c2ccccc2C(=O)c2cc3c(cc21)C3</smiles>

(162)

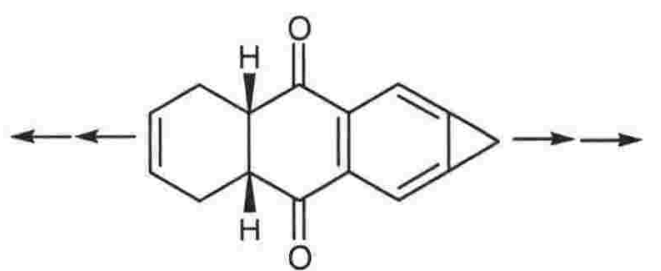

(160)<smiles>COc1c2ccccc2c(OC)c2cc3c(cc12)C3</smiles>

(163)<smiles>[R7]C([R])=C1c2cc3c(cc21)C(=O)c1ccccc1C3=O</smiles>

(164)<smiles>[R7]C([R])=C1c2cc3c(OC)c4ccccc4c(OC)c3cc21</smiles>

(165)

Tetrathiafulvalene (TTF) ${ }^{122}$ (166) is a well known $\pi$ electron donor that has attracted much attention in the field of new organic materials. TTF and its dithiolecontaining derivatives, e.g. (167), ${ }^{123}$ are known ${ }^{124}$ to afford charge-transfer (CT) salts when treated with a $\pi$ electron acceptor, e.g., 2,3-dichloro-5,6-dicyano-1,4-benzoquinone (DDQ). Some of these salts have interesting electrochemical properties, including superconductivity. ${ }^{124}$ However, the impact of strain on the physicochemical properties of these CT salts has yet to be established fully. Attempts to prepare the novel dithiole-containing cycloproparenes (168) and (169) via the Wittig-Horner olefination of cyclopropaquinone 
synthons (154) and (162), respectively, and the appropriate dithiole phosphonate anion will be discussed. In addition, attempts are to be made to convert (168) and (169) into the first known cycloproparene CT salts.<smiles>C1=CSC(=C2SC=CS2)S1</smiles>

(166)<smiles></smiles>

(167)<smiles>C1=CSC(=c2ccc(=C3SC=CS3)c3cc4c(cc23)C4)S1</smiles>

(168)<smiles>C1=CSC(=c2c3ccccc3c(=C3SC=CS3)c3cc4c(cc23)C4)S1</smiles>

(169)

It has been shown by Oda et al. that intermediate (170) which is generated from treating an organolithium with various $\mathrm{N}, \mathrm{N}$-dimethylamides can be intercepted by cyclopentadiene to afford the corresponding pentafulvene (171). ${ }^{125}$ It is hoped that quenching intermediate (170) with cyclopropanaphthalene (58) will afford the corresponding alkylidenecycloproparene compounds (114). Efforts to convert (58) into its methylidene derivatives (114) in situ are described.

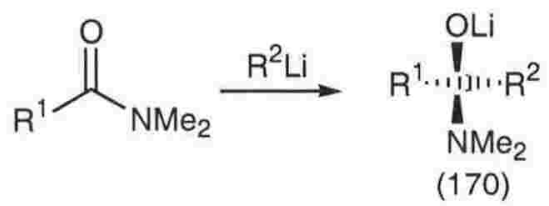

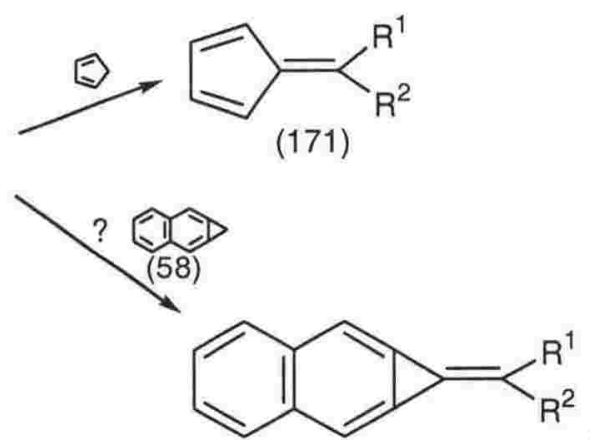

(114) 


\section{Chapter 2}

\section{Chemistry of the Monosilylcycloproparenyl Anion}

The desilylation of the 1,1-bis(trimethylsilyl)- $1 H$-cyclopropa[b]naphthalene (105) is readily accomplished with potassium $t$-butoxide and the ensuing $\alpha$-silyl anion (106) has been captured by a wide variety of aldehydes and ketones to furnish the methylidene compounds (114). ${ }^{24,27,28,98-104}$ However, few examples of $\mathrm{C} 1$ disubstituted cyclopropanaphthalenes derived from (106) were known at the commencement of this study. Stang and his group were able to isolate in good yield nine C1-acetylated cycloproparenes, e.g. (112), from interception of a modified carbonyl-containing compound with anion (106). ${ }^{95}$ Conversely, Halton and Jones only obtained evidence for cycloproparene (113) from the reaction of (106) with methyl iodide. ${ }^{97}$ The purification of (113) from a mixture which contained unchanged disilane (105) and hydrocarbon (58) did not prove possible. Moreover, Halton and Jones encountered even greater difficulties during the attempted preparation of the monosilane (104). ${ }^{97}$ They found that the addition of one molar equivalent of water to anion (106) with slow warming of the mixture to ambient temperature overnight did not afford (104) as anticipated. Instead, the sole product of reaction was cyclopropanaphthalene (58) (89\%). Even when (105) was treated with water and a catalytic amount of base it was hydrocarbon (58) that was generated almost quantitatively. However, evidence was obtained for the monosilane (104) which was formed in trace quantities from this last reaction. ${ }^{97}$<smiles>CCC1(C)c2cc3ccccc3cc21</smiles>

(105)

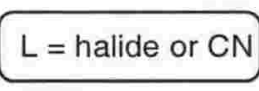

$\downarrow \mathrm{KOBu}^{t}$<smiles>CCC1(C=O)c2cc3ccccc3cc21</smiles>

(112)

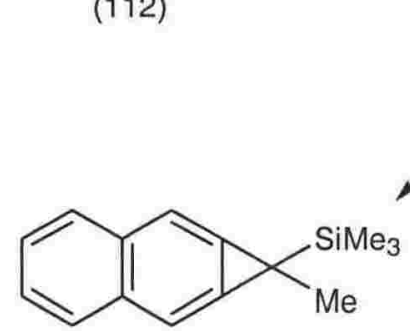

(113)
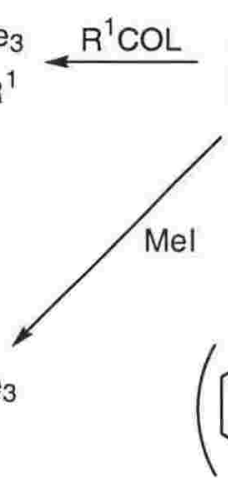<smiles>CC1c2cc3ccccc3cc2C1C</smiles>

(106)

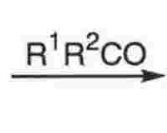

$\downarrow \mathrm{H}_{2} \mathrm{O}$<smiles>[R7]C([Tl])=C1c2cc3ccccc3cc21</smiles>

(114)<smiles>CC(C)C1c2cc3ccccc3cc2C1(C)C</smiles>

(104)

(58) 
Previous attempts by us to prepare and isolate the monosilane (104) by intercepting anion (106) with a proton source other than water were also unsuccessful. ${ }^{97}$ With trifluoroacetic acid, an inseparable mixture of compounds which contained the monosilane (104), unchanged silane (105), cyclopropanaphthalene (58), and an unidentified product thought to be a 2-substituted naphthalene was obtained; this last compound presumably results from opening of the three-membered ring under the acidic conditions.

The nearly quantitative conversion of compound (105) into hydrocarbon (58) with water and catalytic amounts of base can be rationalised if hydroxide ion is capable of effecting C-Si bond cleavage. This was shown ${ }^{90}$ to be the case with 1-(trimethylsilyl)- $1 \mathrm{H}$ cyclopropabenzene (98) and sodium hydroxide (Scheme 18, p. 15) and so such cleavage followed by the interception of the $\alpha$-silyl anion (106) with water would give monosilane (104) and hydroxide ion. The desilyation of (104) with this base then provides trimethylsilanol and the cycloproparenyl anion (103) and interception of this anion with water furnishes hydrocarbon (58) and hydroxide ion. The hydroxide ion is catalytic until conversion to hydrocarbon (58) is complete (Scheme 35). That potassium hydroxide does indeed effect cleavage of the C-Si bond of cycloproparenes (105) and (104) was demonstrated by us in an earlier study ${ }^{97}$ from the isolation of cyclopropanaphthalene (58) after treatment of (105) with aqueous potassium hydroxide. It was also found that treatment of (105) with potassium hydroxide and a carbonyl-containing compound in the presence of 18-crown-6 affords the corresponding methylidenecycloproparene (114). However, in some cases the yields of the exocyclic olefins were $30 \%$ lower than the analogous reactions which employed the desilyating reagent potassium $t$-butoxide.

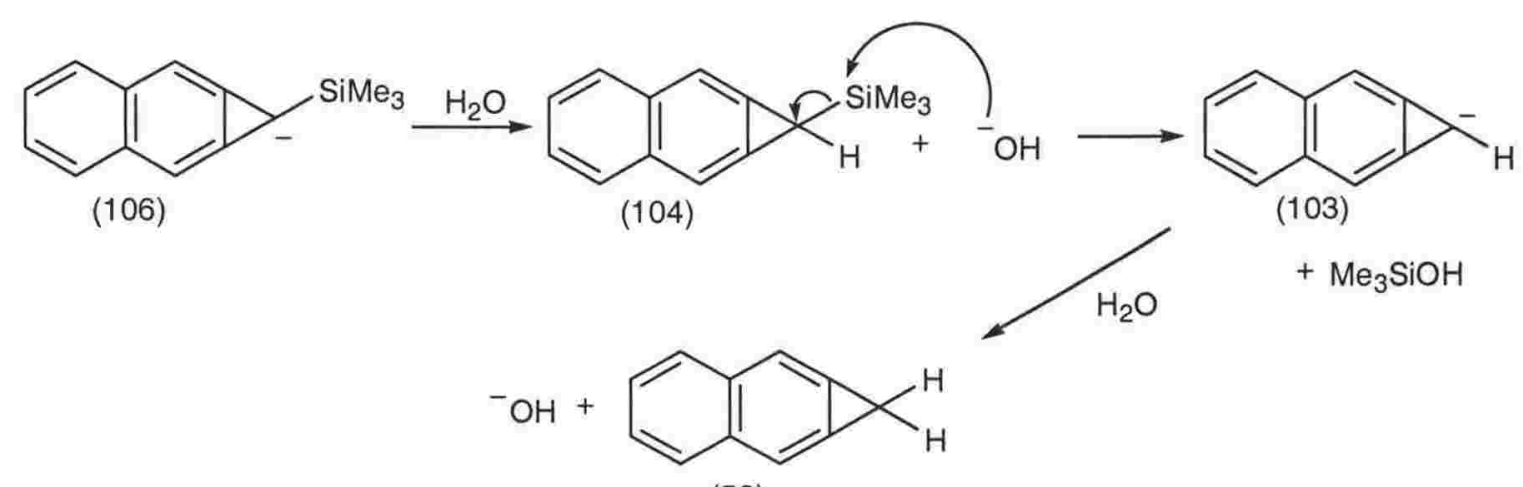

(58)

Scheme 35

In this chapter more recent efforts to refocus the preparation and characterisation of the C1-disubstituted cycloproparenes (104) and (113) which formed the basis of the author's Honours project are presented. These served not only to improve the author's synthetic and manipulation skills but also, to establish whether the size exclusion gel Sephadex LH-20 was an appropriate solid phase support for the separation of cyclopropa- 
renes. In addition, a new and novel dibenzo $[b, g]$ fluorene was discovered and its structural elucidation by extensive spectroscopic studies is presented.

The $\alpha$-silyl anion (106) is generated readily from treatment of 1,1-bis(trimethylsilyl)-1H-cycloprop[$[b]$ naphthalene (105) with potassium $t$-butoxide. In turn, synthon (105) is easily available in four steps from naphthalene in an overall yield of $22 \%$ (Scheme 36). Birch reduction of naphthalene with sodium metal gives 1,4-dihydronaphthalene $(172)^{126}$ in $88 \%$ yield. Subsequent addition of dichlorocarbene using the methodology developed by this author, ${ }^{96}$ and described in the Experimental section, affords the gem-dichloro compound $(67)^{53}$ which, after didehydrochlorination with potassium $t$-butoxide, furnishes the odoriferous cycloproparene (58) in $62 \%$ yield. ${ }^{53}$ Finally, the metalation and silylation of (58) affords silane (105) in $86 \%$ yield. $^{24}$<smiles>CCO[Sb](C)(O)(O)c1ccc2c(c1)CC=CC2</smiles>

(172)

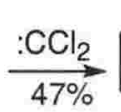

(67)

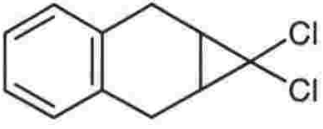

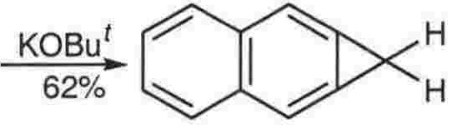

(58)

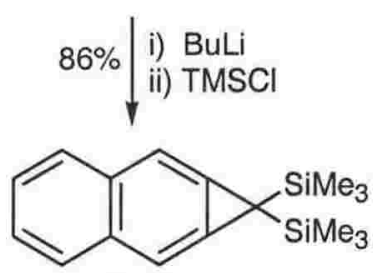

(105)

The preparation and capture of anion (106) with methyl iodide was repeated using the literature precedent ${ }^{97}$ and an oil was obtained that contained the C1-methylcycloproparene (113) and cyclopropanaphthalene (58) in a 9:1 ratio $\left({ }^{1} \mathrm{H}\right.$ n.m.r.). As the two compounds had identical $R_{\mathrm{f}}$ (retention factor) values on t.l.c. (thin layer chromatography), a silica gel column was deemed inappropriate for their separation. Instead, separating these compounds on the basis of their difference in molecular mass $\left(\Delta M_{\mathrm{r}} 86 \mathrm{~g} / \mathrm{mol}\right)$ was attempted using the size exclusion gel Sephadex LH-20. Subjection of part of the cycloproparene-containing mixture to the resin and collection of the faster eluting, higher molecular mass-containing fraction, gave a sample of (113) that was free of hydrocarbon (58) and an analytical sample was obtained in $47 \%$ yield after one further radial chromatogram. The ${ }^{1} \mathrm{H}$ n.m.r. spectrum of (113) shows singlets at $\delta 0.02$ and 1.69 that integrate for nine and three protons and they are assigned to the $\mathrm{SiMe}_{3}$ and $\mathrm{Me}$ group protons, respectively. The $\mathrm{H} 2 / 7$ protons resonate as a singlet at $\delta 7.34$ and an aromatic AA'BB' spin system is observed for the protons $\mathrm{H} 3 / 6$ and $\mathrm{H} 4 / 7$.

The ${ }^{13} \mathrm{C}$ n.m.r. spectrum of (113) shows eight resonances, which is nicely compatible with the symmetry of this compound. The $\mathrm{SiMe}_{3}$ carbons are shielded and 
$\mathrm{C} 2 / 7$ are shielded and resonate at $\delta 109.9$, a value comparable to those of other cyclopropanaphthalenes. $^{35,100}$

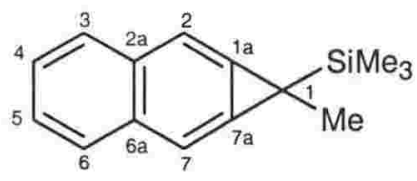

(113)

In view of the success in isolating and characterising the $\mathrm{C} 1$ disubstituted cycloproparene (113) attempts to prepare the monosilane (104) were re-assessed. However, due to the problems associated with mixing the $\alpha$-silyl anion (106) with either water or trifluoroacetic acid for $\sim 16 \mathrm{~h}$ a different approach to (104) was needed. On the assumption that the cleavage of the $\mathrm{C}$-Si bond of monosilane (104) with potassium hydroxide (generated from quenching (106) with water) may not be particularly facile at low temperature over a short period of time, anion (106) was generated, quenched with water at $-78^{\circ} \mathrm{C}$, and the mixture worked up after only two minutes. The resulting product mixture was found to contain predominantly monosilane (104) $\left({ }^{1} \mathrm{H}\right.$ n.m.r. $)$ with traces of cyclopropanaphthalene (58) and a third component that had protons resonating at $\delta 8.10$ (dd, $1 \mathrm{H}, J 8.6,1.7 \mathrm{~Hz}$ ) and 8.61 (broad s, 1H). As these deshielded signals typify ${ }^{109,110}$ a 2substituted naphthalene this last compound is believed to be one that results from opening of the three-membered ring. The proton n.m.r. spectrum of the mixture gave a ratio for the three compounds of $\sim 16: 5: 6$ and, as expected, they proved to be inseparable by t.l.c. However, use of the Sephadex LH-20 size exclusion gel followed by radial chromatography gave an analytical sample of 1-(trimethylsilyl)-1 $H$-cyclopropa[ $b]$ naphthalene (104) (9\%) as an unstable oil that is best stored under argon in the freezer. The ${ }^{1} \mathrm{H}$ n.m.r. data obtained for this pure compound are in good agreement with those previously published by us $^{97}$ for a contaminated sample with only minor chemical shift changes recorded. The most notable feature of the pure compound is the observation of small longrange couplings $(J<1 \mathrm{~Hz})$ between the $\mathrm{H} 1(\delta 3.23)$ and the $\mathrm{H} 2 / 7$ aromatic protons $(\delta 7.33)$. In addition, a small $(J<1 \mathrm{~Hz})$ coupling between $\mathrm{H} 3 / 6$ and $\mathrm{H} 2 / 7$ was observed with the latter appearing as a doublet of doublets centred at $\delta 7.33$ rather than a singlet as observed previously for other cycloproparenes. To the author's knowledge, this provides the first recorded example of such long-range couplings in a cycloproparene.

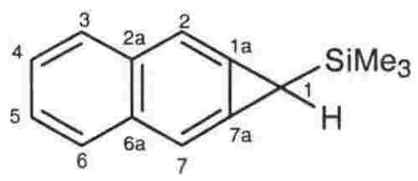

(104) 
As expected, the ${ }^{13} \mathrm{C}$ n.m.r. spectrum of (104) shows seven signals. The $\mathrm{SiMe}_{3}$ carbons resonate at $\delta-3.4$, comparable to those of silane (113), whilst $\mathrm{C} 2 / 7$ met with expectation and show typical shielding $(\delta 110.0)$. The other five carbon resonances for (104) were within the usual ranges.

Although an analytical sample of monosilane (104) has now been obtained, it was decided to test whether treatment of the $\alpha$-silyl anion (106) with a solvent carrying mildly acidic protons, i.e. toluene ( $\left.\mathrm{p} K_{\mathrm{a}} 41\right)$, might also give the $\mathrm{C} 1$-substituted cycloproparene (104). On generation of the requisite $\alpha$-silyl anion, addition of one molar equivalent of toluene followed by conventional workup, including radial chromatography, gave a small quantity of an oily yellow solid. The ${ }^{1} \mathrm{H}$ n.m.r. spectrum of this implicated the desired monosilane (104), hydrocarbon (58), and an unidentified component in a ratio of $\sim 53: 10: 5$. The unidentified product is thought to be a 2 -substituted naphthalene $e^{109,110}$ from its two low field proton resonances at $\delta 8.10(\mathrm{dd}, 1 \mathrm{H}, J 8.6,1.7 \mathrm{~Hz})$ and $8.61($ broad s, $1 \mathrm{H})$ The paucity of sample precluded purification of the three compounds and this route to (104) was not deemed viable. However, in addition to this monosilane-containing fraction the chromatography gave a second, less mobile, component. Analysis of this eluate by ${ }^{1} \mathrm{H}$ n.m.r. spectroscopy revealed the presence of a methyl and a methylene group at $\delta 2.59$ and 4.06 , respectively. Whilst the latter resonated as a singlet the former resonated as a narrow doublet with a small long-range coupling $(J \sim 1 \mathrm{~Hz})$. Resonances in the aromatic region of the proton spectrum were also observed and though complex, six distinct sets of signals were observed. These integrated in a 3:2:2:2:1:1 ratio and corresponded to a total of eleven aromatic protons (see Figs. 1 and 2). The ${ }^{13} \mathrm{C}$ n.m.r. spectrum of the compound contained twenty-two distinct resonances of which twenty were aromatic carbons with chemical shifts in the range 121.1-143.55 ppm It was clear that this compound is asymmetric and does not contain a cycloproparene framework. The IR spectrum of the compound showed only aliphatic and aromatic C-H stretching bands above $1500 \mathrm{~cm}^{-1}$ suggesting it may be a hydrocarbon. The base peak of the mass spectrum is the molecular ion $(\mathrm{m} / \mathrm{z} 280)$ and it corresponds to a molecular mass double that of cyclopropanaphthalene (58) $(\mathrm{m} / \mathrm{z}$ 140). The compound is a formal dimer of (58) but it is formed in only $6 \%$ yield from anion (104) and toluene. Because this dimer contains twenty-two distinct carbon resonances $\left({ }^{13} \mathrm{C}\right.$ n.m.r.) and the proton spectrum integrates for sixteen protons the $m / z$ value of 280 implies a molecular formula for this dimer as $\mathrm{C}_{22} \mathrm{H}_{16}$ and an index of hydrogen deficiency of fifteen. 


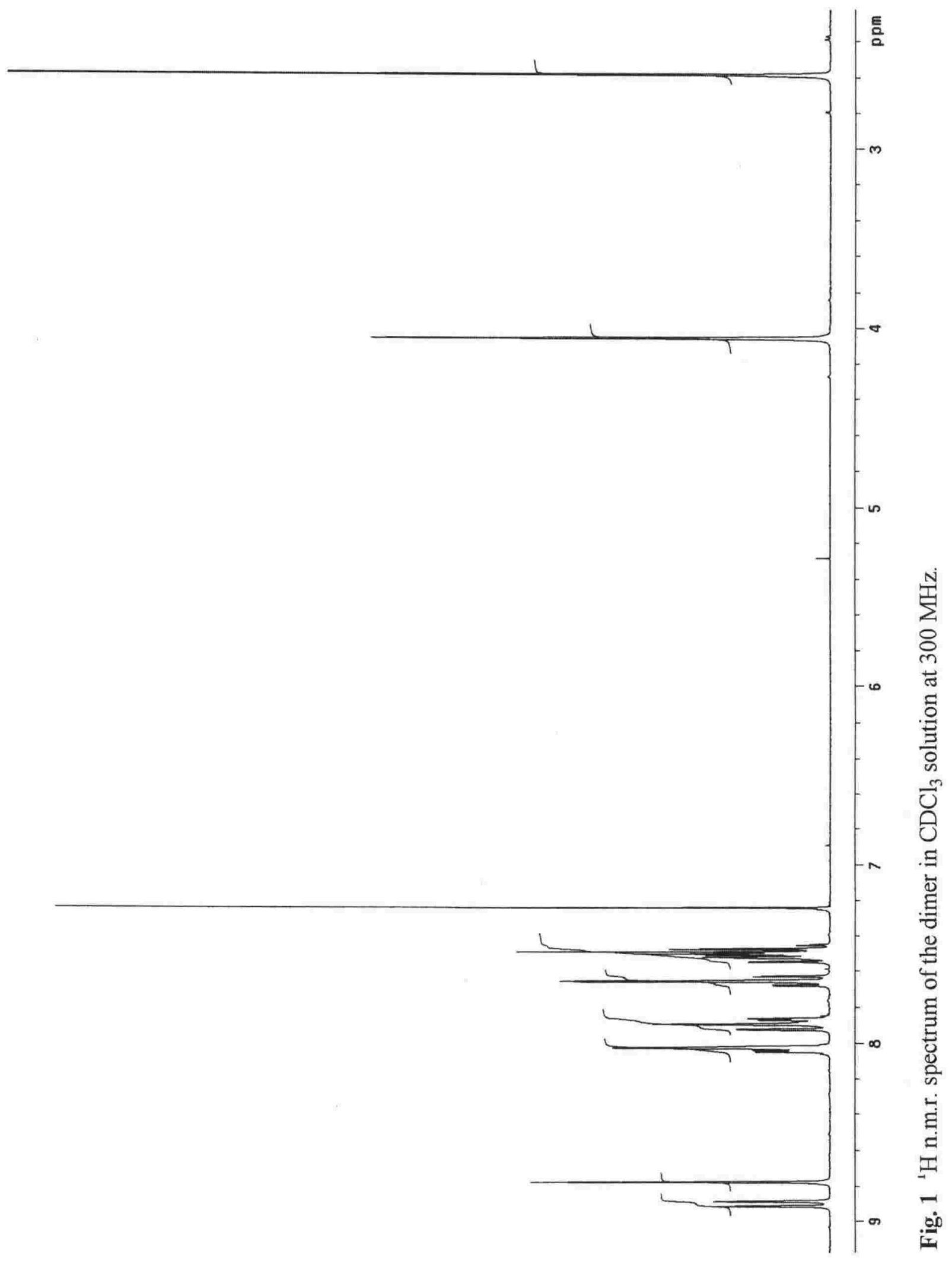




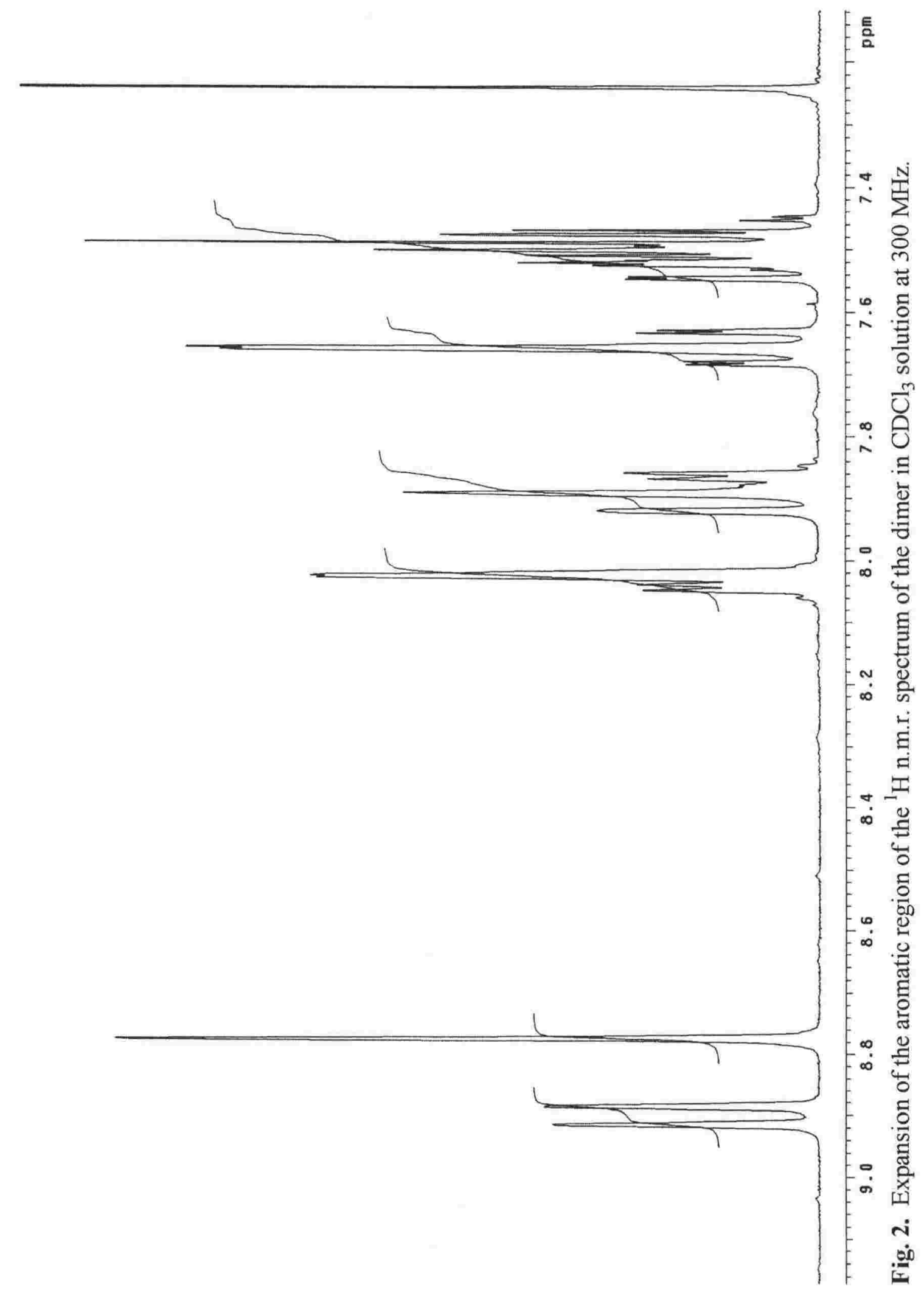


The aromatic region of the ${ }^{1} \mathrm{H}$ n.m.r. spectrum of the dimer reveals a broad oneproton singlet at 8.77 and a one-proton doublet at $8.90 \mathrm{ppm}(J 8.1 \mathrm{~Hz})$. Correlation of the former to the carbon signal at $121.1 \mathrm{ppm}$ and the latter to that at $123.6 \mathrm{ppm}$ was established from use of HSQC (heteronuclear single quantum correlation) techniques. First order analysis of the ${ }^{1} \mathrm{H}$ n.m.r. spectrum indicated a two-proton doublet of triplets to be centred at $~ 7.66 \mathrm{ppm}$. However, a HOMO 2DJ experiment (homonuclear $J$-resolved twodimensional experiment) clearly revealed that a one-proton doublet of triplets $(J 7.5,1.3$ $\mathrm{Hz})$ and a one-proton para-coupled doublet $(J 1 \mathrm{~Hz})$ were independently superimposed and centred at $\delta 7.66$ (see Fig. 3). In similar fashion, the apparent two-proton multiplet at $\delta$ 7.86-7.92 was resolved into a one-proton doublet at $\delta 7.87(J 9.2 \mathrm{~Hz})$ and a one-proton doublet of doublets at $\delta 7.91(J 8.2,2.8 \mathrm{~Hz})$. The two-proton multiplet from $\delta 8.02-8.05$ was dissected into a one-proton para-coupled doublet at $\delta 8.03(\mathrm{~J}<1 \mathrm{~Hz})$ and a one-proton doublet of doublets at $\delta 8.04(J 6.7,2.4 \mathrm{~Hz})$. Unfortunately, the HOMO 2DJ experiment did not resolve the three-proton multiplet between 7.44 and $7.57 \mathrm{ppm}$, but these three protons have been assigned to the carbon resonances at $\delta 125.2,125.3$, and 125.4, from use of HSQC data. Similarly, it was possible to determine unambiguously the one bond ${ }^{1} \mathrm{H}-{ }^{13} \mathrm{C}$ connectivity for all protonated carbons; the values are presented in Table 3.

Table 3. ${ }^{13} \mathrm{C}$ and ${ }^{1} \mathrm{H}$ n.m.r. chemical shifts in ppm for the dimer.

\begin{tabular}{llllll}
\hline${ }^{13} \mathbf{C}$ & DEPT & ${ }^{1} \mathbf{H}($ mult, $\boldsymbol{J}$ in Hz) & ${ }^{13} \mathbf{C}$ & DEPT & ${ }^{1} \mathbf{H}$ (mult, $\boldsymbol{J}$ in Hz) \\
\hline 19.4 & $\mathrm{CH}_{3}$ & $2.59(\mathrm{~d}, \sim 1)$ & 128.46 & $\mathrm{C}^{a}$ & - \\
36.0 & $\mathrm{CH}_{2}$ & $4.06(\mathrm{~s})$ & 128.53 & $\mathrm{CH}$ & $8.04(\mathrm{app} \mathrm{dd}, 6.7,2.4)^{b}$ \\
121.1 & $\mathrm{CH}$ & $8.77(\mathrm{bs})$ & 128.56 & $\mathrm{CH}$ & $7.91(\mathrm{dd}, 8.2,2.8)^{b}$ \\
122.9 & $\mathrm{CH}$ & $8.03(\mathrm{~d},<1)^{b}$ & 132.0 & $\mathrm{C}$ & - \\
123.6 & $\mathrm{CH}$ & $8.90(\mathrm{~d}, 8.1)$ & 132.5 & $\mathrm{C}$ & - \\
125.2 & $\mathrm{CH}$ & $7.44-7.57(\mathrm{~m})$ & 133.2 & $\mathrm{C}$ & - \\
125.3 & $\mathrm{CH}$ & $7.44-7.57(\mathrm{~m})$ & 134.0 & $\mathrm{C}$ & - \\
125.4 & $\mathrm{CH}$ & $7.47-7.57(\mathrm{~m})$ & 135.0 & $\mathrm{C}$ & - \\
125.9 & $\mathrm{CH}$ & $7.66(\mathrm{app} \mathrm{dt}, 7.5,1.3)^{b}$ & 141.78 & $\mathrm{C}$ & - \\
127.4 & $\mathrm{CH}$ & $7.87(\mathrm{~d}, 9.2)^{b}$ & 141.84 & $\mathrm{C}$ & - \\
127.9 & $\mathrm{CH}$ & $7.66(\mathrm{q}, \sim 1)^{b, c}$ & 143.55 & $\mathrm{C}$ & - \\
\hline
\end{tabular}

${ }^{a}$ Quaternary carbon.

${ }^{b}$ Chemical shifts and coupling constants established from HOMO2DJ experiment.

${ }^{C}$ Inner two lines only observed. 


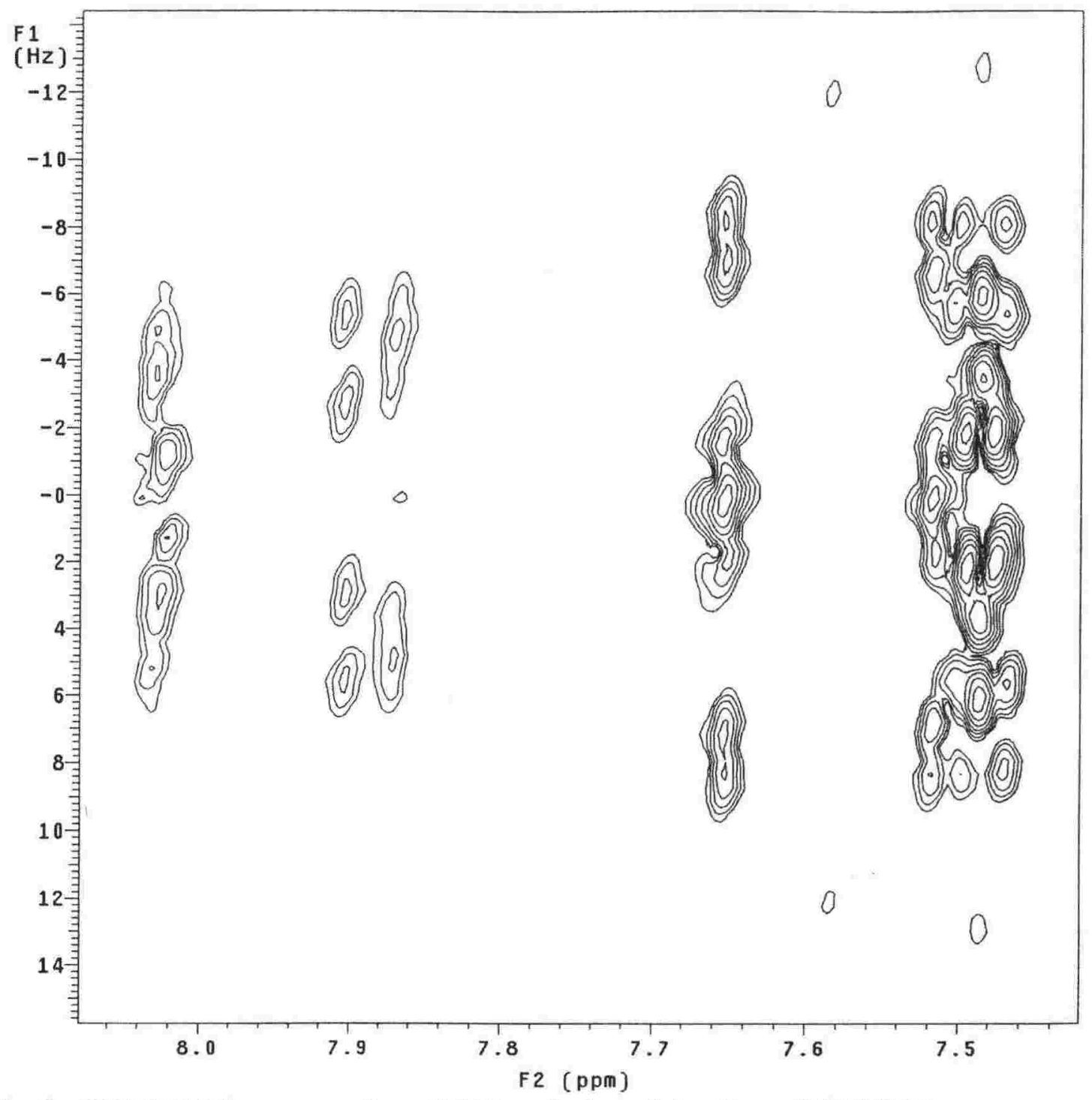

Fig. 3. HOMO $2 \mathrm{DJ}$ spectrum for a $\mathrm{CDCl}_{3}$ solution of the dimer $(300 \mathrm{MHz})$.

The molecular formula of the dimer was established as $\mathrm{C}_{22} \mathrm{H}_{16}$ with an index of hydrogen deficiency of fifteen. Because twenty of the twenty-two carbons are aromatic in nature, ten double bond equivalents are accounted for. Hence, the compound must be polycyclic in nature and comprised of five rings of which four are aromatic. The presence of a deshielded methylene singlet at $\delta 4.06 / 36.0$ is consistent with this centre being doubly benzylic and thus adjacent to two aromatic rings. This suggests that the dimer could be a substituted fluorenyl system, viz. (173) (Fig. 4). Furthermore, the observation that the methyl group shows long-range proton-proton spin-spin coupling $(J \sim 1 \mathrm{~Hz})$ to an adjacent but otherwise isolated aromatic proton at $\delta 7.66$ (q, $J \sim 1 \mathrm{~Hz}$; inner two lines only observed) implies the angular fusion of two rings under consideration, viz. (174). In addition, the presence of a one-proton para-coupled doublet and a one-proton broad singlet at $\delta 8.03$ and 8.77, respectively, that are coupled (COSY spectrum) suggests the presence of a separate benzenoid ring that is linearly fused to two other rings as in (175). 


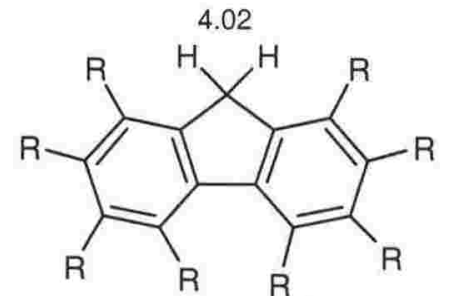

$\mathrm{R}=\mathrm{H}$ or $\mathrm{RR}=$ benzo fusion

(173)

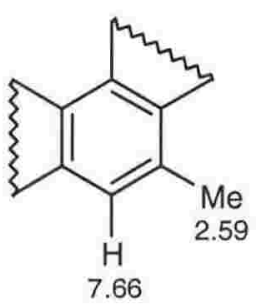

(174)

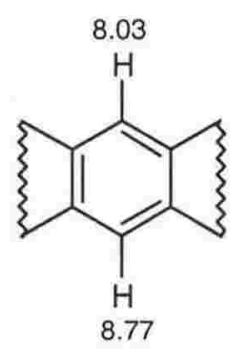

(175)

Fig. 4. Substructures of the dimer with selected ${ }^{1} \mathrm{H}$ n.m.r. chemical shift data (ppm).

The three substructures in Fig. 4 above allow four possible structures to be constructed for the unknown compound. These are the two dibenzo $[a, h]$ fluorenes $(176)$ and (177), and the two isomeric dibenzo[b,g]fluorenes (178) and (179).

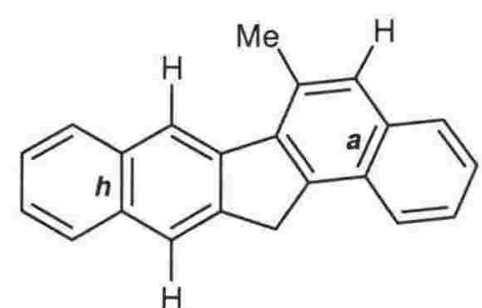

(176)

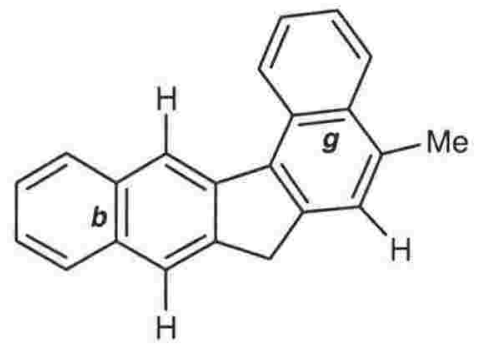

(178)<smiles>Cc1cc2c(c3ccccc13)Cc1cc3ccccc3cc1-2</smiles>

(177)

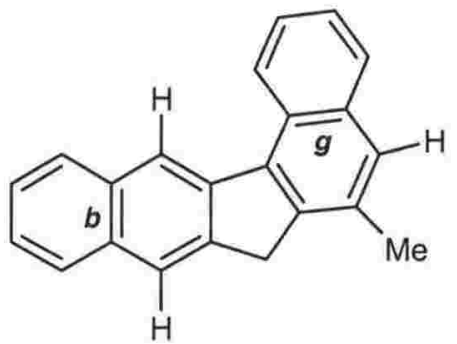

(179)

Differentiation between compounds (176)-(179) and identification of the unknown hydrocarbon as the dibenzo[b,g]fluorene (179) has come from extensive n.m.r. investigations. The observation of an n.O.e (nuclear Overhauser enhancement) (see Fig. 5 below) between the methyl protons and the isolated one-proton doublet at $\delta 7.66$ places the methyl group adjacent to this aromatic proton on the benzenoid ring that is angularly ringfused. However, and more importantly, an n.O.e enhancement was also recorded between the methyl group and the methylene protons. As this requires these hydrogen atoms to be spatially proximal it rules out (176), (177), and (178) as the hydrocarbon product and leaves the dibenzo $[b, g]$ fluorene (179) as the sole contender. An n.O.e. effect between the methylene protons at $\delta 4.06$ and the one-proton para-coupled doublet at $\delta 8.03$ identifies the location of the linear fused ring adjacent to the five-membered ring and, by default, allows for the conventional assignment of the coupled para-proton $\mathrm{H} 13$ as that resonating at $\delta 8.77$. When this last signal is irradiated a positive n.O.e. to the doublet of doublets at $\delta$ 8.04 and the doublet at $\delta 8.90$ is observed. However, these resonances could not be 
assigned unambiguously to $\mathrm{H} 1$ or $\mathrm{H} 12$ without use of TOCSY (total correlation spectroscopy) and HMBC (heteronuclear multiple bond correlation) experimental data.

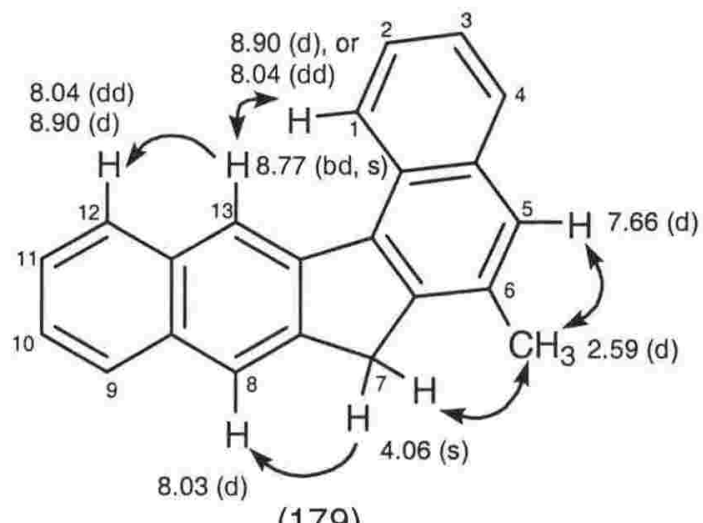

(179)

Fig. 5. ${ }^{1} \mathrm{H}$ n.m.r. chemical shifts (ppm) and nuclear Overhauser enhancements (arrows) recorded for 6-methyl-7H-dibenzo[b,g]fluorene (179) in $\mathrm{CDCl}_{3}$ at $300 \mathrm{MHz}$.

From a series of ID TOSCY experiments with increasing mixing times of 20, 40, and $80 \mathrm{~ms}$, respectively, the selective excitation of the proton with the chemical shift of $\delta$ 8.04 revealed, sequentially, resonances at $\delta 7.44,7.55$, and 7.87 , as multiplets. The fact that an HMBC correlation is observed between the proton at $\delta 7.87$ and the $\mathrm{C} 8$ carbon atom $(\delta 122.9)$, itself identified by and HSQC correlation to H8 ( $\delta 8.03)$, identifies the former proton as $\mathrm{H} 9$ whilst the doublet of doublets at $\delta 8.04$ is confidently assigned to H12; by default the doublet at $\delta 8.90$ must be due to H1. Similarly, a series of 1D TOSCY experiments in which the proton at $\delta 8.90(\mathrm{H} 1)$ was irradiated revealed multiplets at $\delta 7.66$, 7.57, and 7.91 sequentially and provides adequate proof that this ring is ortho-fused. With these data and the use of HMBC correlations it proved possible to assign confidently almost all of the protons and carbons of hydrocarbon (179) (Experimental).

Although the spectroscopic data obtained for the dibenzo[b,g]fluorene (179) argue convincingly for its assignment, unambiguous proof of the structure was sought. That (179) is the hydrocarbon product has been proved from a single crystal X-ray structure obtained and analysed by Professor Boese in Essen, Germany. The results confirm 6methyl-7H-dibenzo[ $b, g]$ fluorene (179) as the new dimer as shown in the ORTEP plot of Fig 6; bond lengths and bond angles are given in Tables 4 and 5, respectively. The results of this study have now been published. ${ }^{127}$ 


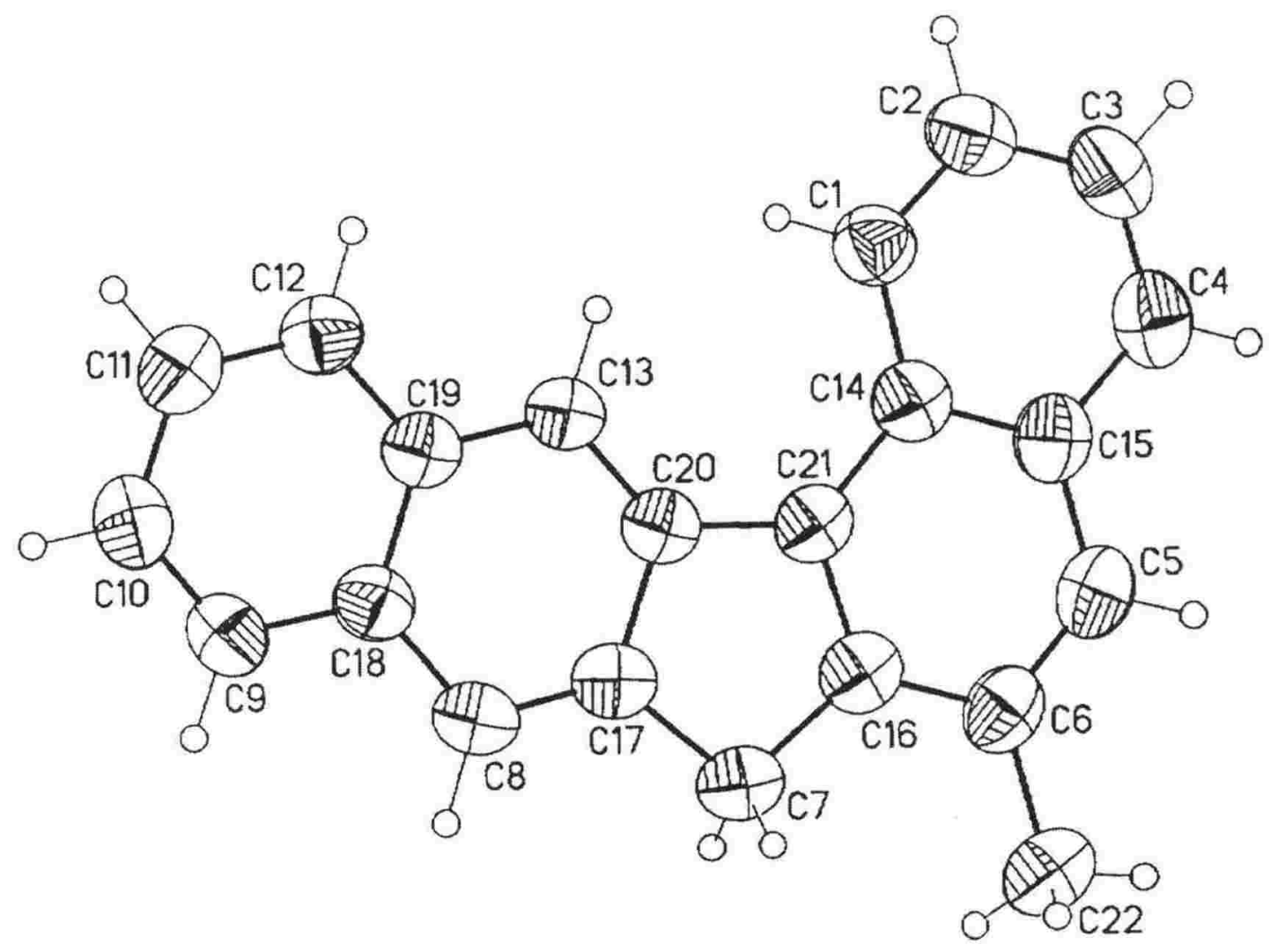

Fig. 6. ORTEP plot of 6-methyl-7- $H$-dibenzo[b,g]fluorene (179) with crystallographic numbering at $50 \%$ probability.

Table 4. Molecular non-hydrogen distances ( $\AA$ ) for (179) employing crystallographic numbering.

\begin{tabular}{cccc}
\hline Atoms $^{a}$ & Distance & Atoms & Distance \\
\hline $\mathrm{C}(1)-\mathrm{C}(2)$ & $1.370(2)$ & $\mathrm{C}(9)-\mathrm{C}(10)$ & $1.365(3)$ \\
$\mathrm{C}(1)-\mathrm{C}(14)$ & $1.419(2)$ & $\mathrm{C}(9)-\mathrm{C}(18)$ & $1.421(2)$ \\
$\mathrm{C}(2)-\mathrm{C}(3)$ & $1.396(3)$ & $\mathrm{C}(10)-\mathrm{C}(11)$ & $1.408(3)$ \\
$\mathrm{C}(3)-\mathrm{C}(4)$ & $1.361(3)$ & $\mathrm{C}(11)-\mathrm{C}(12)$ & $1.365(2)$ \\
$\mathrm{C}(4)-\mathrm{C}(15)$ & $1.425(2)$ & $\mathrm{C}(12)-\mathrm{C}(19)$ & $1.422(2)$ \\
$\mathrm{C}(5)-\mathrm{C}(6)$ & $1.376(3)$ & $\mathrm{C}(13)-\mathrm{C}(20)$ & $1.376(2)$ \\
$\mathrm{C}(5)-\mathrm{C}(15)$ & $1.426(2)$ & $\mathrm{C}(13)-\mathrm{C}(19)$ & $1.419(2)$ \\
$\mathrm{C}(6)-\mathrm{C}(16)$ & $1.409(2)$ & $\mathrm{C}(14)-\mathrm{C}(15)$ & $1.421(2)$ \\
$\mathrm{C}(6)-\mathrm{C}(22)$ & $1.507(2)$ & $\mathrm{C}(14)-\mathrm{C}(21)$ & $1.438(2)$ \\
$\mathrm{C}(7)-\mathrm{C}(16)$ & $1.509(2)$ & $\mathrm{C}(16)-\mathrm{C}(21)$ & $1.397(2)$ \\
$\mathrm{C}(7)-\mathrm{C}(17)$ & $1.512(2)$ & $\mathrm{C}(17)-\mathrm{C}(20)$ & $1.441(2)$ \\
$\mathrm{C}(8)-\mathrm{C}(17)$ & $1.360(2)$ & $\mathrm{C}(18)-\mathrm{C}(19)$ & $1.425(2)$ \\
$\mathrm{C}(8)-\mathrm{C}(18)$ & $1.424(2)$ & $\mathrm{C}(20)-\mathrm{C}(21)$ & $1.479(2)$ \\
\hline
\end{tabular}


Table 5. Interatomic non-hydrogen angles $\left({ }^{\circ}\right)$ for (179) employing crystallographic numbering.

\begin{tabular}{|c|c|c|c|}
\hline Atoms $^{a}$ & Angle & Atoms & Angle \\
\hline $\mathrm{C}(2)-\mathrm{C}(1)-\mathrm{C}(14)$ & $121.3(2)$ & $\mathrm{C}(14)-\mathrm{C}(15)-\mathrm{C}(5)$ & $120.2(2)$ \\
\hline$C(1)-C(2)-C(3)$ & $120.4(2)$ & $\mathrm{C}(4)-\mathrm{C}(15)-\mathrm{C}(5)$ & $120.9(2)$ \\
\hline$C(4)-C(3)-C(2)$ & $120.3(2)$ & $C(21)-C(16)-C(6)$ & $122.5(2)$ \\
\hline $\mathrm{C}(3)-\mathrm{C}(4)-\mathrm{C}(15)$ & $121.1(2)$ & $C(6)-C(16)-C(7)$ & $111.2(2)$ \\
\hline $\mathrm{C}(6)-\mathrm{C}(5)-\mathrm{C}(15)$ & $122.1(2)$ & $C(8)-C(17)-C(20)$ & $126.4(2)$ \\
\hline $\mathrm{C}(5)-\mathrm{C}(6)-\mathrm{C}(16)$ & $117.8(2)$ & $\mathrm{C}(8)-\mathrm{C}(17)-\mathrm{C}(7)$ & $121.3(2)$ \\
\hline $\mathrm{C}(5)-\mathrm{C}(6)-\mathrm{C}(22)$ & $121.9(2)$ & $\mathrm{C}(20)-\mathrm{C}(17)-\mathrm{C}(7)$ & $129.2(2)$ \\
\hline$C(16)-C(7)-C(17)$ & $120.3(2)$ & $\mathrm{C}(9)-\mathrm{C}(18)-\mathrm{C}(8)$ & $109.44(14)$ \\
\hline $\mathrm{C}(17)-\mathrm{C}(8)-\mathrm{C}(18)$ & $103.04(13)$ & $C(9)-C(18)-C(19)$ & $122.8(2)$ \\
\hline $\mathrm{C}(10)-\mathrm{C}(9)-\mathrm{C}(18)$ & $120.6(2)$ & $\mathrm{C}(8)-\mathrm{C}(18)-\mathrm{C}(19)$ & $118.8(2)$ \\
\hline$C(9)-C(10)-C(11)$ & $120.4(2$ & $\mathrm{C}(13)-\mathrm{C}(19)-\mathrm{C}(12)$ & $121.50(14)$ \\
\hline$C(12)-C(11)-C(10)$ & $120.3(2)$ & $\mathrm{C}(13)-\mathrm{C}(19)-\mathrm{C}(18)$ & $120.1(2)$ \\
\hline$C(11)-C(12)-C(19)$ & $120.3(2)$ & $\mathrm{C}(12)-\mathrm{C}(19)-\mathrm{C}(18)$ & $118.4(2)$ \\
\hline$C(20)-C(13)-C(19)$ & $120.60(14)$ & $C(13)-C(20)-C(17)$ & $118.9(2)$ \\
\hline$C(1)-C(14)-C(15)$ & $118.0(2)$ & $C(13)-C(20)-C(21)$ & $133.22(14)$ \\
\hline$C(1)-C(14)-C(21)$ & $124.6(2)$ & $C(17)-C(20)-C(21)$ & $107.84(13)$ \\
\hline$C(15)-C(14)-C(21)$ & $117.4(2)$ & $C(16)-C(21)-C(14)$ & $120.0(2)$ \\
\hline$C(14)-C(15)-C(4)$ & $118.8(2)$ & $C(16)-C(21)-C(20)$ & $108.47(14)$ \\
\hline
\end{tabular}

The internuclear bond lengths obtained for hydrocarbon (179) are largely as expected for a polycyclic fluorenyl system and show some similarities to those of fluorene (180) (Fig. 7). ${ }^{128}$ However, clearly evident is that the C-C bonds of the fluorenyl benzenoid rings fused to the five-membered ring in (179) are no longer similar in length but are significantly distorted.
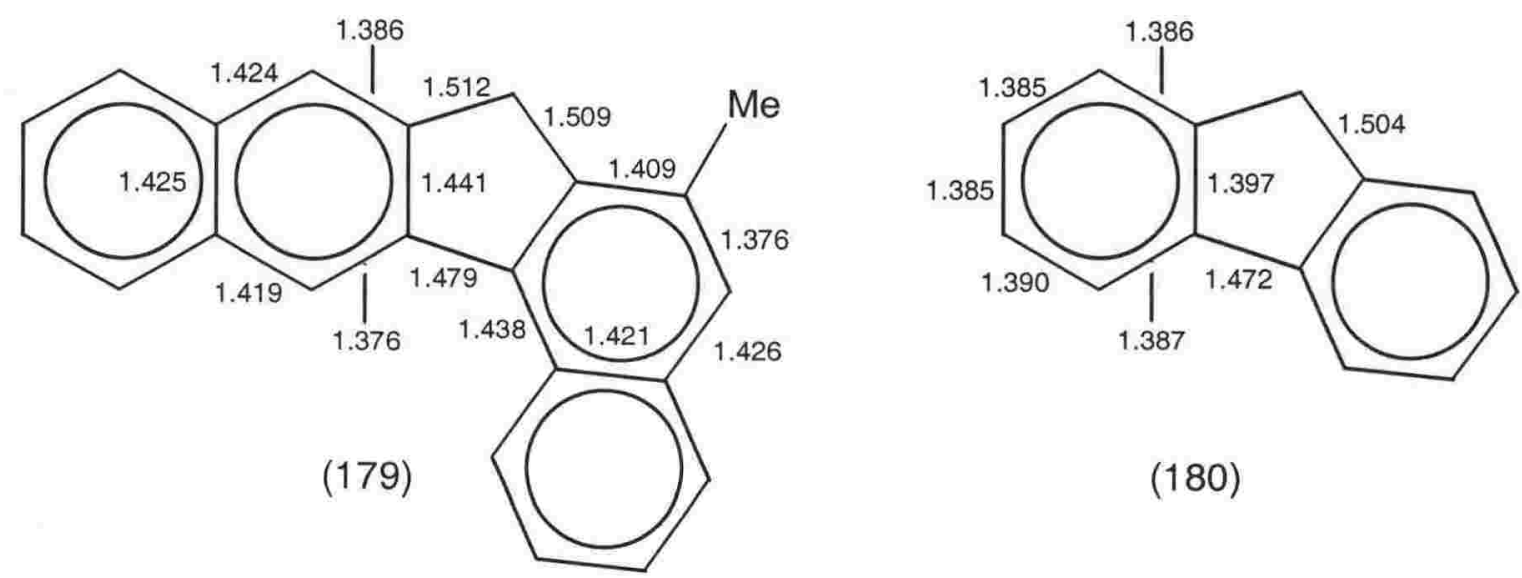

$(180)$

Fig. 7. Selected bond lengths $(\AA)$ of dibenzo[b,g]fluorene (179) and fluorene (180). 
To date, compound (179) represents the first formally characterised dibenzo$[b, g]$ fluorene hydrocarbon. The $5 H$-dibenzo $[b, g]$ fluorene-5,8,13-triones $(181 \mathrm{a}, \mathrm{b})$ have been isolated and characterised ${ }^{129}$ while $7 H$-dibenzo[b,g]fluorene-7-one (182) has been claimed ${ }^{130}$ as an environmental pollutant.

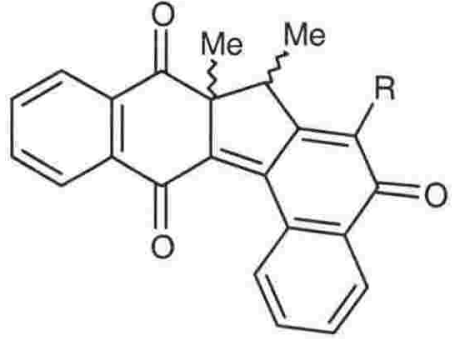

(181a) $\mathrm{R}=\mathrm{Me}$

(181b) $\mathrm{R}=\mathrm{CH}_{2} \mathrm{Ph}$

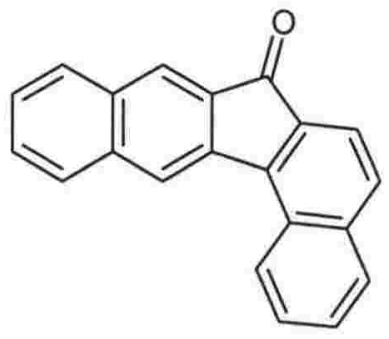

(182)

The mode of formation of hydrocarbon (179) from the $\alpha$-silyl anion (106) (6\%) and toluene is not clear but the same product is formed in $18 \%$ yield when anion (106) is treated with $N, N$-dimethylacetamide (vide infra) (Scheme 37). As toluene and $N, N$ dimethyl acetamide do not appear to have any common features other than very slightly acidic protons it does not seem likely they are directly involved in the formation of dibenzofluorene (179). Because compound (179) corresponds to a dimer of cyclopropanaphthalene (58) is seemed plausible that anion (106) could either dimerise or react with cyclopropanaphthalene (58) to give the dibenzofluorene product (179); cycloproparene (58) could be formed from anion (106) if conditions were not strictly anhydrous. In an attempt to discover whether the latter explanation accounts for the formation of hydrocarbon (179) $\alpha$-silyl anion (106) was treated with cyclopropanaphthalene (58); 6methyldibenzo[ $b, g]$ fluorene (179) was formed in $28 \%$ yield! This clearly implies that formation of hydrocarbon (179) involves the interaction of anion (106) with cycloproparene (58). The pathway from anion (106) to dibenzofluorene (179) is likely complex and it has not yet proved possible to provide an adequate explanation for the formation of dimer (179). However, the coupling of the $\mathrm{C} 1$ centre of one cycloproparenyl system with $\mathrm{C} 2$ of another seems likely.
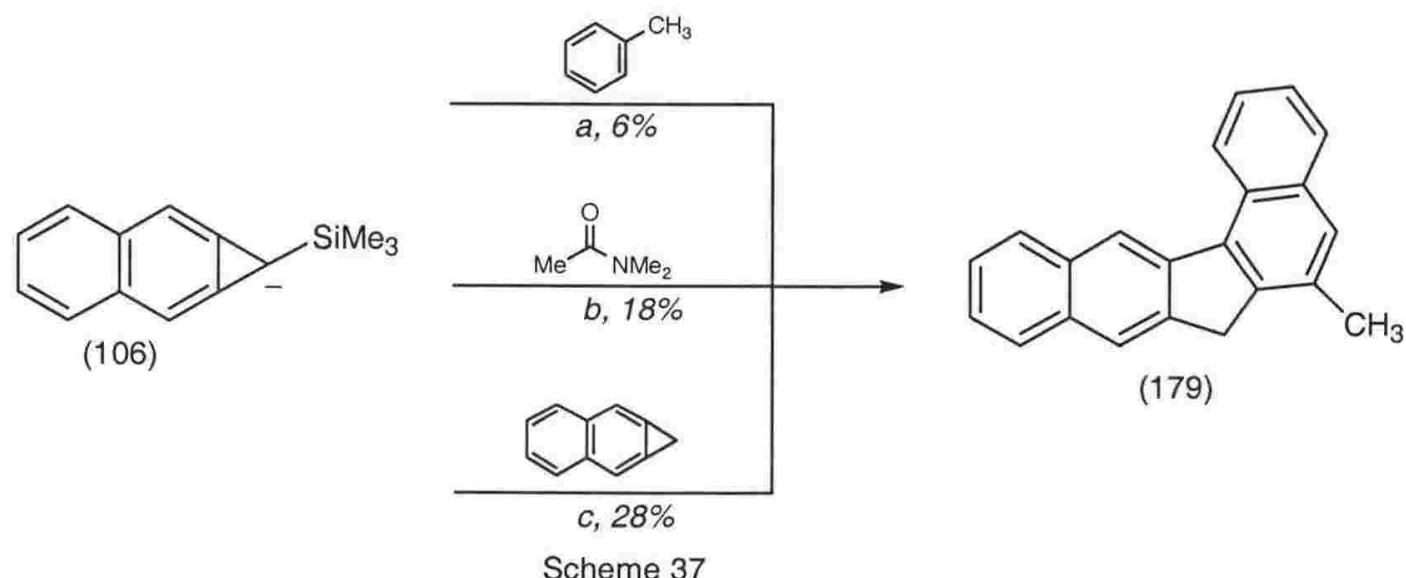

Scheme 37 
In conclusion, the reaction of the $\alpha$-silylcycloproparenyl anion (106) with methyl iodide gives rise to the $\mathrm{C} 1$-methylated cycloproparene (113) and the hydrocarbon (58) as a product mixture that proved inseparable by silica gel chromatography. Use of the lipophilic size exclusion gel Sephadex LH-20 allowed for the separation of these compounds and (113) has been fully characterised.

Quenching anion (106) with water gives a mixture of monosilane (104), cyclopropanaphthalene (58) and a product thought to be a 2-substituted naphthalene. Again, use of Sephadex LH-20 size exclusion gel successfully separates cycloproparene (104) from the mixture and an analytical sample of (104) was obtained.

In an alternative approach to silane (104), anion (106) was treated with toluene but the monosilane product (104) was obtained only in low yield. However, in addition to (104), the novel 6-methyl-7H-dibenzo[b,g]fluorene (179) was isolated in $6 \%$ yield. The structure of this compound was elucidated from extensive n.m.r. investigations and has been confirmed by a single crystal X-ray analysis. Compound (179) is the first dibenzo$[b, g]$ fluorene reported to date and has been the subject of a scientific publication. ${ }^{127}$

Reaction of anion (106) with either $N, N$-dimethylacetamide or cyclopropanaphthalene (58) gives the dibenzofluorene (179) in 18 and $28 \%$ yields, respectively. Although it has not proved possible to provide a rational mechanism for the formation of (179) it seems likely that this compound is formed from the reaction of the $\alpha$-silyl anion (106) with cyclopropanaphthalene (58). The last compound is presumed to have resulted from the reaction of (106) and traces of water. 


\section{Chapter 3}

\section{Approaches to Methylidenecyclopropanaphthalenes}

The novel methylidenecycloproparenes (114) and (116) have attracted considerable attention from both theoretical and experimental chemists since the first derivatives were reported in the literature some seventeen years ago. ${ }^{22-24,98-108}$ In general, these shelf-stable molecules are brightly coloured with measurable polarities and some members of the series are even known to fluoresce in dilute solution with Stokes shifts ${ }^{100,108}$ comparable to that of fluorescein. ${ }^{131}$ Consequently, the compounds have potential application as laser dyes or in the field of non-linear optics. The major route to the alkylidenecycloproparenes is via the Peterson olefination of the $\alpha$-silyl anions (106) and (115) with a carbonyl-containing compound and in excess of eighty derivatives are now available. ${ }^{24,27,28,98-104}$ One drawback of the Peterson olefination procedure is that it is performed under strongly basic conditions. Consequently, the preparation of alkylidenecycloproparenes from anions (106) and (115), and aldehydes or ketones that are base sensitive, is often unsuccessful due to competing enolate formation. ${ }^{100}$

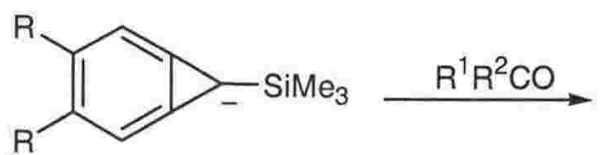

(106) $R R=$ benzo fusion

(115) $\mathrm{R}=\mathrm{H}$

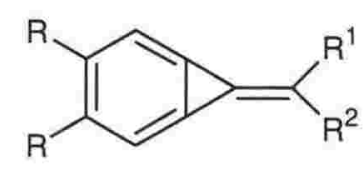

(114) $R R=$ benzo fusion (116) $\mathrm{R}=\mathrm{H}$

A variation of the above method for preparing such methylidenecyclopropanaphthalenes was developed by Stang et al. in 1994 and involves preparation and subsequent interception of $\mathrm{C} 1$ acyl-substituted silanes (112) with a nucleophile. ${ }^{95}$ The last step induces Peterson olefination and nine novel alkylidenecyclopropanaphthalenes (114) have been prepared by this route. Not only has this procedure given some of the simplest alkylidene derivatives reported to date $\left(114 \mathrm{a}, \mathrm{R}^{1}=\mathrm{Me}, \mathrm{R}^{2}=\mathrm{H} ; 114 \mathrm{~b}, \mathrm{R}^{1}=\mathrm{R}^{2}=\mathrm{Me}\right)$, but also it enables unsymmetrical alkylidene derivatives to be prepared. Unfortunately, efforts to replicate the formation of the C1-disubstituted synthon (112) from anion (106) and an acyl halide or cyanide has proved unsuccessful in these laboratories. ${ }^{96,132}$ It is likely that traces of acid contaminate the acyl halide reagent which causes cleavage of the three-membered ring of the cycloproparene precursor. Unfortunately, this has prevented the scope and limitation of this procedure from being assessed fully. 


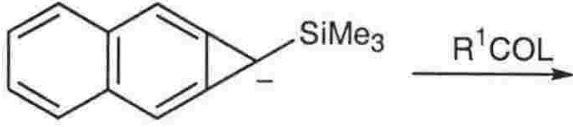

(106)

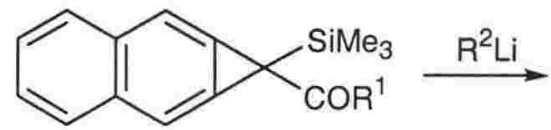

(112)

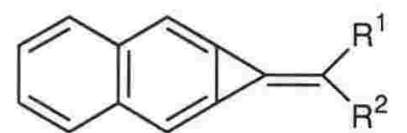

(114)

$\mathrm{L}=$ halide or $\mathrm{CN}$

A standard procedure ${ }^{133}$ for the preparation of 6,6-disubstituted pentafulvenes (171) involves preparation of the cyclopentadienyl anion (183) followed by the addition of a carbonyl compound and subsequent dehydration. However, more recently, Oda and his group $^{125}$ have shown that when intermediate (170) is quenched with cyclopentadiene, a 6,6-disubstituted pentafulvene (171) is produced (Scheme 38). This reaction most likely proceeds by way of the alkoxy moiety of (170) abstracting an acidic methylene proton from cyclopentadiene $\left(\mathrm{p} K_{a} \sim 16\right)^{11}$ to generate the aromatic cyclopentadienyl anion (183) and alcohol (184). Intermediate (184) can then eliminate $N, N$-dimethylamide either directly or stepwise via (185) to give a carbonyl-containing compound and $N, N$-dimethylamide. Interception of this aldehyde or ketone in situ by anion (183) then affords the corresponding exocyclic olefin (171) in a one-pot procedure.

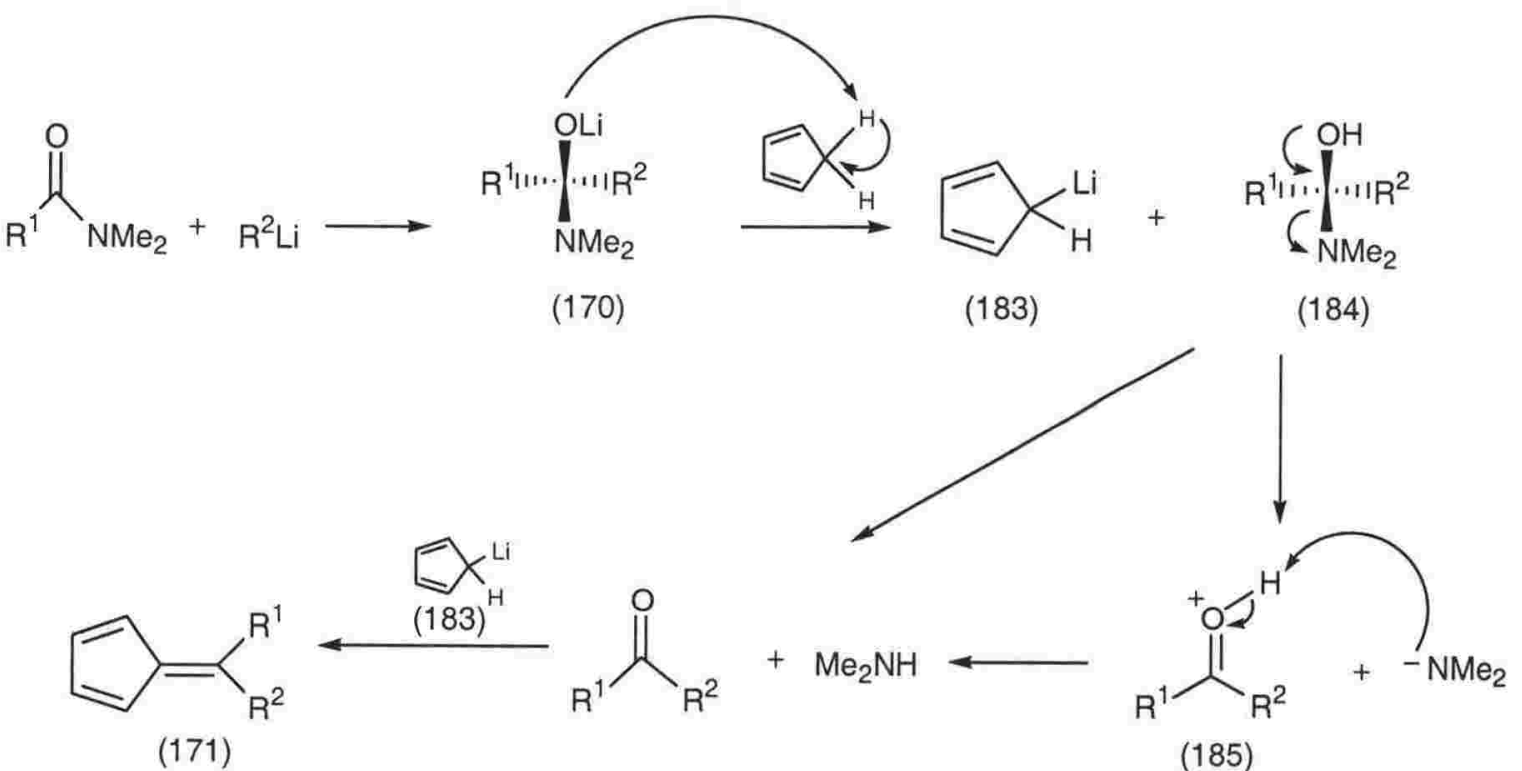

(171)

Scheme 38

The $\mathrm{C} 1$ methylene protons of cyclopropanaphthalene (58) are slightly acidic and have a $\mathrm{p} K_{a}$ of approximately $36^{90}$ and, therefore, they may be sufficiently acidic to be deprotonated by intermediate (170). If so, this would give rise to the cycloproparenyl anion (103) and a carbonyl-containing compound (Scheme 39). The reaction of these last two components could then furnish the corresponding methylidene compound (114). Were this method for synthesising (114) successful, it would provide an alternative means for the preparation of unsymmetrical and possibly otherwise inaccessible derivatives that are not available by other routes. Attempts to prepare such exocyclic olefins (114) by this novel methodology forms the basis of this chapter. 


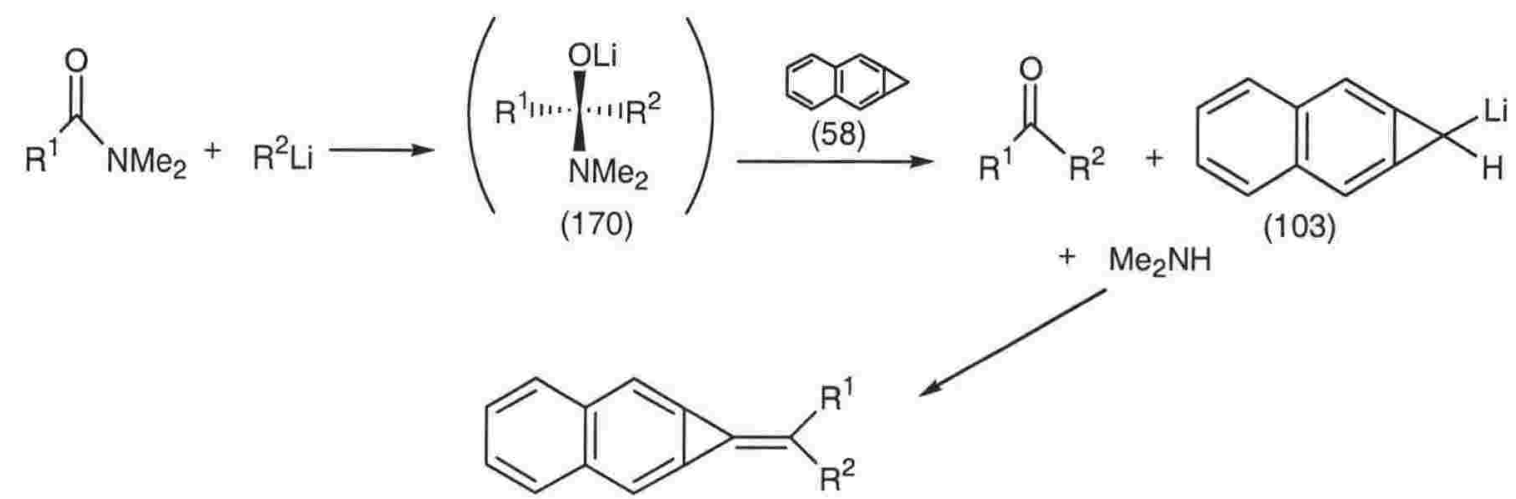

(114)

Scheme 39

Before examining the reaction sequence described above it was decided to prepare the known ${ }^{134}$ diphenylpentafulvene $\left(171 \mathrm{a}, \mathrm{R}^{1}=\mathrm{R}^{2}=\mathrm{Ph}\right)$ in order to gain experience with the Oda procedure. ${ }^{125}$ Thus, intermediate $\left(170 \mathrm{a}, \mathrm{R}^{1}=\mathrm{R}^{2}=\mathrm{Ph}\right)$ was generated from phenyllithium and $N, N$-dimethylbenzamide, and allowed to react with cyclopentadiene. The desired pentafulvene (171a) was isolated in $65 \%$ yield. In light of this success the analogous reaction with cyclopropanaphthalene (58) was examined.

As 1-(diphenylmethylidene)- $1 H$-cyclopropa[b]naphthalene $(1141)^{24}$ is formed in almost quantitative yield from the Peterson olefination of anion (106) with benzophenone this was the first methylidene derivative sought by the Oda procedure. Intermediate (170a) was generated according to the procedure of Oda et al. ${ }^{125}$ but subsequent reaction with hydrocarbon (58) following the literature procedure gave unchanged substrate $(58)(87 \%)$, benzophenone (47\%), and $N, N$-dimethylbenzamide $(17 \%)$. This result implies that the benzylic protons of cycloproparene (58) are insufficiently acidic for abstraction by anion (170a). Protonation of (170a) by water (upon workup) or the ejection of lithium $\mathrm{N}, \mathrm{N}$ dimethylamide from this intermediate would both give rise to benzophenone. Even when (170a) is treated with cyclopropanaphthalene (58) for 7 days at room temperature or for $24 \mathrm{~h}$ at $38^{\circ} \mathrm{C}$ similar outcomes were recorded with hydrocarbon (58) being returned unchanged almost quantitatively; benzophenone and unreacted benzamide were also isolated. More notable, however, is that the reaction at $38^{\circ} \mathrm{C}$ gave a new minor product in addition to these last three compounds. Analysis of the ${ }^{1} \mathrm{H}$ n.m.r. spectrum of this product showed the aromatic region to integrate for sixteen protons but a one-proton singlet at $\delta$ 4.75 was also observed. These data are incompatible with those of alkylidene compound (1141) which have been recorded previously. ${ }^{24}$ However, they are identical to those of 1(diphenylhydroxymethyl)-1H-cyclopropa[b]naphthalene (186) which had been obtained previously by Cutler and Halton ${ }^{93}$ in $27 \%$ yield from the reaction of anion (103) with benzophenone. These authors also isolated the exocyclic olefin (1141) in 17\% yield. This last olefin presumably results from the abstraction of the remaining C1-benzylic proton of 
(188) by unreacted butyllithium to generate anion (187) which furnishes the methylidenecyclopropanaphthalene (1141) (Scheme 40). It would appear, therefore, that either intermediate (170a) or, more likely, unreacted butyllithium deprotonates cyclopropanaphthalene (58) to generate anion (103) and benzophenone. The reaction of these last two entities then affords the alcohol (186) via (188) in $1 \%$ yield. However, at no time was any evidence obtained for the methylidene compound (1141). This suggests that the abstraction of the $\mathrm{C} 1$ benzylic proton from (188) is not facilitated easily by lithiate (170a) and steric factors may be involved.

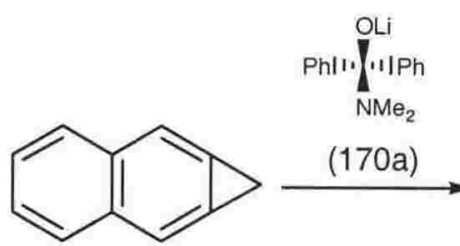

(58)<smiles>[CH2]C1c2cc3ccccc3cc21</smiles>

(103)<smiles>O=C(c1ccccc1)c1ccccc1</smiles>

(188)

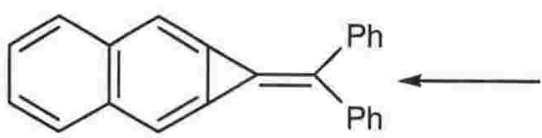

(114l)

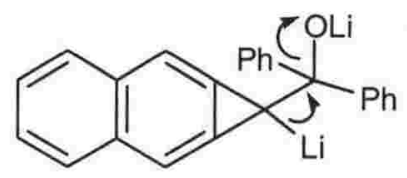

(187)

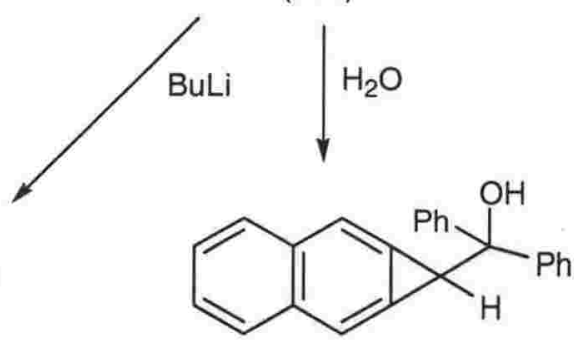

(186)

Scheme 40

In an attempt to improve the outcome of the above reaction it was decided to assess the behaviour of (58) with intermediates other than the diphenyllithiate (170a). With the dimethylated intermediate (170b) unchanged (58) was recovered in $81 \%$ yield. However, in addition to hydrocarbon (58) a minor component was obtained from chromatography. Analysis of this latter compound by ${ }^{1} \mathrm{H}$ n.m.r. spectroscopy revealed that it contained a three-, a one-, and a two-proton singlet at $\delta 1.86,4.52$, and 7.73 , respectively. In addition, an aromatic AA'BB' spin system was observed which integrated for four protons. The relative simplicity of the spectrum implies symmetry with the three-membered ring of the cycloproparene precursor remaining intact. The mass spectrum of the compound gave a molecular ion at $\mathrm{m} / \mathrm{z} 182$ with a base peak at $\mathrm{m} / z 139$ that corresponds to the loss of MeCO. On the basis of these data the compound is tentatively assigned as the 1-acetylcyclopropanaphthalene (189) but it is formed in a mere $2 \%$ yield. Unfortunately, all further attempts to purify this compound resulted in its decomposition.<smiles>CC(=O)C1c2cc3ccccc3cc21</smiles>

(189) 
The formation of (189) can be accounted for if intermediate (170b) abstracts a benzylic $\mathrm{C} 1$ proton from (58) to generate the cycloproparenyl anion (103). The addition of unreacted $N, N$-dimethylacetamide to (103) then gives intermediate (190) which, after ejecting lithium $N, N$-dimethylamide, gives the observed product (189) (Scheme 41). Indeed, generating the cyclopropanaphthalenyl anion (103) from hydrocarbon (58) and butyllithium at low temperature, followed by the addition $N, N$-dimethylacetamide furnishes the hitherto unknown acetylated cyclopropanaphthalene (189) in $26 \%$ yield. The presence of an $\alpha$ hydrogen atom in (189) that is also benzylic augurs well for enolate ion formation. Moreover, capture of enolate at oxygen by appropriate electrophiles would afford the first known examples of alkylidenecycloproparenes bearing an oxygen atom at the $\mathrm{C} 8$ position. Furthermore, electrophilic opening of the three-membered ring may proceed in an atypical fashion to that of other cycloproparenes due to the presence of the electron withdrawing acyl group while upon thermolysis the corresponding naphtho[b]furan may result. The preparation, characterisation, and chemistry of (189) and its derivatives will be discussed in detail in Chapter 4.

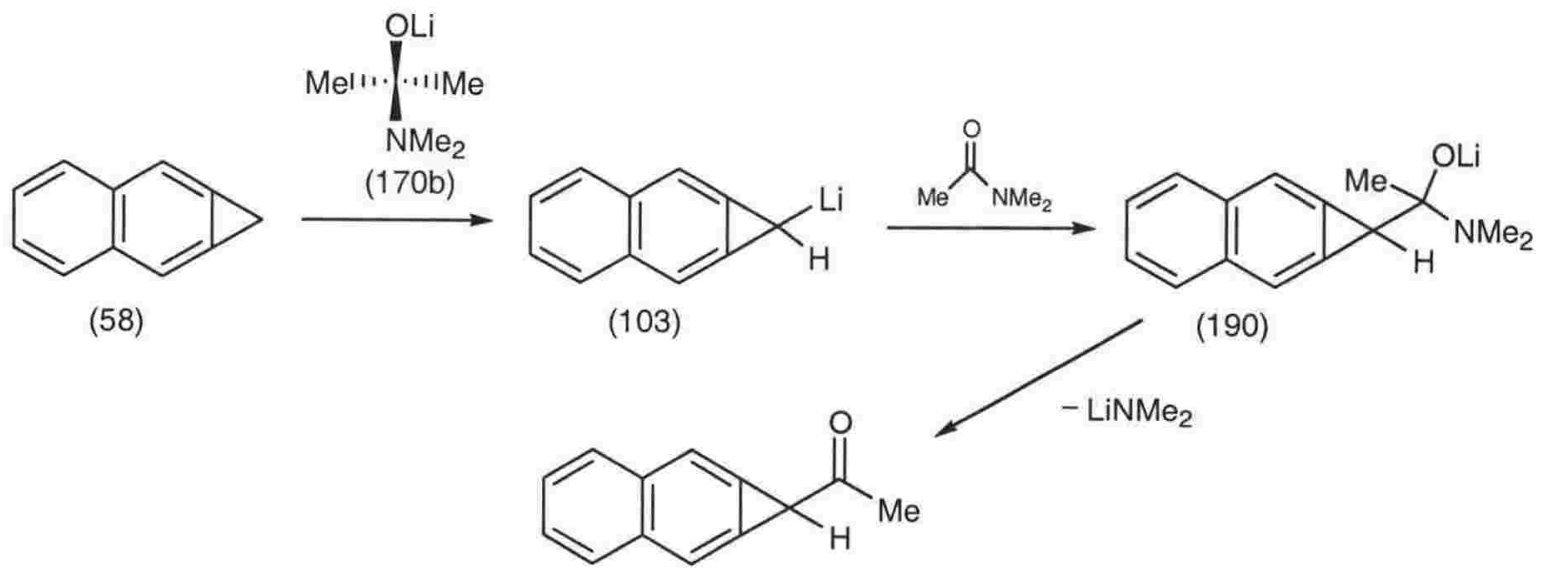

(189)

Scheme 41

The failure to prepare methylidenecyclopropanaphthalenes (114) in situ from anion (170) and cyclopropanaphthalene (58) prompted an examination of the reaction between the lithiate (170) and disilane (105) since alkoxide ions are known to effect desilylation. It was hoped that by treating amide with organolithium for approximately half of the published $^{125}$ time used to generate intermediate (170) the reaction would only go to $~ 50 \%$ completion; a 1:1:1 mixture of the intermediate (170), unchanged amide, and unchanged organolithium should result. Oxyanion (170) may then cleave the C-Si bond of (105) to generate the $\alpha$-silyl anion (106) and the siloxy compound (191) (Scheme 42). Any unreacted amide could then acylate (106) in an addition-elimination sequence, and give rise to the $\mathrm{C} 1$-disubstituted cycloproparene (112). In turn, (112) could be intercepted by 
unreacted organolithium to give the tetrahedral intermediate (192) which could undergo Peterson olefination to afford the exocyclic olefin (114).

$\mathrm{R}^{1} \mathrm{O}_{\mathrm{NMe}_{2}}+\mathrm{R}^{2} \mathrm{Li} \longrightarrow \mathrm{R}^{1} \ldots \ldots \int_{\mathrm{NMe}_{2}}^{\mathrm{OLi}} \cdot \cdots R^{2}$ (170)

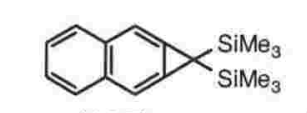

(105)

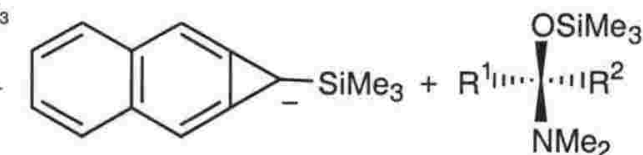

(106)

(191)

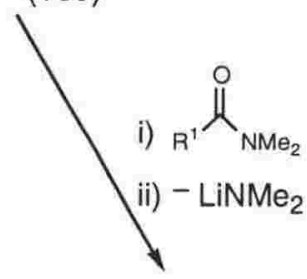

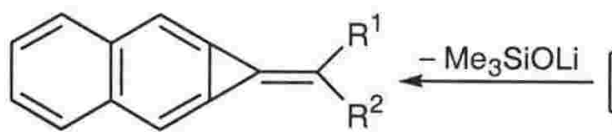

(114)

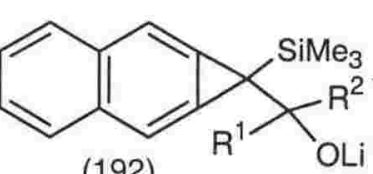

(192)

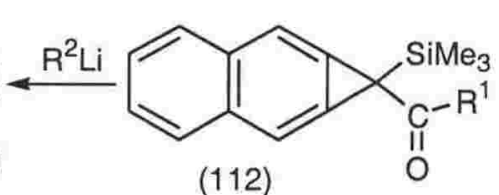

(112)

Scheme 42

The diphenylamide salt $\left(170 \mathrm{a}, \mathrm{R}^{1}=\mathrm{R}^{2}=\mathrm{Ph}\right)$ was generated over $15 \mathrm{~min}$ and allowed to react with disilane (105). Unfortunately, unchanged cycloproparene (105) was recovered almost quantitatively (93\%); benzophenone (76\%) and unchanged amide (22\%) were also isolated. However, in addition to these, the sought after diphenylmethylidene derivative (114l, $\mathrm{R}^{1}=\mathrm{R}^{2}=\mathrm{Ph}$ ) was isolated in $3 \%$ yield. The success prompted efforts to optimise the process, and when the reaction was repeated at $39^{\circ} \mathrm{C}$ over $24 \mathrm{~h}$ the yield of compound (1141) had tripled (9\%) and unchanged silane (105) was recovered in $91 \%$ yield. Unfortunately, the low yield of product negates this route to (1141) as viable. Nonetheless, efforts at preparing other methylidenecyclopropanaphthalenes using this procedure were made.

When attempts were made to prepare the dimethylated alkylidene (114b, $\mathrm{R}^{1}=\mathrm{R}^{2}=\mathrm{Me}$ ) from $\left(170 \mathrm{~b}, \mathrm{R}^{1}=\mathrm{R}^{2}=\mathrm{Me}\right)$, methyllithium, and $N, N$-dimethylacetamide (Scheme 43), a mixture of unchanged silane (105) and the monosilylcyclopropanaphthalene (104) was obtained in a 39:12 ratio. This equates to a $46 \%$ recovery of substrate (105) and a $14 \%$ conversion into cycloproparene (104); no evidence for olefin (114b) was obtained. Presumably, $\alpha$-silyl anion (106) and siloxy compound (191b) are formed from cleavage of the C-Si bond of (105) by (170b) and, if the reaction was not strictly anhydrous, protonation of anion (106) would give monosilane (104). Alternatively, intermediate (170b) could eject lithium $N, N$-dimethylamide to give acetone. Deprotonation of acetone ( $\left.\mathrm{p} K_{a} \sim 19\right)$ by anion (106) would also afford silane (104). Although several attempts were made to ensure strictly anhydrous conditions, the outcome of the reaction remained unchanged suggesting that the latter explanation accounts for the formation of (104). 


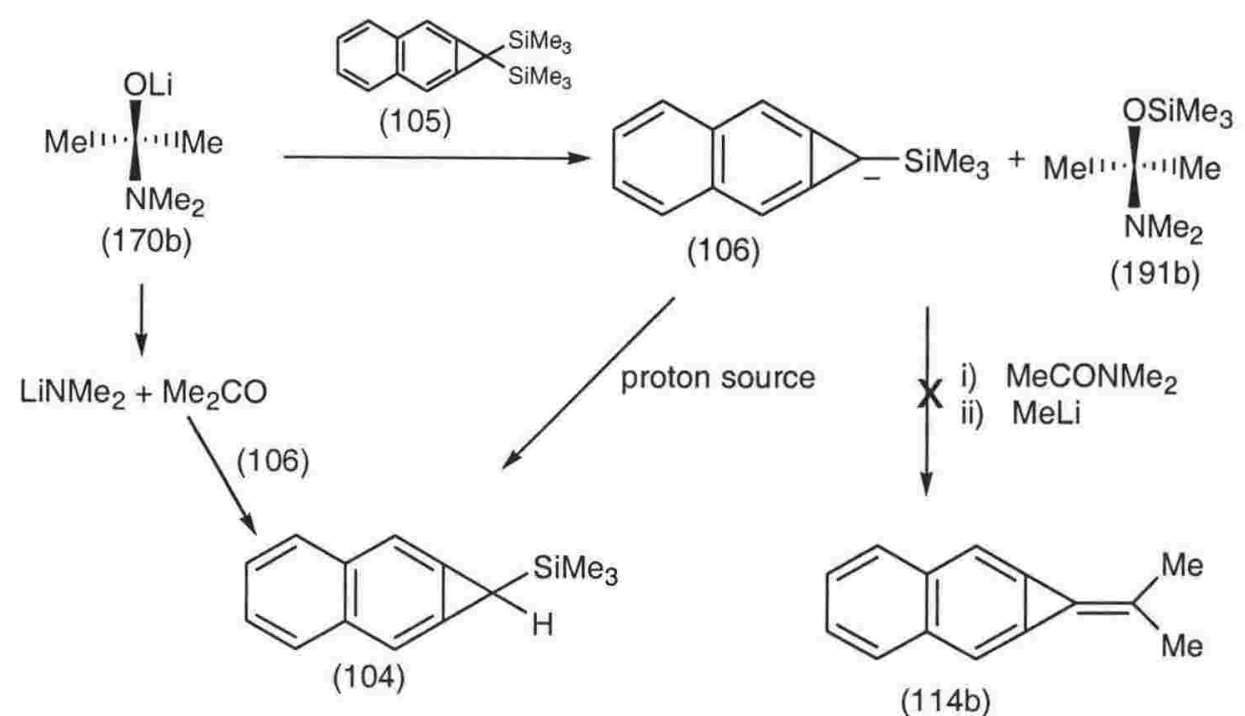

Scheme 43

The preparation of the 2'-propylidene derivative (114b) from disilane (105) using a modified version of Oda's procedure ${ }^{125}$ has not proved possible despite this same methodology affording the diphenyl derivative (1141) in $9 \%$ yield. As we had generated the butylphenyl intermediate (170c) from readily available starting materials it was decided to assess whether the reaction of this intermediate with disilane (105) in the presence of $N, N$-dimethylbenzamide and butyllithium would afford the hitherto unknown 1'-phenylpentylidene (114n) (Scheme 44).

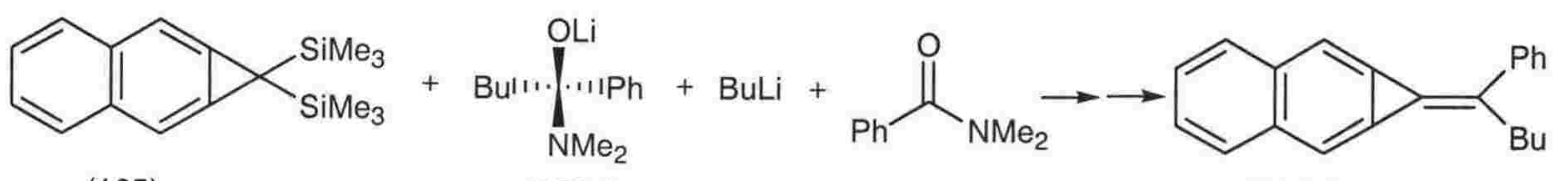

(114n)

Scheme 44

Oxyanion (170c) was prepared from butyllithium and $N, N$-dimethylbenzamide and it was then treated with (105) as described previously. Workup gave unchanged silane (105) (87\%) and a second, brightly coloured, less mobile chromatographic fraction. The ${ }^{1} \mathrm{H}$ n.m.r. spectrum of this material (Fig. 8) showed four aliphatic muliplets between $\delta 0.99$ and 2.95 that integrated in a 3:2:2:2 ratio with multiplicities compatible with those of a butyl group. Although the aromatic region of the spectrum was complex it integrated for eleven protons. Clearly evident was a one-proton para-coupled doublet $(J 1.7 \mathrm{~Hz})$ at $\delta$ 7.58. This suggests that either opening of the three-membered ring to a 2-substituted naphthalene has occurred or that the product of reaction is the desired alkylidenecyclopropanaphthalene (114n) with non-equivalent and para-coupled H2 and H7 protons. A low-resolution mass spectrum of this compound gave a molecular ion at $\mathrm{m} / \mathrm{z} 284$ as required for $\mathrm{C}_{22} \mathrm{H}_{20}$ implying that $(114 \mathrm{n})$ is formed, but only in a $7 \%$ yield. These data alone do not allow for unambiguous assignment of this product as the methylidene 
compound $(114 n)$. Therefore, independent synthesis of $(114 n)$ by Peterson olefination of anion (106) and 1-phenylpentan-1-one (valerophenone) was sought.

The reaction of $\alpha$-silyl anion (106) with valerophenone gave a bright yellow oil $(57 \%)$ with a proton n.m.r. spectrum identical to that of the product previously proposed as the 1'-phenylpentylidene compound $(114 \mathrm{n})$. The ${ }^{13} \mathrm{C}$ n.m.r. spectrum showed four aliphatic and sixteen aromatic carbons and it is clear that the cycloproparenyl framework is not symmetrical. The presence of two shielded carbon resonances at $\delta 106.7$ and 106.8 is consistent with expectation $^{35,100}$ for non-identical C2/7 as required by (114n). An HSQC correlation between the carbon at $\delta 106.8$ and the para-coupled doublet at $\delta 7.58$ and from the carbon at $\delta 106.7$ to the multiplet from $\delta 7.45-7.51$ was recorded. This shows that $\mathrm{H} 2$ and $\mathrm{H} 7$ are indeed non-equivalent but with carbon chemical shifts that differ by only $\sim 0.07 \mathrm{ppm}$. It is notable that the lack of symmetry in the cyclopropanaphthalenyl framework is shown by every carbon atom having a distinct and different chemical shift and, to the author's knowledge, this is the compound for which this effect has been recorded. As the phenylpentylidene compound (114n) was formed in 57\% yield from Peterson olefination protocol and in only $7 \%$ yield using the Oda methodology the preparation of these exocyclic olefins by the latter procedure can be defined as non-viable.

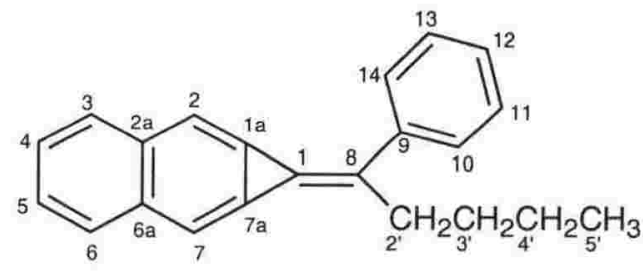

$(114 n)$ 


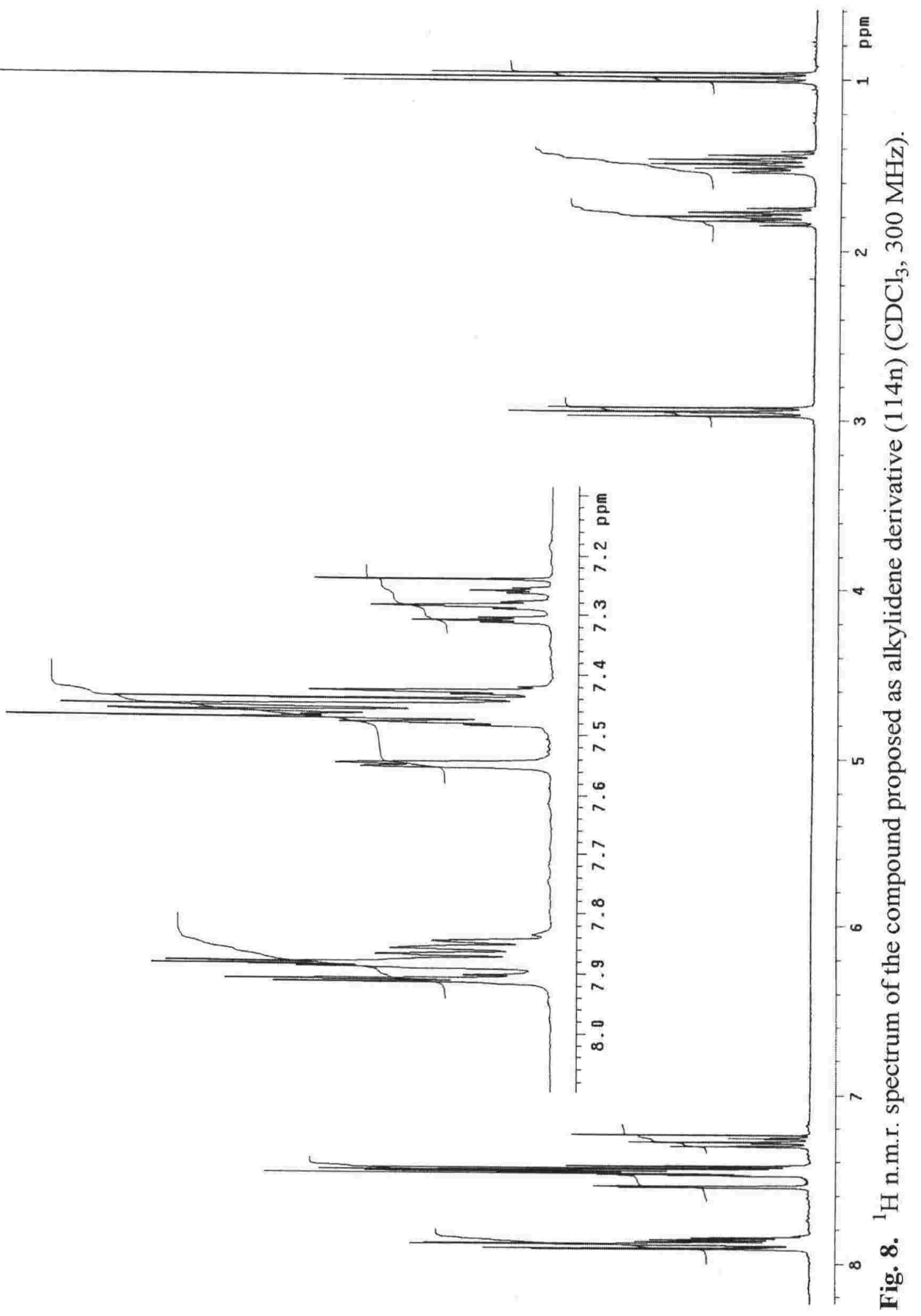


Intermediate (170) (Scheme 39, p. 46) is generated from nucleophilic attack on the carbonyl carbon atom of an $N, N$-dimethylamide by an organolithium and the subsequent treatment of it with cyclopentadiene affords the 6,6-disubstituted pentafulvene (171). One method for generating an organolithium reagent is from metalation of a bromo organic compound with butyllithium at low temperature. As the ketones $p$-bromobenzophenone and $p, p$-dibromobenzophenone were available to us the hitherto unknown brominated cycloproparenes (114o) and (114p) were prepared because treatment of these with butyllithium, $\mathrm{N}, \mathrm{N}$-dimethylamide, and then cyclopentadiene should afford the novel and highly conjugated cycloproparenylpentafulvenes (197) and (198) via organolithium derivatives (193)/(194) and (195)/(196), respectively (Scheme 45).<smiles>Cc1ccc(C(=C2c3cc4ccccc4cc32)c2ccc(I)cc2)cc1</smiles><smiles>[R]c1ccc(C(=C2c3cc4ccccc4cc32)c2ccc(C(C)=C3C=CC=C3)cc2)cc1</smiles>
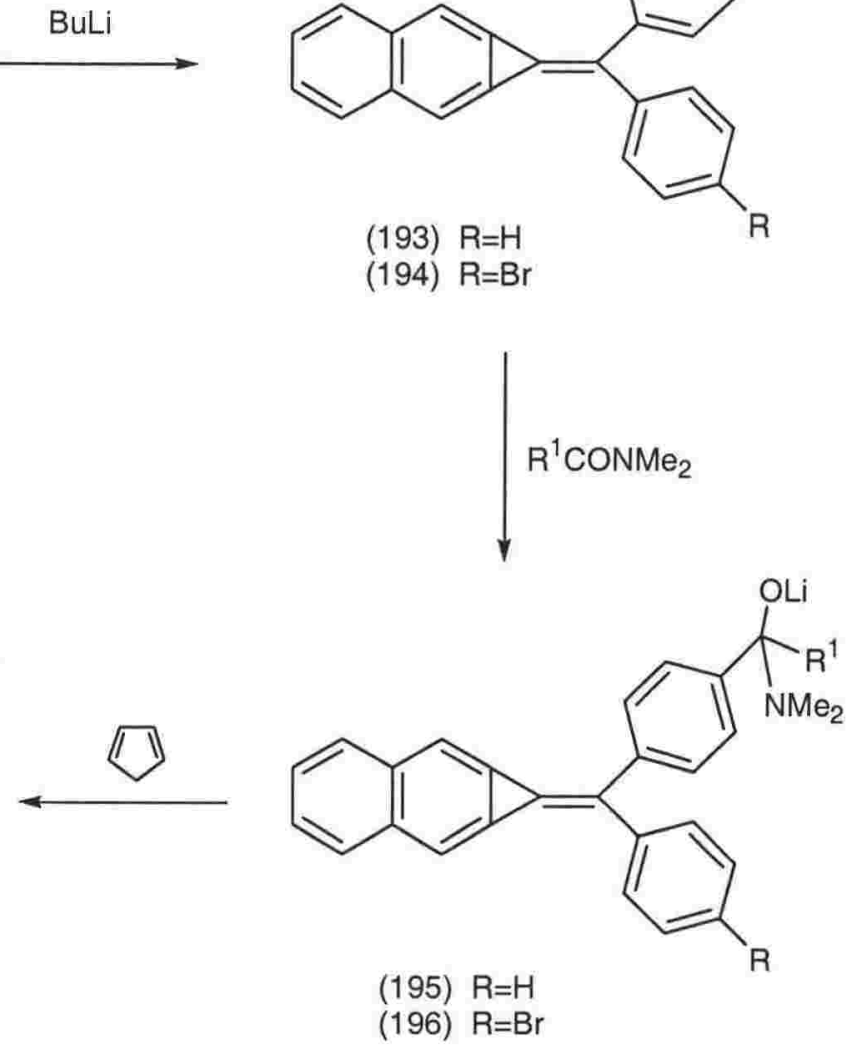

Scheme 45

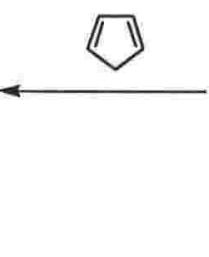

(194) $\mathrm{R}=\mathrm{Br}$

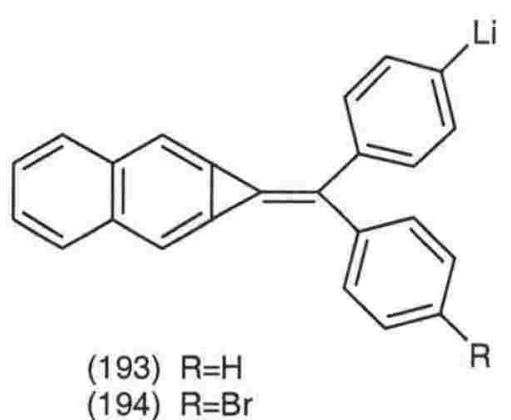

(196) $\mathrm{R}=\mathrm{Br}$

The preparation of monobrominated methylidene compound (1140) was achieved in $90 \%$ yield by Peterson olefination of $p$-bromobenzophenone with $\alpha$-silyl anion (106). The ${ }^{1} \mathrm{H}$ n.m.r. spectrum for this compound is complex but clearly evident are a one-proton apparent triplet of triplets $(J 7.3,1.6 \mathrm{~Hz})$ and a two-proton AA' component of an AA'BB' spin system for $\mathrm{H} 18$ and $\mathrm{H} 3 / 6$, respectively. The ${ }^{13} \mathrm{C}$ n.m.r. spectrum of (114o) contains eighteen signals with the $\mathrm{C} 2 / 7$ shielded as expected ${ }^{35,100}$ and non-equivalent $[\delta 107.4(7)$ and 107.6(0)]. The chemical shifts of $\mathrm{C} 1$ and of all the methine carbons in the cyclopropanaphthalenyl ring are more deshielded by $0.2-0.3 \mathrm{ppm}$ than the analogous carbons of the unsubstituted diphenylmethylidene compound (1141). This suggests that the influence of 
the remote bromine substituent is to withdraw electron density from the cycloproparenyl framework from traditional mesomeric electron donation and inductive electron withdrawal. Indeed, extensive studies have shown that an excellent correlation exists between the chemical shift of each carbon atom of the cycloproparenyl framework as well as the exocyclic olefin centres and the Hammett constant $\sigma_{p}{ }^{+}$for various alkylidenecycloproparenes which bear para-substituted pendant phenyl groups at $\mathrm{C} 8 .^{100,101,104}$ The mass spectrum of (1140) contains a molecular ion cluster at $\mathrm{m} / \mathrm{z} 384\left(\mathrm{C}_{24} \mathrm{H}_{15}{ }^{81} \mathrm{Br}\right)$ and $\mathrm{m} / \mathrm{z} 382$ $\left(\mathrm{C}_{24} \mathrm{H}_{15}{ }^{79} \mathrm{Br}\right)$ in the expected $1: 1$ ratio for a monobrominated compound. In the electronic absorption spectrum the absorption maximum for this brightly coloured polar compound is at $438 \mathrm{~nm}$ in hexane, but on running the UV spectrum in acetonitrile a small hypsochromic shift of $3 \mathrm{~nm}$ was recorded. This behaviour is typical of other polar alkylidenecycloproparenes. $^{35,100}$

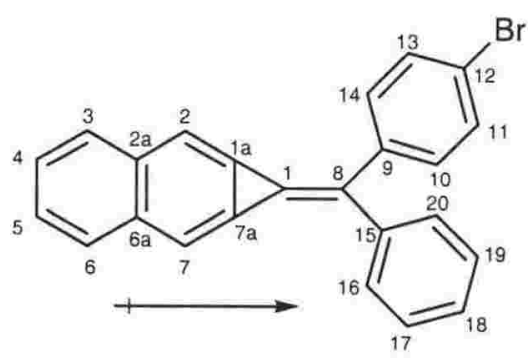

(1140)

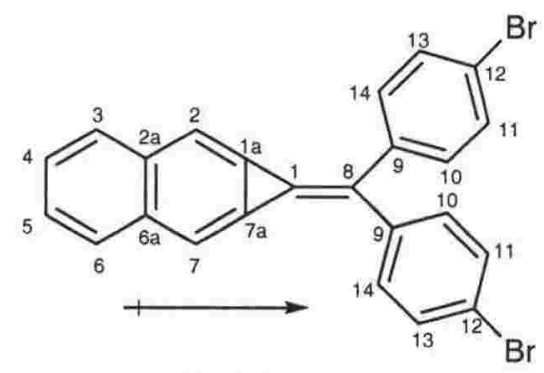

$(114 p)$

In analogy to the reaction discussed above, the dibrominated cycloproparene (114p) was prepared in 94\% yield from 4,4-dibromobenzophenone and the $\alpha$-silyl anion (106). The ${ }^{1} \mathrm{H}$ n.m.r. spectrum of (114p) is simple and contains only an AA'BB' spin system for $\mathrm{H} 3 / 6$ and H4/5 and a ten-proton-containing broad singlet for H2/7, H11/13, and H10/14. The ${ }^{13} \mathrm{C}$ n.m.r. spectrum displays eleven signals with $\mathrm{C} 2 / 7$ shielded as expected ${ }^{35,100}(\delta$ 107.8). Notable, is that the $\mathrm{C} 1$ carbon atom and all of the methine carbons in the cyclopropanaphthalenyl framework are 0.1-0.3 ppm more deshielded than the analogous carbons of the monobrominated derivative (1140) and 0.3-0.6 ppm more deshielded than those of the unsubstituted derivative (1141). Presumably this almost linear incremental increase results from inductive withdrawal of electron density from the cyclopropanaphthalenyl framework by the two bromine atoms polarizing olefin $(114 \mathrm{p})$ to a greater extent than would be the case for the methylidene compound (114o). Indeed, the dipole moment for (114p) has been measured by Dixon and Halton ${ }^{104}$ as $1.5 \mathrm{D}$ which is $0.3 \mathrm{D}$ higher than the measured value of the monobrominated derivative $(114 \mathrm{o})(1.2 \mathrm{D})$, and 1.1 D higher than that the unsubstituted methylidenecyclopropanaphthalene (1141) (0.4 D). ${ }^{24,98}$

With the brominated olefins $(114 \mathrm{o})$ and $(114 \mathrm{p})$ now available their conversion into the $\pi$-extended cycloproparenes (197) and (198) using the method developed by Oda ${ }^{125}$ and co-workers could be examined. It was decided to assess this reaction sequence 
initially with the monohalogenated alkylidene compound (114o). Treating (114o) with butyllithium at low temperature and intercepting the lithiate (193) with $N, N$-dimethylbenzamide (Scheme 45, p. 53) presumably gives rise to intermediate (195a, $\left.\mathrm{R}^{1}=\mathrm{Ph}\right)$. Quenching this mixture with cyclopentadiene gave a complex product mixture which consisted of more than ten components (t.l.c.). Collection of the chromatographic fraction with an $R_{\mathrm{F}}$ of 0.5 (dichloromethane/light petroleum elution; $4: 1$ ) and analysis of the bright red oil by ${ }^{1} \mathrm{H}$ n.m.r. spectroscopy revealed a complex aromatic region which integrated for twenty protons. Although this sample was not pure ( ${ }^{1} \mathrm{H}$ n.m.r. and t.l.c) an isolated twoproton AA' component of an AA'BB' spin system was clearly visible; this suggests that the compound is a cycloproparene. The observation of two one-proton para-coupled doublets at $\delta 7.59$ and $7.63(\mathrm{~J} 1.6 \mathrm{~Hz})$ is consistent with non-identical $\mathrm{H} 2$ and $\mathrm{H} 7$ protons as required for the unsymmetrical methylidene compound $\left(197 \mathrm{a}, \mathrm{R}^{1}=\mathrm{Ph}\right)$. Moreover, in addition to these aromatic resonances three olefinic multiplets that integrated in a 1:1:2 ratio were observed at $\delta 6.32-6.43,6.49-6.51$, and 6.63-6.69, respectively. These last three resonances have comparable chemical shifts to those of the cyclopentadienyl protons of the pentafulvene (171a, $\mathrm{R}^{1}=\mathrm{R}^{2}=\mathrm{Ph}$ ) recorded on the same spectrophotometer and are assigned to $\mathrm{H}^{\prime}, \mathrm{H}^{\prime}$ ', and $\mathrm{H} 2^{\prime} / 3^{\prime}$, respectively. In order to purify further this compound it was resubjected to radial chromatography. Unfortunately, this resulted in its decomposition to a complex mixture of products; it did not prove possible to resolve further this mixture. Additional attempts to prepare (197a) and purify it by crystallisation, sublimation, or by use of Sephadex LH-20 size exclusion gel, all resulted in similar outcomes. The lack of success in isolating compound (197a) prompted efforts towards preparing the monobrominated derivative (198a) in the hope this compound may not prove as difficult to isolate as (197a).

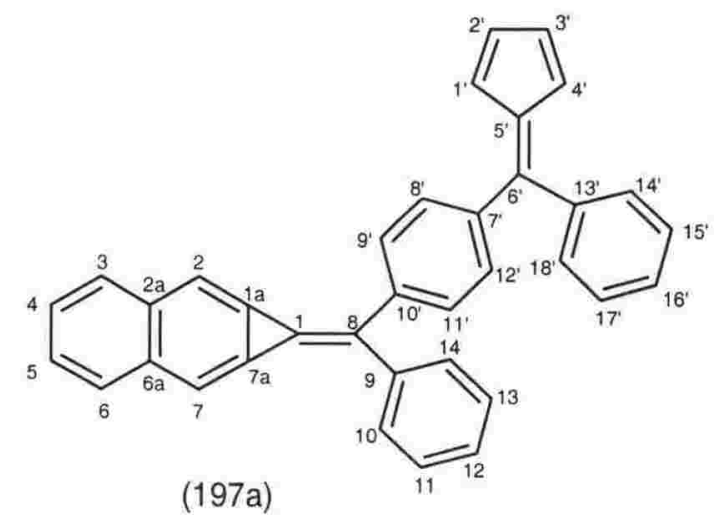

The dibrominated alkylidene compound (114p) was treated with one molar equivalent of butyllithium at low temperature and was then allowed to react with $\mathrm{N}, \mathrm{N}$ dimethylbenzamide. The presumed intermediate $\left(196 \mathrm{a}, \mathrm{R}^{1}=\mathrm{Ph}\right)$ was then quenched with cyclopentadiene. Workup gave a bright orange oil which contained over ten components 
(t.l.c.). However, collection of the chromatographic fraction with an $R_{\mathrm{F}}$ of 0.5 (dichloromethane/light petroleum elution; 4:1) gave a bright orange oil which contained $\left({ }^{1} \mathrm{H}\right.$ n.m.r.) three olefinic resonances at $\delta 6.32-6.36,6.48-6.52$, and $6.63-6.70$ in a $1: 1: 2$ ratio. The aromatic region of the ${ }^{1} \mathrm{H}$ n.m.r. spectrum contained a four-proton AA'BB' spin system and a two-proton singlet; this suggests that this product is symmetrical and that the cyclopropanaphthalenyl framework is intact. However, it is notable that the ratio of the aromatic protons to the olefinic resonances is $24: 8$ and not the $19: 4$ required for (198a, $\mathrm{R}^{1}=\mathrm{Ph}$ ). Based on these data the compound is proposed as the bis-6,6-diphenylpentafulvene-containing cyclopropanaphthalene (199). Indeed, the high-resolution mass spectrum shows an $[\mathrm{M}+1]^{+}$ion at $\mathrm{m} / \mathrm{z}, 609.2679$ as required by the molecular mass $\mathrm{C}_{48} \mathrm{H}_{32}$ of (199). Although attempts to purify further this compound resulted in much decomposition, an analytical sample ultimately was obtained in $1 \%$ yield. The ${ }^{13} \mathrm{C}$ n.m.r. spectrum for (199) is fully consistent with its symmetry and C2/7 met with expectation and are shielded $(\delta 107.8)$. Notable is that $\mathrm{C} 1$ carbon atom and the $\mathrm{C} 2-\mathrm{C} 7$ carbon atoms in the cyclopropanaphthalenyl framework are 0.1-1.1 ppm more deshielded than the analogous carbons of the unsubstituted diphenyl derivative (1141) which implies that these centres contain less electron density than those of (1141). It seems likely that the cyclopentadienyl moieties withdraw electron density from the cyclopropanaphthalenyl framework. Although it was hoped to obtain a single crystal X-ray analysis of this compound it has not proved possible to grow a suitable crystal.

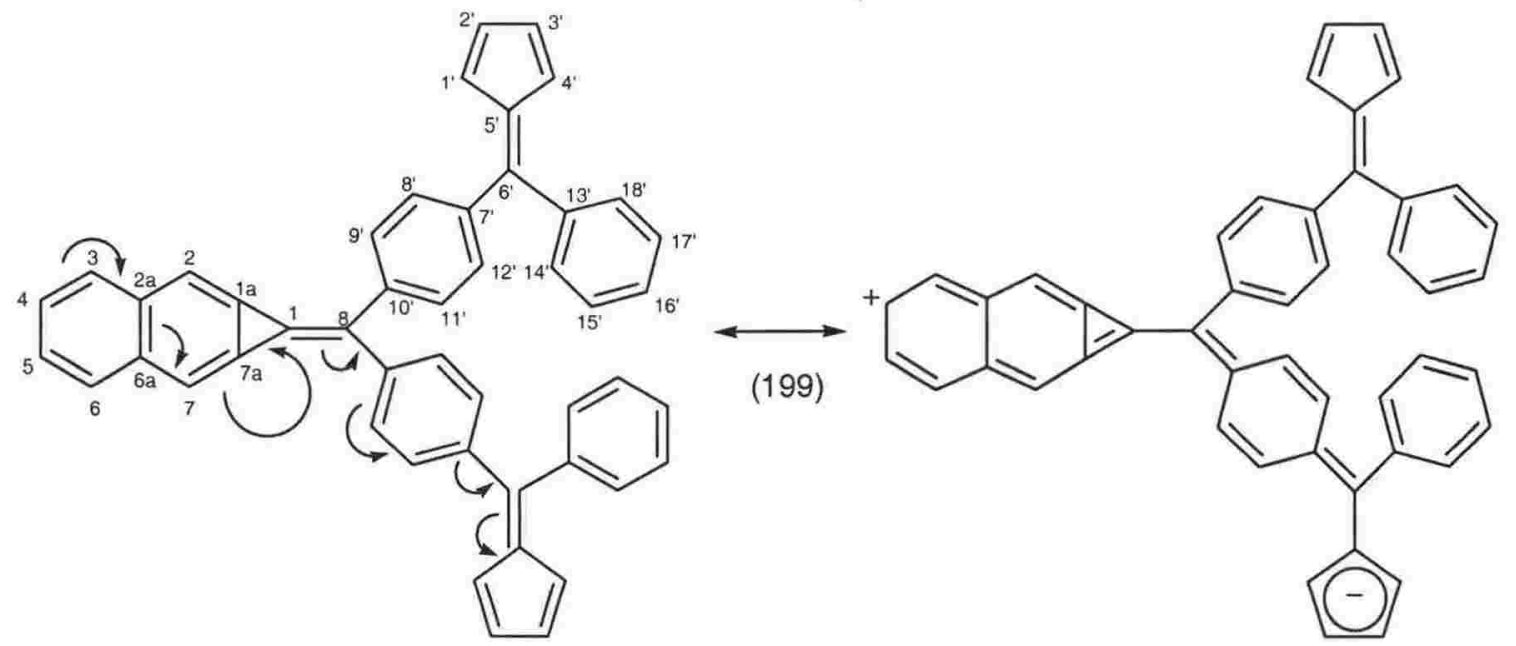

Due to the failure to isolate the pure mono-(6,6-diphenyl)methylidene-containing compound (197a), it was decided to explore its synthesis by interception of the hitherto unknown ketone (200) with cyclopentadiene. The by-products from this reaction are expected to be fewer than those from the Oda methodology and thus it may prove possible to isolate and characterise (197a). Synthon (200) should become available from the reaction of intermediate (195a) with water (Scheme 46). 


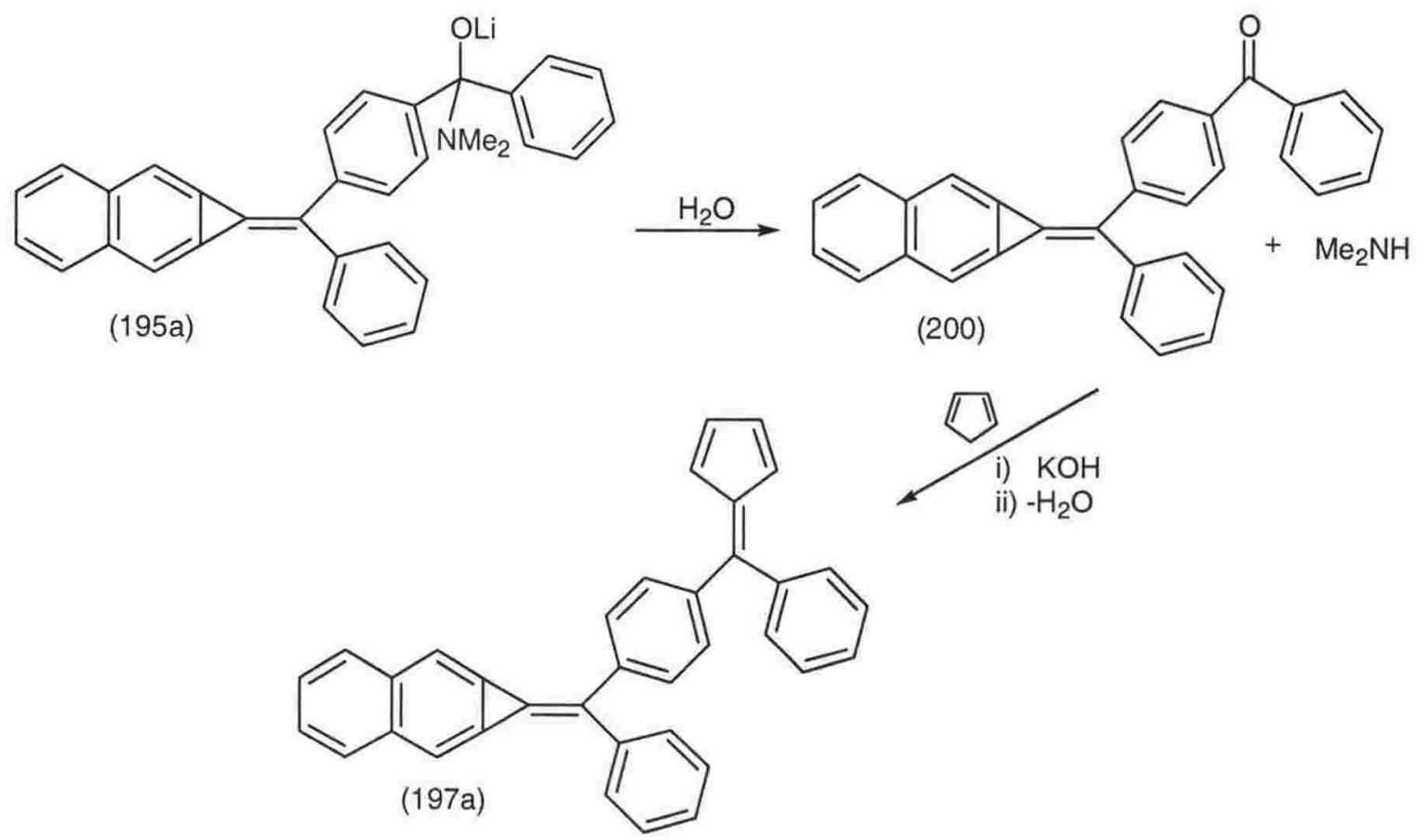

Scheme 46

With a view to preparing the carbonyl-containing methylidenecycloproparene (200), the lithiated anion (193a) (see Scheme 45, p. 53) was generated from the monobrominated methylidene compound (1140) with butyllithium at low temperature. This lithiate was then allowed to react with $N, N$-dimethylbenzamide but, as this amide is sparingly soluble in the reaction solvent (thf), it was added neat under an argon flow. Quenching anion (195a) with water followed by workup gave, not (200), but the diphenylmethylidene compound (114l) in 40\% yield. Moreover, a second, less mobile, chromatographic fraction was isolated. The proton spectrum for the compound shows six sets of resonances between 6.93 and $7.89 \mathrm{ppm}$ which integrates for fifteen protons. Clearly evident is a two-proton $\mathrm{AA}^{\prime}$ component of an $\mathrm{AA}^{\prime} \mathrm{BB}^{\prime}$ spin system $(\delta$ 7.86-7.89) which suggests that the cycloproparenyl framework has remained intact. However, in addition to these aromatic resonances, a broad one-proton singlet at $\delta 5.02$ is also observed. These data are incompatible with those expected for the benzoyl compound (200). The carbon spectrum of the product contains only seventeen resonances and, of the eight that are quaternary, none are compatible with a carbonyl resonance as expected for (200). However, it is notable that the ${ }^{13} \mathrm{C}$ n.m.r. spectrum contains a deshielded quaternary carbon resonance at $\delta 155.0$ which is typical of an ipso $\mathbf{C O H}$ carbon of a phenol. It suggests that the compound is a phenol and the broad one-proton singlet at $\delta 5.02$ in the proton spectrum supports this. Further evidence that this compound is phenolic comes from the observation of the $\mathrm{O}-\mathrm{H}$ bond stretch at $3401 \mathrm{~cm}^{-1}$ in the IR spectrum. The high-resolution mass spectrum for this compound shows an $[\mathrm{M}-\mathrm{H}]^{+}$ion at $\mathrm{m} / \mathrm{z} 319.1131(\Delta 4.4 \mathrm{ppm})$ for the 
molecular formula $\mathrm{C}_{24} \mathrm{H}_{16} \mathrm{O}$. On the basis of these data this compound is assigned confidently as phenol (114q) and it is formed in a $20 \%$ yield.

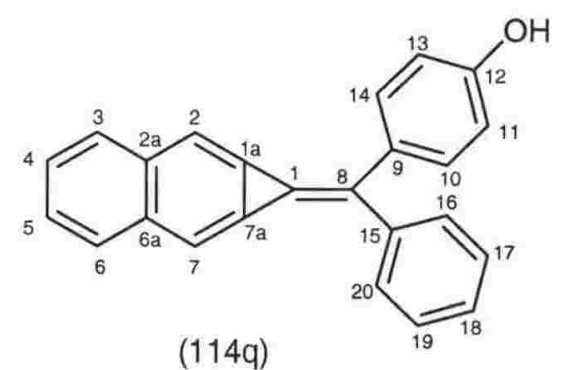

The mode of formation of phenol (114q) from lithiate (193) and $N, N$-dimethylbenzamide is not clear but it seems unlikely that amide is involved. Presumably, interaction with some other species must give rise to (114q). In order to gain insight into how phenol (114q) is produced, anion (193) was generated at $-78^{\circ} \mathrm{C}$ and allowed to react with water after $1 \mathrm{~h}$. Unsubstituted diphenylmethylidenecycloproparene (114l) was obtained in $87 \%$ yield with no evidence for phenol (114q). When anion (193) was warmed slowly to ambient temperature in the absence of electrophile, reaction with water alone gave a small amount of phenol (114q) (4\%) but the major product of reaction was a red oil. The n.m.r. spectra of the oil contains proton and carbon chemical shifts identical to those of the diphenylmethylidenecycloproparene (1141). However, in addition to the signals of (114l), the aromatic region of the ${ }^{1} \mathrm{H}$ n.m.r. spectrum clearly shows two isolated twoproton multiplets and two one-proton para-coupled doublets $(J 2.0 \mathrm{~Hz})$ at $\delta 7.53$ and 7.56 . Also evident is that a new two-proton AA' component of an AA'BB' spin system is superimposible with that of (114l). The data suggest that in addition to (114l) a second methylidenecycloproparene is present. The aliphatic region of the proton spectrum contains four multiplets with resonances and multiplicities compatible with those expected for a butyl group. On this basis the unknown compound is tentatively assigned as the butyl-containing cycloproparene (114r) and it is present to the same extent as (114l). This implies that these two compounds are each formed in a yield of $\sim 45 \%$. Although it did not prove possible to separate (114r) and (114l), a high-resolution mass measurement on this mixture gave an $[\mathrm{M}+1]^{+}$ion for the molecular ion of $(114 \mathrm{r})\left(\mathrm{C}_{28} \mathrm{H}_{24}\right)$ at $\mathrm{m} / z$ z61.1951 ( $\Delta$ $0.1 \mathrm{ppm})$.

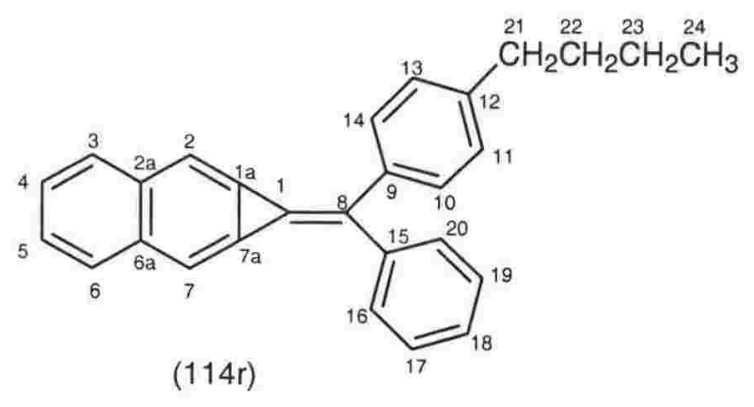


The methylidenecyclopropanaphthalene (114r) most likely results from the lithiate (193) attacking bromobutane in an $\mathrm{S}_{\mathrm{N}} 2$ sense (Scheme 47) while the diphenylmethylidene (1141) can be accounted for by the interception of anion (193) with water upon workup. Phenol (114q) is formed only in low yields from warming anion (193) to room temperature in the absence of electrophile, but in a higher yield by addition of $N, N$-dimethylbenzamide to (193). However, it is unlikely that this amide is involved in the formation of (114q); it is possible that oxygen was inadvertently introduced into the reaction flask upon the addition the amide reagent. In fact, the reactions of organolithium reagents with oxygen are known ${ }^{135}$ to give hydroperoxides which can ultimately afford phenols. Although the mechanism for the formation of alcohols from organolithium reagents is not fully understood $^{135}$ it is believed to involve radicals and likely proceeds by the pathways shown in Scheme 48. ${ }^{135}$ Indeed, aerating lithiate (193) gives the phenol (114q) and the diphenylmethylidene compound (114l) in 20 and $33 \%$ yields, respectively. The methylidene compound (1141) likely results from the reaction of anion (193) with water but it could arise from hydrogen atom capture. If the reactions of anion (193) with oxygen and water are more facile than with $\mathrm{N}, \mathrm{N}$-dimethylbenzamide then this would explain the absence of the benzoylmethylidenecycloproparene (200).<smiles>CC(C)(C)c1ccc2cc3c(cc2c1)C3=C(c1ccccc1)c1ccc(Cl)cc1</smiles>

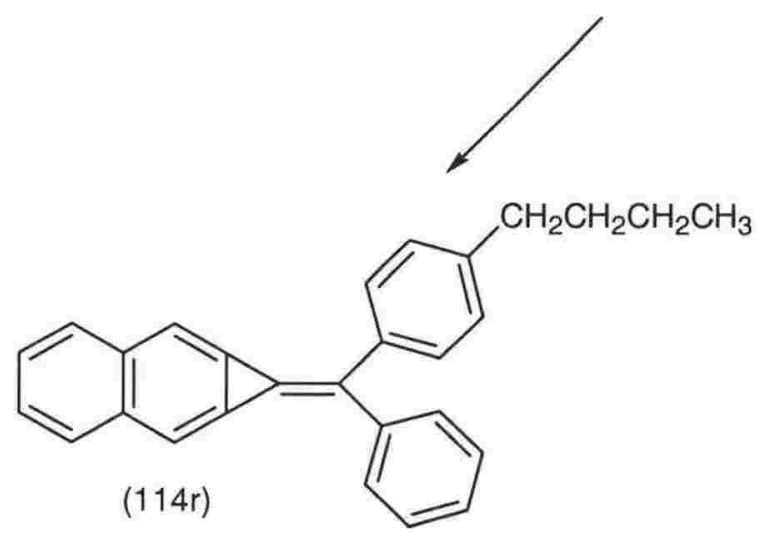

Scheme 47 


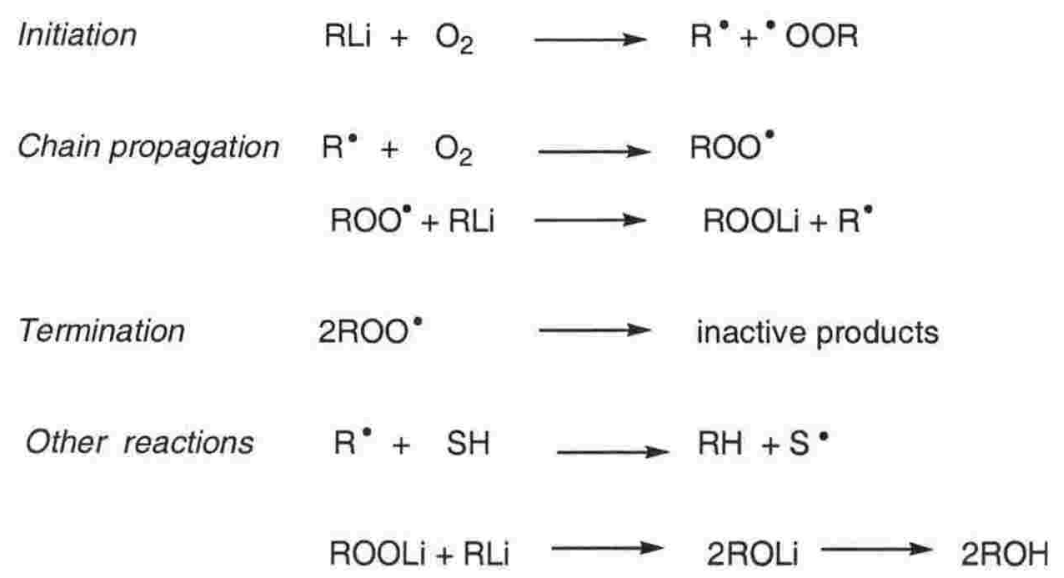

$\mathrm{SH}=$ solvent or other proton source

Scheme 48

As the failure to obtain the benzoyl derivative (200) from lithiate (193) and $N, N$ dimethylbenzamide was attributed to interaction of (193) with molecular oxygen, more stringent conditions to exclude oxygen were made (Experimental). In the event, a small quantity (12\%) of the diphenylmethylidene compound (114l) was isolated but the major product of reaction now became the desired benzoyl derivative (200) in $77 \%$ yield. The n.m.r. data are fully compatible with the assigned structure (Experimental), a conjugated carbonyl stretch is observed in the IR spectrum at $1650 \mathrm{~cm}^{-1}$, and the mass spectrum shows an $[\mathrm{M}+\mathrm{H}]^{+}$ion at $\mathrm{m} / \mathrm{z} 409.1565$ which confirms the molecular formula of $\mathrm{C}_{31} \mathrm{H}_{20} \mathrm{O}$. However, the compound did not give satisfactory microanalytical data with the percentage of carbon nearly $12 \%$ lower than expected. A re-examination of the sample by ${ }^{1} \mathrm{H}$ n.m.r. spectroscopy revealed that it had occulded dichloromethane. The microanalytical data imply that there are eight-and-a-half molecules of dichloromethane for every ten molecules of alkylidene (200). Even after pumping under high vacuum for $48 \mathrm{~h}$ and resubmitting the sample for microanalysis the carbon content remained substantially lower than expected. Subsequently, it was discovered that the compound is hygroscopic and forms 2:1 complex with water. As it did not prove possible to grow a crystal of (200) suitable for an X-ray structure the way in which the solvent is occulded is unknown.

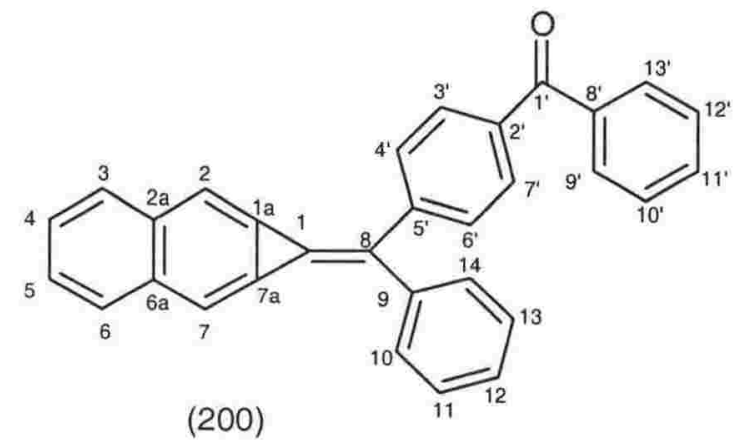


With the ketone (200) now available efforts to prepare the pentafulvene (197a) from the former and cyclopentadienyl anion (183) using the literature methodology were made. $^{136}$ In the event, an analytical sample of (197a) was obtained in $31 \%$ yield. As was the case for the carbonyl precursor (200), pentafulvene (197a) occludes solvent with the ratio of (197a) to hexane as 17:3. The ${ }^{1} \mathrm{H}$ n.m.r. spectrum of (197a) shows the olefinic cyclopentadienyl protons in the usual range ( $\delta 6.32-6.69), \mathrm{H} 3 / 6$ resonate as an $\mathrm{AA}^{\prime}$ multiplet, and $\mathrm{H} 2$ and $\mathrm{H} 7$ as para-coupled doublets ( $\delta 7.59$ and 7.63, $J 1.6 \mathrm{~Hz})$ (Fig. 9). The complex ${ }^{13} \mathrm{C}$ n.m.r. spectrum contains twenty-one resonances between $\delta 107.5$ and 151.8 of which thirteen are quaternary and as usual, C2/7 are shielded (107.5 ppm). As it would be desirable to obtain an X-ray structure of the 6,6-diphenylfulvene (197a) attempts to grow a suitable crystal for analysis were made. Unfortunately, the compound was obtained as a powder and structural analysis was not possible.

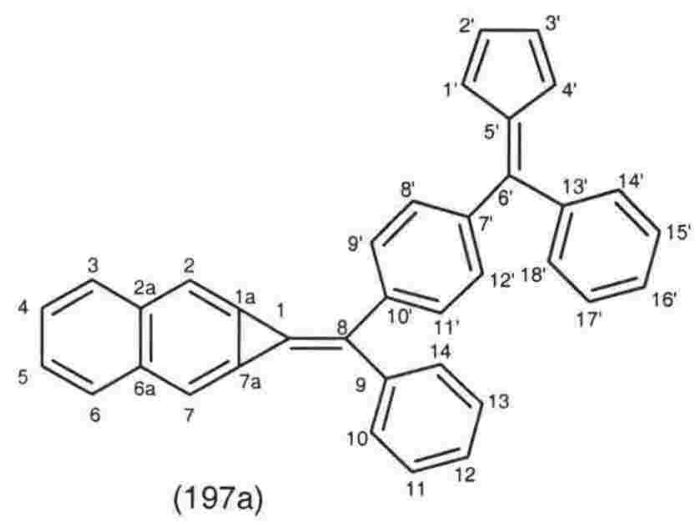

In conclusion, the preparation of alkylidenecycloproparenes (114) from various lithiates (170) and cyclopropanaphthalene (58) using the methodology developed by Oda et $a l .{ }^{125}$ has been examined but no evidence for the desired compounds was obtained despite variations in the reaction conditions. This suggests that the $\mathrm{C} 1$ cyclopropanaphthalenyl protons are insufficiently acidic to be abstracted by lithiate (170).

Reaction of the intermediates (170a) and (170c) with disilane (105) in the presence of amide and (unreacted) organolithium reagent gave the known diphenylmethylidene derivative (1141) and its hitherto unknown analogue (114n) in 9 and $7 \%$ yields, respectively. However, as these olefins are prepared in substantially higher yields (95 and $57 \%$, respectively) using the Peterson olefination protocol this route to methylidenecyclopropanaphthalenes (114) is not useful. 

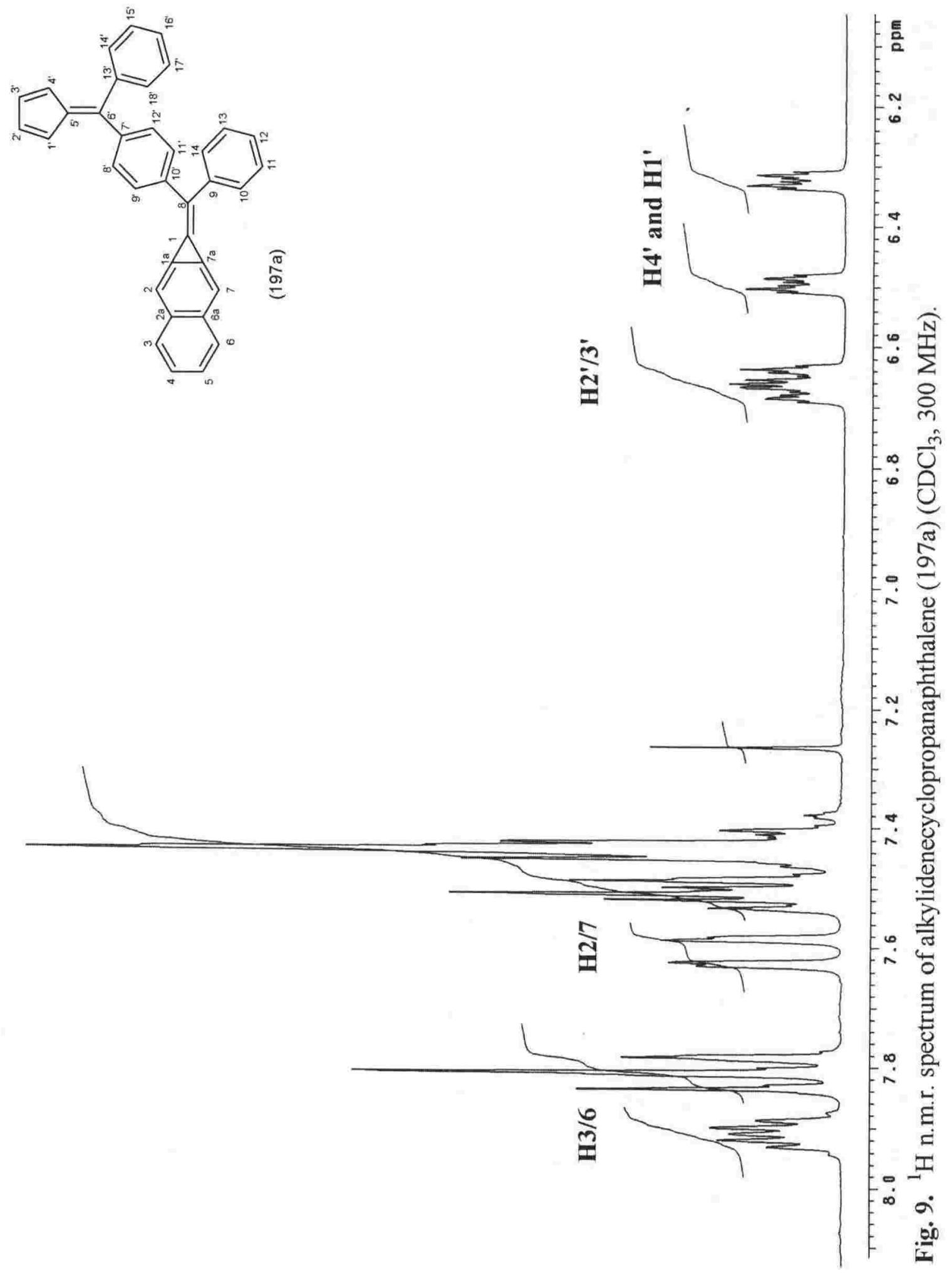
As the preparation of the alkylidenecyclopropanaphthalenes from the Oda procedure $^{125}$ met with only limited success it was decided to prepare the cycloproparenyl lithiates (195) and (196) for reaction with cyclopentadiene. The new brominated synthons (114o) and (114p) were treated with butyllithium, $N, N$-dimethylbenzamide and finally cyclopentadiene. The reactions gave pentafulvene (197a) and the bis-cyclopentadiene derivative (199), respectively. Although it did not prove possible to isolate (197a) prior to its decomposition an analytical sample of (199) was obtained in $1 \%$ yield.

For characterisation purposes the preparation of (197a) from the benzoylmethylidene compound (200) with the cyclopentadienyl anion (183) was attempted. Initial efforts did not give rise to the desired ketone and instead phenol (114q) was isolated. Subsequently, it was found that the reaction of oxygen with lithiate (193) gave phenol (114q) and diphenylmethylidene (1141) in $27 \%$ and $40 \%$ yields, respectively, indicating that the reaction of (193) with oxygen gives rise to phenol.

Ketone (200) was successfully prepared in $77 \%$ yield from $N, N$-dimethylbenzamide and (193) under anaerobic conditions. The addition of the cyclopentadienyl anion (183) to ketone (200) gave the $\pi$-extended compound (197a) in $31 \%$ yield. 


\section{Chapter 4}

\section{1-Acyl-1H-cyclopropa[b]naphthalenes and the Preparation of Cyclopropa $[b]$ naphthaleneylidene Enol Ethers}

To date, few examples of monosubstituted cyclopropa $[b]$ naphthalenes are known. ${ }^{93,97,126}$ Cutler and Halton were able to isolate a few C1-substituted cycloproparenes, albeit in low yields, from treatment of lithiate (103) with methyl iodide, acetone, or benzophenone, (Scheme 21, p. 17), while the interception of anion (103) by p-toluoyl chloride afforded the aryl(dicycloproparenyl)methanol (110). ${ }^{93}$ However, they were unable to prepare C1-acylated cycloproparenes from the interception of anion (103) with acyl chlorides or alkyl benzoates. In the preceding chapter of this thesis the author provided evidence to support the existence of C1-acetylated cyclopropanaphthalene (189) from reaction of hydrocarbon (58) with lithiate (170b). It was proposed that the lithiate deprotonated cycloproparene (58) to generate anion (103) which was then intercepted by unreacted $N, N$-dimethylacetamide to give intermediate (190) (Scheme 41, p. 48). This last compound can, in turn, eject lithium dimethylamide with concomitant formation of ketonecontaining product (189). In this chapter, efforts to prepare unknown C1-acylated cycloproparenes by interception of anion (103) with $N, N$-dimethylamide are discussed. As the former group of compounds contain an $\alpha$ hydrogen atom this augurs well for enolate formation and capture at the oxygen centre should furnish the first examples of cyclopropa $[b]$ naphthalenylidene enol ethers. Furthermore, the thermolysis of acylated cycloproparenes is expected on the basis of earlier studies ${ }^{51,52}$ to afford naphthofurans and, separately upon treatment with mineral acid, 2-substituted naphthalenes may result from atypical opening of the three-membered ring.

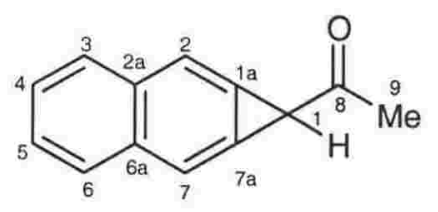

(189)

As evidence for the existence of 1 -acetyl- $1 H$-cyclopropa $[b]$ naphthalene (189) was presented in the preceding chapter this was the first monosubstituted cycloproparene sought. Anion (103) was generated at low temperature as described previously ${ }^{93}$ and then treated with $N, N$-dimethylacetamide. Workup returned a small amount of unchanged (58) $(13 \%)$, in addition to which a second, more polar compound was obtained from chromatography. Analysis of the proton spectrum of this last compound revealed a threeproton singlet at $\delta 1.86$ and a one-proton singlet at $\delta 4.52$. In addition to these resonances, 
a four-proton AA'BB' spin system $(\delta 7.96-7.99$ and 7.53-7.58) and a two-proton singlet $(\delta$ 7.73) were observed in the aromatic region of the spectrum. These data match those of the product proposed as (189) in the previous chapter. The carbon spectrum of this compound contains the eight signals expected for (189) with the methyl and the $\mathrm{C} 1$ methine carbon atoms resonating at $\delta 26.0$ and 40.7, respectively. The carbonyl carbon resonates at $\delta$ 204.0 and the shielded C2/7 carbons appear at $\delta 113.3$. The IR spectrum of (189) shows a carbonyl stretch at $1703 \mathrm{~cm}^{-1}$ and the low-resolution mass spectrum gave a molecular ion at $m / z 182$ as required by the molecular formula $\mathrm{C}_{13} \mathrm{H}_{10} \mathrm{O}$. Although attempts to obtain an analytical sample of the compound were frustrated by its thermal and acid sensitivity (vide infra) ultimately an analytical sample was obtained in $17 \%$ yield (Experimental).

Spurred by the success in isolating the acetylated cycloproparene (189) efforts were made to intercept anion (103) with other readily available $N, N$-dimethylamides. The analogous addition of the $\mathrm{N}, \mathrm{N}$-dimethylbenzamide to (103) should afford 1-benzoyl-1Hcyclopropa[b]naphthalene (202) via the tetrahedral intermediate (201). The reaction gave (202) in a modest $46 \%$ yield and no unchanged (58) was recovered (Scheme 49). The proton spectrum of (202) shows a deshielded H1 proton at $\delta 5.34$ and the aromatic region integrates for eleven protons with the expected four-proton AA'BB' spin system $(\delta 7.94-$ 7.98, 7.53-7.58) and two-proton singlet for $\mathrm{H} 2 / 7$ ( $\delta$ 7.73) clearly evident thereby confirming that the three-membered ring is intact. The carbon spectrum of (202) contains eleven signals five of which are quaternary as required by the symmetry of (202). The benzoyl carbon resonates at $\delta 189.6, \mathrm{C} 2 / 7$ are shielded and resonate at $\delta 109.0$, and the $\mathrm{C} 1$ signal is at $\delta 32.8$. The low resolution mass spectrum of (202) shows a molecular ion at $m / z 244$ for $\mathrm{C}_{18} \mathrm{H}_{12} \mathrm{O}$. In analogy to acetylcycloproparene (189) the 1-benzoyl derivative (202) is both thermally and acid sensitive (vide infra).

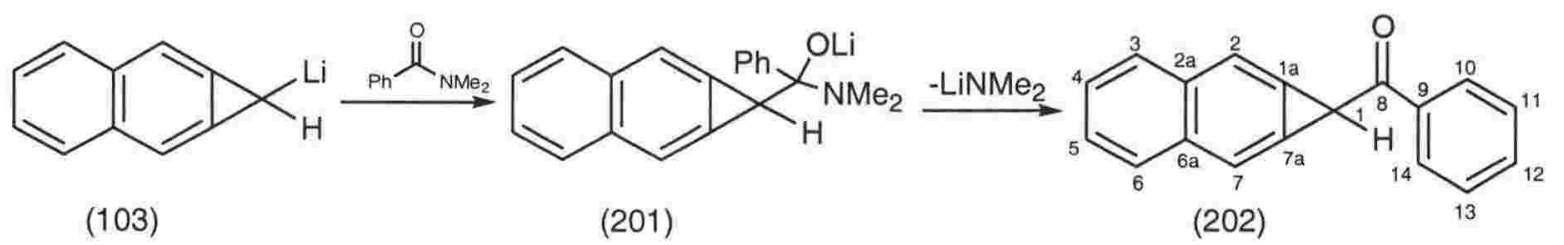

Scheme 49

Several attempts to prepare the C1-formylated cycloproparene (203) from reaction of anion (103) with $N, N$-dimethylformamide were unsuccessful and only complex product mixtures were obtained. However, upon treating (103) with $N, N$-dimethylcarbamoyl chloride $\left(\mathrm{Me}_{2} \mathrm{NCOCl}\right)$, a less mobile chromatographic fraction was collected in $34 \%$ yield, in addition to a $20 \%$ recovery of unchanged (58). The proton spectrum of this component shows a four-proton $\mathrm{AA}^{\prime} \mathrm{BB}^{\prime}$ spin system $(\delta 7.93-7.97,7.49-7.53)$ and a two-proton aromatic singlet $(\delta 7.85)$ which suggests retention of the cycloproparenyl framework (Fig. 
10). However, the aliphatic region of the spectrum is incompatible with the monosubstituted cycloproparene (204) as it contains two six-proton singlets ( $\delta 2.92$ and 3.67 ) and the one-proton resonance expected for $\mathrm{H} 1$ is absent. The symmetry of the compound and the presence of two six-proton aliphatic singlets are consistent with non-identical $N, N$ dimethyl amido protons and suggests that this product is the C1-disubstituted cycloproparene (205). The non-equivalence of the dimethylamido H9 and H9' protons results from the two methyl groups being in different chemical and magnetic environments due to restricted rotation about the CO-N bond ${ }^{137}$ and this accounts for two distinct $\mathrm{N}$ methyl resonances at $\delta 35.9$ and 38.2 in the carbon spectrum. As expected, C2/7 are shielded $(\delta 112.9)$, the carbonyl stretch of the tertiary amide carbonyl in (205) is observed in the typical region at $1643 \mathrm{~cm}^{-1}$, and the molecular ion at $\mathrm{m} / z 282$ in the low-resolution mass spectrum is that required by $\mathrm{C}_{17} \mathrm{H}_{18} \mathrm{~N}_{2} \mathrm{O}_{2}$.<smiles>O=CC1c2cc3ccccc3cc21</smiles>

(203)<smiles>CNC(=O)C1c2cc3ccccc3cc21</smiles>

(204)

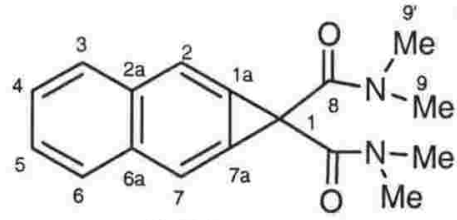

(205)

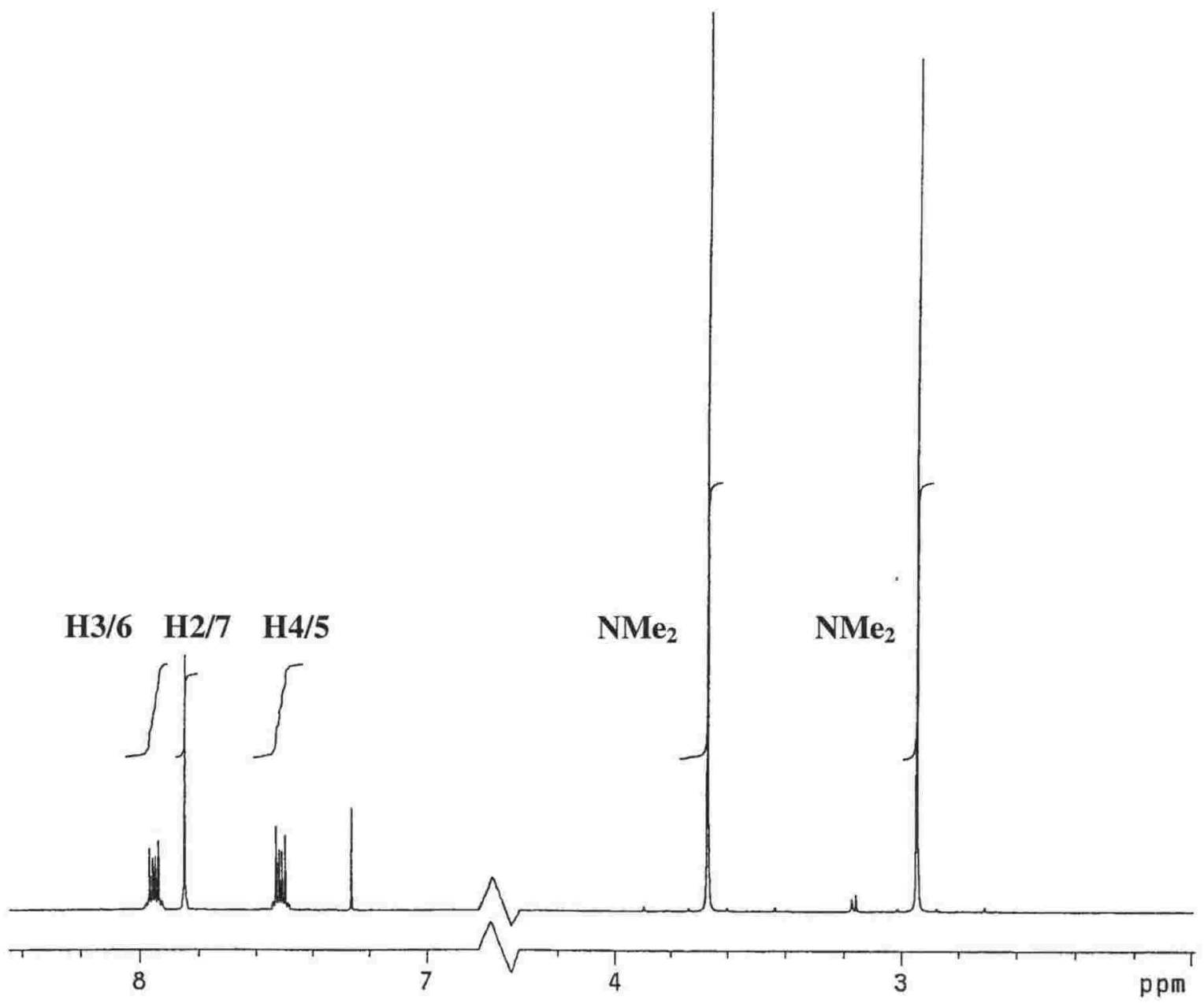

Fig. 10. ${ }^{1} \mathrm{H}$ n.m.r. spectrum of dicarboxamide (205) $\left(\mathrm{CDCl}_{3}, 300 \mathrm{Mz}\right)$. 
The formation of the diamide (205) from (103) with only one molar equivalent of $\mathrm{N}, \mathrm{N}$-dimethylcarbamoyl chloride can be rationalised if unchanged hydrocarbon (58) and butyllithium were present when the amide reagent was added. Any lithiate (103) generated would be intercepted by $\mathrm{N}, \mathrm{N}$-dimethylcarbamoyl chloride to give the monoaddition product (204) via intermediate (206) (Scheme 50). However, as enolate anion (207) is resonance stabilised to a greater extent than lithiate (103), any unreacted base should preferentially abstract the $\mathrm{H} 1$ proton from (204) and generate (207) rather than generate anion (103) from (58). The interception of anion (207) by unreacted amide reagent would then account for the observed disubstituted cycloproparene (205). It seems likely that lithiate (103) deprotonates (204) to give the thermodynamically more stable anion (207) and hydrocarbon (58) (in analogy to the formation of disilane (105) from anion (103) and trimethylsilyl chloride, see Scheme 20, p. 16). Attempts to prepare the monoadduct (204) by varying the proportion of amide between 0.5 and 10 molar equivalents were unsuccessful and only the dicarboxamide (205) and unchanged hydrocarbon (58) were isolated.

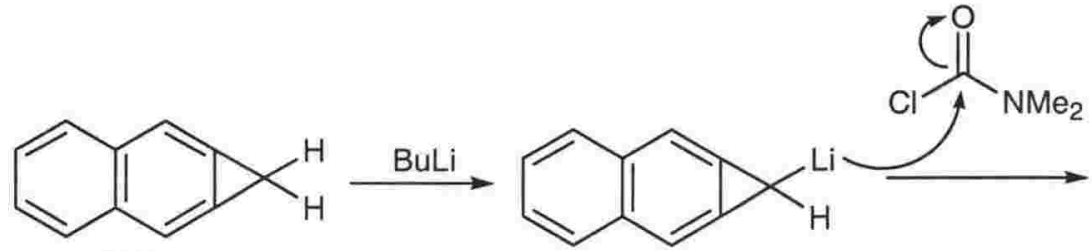

(58)

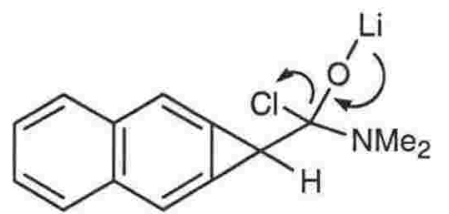

(206)

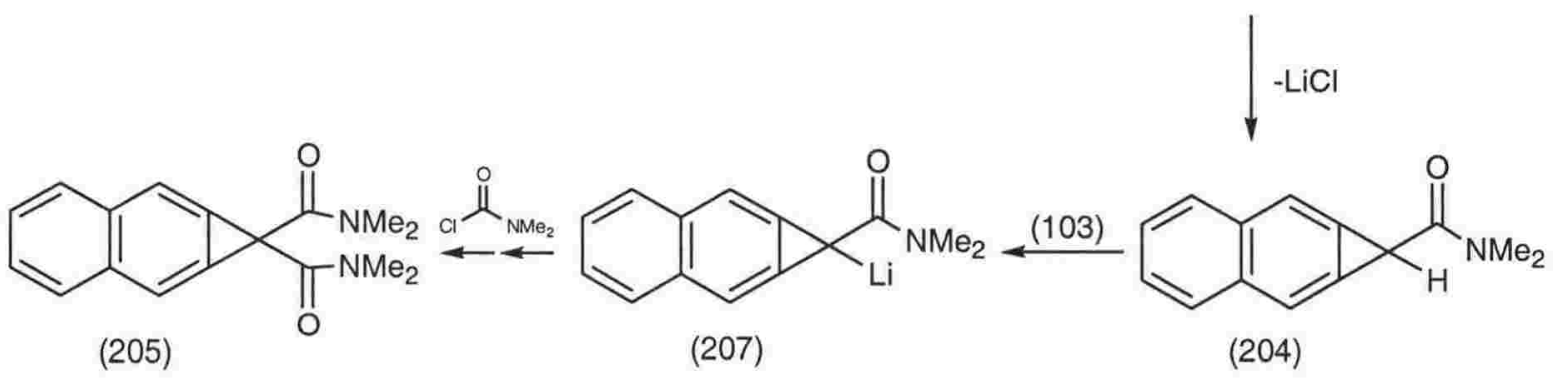

Scheme 50

Spurred by the success of isolating the C1-acylated cycloproparenes (187), (202), and (205), the reactions of the $\alpha$-silyl anion (106) with $N, N$-dimethylbenzamide and $N, N$ dimethylacetamide were examined with a view to obtaining the new C1-disubstituted cycloproparene (112a) and the known acetylated analogue (112b), respectively. Although the latter compound had been prepared by Stang and his group, ${ }^{95}$ attempts to reproduce their work in these laboratories from the reaction of anion (106) with acetyl chloride proved unsuccessful. ${ }^{96,132}$ The $\alpha$-silyl anion (106) was generated from disilane (105) and potassium $t$-butoxide, and treated with $N, N$-dimethylbenzamide. Unfortunately, the only identifiable product of reaction was the 1 -benzoyl- $1 H$-cyclopropa[ $b]$ naphthalene (202) but 
it was obtained in a meagre $6 \%$ yield. This suggests that upon generation (112a) is desilylated by unreacted base to give the enolate (208), protonation of which then affords (202) upon workup (Scheme 51). In similar vein, the reaction of the $\alpha$-silyl anion (106) with $N, N$-dimethylacetamide did not give the desired silane (112b). Instead, cyclopropanaphthalene (58) and the dibenzo[b,g]fluorene (179) were isolated in 20 and $18 \%$ yields, respectively (Scheme 51). While this may suggest that the reaction conditions were not strictly anhydrous, it was not possible to improve upon this outcome despite many attempts.

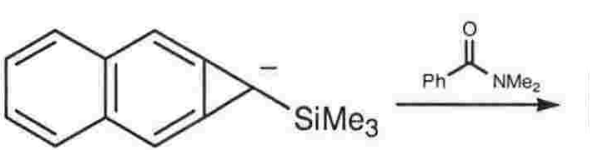

(106)
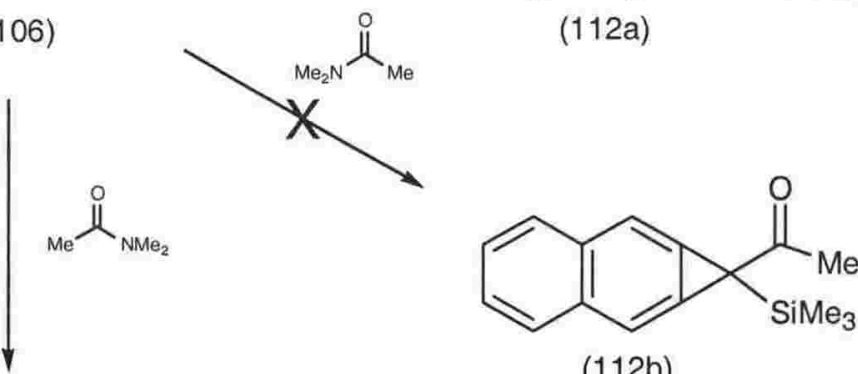

(112b)
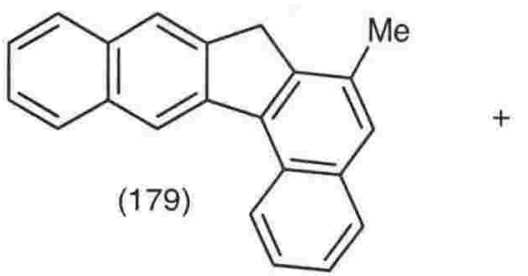

(58)

Scheme 51

Prior to this study the behaviour of cycloproparenes carrying $\mathrm{C} 1$ electron withdrawing groups to electrophiles had not been examined. The reaction of cyclopropabenzene (4) and its alkyl substituted derivatives with mineral acid ${ }^{39,42}$ and halogen ${ }^{16,40-42}$ are known to give rise to 1,2-disubstituted benzenes in high yield by a mechanism thought to proceed by capture of electrophile by the $\pi$-electrons at the strained bridge bond (path $a$, Scheme 5, p. 7). ${ }^{41}$ In strict analogy, the reaction of the C1-substituted cyclopropanaphthalenes (189), (202), and (205) with hydrochloric acid should give the 2,3-disubstituted naphthalenes (209a-c). However, as the bridge bonds of these substrates are less nucleophilic than those of the cycloproparenes previously studied (because of the electron withdrawing functionality at $\mathrm{C} 1$ ), it is possible that the mode by which the three-membered ring opens could be different. In particular, proton capture by the carbonyl oxygen to give the oxonium ions (210a-c) could trigger opening of the three-membered ring to give the aryl cations (211a-c) (path a, Scheme 52). Capture of the latter by chloride ion would then afford the 2-chloro-3-[(substituted)methyl]naphthalenes (212a-c). Alternatively, and more 
likely, the 2,3-disubstitued naphthalenes (212a-c) could arise from direct capture of intermediates $(210 \mathrm{a}-\mathrm{c})$ by chloride ion with ring-opening (path $b$, Scheme 52 ).

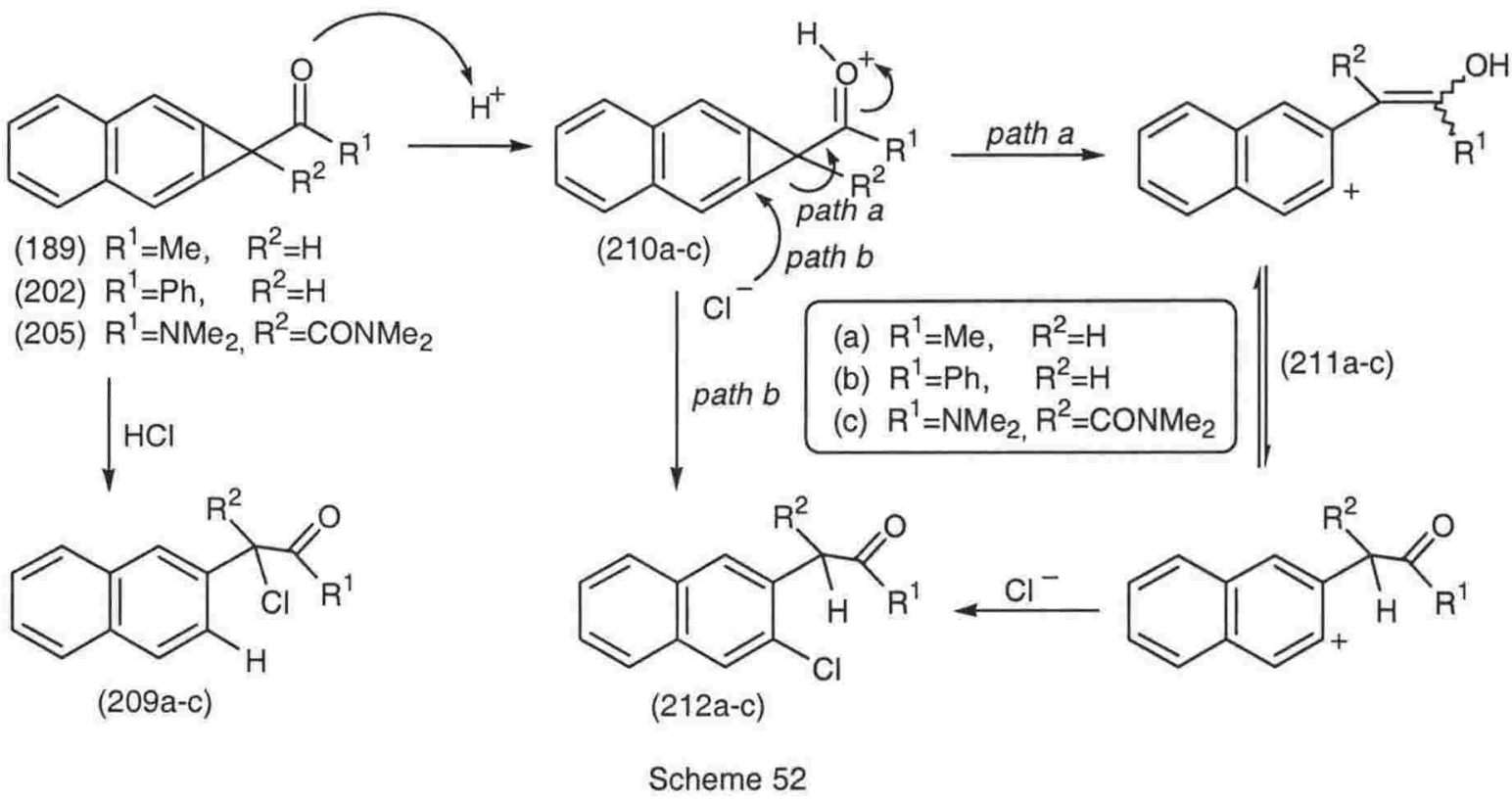

In the event, treatment of 1-acetylcycloproparene (189) with hydrochloric acid gives the previously unknown 1-(3-chloro-2-naphthalenyl)propan-2-one (212a) in 60\% yield. The assignment of structure to this product as (212a) and not the 2-substituted naphthalene (209a) comes from the observation of a deshielded two-proton methylene singlet at $\delta 4.00$ and the observation of six aromatic protons instead of the seven required for (209a). The H1 and H4 protons appear as broad singlets at $\delta 7.70$ and 7.90 , respectively, and the methylene carbon is deshielded and resonates at $\delta 48.6$; the carbonyl carbon is at $\delta 205.3$.<smiles>CC(=O)C(Cl)c1ccc2ccccc2c1</smiles>

(209a)

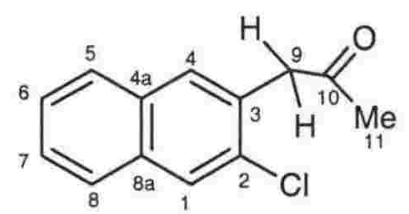

(212a)

In analogy to the reaction discussed above, treatment of 1-benzoylcyclopropanaphthalene (202) and the dicarboxamide derivative (205) with hydrochloric acid affords the new 2-chloro-3[(substituted)methyl]naphthalenes (212b) and (212c) in 79 and $74 \%$ yields, respectively. No evidence was obtained to support the formation of the 2substituted naphthalenes $(209 \mathrm{~b})$ or $(209 \mathrm{c})$. As with the derivative (212a) the proton spectrum of ethanone $(212 \mathrm{~b})$ shows a two-proton methylene singlet $(\delta 4.59)$ and two broad singlets ( $\delta 7.74$ and 7.93$)$ for the aromatic protons $\mathrm{H} 1$ and $\mathrm{H} 4$. The spectral data of $(212 \mathrm{~b})$ are fully compatible with the assigned structure (Experimental) and, as expected, the $\mathrm{NMe}_{2}$ protons ( $\delta 2.94$ and 3.02) and carbons ( $\delta 36.0$ and 37.2 ) have distinct and different chemical shifts as a result of restricted rotation about the $\mathrm{CO}-\mathrm{N}$ bonds. ${ }^{137}$ The preparation 
of these hitherto unknown 2,3-disubstituted naphthalenes (212a-c) is now achieved readily by treatment of the acylated cycloproparenes (189), (202), and (205) with hydrochloric acid thereby demonstrating the synthetic utility of cycloproparenes for preparing new compounds.

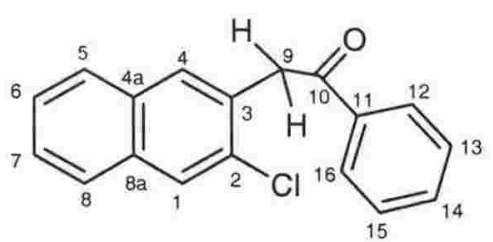

(212b)

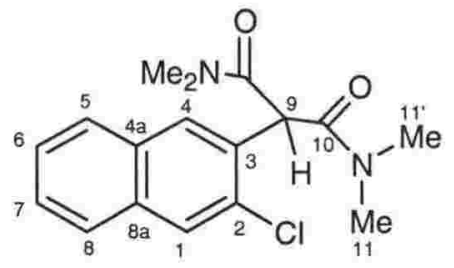

(212c)

A previous study by Dürr and coworkers of the only other cycloproparenes known to carry electron withdrawing substituents at the $\mathrm{C} 1$ centre, namely 1,1-diester substituted cyclopropabenzenes, showed that upon thermolysis and/or photolysis these compounds afforded benzofurans. ${ }^{51,52}$ The reaction most likely involves a diradical intermediate which can ring close to form the observed products, see Scheme 12, p. 10. Therefore, comparable treatment of the C1 acetyl- and benzoylcyclopropanaphthalenes (189), (202), and the C1disubstituted derivative (205) should give the corresponding naphthofurans (214a-c) via diradicals (213a-c) (Scheme 53).

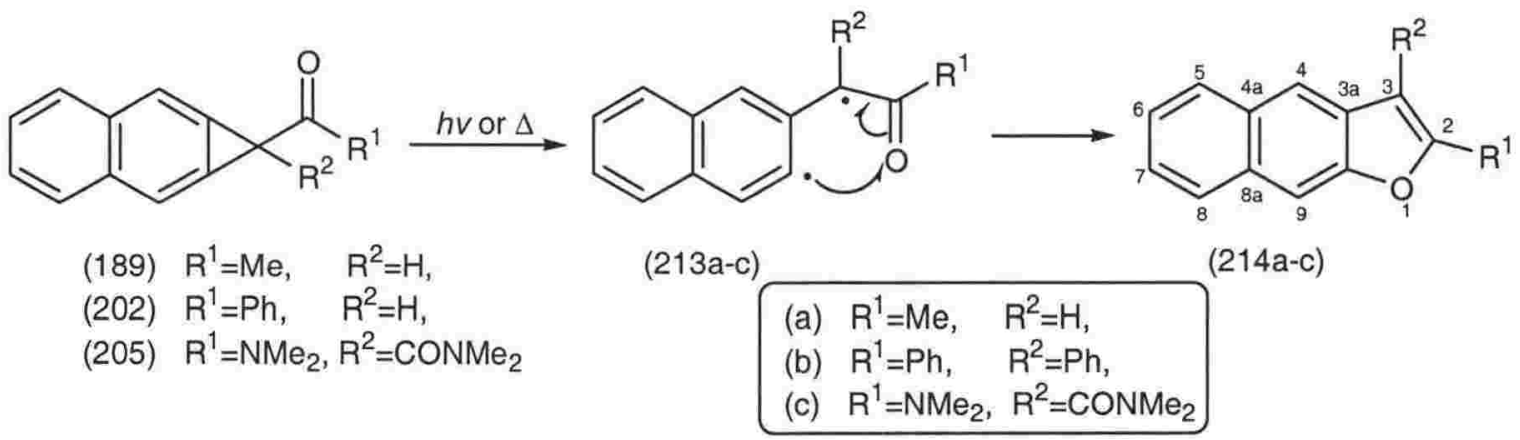

Scheme 53

Upon refluxing dicarboxyamide (205) in benzene the unknown aminofuran (214c) is obtained quantitatively. The ${ }^{1} \mathrm{H}$ n.m.r. spectrum of (212c) shows a two-proton multiplet for the non-equivalent $H 5 / 8$ protons $(\delta 7.79-7.82)$, a two-proton multiplet for $H 6 / 7$ ( $\delta 7.29$ 7.40), two one-proton broad singlets for $\mathrm{H} 4$ and $\mathrm{H} 9$ at $\delta 7.42$ and 7.58 , respectively, and the enamine $\mathrm{NMe}_{2}$ protons resonate as a six-proton singlet at $\delta 3.15$. However, it is notable that the $\mathrm{C} 3-\mathrm{CONMe}$ protons resonate as a broad six-proton singlet rather than the two three-proton singlets expected ${ }^{137}$ from restricted rotation about such CO-N bonds. Moreover, the carbon resonances of the two $\mathrm{CONMe}_{2}$ carbons are not discernible above the baseline of the carbon spectrum and have been located at $\sim 35.2 \mathrm{ppm}$ only from the use of HSQC and gHMBC correlations. These data suggest that at ambient temperature rotation about the $\mathrm{C}(=\mathrm{O}) \mathrm{N}$ bond occurs with the same time scale as that of the n.m.r. measurements. As the carbonyl group is resonance stabilised from $\alpha, \beta$-unsaturation, the 
contribution from the non-bonding electrons of the nitrogen atom is presumed less important, thereby giving the $\mathrm{CO}-\mathrm{NMe}_{2}$ bond less $\pi$ character and a less restricted rotation. Upon re-running the spectra at $-20^{\circ} \mathrm{C}$ rotation is slowed sufficiently that the two $\mathrm{CONMe}$ proton types are resolved as 1:1 singlets at $\delta 3.18$ and 3.19 and the carbon spectrum clearly shows the two $\mathrm{CONMe}_{2}$ carbons at $\delta 35.2$ and 39.3.

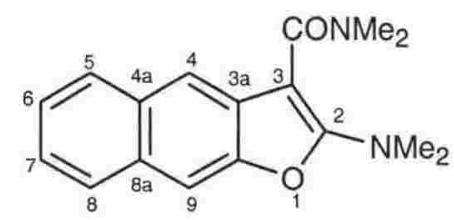

$(214 \mathrm{c})$

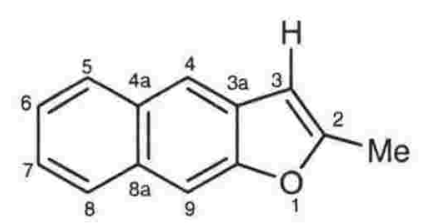

(214a)

In analogy to the thermal rearrangement of the disubstituted cycloproparene (205), the 1-acetylcyclopropanaphthalene (189) gives the known ${ }^{138}$ naphthofuran (214a) in $20 \%$ yield upon reflux in benzene; this equates to an overall yield of $\sim 1 \%$ from naphthalene. While this is very low, it should be noted that only one other synthetic route to furan (214a) occurs with an overall yield of $8 \%$ from 2-methoxynaphthalene. However, this latter route requires the use of expensive reagents, viz. $\mathrm{Pd} / \mathrm{C}$, and so the thermal rearrangement of (189) and other 1-acylated cyclopropanaphthalenes provides an alternative and less expensive method. As the yield of isolated (214a) from the thermolysis of (189) is low it seems likely that the presence of the acyl methyl group $\left(\mathrm{R}^{1}\right)$ in intermediate (213a) provides added scope for alternative radical reactions; it did not prove possible to identify any of the other reaction products.

Upon refluxing the 1-benzoylcyclopropanaphthalene (202) in benzene the hitherto unknown 2-phenylnaphthofuran (214b) was isolated but in a mere 7\% yield. Presumably the low yield of (214b) results because alternative reaction pathways exist including insertion reactions of diradical (213b); a similar outcome results upon irradiating (202) with UV light $(254 \mathrm{~nm})$ whereupon $(214 \mathrm{~b})$ is formed in $5 \%$ yield. However, the thermal decomposition of (202) in the solid state, although violent, gives the naphthofuran (214b) in higher $(17 \%)$ yield suggesting that in solution the diradical (213b) reacts with solvent. The proton spectrum of (214b) is complex but shows a one-proton doublet $(J 1 \mathrm{~Hz})$ at $\delta$ 7.13 and a broad singlet at $\delta 8.04$ in addition to two five-proton multiplets. The carbon spectrum shows ten methine resonances and six quaternary carbons as expected for (214b) and the analytical data establish the molecular formula as $\mathrm{C}_{18} \mathrm{H}_{12} \mathrm{O}$ (Experimental).

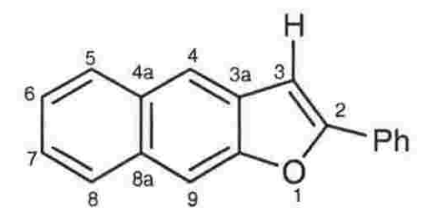

(214b) 
The presence of the $\alpha$ proton adjacent to the carbonyl group in the monosubstituted cycloproparenes (189) and (202) augurs well for the formation of enolates (215) and (216), respectively. These enolates can be expected to be captured to give $\mathrm{C} 1$ disubstituted naphthalenes $\left(217, \mathrm{R}^{1}=\mathrm{Me}\right)$ and $\left(218, \mathrm{R}^{1}=\mathrm{Ph}\right)$ and/or the cycloproparenylidene enol ethers (219, $\left.\mathrm{R}^{1}=\mathrm{Me}\right)$ and $\left(220, \mathrm{R}^{1}=\mathrm{Ph}\right)$. As it is $\mathrm{known}^{94}$ that (trisubstituted)silyl chlorides capture enolates at oxygen in preference to carbon, the treatment of enolates (215) and (216) with these reagents should provide the first examples of alkylidenecycloproparenes which have an ether substituent at C8. Moreover, it could provide a new route for preparing methylidenecycloproparenes in one step from reaction at the benzylic centre of a cycloproparene (Scheme 54).<smiles>O=C(F)C1c2cc3ccccc3cc21</smiles>

(189) $R^{1}=\mathrm{Me}$ (202) $\mathrm{R}^{1}=\mathrm{Ph}$

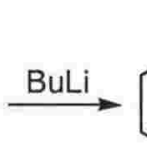<smiles>O=C([Te])C1(P)c2cc3ccccc3cc21</smiles>

(217) $R^{1}=\mathrm{Me}$ (218) $\mathrm{R}^{1}=\mathrm{Ph}$<smiles>CC(O)=C1c2cc3ccccc3cc21</smiles>

(215) $R^{1}=\mathrm{Me}$ (216) $\mathrm{R}^{1}=\mathrm{Ph}$ $\mathrm{R}^{2} \mathrm{Cl}$<smiles>CCC=C1c2cc3ccccc3cc21</smiles>

(219) $R^{1}=M e$ (220) $\mathrm{R}^{1}=\mathrm{Ph}$

\section{Scheme 54}

Treatment of benzoylcycloproparene (202) with butyllithium and subsequent silylation with trimethylsilyl chloride gave an unstable oil that is proposed as the methylidenecyclopropanaphthalene $\left(220 \mathrm{a}, \mathrm{R}^{2}=\mathrm{OSiMe}_{3}\right)$. Unfortunately, this product was 
product is methylidene (220a) and not the C1-disubstituted cyclopropanaphthalene (218a, $\mathrm{R}^{2}=\mathrm{SiMe}_{3}$ ). Unfortunately, attempts to purify further this compound resulted in its decomposition. As trimethylsilyl enol ethers are known ${ }^{141}$ to be highly sensitive to water and acid it seems likely that the compound decomposed upon attempted purification.
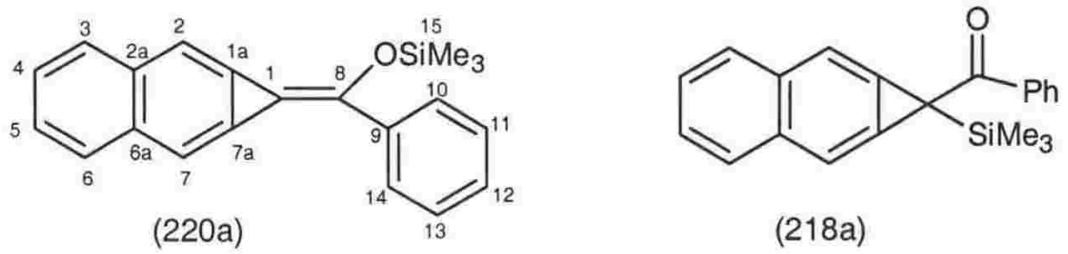

In order to improve the stability of the cycloproparenylidene enol ether the enolate (216) was allowed to react with the more sterically demanding $t$-butyldimethylsilyl chloride with a view to preparing the methylidenecycloproparene (220b). Indeed, a pure sample of (220b) was isolated in 57\% yield as bright yellow plates (Experimental). The proton spectrum of (220b) displays the $\mathrm{OSiMe}_{2}$ and the $\mathrm{OSiBu}^{t}$ resonances at $\delta 0.48$ and 1.19 while a four-proton AA'BB' spin system is observed at $\delta 7.84-7.88$ and 7.57-7.50 thereby providing adequate proof that the cycloproparenyl framework is intact. As expected the $\mathrm{H} 2$ and $\mathrm{H} 7$ protons are non-equivalent and appear as one-proton doublets $(J$ $1.5 \mathrm{~Hz}$ ) at $\delta 7.29$ and 7.40 . The ${ }^{13} \mathrm{C}$ n.m.r. spectrum shows nineteen resonances with all of the carbons of the cycloproparenyl framework having distinct and different chemical shifts as required for the unsymmetrical compound. As usual, C2 and C7 are shielded $(\delta 104.1$ and 104.9) but it is notable that $\mathrm{C} 1(95.9 \mathrm{ppm})$ is $\sim 16 \mathrm{ppm}$ more shielded than $\mathrm{C} 1$ of the diphenylmethylidene $(1141)$ ( $\delta 112.0)$ and the $\mathrm{C} 8$ carbon atom $(135.8 \mathrm{ppm})$ is $\sim 15 \mathrm{ppm}$ more deshielded than of (1141) $(\delta$ 120.8). There can be little doubt that these dramatic changes in the carbon resonances on replacing the C8 phenyl substituent of (1141) with a $t$ butyldimethylsiloxy moiety are caused by the oxygen atom donating electrons towards the cycloproparenyl framework. The electronic absorption spectrum for this brightly coloured compound shows only a small $(2 \mathrm{~nm})$ hypsochromic shift upon changing the solvent from hexane to acetonitrile but there seems little doubt that the cyclopropa $[b]$ naphthalenylidene enol ether (220b) is polar.
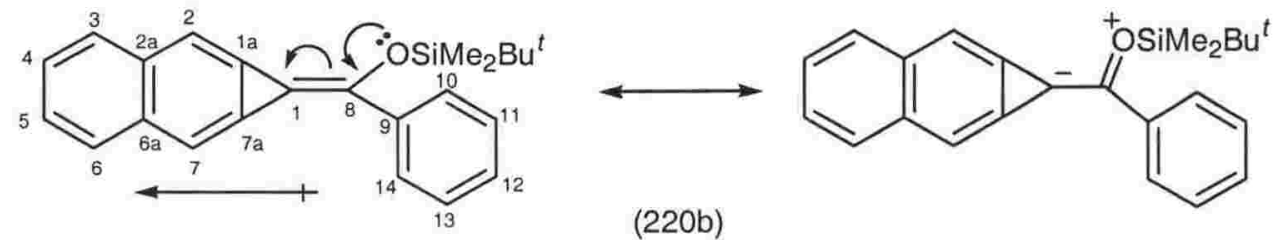

The success in preparing and characterising the novel siloxymethylidene compound (220b) triggered efforts toward preparing the sterically even more congested derivative (220c) which bears a $t$-butyldiphenylsiloxy moiety at C8. Enolate (216) and $t$ butyldiphenylsilyl chloride gave a bright yellow oil (63\%) that was pure $\left({ }^{1} \mathrm{H}\right.$ n.m.r. and 
t.l.c.) and gave an $[\mathrm{M}+\mathrm{H}]^{+}$molecular ion at $\mathrm{m} / \mathrm{z} 483.2130$ as required by the molecular formula of $\mathrm{C}_{34} \mathrm{H}_{30} \mathrm{OSi}$. The proton n.m.r. spectrum shows a nine-proton singlet at $\delta 1.30$ for the $t$-butyl group and, although the aromatic region is complex, a para-coupled doublet $(J 1.2 \mathrm{~Hz})$ at $7.27 \mathrm{ppm}$ is clearly visible. However, the aromatic region integrates only for twenty protons and not twenty-one required for (220c). More notable is a one-proton doublet $(J 1.2 \mathrm{~Hz})$ at $5.78 \mathrm{ppm}$ (Fig. 11). The ${ }^{13} \mathrm{C}$ n.m.r. spectrum shows twenty-two carbons of which nine are quaternary. The $\mathrm{Me}_{3} \mathrm{CSi}$ and $\mathrm{Me}_{3} \mathrm{CSi} t$-butyl carbon atoms resonate at $\delta 19.5$ and 26.5 and a shielded quaternary signal at $\delta 97.5$ is compatible with the olefinic $\mathrm{C} 1$ of methylidene (220c). Two shielded methine carbons at $\delta 103.9$ and 104.9 are observed that compare well with $\mathrm{C} 2$ and $\mathrm{C} 7$ of the $t$-butyldimethylsiloxy analogue (220b). HSQC data show that the carbon with the chemical shift of $\delta 103.9$ correlates with the one-proton para-coupled doublet $\delta 7.27$ whilst the carbon at $\delta 104.9$ correlates with the one-proton doublet at $\delta 5.78$. These protons are assigned as $\mathrm{H} 7$ ( $E$ to the oxygen atom) and $\mathrm{H} 2$ ( $Z$ to the oxygen atom), respectively. These data are compatible with the desired 1[( $t$-butyldiphenylsiloxy)phenylmethylidene $]-1 H$-cyclopropa[ $b]$ naphthalene $(220 c)$ sustaining a large chemical shift difference (1.29 ppm) between the $\mathrm{H} 2$ and $\mathrm{H} 7$ protons by virtue of the sterically very demanding $t$-butyldiphenylsiloxy group. Indeed, Dr. Margetic (Central Queensland University) has performed semi-empirical calculations at the restricted Hartee-Fock (RHF) level of theory which predict that, in its preferred conformer, the $\mathrm{H} 2$ atom of $(220 \mathrm{c})$ is $411 \mathrm{pm}$ from the centre of one of the pendant phenyl rings attached to silicon which places the $\mathrm{H}$ atom in its shielding cone (Fig. 12) ${ }^{142}$ this accounts for the unusual and significant shielding of this atom. The energy of this conformer has been predicted ${ }^{142}$ as $546 \mathrm{~kJ} \mathrm{~mol}^{-1}$ but, a range of isomers can be derived from rotation about the $\mathrm{C} 8-\mathrm{OSi}$ and $\mathrm{Si}-\mathrm{C}_{i p s o}$ bonds of this molecule that fall within a narrow energy range of $12 \mathrm{~kJ} \mathrm{~mol}^{-1}$. The electronic absorption spectrum for this polar methylidene $(220 \mathrm{c})$ shows an absorption maximum at $422 \mathrm{~nm}$ in hexane but only a minimal bathochromic shift of $1 \mathrm{~nm}$ is observed in changing to acetonitrile.

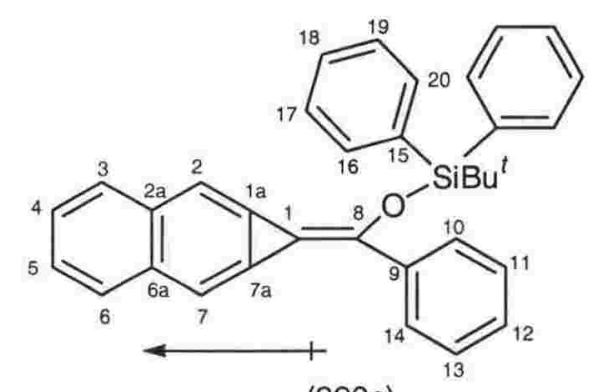

(220c) 


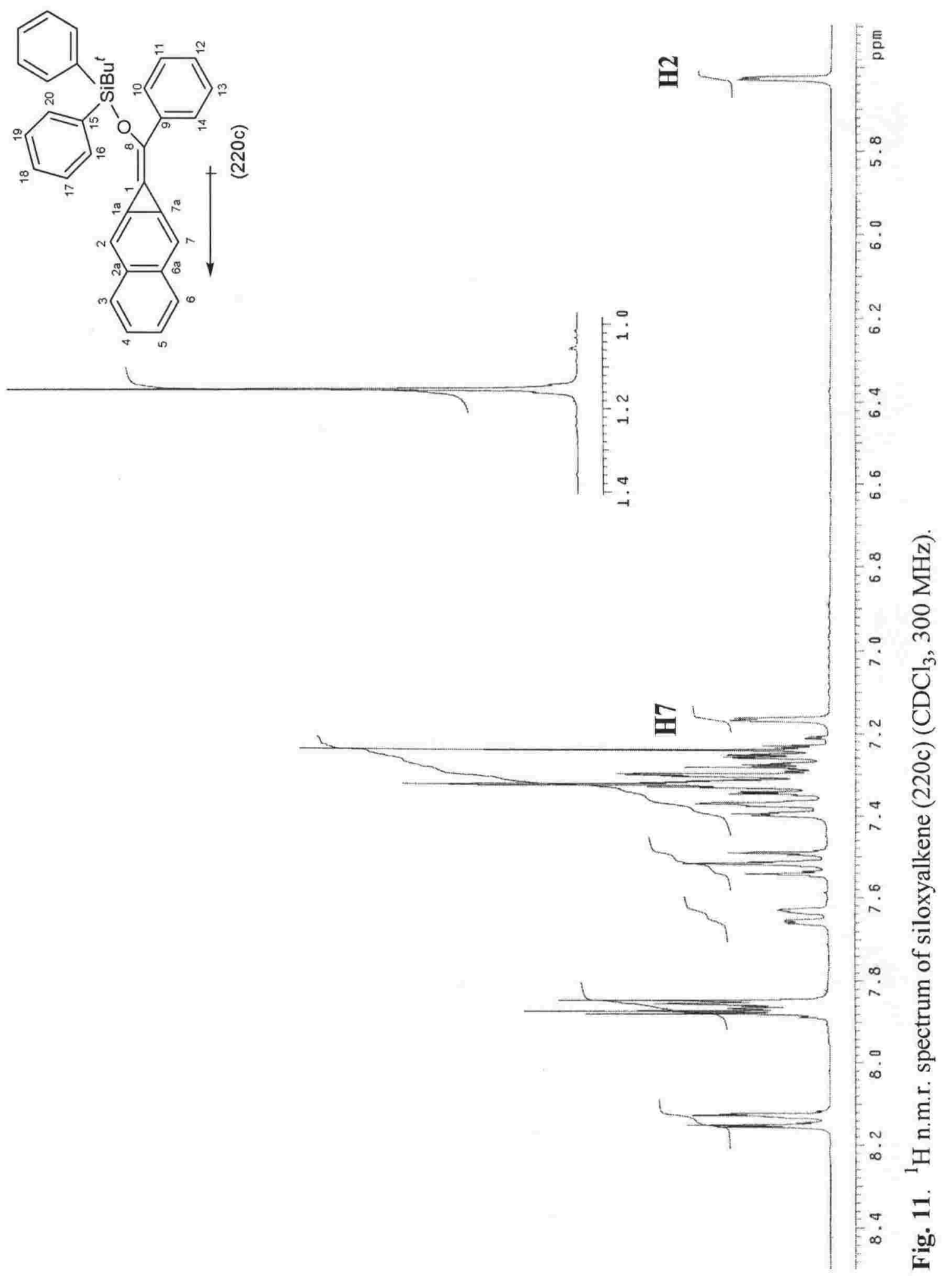



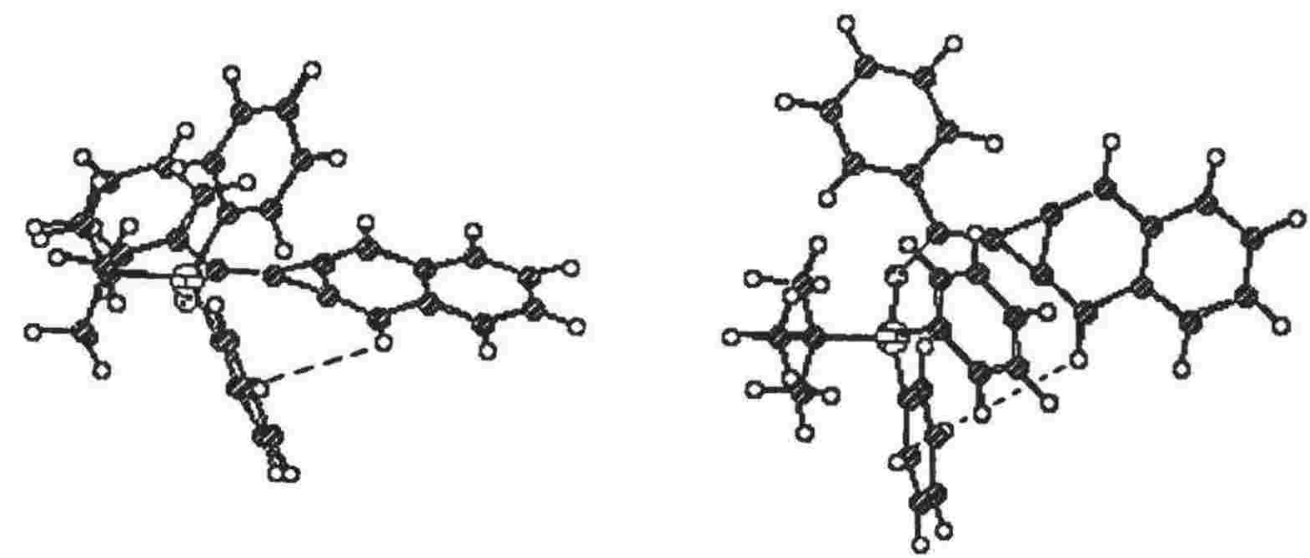

Fig. 12. Two views of the favoured conformer of silyl enol ether (220c) showing the shielding of $\mathrm{H} 2$ by a Si-Ph ring; the distance of $\mathrm{H} 2$ to the centre of the pendant phenyl is $411 \mathrm{pm}$ (calculated).

Since it proved possible to O-silylate enolate (216) with (trisubstituted)silyl chlorides, interception with a disubstituted chlorophosphine was examined as these reagents also are known ${ }^{143}$ to intercept oxyanions. Use of di-t-butylchlorophosphine with (216) gave a bright yellow solid which provided a proton spectrum with an eighteen proton doublet $\left({ }^{3} J_{\mathrm{P}-\mathrm{H}} 14.7 \mathrm{~Hz}\right)$ at $\delta 1.44$ for the $t$-butyl protons and an aromatic region that integrated for eleven protons with a two-proton AA' component of an AA'BB' spin system $(\delta 7.85-7.89)$ and non-equivalent $\mathrm{H} 2$ and $\mathrm{H} 7$ protons $(\delta 7.53$ and 7.65$)$ clearly visible. These data suggest that the three-membered ring is intact and although the carbon spectrum is complicated by the observation of ${ }^{1-4} J_{\mathrm{P}-\mathrm{H}}$ couplings, the shielded $\mathrm{C} 2$ and $\mathrm{C} 7$ carbon atoms are clearly evident ( $\delta 106.6$ and 108.6) and the $\mathrm{C} 1$ and $\mathrm{C} 8$ exocyclic olefinic carbons are observed at $\delta 99.9$ and $132.4\left({ }^{2} J_{\mathrm{P}-\mathrm{H}} 12.6 \mathrm{~Hz}\right)$, respectively. The compound also shows a single phosphorous resonance at $\delta 68.6$ in the ${ }^{31} \mathrm{P}$ n.m.r. spectrum. However, what is most notable is that the high-resolution mass spectrum shows an $[\mathrm{M}+\mathrm{H}]^{+}$ion at $\mathrm{m} / \mathrm{z}$ 405.1978 which implies a molecular formula of $\mathrm{C}_{26} \mathrm{H}_{29} \mathrm{O}_{2} \mathrm{P}$, with one extra oxygen atom to that required for the desired product $(220 \mathrm{~d})$. Furthermore, the microanalytical data confirm $\mathrm{C}_{26} \mathrm{H}_{29} \mathrm{O}_{2} \mathrm{P}$ as the molecular formula of the compound. On this basis, the product of reaction is assigned as phosphonic acid ester (220e) rather than the sought after phosphinous acid ester (220d) (Scheme 55); the yield of (220e) is $16 \%$. It is assumed that the alkylidene compound (220d) is oxidised upon workup to give (220e). This is fully consistent with the known ${ }^{144}$ behaviour of phosphinous acids upon aerial oxidation. 


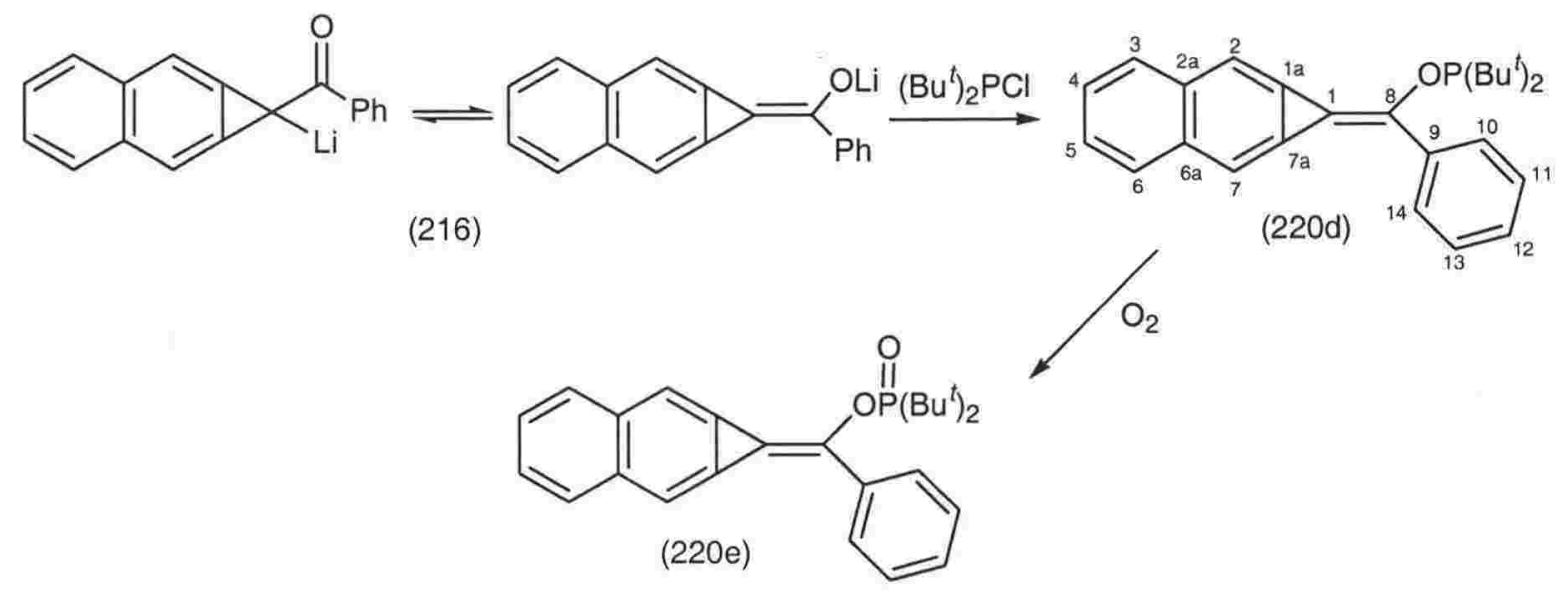

Scheme 55

As 1-benzoylcyclopropanaphthalene (202) can be converted into its corresponding enolate (216) and intercepted to give silyl enol ethers with (trisubstituted)silyl chlorides, it should prove possible to prepare the analogous acetyl derived products from interception of enolate (215) (path $a$, Scheme 56). However, with an acetyl group in synthon (189) it is possible that a proton from the methyl group could be abstracted to give enolate (221) in competition with deprotonation at the benzylic site (path $b$, Scheme 56); the yield of the sought after cycloproparenylidene enol ether may be reduced therefore. Treatment of (189) with butyllithium and interception of the enolate with $t$-butyldimethylsilyl chloride gave an oil which contained predominantly a compound that is tentatively identified as the ( $t$-butyldimethylsiloxy)ethylidene (219a) in $\sim 35 \%$ yield. The proton spectrum for this compound shows a six-, a nine-, and a three-proton singlet at $\delta 0.37,1.05$, and 2.29 for the $\mathrm{SiBu}^{t}, \mathrm{SiMe}_{2}$, and $\mathrm{C} 8$-methyl substituents, respectively. In addition, the aromatic region clearly shows a four-proton AA'BB' spin system $(\delta 7.73-7.77$ and $7.38-7.41)$ and the nonequivalent $\mathrm{H} 2$ and $\mathrm{H} 7$ protons resonate as broad singlets at $\delta 7.09 / 7.10$ confirming that the three-membered ring is intact. The carbon spectrum of (219a) shows sixteen resonances, and, as expected, C2/C7 were shielded and resonate at $\delta 103.4 / 103.6$ while the shielded olefinic $\mathrm{Cl}$ carbon resonates at $\delta 95.4$. Unfortunately, all subsequent attempts to purify the compound resulted in its decomposition. As almost all of the known alkylidenecycloproparenes are stable, ${ }^{100}$ the instability of (219a) likely is due to mesomeric electron donation by the siloxy group increasing electron density at $\mathrm{C} 1$. Protonation at this centre (acid or water) would afford a mono-substituted cyclopropanaphthalene which would then be expected to capture nucleophile with ring-opening to give a 2,3-disubstituted naphthalene (Scheme 56). 


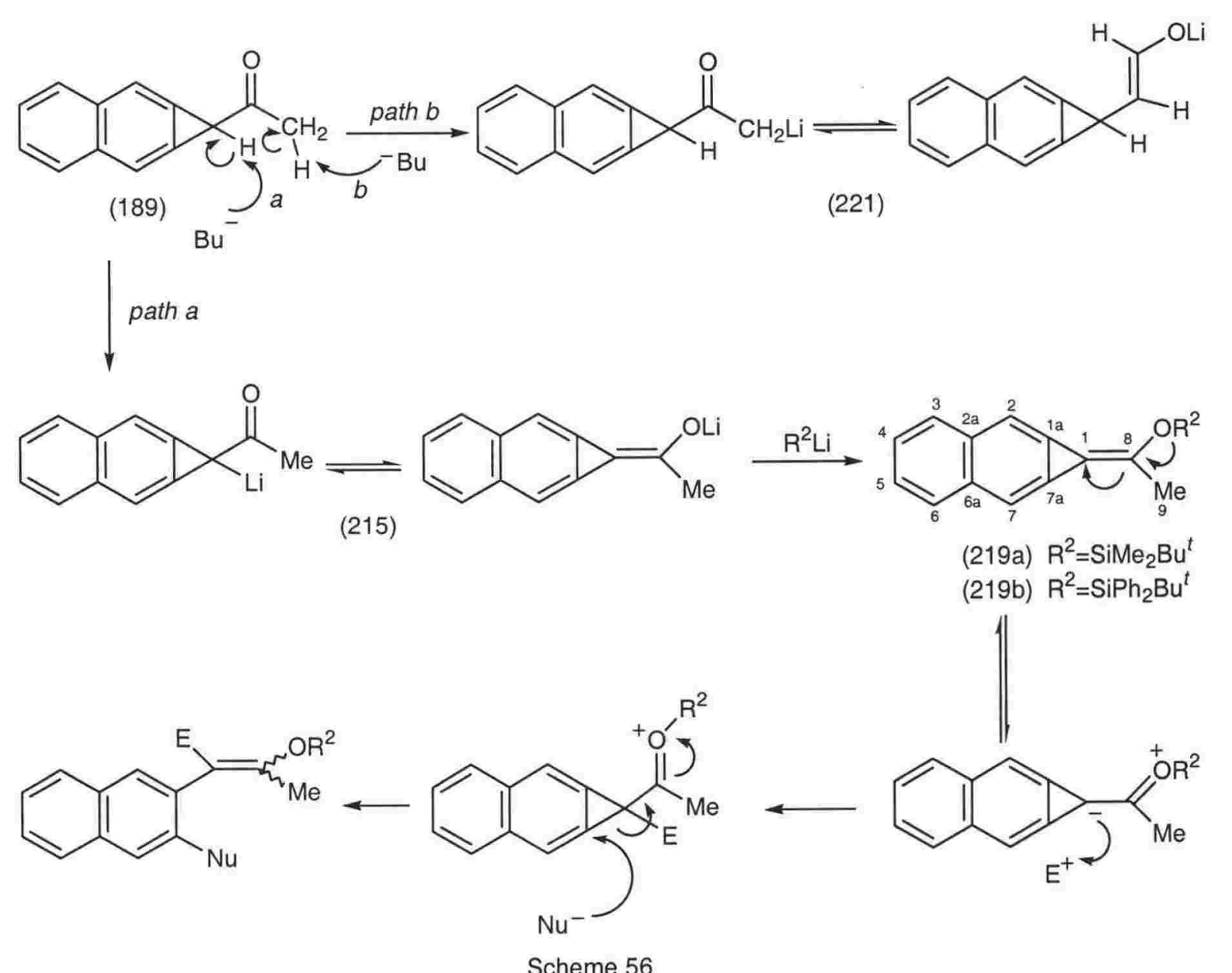

In analogy to the reaction described above, generation of enolate (215) and its treatment with $t$-butyldiphenylsilyl chloride gave a thick yellow tar. Analysis of the tar by ${ }^{1} \mathrm{H}$ n.m.r. spectroscopy showed the presence of two doublets $(J \sim 1 \mathrm{~Hz})$ at $\delta 5.99$ and 7.04 as required for $\mathrm{H} 2$ and $\mathrm{H} 7$ of (219b) in analogy with the lowest energy conformation of the C8 phenyl substituted homologue (220b). Unfortunately, all attempts to isolate (219b) resulted in its decomposition and its identity remains tentative.

In conclusion, the treatment of the cycloproparenyl anion (103) with $\mathrm{N}, \mathrm{N}$-dimethyl -acetamide, -benzamide, or -carbamoyl chloride has afforded the C1-substituted cycloproparenes (189), (202), and the disubstituted derivative (205) in modest yields as the only C1-acylated cycloproparenes so far recorded. ${ }^{142}$

Reaction of these cycloproparenes with hydrochloric acid gave the 2,3-disubstituted naphthalenes (212a-c) and not the 2-substituted analogues (209a-c) that typically ${ }^{39,42}$ arise from proton capture by the $\pi$ bond and benzylic cation interception. The observed products probably result from capture of proton by the carbonyl oxygen atom.

Thermolysis of the acylated cycloproparenes (189), (202), and (205) gives rise to naphthofurans $(214 \mathrm{a}-\mathrm{c})$ as expected and this is consistent with the behaviour of C1-diester substituted cyclopropabenzenes. ${ }^{51,52}$ However, yields are poor except for the dicarboxamide (205) which gave naphthofuran (214c) quantitatively. 
Treatment of 1-benzoylcyclopropanaphthalene (202) with base generates the enolate (216) which is intercepted successfully with trimethylsilyl chloride, $t$-butyldimethylsilyl chloride, and $t$-butyldiphenylsilyl chloride to give the corresponding cyclopropanaphthalenylidene enol ethers (220a-c). With (216) and di-t-butylchlorophosphine the phosphonate (220e) was isolated from aerial oxidation of the initially generated (220d). These compounds are the first methylidenecycloproparenes which bear ether moieties at $\mathrm{C} 8$ and their synthesis represents the first case of alkylidenecycloproparene preparation in one step from the benzylic centre of a cycloproparenyl synthon.

The proton spectrum of the sterically congested $t$-butyldiphenylsiloxy derivative (220b) shows $\mathrm{H} 2$ and $\mathrm{H} 7$ to be separated by $1.49 \mathrm{ppm}$; this is the largest separation so far recorded in the alkylidenecycloproparene series. Semi-empirical calculations show that $\mathrm{H} 2$ ( $\mathrm{Z}$ to the $\mathrm{O}$ atom) is $411 \mathrm{pm}$ from the centre of one of the $\mathrm{Si}-\mathrm{Ph}$ groups which places it in its shielding cone and accounts for the anomalous chemical shift.

The enolate (215) derived from acetylcyclopropanaphthalene (189) is likewise intercepted by $t$-butyldimethylsilyl chloride and $t$-butyldiphenylsilyl chloride to give the siloxyalkylidene compounds (219a) and (219b). However, it was not possible to isolate these compounds prior to their decomposition. 


\section{Chapter 5}

\section{Approaches to Oxygenated Cyclopropanthracenes}

Until recently ${ }^{104}$ the number of oxygenated cycloproparenes in existence was few. ${ }^{35}$ The methoxy-containing cycloproparenes $(70)^{116}$ and $(222),{ }^{145}$ and the alkylidene compounds $(150)^{104,116}$ derived from the former, are isolable under normal conditions. The ether $(223)^{146}$ has been proposed as a reactive intermediate in solution whilst the 4,5-dioxacycloproparene (224) has been generated but is stable only below $-45^{\circ} \mathrm{C}$. ${ }^{147}$ An even rarer class of compounds is the cyclopropaquinones. ${ }^{35,148}$ The simplest of these, cyclopropa-2,5benzoquinone (225), has been generated by fvp methods but was too reactive to be isolated. The naphtho analogue, cyclopropanaphthalene-2,7-dione (226), has also been generated but it too, is capable only of transient existence. ${ }^{149}$ However, compounds (225) and (226) add anthracene and furan, respectively, across the strained bridge bond to give the corresponding Diels-Alder cycloadducts, thereby demonstrating their transient existence. $^{149,150}$<smiles>COc1ccc(OC)c2cc3c(cc12)C3</smiles>

(70)<smiles>c1ccc2c(c1)Oc1cc3c(cc1O2)C3</smiles>

(224)<smiles>COc1cccc2c1C2</smiles>

(222)<smiles>O=C1C=CC(=O)C2=C1C2</smiles>

(225)<smiles>[R7]C([R])=C1c2cc3c(OC)ccc(OC)c3cc21</smiles>

(150)<smiles>O=C1C2=C(C2)C(=O)c2ccccc21</smiles>

(226)<smiles>COc1ccc(OC)c2c1C2(c1ccccc1)c1ccccc1</smiles>

(223)<smiles>O=C1C=CC(=O)c2cc3c(cc21)C3</smiles>

(154)

The only stable cyclopropaquinone reported to date is cyclopropa $[b]$ naphthalene3,6-dione (154). ${ }^{16,118,148}$ The compound is formed in excellent yield (85\%) from the oxidative demethylation of 3,8-dimethoxycyclopropanaphthalene (70) and its structure has been determined by X-ray crystallography. Unfortunately, all attempts to prepare the derived methylidene compounds (153) proved unsuccessful. ${ }^{116}$<smiles>O=C1C=CC(=O)c2cc3c(cc21)C3</smiles>

(154)

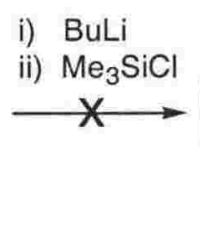

ii) $\mathrm{Me}_{3} \mathrm{SiCl}$
(151)<smiles>CC1(C)c2cc3c(cc21)C(=O)C=CC3=O</smiles><smiles>[R7]C([R])=C1c2cc3c(cc21)C(=O)C=CC3=O</smiles>

(153) 
The attempted metalation of (154) with an excess of either butyllithium or lithium diisopropylamide (LDA) at low temperature followed by the addition of trimethylsilyl chloride did not provide the disilane (151) as expected. Instead, a complex mixture of products was obtained whose components have eluded isolation; no evidence to support the formation of (151) was obtained. ${ }^{116}$ It seems likely that the failure to prepare (151) is caused by competing reaction pathways. Thus, instead of base abstracting a methylene proton to generate the $\mathrm{C} 1$ benzylic anion, it could add to the bridgehead carbon $\mathrm{C} 1 \mathrm{a}$ (or C7a) to generate the ring-opened anion (227). Alternatively, Michael addition of base to the enedione moiety of (154) would afford the oxyanion (228). Thus, cyclopropaquinone appears to be unsuitable as a synthon for preparing methylidenecyclopropaquinones.<smiles>[B]Cc1cc2c(cc1C)C(=O)C=CC2=O</smiles><smiles>[B]C1C=C([O-])c2cc3c(cc2C1=O)C3(C)C</smiles>

In this chapter approaches to the synthesis of the first known methylidenecyclopropaquinones, e.g. (164), via the unknown cyclopropanthraquinone (162) will be described. In addition, efforts directed towards the preparation of the new ether (163) and its methylidene derivatives (165) are addressed.<smiles>O=C1c2ccccc2C(=O)c2cc3c(cc21)C3</smiles>

(162)<smiles>COc1c2ccccc2c(OC)c2cc3c(cc12)C3</smiles>

(163)<smiles>[R7]C([R7])=C1c2cc3c(cc21)C(=O)c1ccccc1C3=O</smiles>

(164)<smiles>[R7]C([R7])=C1c2cc3c(OC)c4ccccc4c(OC)c3cc21</smiles>

(165)

Possible routes to quinone (162) are via the synthon (160) as illustrated in Scheme 57. Thus, treating (160) with potassium carbonate in the presence of dimethyl sulfate $\left[(\mathrm{MeO})_{2} \mathrm{SO}_{2}\right]$ should afford the dihydrocyclopropanthracene (229), which should give ether 
(163) on aromatisation. The preparation of (162) could then be accomplished via the oxidative demethylation of (163) with cerium ammonium nitrate (CAN). An alternative route to (162) is via the oxidation of dihydroquinone (230) with, for example, 2,3-dichloro5,6-dicyano-1,4-benzoquinone (DDQ). Compound (230) itself is likely available from either treating ether (229) with CAN or oxidation of Diels-Alder adduct (160).

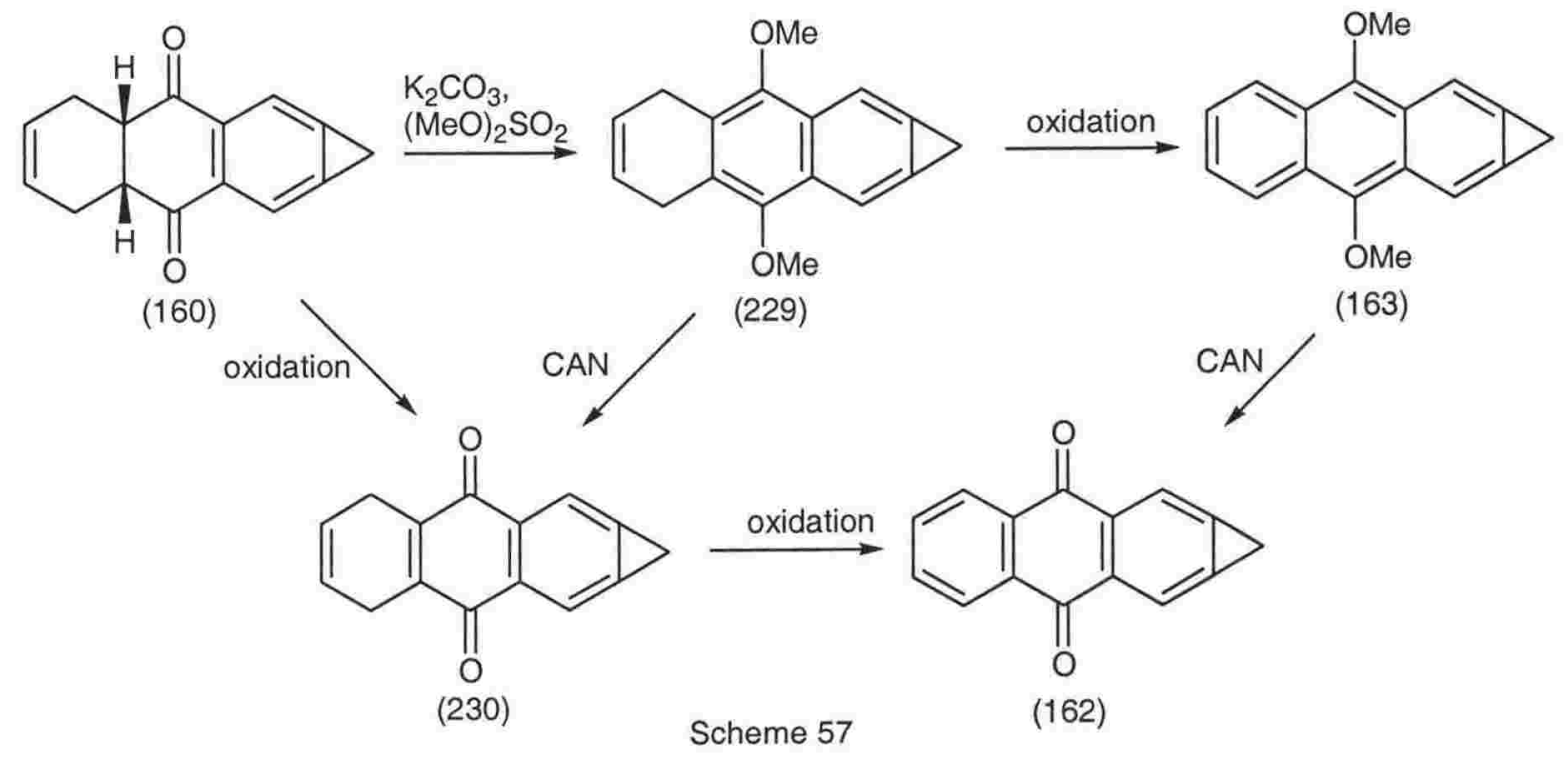

The precursor for tetrahydrocyclopropanthraquinone (160) is quinone (154), itself readily available from common reagents in five steps (Scheme 58). ${ }^{116,118,151-153}$ The $\left[\pi_{\mathrm{s}} 2^{+}\right.$ $\pi^{4}$ s addition of butadiene to benzoquinone for $4-5$ days at $40^{\circ} \mathrm{C}$ gives the Diels-Alder adduct (231) in $89 \%$ yield. $^{151}$ The enolate ion of (231) is generated with potassium carbonate and use of dimethyl sulfate provides ether (232) in excellent yield (97\%). ${ }^{152}$ The addition of dichlorocarbene to (232) provides compound $(233)^{153,116}(72 \%)$ which, after didehydrohalogenation with an excess of base provides diether (70) in $25 \%$ yield. ${ }^{116}$ Finally, treating (70) with CAN as described by Halton et al. provides cyclopropaquinone (154) as bright yellow needles $(75 \%) .{ }^{116,118}$

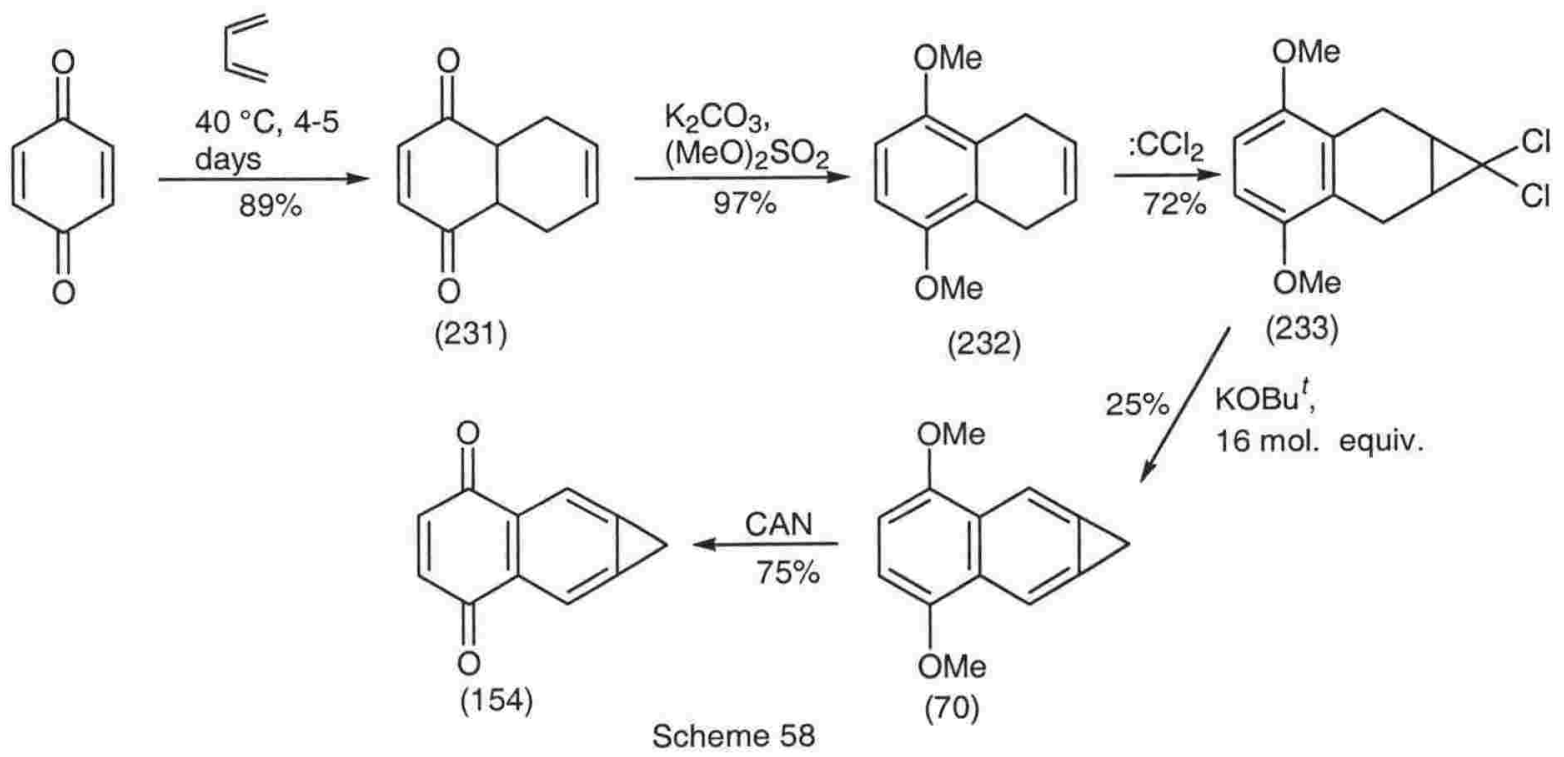


It was proposed by Sretenovic ${ }^{121}$ that the addition of butadiene to (154) gives rise to the $1: 1$ adduct (160) that is necessary for the conversion to quinone (162). However, the 1:2 adduct (161) and unchanged (154) were also obtained; the ratio of these three compounds was reported as being both temperature and time dependent. Thus, when (154) was treated with an excess of butadiene at $100^{\circ} \mathrm{C}$ for two days the $1: 2$ adduct (161) was the sole reaction product; the compound gave data that were fully compatible with the assigned structure. ${ }^{121}$ By performing the reaction for two days at $40^{\circ} \mathrm{C}$ the crude product gave signals in the ${ }^{1} \mathrm{H}$ n.m.r. spectrum that were compatible with unchanged quinone (154). However, in addition to the signals of (154) the spectrum showed resonances at $\delta$ 2.20 (bd d, $2 \mathrm{H}), 2.50$ (bd d, 2H), $3.33(\mathrm{~s}, 2 \mathrm{H}), 3.36-3.38(\mathrm{~m}, 2 \mathrm{H}), 5.71(\mathrm{~s}, 2 \mathrm{H})$, and 7.86 (s, $2 \mathrm{H})$ that were compatible with, and tentatively assigned to, compound (160). Examination of the integrals for $\mathrm{H} 2 / 9$ of $(160)(\delta 7.86)$ and $\mathrm{H} 2 / 7$ of $(154)(\delta 7.90)$ suggested that compounds (154) and (160) were present in $\sim 1: 3$ ratio. Carrying out the reaction at $56^{\circ} \mathrm{C}$ for two days gave the cycloadducts (160) and (161) in $\sim 6: 1$ ratio. Despite obtaining evidence for (160) Sretenovic was unable to obtain samples of (160) suitable for characterisation purposes or for subsequent transformations. ${ }^{121}$<smiles>C=CC1c2cc3c(cc21)C(=O)C=CC3=O</smiles>

(154)

(154)

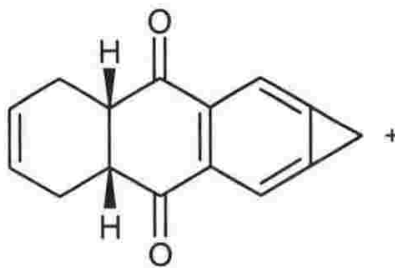

(160)

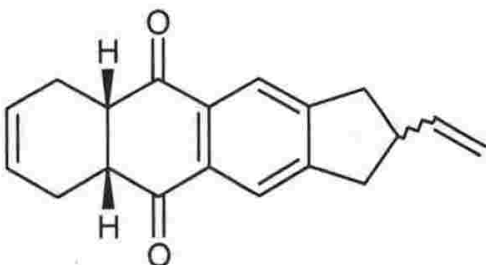

(161)

In order to isolate and characterise the monoadduct (160) the author elected to treat quinone (154) with an excess of butadiene at $45^{\circ} \mathrm{C}$ over the longer period of four days. The ${ }^{1} \mathrm{H}$ n.m.r. spectrum of the crude product gave signals that were compatible with unchanged (154) and the 1:1 Diels-Alder adduct (160) in a ratio of $\sim 3: 17$ and this equates to $\sim 15 \%$ recovery of (154) and an $\sim 85 \%$ yield of (160); only a trace amount of the $1: 2$ adduct (161) was detected. Recrystallisation of the residue from benzene gave a mixture of crystals that were yellow together with a small quantity of green ones; these were easily separated by hand. The yellow crystals were found to contain ( ${ }^{1} \mathrm{H}$ n.m.r.) $~ 98 \%$ of (160) $(27 \%)$ with unchanged (154) while the green crystals contained these same two compounds in a 2:1 ratio. A further two recrystallisations of the yellow crystals provided an analytical sample of (160). 


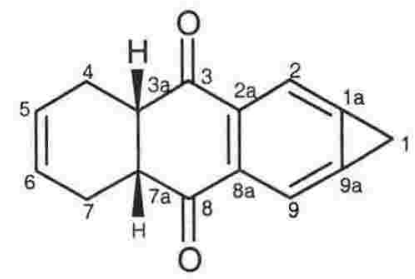

(160)

The proton spectrum of (160) displays a complex AMX spin system between 2.19$2.29,2.44-2.57$, and $3.36-3.41 \mathrm{ppm}$, that are assigned to the non-equivalent methylene protons $\mathrm{H} 4 / 7$, and the methine protons $\mathrm{H} 3 \mathrm{a} / 7 \mathrm{a}$, respectively. The olefinic protons are observed as a triplet $(J 1.5 \mathrm{~Hz})$ at $5.73 \mathrm{ppm}$, the $\mathrm{C} 1$ methylene protons as a broad singlet at $3.36 \mathrm{ppm}$, and the aromatic protons as a singlet at $7.87 \mathrm{ppm}$.

The ${ }^{13} \mathrm{C}$ n.m.r. spectrum of (160) shows eight signals as expected from its symmetry. The $\mathrm{C} 2 / 9$ resonance is shielded as expected ${ }^{35}(113.2 \mathrm{ppm})$ and the quinone carbons resonate at $\delta 198.2$. The carbonyl stretching frequency of $(160)$ is at $1678 \mathrm{~cm}^{-1}$ in the IR spectrum.

Molecular orbital calculations ${ }^{119}$ at the $a b$ initio $6-31 \mathrm{G}^{*}$ level of theory (without geometrical constraints) show the preferred conformation of the cyclohexene ring of (160) to be that of the half chair with an $\mathrm{H} 3 \mathrm{a}-\mathrm{C} 3 \mathrm{a}-\mathrm{H} 7 \mathrm{a}-\mathrm{C} 7 \mathrm{a}$ dihedral angle of $58.2^{\circ}$ (Fig. 13a). When the $\mathrm{H} 3 \mathrm{a}-\mathrm{C} 3 \mathrm{a}-\mathrm{H} 7 \mathrm{a}-\mathrm{C} 3 \mathrm{a}$ dihedral angle is constrained to $0^{\circ}$ four distinct conformers (Fig. 13b-e ) are detected and these have energies $\sim 32-37 \mathrm{~kJ} \mathrm{~mol}^{-1}$ above that of the energy minimum (Fig.13a). Upon running the ${ }^{1} \mathrm{H}$ n.m.r. spectrum of $(160)$ at $-50^{\circ} \mathrm{C}$, the resonances and their multiplicities remain unchanged. This suggests that either interconversion between the conformers of $(160)$ is rapid even at $-50^{\circ} \mathrm{C}$ or, more likely, that conformational change within the cyclohexene ring does not occur in solution at ambient temperature. In order to ascertain this, the sample would need to be heated in the n.m.r. spectrometer and the signals monitored. Unfortunately, the necessary facilities to carry out this experiment were unavailable thus precluding the appropriate experiment from being run.

The calculated ${ }^{119}$ bond lengths and interbond angles of (160) in its energy minimum are shown in Fig. 14. Clearly evident are the bond deformations around the points of ring-fusion. The bonds in the aromatic ring, with the exception of the quinone $\mathrm{C}-\mathrm{C}$ double bond, are calculated to be shorter than those measured crystallographically for quinone (154) and the agreement between theory for $(160)^{119}$ and literature ${ }^{116}$ for (154) is excellent. Calculated bond angles are similar to those in (154). 
(a)

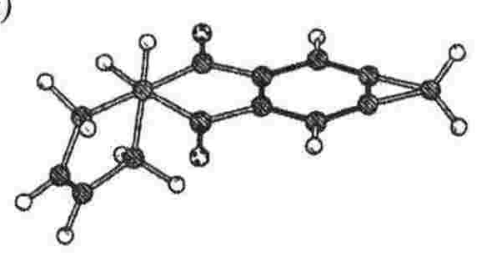

(b)

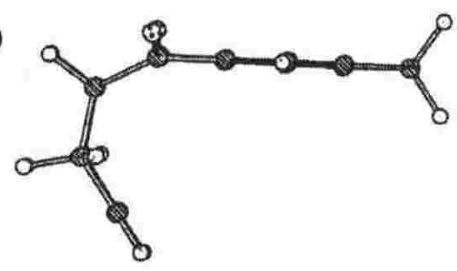

(c)

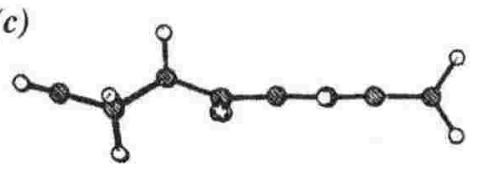

(d)

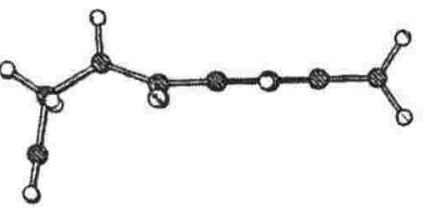

(e)

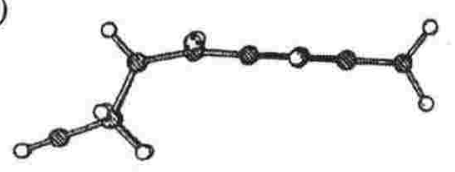

Rel. energy/

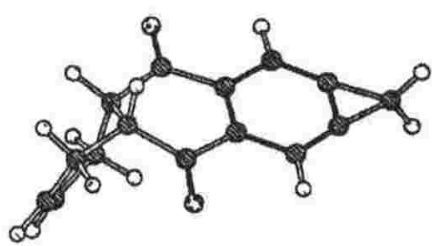

$\mathrm{KJ} \mathrm{mol}^{-1}$

0.0

31.9

32.2
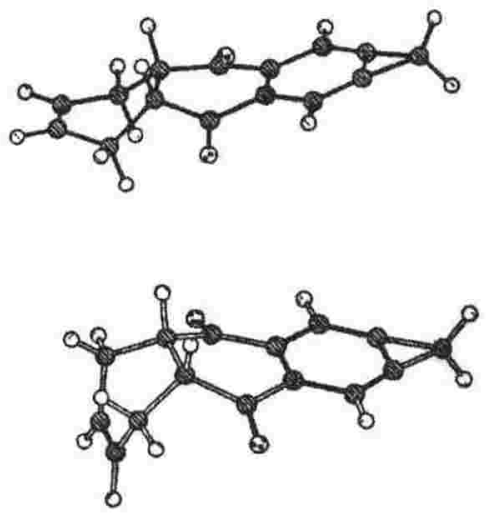

35.5

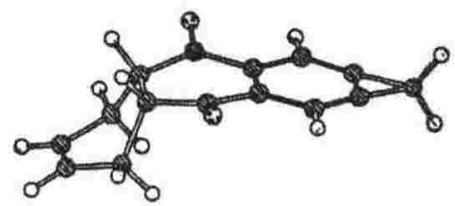

37.0

Fig. 13. Calculated $\left(6-31 \mathrm{G}^{*}\right)$ conformation of $(160)(a)$ without geometrical constraints and $(\boldsymbol{b})-(\boldsymbol{e})$ with the $\mathrm{H} 3 \mathrm{a}-\mathrm{C} 3 \mathrm{a}-\mathrm{C} 7 \mathrm{a}-\mathrm{H} 7 \mathrm{a}$ dihedral angle constrained to $0^{\circ} \mathrm{C}$. Data taken from reference 119 . 


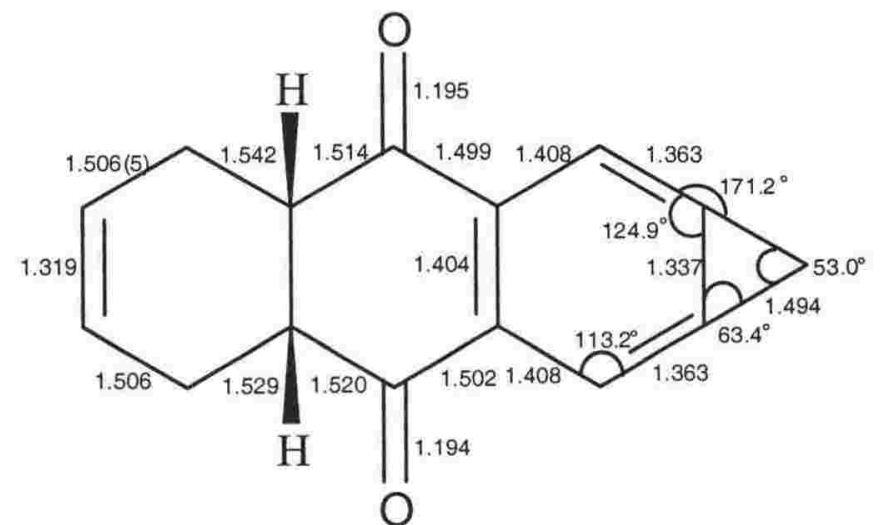

(160)

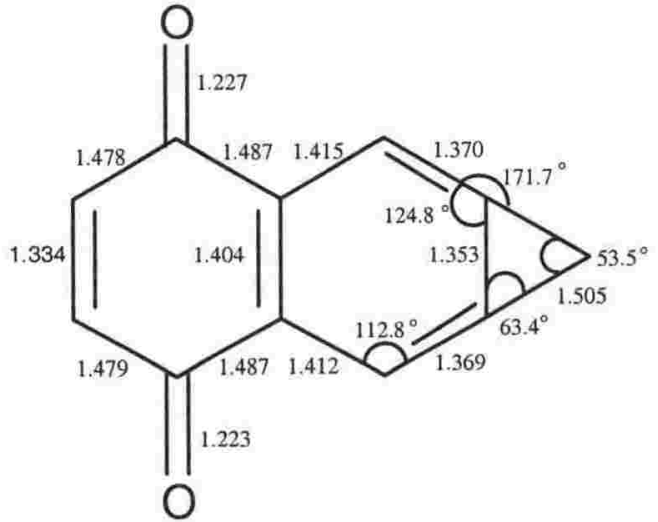

(154)

Fig. 14 Calculated (6-31G*) geometry of butadiene adduct (160) and X-ray geometry of quinone (154). Angles are shown in degrees $\left({ }^{\circ}\right)$ and bond lengths are in $\AA$. Data for $(160)$ and (154) are taken from references 119 and 116, respectively.

Although an analytical sample of tetrahydrocyclopropanthracene (160) was obtained from the successive recrystallisations of the crude residue from the addition of butadiene to quinone (154), the majority of unreacted cyclopropaquinone (154) can also be removed by radial chromatography to give $(160)$ in $~ 45 \%$ yield. However, examination of the enedione (160)-containing fraction by ${ }^{1} \mathrm{H}$ n.m.r. spectroscopy revealed the presence of a minor component not previously detected in addition to unchanged (154). The ${ }^{1} \mathrm{H}$ n.m.r. data abstracted for this new compound were $\delta 3.25(\mathrm{~d}), 3.34(\mathrm{~s}), 5.87(\mathrm{t})$, and $7.94(\mathrm{~s})$ in a 2:1:1:1 ratio. Clearly absent are the $\mathrm{H} 3 \mathrm{a} / 7 \mathrm{a}$ protons but still present are the $\mathrm{H} 4 / 7$ methylene and $\mathrm{H} 5 / 6$ olefinic protons in the ring remote from three-membered ring fusion; the aromatic and $\mathrm{C} 1$ methylene protons remain intact. On the basis of the symmetrical nature of this compound and the absence of $\mathrm{H} 3 \mathrm{a} / 7 \mathrm{a}$ bridge protons it appears that oxidation has occurred and the compound is tentatively assigned as the dihydrocyclopropaquinone (230). Quinone (230) presumably results from the oxidation of (160) by molecular oxygen during chromatography with the silica gel providing an active surface ${ }^{154,155}$. In an attempt to bring about this conversion more efficiently (160) and silica gel were suspended in dichloromethane and stirred but, even after several days, unchanged (160) was recovered quantitatively with no evidence for quinone (230).

In order to oxidise (160) a more conventional reagent was used to effect the dehydrogenation, namely, DDQ. When compound (160) was stirred with an excess of the reagent for several days at ambient temperature workup gave a red multi-component $\left({ }^{1} \mathrm{H}\right.$ n.m.r.) oil that contained a small amount of unchanged enedione (160) but none of the sought after quinone (230). It would appear therefore, that DDQ is not a suitable reagent for the dehydrogenation of (160). 
The lack of success in preparing quinone (230) directed efforts towards preparing its derived ether (229) with an aromatic B-ring. As the enedione (231) is readily converted into the aromatic ether (232) (Scheme 58, p. 82) from reflux with potassium carbonate and dimethyl sulfate in acetone, this procedure was followed for dihydroanthracene (229). However, as the three-membered ring of cycloproparenes is known ${ }^{39}$ to open on heating, the reaction was performed at ambient temperature. The sought after ether was obtained in $63 \%$ yield as a colourless solid that slowly became pink on standing; it is best to store the ether under an inert atmosphere. The ${ }^{1} \mathrm{H}$ and ${ }^{13} \mathrm{C}$ n.m.r. spectra of (229) show five and nine resonances, respectively, as expected from the symmetrical nature of the compound. The $\mathrm{H} 3 \mathrm{a} / 7 \mathrm{a}$ protons of enedione (160) are absent and the now aromatic $3 \mathrm{a} / 7 \mathrm{a}$ carbons are observed at $124.8 \mathrm{ppm}$ in the ${ }^{13} \mathrm{C}$ n.m.r. spectrum. The quinone resonances for synthon (160) were absent and instead, ${ }^{13} \mathrm{C}$ n.m.r. resonances at $\delta 150.0$ for the C-OMe ipso carbons were present; these compare well with those for 3,8-dimethoxycyclopropanaphthalene (70) at $150.4 \mathrm{ppm} .{ }^{116}$ The $\mathrm{OCH}_{3}$ protons for (229) resonate at $\delta 3.91$ and the $\mathrm{OCH}_{3}$ carbons are at $61.0 \mathrm{ppm}$; again, these are in excellent agreement with ether (70). ${ }^{116}$ Unfortunately, all subsequent attempts to prepare further samples of ether (229) were unsuccessful with a complex mixture of products being obtained instead; the reason for this is not known and the observations may be an experimental artifact.

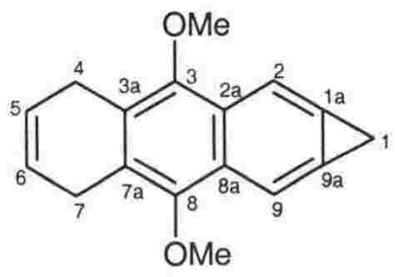

(229)

During the initial preparation of (229) a second, and more minor fraction, was obtained from chromatography. Analysis of this fraction by n.m.r. gave signals that were identical to the ether (229) but were contaminated with dimethyl sulfate ( $\delta 3.91)$. In addition to these a third set of signals at $\delta 3.41(\mathrm{~s}, 2 \mathrm{H}), 7.79-7.81\left(\mathrm{BB}^{\prime}, 2 \mathrm{H}\right), 8.16(\mathrm{~s}, 2 \mathrm{H})$, and 8.31-8.34 (AA', $2 \mathrm{H})$ were observed. The AA'BB' spin system is consistent with the ring remote from three-membered ring fusion being aromatic in character and the absence of the H4/7 methylene resonances suggests that this is so. Due to the simplicity of these spectral data the compound is tentatively assigned as 3,8-dimethoxycyclopropa $[b]$ anthracene (163) providing that the $\mathrm{OCH}_{3}$ protons fortuitously resonate at the same frequency as those of dimethyl sulfate. The three compounds were present in the mixture in a ratio of 20:6:1 from integration. The small amount of this oil and the presence of (163) only to an extent of $\sim 4 \%$ argued against further separation. 
It is possible that during the attempted preparation of ether (229) the dimethoxycycloproparene (163) was not generated, but that instead, quinone (162) was formed. The cycloproparene (162) could result from either aerial oxidation of (163) or oxidation of the dihydrodione (230) (Scheme 59), itself generated from the base-facilitated dehydrogenation of (160). In order to ascertain whether the enedione (160) could be dehydrogenated with base the said solid was treated with anhydrous potassium carbonate; the mixture developed a dark red colouration over this time. Filtration of the mixture and solvent removal gave a solid which was sparingly soluble in all common solvents. It is possible that this solid was the dipotassium salt (234) or the phenol (235). A mechanism for the formation of salt (234) is shown in Scheme 60 and likely involves the intermediate (236). If salt (234) is moisture sensitive then phenol (235) will likely result from exposure to air.<smiles>O=C1c2cc3c(cc2C(=O)[C@H]2CC=CC[C@H]12)C3</smiles>

(160)

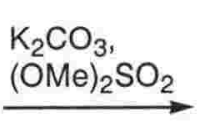

$? \mathrm{~K}_{2} \mathrm{CO}_{3}$<smiles>COc1c2c(c(OC)c3cc4c(cc13)C4)CC=CC2</smiles>

(229)

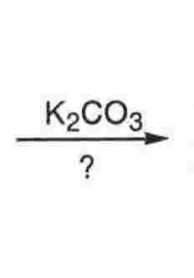<smiles>COc1c2ccccc2c(OC)c2cc3c(cc12)C3</smiles>

(163)<smiles>O=C1C2=C(CC=CC2)C(=O)c2cc3c(cc21)C3</smiles>

(230)

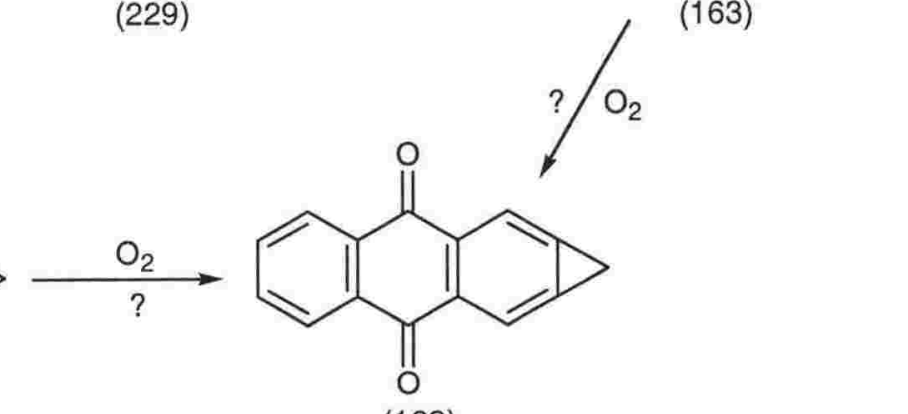

(162)

Scheme 59

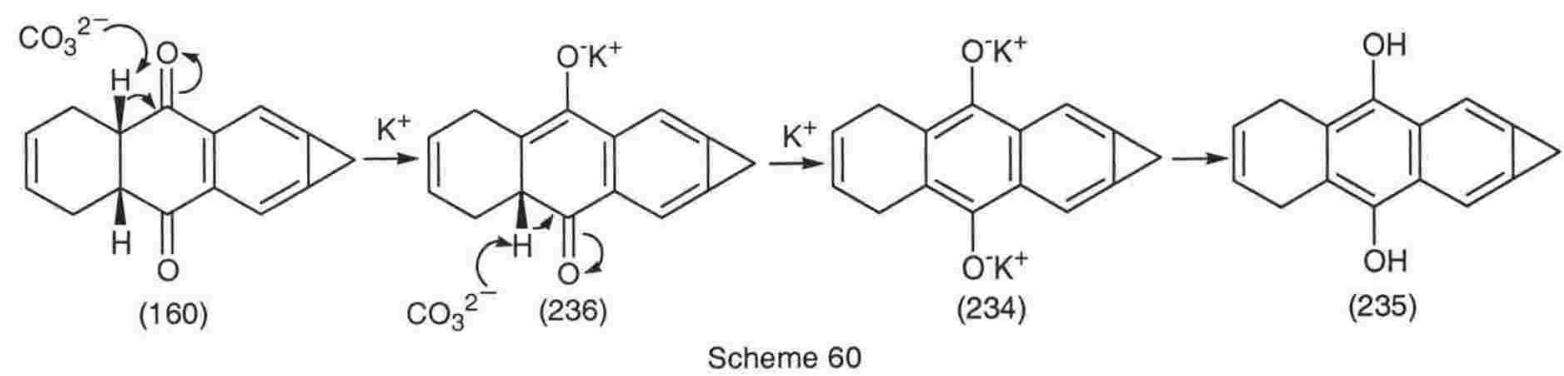

In order to gain appropriate evidence for the formation of potassium salt (234) in solution, compound (160) was treated with potassium carbonate in $d_{6}$-acetone and the reaction monitored by ${ }^{1} \mathrm{H}$ n.m.r. spectroscopy. After $1 \mathrm{~h}$, cycloproparene (160) remained unchanged. Sonication of the n.m.r. tube provided new signals at $\delta 3.50,5.96$, and 7.95 after $30 \mathrm{~min}$ and after a further $30 \mathrm{~min}$ the complete disappearance of enedione (160) was coupled with clear ${ }^{1} \mathrm{H}$ n.m.r. data for (234). Of the signals at $\delta 3.42$ (s, 2H), 3.50 (d, $J 1.0$ $\mathrm{Hz}, 4 \mathrm{H}) .5 .96(\mathrm{t}, J 1.3 \mathrm{~Hz})$, and $7.95(\mathrm{~s}, 2 \mathrm{H})$, that at $\delta 3.42$ is assigned to the $\mathrm{C} 1$ methylene 
protons and the doublet at $\delta 3.50$ to the methylene $\mathrm{H} 4 / 7$ protons in the remote cyclohexadiene ring. The resonances at $\delta 5.96$ and 7.95 are assigned to the olefinic H5/6 and aromatic protons $\mathrm{H} 2 / 9$, respectively. The ${ }^{13} \mathrm{C}$ n.m.r. data no longer display the enedione carbonyl resonances present in (160) and instead, the phenolate C3/8 carbon resonances are observed at $144.2 \mathrm{ppm}$; these are comparable to the $\mathrm{C}$-OMe resonances of ether (229) $\left(\delta\right.$ 150.0) [see Figs. 15 and 16 for ${ }^{1} \mathrm{H}$ n.m.r. and ${ }^{13} \mathrm{C}$ n.m.r. data, respectively, for the conversion of $(160) \rightarrow(234) \rightarrow(229)$ ]. When 1,4-dihydroxybenzene is treated with potassium carbonate under argon it, too, is converted into its red dipotassium salt (237) and the $\mathrm{C}^{-\mathrm{O}^{-}} \mathrm{K}^{+13} \mathrm{C}$ n.m.r. resonance is observed at $\delta 151$.

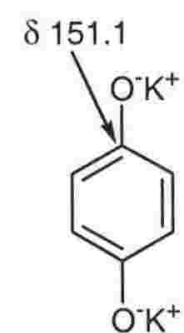

(237)

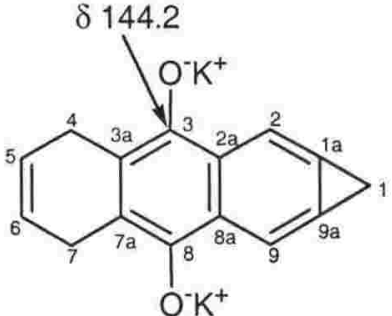

(234)

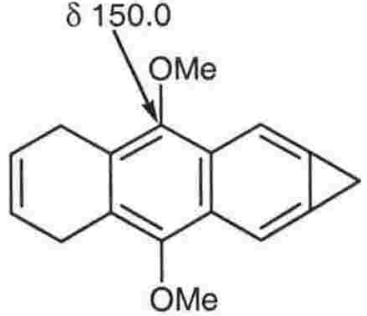

(229)

The solid proposed as diphenolate (234) is unstable in air and after $24 \mathrm{~h}$ its colour changes from purple to yellow with a concomitant increase in its solubility in common solvents. This process can be accelerated by suspending the solid in dichloromethane and bubbling oxygen gas through the mixture. Purification of the yellow solid by radial chromatography gives the dihydroquinone (230) in $41 \%$ yield together with a small amount of a second compound; recrystallisation gave an analytical sample of (230). As expected, the ${ }^{1} \mathrm{H}$ n.m.r. spectrum of (230) displays singlets for the $\mathrm{H} 1$ methylene and $\mathrm{H} 2 / 9$ aromatic protons and the ${ }^{13} \mathrm{C}$ n.m.r. spectrum shows the resonance of the latter at $\delta 112.7$. The quinone carbons are observed at $\delta 184.8$ and the respective stretching frequency is $1655 \mathrm{~cm}^{-1}$ in the IR spectrum. It should be noted that the ${ }^{1} \mathrm{H}$ n.m.r. resonances for (230) are identical to the minor component in the residue obtained from the purification of (160) on silica gel.<smiles>O=C1C2=C(CC=CC2)C(=O)c2cc3c(cc21)C3</smiles>

(230) 
(a)

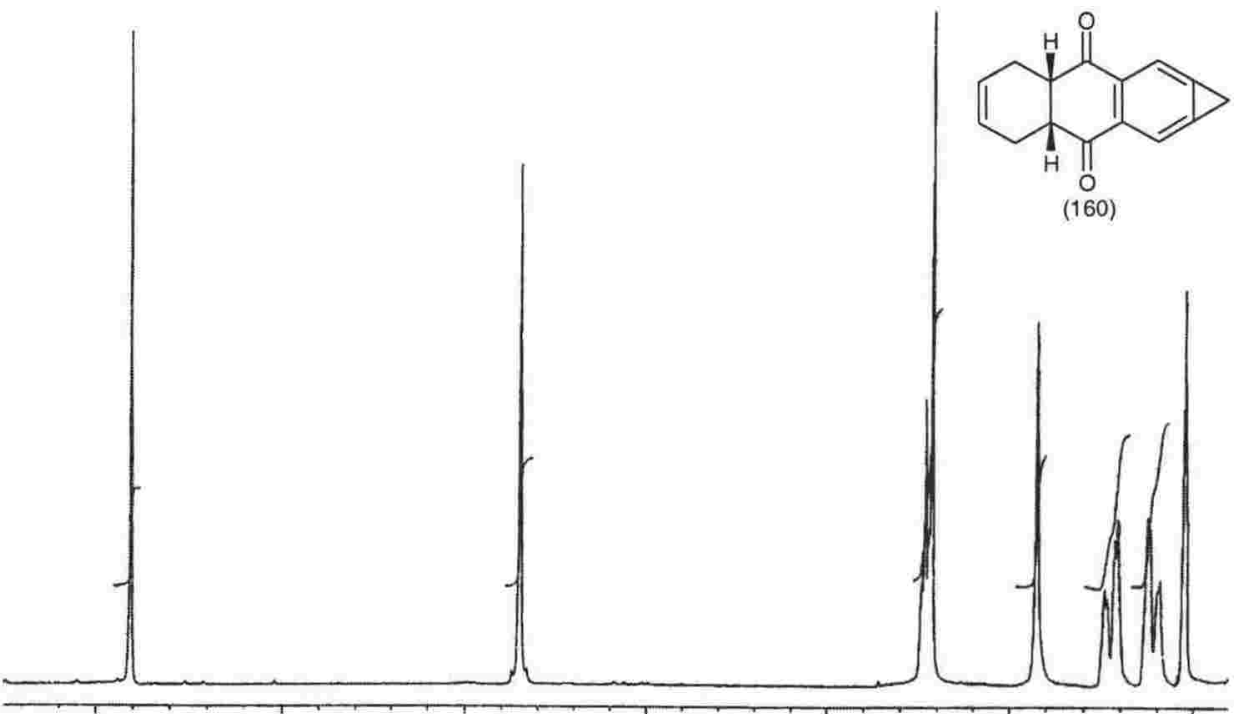

(b)

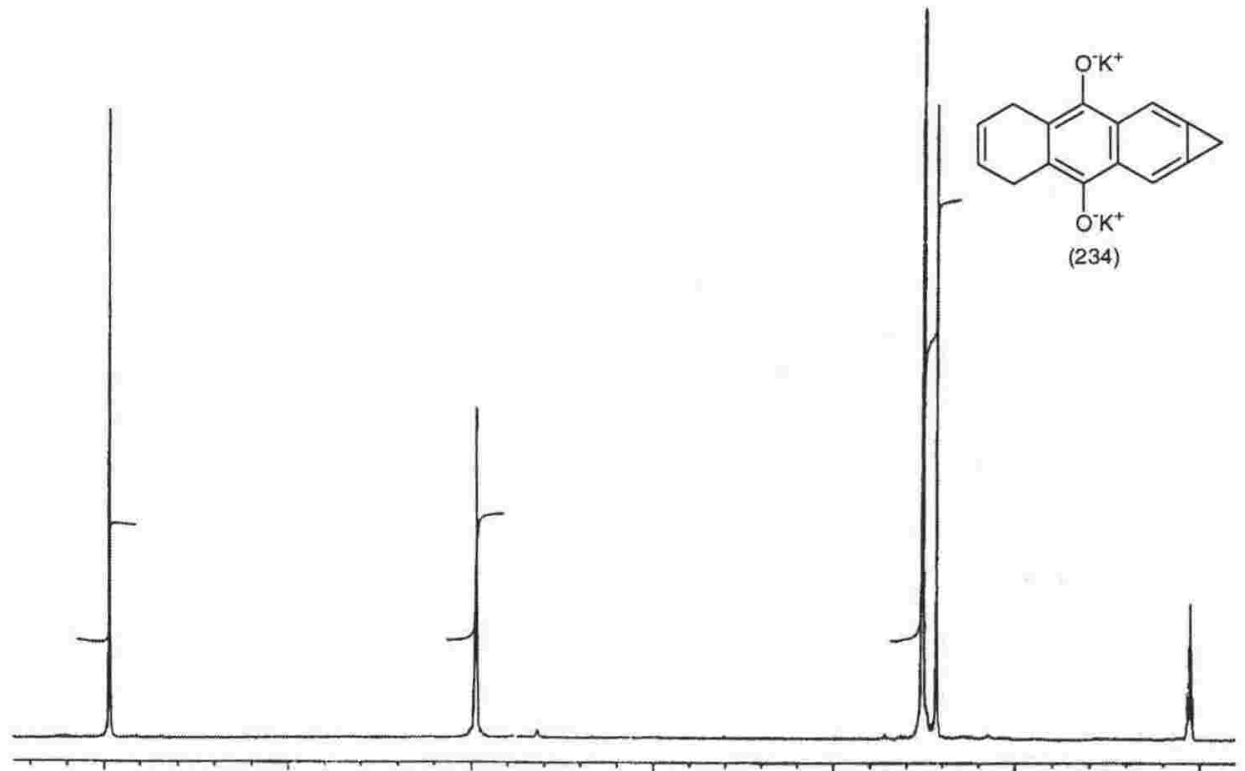

(c)

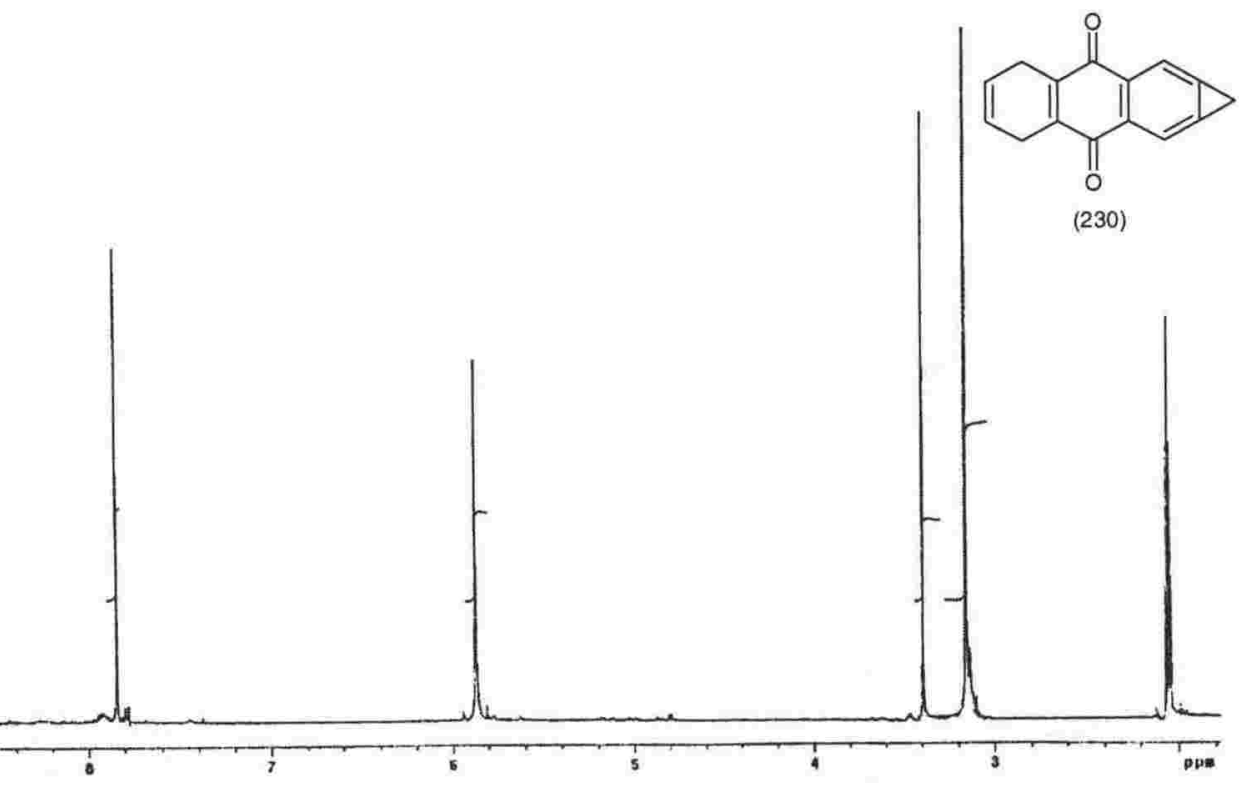

Fig. 15. ${ }^{1} \mathrm{H}$ n.m.r. spectra for compounds $(\boldsymbol{a})(160),(\boldsymbol{b})(234)$ and (c) (230). Spectra were recorded in $d_{6}$-acetone at $300 \mathrm{MHz}$. 
(a)<smiles>O=C1c2ccccc2C(=O)[C@@H]2CC=CC[C@H]12</smiles>

(160)

$\mathrm{C} 3 / 8$
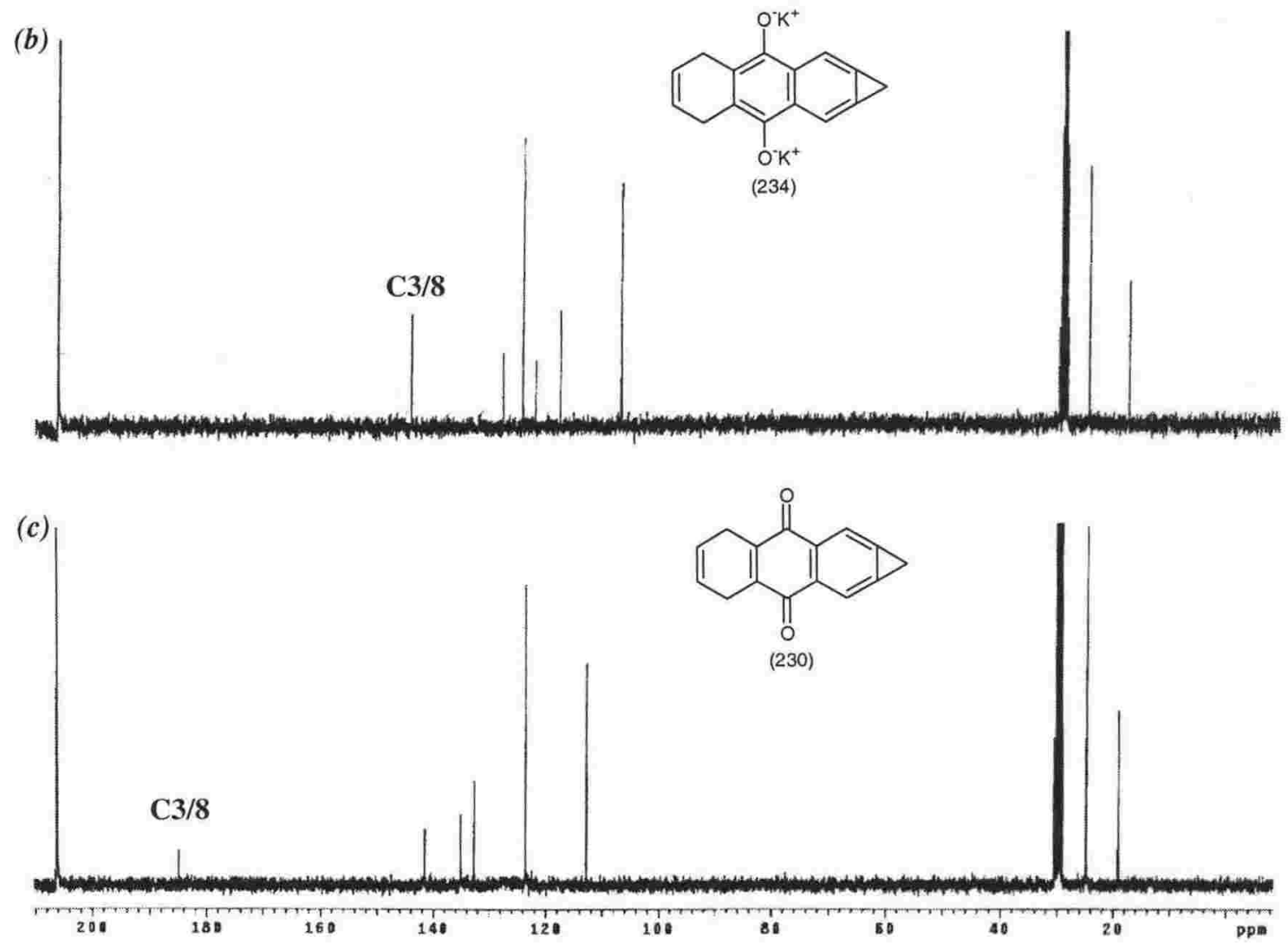

Fig. 16. ${ }^{13} \mathrm{C}$ n.m.r. spectra for compounds (a) (160), (b) (234), and (c) (230). Spectra were recorded in $d_{6}$-acetone at $300 \mathrm{MHz}$. 
Attempts to effect dehydrogenation of (160) with a base other than carbonate were explored. With pyridine a brown solid was obtained that showed ( ${ }^{1} \mathrm{H}$ n.m.r.) a small amount of (160) to be present along with many new signals in the aliphatic and aromatic regions of the spectrum. None of these corresponded to the quinone (230) and decomposition of either (160) or (230) seems likely. A similar outcome was obtained from (160) with potassium hydroxide. Therefore, it would appear that the dehydrogenation of (160) is best effected with potassium carbonate. However, it should be noted, that the reaction is extremely moisture sensitive and particular care is needed to ensure all reagents are strictly anhydrous; significant decomposition occurs even when the slightest traces of water are present and neither (160) nor (230) are isolated.

The minor component obtained from the purification of (230) on silica gel shows singlets in the ${ }^{1} \mathrm{H}$ n.m.r. spectrum at 3.55 and 8.14 ppm, and an AA'BB' system at 8.288.32 and 7.76-7.82 ppm; these integrate in a 1:1:1:1 ratio, respectively, and are consistent with cyclopropanthraquinone (162). It is possible therefore, that (230) is oxidised to quinone (162) on silica gel. In fact, storing (230) in the freezer for 17 days results in significant darkening. A re-examination of the solid by ${ }^{1} \mathrm{H}$ n.m.r. spectroscopy shows quinones (230) and (162) to be present in $\sim 9: 1$ ratio! This suggests that the aerial oxidation of dihydroquinone (230) to quinone (162) is reasonably facile.

In order to effect the conversion of (230) into (162) more efficiently, a solution of (230) was oxygenated with reaction progress being periodically monitored by ${ }^{1} \mathrm{H}$ n.m.r. spectroscopy. After 12 days only trace amounts of quinone (230) remained and conventional workup gave cyclopropanthraquinone $(162)^{119,156}$ as a yellow powder in $80 \%$ yield that gave yellow crystals (dichloromethane) which darkened on standing.

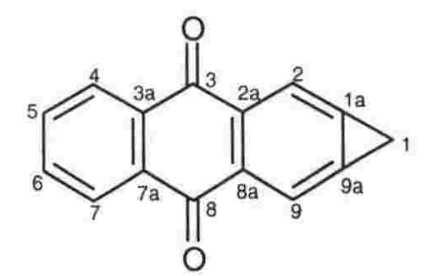

(162)

Cyclopropanthraquinone (162) provides spectral data (Figs. 17 and 18) that are fully consistent with its assigned structure. The ${ }^{1} \mathrm{H}$ n.m.r. spectrum shows the $\mathrm{C} 1$ methylene protons as a singlet at $\delta 3.42$ which is comparable to that of cyclopropanaphthoquinone (154) ( $\delta 3.36){ }^{116,118}$ The aromatic H2/7 protons are observed at $\delta 8.14$, a value somewhat more deshielded than for quinone (154) and the AA'BB' coupled system for $\mathrm{H} 4 / 7$ and $\mathrm{H} 5 / 6$ is observed at 8.31-8.34 and 7.79-7.81, respectively, almost identical with those measured for anthraquinone. This shows that the linear fusion 
of a three-membered ring to anthraquinone does not affect the ${ }^{1} \mathrm{H}$ n.m.r. chemical shifts of the protons most remote from the site of three-membered ring fusion.

Eight signals are observed in the ${ }^{13} \mathrm{C}$ n.m.r. spectrum which is nicely compatible with the anthraquinone (162). The $\mathrm{C} 1{ }^{13} \mathrm{C}$ n.m.r. resonance is observed at $\delta 19.2$ while $\mathrm{C} 2$ and C9 met with expectation ${ }^{35}$ and are shielded $(\delta 113.5)$. The carbonyl carbons are at $\delta$ 183.3 and are slightly more shielded than those of cyclopropanaphthoquinone $(154)(\delta$ 185.2) but are in excellent agreement with those of anthraquinone at $\delta 183.2$; the carbonyl stretching frequency for (162) is at $1668 \mathrm{~cm}^{-1}$ in the IR spectrum. The ${ }^{13} \mathrm{C}$ n.m.r. resonances for $\mathrm{C} 4 / 7$ and $\mathrm{C} 5 / 6$ are at $\delta 127.2$ and 134.0, respectively, and match well with those recorded for anthraquinone at 127.7 and 133.5 . The quaternary ${ }^{13} \mathrm{C}$ n.m.r. resonances at $\delta 132.8,133.1$, and 137.0 were assigned with confidence to $\mathrm{C} 1 \mathrm{a} / 9 \mathrm{a}, \mathrm{C} 3 \mathrm{a} / 7 \mathrm{a}$, and $\mathrm{C} 2 \mathrm{a} / 8 \mathrm{a}$, respectively, from use of gHMBC (gradient heteronuclear multiple bond connectivity) experiments. The chemical shift for $\mathrm{Cla} / 7 \mathrm{a}$ is comparable to that of the analogous carbons for quinone (154) $(\delta$ 132.5) whilst $\mathrm{C} 2 \mathrm{a} / 8 \mathrm{a}$ and $\mathrm{C} 3 \mathrm{a} / 7 \mathrm{a}$ have similar chemical shifts to the quaternary carbons at the sites of ring fusion for anthraquinone $(\delta 133.5)$. This shows that although the impact of three-membered ring fusion to anthraquinone is clearly felt in the cyclopropabenzenoid ring, it has little influence on the chemical shift values at the sites more remote than this.

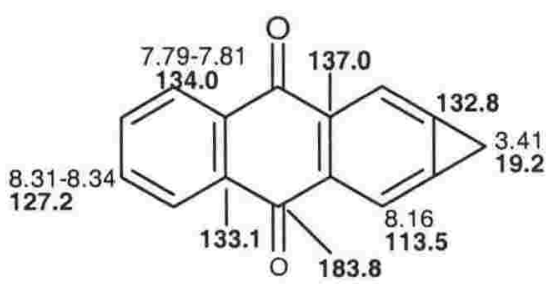

(162)

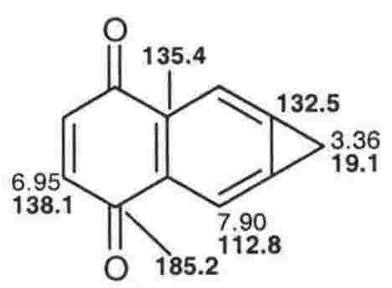

(154)

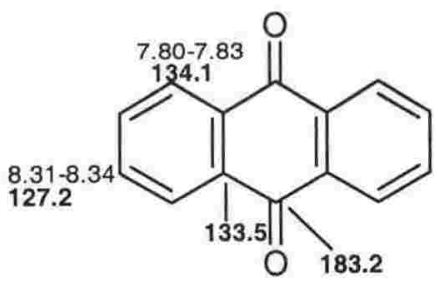

Fig. 17. ${ }^{1} \mathrm{H}$ and ${ }^{13} \mathrm{C}$ n.m.r. (bold) chemical shifts (ppm) for cyclopropanthraquinone (162), cyclopropanaphthalene (154), and anthraquinone. The ${ }^{1} \mathrm{H}$ n.m.r. data for (154) were taken from reference 116 . 


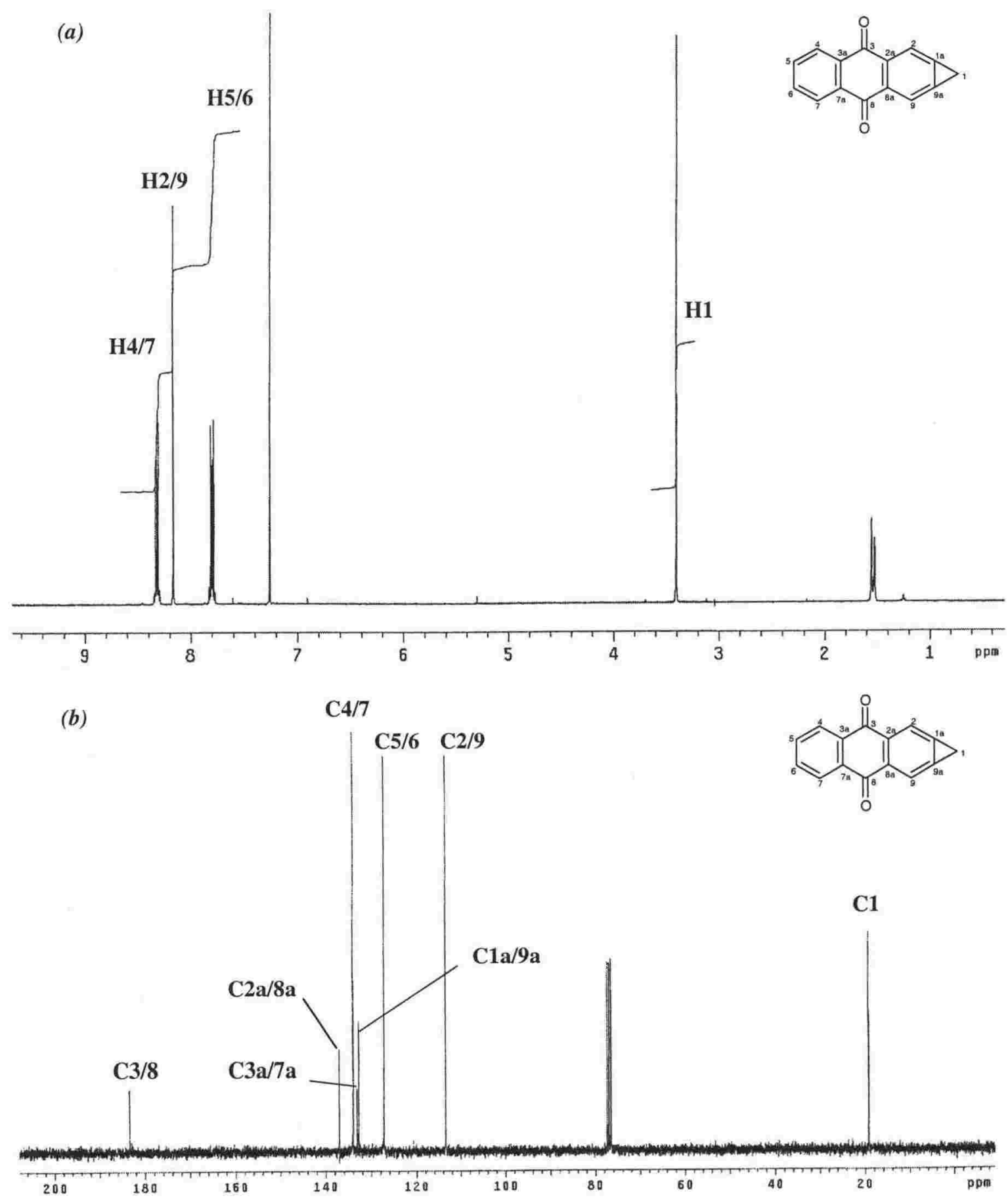

Fig. 18 (a) ${ }^{1} \mathrm{H}$ n.m.r. and $(\boldsymbol{b}){ }^{13} \mathrm{C}$ n.m.r. spectra for cyclopropanthraquinone (162). Spectra were recorded in $\mathrm{CDCl}_{3}$ at $300 \mathrm{MHz}$.

In order to ascertain whether the fusion of a three-membered ring to anthraquinone effects its redox characteristics a cyclic voltammogram of (162) was obtained and compared with that of anthraquinone measured under identical conditions. The first wave potential $\left(E_{1}\right)$ (which corresponds to the addition of one electron to the quinone) and the second wave potential $\left(E_{2}\right)$ (which corresponds to the addition of a second electron to produce the corresponding aromatic dianion) for anthraquinone are in good agreement with the literature ${ }^{155}$ and have an experimentally determined uncertainty of $\pm 3 \mathrm{mV}( \pm 3 \%)$. With $(162)$, the first $\left(E_{1}\right)$ and second $\left(E_{2}\right)$ potentials are almost identical to those for 
anthraquinone and are within $\pm 3 \%$ of the literature ${ }^{155}$ values for anthraquinone. It can be concluded therefore, that the linear fusion of a three-membered ring to anthraquinone has no significant effect on its redox characteristics. This behaviour contrasts with that of cyclopropaquinone (154) which was found to be slightly more difficult to reduce than 1,4naphthoquinone. The difference in the reduction potentials for the first and second waves for (154) was found to lie midway between those for 1,4-naphthoquinone and 9,10anthraquinone (Table 6). ${ }^{116}$

Table 6. Electrochemical reduction potentials for anthraquinone and cyclopropanthraquinone (162) in Volts.

\begin{tabular}{|c|c|c|c|c|}
\hline Compound & $E_{1}$ & $E_{2}$ & $E_{1}+E_{2}$ & $E_{I}-E_{2}$ \\
\hline 1,4-Benzoquinone ${ }^{a}$ (literature) & -0.50 & -1.41 & 0.91 & -1.91 \\
\hline 1,4-Naphthoquinone ${ }^{a}$ (literature) & -0.67 & -1.50 & 0.83 & -2.17 \\
\hline $\begin{array}{l}1 H \text {-Cyclopropa }[b] \text { naphthalene-3,6- } \\
\text { dione }(154)^{b}\end{array}$ & -0.72 & -1.49 & 0.77 & -2.21 \\
\hline 9,10-Anthraquinone (measured) & -0.97 & -1.68 & 0.71 & -2.65 \\
\hline 9,10 -Anthraquinone (literature) ${ }^{a}$ & -0.94 & -1.66 & 0.72 & -2.60 \\
\hline $\begin{array}{l}\text { 1H-Cyclopropa }[b] \text { anthracene- } 3,8 \text { - } \\
\text { dione }(162)\end{array}$ & -0.96 & -1.68 & 0.72 & -2.64 \\
\hline
\end{tabular}

${ }^{a}$ Data taken from reference 155 .

${ }^{b}$ Data taken from reference 116 .

Although quinone (162) is available from the oxygenation of a solution of (230) over 17 days, the conversion can also be effected by use of DDQ over 6.5 days (58\%). However, the reaction must be conducted at room temperature because even at $40^{\circ} \mathrm{C}$, significant decomposition occurs and a white solid is obtained that contains only trace amounts of (162). Unfortunately, all attempts to purify the solid and elucidate its structure were unsuccessful.

The oxidation of (230) can also be effected by use of potassium carbonate over $24 \mathrm{~h}$ and quinone (162) is obtained in $69 \%$ yield. However, if the reaction is left uninterrupted for $48 \mathrm{~h}$ a yellow oil is obtained ( $48 \%$ mass return) that contains a complex mixture of components with only trace amounts of cyclopropanthraquinone (162) $\left({ }^{1} \mathrm{H}\right.$ n.m.r.); a similar outcome is obtained from (230) with potassium hydroxide.

A mechanism that explains the formation of (162) from (230) and potassium carbonate is shown in Scheme 61. Base abstraction of an allylic proton followed by electron migration results in phenolate (238) which can then be protonated by bicarbonate. An analogous proton abstraction/reprotonation sequence from a remaining allylic centre would then give the bis-phenol. Quinone (162) can be expected upon aerial oxidation. 


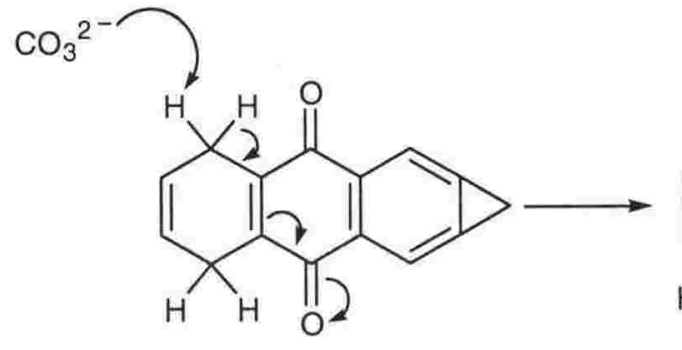

(230)<smiles>CC1C(=O)C2=CC=CCC2C([O-])=C2C=C3C1=CC32</smiles>

(238)<smiles>O=C1C2=CC=CCC2=C(O)c2cc3c(cc21)C3</smiles><smiles>O=C1c2ccccc2C(=O)c2cc3c(cc21)C3</smiles>

(162)

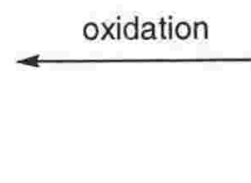

Scheme 61

With quinone (162) now available it would appear that it could be an appropriate synthon for novel alkylidenecyclopropaquinones, e.g. (164), in which the electron accepting quinone moieties polarise these compounds in an opposite sense to those containing methoxy functionality, e.g. (150). The metalation and silylation of (162) should provide either monosilane (239) or the disilane (240) that can be used for subsequent $\alpha$ silyl anion generation. Peterson olefination with an appropriate carbonyl-containing compound should then afford the desired methylidene compound (164) (Scheme 62).

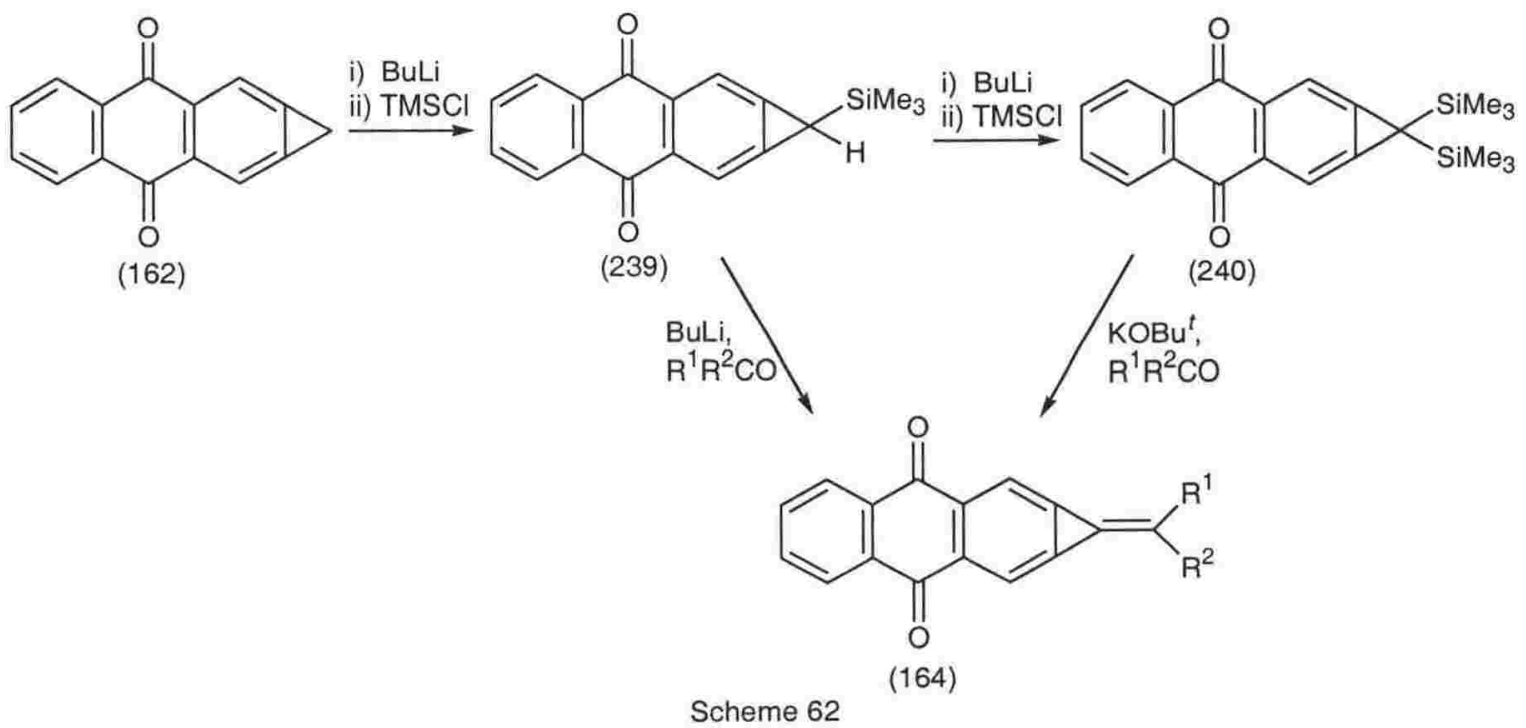

With a view to preparing the disilane (240) quinone (162) was treated with butyllithium then trimethylsilyl chloride using the methodology developed in these laboratories for the disilylation of cyclopropanaphthalene (58). ${ }^{24}$ Workup provided a yellow oil that contained a complex mixture of products $\left({ }^{1} \mathrm{H}\right.$ n.m.r. $)$ none of which corresponded to the monosilane (239), the disilane (240), or unchanged quinone (162). A similar outcome was obtained on treating (162) with butyllithium and the sterically 
congested $t$-butyldiphenylsilyl chloride. These results suggest that the failure to furnish the desired silanes likely results from competing reaction pathways involving the enedione as was the case for (154). Therefore, due to the lack of success in preparing the requisite silyl compounds (239) or (240) that are synthons to the methylidene compounds (164), it may be concluded that, like cyclopropanaphthoquinone (154), cyclopropanthraquinone (162) is not an appropriate synthon for methylidenecyclopropaquinones.

Although cyclopropanthraquinone (162) is now available in $\sim 2 \%$ yield from a seven step synthesis that commences with 1,4-benzoquinone, the compound did not prove to be a viable synthon for methylidenecyclopropaquinones (164). An alternative approach for synthesising (164) involves preparing the dimethoxymethylidene compounds (165) and oxidatively demethylating these with CAN (Scheme 63). The alkylidene compounds (165) should be available from treatment of the disilane (241) with potassium $t$-butoxide and subsequent Peterson olefination of the so-generated $\alpha$-silyl anion and an aldehyde or ketone. The disilane (241) itself should be available from the metalation and silylation of the unknown cycloproparene (163). As the attempted disilylation of the $\mathrm{C} 1$ centre of cyclopropanaphthoquinone (154) proved unsuccessful, but the analogous reaction with 3,8dimethoxycyclopropanaphthalene (70) afforded the desired disilylcyclopropanaphthalene (151) (Scheme 33, p. 26), it was hoped that preparation of the anthracene homologue (241) via this protocol would prove uneventful.<smiles>COc1c2ccccc2c(OC)c2cc3c(cc12)C3</smiles>

(163)

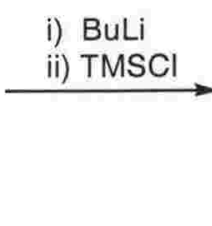

Scheme 63<smiles>[R7]C([R])=C1c2cc3c(cc21)C(=O)c1ccccc1C3=O</smiles><smiles>COc1c2ccccc2c(OC)c2cc3c(cc12)C3(C)C</smiles>

(241)

The synthon for preparing cycloproparene (163) is the dihydroether (229). However, due to the small amount of the dihydroether (229) available and our inability to prepare more of the compound, the above route for preparing (163) was not felt viable. Despite this, it was decided to determine whether (229) would dehydrogenate with DDQ to give dimethoxycyclopropanthracene (163). The reaction gave a yellow solid that was 
identified as cyclopropanthraquinone (162) (49\%) and not the sought after ether (163)! All of the spectral data for this solid were identical to those of the authentic samples of (162) already prepared. It seems likely that the ether (163) is initially formed but is readily oxidatively demethylated. By performing the reaction in an n.m.r. tube under an inert atmosphere it may be possible to gain evidence for ether (163). However, insufficient material was available to conduct this experiment.<smiles></smiles>

(229)<smiles>COc1c2ccccc2c(OC)c2cc3c(cc12)C3</smiles>

(163)

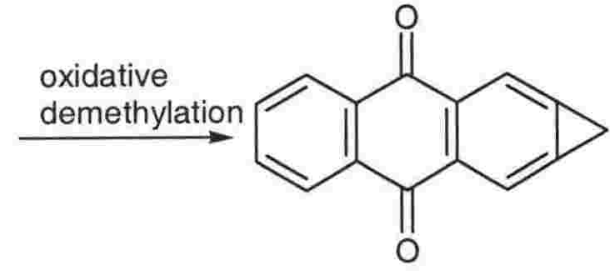

(162)

Due to the lack in success in preparing ether (163) by the above methodology an alternative route for preparing it was required; this is shown in Scheme 64. Thus, the cycloaddition of 1,4-naphthoquinone and butadiene should afford the known ${ }^{157}$ DielsAlder cycloadduct (242) which, after treatment with potassium carbonate/dimethyl sulfate should give the ether (243). ${ }^{158}$ The addition of dichlorocarbene to (243) is expected to afford the unknown gem-dichloro compound (244) which, after being treated with potassium $t$-butoxide may afford the 3,8-dimethoxy-1H-cyclopropa[b]anthracene (163) (Scheme 64).<smiles>CC1C=CC(=O)c2ccccc21</smiles><smiles>O=C1c2ccccc2C(=O)C2CC=CCC12</smiles>

(242)<smiles>COc1c2ccccc2c(OC)c2cc3c(cc12)C3</smiles>

(163)

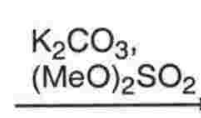<smiles>COc1c2c(c(OC)c3ccccc13)CC=CC2</smiles>

(243)

\section{Scheme 64}<smiles>[3H]C([3H])([3H])[3H]</smiles><smiles>COc1c2c(c(OC)c3ccccc13)CC1C(C2)C1(Cl)Cl</smiles>

(244)

Treatment of naphthoquinone with buta-1,3-diene at $75^{\circ} \mathrm{C}$ gives the known ${ }^{157}$ Diels-Alder adduct (242) in $75 \%$ yield which is easily converted into the dimethoxy ether $(243)^{158}(62 \%)$. Attempts to bring about the cheleotropic addition of dichlorocarbene to (243) from use of potassium hydroxide/chloroform and phase transfer catalyst were unsuccessful and (243) was recovered quantitatively. However, when sodium methoxide/ethyl trichloroacetate $\left(\mathrm{CCl}_{3} \mathrm{COOEt}\right)$ is used as source of dichlorocarbene, 
workup provides a red/brown solid, the analysis of which suggests $\left({ }^{1} \mathrm{H}\right.$ n.m.r.) that unchanged ether (243) and product (244) are present in a 1:1 ratio. Further treatment of the brown residue with dichlorocarbene under the same conditions results in the complete consumption of (243) and the hitherto unknown carbene adduct (244) is isolated in $62 \%$ yield. The ${ }^{1} \mathrm{H}$ n.m.r. spectrum of (244) shows an AMX spin system for the bridge and methylene protons in the cyclopropa-fused ring, an AA'BB' multiplet for the aromatic protons, the OMe protons resonate as a singlet at $\delta 3.90$. The olefinic protons of precursor (243) at $\delta 6.02$ are clearly absent. The ${ }^{13} \mathrm{C}$ n.m.r. spectrum of (244) shows nine signals with the dichloro $\mathrm{C} 1$ quaternary carbon deshielded and at $\delta 66.5$. The C-OMe ipso carbons are at $\delta 149.1$ and those for the $\mathrm{OCH}_{3}$ group are at $\delta 61.5$; these last two chemical shifts match well those of 3,8-dimethoxy-4,7-dihydrocyclopropanthracene (229) and 3,6dimethoxycyclopropanaphthalene (70). ${ }^{116}$

In an attempt to prepare the sought after cyclopropanthracene (163) the didehydochlorination of (244) was attempted with twelve equivalents of potassium $t$-butoxide at $0^{\circ} \mathrm{C}$. Workup gave a bright yellow solid that contained ( ${ }^{1} \mathrm{H}$ n.m.r.) singlets at $\delta 1.36,4.09$, 4.11, and 4.65 that integrated as 9:3:3:2. In addition to these singlets, two complex multiplets were observed in the aromatic region of the spectrum. The absence of a two proton methylene resonance between 3 and 4 ppm of the ${ }^{1} \mathrm{H}$ n.m.r. spectrum as expected ${ }^{35}$ for the $\mathrm{C} 1$ methylene protons of (163) and the shielded nine proton singlet at $\delta 1.36$, typical of a $t$-butoxy group, suggests that the three-membered ring is no longer intact and that the product is a ring-opened $t$-butoxy ether. Comparison of the spectral data with those of the known ${ }^{153}$ ether (245) confirms the two compounds as identical. The outcome of the didehydrohalogenation reaction is unchanged at $-25^{\circ} \mathrm{C}$ or even $-80^{\circ} \mathrm{C}$ and ether (245) is still isolated (49\%). At $-80^{\circ} \mathrm{C}$ with only two molar equivalents of base an $8: 1$ mixture of unchanged (244) and ring-opened (245) is obtained with no evidence for the desired cycloproparene (163).

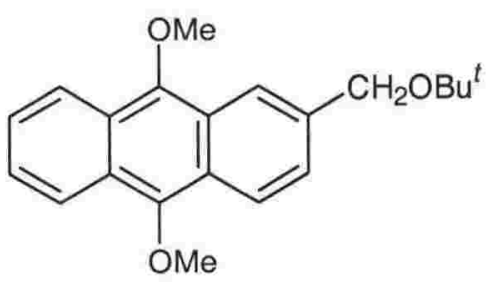

(245)

The formation of (245) can be accounted for as shown in Scheme 65. Thus the initial loss of hydrochloric acid followed by rearrangement (Scheme 13, p. 11) gives the intermediate (246). Subsequent abstraction of an allylic proton followed by electron migration then gives the ring-opened anion (247) which, after protonation, affords the halide (248). The solvolysis of (248) with $t$-butanol (or $\mathrm{Bu}^{t} \mathrm{O}^{-}$) then affords the ether (245) 
(Scheme 65). In analogy, earlier attempts ${ }^{66}$ to prepare the unsubstituted cyclopropanthracene (60) from the base induced didehydrochlorination of (72) were also unsuccessful and a mixture of the halide (75) and the $t$-butoxy ether (76) was furnished, see Scheme 15, p. 12. In the present case no evidence for the halide (248) was obtained at any time. This suggests that the alcoholysis of (248) is more facile than the unsubstituted anthracene (75). Presumably (248) is more susceptible to solvolysis as a result of electron donation by the two methoxy groups.<smiles>COc1c2c(c(OC)c3ccccc13)CC1C(C2)C1(Cl)Cl</smiles>

(244)

\section{$\underset{\mathrm{KOBu}}{\stackrel{\mathrm{K}^{t}}{-}-\mathrm{HCl}}$} ii) $\mathrm{H}$ transfer

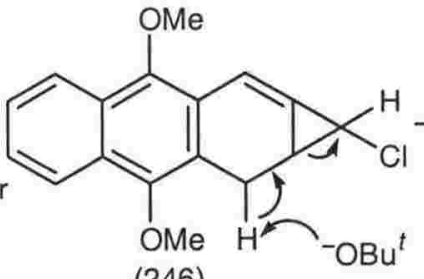
(246)<smiles>COc1c2ccccc2c(OC)c2cc([CH]Cl)ccc12</smiles>
$\mathrm{Bu}{ }^{t} \mathrm{OH}$<smiles>CCCOCc1ccc2c(OC)c3ccccc3c(OC)c2c1</smiles>

(245)

Scheme 65

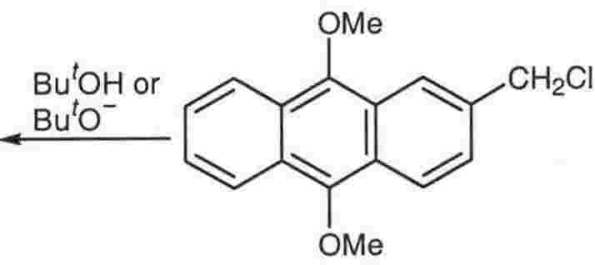

(248)

Due to the inability to prepare cycloproparene (163) from the didehydrochlorination of (243) with potassium $t$-butoxide it was decided to test whether (244) could be treated with CAN to afford the new quinone (249). The didehydrochlorination of this last compound may afford cyclopropanthraquinone (162) in a higher overall yield than that obtained from the seven step preparation that commences with 1,4-benzoquinone $(\sim 2 \%)$. When the diether (244) was treated with CAN the new quinone (249) was obtained in 50\% yield. The ${ }^{1} \mathrm{H}$ n.m.r. spectrum for (249) shows an AMX spin system for the bridge and methylene protons of the cyclopropa-fused ring and an AA'BB' spin system for the aromatics; the OMe protons in the synthon (244) are absent. The ${ }^{13} \mathrm{C}$ n.m.r spectrum shows eight resonances with $\mathrm{C} 1$ at $\delta 64.3$ and the carbonyl groups at $\delta 183.8$. The carbonyl stretching frequency of (249) is at $1660 \mathrm{~cm}^{-1}$ in the IR spectrum.<smiles>COc1c2c(c(OC)c3ccccc13)CC1C(C2)C1(Cl)Cl</smiles>

(244)<smiles>O=C1C2=C(CC3C(C2)C3(Cl)Cl)C(=O)c2ccccc21</smiles>

(249)<smiles>O=C1c2ccc([N+]([O-])([O-])[O-])cc2C(=O)c2cc3c(cc21)C3</smiles>

(162)

The availability of (249) provides for an alternative approach to quinone (162) by the dehydrohalogenation of (249) with potassium $t$-butoxide. Hence, reaction with an 
excess of the base gave a brown oil but only with low (20\%) mass return. Analysis of the oil showed it to contain a complex mixture of components ( ${ }^{1} \mathrm{H}$ n.m.r.) none of which corresponded to cyclopropanthraquinone (162) or unchanged quinone (249). The reaction was not examined further.

When the C1-dihalogenated cycloproparene (85a) is treated with butyllithium at low temperature carbene is generated which subsequently dimerises to afford the novel symmetrical alkylidene $(250) .{ }^{160}$ In view of this, it was hoped to prepare the $\mathrm{C} 1$ dichlorinated cycloproparenes (251) and (252) and their analogous methylidenecycloproparenes from subsequent carbene generation and dimerisation. When tetrahydrocycloproparene (244) was treated with DDQ workup provided a complex mixture of products ( ${ }^{1} \mathrm{H}$ n.m.r.) none of which corresponded to the halogenated compound (251); decomposition seems likely. As the $\mathrm{C} 1$ dichlorocycloproparenes are known ${ }^{35,84,85}$ to be moisture sensitive it is possible that (251) is generated but is too sensitive to survive workup; a similar outcome was obtained from quinone (249) with DDQ.<smiles>ClC1(Cl)c2c(-c3ccccc3)ccc(-c3ccccc3)c21</smiles>

(85a)<smiles>c1ccc(-c2ccc(-c3ccccc3)c3c2C3=C2c3c(-c4ccccc4)ccc(-c4ccccc4)c32)cc1</smiles>

(250)<smiles>COc1c2ccccc2c(OC)c2cc3c(cc12)C3(Cl)Cl</smiles>

(251)<smiles>O=C1c2ccccc2C(=O)c2cc3c(cc21)C3(Cl)Cl</smiles>

(252)

A final approach to dimethoxycyclopropanthracene (163) that could be used involves the addition of methylene to (243) followed by the subsequent aromatisation of the carbene adduct (253) with DDQ (Scheme 66).

When a solution of olefin (243) is added to methylene, the later generated by the addition of diethylzinc $\left(\mathrm{Et}_{2} \mathrm{Zn}\right)$ to diiodomethane in accord with the Simmons-Smith ${ }^{159}$ protocol, unchanged (243) is recovered in $85-100 \%$ yield. The outcome of this reaction remains unchanged even in refluxing ether. The addition of diazomethane to ether (243) also resulted in the recovery of (243) $(70 \%)$ but in this case a small amount of anthraquinone $(8 \%)$ was also isolated. These two compounds were the only characterisable products of reaction. Thus, due to difficulties in preparing the necessary carbene adduct (253), this route for preparing the cycloproparene (164) was not felt to be viable.<smiles>COc1c2c(c(OC)c3ccccc13)CC=CC2</smiles>

(243)<smiles>C#CCCCC</smiles><smiles>c1ccccc1</smiles>

(253)<smiles>COc1c2ccccc2c(OC)c2cc3c(cc12)C3</smiles>

(164)

Scheme 66 
In conclusion, cyclopropanthraquinone (162) has been prepared from both aerial oxygenation of quinone (230) and its treatment with either DDQ or potassium carbonate. ${ }^{119,156}$ The quinone synthon (230) itself was prepared readily by base induced dehydrogenation of the Diels-Alder adduct (160) via the diphenolate (234). Quinone (162) is the second stable cyclopropaquinone and only the seventh known cyclopropanthracene derivative known. Unfortunately, attempts to silylate (162) and ultimately prepare alkylidenecyclopropaquinones (164) have proved unsuccessful.

The 4,7-dihydroether (229) was prepared and characterised but all subsequent attempts to resynthesise it for further transformations proved unsuccessful. Treatment of the single sample of (229) with DDQ gave rise to cyclopropanthraquinone (162) and not the sought after cycloproparene (163).

Attempts to prepare the 3,8-dimethoxy ether (163) from the base induced didehydrochlorination of the gem-dichloro compound (244) were unsuccessful with opening of the three-membered ring occurring instead; the solvolysis product (245) was isolated. With quinone (249) and DDQ an uncharacterisable material was isolated in low mass return.

Attempts to prepare the $\mathrm{C} 1$ dichlorinated cycloproparenes (251) and (252) from (244) and (249) and DDQ proved unsuccessful. As other C1 dihalogenated compounds, e.g. ( $85 \mathrm{a})$ and $(85 \mathrm{~b})$, are known $\mathrm{n}^{35,84,85}$ to be highly sensitive to water it seems likely that compounds (244) and (249) are too sensitive to survive the workup conditions.

Finally, the addition of methylene to the ether (243) to give the carbene adduct (253) which could subsequently be aromatised was seen as an alternate route to the ether (163). Unfortunately, attempts to prepare the adduct (253) by Simmons-Smith ${ }^{159}$ protocol or from the addition of diazomethane were unsuccessful and unchanged starting material (243) was recovered in yields of $70-100 \%$. 


\section{Chapter 6}

\section{Approaches to Dithiole-Containing Cycloproparenes}

Tetrathiafulvene (TTF) (166) was first reported in the literature some thirty-one years ago. ${ }^{122}$ Since then this sulfur-containing compound and its derivatives have been the subject of numerous reviews ${ }^{124,161}$ and have attracted considerable attention from physicists and chemists alike. In its neutral form TTF is a non-aromatic $14 \pi$-electron system, however, it is readily oxidised $\left(E_{1} 0.37 \mathrm{~V}\right)$ to corresponding aromatic dithiolium cation (254). Subsequent oxidation $\left(E_{2} 0.67 \mathrm{~V}\right.$ ) of radical cation (254) then affords the heteroaromatic dication (255). ${ }^{161}$ The stability of these dithiolium ions is such that when TTF is treated with an appropriate $\pi$-electron acceptor, $e$.g. tetracyano-p-quinodimethane (256) or DDQ, charge-transfer (CT) complexes result. In the solid state these salts often pack either as two-dimensional sheets or in highly ordered stacks which are stabilised by non-bonded sulfur-sulfur and intermolecular $\pi-\pi$ interactions. ${ }^{124,161}$ Consequently, these materials have strongly anisotropic transport properties and often exhibit interesting electro-conduction properties including superconductivity. ${ }^{162}$<smiles>C1=CSC(=C2SC=CS2)S1</smiles>

(166)
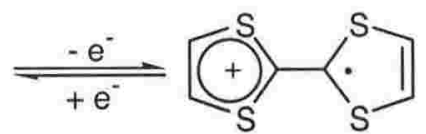

(254)
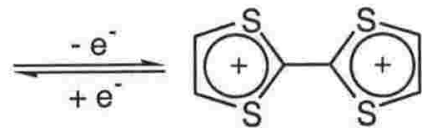

(255)

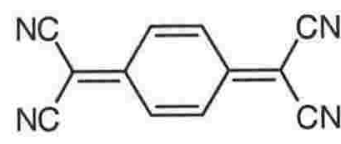

(256)

Much current attention focuses on modifying the TTF core in order to enhance its electron donating power and improve the conductivity of the variously derived CT salts. Usually, this is achieved by incorporating quinonoid, ${ }^{163-166}$ vinylogous, ${ }^{167,168}$ or hetero$\operatorname{aromatic}^{169,170}$ spacers between the dithiole units as illustrated by compounds (167), ${ }^{164}$ (257), ${ }^{168}$ and (258), ${ }^{170}$ respectively.

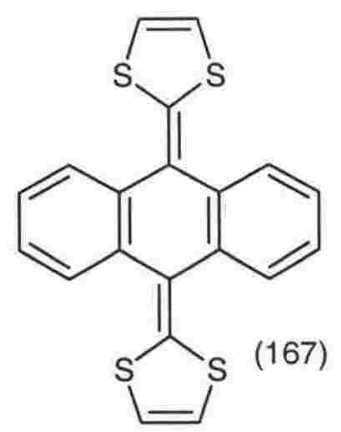

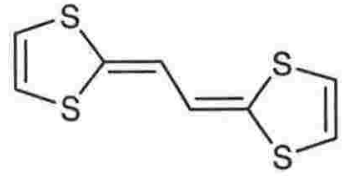

(257)

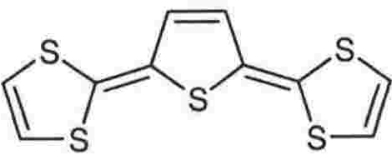

(258)

In contrast to the many examples of $\pi$-electron donors that carry at least two dithiole rings, few examples of compounds bearing just one dithiole unit are known $^{164,171,172}$ even though they form CT salts with comparable properties to those derived from TTF. ${ }^{171,172}$ For example, the thiophene derivative (259) ${ }^{172}$ and the 1,3 - 
dithiole-2-ylidenes $(260 \mathrm{a}-\mathrm{c})^{171}$ are found to have electron-donating abilities comparable to TTF (166).<smiles>C1=CSC(=C2c3ccsc3Sc3sccc32)S1</smiles>

(259)<smiles>[R]C1=C([R])SC(=c2c3ccccc3c3cccc4cccc2c43)S1</smiles>

An even rarer class of dithiole-containing compounds is that involving strained molecules. Nonetheless, the strained cyclophane $(261)^{173}$ and the crown ether $(262)^{174}$ are known and their structures have been determined unambiguously by X-ray crystallography. In each case, the chain that links the exocyclic sulfur atoms causes significant deformation to the component dithiole rings. Attempts to prepare CT salts from (261) have not been reported in the literature whilst attempts to form CT salts from (262) proved unsuccessful. ${ }^{174}$ Very recently, Bryce et al. ${ }^{175}$ described the synthesis of the strained cyclophane (263) that does form a CT salt upon complexation with the triiodide ion. To the author's best knowledge, this is the sole example of a CT salt derived from a strained dithiole-containing entity.

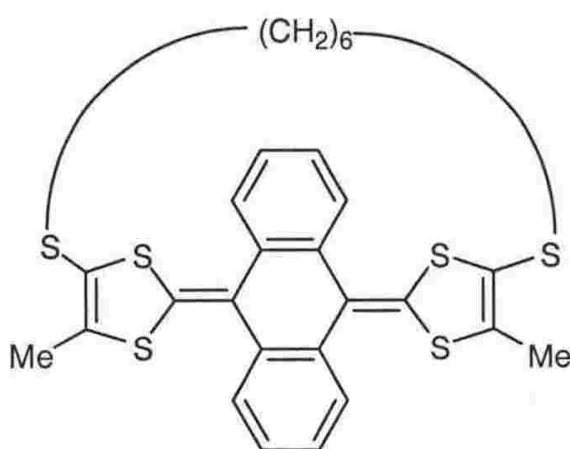

(261)

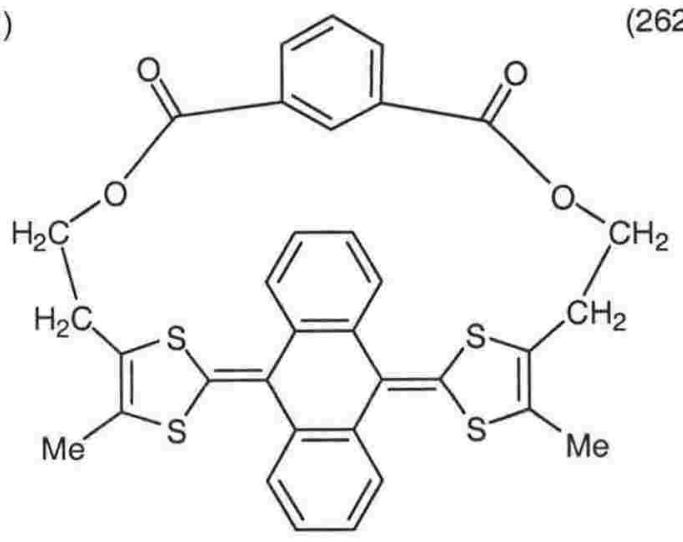

(263)

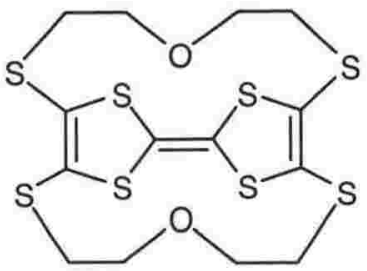

(262)

As few examples of strained dithiole-containing compounds are known, the impact that stress and strain has on the physicochemical properties of the dithiole moiety has yet 
to be established. Consequently, the aim of the present study has been to couple the chemistry of the strained cycloproparenes with that of dithiole-containing compounds in order to gain insight into how strain impacts upon the latter. Furthermore, it was also hoped that the study could furnish both the first examples of cycloproparenyl-CT salts and potentially interesting $\pi$-extended alkylidenecycloproparenes bearing at least one dithiole moiety.

A common method for incorporating an unsubstituted dithiole moiety into an organic molecule is by way of Wittig-Horner olefination of organolithium (264) with an appropriate carbonyl-containing compound. For example, when lithiate (264) is treated with only 0.5 molar equivalents of anthraquinone the dithiole-bearing products (167) and (265) are obtained in 62 and $10 \%$ yields, respectively, with the bis-adduct clearly the major product. $^{164}$ Consequently, analogous reactions of anion (264) with cyclopropanaphthoquinones (154) and (162) could be expected to afford then novel cross-conjugated dithioles $(168) /(266)$, and (169)/(267), respectively (Scheme 67).

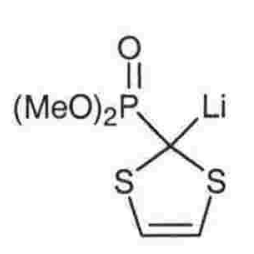

(264)

(1)
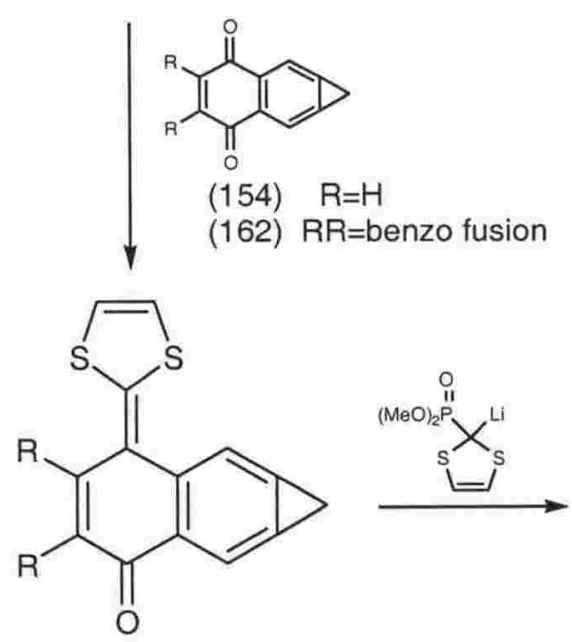

(266) $\mathrm{R}=\mathrm{H}$

(267) RR=benzo fusion<smiles>O=C1c2ccccc2C(=C2SC=CS2)c2ccccc21</smiles>

(265)

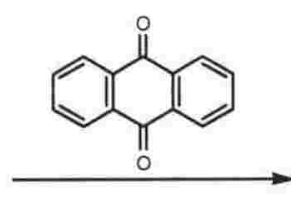

(1)<smiles></smiles><smiles>[R]c1c([2H])c(=C2SC=CS2)c2cc3c(cc2c1=C1SC=CS1)C3</smiles>

Scheme 67

The essential carbanion (264) for Wittig-Horner olefination is generated easily by metalation of the phosphonate ester (268) with butyllithium at low temperature. ${ }^{164}$ In turn, ester (268) is prepared from the commercially available vinylene trithiocarbonate (269) in four steps in an overall yield of $66 \%$ (Scheme 68). Thus, the methylation of (269) 
followed by the addition of tetrafluoroboric acid affords the known ${ }^{176}$ salt (270) in $90 \%$ yield. Subsequent reduction of (270) gives the malodorous $(271)^{177}(94 \%)$ which, after treatment with acetic anhydride and tetrafluoroboric acid, provides the dithiolium salt $(272)^{177}(89 \%)$. Finally, the reaction of (272) with trimethyl phosphite in the presence of sodium iodide furnishes synthon $(268)$ in $88 \%$ yield. ${ }^{164}$<smiles>S=c1sccs1</smiles>

(269)

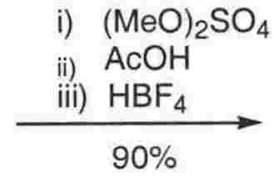

$90 \%$

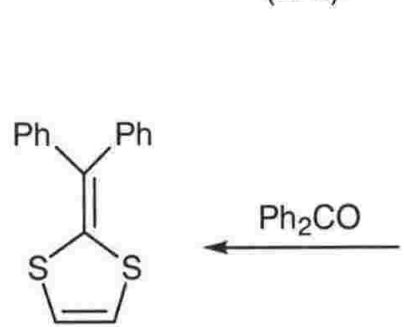

(273)

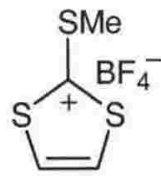

(270)

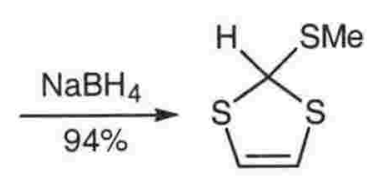

(271)

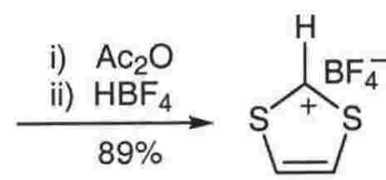

(272) $88 \%(\mathrm{MeO})_{3} \mathrm{P} / \mathrm{Nal}$<smiles>CO[PH](=O)C1(Cl)SC=CS1</smiles>

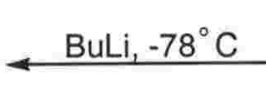<smiles>CO[PH](=O)C1SC=CS1</smiles>

(268)

Scheme 68

Before assessing the reaction of anion (264) with cyclopropaquinones (154) and (162), the known ${ }^{164}$ 2-(diphenylmethylidene)-1,3-dithiole (273) was prepared from interception of anion (264) with benzophenone in order to provide experience in handling the Wittig-Horner protocols. Dithiol-2-ylidene (273) was obtained in 74\% yield and this compares more than favourably with the literature value of $68 \% .^{164}$ Because of this interception of (264) with cyclopropaquinones (154) and (162) was investigated.

Several attempts to prepare the dithiol-2-ylidenes (168) and (266) from the reactions of (264) and 0.5 or 1 molar equivalents of cyclopropanaphthoquinone (154) gave a complex mixture of products and no evidence to support the formation of the sought-after products was gained. Moreover, there was no recovery of unchanged quinone (154) and the identity of the reaction products has not been resolved. It is possible that the failure to obtain compounds (168) and (266) is caused by electron transfer from the electron donating phosphonate anion (264) and acceptor quinone (154). Indeed, such reasoning ${ }^{178}$ has been suggested to account for the failure of the Wittig-Horner olefination of anion (264) with a 2,3-disubstituted naphthoquinone derivative that has a first reduction potential $\left(E_{1}\right)$ similar to that of quinone (154).

The failure to provide the dithioles (168) and (266) from Wittig-Horner olefination of anion (264) with cyclopropanaphthoquinone (154) triggered attempts to effect interception with cyclopropanthraquinone (162). In this case the reaction with a restricted amount of (162), viz. 0.5 molar equivalents, gave in $\sim 23 \%$ yield and as an unstable yellow powder, the bis-substituted cyclopropanthracene derivative (169) that bears two dithiole moieties. Although it was not possible to obtain an analytical sample of (169) the ${ }^{1} \mathrm{H}$ 
n.m.r. spectrum of the somewhat impure sample clearly showed an AA'BB' spin system $(\delta$ 7.68-7.73, 7.29-7.32) for the $\mathrm{H} 3 / 6$ and $\mathrm{H} 4 / 5$ protons and a two-proton singlet for $\mathrm{H} 2 / 9$ at $\delta$ 7.65. The formally non-equivalent olefinic protons of the dithiole moiety resonate as a broad singlet at $\delta 6.27$ and this compares well with that for the analogous protons of the parent anthracene derivative $(167)(\delta 6.30) .{ }^{164}$ Presumably, these protons are sufficiently remote from the sites of cyclopropa-fusion such that there is little effect on their magnetic environments. However, the most notable feature of the spectrum of (169) is the nonequivalence of the $\mathrm{C} 1$ geminal protons which resonate as two one-proton doublets ( $J$ $4.2 \mathrm{~Hz}$ ) at $\delta 3.14$ and 3.60 rather than the two-proton singlet characteristic of the planar cycloproparenes. In its lowest energy conformer compound (169) can be expected ${ }^{165,166}$ to adopt a non-planar, saddle-like conformation in which the two 1,3-dithiole rings assume a U-shape with the bridging ring such that the steric repulsions between the sulfur atoms and the peri hydrogen atoms of the anthracene moiety at C2/4 and C7/9 are minimised (Fig. 19). In turn, this causes the non-equivalence of the $C 1$ methylene protons. However, the molecule should be capable of boat-to-boat inversion such that $\mathrm{H}_{a}$ and $\mathrm{H}_{b}$ become equivalent. Clearly, the inversion at ambient temperature in $\mathrm{CDCl}_{3}$ solution is slower than the time scale of the n.m.r. instrument. By warming a solution of (169) in the spectrophotometer the aforementioned signals should coalesce as the energy barrier to inversion is approached. However, the non-availability of the necessary facilities to us prevented the energetics of the process from being examined.

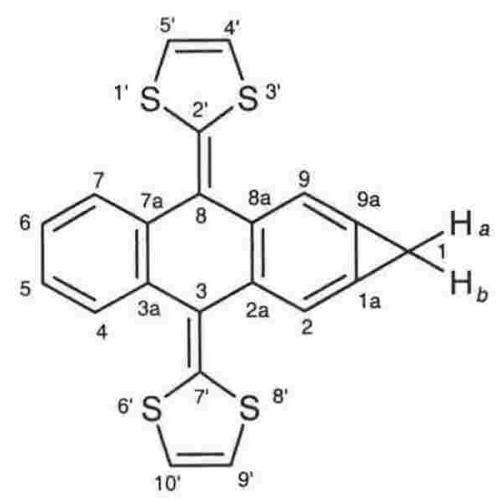

(169) 


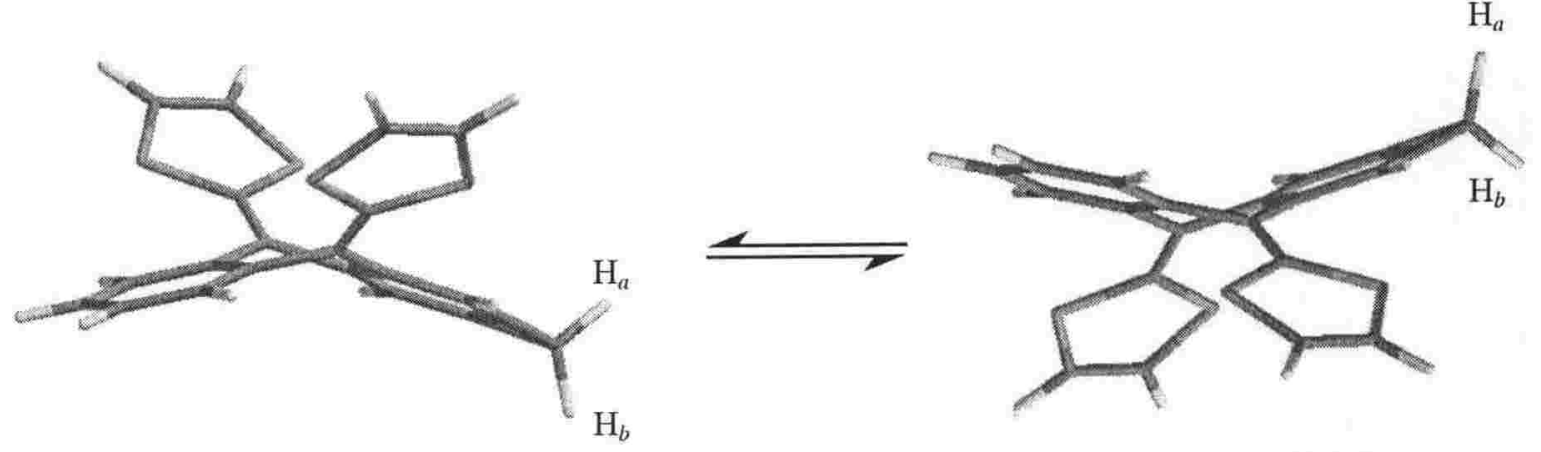

Fig. 19. Projected minimum energy conformers of dithiole cycloproparene (169).

The carbon n.m.r. spectrum of (169) clearly shows the equivalent methine carbon pairs of $\mathrm{C} 4 / 7$ and $\mathrm{C} 5 / 6$ at $\delta 124.8$ and 125.9 , respectively, while the non-equivalent olefinic C4'/5' carbons of the dithiole ring appear are nearly identical [ $\delta 117.0(3)$ and 117.0(5)]. These three resonances are essentially at the same chemical shifts as those in the parent anthracene analogue $(167)(\delta 124.9,125.9$, and 117.1). These data indicate that the linear fusion of a three-membered ring to (167) does not have any significant impact upon the magnetic environments of the carbon atoms remote from the sites of cyclopropa-fusion. However, the adjacent $\mathrm{C} 2 / 9$ carbons are shielded ( $\delta 113.1$ ), as expected, ${ }^{35,100}$ and the mass spectrum shows an $[\mathrm{M}+\mathrm{H}]^{+}$ion at $m / z 392.9890(\Delta-1.1 \mathrm{ppm})$ confirming the $\mathrm{C}_{21} \mathrm{H}_{12} \mathrm{~S}_{4}$ composition. As cycloproparene (168) is both acid and light sensitive and decomposes upon attempted purification [chromatography (alumina gel, basic) or recrystallisation] it was neither possible to obtain a pure sample nor to assess its chemistry further.

When phosphonate ester (264) is treated with a stoichiometric quantity of cyclopropanthraquinone (162) it is the mono 1,3-dithiol-2-ylidene derivative (267) that is obtained as the major product but only in $23 \%$ yield. The proton spectrum of (267) is fully compatible with its structure and the impact of the dithiole ring is notable upon $\mathrm{H} 2$ and $\mathrm{H} 9$ that resonate as well separated para-coupled doublets $(J 1.8 \mathrm{~Hz})$ at $\delta 8.10$ and 7.81 , respectively. The olefinic protons of the dithiole moiety resonate as a two-proton singlet ( $\delta 6.42)$ (Fig. 20$)$ as do the benzylic hydrogen atoms ( $\delta 3.39)$. In contrast to the non-planar saddle shape of the bis(1,3-dithiol-2-ylidene) derivative (169) that creates non-equivalent benzylic $\mathrm{H}_{a}$ and $\mathrm{H}_{b}$ atoms, (267) must invert rapidly in $\mathrm{CDCl}_{3}$ solution at room temperature. One must presume that the energy barrier for inversion is lower than that for the bis-dithiole derivative (169) because the planar transition state only has two perihydrogen atom sulfur contacts as opposed to the four of (169). 


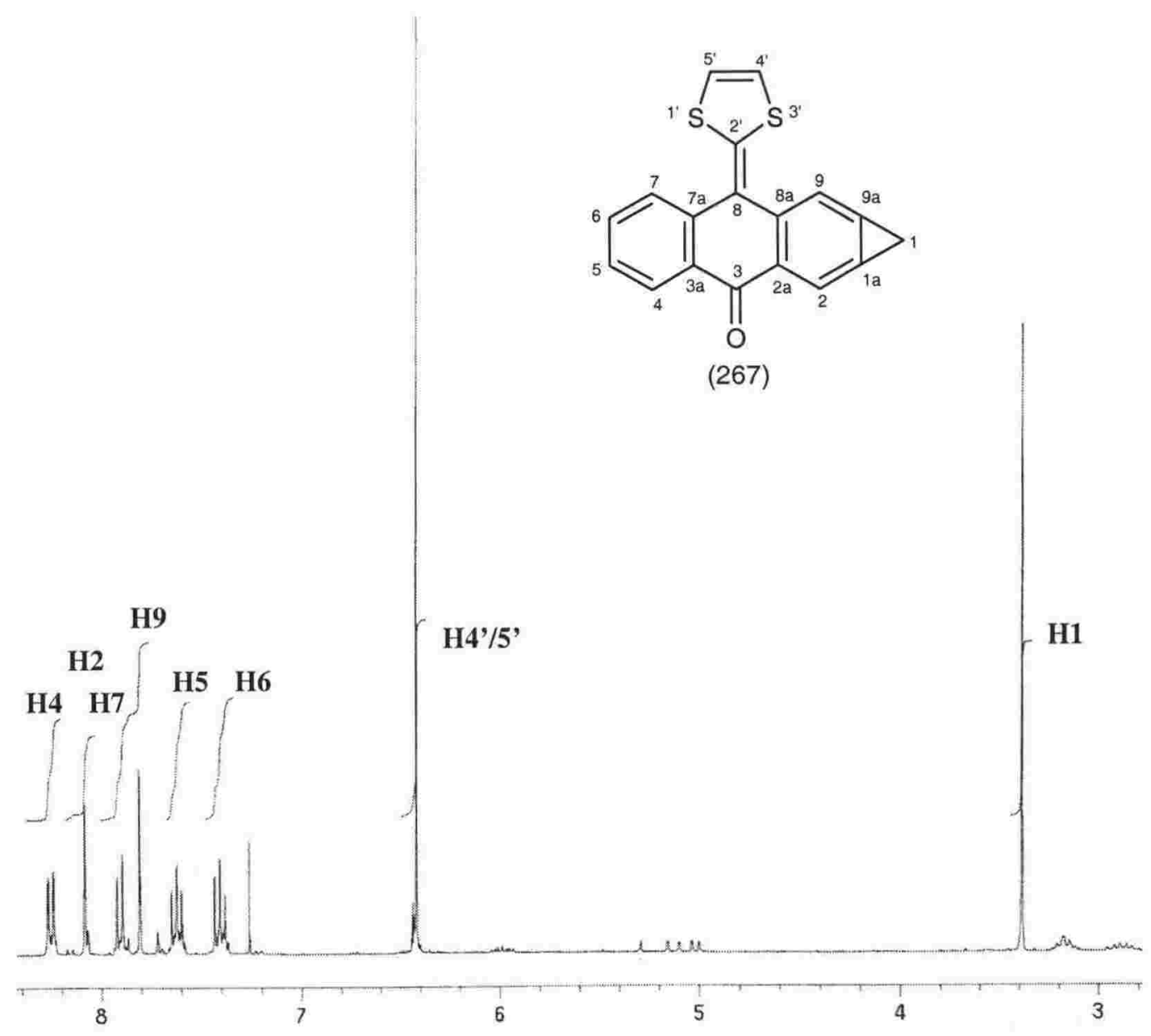

Fig. 20. Proton spectrum of the dithiole-containing cyclopropanthracene (267) $\left(\mathrm{CDCl}_{3}\right.$, $300 \mathrm{MHz}$ ).
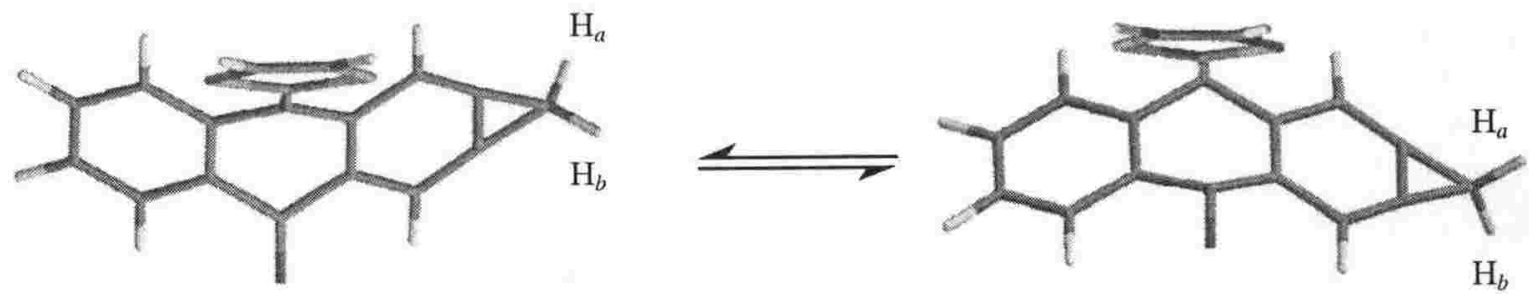

Fig. 21. Proposed minium energy of conformers of dithiole (267).

The carbon n.m.r spectrum of cyclopropanthracene (267) is also fully compatible with the assigned structure with the diagnostic $\mathrm{C} 2 / 9$ carbons adjacent to the threemembered ring shielded at $\delta 113.0 / 113.8$ while the mass spectrum shows a $[\mathrm{M}+\mathrm{H}]^{+}$ion at $m / z 307.0253\left(\Delta 2.4 \mathrm{ppm}\right.$ ) for $\mathrm{C}_{18} \mathrm{H}_{10} \mathrm{~S}_{2} \mathrm{O}$. Attempts to grow crystals of (267) suitable for a single X-ray analysis were made and, although these resulted in significant decomposition of the sample, a few analytical truncated cones were obtained. These were sent to Professor R. Boese in Essen (Germany) for analysis, but sadly, none of the crystals defracted at all. ${ }^{179}$ 
The first recorded dithiole-containing cycloproparenes (169) and (267) have limited stability in solution ( $\sim 1$ day) and slowly decompose when exposed to light ( 1 week). Because of this an examination of their physicochemical properties, including the formation of CT salts, was not deemed viable in the limited time available time of this $\mathrm{PhD}$ study. Therefore, efforts were directed towards preparing other dithiole-bearing cycloproparenes in the hope that they would be more stable and hence more amenable to study. As the carbonyl-containing methylidenecycloproparene (200) was prepared in the earlier part of this study and is now readily available (Scheme 46, p. 57) it was used to prepare the $\pi$-extended dithiole-2-ylidene (274) via Wittig-Horner olefination with phosphonate ester (264) (Scheme 69).

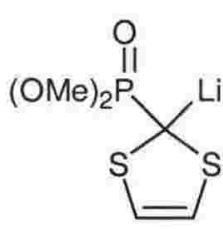

(264)

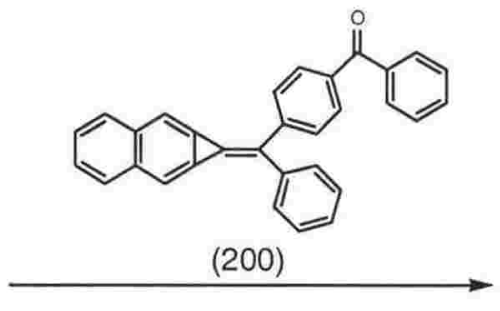

Scheme 69

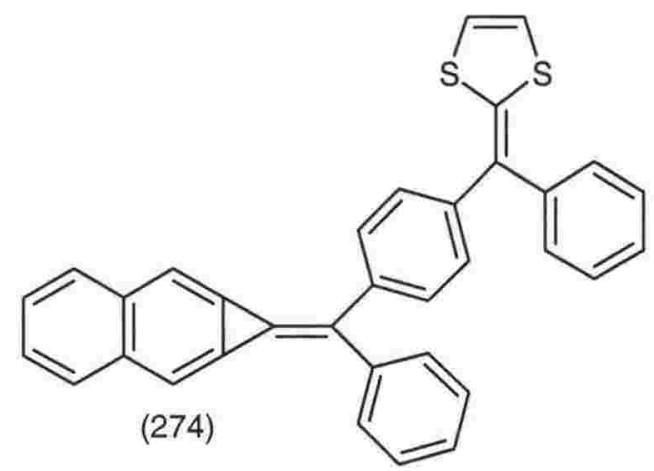

(274)

In the event, treatment of lithiate (264) with ketone (200) gave an analytical sample of methylidene compound (274) in excellent $66 \%$ yield as a bright powder that is both light and acid sensitive. Moreover, the compound occludes solvent but its structure is secure since the aromatic region of the proton n.m.r. spectrum integrates for twenty protons of which a single one-proton para coupled doublet $(\delta 7.54, J 1.8 \mathrm{~Hz})$ for either $\mathrm{H} 2 \mathrm{or} \mathrm{H} 7$ is clearly visible and a two-proton AA' component of an AA'BB' spin system $(\delta 7.86-7.90)$ for $\mathrm{H} 3 / 6$ is also evident. In addition, the two non-equivalent olefinic protons for the now incorporated dithiole ring resonate as doublets $(J 6.6 \mathrm{~Hz})$ at $\delta 6.24$ and 6.28 . The carbon n.m.r. spectrum of (274) displays twenty-five resonances with the $\mathrm{C} 2 / 7$ carbons shielded $[\delta$ 107.0(6)/107.1(6)] while C1 and the C3/6 methine carbons are slightly more shielded than the analogous carbons of diphenylmethylidene (114l, p 22), see Table 7. The dithiole moiety can mesomerically donate $\pi$-electrons into the cyclopropanaphthalenyl ring such it is more anionic than the unfunctionalised diphenyl analogue (1141). If this is true it accounts for the higher sensitivity of this methylidenecycloproparene to acid than (1141). 
Table 7. Selected ${ }^{13} \mathrm{C}$ data $(\delta)$ of diphenylmethylidene $(1141)^{24}$ and its dithiole-containing analogue (274) $\left(\mathrm{CDCl}_{3}, 300 \mathrm{MHz}\right)$.

\begin{tabular}{ccc}
\hline Carbon & $(1141)$ & $(274)$ \\
& $\delta$ & $\delta$ \\
\hline 1 & 112.0 & 111.8 \\
$2 / 7$ & 107.3 & $107.0(6) / 107.1(6)$ \\
$3 / 6$ & 128.8 & $126.7(0) / 126.7(2)$ \\
$4 / 5$ & 126.7 & 126.7 \\
8 & 120.8 & 119.5 \\
\hline
\end{tabular}
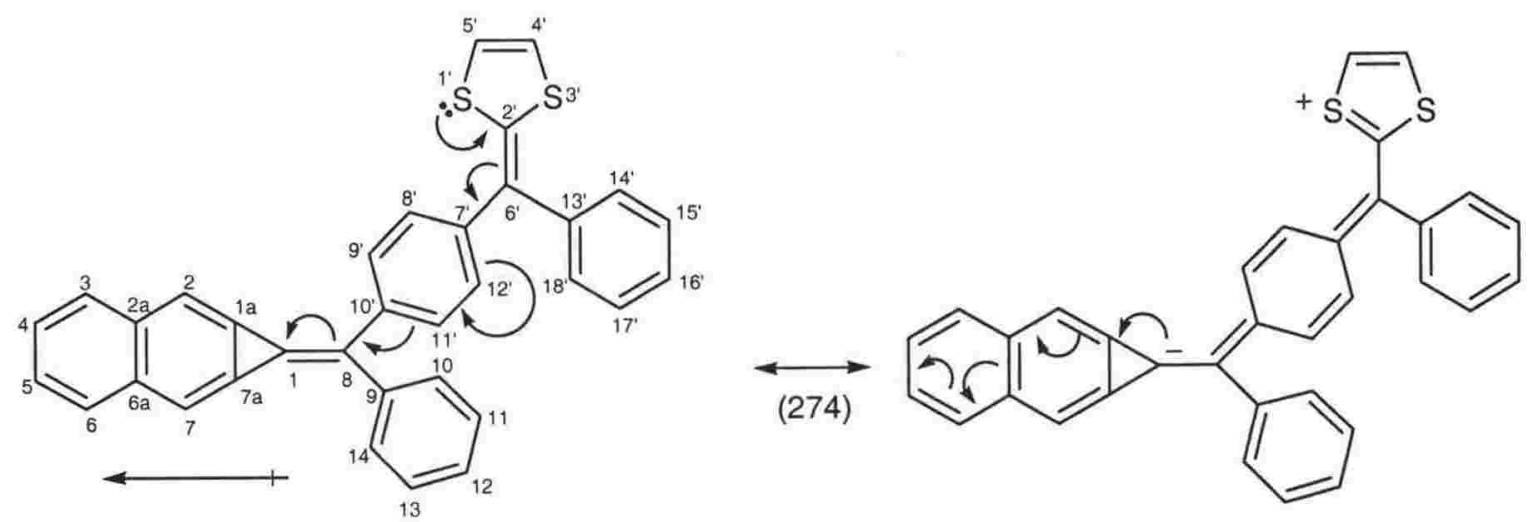

The good yield in which (274) was obtained provided sufficient material for a preliminary study of its ability to act as a donor and form a CT salt with an appropriate electron acceptor. In the event, an acetonitrile solution of (274) gave an instantaneous colour change from red/yellow to dark green when two molar equivalents of DDQ were added. The electronic absorption spectrum of the green solution showed that the absorption bands of donor (274) and acceptor DDQ had been replaced by new absorptions (Table 8). However, most notable was the appearance of a broad, weak absorption band between 540 to $620 \mathrm{~nm} \mathrm{viz}$. at longer wavelengths than the absorption maxima of the individual components and this typifies ${ }^{180}$ intermolecular CT transition. Unfortunately, all attempts to isolate the solid CT complex were unsuccessful and it is presumed to have decomposed since upon standing in acetonitrile at either room temperature or at $-16^{\circ} \mathrm{C}$ for $16 \mathrm{~h}$ it became brown.

Table 8. Electronic absorption data for DDQ, (274), and (274) + DDQ (1:2) ${ }^{a}$.

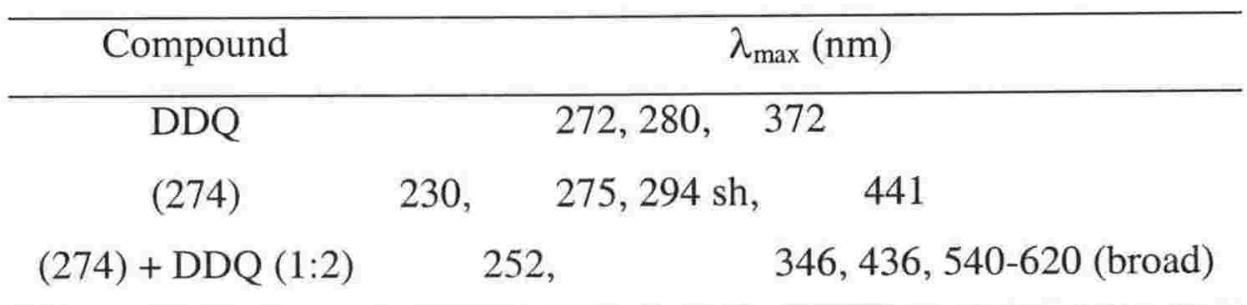

${ }^{a}$ Spectra were recorded on a Hewlett-Packard 8452A Diode array spectrophotometer for an acetonitrile solution. 
Because evidence was obtained to support the formation of a DDQ/CT complex derived from cycloproparene (274), efforts to prepare a range of other dithiole-bearing alkylidenecycloproparenes were made in the hope that a stable and isolable CT salts may ensue. An alternative strategy for preparing such novel $\pi$-electron donors involves the Peterson olefination of the $\alpha$-silyl anion (106) with a carbonyl-containing compound that already had a dithiole moiety incorporated, e.g., by treatment of cycloproparenyl anion (106) with the known 1,3-dithiol-2-ylidene (265) the linear $\pi$-extended alkylidenecycloproparene (275) should result (Scheme 70).

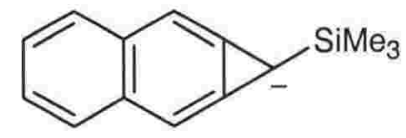

(106)

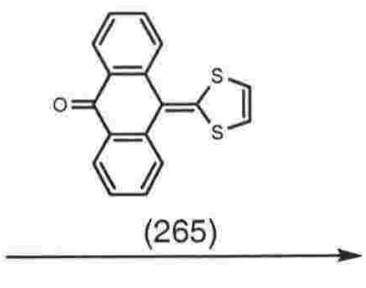

Scheme 70

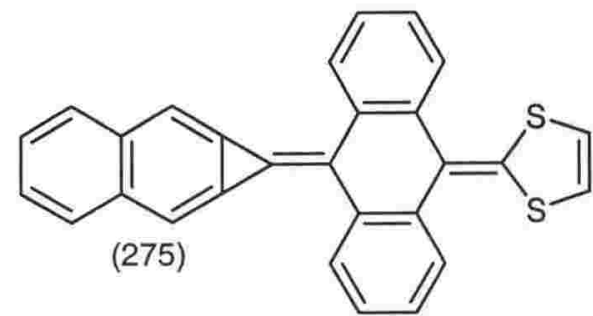

(275)

Synthon (265) has already been described and is available from the Wittig-Horner olefination of phosphonate ester (264) and anthraquinone but unfortunately it is obtained in only low (10\%) yield. ${ }^{164}$ However, an alternative synthesis employs the dithiolium salt (170) and anthrone. Upon refluxing the substrates in pyridine and acetic acid for $30 \mathrm{~min}$ the sought after (265) is obtained in $85 \%$ yield (Scheme 71 ). ${ }^{181}$ This reaction in these laboratories gave the dithiol-2-ylidene (265) in $79 \%$ yield.

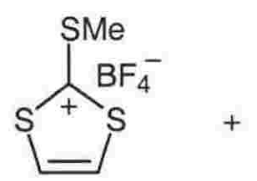

(170)<smiles>O=C1c2ccccc2Cc2ccccc21</smiles>

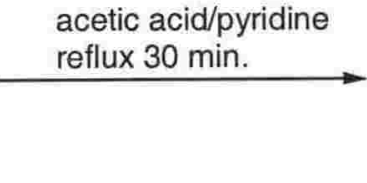

Scheme 71<smiles>O=C1c2ccccc2C(=C2SC=CS2)c2ccccc21</smiles>

265)

Treatment of ketone (265) with the $\alpha$-silyl anion (106) gave a complex mixture of products. Separation of these by chromatography and collection of the most mobile component gave a red residue which, after crystallisation, gave an analytical sample of the sought after alkylidenecycloproparene (275) as dark red/black needles but only in $3 \%$ yield. Subsequent experiments provided (275) in yields that varied from 1-6\%. The proton n.m.r. spectrum of (275) is fully compatible with its structure as it shows a fourproton AA'BB' spin system ( $87.89-7.92 / 7.47-7.50)$ for the aromatic $\mathrm{H} 3 / 6$ and $\mathrm{H} 4 / 5$ protons that is compatible with the molecular symmetry. Indeed, H2/7 and the olefinic methine protons of the dithiole ring resonate as two-proton singlets at $\delta 7.65$ and 6.28 , respectively, while the carbon n.m.r. spectrum contains the characteristically ${ }^{35,100}$ shielded 
C2/7 signals at $\delta 107.8$. The low yields of (275) precluded an examination of CT salt formation. However, it was possible to obtain crystals of (275) which were flake-like but appeared suitable for a single crystal structural analysis. Preliminary results from Essen, Germany, show that (275) packs in highly ordered sheet-like arrays (Fig. 22). ${ }^{179}$ Unfortunately, the crystals were too flake-like and thin to provide the quality needed for an accurate data set for detailed analyses. Nonetheless, the preliminary data are adequate to assess the crystal packing despite the obvious deficiencies in the intermolecular bond lengths and angles. ${ }^{179}$
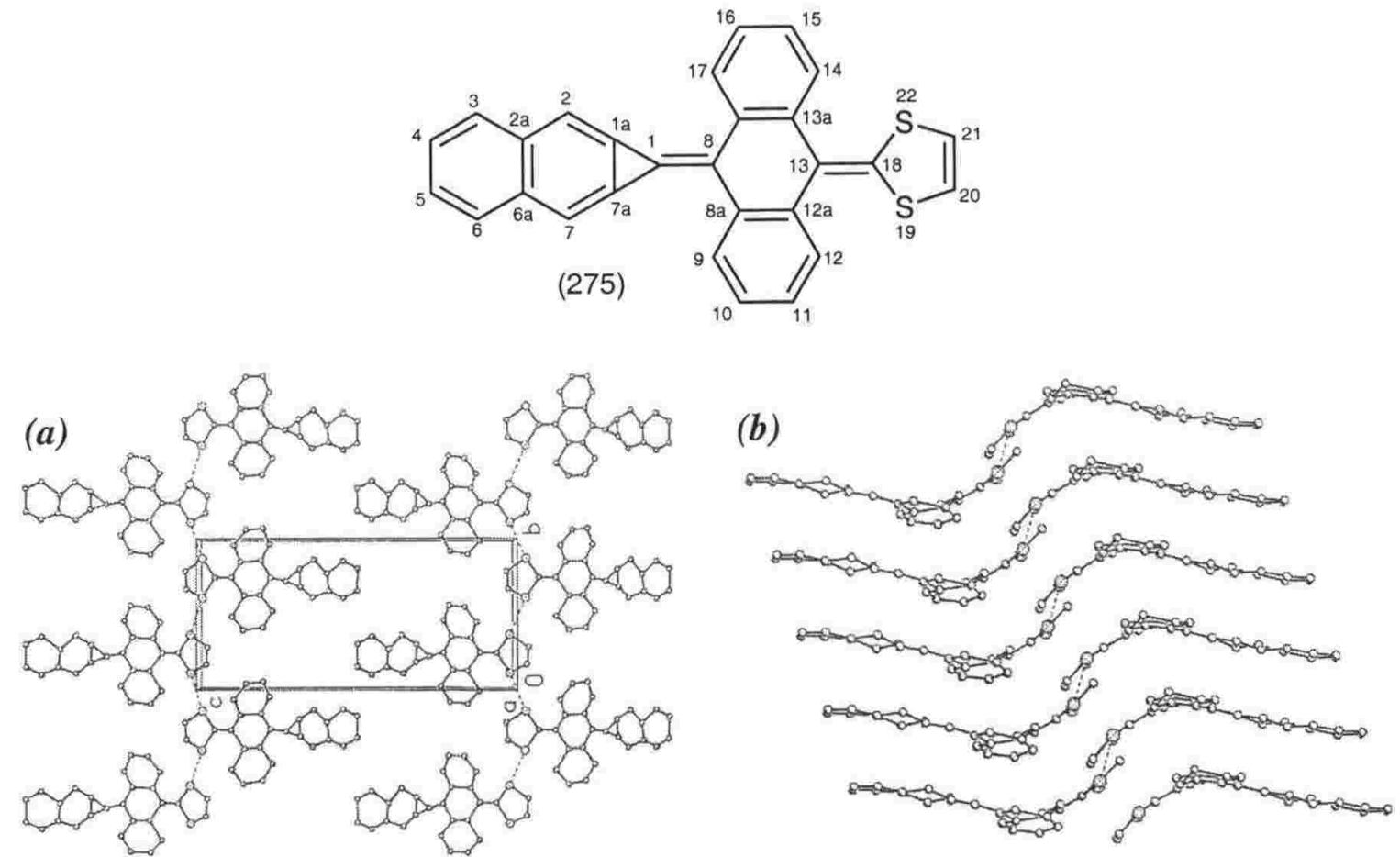

Fig. 22. Alternate views of the sheet-like packing array of (275) in its crystalline form (a) view of the array in which compound (275) packs and $(\boldsymbol{b})$ an alternative view of the packing of (275) ( $\mathrm{S} \cdots \mathrm{S}$ contacts shown as broken lines).

The availability of 1,2-diphenylethanone augured well for the formation of the hitherto unknown dithiole-bearing ketone (276) (Scheme 72). Peterson olefination of this last compound with $\alpha$-silyl anion (106) could well then afford the novel dithiolecontaining methylidenecycloproparene (277). 


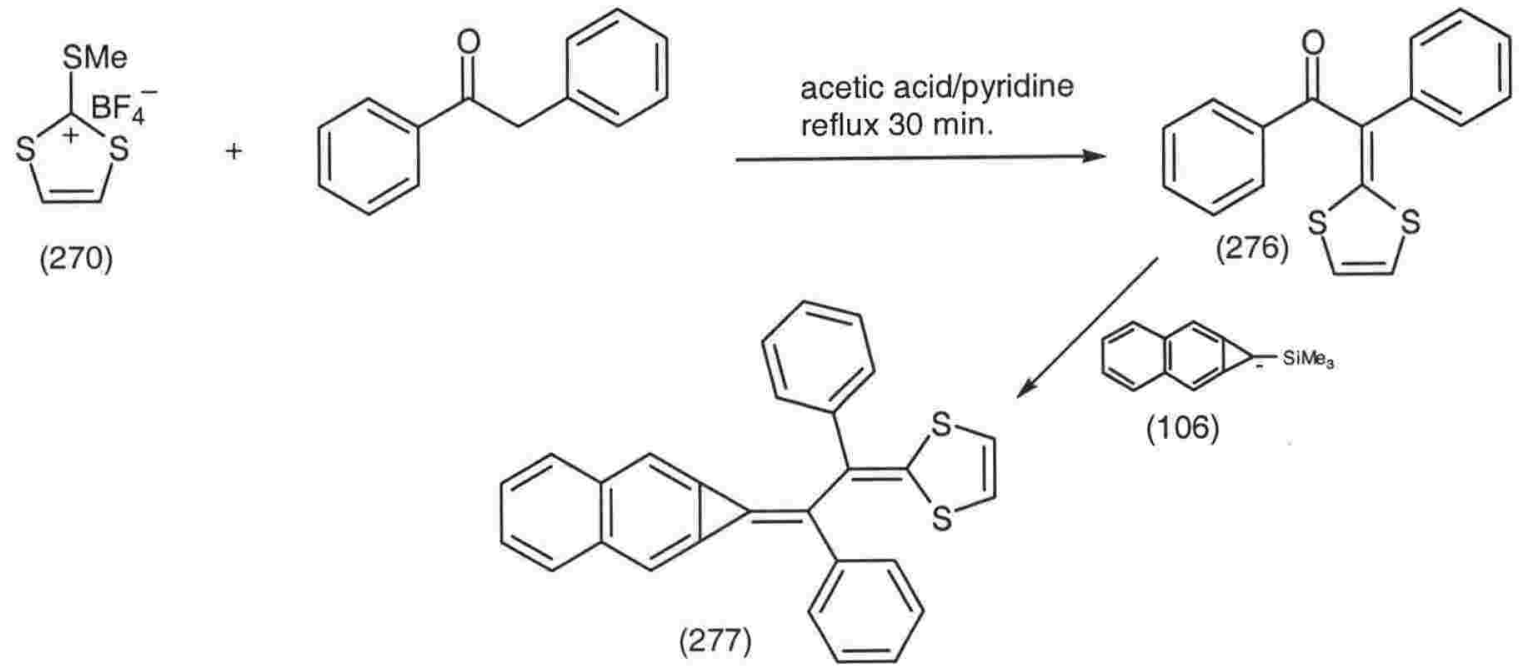

(277)

Scheme 72

The reaction of (270) with 1,2-diphenylethanone under the conditions described previously gave a 1:1 mixture of unchanged ketone and the desired product (276) as evidenced by ${ }^{1} \mathrm{H}$ n.m.r. spectroscopy. Resubjection of this residue to salt (270) resulted in the complete consumption of the ethanone and the formation of the new dithiol-2-ylidene (276) that was subsequently isolated in $90 \%$ yield; all spectral data were in agreement with the assigned structure (Experimental). Treatment of ketone (276) with the $\alpha$-silyl anion (106) gave the sought after $\pi$-extended ethylidenecycloproparene as a bright red oil that is acid and light sensitive and decomposes on alumina gel (basic). Nonetheless, an analytical sample of (277) was eventually obtained in $13 \%$ yield. The proton n.m.r. spectrum of (277), although complex, shows the olefinic protons of the dithiole ring as two one-proton doublets $(J 6.7 \mathrm{~Hz})$ at $\delta 6.26$ and 6.31 while a one-proton para-coupled doublet $(J 1.6 \mathrm{~Hz})$ at $\delta 7.68$ can only be due to either $\mathrm{H} 2$ or $\mathrm{H} 7$ and a four-proton multiplet $(\delta 7.82-7.92)$ clearly contains a distorted AA' component of an AA'BB' spin system. The carbon spectrum of (277) contains twelve methine resonances as required with the non-identical $\mathrm{C} 2 / 7$ resonating at $\delta 107.7 / 109.0$. A high-resolution mass measurement shows $[\mathrm{M}+\mathrm{H}]^{+}$at $\mathrm{m} / \mathrm{z} 419.0925(\Delta 0.4 \mathrm{ppm})$ as required for $\mathrm{C}_{28} \mathrm{H}_{18} \mathrm{~S}_{2}$. Once again this dithiole derivative was unstable and this, complicated by the low yield of formation, precluded an examination of its physicochemical properties.

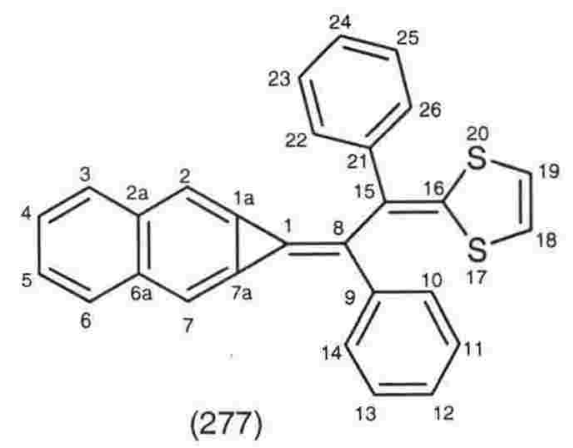


Despite the low stability of the compounds the novel cross-conjugated dithiolecontaining cyclopropanthracene derivatives (169) and (267) have been prepared from cyclopropanthraquinone (162) with the requisite quantity of phosphonate ester (264). Analogous reactions of ester (264) with cyclopropanaphthoquinone (154) were unsuccessful and no evidence to support the formation of the desired compounds or unchanged quinone was obtained.

Treatment of the carbonyl-containing methylidenecycloproparene (200) with lithiate (264) afforded the $\pi$-extended dithiole-containing methylidene compound (274) in $66 \%$ yield. With DDQ this last compound gave evidence for a CT complex (UV-vis spectrum). Unfortunately, the salt proved to be unstable and eluded isolation.

Finally, treatment of $\alpha$-silyl anion (106) with the known dithiole-containing carbonyl compound (265) and the hitherto unknown ketone (276) gave the sought after alkylidenecycloproparenes (275) and (277) which bear one dithiole moiety. The isolation of these compounds was only in 1-6 and 13\% yields, respectively, and this prevented assessment of their physicochemical properties. 


\section{Chapter 7}

\section{Experimental}

\section{General methods.}

Microanalyses were performed by the Analytical Facility at Otago University, Dunedin. Accurate mass measurements were recorded by $\mathrm{Mr} \mathrm{O}$. Zubkov on a PE Biosystems Mariner spectrometer operating in electrospray mode unless stated otherwise and low-resolution mass measurements were recorded a Hewlett-Packard 5595 instrument at $70 \mathrm{eV}$. ${ }^{1} \mathrm{H}$ and ${ }^{13} \mathrm{C}$ n.m.r. spectra were recorded on a Varian INOVA instrument at $\sim 300$ and $\sim 75 \mathrm{MHz}$, respectively, in $\mathrm{CDCl}_{3}$ solution unless stated otherwise with the residual solvent peak used as internal standard. Multiplicities are defined by the usual notation and quaternary carbons donated by $4^{\circ} .{ }^{31} \mathrm{P}$ n.m.r. spectra were recorded on the same instrument using $10 \%$ phosphoric acid in $\mathrm{D}_{2} \mathrm{O}$ as internal standard $(\delta 0.00)$. The assignments of ${ }^{1} \mathrm{H}$ and ${ }^{13} \mathrm{C}$ resonances were made with the aid of DEPT, ${ }^{1} \mathrm{H}-{ }^{1} \mathrm{H}$ COSY, ${ }^{13} \mathrm{C}-{ }^{1} \mathrm{H}$ HSQC, and HMBC correlations. For the dibenzofluorene (179) HOMO 2DJ, n.O.e and 1D TOSCY experiments were also used; the mixing times of the last experiments were 20,40, and $80 \mathrm{~ms}$, respectively. IR spectra were recorded for $\mathrm{KBr}$ disks on a BIORAD FTS-7 spectrophotometer $\left( \pm 1 \mathrm{~cm}^{-1}\right)$, and UV measurements were obtained on a Hewlett-Packard $8452 \mathrm{~A}$ Diode array spectrometer in quartz cells to $\pm 1 \mathrm{~nm}$. Melting points were determined using a Reichert Thermovar hot-stage melting point apparatus and are uncorrected. Cyclic voltammetry measurements were conducted using a Princeton Applied Research Model 362 scanning potentiostat and are accurate to $\pm 3 \mathrm{~V}$.

Thin layer chromatographic analyses were performed using Merck Kieselgel (Alufoilen) $60 \mathrm{~F}_{254}$ to a thickness of $0.2 \mathrm{~mm}$ and components detected under a UV lamp at 254 or $350 \mathrm{~nm}$. Radial chromatography plates were coated with silica gel $\mathrm{DGF}_{254}$ to a thickness of 1,2, 3 or $4 \mathrm{~mm}$, and the total amount of material loaded onto the plate was $0.25 \mathrm{~g}, 0.75 \mathrm{~g}, 1.12 \mathrm{~g}$, and $1.5 \mathrm{~g}$, respectively. Column chromatography employed Kieselgel 60 (230-400 ASTM) as the stationary phase unless stated otherwise.

Solvents were purified before use according to the procedures given in Perrin, Armarego, and Perrin. ${ }^{182}$ Technical grade $p$-benzoquinone was purified by sublimation using an Esoteric Chemicals temperature gradient sublimator, while technical grade 1,4naphthoquinone was used without purification. Potassium $t$-butoxide was sublimed using a 
Büchi GKI-51 ball oven in sublimation mode. The concentrations of organolithium reagents were checked periodically by titration with 2,5-dimethoxybenzyl alcohol employing the method of Winkle, Lansinger, and Ronald. ${ }^{183}$ The silver/silver chloride electrodes were prepared by a standard method. ${ }^{184}$ Silver electrodes were cleaned by boiling in saturated sodium bicarbonate solution containing aluminium foil and were rinsed thoroughly with distilled water, whilst platinum electrodes were boiled in aqua regia for $5 \mathrm{~min}$, rinsed with distilled water, and dried. When low temperatures $\left(<-16^{\circ} \mathrm{C}\right)$ needed to be maintained for longer than $1 \mathrm{~h}$, a Haake EK101 cryostat employing either drum acetone or ethanol as bath coolant was used otherwise cold baths were prepared from dry ice/ethanol or acetone $\left(-78^{\circ} \mathrm{C}\right)$, dry ice/acetonitrile $\left(-43^{\circ} \mathrm{C}\right)$ or from a slurry of liquid nitrogen/ethanol $\left(-16--110^{\circ} \mathrm{C}\right)$.

The argon used throughout this study was zero grade whilst nitrogen was oxygenfree. Reactions performed under a dry inert atmosphere were performed in flasks equipped with rubber septa and syringe needle inlets and outlets and purged and dried as follows. For a dry nitrogen atmosphere the gas was passed first through two drying towers $(\sim 100 \times 4$ $\mathrm{cm}$ ) comprising of 3 and $4 \AA$ molecular sieves and then through a cold trap submerged in liquid nitrogen. Glassware was heated with a Bunsen burner while simultaneously purging it with dry nitrogen then cooled under a gently flow of the same gas. To obtain a dry argon atmosphere the reaction flask was evacuated (rotary vacuum pump, $\sim 10 \mathrm{~mm} / \mathrm{Hg}$ ) and purged with this gas using Schlenk techniques; the procedure was repeated five times. The flask was then evacuated and flame dried (Bunsen burner). After cooling, it was purged with argon and then flushed with this gas for a further 3 min. Syringes and needles were oven-baked $\left(120^{\circ} \mathrm{C}\right)$ for at least $24 \mathrm{~h}$ prior to use, and were cooled over dry silica gel in an evacuated dessicator (vacuum pump). Prior to use, the syringes and needles were purged five times with dry, inert gas. To obtain strictly anhydrous tetrahydrofuran (thf) the analytical reagent was pre-dried over potassium metal for $24 \mathrm{~h}$ under argon and was then cautiously decanted into a dry round bottomed flask purged with argon and containing benzophenone $(\sim 300 \mathrm{mg})$, and five glass beads. Potassium metal was added and the mixture refluxed in a self-contained distillation/reflux apparatus until the purple benzophenone ketyl persisted whereupon the mixture was cooled to room temperature under argon and the distillation unit sealed from the atmosphere. Anhydrous thf was distilled immediately prior to use and was collected via a dry syringe and needle that had been purged with argon. Dry diethyl ether used throughout this study was anhydrous analytical 
grade (Univar) and was used without further purification but was handled and stored under argon.

\section{Chapter 2.}

\section{1,4-Dihydronaphthalene (172).}

Naphthalene $(80 \mathrm{~g}, 0.61 \mathrm{mmol})$, sodium metal $(60 \mathrm{~g}, 2.6 \mathrm{~mol})$, and benzene $(200 \mathrm{ml})$ were placed in a round bottomed flask $(3 \mathrm{~L})$ equipped with a pressure equalised dropping funnel, condenser, and magnetic stir bar, and heated to reflux whilst stirring. Absolute ethanol $(750 \mathrm{ml})$ in benzene $(200 \mathrm{ml})$ was added carefully in $5 \mathrm{ml}$ portions such that moderate reflux was maintained. About half-way though the addition $(\sim 1.5 \mathrm{~h})$ the reaction became violent, , copious amounts of foam were produced, and stirring was ceased. After $\sim 15$ min the reaction was less vigorous, most of the sodium metal had been consumed, and the addition of small aliquots of the ethanolic solution were recommenced. When the addition ethanol did not result in a vigorous reaction the remainder was added in one portion. The mixture was then refluxed overnight, cooled to ambient temperature, poured into a beaker containing ice ( $2 \mathrm{~L}$ ) and hydrochloric acid $(2 \mathrm{M}, 200 \mathrm{ml})$, and the phases separated. The aqueous phase was extracted (benzene, $3 \times 80 \mathrm{ml}$ ), and the combined organic layers washed (water, $2 \times 80 \mathrm{ml}$ ), dried $\left(\mathrm{MgSO}_{4}\right)$, and concentrated under reduced pressure to a red/orange oil. Fractional distillation gave the title compound (172) as an oily yellow solid (66 g, $88 \%$ ), b.p. $210-212^{\circ} \mathrm{C}$ (lit. ${ }^{126} 94 \%$, b.p. $210-212^{\circ} \mathrm{C}$ ).

\section{1,1-Dichloro-1a,2,7,7a-tetrahydro-1H-cyclopropa[b]naphthalene (67).}

1,4-Dihydronaphthalene (183) (20.5 g, $0.16 \mathrm{~mol}$ ), powdered sodium hydroxide (21 g, $0.53 \mathrm{~mol})$ and benzyltriethylammonium bromide $(2.74 \mathrm{~g}, 0.01 \mathrm{~mol})$ were suspended in diethyl ether $(100 \mathrm{ml})$ in a round bottomed flask $(1 \mathrm{~L})$ equipped with a mechanical stirrer, and a pressure equalised dropping funnel. The apparatus was cooled in an ice bath and, whilst stirring vigorously, chloroform $(80 \mathrm{ml}, 0.99 \mathrm{~mol})$ was added dropwise over $20 \mathrm{~min}$. The cloudy yellow solution was left stirring in the cold bath, which attained room temperature slowly, for $48 \mathrm{~h}$. The crude product was filtered though a pad of silica gel $(\sim 20 \mathrm{~g})$ under vacuum and the inorganic residue washed (diethyl ether, $2 \times 100 \mathrm{ml}$ ). The combined filtrates were washed (water, $200 \mathrm{ml}$ ), dried $\left(\mathrm{MgSO}_{4}\right)$, and concentrated under reduced pressure to an orange oil. Trituation (diethyl ether, $-16^{\circ} \mathrm{C}$ ) gave the title 
compound (67) as pale yellow crystals $\left(15.45 \mathrm{~g}, 47 \%\right.$ ), m.p. $52-55^{\circ} \mathrm{C}$ (lit. ${ }^{53} 49-51^{\circ} \mathrm{C}$ ). Spectral data were in agreement with those reported..$^{53}$

1H-Cyclopropa[b]naphthalene (58).

To a stirred solution of commercial grade potassium $t$-butoxide $(130 \mathrm{~g}, 1.2 \mathrm{~mol})$ (Lancaster, 97\%), in anhydrous thf $(450 \mathrm{ml})$, cooled to $0^{\circ} \mathrm{C}$ and under a nitrogen atmosphere, was added dropwise over $30 \mathrm{~min}$ 1,1-dichloro-1a,2,7,7a-tetrahydro- $1 \mathrm{H}$-cyclopropa $[b]$ naphthalene $(67)(20 \mathrm{~g}, 0.094 \mathrm{~mol})$ in the same solvent $(100 \mathrm{ml})$. The mixture was allowed to stir overnight in the cold bath, which slowly attained ambient temperature, the solvent was removed under reduced pressure and the resultant orange residue was dissolved (water/light petroleum; $500 \mathrm{ml}, 1: 1$ ). After separation of the phases the aqueous layer was extracted (light petroleum, $2 \times 125 \mathrm{ml}$ ) and the combined organic layers were washed (water, $4 \times 125 \mathrm{ml}$ ), dried $\left(\mathrm{MgSO}_{4}\right)$, and concentrated under reduced pressure to yellow residue. Flash column chromatography (light petroleum elution), collection of the major component and recrystallisation (light petroleum) gave the title compound (58) as odoriferous colourless crystals $\left(8.2 \mathrm{~g}, 62 \%\right.$ ), m.p. $86-87^{\circ} \mathrm{C}$ (lit. ${ }^{53} 38 \%$, m.p. $86-87^{\circ} \mathrm{C}$ ). N.m.r. data were in excellent agreement with those published. ${ }^{53}$

\section{1,1-Bis(trimethylsilyl)-1H-cyclopropa[b]naphthalene (105).}

To a stirred solution of $1 H$-cyclopropa $[b]$ naphthalene $(58)(4 \mathrm{~g}, 28.7 \mathrm{mmol})$ in anhydrous thf $(50 \mathrm{ml})$, under argon and cooled to $-78^{\circ} \mathrm{C}$, was added slowly by syringe butyllithium $(2.2 \mathrm{M}, 12.9 \mathrm{ml}, 28.3 \mathrm{mmol})$. The resultant dark solution was stirred for $20 \mathrm{~min}$ at this temperature and then trimethylsilyl chloride $(3.6 \mathrm{ml}, 28.3 \mathrm{mmol})$ was added. After stirring for a further $20 \mathrm{~min}$, a second portion of butyllithium $(2.2 \mathrm{M}, 6.5 \mathrm{ml}$, $14.2 \mathrm{mmol})$ was added, followed by trimethylsilyl chloride $(1.8 \mathrm{ml}, 14.2 \mathrm{mmol})$ after $20 \mathrm{~min}$ had elapsed. The last two steps were repeated and the dark solution was then left to slowly attain ambient temperature overnight in the cold bath. The mixture was quenched (water, $100 \mathrm{ml}$ ), light petroleum $(100 \mathrm{ml})$ was added, and the phases were separated and the aqueous extracted (light petroleum, $2 \times 100 \mathrm{ml}$ ). The combined organic layers were washed (water, $100 \mathrm{ml})$, dried $\left(\mathrm{MgSO}_{4}\right)$, and concentrated under reduced pressure to a yellow solid. Flash column chromatography (light petroleum elution) afforded the title compound (105) as colourless prisms (7 g, 86\%), m.p. $95-97^{\circ} \mathrm{C}$ (lit. ${ }^{24} 66 \%$, m.p. $95-96^{\circ} \mathrm{C}$ ). Spectral data were in accord with the literature values. ${ }^{24}$ 
To a stirred solution of disilylcyclopropanaphthalene (105) (200 mg, $0.7 \mathrm{mmol})$ in thf $(10 \mathrm{ml})$, under an argon atmosphere and cooled to $-70^{\circ} \mathrm{C}$, was added potassium $t$ butoxide $(79 \mathrm{mg}, 0.7 \mathrm{mmol})$ in the same solvent $(10 \mathrm{ml})$. The mixture was warmed to $40^{\circ} \mathrm{C}$ for $90 \mathrm{~min}$ then re-cooled to $-70^{\circ} \mathrm{C}$ whereupon the dark yellow-black mixture was quenched (water, $1 \mathrm{ml}, 55.6 \mathrm{mmol}$ ). The now turbid and lighter coloured mixture was stirred at this temperature for $2 \mathrm{~min}$ and then poured into water/dichloromethane $60 \mathrm{ml}$, $1: 1$ ) and the phases separated. The aqueous phase was extracted (dichloromethane, $3 \times 15 \mathrm{ml}$ ) and the combined organic layers washed (water, $50 \mathrm{ml}$ ), dried $\left(\mathrm{MgSO}_{4}\right)$, and concentrated under reduced pressure to a pale yellow oil. Radial chromatography (light petroleum elution) gave a yellow oil ( $84 \mathrm{mg}$ ) as the major and most mobile component. ${ }^{1} \mathrm{H}$ n.m.r. analysis of the oil indicated a mixture of monosilylcyclopropanaphthalene (113), cyclopropanaphthalene (58), and an unidentified component thought ${ }^{109,110}$ to be ring opened [(dd, $1 \mathrm{H}, J 8.6,1.7 \mathrm{~Hz})$ and $8.61(\mathrm{bd} \mathrm{s}, 1 \mathrm{H})]$ in a ratio of $c .16: 5: 6$.

A sample $(c .40 \mathrm{mg})$ of the mixture was chromatographed over the size exclusion gel Sephadex LH-20 (thf elution) to give an oil that contained two components on t.l.c. A second radial chromatography (hexane elution) afforded 1-trimethylsilyl-1H-cyclopropa[b] naphthalene (104) as a colourless oil $(6.2 \mathrm{mg}, 9 \%)$ that solidified at $-16^{\circ} \mathrm{C}$ and turned yellow on standing. (Found $M$ 212.1026. $\mathrm{C}_{14} \mathrm{H}_{16} \mathrm{Si}$ requires $\mathrm{M}^{+} m / z$ 212.1021). IR $v_{\max }$ $3069,3038,2955,2895,1248,1227,1157,1046,1020,924,916,891,860,839,743,698$, $671 \mathrm{~cm}^{-1}$. UV $\lambda_{\max }$ (hexane) $228 \mathrm{sh}$ (4.42), 240 (4.23), $246 \mathrm{sh}$ (4.11), 262 (3.72), 272 (3.69), 2.84 (3.52), 311 (3.35), 324 nm (log ع 3.54). ${ }^{1} \mathrm{H}$ n.m.r. $\delta 0.02$ (s, 9H, $\mathrm{SiMe}_{3}$ ), 3.23 (t, $J<1 \mathrm{~Hz}, 1 \mathrm{H}, \mathrm{H1}$ ), 7.33 (dd, $J<1,<0.5 \mathrm{~Hz}, 2 \mathrm{H}, \mathrm{H} 2 / 7$ ), 7.37-7.40 (BB', 2H, H4/5), 7.777.80 (AA', 2H, H3/6); ${ }^{13} \mathrm{C}$ n.m.r. -2.4 ( $\left.\mathrm{SiMe}_{3}\right), 24.3$ (C1), 110.0 (C2/7), 125.0 (C4/6), 127.1 (C1a/7a), $127.9(\mathrm{C} 3 / 6), 136.2(\mathrm{C} 2 \mathrm{a} / 6 \mathrm{a})$. Mass spectrum (relative intensity): $\mathrm{m} / \mathrm{z}(70$ eV) 213 (M+1, 6), 212 (M, 26), 211 (M-H, 9), 198 (20), 197 (M-Me, 100), 196 (20), 195 (78), 181 (12), 171 (20), 169 (17), 167 (13), 165 (10), 155 (15), 154 (13), 153 (14), 152 (12), $139\left(\mathrm{M}-\mathrm{SiMe}_{3}, 45 \%\right)$.

\section{1-Trimethylsilyl-1-methyl-1H-cyclopropa[b]naphthalene (113).}

The silylcycloproparenyl anion (106) was prepared from disilylcyclopropanaphthalene $(105)$ (300 mg, $1.06 \mathrm{mmol})$ and potassium $t$-butoxide $(118.5 \mathrm{mg}, 1.06 \mathrm{mmol})$ in the manner described in the preparation of 1-trimethylsilyl-1H-cyclopropa[b]naphthalene (104) and was treated with methyl iodide $(0.066 \mathrm{ml}, 1.06 \mathrm{mmol})$. The red/orange mixture was 
then allowed to warm to room temperature overnight and was then poured into water/dichloromethane $(60 \mathrm{ml}, 1: 1)$ and the phases separated. The aqueous phase was extracted (dichloromethane, $3 \times 15 \mathrm{ml}$ ) and the combined organic layers washed (water, $50 \mathrm{ml})$, dried $\left(\mathrm{MgSO}_{4}\right)$, and concentrated under reduced pressure to a red oil. Radial chromatography (light petroleum elution) gave a pale yellow oil (204 mg) as the major and most mobile component that contained ( ${ }^{1} \mathrm{H}$ n.m.r.) the title compound (113) and cyclopropanaphthalene (58) in a ratio of $c .9: 1$.

A sample of the oil $(59 \mathrm{mg})$ was subjected to chromatography over Sephadex LH20 (thf elution). Concentration of the product-containing fractions in vacuum and resubjection to radial chromatography (hexane elution) gave an analytically pure sample of 1 trimethylsilyl-1-methyl-1H-cyclopropa/b]naphthalene (113) (32.6 mg, 47\%) as a colourless oil that solidified at $-16^{\circ} \mathrm{C}$, m.p. $46-50^{\circ} \mathrm{C}$ (Found: $\mathrm{C}, 79.8 ; \mathrm{H}, 8.3 . \mathrm{C}_{15} \mathrm{H}_{18} \mathrm{Si}$ requires $\mathrm{C}, 79.6 ; \mathrm{H}, 8.0 \%$ ). IR $v_{\max } 3073,3038,2955,2917,2893,2859,1447,1248,870$, $839,743,692,648 \mathrm{~cm}^{-1}$. UV $\lambda_{\max }$ (hexane) 226 (4.59), $264 \mathrm{sh}$ (3.82), 272 (3.75), 284 (3.55), 312 (3.42), $326 \mathrm{~nm}\left(\log \varepsilon\right.$ 3.55). ${ }^{1} \mathrm{H}$ n.m.r. $\delta 0.02$ (s, 9H, $\mathrm{SiMe}_{3}$ ), 1.69 (s, 3H, Me), 7.34 (s, 2H, H2/7), 7.39-7.42 (BB', 2H, H4/5), 7.79-7.82 (AA', 2H. H3/6); ${ }^{13} \mathrm{C}$ n.m.r. $\delta$ $3.3\left(\mathrm{SiMe}_{3}\right), 22.8$ (Me), 31.9 (C1), 109.9 (C2/7), 124.9 (C4/5), 128.0 (C3/6), 134.2 (C1a/7a), 135.9 (C2a/6a). Mass spectrum (relative intensity): $\mathrm{m} / \mathrm{z}(70 \mathrm{eV}) 227(\mathrm{M}+1,4)$, 226 (M, 20), 212 (21), 211 (M-Me, 100), 210 (10), 209 (34), 195 (41), 185 (23), 183 (21), 171 (15), 167 (13), 165 (24), 155 (11), 153 (M-SiMe, 24$), 152$ (34), 151 (13), 74 (10), 73 $(64 \%)$.

\section{6-Methyl-7H-dibenzo[b,g]fluorene (179).}

The monosilylcyclopropanaphthalenyl anion (106) was prepared from disilylcyclopropanaphthalene (105) (100 mg, $0.35 \mathrm{mmol})$ and potassium $t$-butoxide $(40 \mathrm{mg}$, $0.35 \mathrm{mmol}$ ) in the same manner as that described above. After returning the temperature to $-70^{\circ} \mathrm{C}$ one of the procedures $a$ ) $-c$ ) below was followed.

a) From the addition of toluene:- dry toluene $(0.04 \mathrm{ml}, 0.35 \mathrm{mmol})$ was added dropwise over $5 \mathrm{~min}$ by syringe and the dark mixture was allowed to attain room temperature over $16 \mathrm{~h}$. The resultant red mixture was then workedup in a conventional manner to give a bright orange oil. Radial chromatography (light petroleum elution) gave a yellow oily solid $(10.2 \mathrm{mg})$ as the most mobile fraction which was shown ( ${ }^{1} \mathrm{H}$ n.m.r.) to contain monosilylcyclopropanaphthalene (104), cyclopropanaphthalene (58), and an unidentified component thought ${ }^{109,110}$ to be ring-opened $[(8.10(\mathrm{dd}, 1 \mathrm{H} J 8.6,1.7 \mathrm{~Hz})$ and $8.61(\mathrm{bd} \mathrm{s}, 1 \mathrm{H})]$ in a ratio 
of c. 53:10:5. Continued elution over c. 20-30 min provided a further component as a solid (20.4 mg) that was markedly fluorescent on UV irradiation. Recrystallisation of this (dichloromethane/light petroleum; $1: 1)$ at $-20^{\circ} \mathrm{C}$ afforded 6 -methyl-7H-dibenzo $[\mathrm{b}, \mathrm{g}]$ fluorene (179) $(5.9 \mathrm{mg}, 6 \%)$ - see below.

b) From the addition of $\mathrm{N}, \mathrm{N}$-dimethylacetamide:- $N, N$-dimethylacetamide $(0.033 \mathrm{ml}$, $0.35 \mathrm{mmol})$ in thf $(1 \mathrm{ml})$ was added and the mixture allowed to attain room temperature slowly overnight. Workup as for method $a$ above gave a dark red-brown oil. Radial chromatography (light petroleum elution) gave cyclopropanaphthalene (58) as a white solid (9.6 mg, 20\%) as the most mobile component. Further elution and recrystallisation of the resultant yellow solid as in $a$ above afforded dibenzofluorene (179) (17.7 mg, 18\%).

The product sample was combined with that from $a$ above, dissolved in dichloromethane/hexane (1:1) and recrystallised by solvent diffusion in a sealed container holding hexane to afford 6-methyl-7H-dibenzo[b,glfluorene (179) as straw coloured blocks suitable for analysis and crystal structure determination, m.p. $174^{\circ} \mathrm{C}$ (sub.), $212-213^{\circ} \mathrm{C}$ (sublimate melts). (Found: C, 94.3; H, 5.7(5). $\mathrm{C}_{22} \mathrm{H}_{16}$ requires $\left.\mathrm{C}, 94.2(5) ; \mathrm{H}, 5.7(5) \%\right)$. IR $v_{\max } 3050,3019,2918,2878,1686,1516,1425,1414,1213,1024,951,934,862,762$, $741,650 \mathrm{~cm}^{-1}$. UV $\lambda_{\max }$ (acetonitrile) 249 (4.70), $254 \mathrm{sh}$ (4.63), 269 (3.89), 280 (4.02), 292 (4.07), $316 \mathrm{sh}$ (4.13), 328 (4.27), 335 (4.24), 344 (4.26), $350 \mathrm{~nm}$ (log ع 4.38). ${ }^{1} \mathrm{H}$ n.m.r. $\delta 2.59$ (d, $J 1 \mathrm{~Hz}, \mathrm{Me}$ ), 4.06 (s, $\mathrm{CH}_{2}$ ), 7.44-7.57 (m, 3H, H3/10/11), 7.66* (q, J c. 1 $\mathrm{Hz}, 1 \mathrm{H}, \mathrm{H} 5$ ), $7.66^{*}$ (app dt, $J 7.6,1.3 \mathrm{~Hz}, 1 \mathrm{H}, \mathrm{H} 2$ ), $7.87^{*}$ (d, $J 9.2 \mathrm{~Hz}, 1 \mathrm{H}, \mathrm{H} 9$ ), 7.91* (app dd, $J 8.2,2.8 \mathrm{~Hz}, 1 \mathrm{H}, \mathrm{H} 4$ ), 8.03 (d, J<1 Hz, 1H, H8), 8.04* (app dd, $J 6.7,2.4 \mathrm{~Hz}, 1 \mathrm{H}$, H12), 8.77 (broad s, $1 \mathrm{H}, \mathrm{H} 13$ ), 8.90 (d, $J 8.1 \mathrm{~Hz}, 1 \mathrm{H}, \mathrm{H} 1$ ); ${ }^{13} \mathrm{C}$ n.m.r. $\delta 19.4$ (Me), 36.6 $\left(\mathrm{CH}_{2}\right), 121.1$ (C13), $122.9(\mathrm{C} 8), 123.6$ (C12 or C1), 125.2 (C10 or C3), 125.3 (C3 or $\left.\mathrm{C} 10\right)$, 125.4 (C2 or C11), 125.9 (C11 or C2), 127.4 (C4 or C9), 127.9 (C5), 128.5(2) (C1 or C12), 128.5(6) (C9 or C4); 128.4(6), $132.0\left(4^{\circ}\right), 132.5\left(4^{\circ}\right), 133.2\left(4^{\circ}\right), 134.0\left(4^{\circ}\right), 135.0\left(4^{\circ}\right)$, 141.7(8) $\left(4^{\circ}\right), 141.8(4)\left(4^{\circ}\right), 143.6\left(4^{\circ}\right)$. Mass spectrum (relative intensity): $\mathrm{m} / \mathrm{z}(70 \mathrm{eV}) 281$ (M+1, 21), 280 (M, 100), 279 (27), 278 (12), 276 (14), 266 (14), 265 (M-Me, 64), 263 (10), 140 (15), 139 (17), 138 (17), 133 (29), 132 (17), 126 (19), 125 (11\%).

c) From addition of $1 \mathrm{H}$-cyclopropa[b]naphthalene:- cyclopropanaphthalene (58) (49 mg, $0.35 \mathrm{mmol})$ in thf $(4 \mathrm{ml})$ was added and the dark mixture warmed to room temperature overnight. Workup and radial chromatography as described above returned cyclopropanaphthalene (58) (1.1 mg, 2\%) as the most mobile component and fluorene (179) (36.4 mg)

\footnotetext{
${ }^{*}$ Chemical shifts, apparent peak multiplicities, and coupling constants were obtained from HOMO-2DJ experiments and are not from expanded ${ }^{1} \mathrm{H}$ n.m.r. spectra.
} 
as the second component. Recrystallisation of this latter component (dichloromethane/ light petroleum; $1: 1)$ at $-20^{\circ} \mathrm{C}$ afforded 6-methyl-7H-dibenzo[b,g]fluorene (179) (28 mg, $28 \%$ ) as pale straw coloured needles, identical to the samples described above.

\section{Chapter 3.}

Preparation of 6,6-diphenylpentafulvene (171a) from cyclopentadiene-Oda's procedure.

To a stirred solution of bromobenzene $(0.54 \mathrm{ml}, 5.1 \mathrm{mmol})$ in anhydrous thf $(10 \mathrm{ml})$, cooled to $-50^{\circ} \mathrm{C}$ and under a nitrogen atmosphere, was added butyllithium $(2.5 \mathrm{M}$, $2.12 \mathrm{ml}, 5.3 \mathrm{mmol}$ ). The colourless mixture was stirred at this temperature for $1 \mathrm{~h}$ and then $N, N$-dimethylbenzamide $(895 \mathrm{mg}, 6 \mathrm{mmol}$ ) was added under a steady stream of dry nitrogen. The mixture was stirred at $0^{\circ} \mathrm{C}$ for $1 \mathrm{~h}$ then cyclopentadiene $(1 \mathrm{ml}, 12.1 \mathrm{mmol})$ was added dropwise. After being stirred at the same temperature for a further $3 \mathrm{~h}$ the now bright orange mixture was poured into water/dichloromethane $(100 \mathrm{ml}, 1: 1)$. The phases were separated, and the aqueous extracted (dichloromethane, $3 \times 15 \mathrm{ml}$ ). The combined organic layers were washed (water, $50 \mathrm{ml}$ ), dried $\left(\mathrm{MgSO}_{4}\right)$, and concentrated under reduced pressure to a red oil. Trituration (hexane, $-16^{\circ} \mathrm{C}$ ) afforded the 6,6 -diphenylpentafulvene (171a) as bright red plates (754 mg, 65\%), m.p. $81-82^{\circ} \mathrm{C}$ (lit. ${ }^{125} 85 \%$, m.p. ${ }^{134} 82^{\circ} \mathrm{C}$ ).

General procedure for the attempted preparation of 1-(diphenylmethylidene)-1H-cyclopropa[b]naphthalene (114l) from cyclopropanaphthalene (58) employing the Oda procedure.

To a stirred solution of bromobenzene $(0.16 \mathrm{ml}, 1.53 \mathrm{mmol})$ in thf $(10 \mathrm{ml})$, cooled to $-50 \mathrm{C}$ and under a nitrogen atmosphere, was added dropwise butyllithium in hexanes ( $2.5 \mathrm{M}, 0.64 \mathrm{ml}, 1.59 \mathrm{mmol})$. The colourless mixture was stirred at this temperature for $1 \mathrm{~h}$ and then $N, N$-dimethylbenzamide $(269 \mathrm{mg}, 1.8 \mathrm{mmol}$ ) was added under a stream of nitrogen. The colourless mixture was stirred at $0^{\circ} \mathrm{C}$ for $1 \mathrm{~h}$, cyclopropanaphthalene (58) (500 mg, $3.6 \mathrm{mmol}$ ) was added as a solution in thf $(4 \mathrm{ml})$, and the mixture was then allowed to react in accord with one of the procedures $1 a-1 c$ below:

1a) the mixture was allowed to warm slowly to ambient temperature over $24 \mathrm{~h}$ whereupon the now pale yellow mixture was poured into water/dichloromethane $(100 \mathrm{ml}, 1: 1)$. The phases were separated and the aqueous phase extracted (dichloromethane, $3 \times 15 \mathrm{ml}$ ). The combined organic layers were washed (water, $50 \mathrm{ml}$ ), dried $\left(\mathrm{MgSO}_{4}\right)$, and concentrated under reduced pressure to a pale yellow oily solid. Radial chromatography (light petroleum 
elution) returned unchanged cyclopropanaphthalene $(58)(436 \mathrm{mg}, 87 \%)$ as the most mobile component. Further elution (dichloromethane) afforded benzophenone (132 mg, $47 \%$ based on bromobenzene) as a pale yellow oil; spectral data were identical to those of an authentic sample. Finally, unchanged $N, N$-dimethylbenzamide (48 mg, 18\%) was returned (acetone elution) as a pale brown oil. No attempts were made to purify further the ketone or amide.

$1 b)$ the mixture was allowed to warm to ambient temperature whereupon it was stirred for $7 \mathrm{~d}$, after this time the turbid mixture had developed a yellow colouration. Workup and chromatography as for method $1 a$ above returned unchanged cyclopropanaphthalene (58) (435 mg, 87\%) as the major and most mobile component. Elution with dichloromethane gave benzophenone $(140 \mathrm{mg}, 77 \%)$ as a pale yellow oil while further elution (acetone) returned unchanged amide $(48 \mathrm{mg}, 18 \%)$ as a brown oil. The benzophenone and $N, N$ dimethylbenzamide were not purified further.

1c) the mixture was warmed to $38^{\circ} \mathrm{C}$ where it was stirred for $24 \mathrm{~h}$. The resultant turbid yellow mixture was subjected to workup and chromatography as for method $1 a$ above whereupon unchanged cyclopropanaphthalene (58) (449 mg, 90\%) was returned as the major and most mobile component. Continued elution (dichloromethane/light petroleum; 1:1) gave 1-(diphenylhydroxymethyl)- $1 H$-cyclopropa $[b]$ naphthalene $^{93}$ (186) (6.6 mg, 1\%) as an oily white solid and, with dichloromethane, benzophenone ( $94 \mathrm{mg}, 34 \%$ based on bromobenzene) as a pale yellow oil. Further elution (acetone) resulted in the recovery of unchanged amide (48 mg, 17\%).

Attempted preparation of 2'-propenylidene-1H-cyclopropa[b]naphthalene (114b) from cyclopropanaphthalene (58) via the Oda procedure.

To a stirred solution of $N, N$-dimethylacetamide $(0.167 \mathrm{ml}, 1.80 \mathrm{mmol})$ in dry thf $(10 \mathrm{ml})$, cooled to $0^{\circ} \mathrm{C}$ and under a nitrogen atmosphere, was added dropwise methyllithium $(1.2 \mathrm{M}, 1.33 \mathrm{ml}, 1.59 \mathrm{mmol})$. The mixture was stirred at this temperature for $1 \mathrm{~h}$ and then cyclopropanaphthalene (58) (500 mg, $3.6 \mathrm{mmol}$ ) was added slowly in the same solvent $(4 \mathrm{ml})$. The yellow mixture was allowed to warm to ambient temperature slowly over $24 \mathrm{~h}$ at which time the now dark yellow/brown mixture was subjected to workup as for method $1 a$ above to give a pale yellow solid. Radial chromatography (light petroleum elution) returned unchanged cyclopropanaphthalene (58) (405 $\mathrm{mg}, 81 \%$ ) as the major and most mobile component. Further elution (dichloromethane/light petroleum; 1:1) gave 1 - 
acetyl-1H-cyclopropa[b]naphthalene (189) $(6 \mathrm{mg}, 2 \%)$ as an unstable yellow oil, vide infra.

General procedure for the attempted preparation of 1-(diphenylmethylidene)-1H-cyclopropa[b]naphthalene (114l) from disilylcyclopropanaphthalene (105) via the Oda procedure.

To a stirred solution of bromobenzene $(0.08 \mathrm{ml}, 0.77 \mathrm{mmol})$ in dry thf $(10 \mathrm{ml})$, cooled to $-50^{\circ} \mathrm{C}$ and under dry nitrogen, was added dropwise butyllithium $(2.5 \mathrm{M}, 0.32 \mathrm{ml}$, $0.80 \mathrm{mmol}$ ). The colourless mixture was stirred at this temperature for $30 \mathrm{~min}$ and then $\mathrm{N}, \mathrm{N}$-dimethylbenzamide $(130 \mathrm{mg}, 0.88 \mathrm{mmol}$ ) was added in one portion as a solid under a stream of nitrogen. The mixture was subsequently stirred at $0^{\circ} \mathrm{C}$ for $1 \mathrm{~h}$ and then disilylcyclopropanaphthalene (105) (500 mg, $1.76 \mathrm{mmol}$ ) was added in one portion as a solution in thf $(8 \mathrm{ml})$. The pale yellow mixture was then allowed to react in accord with one of the procedures $2 a$ or $2 b$ below:

2a) The mixture was allowed to warm slowly to ambient temperature over $24 \mathrm{~h}$ and then the bright yellow/green mixture was poured into water/dichloromethane $(100 \mathrm{ml} ; 1: 1)$. Workup as for method $1 a$ above gave a bright yellow oil. Radial chromatography (light petroleum elution) returned unchanged disilane (105) (465 mg, 93\%) as the most mobile component. Further elution (light petroleum) gave 1-(diphenylmethylidene)- $1 H$-cyclopropa[b]naphthalene (114l) (7.1 mg, 3\%; based on bromobenzene) spectral data were identical to those of an authentic sample. ${ }^{24}$ Further elution (dichloromethane) afforded benzophenone as a pale yellow oil (107 mg, $76 \%$ based on bromobenzene); spectral data were identical to those of an authentic sample. Finally, the least mobile component (acetone elution) was found to contain unchanged $N, N$-dimethylbenzamide (28 mg, 22\%).

$2 b$ ) the mixture was stirred for $24 \mathrm{~h}$ at $38^{\circ} \mathrm{C}$. After cooling, the bright yellow/green mixture was subjected to workup and chromatography as in method $1 a$ above. Unchanged disilane (105) (455 mg, 91\%), 1-(diphenylmethylidene)-1H-cyclopropa[ $b]$ naphthalene (1141) (22 mg, 9\%; based on bromobenzene), benzophenone ( $95 \mathrm{mg}, 68 \%$ based on bromobenzene), and unchanged amide (32 $\mathrm{mg}, 25 \%$ ) were obtained as above.

Attempted preparation of 2'-propenylidene- $1 \mathrm{H}$-cyclopropa[b]naphthalene (114b) from disilylcyclopropanaphthalene (105) via the Oda procedure.

To a stirred solution of $N, N$-dimethylacetamide $(0.081 \mathrm{ml}, 0.88 \mathrm{mmol})$ in dry thf, (30 ml) cooled to $0^{\circ} \mathrm{C}$ and under a nitrogen atmosphere, was added methyllithium $(1.2 \mathrm{M}$, 
$0.67 \mathrm{ml}, 0.8 \mathrm{mmol}$ ). The colourless mixture was stirred at this temperature for $30 \mathrm{~min}$ then disilylcyclopropanaphthalene (105) (500 mg, $1.75 \mathrm{mmol}$ ) was added in the same solvent ( $8 \mathrm{ml}$ ). The mixture was heated to $42^{\circ} \mathrm{C}$ for $24 \mathrm{~h}$ over which time it developed a green and then finally a dark yellow/brown colouration. After cooling, the mixture was poured into water/dichloromethane $(100 \mathrm{ml} ; 1: 1)$. Workup as for method $1 a$ above gave a bright yellow oil. Radial chromatography (light petroleum elution) gave a yellow solid $(280 \mathrm{mg})$ as the most mobile component that contained silanes (104) and (105) as an inseparable mixture on t.l.c in a c. $12: 39$ ratio $\left({ }^{1} \mathrm{H}\right.$ n.m.r.). This equates to 14 , and $46 \%$ yields, respectively.

Preparation of 1'-(phenylpentylidene)-1H-cyclopropa[b]naphthalene (114n) from disilylcyclopropanaphthalene (105) via the Oda procedure.

To a stirred suspension of $N, N$-dimethylbenzamide $(131 \mathrm{mg}, 0.88 \mathrm{mmol})$ in thf, $(10 \mathrm{ml})$ cooled to $0^{\circ} \mathrm{C}$ and under nitrogen, was added dropwise butyllithium $(1.77 \mathrm{M}$, $0.45 \mathrm{ml}, 0.80 \mathrm{mmol}$ ). The resultant mixture was stirred at this temperature for $30 \mathrm{~min}$ and then disilylcyclopropanaphthalene $(105)(500 \mathrm{mg}, 1.76 \mathrm{mmol})$ in thf $(4 \mathrm{ml})$ was added. The resultant bright yellow mixture was heated to $38^{\circ} \mathrm{C}$ for $24 \mathrm{~h}$ over which time the mixture became dark yellow/brown in colour. Conventional workup gave a red/orange oily solid. Radial chromatography (light petroleum elution) returned unchanged (105) (435 mg, $87 \%$ ) as the most mobile component. Further elution (light petroleum) gave 1'(phenylpentylidene)- $1 H$-cyclopropa $[b]$ naphthalene $(114 \mathrm{n})(16 \mathrm{mg}, 7 \%)$ as a bright yellow oil, vide infra.

\section{Preparation of 1-phenylpentan-1-one (valerophenone).}

To a suspension of $N, N$-dimethylbenzamide $(1 \mathrm{~g}, 6.7 \mathrm{mmol})$ in dry thf $(30 \mathrm{ml})$, cooled to $-70^{\circ} \mathrm{C}$ and under a dry nitrogen atmosphere, was added dropwise butyllithium (2.02 M, $3.32 \mathrm{ml}, 6.7 \mathrm{mmol})$. The mixture, which developed a pale yellow colouration, was stirred at this temperature for $1 \mathrm{~h}$ and then was slowly warmed to ambient temperature overnight. The pale pink/orange mixture was poured into water $(100 \mathrm{ml})$ and the aqueous mixture extracted (dichloromethane, $3 \times 15 \mathrm{ml}$ ). The combined organic layers were washed (water, $200 \mathrm{ml}$ ), dried $\left(\mathrm{MgSO}_{4}\right)$, and concentrated under reduced pressure to a pale yellow liquid that was pumped under vacuum $(c .0 .1 \mathrm{mmHg})$ for $2 \mathrm{~h}$. The title ketone $(1.05 \mathrm{~g}$, $97 \%$ ) was obtained as a fruity smelling yellow liquid and was used without further purification. ${ }^{1} \mathrm{H}$ n.m.r. $\delta .0 .88$ (t, $J 7.6 \mathrm{~Hz}, \mathrm{Me}$ ), 1.35 (sextet, $J 7,6 \mathrm{~Hz}, \mathrm{CH}_{2} \mathrm{CH}_{3}$ ), 1.66 
(pentet, J 7.6 Hz, $\mathrm{CH}_{2} \mathrm{CH}_{2} \mathrm{CH}_{3}$ ), $2.89\left(\mathrm{t}, J 7.8 \mathrm{~Hz}, \mathrm{CH}_{2} \mathrm{CH}_{2} \mathrm{CH}_{2} \mathrm{CH}_{3}\right.$ ), 7.34-7.40 (m, 2H), 7.44-7.50 (app tt, J7.7, 1.7, 1H), 7.88-7.92 (m, 2H).

\section{I'-(Phenylpentylidene)-1H-cyclopropa[b]naphthalene (114n).}

To a stirred solution of disilylcyclopropanaphthalene (105) (200 mg, $0.7 \mathrm{mmol})$ in anhydrous thf $(10 \mathrm{ml})$, cooled to $-70^{\circ} \mathrm{C}$ and under a dry nitrogen atmosphere, was added potassium $t$-butoxide $(79 \mathrm{mg}, 0.7 \mathrm{mmol})$ in the same solvent $(8 \mathrm{ml})$. The mixture was warmed to $-43^{\circ} \mathrm{C}$ where it was stirred for $1.5 \mathrm{~h}$, recooled to $-70^{\circ} \mathrm{C}$ and valerophenone $(0.115 \mathrm{ml}, 0.7 \mathrm{mmol})$ was added dropwise. The initially dark yellow/brown mixture, which became dark maroon during the addition, was allowed to warm slowly to ambient temperature overnight. After pouring into water $(50 \mathrm{ml})$, the aqueous mixture was extracted (dichloromethane, $3 \times 15 \mathrm{ml}$ ). The combined organic layers were washed (water, $50 \mathrm{ml}$ ), dried $\left(\mathrm{MgSO}_{4}\right)$, and concentrated under reduced pressure to a bright yellow oil. Radial chromatography (light petroleum elution) gave cyclopropanaphthalene (58) (11.6 mg, 12\%) as the most mobile component. Further elution (light petroleum) furnished a bright yellow oil which on trituration (hexane, $-16^{\circ} \mathrm{C}$ ) afforded $1^{\prime}$-(phenylpentylidene)$1 \mathrm{H}$-cyclopropa[b]naphthalene $(114 \mathrm{n})(113.5 \mathrm{mg}, 57 \%)$ as bright yellow plates, m.p. 66$68^{\circ} \mathrm{C}$ (Found: C, 92.9; H, 7.3. $\mathrm{C}_{22} \mathrm{H}_{20}$ requires $\mathrm{C}, 92.9 ; \mathrm{H}, 7.1 \%$ ). IR $v_{\max } 3079,3053$, 2949, 2866, 2855, 1590, 1491, 1450, 1441, 1420, 1350, 1172, 1145, 851, 765, 748, 689, $600 \mathrm{~cm}^{-1}$. UV $\lambda_{\max }$ (hexane) 230 (4.71), $244 \mathrm{sh}$ (4.31), $253 \mathrm{sh}$ (4.20), 288 (4.36), $374 \mathrm{sh}$ (4.32), 394 (4.59), $421 \mathrm{~nm}$ (log ع 4.63); $\lambda_{\max }$ (acetonitrile) 230 (4.70), $244 \mathrm{sh}$ (4.31), $254 \mathrm{sh}$ (4.17), 288 (4.36), $370 \mathrm{sh}$ (4.30), 393 (4.61), $418 \mathrm{~nm}$ (log $\varepsilon 4.64) .{ }^{1} \mathrm{H}$ n.m.r. $\delta 0.99$ (t, $J$ $7.3 \mathrm{~Hz}, \mathrm{CH}_{3}$ ), 1.48 (hexet, J 7.4 Hz, $\mathrm{CH}_{3} \mathrm{CH}_{2}$ ), 1.80 (pentet, $J 7.5 \mathrm{~Hz}, \mathrm{CH}_{3} \mathrm{CH}_{2} \mathrm{CH}_{2}$ ), 2.95 (t, $\left.J 7.6 \mathrm{~Hz}, \mathrm{CH}_{2}-\mathrm{C}=\right), 7.31$ (app tt, $\left.1 \mathrm{H}, J 7.3 \mathrm{~Hz}, J 1.6 \mathrm{~Hz}, \mathrm{H} 12\right), 7.45-7.51$ (m, 5H, H2 or $\mathrm{H} 7, \mathrm{H} 4 / 5$, and $\mathrm{H} 11 / 13), 7.58$ (d, $1 \mathrm{H}, J 1.7 \mathrm{~Hz}, \mathrm{H} 7$ or $\mathrm{H} 2), 7.87-7.94$ (m, 4H, H3/6 and H10/14); ${ }^{13}$ C n.m.r. $\delta 14.0$ (C18), 22.6 (C17), 30.7 (C16), 32.4 (C15), 106.7 (C7 or C2), 106.8 (C2 or C7), 110.0 (C1), 118.5 (C8), 125.3 (C10/14), 126.3(5) (C4 or C5), 126.4(1) (C4 or C5), 126.75 (C12), 126.77 (C1a or C7a), 128.4 (C7a or C1a), 128.5(0) (C11/13), 128.5(4) (C3 or C6), 128.7 (C6 or C3), 138.0 (C2a or C6a), 138.5 (C2a, or C6a or C9), 139.0 (C6a or C2a or C9). Mass spectrum (relative intensity): $m / z(70 \mathrm{eV}) 285(14, \mathrm{M}+1)$, 284 (57, M), 269 (5, M-Me), 255 (14, M-Et), 254 (12), 253 (12), 252 (11), 242 (27), 241 (100, M-Pr), 240 (43), 239 (71), 237 (10), 228 (20), 227 (7, M-Bu), 226 (18), 215 (15), 120 (12), 91 (10), 63 (12), 51 (16), 43 (26, Pr), 42 (10), 41 (43), 39 (31), 29 (29, Et), 27 (71\%). 
1-[(p-Bromophenyl)phenylmethylidene]-1H-cyclopropa[b]naphthalene (114o).

To a stirred solution of disilylcyclopropanaphthalene (105) (284 mg, $1 \mathrm{mmol}$ ) and p-bromobenzophenone $(261 \mathrm{mg}, 1 \mathrm{mmol})$ in dry thf $(10 \mathrm{ml})$, cooled to $-70^{\circ} \mathrm{C}$ and under a dry nitrogen atmosphere, was added potassium $t$-butoxide $(112 \mathrm{mg}, 1 \mathrm{mmol})$ in the same solvent $(10 \mathrm{ml})$. The bright yellow mixture was allowed to warm slowly to ambient temperature overnight with stirring and then was poured into water/dichloromethane $(100 \mathrm{ml} ; 1: 1)$. The aqueous phase was extracted (dichloromethane, $3 \times 15 \mathrm{ml})$ and the combined organic layers washed (water, $50 \mathrm{ml}$ ), dried $\left(\mathrm{MgSO}_{4}\right)$, and concentrated under reduced pressure to a bright yellow oil. Trituration (hexane, $-16^{\circ} \mathrm{C}$ ) afforded $1-[(p$-bromophenyl)phenylmethylidene]-1H-cyclopropa[b]naphthalene (1140) (345.2 mg, 90\%) as bright yellow needles, m.p. $100-101^{\circ} \mathrm{C}$ (Found: C, 75.4; H, 3.7(5); Br, 20.7. $\mathrm{C}_{24} \mathrm{H}_{15} \mathrm{Br}$ requires $\mathrm{C}, 75.2 ; \mathrm{H}, 3.9(5) ; \mathrm{Br}, 20.8(5) \%)$. IR $v_{\max } 3036,3007,1777,1588,1478,1443$, $1418,1345,1136,1069,1009,841,760,748,741,698,687,536,455 \mathrm{~cm}^{-1}$. UV $\lambda_{\max }$ (hexane) 230 (4.71), 276 (4.47), $292 \mathrm{sh}$ (4.27), 412 (4.62), $438 \mathrm{~nm}$ (log $\varepsilon$ 4.64); $\lambda_{\max }$ (acetonitrile) 230 (4.73), 273 (4.49), $288 \mathrm{sh}$ (4.36), 412 (4.64), $435 \mathrm{~nm}(\log \varepsilon 4.65) .{ }^{1} \mathrm{H}$ n.m.r. $\delta 7.37$ (app tt, $J 7.3 \mathrm{~Hz}, J 1.6 \mathrm{~Hz}, 1 \mathrm{H}, \mathrm{H} 18$ ), 7.44-7.51 (m, 4H, H4/5 and H17/19), 7.53-7.63 (m, 6H, H2/7, H10/14, and H11/13), 7.70-7.74 (m, 2H, H16/20), 7.86-7.89 (m, $2 \mathrm{H}, \mathrm{H} 3 / 6) ;{ }^{13} \mathrm{C}$ n.m.r. $\delta 107.4(7)(\mathrm{C} 2$ or $\mathrm{C} 7), 107.6(0)$ (C7 or $\left.\mathrm{C} 2\right), 112.3(\mathrm{C} 1), 118.5(\mathrm{C} 8)$, 121.1 (C12), 126.9(0) (C4/5), 126.9(4) (C1a or C7a), 126.9(9) (C7a or C1a), 127.6 (C18), 128.1 (C16/20), 128.6 (C17/19), 128.8 (C3/6), 129.7 (C10/14 or C11/13), $131.6(\mathrm{C} 11 / 13$ or C10/14), 138.5 (C9), 138.7(8) (C2a or C6a), 138.8(1) (C6a or C2a), 139.0 (C15). Mass spectrum (relative intensity): $m / z(70 \mathrm{eV}) 385\left(16, \mathrm{C}_{24} \mathrm{H}_{15}{ }^{81} \mathrm{Br}+1\right), 384\left(63, \mathrm{C}_{24} \mathrm{H}_{15}{ }^{81} \mathrm{Br}\right.$ ), 382 (57, $\mathrm{C}_{24} \mathrm{H}_{15}{ }^{79} \mathrm{Br}$ ), 304 (10), 303 (37, M-Br), 302 (100, M-HBr), 301 (18), 300 (47), 298 (10), 151 (19) 150 (19\%).

\section{1-[Di(p-bromophenyl)methylidene]-1H-cyclopropa[b]naphthalene (114p).}

To a stirred solution of disilylcyclopropanaphthalene (105) (500 mg, $1 \mathrm{mmol})$ and p,p'-dibromobenzophenone $(598 \mathrm{mg}, 1.76 \mathrm{mmol})$ in thf $(20 \mathrm{ml})$, cooled to $-70^{\circ} \mathrm{C}$ and under a dry nitrogen atmosphere, was added potassium $t$-butoxide $(198 \mathrm{mg}, 1.76 \mathrm{mmol})$ in the same solvent $(20 \mathrm{ml})$. The bright yellow mixture was allowed to warm slowly to ambient temperature overnight with stirring and then was poured into water/dichloromethane $(100 \mathrm{ml} ; 1: 1)$. The aqueous phase was extracted (dichloromethane, $3 \times 15 \mathrm{ml})$ and the combined organic layers washed (water, $50 \mathrm{ml}$ ), dried $\left(\mathrm{MgSO}_{4}\right)$, and concentrated under reduced pressure to a bright orange solid. Trituration (hexane, $-16^{\circ} \mathrm{C}$ ) afforded 1 - 
[di(p-bromophenyl)methylidene]-1H-cyclopropa[b]naphthalene (114p) $(759 \mathrm{mg}, 94 \%)$ as lustrous orange crystals, m.p. $193-194^{\circ} \mathrm{C}$ (Found: C, 62.3; H, 3.0; $\mathrm{C}_{12} \mathrm{H}_{15} \mathrm{Br}_{2}$ requires $\mathrm{C}$, 62.4; H, 3.0(5)\%; Found $[\mathrm{M}+\mathrm{H}]^{+} 460.9549 ; \mathrm{C}_{12} \mathrm{H}_{15}{ }^{79} \mathrm{Br}_{2}$ requires $\left.[\mathrm{M}+\mathrm{H}]^{+} 460.9541\right)$. IR $v_{\max } 3046,3034,1777,1482,1423,1392,1346,1248,1176,1136,1071,1005,967,835$, $825,751,721,703 \mathrm{~cm}^{-1}$. UV $\lambda_{\max }$ (hexane) 230 (4.68), $276 \mathrm{sh}(4.50), 284$ (4.53), 414 (4.59), $440 \mathrm{~nm}$ (log $\varepsilon 4.60) ; \lambda_{\max }$ (acetonitrile) 230 (4.68), $274 \mathrm{sh}$ (4.49), 281 (4.52), 412 (4.59), 438 nm (log ع 4.59). ' H n.m.r. $\delta 7.50-7.53$ (BB', 2H, H4/5), 7.59 (bs, s, 10H, H2/7, $\mathrm{H} 9 / 13$ and $\mathrm{H} 10 / 12), 7.89-7.93$ (AA', H3/6); ${ }^{13} \mathrm{C}$ n.m.r. $\delta 107.8(\mathrm{C} 2 / 7), 112.6(\mathrm{C} 1), 117.3$ (C8), 121.3 (C12), 126.6 (C1a/7a), 127.1 (C4/5), 128.9 (C3/6), 129.5 (C10/14 or C11/13), 131.7 (C11/13 or C10/14), 138.0 (C9), 138.9 (C2a/6a).

Attempted preparation of 1-\{[10'-(6,6-diphenylpentafulvalenyl)]phenylmethylidene $\}-1 \mathrm{H}$ cyclopropa[b]naphthalene (197a) from 1-[(p-bromophenyl)phenylmethylidene]-1H-cyclopropa[b]naphthalene $(1140)$ via the Oda procedure.

To a stirred solution of 1-[(p-bromophenyl)phenylmethylidene $]-1 H$-cyclopropa $[b]-$ naphthalene $(1140)(550 \mathrm{mg}, 1.44 \mathrm{mmol})$ in dry thf $(35 \mathrm{ml})$ cooled to $-78^{\circ} \mathrm{C}$ and under an argon atmosphere was added butyllithium $(1.92 \mathrm{M}, 0.78 \mathrm{ml}, 1.5 \mathrm{mmol})$. The mixture which developed a dark yellow/brown colouration was stirred at this temperature for $1 \mathrm{~h}$ and the $N, N$-dimethylbenzamide $(245 \mathrm{mg}, 1.64 \mathrm{mmol})$ was added under a stream of argon. The mixture was then stirred at $0^{\circ} \mathrm{C}$ for $1 \mathrm{~h}$ and was then quenched with cyclopentadiene $(0.24 \mathrm{ml}, 2.88 \mathrm{mmol})$. The dark mixture was then allowed to attain ambient temperature overnight and was then poured into water/dichloromethane $(400 \mathrm{ml} ; 1: 1)$. The phases were separated and the aqueous mixture extracted (dichloromethane; $2 \times 100 \mathrm{ml}$ ) and the combined organic layers washed (water, $100 \mathrm{ml})$, dried $\left(\mathrm{MgSO}_{4}\right)$ and concentrated under reduced pressure to a red oil. Radial chromatography (dichloromethane/light petroleum elution; $1: 4)$ and collection of the component with an $R_{\mathrm{f}}$ of 0.5 gave a red oil that contained the title compound (197a) ( ${ }^{1} \mathrm{H}$ n.m.r.), vide infra. Attempts to further purify this oil by radial chromatography, trituration, sublimation, or by use of the Sephadex LH-20 size exclusion gel all resulted in the decomposition of this compound. The identity of the decomposition products remains unknown.

Preparation of 1-\{di[(10'-[6,6-diphenylpentafulvalenyl])]methylidene $\}-1 \mathrm{H}$-cyclopropa[b]naphthalene (199) from 1-[di(p-bromophenyl)lmethylidene]-1H-cyclopropa[b]naphthalene (114p) via the Oda procedure. 
To a stirred solution of $(114 \mathrm{p})(461 \mathrm{mg}, 1.0 \mathrm{mmol})$ in dry thf $(35 \mathrm{ml})$ cooled to $-78^{\circ} \mathrm{C}$ and under an argon atmosphere was added butyllithium $(1.92 \mathrm{M}, 0.52 \mathrm{ml}$, $1.14 \mathrm{mmol}$ ). The mixture which developed a dark yellow/brown colouration was stirred at this temperature for $1 \mathrm{~h}$ and the $N, N$-dimethylbenzamide $(169 \mathrm{mg}, 1.14 \mathrm{mmol}$ ) was added under a stream of argon. The mixture was then stirred at $0^{\circ} \mathrm{C}$ for $1 \mathrm{~h}$ and was then quenched with cyclopentadiene $(0.17 \mathrm{ml}, 2.0 \mathrm{mmol})$. The dark mixture was then allowed to slowly attain ambient temperature slowly overnight and was then poured into water/dichloromethane $(400 \mathrm{ml} ; 1: 1)$. The phases were separated and the aqueous mixture extracted (dichloromethane; $2 \times 100 \mathrm{ml}$ ). The combined organic layers were washed (water, $100 \mathrm{ml})$, dried $\left(\mathrm{MgSO}_{4}\right)$, and concentrated under reduced pressure to an orange oil that contained in excess of ten components by t.l.c. Radial chromatography (dichloromethane/ light petroleum elution; $1: 4)$ and collection of the band with $R_{\mathrm{f}} 0.5$ gave an impure sample of (199). Recrystallisation (dichloromethane, $-16^{\circ} \mathrm{C}$ ) followed by purification on Sephadex LH-20 size exclusion gel (thf elution) and one further radial chromatography (dichloromethane elution) gave an analytical sample proposed as $1\{[$ di(10'-[6,6-diphenylpentafulvalenyl]])methylidene $\}$ - $1 \mathrm{H}$-cyclopropa[b]naphthalene (199) as a bright orange oil (6 mg, $1 \%$ ), m.p. $>305^{\circ} \mathrm{C}$ (Found: $[\mathrm{M}+\mathrm{H}]^{+} 609.2679 . \mathrm{C}_{48} \mathrm{H}_{32}$ requires $[\mathrm{M}+\mathrm{H}]^{+} 609.2577$ ). IR $v_{\max } 3052,2922,1773,1584,1489,1466,1442,1425,1361,1347,1323,1176,1137$, $1084,1073,1029,1017,997,849,824,780,745,697,676,645 \mathrm{~cm}^{-1}$. UV $\lambda_{\max }$ (hexane) 232 (4.83), 257, (4.65), 288 (4.59), 350 (4.51), 444 nm (log ع 4.68); UV $\lambda_{\max }$ (acetonitrile) 232 (4.74), 254, (4.56), 286 (4.51), 337 (4.38), 441 nm (log ع 4.58) ${ }^{1}$ H n.m.r. $\delta$ 6.32-6.36 (m, 2H, H1' or H4'), 6.48-6.52 (m, 2H, H4' or H1'), 6.63-6.70 (m, 4H, H2'/H3'), 7.427.48 (m, 14H, H8'/12', H14'/18', H15'/17' and H16'), 7.49-7.54 (BB', 2H, H4/5), 7.65 (s, 2H, H2/7), 7.82-7.87 (m, 4H, H9'/11'), 7.91-7.95 (AA', 2H, H3/6); ${ }^{13}$ C n.m.r. $\delta 107.8$ (C2/7), 113.3 (C1), 118.4 (C8), 124.3 (C1' or C4'), 124.5 (C4' or C1'), 127.0(3) (C4/5), 127.0(6) (C1a/7a), 127.3 (C8'/12', C14'/18' or C15'/17'), 127.7 (C9'/11'), 128.7 (C16'), 128.9 (C3/6), 132.1 (C2' or C3'), 132.2 (C8'/12', C14'/18' or C15'/17'), 132.4 (C2' or C3'), 132.5 (C8'/12' or C14'/18' or C15'/17'), 139.0 (C2a/6a), 139.7 (C6', C7',C10', C13'), 140.2 (C10' or C7'), 141.3 (C6', or C7', or C10', or C13'), 143.9 (C5'), 151.7 (C7', C6', C10' or C13').

1-[(p-Hydroxyphenyl)phenylmethylidene $]-1 \mathrm{H}$-cyclopropa[b]naphthalene $(114 q)$ from the attempted preparation of 1 -[p-benzoylphenyl)phenylmethylidene]-1H-cyclopropa[b]naphthalene (200). 
To a stirred solution of 1-[(p-bromophenyl)phenylmethylidene $]-1 H$-cyclopropa $[b]-$ naphthalene (114o) $(100 \mathrm{mg}, 0.26 \mathrm{mmol})$ in dry thf $(2 \mathrm{ml})$, cooled to $-78^{\circ} \mathrm{C}$ and under an argon atmosphere was added butyllithium $(2.5 \mathrm{M}, 0.1 \mathrm{ml}, 0.26 \mathrm{mmol})$. The dark mixture was stirred at this temperature for $1 \mathrm{~h}$ and then $N, N$-dimethylbenzamide (39 mg, $0.26 \mathrm{mmol}$ ) was added under a stream of argon. The mixture was then allowed to warm to ambient temperature overnight and was poured into water/dichloromethane $(100 \mathrm{ml} ; 1: 1)$. After separating the phases the organic layer was washed (water, $2 \times 25 \mathrm{ml}$ ), dried $\left(\mathrm{MgSO}_{4}\right)$, and concentrated under reduced pressure to an orange oil. Radial chromatography (light petroleum elution) and collection of the major component gave 1-(diphenylmethylidene)$1 H$-cyclopropa[b]naphthalene $(1141)(31 \mathrm{mg}, 40 \%)$ as a bright yellow oil the n.m.r. data of which were identical to those of authentic sample. ${ }^{24}$ Further elution (dichloromethane/ light petroleum; 1:1) gave 1-[(p-hydroxyphenyl)phenylmethylidene]-1H-cyclopropa[b]naphthalene (114q) (17 mg, 20\%) as a dark green/brown solid, m.p. $85-88^{\circ} \mathrm{C}$ (Found: [M$\mathrm{H}^{+}$(electrospray) 319.1131. $\mathrm{C}_{24} \mathrm{H}_{16} \mathrm{O}$ requires $[\mathrm{M}-\mathrm{H}]^{+}$319.1137). IR $v_{\max } 3401,2975$, 2953, 1633, 1608, 1445, 1413, 1384, 1267, 1173, 1138, 1089, 1049, 880, 845, $698 \mathrm{~cm}^{-1}$. UV $\lambda_{\max }$ (hexane) 230 (4.58), 275, (4.28), 413 (4.43), $441 \mathrm{~nm}$ (log $\left.\varepsilon 4.50\right)$; UV $\lambda_{\max }$ (acetonitrile) 230 (4.58), 274 (4.29), 275 (4.29) 414 (4.43), 440 nm (log $\varepsilon 4.48){ }^{1} \mathrm{H}$ n.m.r. $\delta$ 5.02 (bd s, OH), 6.93-6.97 (m, 2H, H10/14), 7.37 (app tt, $J 7.3, J 1.7 \mathrm{~Hz}, \mathrm{H} 18$ ), 7.45-7.52 (m, 6H, H2/7, H4/5 and H17/19), 7.63-7.68 (m, 2H, H11/13), 7.74-7.77 (m, 2H, H16/20), 7.86-7.89 (AA', 2H, H3/6); ${ }^{13} \mathrm{C}$ n.m.r. $\delta$ 106.6(8) (C2 or C7), 106.7(1) (C7 or C2), 110.7 (C1), 115.4 (C10/14), 119.6 (C8), 126.5(6) (C4/5), 126.5(8) (C4/5), 127.4 (C18), 127.5 (C1a/7a), 128.2 (C16/20), 128.4 (C17/19), 128.7 (C3/6), 129.6 (C11/13), 132.1 (C9), 138.5(8), (C2a or C6a), 138.6(2) (C6a or C2a), 139.6 (C15), 155.0 (C12).

Preparation of 1-[(p-hydroxyphenyl)phenylmethylidene]-1H-cyclopropa[b]naphthalene (114q) from lithiate (193) and oxygen.

To a stirred solution of 1-[( $p$-bromophenyl)phenylmethylidene]-1H-cyclopropa[b]naphthalene $(114 \mathrm{o})(100 \mathrm{mg}, 0.26 \mathrm{mmol})$ in dry thf $(2 \mathrm{ml})$, cooled to $-78^{\circ} \mathrm{C}$ and under an argon atmosphere was added butyllithium $(2.5 \mathrm{M}, 0.1 \mathrm{ml}, 0.26 \mathrm{mmol})$. The dark mixture was stirred at this temperature for $1 \mathrm{~h}$ and then $10 \mathrm{ml}$ of air was injected into the flask. The mixture was then allowed to warm to ambient temperature overnight and was poured into water/dichloromethane $(100 \mathrm{ml} ; 1: 1)$, the phases were separated, the organic layer was washed (water, $2 \times 25 \mathrm{ml}$ ), dried $\left(\mathrm{MgSO}_{4}\right)$, and concentrated under reduced pressure to an orange oil. Radial chromatography (light petroleum elution) and collection of the major 
component gave 1-(diphenylmethylidene)-1H-cyclopropa[b]naphthalene (114l) (26 mg, $33 \%)$ as a bright yellow oil; the n.m.r. data were identical to those of authentic sample. ${ }^{24}$ Further elution (dichloromethane/light petroleum; 1:1) gave 1-[( $p$-hydroxyphenyl)phenylmethylidene]- $1 H$-cyclopropa[ $b]$ naphthalene $(114 \mathrm{q})(16 \mathrm{mg}, 20 \%)$ as a dark green/brown solid that was identical in all respects to the sample obtained above.

Reaction of the anion (193) derived from 1-[(p-bromophenyl)phenylmethylidene]-1Hcyclopropa[b]naphthalene (1140) with water.

To a stirred solution of 1-[(p-bromophenyl)phenylmethylidene $]-1 H$-cyclopropa $[b]$ naphthalene (1140) $(100 \mathrm{mg}, 0.26 \mathrm{mmol})$ in dry thf $(2 \mathrm{ml})$, cooled to $-78^{\circ} \mathrm{C}$ and under an argon atmosphere was added butyllithium $(2.5 \mathrm{M}, 0.1 \mathrm{ml}, 0.26 \mathrm{mmol})$. The dark mixture was stirred at this temperature for $1 \mathrm{~h}$ and then was quenched with water $(0.1 \mathrm{ml}$, $5.6 \mathrm{mmol}$ ). The mixture was mixture was stirred at this temperature for a further $1 \mathrm{~h}$ and then was poured into water/dichloromethane $(100 \mathrm{ml} ; 1: 1)$. After separating the phases the organic layer was washed (water, $2 \times 25 \mathrm{ml}$ ), dried $\left(\mathrm{MgSO}_{4}\right)$, and concentrated under reduced pressure to an orange oil. Radial chromatography (light petroleum elution) and collection of the major component gave 1-(diphenylmethylidene)-1H-cyclopropa[b]naphthalene $(1141)(68 \mathrm{mg}, 87 \%)$ as a bright yellow oil as the only chromatographically mobile component; all n.m.r. data were identical to those of authentic sample. ${ }^{24}$

Warming the anion (193) derived from 1-[(p-bromophenyl)phenylmethylidene]-1H-cyclopropa $[\mathrm{b}]$ naphthalene $(1140)$ to room temperature in the absence of an electrophile.

To a stirred solution of 1-[( $p$-bromophenyl)phenylmethylidene]-1H-cyclopropa[b]naphthalene $(1140)(100 \mathrm{mg}, 0.26 \mathrm{mmol})$ in dry thf $(2 \mathrm{ml})$, cooled to $-78^{\circ} \mathrm{C}$ and under an argon atmosphere was added butyllithium $(2.5 \mathrm{M}, 0.1 \mathrm{ml}, 0.26 \mathrm{mmol})$. The dark mixture was allowed to warm slowly to ambient temperature $(2 \mathrm{~h})$ and was poured into water/ dichloromethane $(100 \mathrm{ml} ; 1: 1)$. After separating the phases the organic layer was washed (water, $2 \times 25 \mathrm{ml}$ ), dried $\left(\mathrm{MgSO}_{4}\right)$, and concentrated under reduced pressure to an orange oil. Radial chromatography (light petroleum elution) and collection of the major component gave an inseparable mixture $(79 \mathrm{mg})$ of 1 -(diphenylmethylidene)- $1 H$-cyclopropa $[b]$ naphthalene $(114 \mathrm{l})^{24}$ and a product proposed as 1-[( $p$-butylphenyl)phenylmethylidene $]-1 H$-cyclopropa $[b]$ naphthalene $(114 \mathrm{r})$ in a $1: 1$ ratio $\left({ }^{1} \mathrm{H}\right.$ n.m.r.) (45\% each). (Found: $[\mathrm{M}+\mathrm{H}]^{+}$361.19511. $\mathrm{C}_{28} \mathrm{H}_{24}$ requires $[\mathrm{M}+\mathrm{H}]^{+}$361.1951). N.m.r data abstracted for (114r) from the mixture are: ${ }^{1} \mathrm{H}$ n.m.r. $\delta 1.00\left(\mathrm{t}, 3 \mathrm{H}, J 7.3 \mathrm{~Hz}, \mathrm{CH}_{3}\right), 1.45$ (hextet, $J$ 
$7.57 \mathrm{~Hz}, \mathrm{CH}_{3} \mathrm{CH}_{2}$ ), 1.71 (pentet, $J 7.8 \mathrm{~Hz}, 2 \mathrm{H}, \mathrm{CH}_{3} \mathrm{CH}_{2} \mathrm{CH}_{2}$ ), 2.72 (t, J 7.81 Hz, 2H, $\mathrm{CH}_{2^{-}}$ $\mathrm{C}=), 7.29-7.32(\mathrm{~m}, 2 \mathrm{H}), 7.35-7.40(\mathrm{~m}, 2 \mathrm{H}), 7.48-7.52(\mathrm{~m}, 5 \mathrm{H}), 7.53(\mathrm{~d}, J 2.0 \mathrm{~Hz}, 1 \mathrm{H}, \mathrm{H} 2$ or H7), 7.56 (d, J $2.0 \mathrm{~Hz}, 1 \mathrm{H}, \mathrm{H} 2$ or H7), 7.69-7.72 (m, 2H), 7.86-7.92 (AA', 2H, H3/6); ${ }^{13} \mathrm{C}$ n.m.r. $\delta 14.0\left(\mathrm{CH}_{3}\right), 22.5\left(\mathrm{CH}_{2}\right), 33.6\left(\mathrm{CH}_{2}\right), 35.5\left(\mathrm{CH}_{2}\right), 106.8(9)(\mathrm{C} 2$ or $\mathrm{C} 7), 106.9(6)(\mathrm{C} 2$ or $\mathrm{C} 7), 111.3(\mathrm{C} 1), 119.8(\mathrm{C} 8), 127.3(\mathrm{CH}), 127.4(\mathrm{CH}), 127.5\left(4^{\circ}\right), 127.6\left(4^{\circ}\right), 128.0$ $(\mathrm{CH}), 128.2(\mathrm{CH}), 128.4(\mathrm{CH}), 128.5(\mathrm{CH}), 128.7(\mathrm{CH}), 136.7\left(4^{\circ}\right), 138.6\left(4^{\circ}\right), 139.4\left(4^{\circ}\right)$, $142.3\left(4^{\circ}\right)$. Further elution (dichloromethane/light petroleum; 1:1) gave 1-[(p-hydroxyphenyl)phenylmethylidene]-1H-cyclopropa[$[b]$ naphthalene $(114 \mathrm{q})(3.3 \mathrm{mg}, 4 \%)$ as a dark green/brown solid that was identical in all respects to the samples obtained above.

1-[p-Benzoylphenyl)phenylmethylidene]-1H-cyclopropa[b]naphthalene-0.9 $\mathrm{CH}_{2} \mathrm{Cl}_{2}$ (200).

To a stirred solution of 1-[(p-bromophenyl)phenylmethylidene $]-1 H$-cyclopropa[ $b]-$ naphthalene $(114 \mathrm{o})(700 \mathrm{mg}, 1.82 \mathrm{mmol})$ in dry thf $(20 \mathrm{ml})$, cooled to $-78^{\circ} \mathrm{C}$ and under an argon atmosphere was added butyllithium $(2.30 \mathrm{M}, 0.79 \mathrm{ml}, 1.82 \mathrm{mmol})$. The dark mixture was stirred at this temperature for $1 \mathrm{~h}$ and then solid $N, N$-dimethylbenzamide $(411 \mathrm{mg}$, $1.82 \mathrm{mmol}$ ) was quickly added in one portion under a steady flow of dry argon. The mixture was then allowed to warm to ambient temperature overnight and was then poured into water/ dichloromethane $(200 \mathrm{ml} ; 1: 1)$. After separating the phases the organic layer was washed (water, $2 \times 50 \mathrm{ml}$ ), dried $\left(\mathrm{MgSO}_{4}\right)$, and concentrated under reduced pressure to an orange oil. Radial chromatography (light petroleum elution) and collection of the major compon-ent gave 1-(diphenylmethylidene)-1H-cyclopropa[b]naphthalene (114l) (68 mg, $12 \%$ ) as a bright yellow oil; all n.m.r. data were identical to those of authentic sample. ${ }^{24}$ Further elution (dichloromethane/petroleum ether; 1:1) and recrystallisation (diethyl ether) of the yellow residue gave 1 -[p-benzoylphenyl)phenylmethylidene]-1H-cyclopropa[b] naphthalene $-0.85 \mathrm{CH}_{2} \mathrm{Cl}_{2}(200)(570 \mathrm{mg}, 77 \%)$ as bright yellow crystals, m.p. $76-78^{\circ} \mathrm{C}$ (Found: C, 79.0; H, 4.6; $\mathrm{C}_{31} \mathrm{H}_{20} \mathrm{O}-0.85 \mathrm{CH}_{2} \mathrm{Cl}_{2}$ requires $\mathrm{C}, 79.5 ; \mathrm{H}, 4.5$; Found: $[\mathrm{M}+\mathrm{H}]^{+}$ (electrospray) 409.1565; $\mathrm{C}_{31} \mathrm{H}_{20} \mathrm{O}$ requires $[\mathrm{M}+\mathrm{H}]^{+}$409.1587). IR $v_{\max } 3049,2923,1774$, $1650,1594,1444,1346,1314,1277,1174,1135,936,923,850,763,747,698,664 \mathrm{~cm}^{-1}$. UV $\lambda_{\max }$ (hexane) 230 (4.99), 260 (4.93), 278 (4.95), 422 (4.81), $446 \mathrm{~nm}$ (log $\left.\varepsilon 4.80\right)$; UV $\lambda_{\max }$ (acetonitrile) 230 (5.09), 260 (4.91), 280 (4.79), 423 (4.99), $442 \mathrm{~nm}(\log \varepsilon 4.97){ }^{1} \mathrm{H}$ n.m.r. $\delta 7.41$ (app tt, $J 7.3, J 1.7 \mathrm{~Hz}, 1 \mathrm{H}, \mathrm{H} 17$ or $\mathrm{H} 25), 7.48-7.56(\mathrm{~m}, 6 \mathrm{H}), 7.60-7.65$ (m, $3 \mathrm{H}, \mathrm{H} 2 / 7$ and $\mathrm{H} 17$ or $\mathrm{H} 25), 7.75-7.79(\mathrm{~m}, 2 \mathrm{H}), 7.88-7.96(\mathrm{~m}, 8 \mathrm{H}) ;{ }^{13} \mathrm{C}$ n.m.r. $\delta 107.9(\mathrm{C} 2$ or C7), 108.0 (C2 or C7), $113.7(\mathrm{C} 1), 118.5(\mathrm{C} 8), 126.8\left(4^{\circ}\right), 127.0(\mathrm{CH}), 127.5(\mathrm{CH})$, $127.6(\mathrm{C} 17$ or $\mathrm{C} 25), 128.2(\mathrm{CH}), 128.3(\mathrm{CH}), 128.6(\mathrm{CH}), 128.9(\mathrm{CH}), 130.0(\mathrm{CH}), 130.5$ 
$(\mathrm{CH}), 132.3(\mathrm{C} 25$ or $\mathrm{C} 17), 135.3\left(4^{\circ}\right), 135.9\left(4^{\circ}\right), 137.9\left(4^{\circ}\right), 138.8\left(4^{\circ}\right), 138.9\left(4^{\circ}\right), 139.0$ $\left(4^{\circ}\right), 143.9\left(4^{\circ}\right), 196.1(\mathrm{C} 13)$. After pulverising the sample and pumping it under high vacuum 1-[p-benzoylphenyl)phenylmethylidene]-1H-cyclopropa[b]naphthalene-0.5 $\mathrm{H}_{2} \mathrm{O}$ was obtained, m.p. $66-69^{\circ} \mathrm{C}$ (Found: $\mathrm{C}, 89.4 ; \mathrm{H}, 5.0 ; \mathrm{C}_{31} \mathrm{H}_{20} \mathrm{O}-0.5 \mathrm{H}_{2} \mathrm{O}$ requires $\mathrm{C}, 89.2$; H, 5.1).

Preparation of 1-\{[10'-(6,6-Diphenylpentafulvalenyl)]phenylmethylidene $\}-1 \mathrm{H}-$ cyclopropa[b]naphthalene-0.18 $\mathrm{C}_{6} \mathrm{H}_{14}(197 a)$.

To a stirred solution of 1-[p-benzoylphenyl)phenylmethylidene]-1H-cyclopropa[b]naphthalene-0.9 $\quad \mathrm{CH}_{2} \mathrm{Cl}_{2} \quad(200) \quad(100 \mathrm{mg}, \quad \sim 0.83 \mathrm{mg}$ unsolvated 1-[(p-benzoylphenyl)phenylmethylidene]-1H-cyclopropa[b]naphthalene, $\sim 0.20 \mathrm{mmol}$ ) and 18-crown-6 (4 mg, $0.01 \mathrm{mmol})$ in dry thf $(10 \mathrm{ml})$, at $0^{\circ} \mathrm{C}$ and under argon, was added cyclopentadiene $(0.02 \mathrm{ml}, 0.25 \mathrm{mmol})$ and then freshly ground potassium hydroxide $(14 \mathrm{mg}, 0.25 \mathrm{mmol})$. The now dark red mixture was then allowed to stir overnight at room temperature and then poured into water/dichloromethane $(100 \mathrm{ml} ; 1: 1)$. After separating the phases the aqueous layer was extracted (dichloromethane, $2 \times 25 \mathrm{ml}$ ) and the combined organic layers washed (water; $2 \times 25 \mathrm{ml}$ ), dried $\left(\mathrm{MgSO}_{4}\right)$, and concentrated under reduced pressure to a red oil. Radial chromatography (dichloromethane/light petroleum elution; 4:1) and collection of the only mobile component gave an orange oil that on trituation (dichloromethane/hexane $\left.1: 1,-16^{\circ} \mathrm{C}\right)$ gave $1-\left\{\left[10^{\prime}-(6,6\right.\right.$-diphenylpentafulvalenyl $\left.)\right]$ phenylmethylidene $\}-1 \mathrm{H}$-cyclopropa/b]naphthalene-0.18 $\mathrm{C}_{6} \mathrm{H}_{14}$ (197a) (30 mg, 31\%; based on the amount of unsolvated alkylidenecyclopropanaphthalene used) as an orange power, m.p. $122-124^{\circ} \mathrm{C}$ (Found: $[\mathrm{M}+\mathrm{H}]^{+}$457.1941; $\mathrm{C}_{36} \mathrm{H}_{24}$ requires $[\mathrm{M}+\mathrm{H}]^{+}$457.1951). IR $v_{\max } 3049,2952,2922,1776$, $1581,1488,1442,1360,1325,1137,966,848,822,696,674,647 \mathrm{~cm}^{-1}$. UV $\lambda_{\max }$ (hexane) 232 (4.62), 256 (4.46), 280 (4.42), 346 (4.19), 431 (4.53), $448 \mathrm{~nm}$ (log $\varepsilon 4.53)$; UV $\lambda_{\max }$ (acetonitrile) 230 (4.68), 254 (4.48), 283 (4.41), 344 (4.25), 437 nm (log ع 4.59) ${ }^{1} \mathrm{H}$ n.m.r. 6.32-6.34 (m, 1H, H1' or H5'), 6.49-6.51 (m, 1H, H5' or H1'), 6.63-6.69 (m, 2H, H3'/4'), $7.40-7.53(\mathrm{~m}, 12 \mathrm{H}), 7.59$ (d, $J 1.6 \mathrm{~Hz}, 1 \mathrm{H}, \mathrm{H} 2$ or H7), 7.63 (d, $J 1.6 \mathrm{~Hz}, 1 \mathrm{H}, \mathrm{H} 7$ or H2), 7.78-7.84 (m, 4H), 7.89-7.93 (AA', 2H, H3/6); ${ }^{13}$ C n.m.r. $\delta 107.5$ (C2/7), 112.7 (C1), 119.0 (C8), 124.3 (C5' or C1'), 124.4 (C1' or C5'), 126.8(4) (CH), 126.8(6) (CH), 127.0 $\left(4^{\circ}\right), 127.1(\mathrm{CH}), 127.4\left(4^{\circ}\right), 127.5(\mathrm{CH}), 127.7(\mathrm{CH}), 128.3(\mathrm{CH}), 128.5(\mathrm{CH}), 128.7(2)$ (CH), 128.7(9), (CH), 128.9 (C3/6), 132.1 (C3' or C4'), $132.2(\mathrm{CH}), 132.3$ (C3' or C4'), $132.5(\mathrm{CH}), 138.8\left(4^{\circ}\right), 138.9\left(4^{\circ}\right), 139.1\left(4^{\circ}\right), 140.1\left(4^{\circ}\right), 141.3\left(4^{\circ}\right), 143.8\left(4^{\circ}\right), 151.8$ $\left(4^{\circ}\right)$. 


\section{Chapter 4.}

\section{1-Acetyl-1H-cyclopropa[b]naphthalene (189).}

To a stirred solution of cyclopropa $[b]$ naphthalene $(58)(500 \mathrm{mg}, 3.6 \mathrm{mmol})$ in thf $(15 \mathrm{ml})$, cooled to $-70^{\circ} \mathrm{C}$ and under a nitrogen atmosphere, was added dropwise butyllithium $(1.77 \mathrm{M}, 2.02 \mathrm{ml}, 3.6 \mathrm{mmol})$. The dark mixture was stirred at this temperature for $30 \mathrm{~min}$ and then $\mathrm{N}, \mathrm{N}$-dimethylacetamide $(0.33 \mathrm{ml}, 3.6 \mathrm{mmol})$ was added. The mixture was allowed to warm slowly to ambient temperature overnight and then poured into water/ dichloromethane $(60 \mathrm{ml} ; 1: 1)$. The phases were separated, the aqueous phase was extracted (dichloromethane, $3 \times 15 \mathrm{ml}$ ), and the combined organic layers were washed (water, $50 \mathrm{ml}$ ), dried $\left(\mathrm{MgSO}_{4}\right)$, and concentrated under reduced pressure to a yellow oil. Radial chromatography (light petroleum elution) returned unchanged cyclopropanaphthalene (58) (64 mg, 13\%) as the most mobile component. Further elution (dichloromethane/light petroleum elution; $1: 4)$ gave an orange oil $(203 \mathrm{mg})$. Resubjection of the oil to radial chromatography (dichloromethane/light petroleum elution, 1:4) gave (189) (168 mg, 26\%) as a yellow oil. An analytical sample of 1-acetyl-1H-cyclopropa/b]naphthalene (189) (110 mg, 17\%) was obtained as a yellow oil of limited stability from one further radial chromatography. (Found: C, 84.6; H, 5.6(5). $\mathrm{C}_{13} \mathrm{H}_{10} \mathrm{O}$ requires $\mathrm{C}, 85.7 ; \mathrm{H}, 5.5, \mathrm{O}, 8.8 \%$ ). IR $v_{\max } 3048,2973,1703,1524,1357,1253,1222,1179,1164,1140,863,748 \mathrm{~cm}^{-1}$. UV $\lambda_{\max }$ (acetonitrile) $227 \mathrm{sh}$ (4.57), 268 (3.73), 278 (3.77), $288 \mathrm{sh}$ (3.68), 308 (3.27), $320 \mathrm{sh}$ (3.27), 322 nm (log ع 3.31). ' H n.m.r. $\delta 1.86$ (s, 3H, Me), 4.52 (s, 1H, H1), 7.53-7.58 (BB', 2H, H4/5), 7.73 (s, 2H, H2/7), 7.96-7.99 (AA', 2H, H3/6); ${ }^{13} \mathrm{C}$ n.m.r. $\delta 26.0(\mathrm{Me}), 40.7$ (C1), 113.3 (C2/7), 122.3 (C1a/7a), 126.1 (C4/5), 128.7 (C3/6), 137.3 (C2a/6a), 204.0 (CO). Mass spectrum (relative intensity): $m / z(70 \mathrm{eV}) 183(\mathrm{M}+1,9), 182(\mathrm{M}, 63), 181(\mathrm{M}-$ H, 32), 153 (16), 152 (18), 140 (14), 139 (M-MeCO, 100), 63 (17) 43 (MeCO, 21\%).

\section{1-Benzoyl-1H-cyclopropa[b]naphthalene (202)}

The cycloproparenyl anion (103) was prepared from cyclopropa $[b]$ naphthalene (58) (141 mg, $1.0 \mathrm{mmol}$ ) and butyllithium $(2.5 \mathrm{M}, 0.4 \mathrm{ml}, 1 \mathrm{mmol})$ as described for the preparation of 1 -acetyl-1H-cyclopropa[b]naphthalene (189) above and allowed to react with $\mathrm{N}, \mathrm{N}$-dimethylbenzamide $(149 \mathrm{mg}, 1 \mathrm{mmol})$. The mixture was allowed to warm slowly to ambient temperature overnight and then poured into water/dichloromethane $(60 \mathrm{ml} ; 1: 1)$. The phases were separated, the aqueous phase was extracted (dichloro- 
methane, $3 \times 15 \mathrm{ml}$ ), and the combined organic layers were washed (water, $50 \mathrm{ml}$ ), dried $\left(\mathrm{MgSO}_{4}\right)$, and concentrated under reduced pressure to a yellow solid. Recrystallisation (dichloromethane/light petroleum; 1:1) gave heat and acid sensitive pale yellow plates (111 mg, 46\%) of (202). An analytical sample of 1-benzoyl-1H-cyclopropa[b]naphthalene (202) (26 mg, 11\%) was obtained from one further radial chromatography and recrystallisation (dichloromethane, $-16^{\circ} \mathrm{C}$ ) as colourless plates that slowly turn brown on standing, m.p. $101^{\circ} \mathrm{C}$ (decomp.) (Found: C, 88.3(5); H, $4.8 \mathrm{C}_{18} \mathrm{H}_{12} \mathrm{O}$ requires C, 88.5; H, 4.9(5), O, $6.5(5) \%)$. IR $v_{\max } 3065,3040,3021,1684,1674,1591,1578,1522,1446,1323,1213$, $1179,1065,1009,868,816,754,732,686 \mathrm{~cm}^{-1}$. UV $\lambda_{\max }$ (hexane) 244 (4.13), $252 \mathrm{sh}$ (3.97), 270 (3.72), $280 \mathrm{sh}$ (3.69), $290 \mathrm{sh}$ (3.51), 310 (3.18), $322 \mathrm{~nm}\left(\log \varepsilon\right.$ 3.29). ${ }^{1} \mathrm{H}$ n.m.r. $\delta 5.34$ (s, 1H, H1), 7.49-7.53 (BB', 2H, H4/5), 7.57 (app t, J, 7.3 Hz, 2H, H11/13), 7.66 (app tt, $J, 7.3,1.7 \mathrm{~Hz}, 1 \mathrm{H}, \mathrm{H} 12$ ), 7.78 (s, 2H, H2/7), 7.94-7.98 (AA', 2H, H3/6), 8.23-8.26 (m, 2H, H10/14); ${ }^{13} \mathrm{C}$ n.m.r. $\delta 32.8$ (C1), 109.0 (C2/7), 118.0 (C1a/7a), $121.5(\mathrm{C} 4 / 5), 124.3$ (C11/13), 124.4 (C3/6), 124.5 (C10/14), 129.1 (C12), 131.5 (C9), 132.9 (C2a/6a), 189.6 (C8). Mass spectrum (relative intensity): $\mathrm{m} / z$ (70 eV) $245(\mathrm{M}+1,20), 244(\mathrm{M}, 100), 215$ (33), 139 (M-PhCO, 60) 105 (PhCO, 16), 77 (12\%).

\section{Attempted preparation of 1-formyl-1H-cyclopropa[b]naphthalene (203).}

The cycloproparenyl anion (103) was prepared from cyclopropa[b]naphthalene (58) (139 $\mathrm{mg}, 0.99 \mathrm{mmol})$ and butyllithium $(2.5 \mathrm{M}, 0.40 \mathrm{ml}, 0.99 \mathrm{mmol})$ as described for the preparation of 1-acetyl-1H-cyclopropa[b]naphthalene above and allowed to react with $N, N$ dimethylformamide $(0.077 \mathrm{ml}, 0.99 \mathrm{mmol})$. Conventional workup afforded a yellow oil that contained a complex mixture of components $\left({ }^{1} \mathrm{H}\right.$ n.m.r. and t.l.c), none of which provided data compatible with (203) or unchanged hydrocarbon (58). Attempts to purify the mixture were not made.

\section{Bis(N,N-dimethyl)-1H-cyclopropa[b]naphthalene-1,1-dicarboxamide (205).}

The cycloproparenyl anion (103) was prepared from cyclopropa[b]naphthalene (58) (140 mg, $1.0 \mathrm{mmol})$ and butyllithium $(1.77 \mathrm{M}, 0.56 \mathrm{ml}, 1.0 \mathrm{mmol})$ as described for the preparation of 1-acetyl-1H-cyclopropa[b]naphthalene above and allowed to react with $N, N$ dimethylcarbamoyl chloride $(0.092 \mathrm{ml}, 1.0 \mathrm{mmol})$. Conventional workup afforded an oil which upon radial chromatography (light petroleum elution) returned unchanged cyclopropanaphthalene (58) (28 mg, 20\%) as the most mobile component. Further elution (ethyl acetate) gave a pale brown solid. Recrystallisation (dichloromethane, $-16^{\circ} \mathrm{C}$ ) of the 
brown residue obtained provided light brown rectangular prisms of bis $(\mathrm{N}, \mathrm{N}-$ di-methyl)-lHcyclopropa[b]naphthalene-1,1-dicarboxamide (205) (97 mg, 34\%), m.p. $126-130^{\circ} \mathrm{C}$ (Found: C, 72.3; H, 6.4; N, 9.9. $\mathrm{C}_{17} \mathrm{H}_{18} \mathrm{~N}_{2} \mathrm{O}_{2}$ requires $\mathrm{C}, 72.3 ; \mathrm{H}, 6.4 ; \mathrm{N}, 9.9 ; \mathrm{O}, \%$ ). IR $v_{\max } 3044,3005,2925,2855,1643,1498,1460,1390,1256,1235,1166,1136,1057,1020$, $891,839,747,696 \mathrm{~cm}^{-1}$. UV $\lambda_{\max }$ (acetonitrile) 239 (4.72), $244 \mathrm{sh}$ (4.49), 271 (3.76), 279 (3.77), 290 sh (3.67), 310 (3.46), 314 sh (3.42), 323 nm (log ع 3.55); ${ }^{1}$ H n.m.r. $\delta 2.94$ (s, $6 \mathrm{H}, 2 \times \mathrm{Me}$ ), 3.67 (s, 6H, 2×Me), 7.49-7.53 (BB', 2H, H4/5), 7.85 (s, 2H, H2/7), 7.93-7.97 (AA', 2H, H3/6); ${ }^{13}$ C n.m.r. $\delta 35.9$ (2×Me), 38.2 (2×Me), 46.6 (C1), 112.9 (C2/7), 124.3 (C1a/7a), $126.3(\mathrm{C} 4 / 5), 128.9(\mathrm{C} 3 / 6), 137.7$ (C2a/6a), 165.7 (2×CO). Mass spectrum (relative intensity): $\mathrm{m} / \mathrm{z}(70 \mathrm{eV}) 283(\mathrm{M}+1,16), 282(\mathrm{M}, 82), 265(8), 239(20), 238(\mathrm{M}-$ $\left.\mathrm{NMe}_{2}, 100\right), 211$ (33), 210 (31), 196 (17), 195 (30), 182 (13), 168 (13), 167 (12), 166 (13), 153 (23), 152 (26), 141 (20), 140 (20), 139 (38), 127 (13), 126 (28), 72 ( $\left.\mathrm{Me}_{2} \mathrm{NCO}, 46\right), 58$ (60), 44 (36), 43 (25), $42(92 \%)$.

Variations in the proportion of carbamoyl chloride from $0.5-10$ molar equivalents had little impact on the yield of diamide isolated and no evidence was obtained to support the presence of a monoamide.

General procedure for the reaction of the 1-acyl-1H-cyclopropa[b]naphthalenes with mineral acid.

To the stirred of the 1-acyl- $1 H$-cyclopropa $[b]$ naphthalene $(57-100 \mathrm{mg}, 0.31$ $0.41 \mathrm{mmol})$ in thf $(5 \mathrm{ml})$ and under nitrogen was added dilute hydrochloric acid $(2 \mathrm{M}, 1 \mathrm{ml}$, $2 \mathrm{mmol}$ ) and the stirring continued until the reaction was complete (t.l.c.). The yellow product mixture was poured into water/dichloromethane $(100 \mathrm{ml} ; 1: 1)$, the phases were separated, and the aqueous phase extracted (dichloromethane, $3 \times 15 \mathrm{ml}$ ). The combined organic layers were washed (water, $50 \mathrm{ml}$ ), dried $\left(\mathrm{MgSO}_{4}\right)$, and concentrated under reduced pressure to an oily yellow solid that was purified by radial chromatography.

a) from 1-acetyl-1H-cyclopropa[b]naphthalene (189) $(57 \mathrm{mg}, 0.31 \mathrm{mmol})$ after $36 \mathrm{~h}$ was obtained 1-(3-chloro-2-naphthalenyl)propan-2-one (212a) $(41 \mathrm{mg}, 60 \%)$ as a pale yellow solid from radial chromatography (dichloromethane/light petroleum elution; 1:1). Recrystallisation (dichloromethane/light petroleum; 1:1) gave an analytical sample as colourless plates, m.p. $87-88^{\circ} \mathrm{C}$ (Found: C, 71.5; H, 5.1; [M+H] $]^{+}$(electrospray) 219.0571. $\mathrm{C}_{13} \mathrm{H}_{11} \mathrm{OCl}$ requires C, 71.4; $\mathrm{H} \mathrm{5.1 \%}$; $\left.[\mathrm{M}+\mathrm{H}]^{+} 219.0571\right)$. IR $v_{\max } 3045,2922,2903,2853$, $1721,1588,1492,1402,1361,1334,1319,1165,1135,1006,966,888,763,688,485$ 
$\mathrm{cm}^{-1}$. UV $\lambda_{\max }$ (acetonitrile) 229 (5.00), 263 (3.58), 272 (3.67), 281 (3.67), $290 \mathrm{~nm}$ (log $\varepsilon$ 3.49). ${ }^{1} \mathrm{H}$ n.m.r. $\delta 2.26$ (s, 3H, Me), 4.00 (s, 2H, $\mathrm{CH}_{2}$ ), 7.47-7.50 (m, 2H, H6/7), 7.70 (bs, 1H, H1), 7.73-7.80 (m, 2H, H5/8), 7.90 (bs, $1 \mathrm{H}, \mathrm{H} 4) ;{ }^{13} \mathrm{C}$ n.m.r. $\delta 29.7(\mathrm{Me}), 48.6\left(\mathrm{CH}_{2}\right)$, 126.4 (C6 or C7), 126.7(2) (C6 or C7), 126.7(7) (C5 or C8), 127.4 (C5 or C8), 127.7 (C4), 130.6 (C1), 130.7 (C2 or C3), 131.9 (C8a or C4a), 132.2 (C2 or C3), 133.2 (C8a or C4a), $205.3(\mathrm{CO})$.

b) from 1-benzoyl-1H-cyclopropa[b]naphthalene (202) (100 mg, $0.41 \mathrm{mmol})$ after $40 \mathrm{~h}$ was obtained 2-phenylnaphtho[2,3-b]furan (214b) $(3.8 \mathrm{mg}, 4 \%)$ as a white solid from radial chromatography (light petroleum elution), vide infra. Further elution (dichloromethane) gave 2-(3-chloro-2-naphthalenyl)-1-phenylethanone (212b) (90 mg, 79\%) as a white solid and the major component. Recrystallisation (thf) gave an analytical sample as lustrous fluffy white needles, m.p. $164-166^{\circ} \mathrm{C}$ (Found: C, 76.8; H, 4.6; Cl, 12.6; $[\mathrm{M}+\mathrm{H}]^{+}$ (electrospray) 281.0728. $\mathrm{C}_{18} \mathrm{H}_{13} \mathrm{ClO}$ requires $\mathrm{C}, 77.0 ; \mathrm{H}, 4.7 ; \mathrm{Cl}, 12.6 \%$; $[\mathrm{M}+\mathrm{H}]^{+}$ 281.0739). IR $v_{\max } 3055,3034,3025,1694,1591,1578,1447,1404,1339,1319,1209$, $1177,1134,1009,999,976,965,885,864,764,750,739,687,627,602,571,480 \mathrm{~cm}^{-1}$. UV $\lambda_{\max }$ (acetonitrile) 229 (4.80), $250 \mathrm{sh}$, (4.26), 271 (3.84), 280 (3.82), $290 \mathrm{~nm}$ (log $\varepsilon$ 3.62). ${ }^{1} \mathrm{H}$ n.m.r. $\delta 4.59$ (s, $\mathrm{CH}_{2}$ ), 7.44-7.54 (m, 4H, H6/7 and H13/15), 7.61 (app tt, J 7.3, $1.7 \mathrm{~Hz}, 1 \mathrm{H}, \mathrm{H} 14), 7.74$ (bs, 1H, H1), 7.75-7.79 (m, 2H, H5 and H8), 7.93 (bs, 1H, H4), 8.08-8.12 (m, 2H, H12/16); ${ }^{13} \mathrm{C}$ n.m.r. $\delta 43.5\left(\mathrm{CH}_{2}\right), 126.3$ (C6 or C7), 126.6 (C6 or C7), 126.7 (C5 or C8), 127.5 (C5 or C8), 127.6 (C4), 128.4 (C12/16), 128.7 (C13/15), 130.6 (C1), 131.0 (C2 or C3 or C4a or C8a), 132.0 (C2 or C3 or $\mathrm{C} 4 \mathrm{a}$ or $\mathrm{C} 8 \mathrm{a}), 132.3$ (C2 or C3 or $\mathrm{C} 4 \mathrm{a}$ or $\mathrm{C} 8 \mathrm{a}), 133.2(5)$ (C2 or $\mathrm{C} 3$ or $\mathrm{C} 4 \mathrm{a}$ or $\mathrm{C} 8 \mathrm{a}), 133.3(2)$ (C14), 136.5 (C11), 196.6 (CO). c) from $\operatorname{bis}(N, N$-dimethyl)-1H-cyclopropa $[b]$ naphthalene-1,1-dicarboxamide (205) (100 mg, $0.35 \mathrm{mmol})$ after $168 \mathrm{~h}$ gave 2-chloro-3-[bis(N,N-dimethylcarboxamido)methyl]naphthalene (212c) $(84 \mathrm{mg}, 74 \%)$ as a pale yellow solid from radial chromatography (ethyl acetate elution). Recrystallisation (dichloromethane/light petroleum; 1:1) gave an analytical sample as colourless blocks, m.p. $145-146^{\circ} \mathrm{C}$ (Found: C, 63.7; H, 6.0; N, 8.8; Cl, 10.9; $[\mathrm{M}+\mathrm{H}]^{+}$(electrospray) 319.1223. $\mathrm{C}_{17} \mathrm{H}_{19} \mathrm{O}_{2} \mathrm{~N}_{2} \mathrm{Cl}$ requires $\mathrm{C}, 64.0 ; \mathrm{H}, 6.0 ; \mathrm{N}, 8.8 ; \mathrm{Cl}$, $10.0 \% ;[\mathrm{M}+\mathrm{H}]^{+}$319.1208). IR $v_{\max } 3059,2924,2854,1656,1495,1455,1439,1385$, $1352,1271,1205,1129,1054,1017,959,880,851,755,606,595,479 \mathrm{~cm}^{-1}$. UV $\lambda_{\max }$ (acetonitrile) 231 (4.67), $276 \mathrm{~nm}(\log \varepsilon 3.55) .{ }^{1} \mathrm{H}$ n.m.r. $\delta 2.94(\mathrm{~s}, 2 \times \mathrm{NMe}), 3.02(\mathrm{~s}, 6 \mathrm{H}, 2$ $\times \mathrm{NMe}), 5.56(\mathrm{~s}, 1 \mathrm{H}, \mathrm{ArCH}), 7.42-7.49$ (m, 2H, H6/7), 7.70-7.73 (m, 1H, H5), 7.79-7.83 (m, 1H, H8), 7.84 (bs, 1H, H1), 7.88 (bs, $1 \mathrm{H}, \mathrm{H} 4) ;{ }^{13} \mathrm{C}$ n.m.r. $\delta 36.0(2 \times \mathrm{NMe}), 37.2(2 \times$ NMe), 51.3 (ArCH), 126.3 (C6 or C7), 126.4 (C5), 126.9 (C6 or C7), 127.4 (C4), 128.1 
(C8), 129.9 (C2 or C3), 130.2 (C1), 131.0 (C2 or C3), 131.9 (C8a or C4a), 133.3 (C8a or $\mathrm{C} 4 \mathrm{a}), 167.7(2 \times \mathrm{CO})$.

General procedure for the preparation of naphtho[2,3-b]furans from 1 -acyl-1Hcyclopropa[b]naphthalenes.

A solution of the requisite 1-acyl-1H-cyclopropa[b]naphthalene (100-340 mg, $0.35-1.87 \mathrm{mmol})$ in dry benzene $(5 \mathrm{ml})$ was refluxed under argon for $16 \mathrm{~h}$. The residue obtained by concentration to dryness was purified as described:

i) from 1-acetyl-1H-cyclopropa[ $b]$ naphthalene $(189)(340 \mathrm{mg}, 1.87 \mathrm{mmol})$ was obtained from radial chromatography (dichloromethane/light petroleum elution; 1:1) 2-methylnaphtho[2,3-b]furan (214a) $(67 \mathrm{mg}, 20 \%)$ as a white solid, m.p. $73-74^{\circ} \mathrm{C}$ (lit. ${ }^{138} 71-72^{\circ} \mathrm{C}$ ). ${ }^{1} \mathrm{H}$ n.m.r. $\delta 2.54$ (d, $\left.J 1 \mathrm{~Hz}, \mathrm{Me}\right), 6.50$ (t, $J 1 \mathrm{~Hz}, \mathrm{H} 3$ ), 7.43-7.47 (m, 2H, H6/7), 7.84 (bs, 1H, H9), 7.93-7.96 (m, 3H, H4 and H5/8); ${ }^{13} \mathrm{C}$ n.m.r. $\delta 14.3$ (Me), 102.2 (C3), 106.0 (C9), 117.4 (C4), 123.7 (C6 or C7), 124.3 (C7 or C6), 127.7 (6) (C5 or C8), 127.7 (9) (C8 or C5), 130.4 (C3a or C9a and C4a or C8a), 130.8 (C8a or C4a), 154.0 (C9a or C3a), 158.1 (C2).

ii) from 1-benzoyl-1H-cyclopropa[b]naphthalene (202) (120 mg, $0.49 \mathrm{mmol})$ was obtained from radial chromatography (light petroleum elution) 2-phenylnaphtho[2,3-b]furan (214b) $(8 \mathrm{mg}, 7 \%)$ as cream plates, m.p. $204^{\circ} \mathrm{C}$ (subl.), $225-233^{\circ} \mathrm{C}$ (sublimate vapourises). (Found: C, 88.8; H, 4.8; $[\mathrm{M}+\mathrm{H}]^{+}$(electrospray) 245.0953. $\mathrm{C}_{18} \mathrm{H}_{12} \mathrm{O}$ requires $\mathrm{C}, 88.5 ; \mathrm{H}$, 4.9(5)\%; [M+H] $\left.]^{+} 245.0961\right)$. IR $v_{\max } 3059,3039,1564,1490,1448,1264,1018,902,867$, $761,742,719,689,478 \mathrm{~cm}^{-1}$. UV $\lambda_{\max }$ (acetonitrile) 222 (4.77), 272 (4.77) 279 (4.71), 330 sh (4.59), 340 (4.64), 356 sh nm (log ع 4.25). ${ }^{1} \mathrm{H}$ n.m.r. $\delta 7.15$ (d, $\left.J 1 \mathrm{~Hz}, 1 \mathrm{H}\right), 7.41-7.52$ $(\mathrm{m}, 5 \mathrm{H}), 7.92-7.97(\mathrm{~m}, 5 \mathrm{H}), 8.04$ (bs, $1 \mathrm{H}) ;{ }^{13} \mathrm{C}$ n.m.r. $\delta 100.7(\mathrm{CH}), 106.6(\mathrm{CH}), 118.5$ $(\mathrm{CH}), 124.0(\mathrm{CH}), 124.7(\mathrm{CH}), 125.3(2 \times \mathrm{CH}), 127.8(\mathrm{CH}), 127.9(\mathrm{CH}), 128.8(2 \times \mathrm{CH})$, $129.1(\mathrm{CH}), 130.1\left(4^{\circ}\right), 130.2\left(4^{\circ}\right), 130.7\left(4^{\circ}\right), 131.5\left(4^{\circ}\right), 153.9\left(4^{\circ}\right), 158.1\left(4^{\circ}\right)$.

When benzoyl-1H-cyclopropa[b]naphthalene (202) (50 mg, $0.20 \mathrm{mmol}$ ) was slowly heated to its melting point $\left(101^{\circ} \mathrm{C}\right)$ the sample decomposed violently. After cooling, the black residue was dissolved in acetone and filtered though a Pasteur pipette containing silica gel. The eluent was then purified by radial chromatography (light petroleum elution) to give napthofuran $(214 \mathrm{~b})(8.6 \mathrm{mg}, 17 \%)$ identical in all respects to the sample obtained above.

A solution of benzoyl-1H-cyclopropa[b]naphthalene (202) $(80 \mathrm{mg}, 0.33 \mathrm{mmol})$ in acetonitrile $(30 \mathrm{ml})$ was placed in a quartz tube fitted with a reflux condenser and irradiated 
at $254 \mathrm{~nm}$ for $16 \mathrm{~h}$. The orange residue obtained after removal of the solvent was purified by radial chromatography (light petroleum elution) to give the phenylnaphtho[2,3- $b$ ]furan (214b) (3.6 mg, 5\%) as a cream solid identical to that obtained above.

iii) from bis( $N, N$-dimethyl)-1H-cyclopropa[b]naphthalene-1,1-dicarboxamide (205) (100 $\mathrm{mg}, 0.35 \mathrm{mmol}$ ) was obtained 2-N,N-dimethylamino-3-N,N-dimethylamidobenzo[2,3-b]furan (214c) (100 mg, 100\%) as a brown wax. Recrystallisation (dichloromethane/light petroleum; 1:1) gave an analytical sample as brown plates, m.p. $135-136^{\circ} \mathrm{C}$ (Found: C, 71.5; H, 6.6; N, 9.9(5); $[\mathrm{M}+\mathrm{H}]^{+}$(electrospray) 283.1442. $\mathrm{C}_{17} \mathrm{H}_{18} \mathrm{O}_{2} \mathrm{~N}_{2}$ requires $\mathrm{C}, 72.3 ; \mathrm{H}$, 6.4; N 9.9\%; [M+H] $\left.]^{+} 283.1441\right)$. IR $v_{\max } 3053,2924,1643,1633,1607,1579,1430,1416$, 1370, 1042, $857 \mathrm{~cm}^{-1}$. UV $\lambda_{\max }(\mathrm{MeCN}) 222$ (4.56), 272 (4.56), 292 (3.83), 305 (4.01), 318 (4.15), 353 nm (log ع 4.11). ${ }^{1} \mathrm{H}$ n.m.r. $\delta 3.15$ (s, 6H, C=CNMe $), 3.17$ (bs, 6H, $\mathrm{CONMe}_{2}$ ), 7.29-7.40 (m, 2H, H6/7), 7.42 (bs, 1H, H4), 7.58 (bs, 1H, H9), 7.79-7.82 (m, 2H, H5/8); ${ }^{13} \mathrm{C}$ n.m.r. $\delta 35.2^{*}$ (CONMe $), 39.4$ (C-NMe $), 85.7$ (C3), 104.9 (C9), 113.2 (C4), 123.2 (C6 or C7), 124.1 (C7 or C6), 126.9 (C5), 127.6 (C8), 129.1 (C4a or C8a), 130.8 (C3a or C9a), 131.3 (C8a or C4a), 149.0 (C9a or C3a), 160.6 (C2), 166.9 (CO).

The chemical shift data for the two formally non-equivalent $\mathrm{CONMe}_{2}$ methyl groups were resolved when the spectrum was re-run at $-20^{\circ} \mathrm{C}$ giving: ${ }^{1} \mathrm{H}$ n.m.r. $\delta 3.18(\mathrm{~s}, 3 \mathrm{H}$ ), 3.19 (s, $3 \mathrm{H}) ;{ }^{13} \mathrm{C}$ n.m.r. $\delta 35.2,39.3$.

General procedure for the preparation of enol ethers from 1-acyl-1H-cyclopropa[b]naphthalenes.

To a stirred solution of the 1-acyl-1H-cyclopropa[b]naphthalene (100-150 mg, $0.41-0.61 \mathrm{mmol})$ in anhydrous thf $(15 \mathrm{ml})$, at $-78^{\circ} \mathrm{C}$ under argon, was added butyllithium 
i) with $t$-butyldimethylsilyl chloride $(84 \mathrm{mg}, 0.55 \mathrm{mmol})$ in thf $(2 \mathrm{ml})$ was obtained after radial chromatography (dichloromethane/light petroleum elution; 1:4) a bright yellow oil $(60 \mathrm{mg})$ that predominantly contained a product proposed as 1-[(1-t-butyldimethylsiloxy)ethylidene]-1H-cyclopropa[b]naphthalene (219a) $\quad(55 \mathrm{mg}, \sim 35 \%)$. Subsequent attempts to purify (219a) resulted in its decomposition. N.m.r. data abstracted from the spectra of the crude mixture for (279a) are: ${ }^{1} \mathrm{H}$ n.m.r. $\delta 0.37$ (s, $\mathrm{Me}_{2} \mathrm{Si}$ ), 1.05 (s, $\mathrm{Me}_{3} \mathrm{CSi}$ ), 2.29 (s, =CMe), 7.09/7.10 (2×bs, H2 or H7), 7.38-7.41 (BB', H4/5), 7.73-7.77 (AA', H3/6); ${ }^{13} \mathrm{C}$ n.m.r. $\delta$-3.9 ( $\mathrm{Me}_{2} \mathrm{Si}$ ), $18.0\left(\mathrm{Me}_{3} \mathrm{CSi}\right), 22.3$ (=CMe), $25.6\left(\mathrm{Me}_{3} \mathrm{CSi}\right), 95.4$ (C1), 103.4/103.6 (C2/7), 125.6/12.7 (C4/5), 127.9/128.1 (C3/6), $126.2\left(4^{\circ}\right), 128.4\left(4^{\circ}\right), 136.7$ $\left(4^{\circ}\right), 137.3\left(4^{\circ}\right), 137.5\left(4^{\circ}\right)$.

ii) with $t$-butyldiphenylsilyl chloride $(0.143 \mathrm{ml}, 0.55 \mathrm{mmol})$ was obtained a thick yellow $\operatorname{tar}(150 \mathrm{mg})$ as the crude product of reaction. ${ }^{1} \mathrm{H}$ n.m.r. analysis indicated the presence of a product proposed as 1-[(1-t-butyldiphenylsiloxy)ethylidene $]-1 H$-cyclopropa $[b]$ naphthalene (219b) from the presence of doublets $(J \sim 1 \mathrm{~Hz})$ at $\delta 5.99(\mathrm{H} 2)$ and $7.04(\mathrm{H} 7)$ with the shielding caused by a pendant phenyl group in analogy with the lowest energy conformer of (220c). Attempts to isolate this product resulted in its decomposition.

b) from 1-benzoyl-1H-cyclopropa[b]naphthalene (202):

i) with trimethylsilyl chloride $(0.08 \mathrm{ml}, 0.61 \mathrm{mmol})$ was obtained a bright yellow solid (84 mg) after radial chromatography (dichloromethane/light petroleum; 1:1) that contained $\left({ }^{1} \mathrm{H}\right.$ n.m.r. $)$ a compound proposed as $1-[($ trimethylsiloxy)phenylmethylidene $]-1 H$-cyclopropa[ $b]$ naphthalene (220a) and unchanged hydrocarbon (202) in a 17:3 ratio. Attempts to purify (220a) resulted in its decomposition. Partial n.m.r.data for (220a) abstracted from spectra are: ${ }^{1} \mathrm{H}$ n.m.r. $\delta 0.46$ (s, 9H, $\mathrm{Me}_{3} \mathrm{Si}$ ), 7.30-7.31 (d, $J 1 \mathrm{~Hz}, \mathrm{H} 2$ or H7), 7.41 (app tt, $J$ 7.3, 1.7 Hz, 1H, H12), 7.42 (d, J1 Hz, H2 or H7), 7.54 (BB', 2H, H4/5), 7.51-7.57 (app t, J $7.6 \mathrm{~Hz}, 2 \mathrm{H}, \mathrm{H} 11 / 13$ ), 7.84-7.89 (AA', 2H, H3/6), 8.07 (app d, $J 8.5 \mathrm{~Hz}, \mathrm{H} 10 / 14$ ); ${ }^{13} \mathrm{C}$ n.m.r. $\delta 0.08\left(\mathrm{Me}_{3} \mathrm{Si}\right), 96.0(\mathrm{C} 1), 104.2$ (C2 or C7), 104.9 (C2 or C7), $124.7(2 \times \mathrm{CH}), 127.0$ $(\mathrm{CH}), 126.2(\mathrm{CH}), 127.8(\mathrm{CH}), 128.1(5)(\mathrm{CH}), 128.2(6)(\mathrm{CH}), 128.3(2 \times \mathrm{CH})$.

ii) with $t$-butyldimethylsilyl chloride $(93 \mathrm{mg}, 0.61 \mathrm{mmol})$ in thf $(2 \mathrm{ml})$ was obtained 1 - $[(\mathrm{t}$ butyldimethylsiloxy)phenylmethylidene]-1H-cyclopropa[b]naphthalene (220b) (125 mg, $57 \%$ ) as a bright yellow solid from radial chromatography (dichloromethane/light petroleum; 1:4). Recrystallisation (dichloromethane/light petroleum; 1:1) gave an analytical sample as bright yellow plates, m.p. $104-106^{\circ} \mathrm{C}$ (Found: C, 80.5; H, 7.2(5); $[\mathrm{M}+\mathrm{H}]^{+}$ 359.1826. $\mathrm{C}_{24} \mathrm{H}_{27} \mathrm{OSi}$ requires $\mathrm{C}, 80.4 ; \mathrm{H}, 7.4 \%$; $\left.[\mathrm{M}+\mathrm{H}]^{+} 359.1807\right)$. IR $v_{\max } 3041,2948$, 2925, 2854, 1785, 1592, 1549, 1509, 1487, 1419, 1311, 1292, 1251, 1177, 1160, 1138, 
$1074,841,783,763,747,689,608,553 \mathrm{~cm}^{-1}$. UV $\lambda_{\max }$ (hexane) 230 (4.58), $246 \mathrm{sh}$ (4.25), 254 (4.15), 291 (4.28), 368 (4.07), 374 (4.14), 393 (4.56), $422 \mathrm{~nm}(\log \varepsilon 4.82)$; UV $\lambda_{\max }$ (acetonitrile) 230 (4.56), $246 \mathrm{sh}$ (4.25), 254 (4.14), 291 (4.26), 368 (4.03), 372 (4.07), 392 (4.49), 420 nm (log ع 4.72). ${ }^{1} \mathrm{H}$ n.m.r. $\delta 0.48$ (s, 6H, $\mathrm{Me}_{2} \mathrm{Si}$ ), 1.19 (s, 9H, Me ${ }_{3} \mathrm{CSi}$ ), 7.29 (d, $J 1.5 \mathrm{~Hz}, \mathrm{H} 2$ or H7), 7.40 (app tt, $J 7.3,1.7 \mathrm{~Hz}, 1 \mathrm{H}, \mathrm{H} 12$ ), 7.40 (d, $J 1.5 \mathrm{~Hz}, \mathrm{H} 7$ or H2), 7.45-7.50 (BB', 2H, H4/5), 7.54 (t, J 7.6 Hz, 2H, H11/13), 7.84-7.88 (AA', 2H, H3/6), 8.09 (app d, $J 8.6 \mathrm{~Hz}, 2 \mathrm{H}, \mathrm{H10} / 14) ;{ }^{13} \mathrm{C}$ n.m.r. $\delta-3.7\left(\mathrm{Me}_{2} \mathrm{Si}\right), 18.4\left(\mathrm{Me}_{3} \mathrm{CSi}\right), 25.9\left(\mathrm{Me}_{3} \mathrm{CSi}\right)$, 95.9 (C1), 104.1 (C7 or C2), 104.9 (C2 or C7), 114.6 (C1a or C7a), 124.7 (C10/14), 126.1(5) (C4 or C5), 126.2 (C4 or C5), 126.4 (C1a or C7a), 127.8 (C12), 128.1 (C3 or C6), 128.2 (C3 or C6), 128.3 (C11/13), 135.8 (C8), 137.1 (C9), 137.7 (C2a or C6a), 137.8 (C2a or C6a).

iii) with $t$-butyldiphenylsilyl chloride $(0.13 \mathrm{ml}, 0.49 \mathrm{mmol})$ was obtained from radial chromatography (dichloromethane/light petroleum; 1:4) an analytical sample of 1-[(t-butyldiphenylsiloxy)phenylmethylidene]-1H-cyclopropa[b]naphthalene (220c) (150 mg, 63\%) as a viscous tar which solidified upon scratching, m.p. $134-140^{\circ} \mathrm{C}$ (Found: C, 84.5(5); H, 6.5; $[\mathrm{M}+\mathrm{H}]^{+}$483.2139. $\mathrm{C}_{34} \mathrm{H}_{30} \mathrm{OSi}$ requires $\mathrm{C}, 84.6 ; \mathrm{H}, 6.3 \%$; $\left.[\mathrm{M}+\mathrm{H}]^{+} 483.2140\right)$. IR $v_{\max }$ $3071,3050,2927,2855,1589,1547,1506,1486,1471,1445,1418,1386,1287,1151$, $1132,1114,1073,1025,886,828,762,742,701,620,602,552,503,490,469 \mathrm{~cm}^{-1}$. UV $\lambda_{\max }$ (hexane) 227 (4.73), 254 sh (4.33), 282 (4.23), 292 (4.39), 369 (4.16), 374 (4.22), 394 (4.64), $422 \mathrm{~nm}(\log \varepsilon 4.90)$; UV $\lambda_{\max }$ (acetonitrile) 228 (4.58), $254 \mathrm{sh}$ (4.19), 282 (4.09), 292 (4.24), 368 (4.01), 374 (4.08), 393 (4.47) 421 nm (log ع 4.71). ${ }^{1} \mathrm{H}$ n.m.r. $\delta 1.30$ (s, 9H, $\mathrm{Me}_{3} \mathrm{CSi}$ ), 5.78 (d, J 1.2 Hz, 1H, H2 or H7), 7.27 (d, J1.2 Hz, 1H, H7 or H2), 7.33-7.52 (m, $10 \mathrm{H}, \mathrm{H} 3, \mathrm{H} 4 / 5, \mathrm{H} 12,2 \times \mathrm{H} 16 / 20$ or $2 \times \mathrm{H} 17 / 19$, and $2 \times \mathrm{H} 18$ ), 7.64 (bd t, $J 7.7 \mathrm{~Hz}, 2 \mathrm{H}$, H1 1/13), 7.74 (bd d, J 8.1 Hz, 1H, H6), 7.99-8.03 (m, 4H, H16/20 or H17/19), 8.29 (bd d, J 7.3 Hz, 2H, H10/14); ${ }^{13} \mathrm{C}$ n.m.r. $\delta 19.5\left(\mathrm{Me}_{3} \mathrm{CSi}\right), 26.5\left(\mathrm{Me}_{3} \mathrm{CSi}\right), 97.5$ (C1), 103.9 (C7 or C2) 104.9 (C2 or C7), 124.6 (C10/14), 125.5 (C1a or C7a), 125.7 (C4 or C5), 125.8 (C4 or C5), 126.4 (C1a or C7a), 127.7 (C16/20 or C17/19), 127.8 (C3 or C6 or C12), 127.9 (C3 or C6 or C12), 128.2 (C3 or C6 or C12), 128.5 (C11/13), 129.8 (C18), 133.3 (C15), 134.6 (C8), 135.1 (C16/20 or C17/19), 136.9 (C9), 137.5 (C2a or C6a), 137.7 (C2a or C6a).

iv) with di-t-butylchlorophosphine $(0.09 \mathrm{ml}, 0.49 \mathrm{mmol})$ was obtained after radial chromatography (ethyl acetate elution) a mixture of di-t-butylphosphinic acid 8-[1-(phenylmethylidene)-1H-cyclopropa[b]naphthalene]-O ether (220e) together with an unidentified phosphorus-containing compound as a yellow oil. Subsequent radial chromatography (ethyl acetate/dichloromethane elution; 6:1) gave an analytical sample of (220e) (32 mg, 
$16 \%$ ) as a bright yellow oil which solidified on standing, m.p. $158-161^{\circ} \mathrm{C}$ (Found: C, 76.7 ; $\mathrm{H}, 7.3 ;[\mathrm{M}+\mathrm{H}]^{+}$(electrospray) 405.1978. $\mathrm{C}_{26} \mathrm{H}_{29} \mathrm{O}_{2} \mathrm{P}$ requires $\mathrm{C}, 77.2 ; \mathrm{H}, 7.2 \% ;[\mathrm{M}+\mathrm{H}]^{+}$ 405.1990). IR $v_{\max } 3053,2924,2854,1770,1742,1713,1632,1469,1448,1423,1384$, $1274,1231,1143,1115,1074,1011,939,889,822,748,692,672,594,476 \mathrm{~cm}^{-1}$. UV $\lambda_{\max }$ (hexane) 230 (4.73), $252 \mathrm{sh}$ (4.31), 290 (4.43), $3.71 \mathrm{sh}(4.25), 392$ (4.63) $420 \mathrm{~nm}(\log$ $\varepsilon$ 4.75); UV $\lambda_{\max }$ (acetonitrile) 230 (4.73), 252 sh (4.30), 290 (4.41), 3.69 sh (4.22), 391 (4.57), $418 \mathrm{~nm}(\log \varepsilon 4.71)$. ${ }^{1} \mathrm{H}$ n.m.r. $\delta 1.44$ (d, $J 14.7 \mathrm{~Hz}, 18 \mathrm{H}, 2 \times \mathrm{Me}_{3} \mathrm{C}$ ), 7.35 (app tt, $J$ 7.3, 1.2 Hz, 1H, H12), 7.42-7.47 (BB', 2H, H4/5), 7.51 (app t, J 7.7 Hz, 2H, H11/13), 7.53 (d, $J 1.7 \mathrm{~Hz}, 1 \mathrm{H}, \mathrm{H} 2$ or H7), 7.65 (d, $J 1.7 \mathrm{~Hz}, 1 \mathrm{H}, \mathrm{H} 7$ or H2), 7.85-7.89 (AA', 2H, H3/6), 7.97 (app d, $J 8.5 \mathrm{~Hz}, 2 \mathrm{H}, \mathrm{H} 10 / 14)$ ) ${ }^{13} \mathrm{C}$ n.m.r. $\delta .26 .7(6 \times \mathrm{Me}), 37.2(\mathrm{~d}, J 80.6 \mathrm{~Hz}, 2 \times$ $\mathrm{Me}_{3} \mathrm{C}$ ), 99.9 (C1), 106.6 (C2 or C7), 108.6 (C7 or C2), 124.0 (C10/14), 126.0 (d, J 1.0 Hz, C1a or C7a), 126.3(3) (C4 or C5), 126.3(8) (C4 or C5), 127.0 (d, J 1.0 Hz C1a or C7a), 127.7 (C12), 128.4 (C3 or C6), 128.6 (C11/13), 128.9 (C3 or C6), 132.4 (d, J 12.6 Hz, C8), 135.5 (d, J 4.5 Hz, C9), 138.3 (C2a or C6a), 138.9 (C2a or C6a); ${ }^{31} \mathrm{P}$ n.m.r. $\delta 68.6$.

\section{Chapter 5 .}

\section{4a,5,8,8a-Tetrahydro-1,4-naphthoquinone (231)}

Into a $300 \mathrm{ml}$ pre-calibrated Carius tube were placed freshly sublimed 1,4-benzoquinone $(15 \mathrm{~g}, 0.14 \mathrm{~mol})$, benzene $(60 \mathrm{ml})$ and a magnetic follower. The mixture was frozen to $-70^{\circ} \mathrm{C}$ (ethanol/liquid nitrogen bath) and buta-1,3-diene (15 g, $24 \mathrm{ml}, 0.28 \mathrm{~mol}$ ) added by vapour-phase transfer. The tube was sealed, warmed slowly to room temperature, and then stirred for $4-5$ days at $40^{\circ} \mathrm{C}$. The mixture was frozen to liquid nitrogen temperature, opened, and the solvent removed to give a green solid. Recrystallisation (light petroleum) gave the title compound (231) $(20.2 \mathrm{~g}, 89 \%)$ as pale yellow needles, m.p. 57$58^{\circ} \mathrm{C}$ (lit. $\left.{ }^{151} 58^{\circ} \mathrm{C}\right)$.

\section{5,8-Dimethoxy-1,4-dihydronaphthalene (232).}

Into a $1 \mathrm{~L}$ round bottomed flask fitted with a pressure equalised dropping funnel and a reflux condenser with a calcium chloride guard tube attached, and were placed 4a,5,8,8a-tetrahydro-1,4-naphthoquinone (231) (26 g, 0.16 mol), anhydrous potassium carbonate $(60 \mathrm{~g}, 0.36 \mathrm{~mol})$, dry acetone $(400 \mathrm{ml})$, and a magnetic stirring bar. The mixture was cooled to $0^{\circ} \mathrm{C}$ and dimethyl sulfate $(50.4 \mathrm{~g}, 38 \mathrm{ml}, 0.4 \mathrm{~mol})$ was added dropwise over $10 \mathrm{~min}$ with vigorous stirring. The mixture was then refluxed for $16 \mathrm{~h}$, cooled, filtered 
under vacuum through a sintered glass filter funnel and the residual base washed (acetone, $2 \times 50 \mathrm{ml}$ ). The combined organic filtrates were concentrated under reduced pressure to a pale brown oil to which water $(100 \mathrm{ml})$ was added and resultant precipitate collected by filtration. After washing the brown solid (water, $3 \times 50 \mathrm{ml}$ ), it dried under high vacuum to give the title compound (232) as a pink/brown solid $(30 \mathrm{~g}, 97 \%)$, m.p. $52-54^{\circ} \mathrm{C}$ (lit. ${ }^{152}$ $\left.51^{\circ} \mathrm{C}\right)$.

\section{1,1-Dichloro-3,6-dimethoxy-1a,2,7,7a-tetrahydro-1H-cyclopropa[b]naphthalene (233).}

To a stirred solution of 5,8-dimethoxy-1,4-dihydronaphthalene (232) (13 g, $0.068 \mathrm{~mol})$ and sodium methoxide $(11 \mathrm{~g}, 0.20 \mathrm{~mol})$ in light petroleum/benzene $(100 \mathrm{ml}$, $1: 1)$, cooled to $0^{\circ} \mathrm{C}$, was added ethyl trichloroacetate $(26.8 \mathrm{~g}, 19.5 \mathrm{ml}, 0.14 \mathrm{~mol})$ dropwise over $1 \mathrm{~h}$. The mixture was then allowed to warm slowly to room temperature over $16 \mathrm{~h}$ and water $(100 \mathrm{ml})$ was added. The phases were separated, the aqueous extracted (benzene, $2 \times 50 \mathrm{ml}$ ), and the combined organic layers washed (water, $100 \mathrm{ml}$ ), dried $\left(\mathrm{MgSO}_{4}\right)$, filtered through cotton wool and concentrated under reduced pressure to an oily brown solid. Recrystallisation (light petroleum) gave the title compound (233) (13.5 g, $72 \%$ ) as a pale yellow solid, m.p. $96-97^{\circ} \mathrm{C}$ (lit. ${ }^{116} 98-98.5^{\circ} \mathrm{C}$ ). The n.m.r. data of (233) were in excellent agreement with the literature values. ${ }^{116}$

\section{3,6-Dimethoxy-1H-cyclopropa[b]naphthalene (70).}

To a stirred solution of commercial grade potassium $t$-butoxide $(54 \mathrm{~g}, 0.46 \mathrm{~mol})$ (Lancaster, $97 \%)$ in dry thf $(200 \mathrm{ml})$, cooled to $0^{\circ} \mathrm{C}$ and under nitrogen was added dropwise over $40 \mathrm{~min}$ 1,1-dichloro-3,6-dimethoxy-1a,2,7,7a-tetrahydro-1 $H$-cyclopropa[ $b]$ naphthalene $(233)(8 \mathrm{~g}, 29.4 \mathrm{~mol})$ in the same solvent $(100 \mathrm{ml})$. The mixture was then allowed to warm slowly warm to ambient temperature where it was stirred for a further $48 \mathrm{~h}$. The solvent was removed under reduced pressure and the resultant orange solid dissolved in water/light petroleum $(800 \mathrm{ml}, 1: 1)$. The phases were separated and the aqueous extracted (light petroleum, $2 \times 400 \mathrm{ml}$ ). The combined organic layers were washed (water, $2 \times$ $400 \mathrm{ml})$, dried $\left(\mathrm{MgSO}_{4}\right)$, and concentrated under reduced pressure to a yellow solid. Radial chromatography (dichloromethane/light petroleum; 1:4 elution) and collection of the major component followed by crystallisation (same solvent mixture) gave 3,6-dimethoxy-1Hcyclopropa $b]$ naphthalene $(70)(1.46 \mathrm{~g}, 25 \%)$ as a white solid, m.p. $130-132^{\circ} \mathrm{C}$ (lit. ${ }^{116} 51 \%$, m.p. $\left.130-131.5^{\circ} \mathrm{C}\right)$. Spectral data were in excellent agreement with the literature values. ${ }^{116}$ 
To a stirred solution of 3,6-dimethoxy-1H-cyclopropa[b]naphthalene (70) (3 g, 15 $\mathrm{mmol})$ in acetonitrile/dichloromethane $(3: 2,145 \mathrm{ml})$ was added in $\sim 5 \mathrm{ml}$ portions cerium (IV) ammonium nitrate $(25 \mathrm{~g}, 46 \mathrm{mmol})$ in water $(115 \mathrm{ml})$. The addition was performed in such a way as to minimise, but to observe, a transient blue/black colouration which rapidly changes to bright orange. Following the addition the mixture was stirred for $1 \mathrm{~h}$ and then extracted (dichloromethane, $2 \times 300 \mathrm{ml}$ ). The organic layers were combined, washed (water, $2 \times 300 \mathrm{ml}$ ), dried $\left(\mathrm{MgSO}_{4}\right)$, and concentrated under reduced pressure to a green solid. Column chromatography (dichloromethane/light petroleum elution; $1: 1$ ) and collection of the major component gave a pure sample of $1 H$-cyclopropa[b]naphthalene3,6-dione (154) $(1.9 \mathrm{~g}, 75 \%)$ as a yellow powder that melts then resolidifies from 103 to $113^{\circ} \mathrm{C}$, re-melts $127-128^{\circ} \mathrm{C}$ [lit. ${ }^{116} 85 \%$, m.p. $130-132^{\circ} \mathrm{C}$ (decomp.)]. All spectral data were in good agreement with those reported in the literature. ${ }^{116}$

\section{[3 $\alpha, 7 \alpha]-3 a, 4,7,7 a-T e t r a h y d r o-1 \mathrm{H}$-cyclopropa[b]anthracene-3,8-dione (160).}

Into a $300 \mathrm{ml}$ pre-calibrated Carius tube $(300 \mathrm{ml})$ were placed cyclopropanaphthoquinone (154) $(1.5 \mathrm{~g}, 8.8 \mathrm{mmol})$, benzene $(30 \mathrm{ml})$, and a magnetic follower. The mixture was cooled to $-50^{\circ} \mathrm{C}$ and buta-1,3-diene $(5 \mathrm{ml}, 56.8 \mathrm{mmol})$ was added by vapour transfer. The tube was then sealed, warmed to $45^{\circ} \mathrm{C}$, and the contents stirred at this temperature for 4 days. The tube and its contents were cooled to liquid nitrogen temperature, opened, and the thawed contents filtered though a plug of cotton wool. The filtrate was concentrated under reduced pressure to a yellow/brown oily solid $(1.90 \mathrm{~g})$ that contained $\left({ }^{1} \mathrm{H}\right.$ n.m.r.) quinone (154) and tetrahydrocycloproparene (160) in 3:17 ratio. The solid was purified by one of the following methods:

i) recrystallisation from benzene at $5^{\circ} \mathrm{C}$ predominantly gave pale yellow crystals $(565 \mathrm{mg})$ together with some green crystals $(50 \mathrm{mg})$ that were separated by hand. The pale yellow crystals contained $\left({ }^{1} \mathrm{H}\right.$ n.m.r.) tetrahydrocycloproparene (160) with $<2 \%$ of quinone (154) and the green crystals contained these two compounds in a 3:6 ratio. The colourless crystals, suitable for subsequent use, were recrystallised first from benzene and then from ether/tetrahydrofuran $(1: 1)$ to give an analytical sample $(19.4 \mathrm{mg}, 1 \%)$ of $[3 \alpha, 7 \alpha]$ 3a,4,7,7a-tetrahydro-1H-cyclopropa[b]anthracene-3,8-dione (160) as pale yellow, diamond shaped crystals, m.p. $96-97^{\circ} \mathrm{C}$ (Found $\mathrm{C}, 80.2 ; \mathrm{H}, 5.5 . \mathrm{C}_{15} \mathrm{H}_{12} \mathrm{O}_{2}$ requires C, 80.3; H, 5.4\%). IR $v_{\max } 3029,2974,2938,2900,2851,1678,1556,1431,1246,1178,1053$, $878,706 \mathrm{~cm}^{-1}$. UV $\lambda_{\max }$ (acetonitrile) 235 (4.42), $273 \mathrm{~nm}(\log \varepsilon 3.81) .{ }^{1} \mathrm{H}$ n.m.r. $\left(\mathrm{CDCl}_{3}\right)$ 
反 2.19-2.29 (A of $\mathrm{ABX}, 2 \mathrm{H}, \mathrm{H} 4 \mathrm{a} / 7 \mathrm{a}$ or $\mathrm{H} 4 \mathrm{~b} / 7 \mathrm{~b}$ ), 2.47-2.57 (B of $\mathrm{ABX}, 2 \mathrm{H}, \mathrm{H} 4 \mathrm{a} / 7 \mathrm{a}$ or H4b/7b), 3.36 (s, 2H, H1), 3.36-3.41 (m, 2H, H3a/H7a), 5.73 (t, J 1.5 Hz, 2H, H5/6), 7.87 (s, 2H, H2/9); ${ }^{1} \mathrm{H}$ n.m.r. $\left(\mathrm{CD}_{3} \mathrm{CO}\right) \delta 2.19-2.25$ (A of $\mathrm{ABX}, 2 \mathrm{H}, \mathrm{H} 4 \mathrm{a} / 7 \mathrm{a}$ or $\mathrm{H} 4 \mathrm{~b} / 7 \mathrm{~b}$ ), 2.422.49 (B of ABX, 2H, H4a/7a or H4b/7b), 3.43 (s, 2H, H1), 3.43-3.49 (m, 2H, H3a/H7a), 5.70 (bs, 2H, H5/6), 7.81 (s, 2H, H2/9). ${ }^{13} \mathrm{C}$ n.m.r. $\left(\mathrm{CDCl}_{3}\right) \delta 19.5(\mathrm{C} 1), 24.4(\mathrm{C} 4 / 7), 46.5$ (C3a/7a), 113.2 (C2/9), 124.6 (C5/6), 133.0 (C1a/9a), $137.7(\mathrm{C} 2 \mathrm{a} / 8 \mathrm{a}), 198.2(\mathrm{C} 3 / 8) ;{ }^{13} \mathrm{C}$ n.m.r. $\left(\mathrm{CD}_{3} \mathrm{CO}\right) \delta 19.5(\mathrm{C} 1), 25.0(\mathrm{C} 4 / 7), 46.9(\mathrm{C} 3 \mathrm{a} / 7 \mathrm{a}), 113.3(\mathrm{C} 2 / 9), 125.5(\mathrm{C} 5 / 6), 133.6$ (C1a/9a), 138.7 (C2a/8a), 198.1 (C3/8). Mass spectrum (relative intensity): $\mathrm{m} / \mathrm{z}(70 \mathrm{eV})$ $225(\mathrm{M}+1,10), 224(\mathrm{M}, 60), 196\left(\mathrm{M}-\mathrm{CO}\right.$ or $\left.\mathrm{M}-\mathrm{C}_{2} \mathrm{H}_{4}, 69\right), 178$ (52), 152 (31), 116 (30), 105 (38), 89 (51), 88 (82), 87 (33), 77 (52), 63 (43), 62 (100), 51 (38), 42 (23), 39 (57), 27 $(47 \%)$.

The combined filtrates from the recrystallisations were combined and subjected to radial chromatography (dichloromethane/light petroleum elution; 1:4) to give a green solid (987 mg) that contained $\left({ }^{1} \mathrm{H}\right.$ n.m.r.) quinone (154) and enedione (160), in 1:9 ratio, as well as a significant decomposition material of unknown identity.

ii) the solid was subjected to radial chromatography (dichloromethane/light petroleum elution; $1: 1)$ to give a yellow solid (1.3-1.6 g) that contained enedione (160), naphthoquinone (154), and dihydroquinone (230). The product ratio varied from reaction to reaction and was either $1: 15: 0,1: 3: 0$, or $1: 2: 30$.

\section{3,8-Dimethoxy-4,7-dihydro-1H-cyclopropa[b]anthracene (229).}

To a stirred solution of tetrahydrocycloproparene (160) (100 mg, $0.45 \mathrm{mmol})$ and potassium carbonate $(222 \mathrm{mg}, 1.62 \mathrm{mmol})$ in anhydrous acetone $(7 \mathrm{ml})$ under argon at $0^{\circ} \mathrm{C}$, was added dimethyl sulfate $(0.106 \mathrm{ml}, 1.13 \mathrm{mmol})$ over $30 \mathrm{sec}$ by syringe. The mixture was allowed to stir while attaining room temperature overnight. The resultant lemon coloured mixture was filtered though a pad of celite under vacuum, the residue was washed (acetone, $4 \times 25 \mathrm{ml}$ ), and the filtrate concentrated under reduced pressure to a dark yellow solid. Radial chromatography (dichloromethane/light petroleum elution; $1: 1$ ) gave as the major and most mobile component a white solid $(72 \mathrm{mg}, 63 \%)$ that strongly fluoresced in UV light. Recrystallisation (dichloromethane/light petroleum; $1: 1,-16^{\circ} \mathrm{C}$ ) gave 3,8 dimethoxy-4,7-dihydro-1H-cyclopropa[b]anthracene (229) (22 mg, 20\%) as colourless plates, m.p 126-130 (Found $\mathrm{C}, 80.7$; $\mathrm{H}$ 6.3. $\mathrm{C}_{15} \mathrm{H}_{12} \mathrm{O}_{2}$ requires $\mathrm{C}, 80.9 ; \mathrm{H}, 6.4 \%$ ). IR $v_{\max }$ 3072, 3029, 2993, 2932, 2876, 2827, 1744, 1671, 1600, 1451, 1423, 1330, 1297, 1063,

$1052,995,950,853,665,598 \mathrm{~cm}^{-1}$. UV $\lambda_{\max }$ (acetonitrile) $216(4.45), 246(4.62), 300 \mathrm{~nm}$ 
(log ع 3.69). ${ }^{1} \mathrm{H}$ n.m.r. . $\left(\mathrm{CDCl}_{3}\right) \delta 3.49$ (s, 2H, H1), 3.56 (d, J $\left.1.2 \mathrm{~Hz}, 4 \mathrm{H}, \mathrm{H} 4 / 7\right), 3.91$ (s, $6 \mathrm{H}, 2 \times \mathrm{OMe}$ ), 6.02 (t, $J 1.4 \mathrm{~Hz}, 2 \mathrm{H}, \mathrm{H} 5 / 6), 7.82$ (s, 2H, H2/9); ${ }^{1} \mathrm{H}$ n.m.r. $\left(d_{6}\right.$-acetone) $\delta 3.45$ (s, 2H, H1), 3.52 (d, J $1.2 \mathrm{~Hz}, 4 \mathrm{H}, \mathrm{H} 4 / 7$ ), 3.88 (s, 6H, 2×OMe), 6.00 (t, J $1.4 \mathrm{~Hz}, 2 \mathrm{H}$, $\mathrm{H} 5 / 6), 7.79$ (s, 2H, H2/9); ${ }^{13} \mathrm{C}\left(\mathrm{CDCl}_{3}\right)$ n.m.r. $\delta 18.4$ (C1), 24.3 (C4/7), $61.0(2 \times \mathrm{OMe})$; 106.8 (C2/9), 122.9 (C1a/9a), 124.1 (C5/6), 124.5 (C3a/7a), 130.0 (C2a/8a), 150.0 (C3/8); ${ }^{13} \mathrm{C}$ ( $d_{6}$-acetone) n.m.r. $\delta 18.3(\mathrm{C} 1), 24.8(\mathrm{C} 4 / 7), 61.2(2 \times \mathrm{OMe}), 107.4(\mathrm{C} 2 / 9), 123.6$ (C1a/9a), 124.8 (C5/6), 125.4 (C3a/7a), 130.9 (C2a/8a), 150.9 (C3/8). Mass spectrum (relative intensity): $m / z$ (70 eV) $253(\mathrm{M}+1,19), 252(\mathrm{M}, 100), 237$ (M-Me, 62), 206 (70), 205 (38), 178 (69), 177 (34), 176 (44), 165 (77), 163 (30\%). Further elution of the plate with the same solvent mixture gave an oily yellow solid ( $24 \mathrm{mg}$ ) that contained ( ${ }^{1} \mathrm{H}$ n.m.r.) ether (229) together with cyclopropanthraquinone (162) (vide infra) and unreacted dimethyl sulfate in a 20:6:1 ratio. Further purification of this solid was not attempted.

All further attempts to prepare the title compound (229) proved unsuccessful.

Attempted preparation of 3,8-dimethoxy-1H-cyclopropa[b] anthracene (163) from treating 3,8-dimethoxy-4,7-dihydro-1H-cyclopropa[b]anthracene (229) with $D D Q$.

A suspension of dihydroether (229) (20 mg, $0.079 \mathrm{mmol})$ and DDQ (45 mg, $0.198 \mathrm{mmol}$ ) in dry benzene was stirred under argon at ambient temperature for $12 \mathrm{~h}$. The bright yellow mixture was then filtered though a sintered glass funnel and the solution concentrated under reduced pressure to give a green solid. Column chromatography (dichloromethane elution) and collection of the most mobile fraction gave $1 H$-cyclopropa[b]anthracene-3,8-dione $(162)(8.5 \mathrm{mg}, 49 \%)$ as a yellow solid that was identical in all respects to the other samples, vide infra.

\section{4,7-Dihydro-1H-cyclopropa[b]anthracene-3,8-dione (230).}

To a stirred solution of the butadiene adduct (160) (100 $\mathrm{mg}, 0.45 \mathrm{mmol}$ ) in anhydrous acetone $(10 \mathrm{ml})$ under argon, was added potassium carbonate $(620 \mathrm{mg}$, $4.49 \mathrm{mmol}$ ) under a stream of argon. The mixture which instantly darkened, was stirred for a further $2.5 \mathrm{~h}$ then filtered though a pad a celite under vacuum. The solid residue was washed (acetone, $4 \times 25 \mathrm{ml}$ ) and the filtrate concentrated under reduced pressure to a purple/ blue solid (97 mg) that contained $\left({ }^{1} \mathrm{H}\right.$ n.m.r.) quinone (230) and cyclopropanthraquinone (162) (vide infra) in a ratio of either $37: 2$, or $32: 7$. The solid, which was only sparingly soluble in all common solvents, was either left on the bench until it became yellow, or it was stirred as a suspension in dichloromethane until it had dissolved. Use of an oxygen 
atmosphere can accelerate the process. Radial chromatography (dichloromethane/light petroleum elution; 1:4) gave as the major and most mobile component the title compound (230) as a yellow solid $(41 \mathrm{mg}, 41 \%)$ that rapidly darkens on standing. Recrystallisation (diethyl ether, $-16^{\circ} \mathrm{C}$ ) gave an analytical sample of 4,7-dihydro-1H-cyclopropa/b]anthracene-3,8-dione (230) (23 mg, 23\%) as yellow crystals, m.p. $122-124^{\circ} \mathrm{C}$ (decomp.) after subl. at $111^{\circ} \mathrm{C}$ (Found $\mathrm{C}, 81.2 ; \mathrm{H} 4.5(5) . \quad \mathrm{C}_{15} \mathrm{H}_{10} \mathrm{O}_{2}$ requires $\mathrm{C}, 81.1 ; \mathrm{H}, 4.5 \%$ ). IR $v_{\max }$ 3037, 3010, 2948, 2923, 2852, 1655, 1626, 1557, 1416, 1294, 1123, 1058, 980, 933, 875, $856,723,715,649,625,570 \mathrm{~cm}^{-1}$. UV $\lambda_{\max }$ (acetonitrile) 260 (4.41), $326 \mathrm{~nm}$ (log $\left.\varepsilon 3.30\right)$. ${ }^{1} \mathrm{H}$ n.m.r. $\left(\mathrm{CDCl}_{3}\right) \delta 3.25$ (d, J $\left.1.2 \mathrm{~Hz}, 4 \mathrm{H}, \mathrm{H} 4 / 7\right), 3.34$ (s, 2H, H1), 5.87 (t, J $1.2 \mathrm{~Hz}, \mathrm{H} 5 / 6$ ), 7.94 (s, 2H, H2/9); ${ }^{1} \mathrm{H}$ n.m.r. $\left(\mathrm{CD}_{3} \mathrm{CO}\right) \delta 3.16$ (d, J $\left.1.2 \mathrm{~Hz}, 4 \mathrm{H}, \mathrm{H} 4 / 7\right), 3.39$ (s, 2H, H1), $5.88(\mathrm{t}, J 1.2 \mathrm{~Hz}, \mathrm{H} 5 / 6), 7.84$ (s, 2H, H2/9); ${ }^{13} \mathrm{C}$ n.m.r. $\left(\mathrm{CDCl}_{3}\right) \delta 19.1(\mathrm{C} 1), 24.4(\mathrm{C} 4 / 7)$, 112.7 (C2/9), 122.7 (C5/6), 131.9 (C1a/9a), 135.4 (C2a/8a), 141.1 (C3a/7a), 184.8 (C3/8); ${ }^{13} \mathrm{C}$ n.m.r. $\left(\mathrm{CD}_{3} \mathrm{CO}\right) \delta 19.1$ (C1), 24.8 (C4/7), 112.8 (C2/9), $123.6(\mathrm{C} 5 / 6), 132.7$ (C1a/9a), 135.0 (C2a/8a), 141.5 (C3a/7a), 184.9 (C3/8). Mass spectrum (electrospray) found: 223 $\left(\mathrm{M}+\mathrm{H}^{+}\right)$.

Quinone (230) is readily oxidised to cyclopropanthracene (162) and even after storage in the freezer $\left(-16^{\circ} \mathrm{C}\right)$ for 17 days some $10 \%$ conversion to compound $(162)$ is observed. It is advisable, therefore, that compound (230) be stored under an inert atmosphere.

Attempted preparation of 4,7-dihydro-1H-cyclopropa[b]anthracene-3,8-dione (230) from (160) and silica gel.

The enedione (160) (20 mg, $0.089 \mathrm{mmol})$ and silica gel $(500 \mathrm{mg})$ were suspended in dichloromethane $(40 \mathrm{ml})$ and the mixture stirred at room temperature for 3 days. The pale yellow mixture was filtered though a sintered glass frit and the filtrate concentrated under reduced pressure to a cream solid $(20 \mathrm{mg})$. Analysis of the solid by ${ }^{1} \mathrm{H}$ n.m.r. spectroscopy showed it be unchanged enedione (160) (100\%).

Attempted preparation of 4,7-dihydro-1H-cyclopropa[b] anthracene-3,8-dione (230) from (160) and potassium hydroxide.

To a stirred solution of (160) $(50 \mathrm{mg}, 0.22 \mathrm{mmol})$ in dry benzene at room temperature and under argon was added freshly ground potassium hydroxide pellets (125 mg, $2.23 \mathrm{mmol}$ ). The dark mixture was then stirred at this temperature for $50 \mathrm{~min}$ and then poured into water/benzene $(50 \mathrm{ml} ; 1: 1)$. The phases were separated, the aqueous 
layer extracted (benzene, $2 \times 20 \mathrm{ml}$ ) and the combined organic layers were washed (water, $100 \mathrm{ml})$, dried $\left(\mathrm{MgSO}_{4}\right)$, and concentrated under reduced pressure to a brown solid. The solid contained a complex mixture of products $\left({ }^{1} \mathrm{H}\right.$ n.m.r.) none of which corresponded to the enedione (160) or quinone (230).

Attempted preparation of 4,7-dihydro-1H-cyclopropa[b] anthracene-3,8-dione (230) from (160) and $D D Q$.

To a stirred solution of enedione $(160)(50 \mathrm{mg}, 0.22 \mathrm{mmol})$ in dry dichloromethane $(20 \mathrm{ml})$ under a nitrogen atmosphere and at room temperature was added DDQ (507 mg, $2.23 \mathrm{mmol}$ ). The mixture was stirred for $44 \mathrm{~h}$ and then filtered though silica gel under vacuum. The solid residue was washed (dichloromethane, $2 \times 20 \mathrm{ml}$ ) and the combined filtrates concentrated under reduced pressure to a yellow foam. Radial chromatography (dichloromethane/light petroleum elution; 1:4) gave as the major and most mobile component a red oil $(38 \mathrm{mg})$ that contained many components $\left({ }^{1} \mathrm{H}\right.$ n.m.r. $)$ and only a trace amount of unchanged (160); no evidence for quinone (230) was obtained.

Attempted preparation of 4,7-dihydro-1H-cyclopropa[b] anthracene-3,8-dione (230) from (160) and pyridine.

To a stirred solution of $(160)(25 \mathrm{mg}, 0.11 \mathrm{mmol})$ in dry chloroform at room temperature and under an argon atmosphere, was added pyridine $(0.045 \mathrm{ml}, 0.55 \mathrm{mmol})$. The dark mixture was then stirred at this temperature for $1 \mathrm{~h}$ and then poured into water/ dichloromethane $(50 \mathrm{ml} ; 1: 1)$. The phases were separated, the aqueous layer extracted (dichloromethane, $2 \times 20 \mathrm{ml}$ ) and the combined organic layers were washed (water, $100 \mathrm{ml}$ ), dried $\left(\mathrm{MgSO}_{4}\right)$, and concentrated under reduced pressure to a brown solid. The solid contained a complex mixture of products $\left({ }^{1} \mathrm{H}\right.$ n.m.r.) and only traces of enedione (160) were detected. Further purification of the solid was not attempted.

\section{3,8-Dihydroxy-4,7-dihydro-1H-cyclopropa[b] anthracene dipotassium salt (234).}

Into an n.m.r. tube flushed with argon was placed solid potassium carbonate (24 mg, $0.17 \mathrm{mmol}$ ) followed by the tetrahyrocycloproparene (160) $(24 \mathrm{mg}, 0.17 \mathrm{mmol}$ ) in $d_{6}$-acetone $(0.45 \mathrm{ml})$. After $1 \mathrm{~h}$ only unchanged cycloproparene $(160)$ was present $\left({ }^{1} \mathrm{H}\right.$ n.m.r.). The n.m.r tube and its contents were then sonicated for $30 \mathrm{~min}$ after which time substrate (160) and diphenolate salt (234) were present in a 10:1 ratio. Sonication for a further $30 \mathrm{~min}$ showed the presence of a compound proposed as 3-8-dihydroxy-4,7-di- 
hydro- $1 H$-cyclopropa $[b]$ anthracene dipotassium salt $(234)$ to be the sole organic product present. ${ }^{1} \mathrm{H}$ n.m.r. ( $d_{6}$-acetone) $\delta 3.42(\mathrm{~s}, 2 \mathrm{H}, \mathrm{H1}), 3.50$ (d, $\left.J 1.0 \mathrm{~Hz}, 4 \mathrm{H}, \mathrm{H} 4 / 7\right), 5.96$ (t, $J$ $1.3 \mathrm{~Hz}, 2 \mathrm{H}, \mathrm{H} 5 / 6), 7.95$ (s, 2H. H2/9); ${ }^{13} \mathrm{C}$ n.m.r. (d6-acetone) $\delta 18.2(\mathrm{C} 1), 25.3(\mathrm{C} 4 / 7)$, 107.0 (C2/9), 117.7 (C3a/7a), 122.1 (C1a/9a), 124.4 (C5/6), 127.8 (C2a/8a), 144.2 (C3/8).

Dianion formation for 1,4-dihydroxybenzene was recorded under the same conditions.

\section{H-Cyclopropa[b]anthracene-3,8-dione (162).}

a) A mixture of quinones (230) and (162) (32:7) (103 mg, c. $0.46 \mathrm{mmol}$ ) was suspended in dichloromethane $(15 \mathrm{ml})$ and a slow stream of oxygen bubbled through. After $2 \mathrm{~h}$ all the solid had dissolved but oxygen flow was continued for 12 days; constant volume was maintained by solvent addition as required and the reaction periodically monitored by ${ }^{1} \mathrm{H}$ n.m.r.. The solvent was removed under reduced pressure to give a yellow solid that was purified by column chromatography (dichloromethane elution) to give $1 H$-cyclopropa[b]anthracene-3,8-dione (162) as a yellow powder ( $82 \mathrm{mg}, 80 \%)$. Recrystallisation (dichloromethane, $\left.-16^{\circ} \mathrm{C}\right)$ gave the title anthracene-3,8-dione $(162)(28 \mathrm{mg}, 27 \%)$ as yellow crystals, m.p. $132^{\circ} \mathrm{C}$ (subl.), sublimate m.p. $>350^{\circ} \mathrm{C}$ (Found C, 81.7; H 3.7; m/z 221.0601. $\mathrm{C}_{15} \mathrm{H}_{8} \mathrm{O}_{2}$ requires $\mathrm{C}, 81.8 ; \mathrm{H}, 3.7 \%$; $[\mathrm{M}+\mathrm{H}]^{+}$221.0603). IR $v_{\max } 2924,2853,1668,1592$, $1581,1557,1317,1292,1123,709,601 \mathrm{~cm}^{-1}$. UV $\lambda_{\max }$ (acetonitrile) 206 (4.27), 259 (4.65), $280 \mathrm{sh}$ (4.04), $324 \mathrm{~nm}(\log \varepsilon 3.61) .{ }^{1} \mathrm{H}$ n.m.r. $\left(\mathrm{CDCl}_{3}\right) \delta 3.41$ (s, $\left.2 \mathrm{H}, \mathrm{H1}\right), 7.79-7.81$ (BB', 2H, H5/6), 8.16 (s, 2H, H2/9), 8.31-8.34 (AA', 2H, H4/7); ${ }^{1} \mathrm{H}$ n.m.r. $\left(\mathrm{CD}_{3} \mathrm{CO}\right) \delta 3.46$ (s, 2H, H1), 7.89-7.92 (BB', 2H, H5/6), 8.07 (s, 2H, H2/9), 8.24-8.27 (AA', 2H, H4/7); ${ }^{13} \mathrm{C}$ n.m.r. $\left(\mathrm{CDCl}_{3}\right) \delta 19.2(\mathrm{C} 1), 113.5(\mathrm{C} 2 / 9), 127.2(\mathrm{C} 4 / 7), 132.8(\mathrm{C} 1 \mathrm{a} / 9 \mathrm{a}), 133.1(\mathrm{C} 3 \mathrm{a} / 7 \mathrm{a})$, 134.0 (C5/6), 137.0 (C2a/8a), $183.3(\mathrm{C} 3 / 8)$; ${ }^{13} \mathrm{C}$ n.m.r. $\left(\mathrm{CD}_{3} \mathrm{CO}\right) \delta 19.3(\mathrm{C} 1), 113.6(\mathrm{C} 2 / 9)$, 127.7 (C4/7), 133.6 (C1a/9a), 133.9 (C3a/7a), 135.0 (C5/6), 137.9 (C2a/8a), 183.5 (C3/8);.

b) The quinones (230) and (162) (32:7) (50 mg, 0.22 mmol) and DDQ (128 mg, $0.56 \mathrm{mmol}$ ) were suspended in anhydrous benzene $(5 \mathrm{ml})$ at ambient temperature for 6.5 days under argon. Workup as in $a$ above gave a mixture of the anthracenedione (162) and DDQ. Further chromatography provided compound (162) (29 mg, 58\%) identical to that described in $a$ above. When the reaction is conducted at $40^{\circ} \mathrm{C}$ for $17 \mathrm{~h}$ a white solid is obtained from chromatography (dichloromethane/light petroleum elution; 1:1). All subsequent attempts to further purify this solid were unsuccessful.

c) To the mixture of the quinones (230) and (162) (32:7) $(50 \mathrm{mg}, 0.22 \mathrm{mmol})$ in dry acetone $(5 \mathrm{ml})$ under argon was added potassium carbonate $(311 \mathrm{mg}, 2.26 \mathrm{mmol})$ and the suspension stirred for $24 \mathrm{~h}$. Workup as in $a$ above gave $\left({ }^{1} \mathrm{H}\right.$ n.m.r.) quinones (230) and 
(162) in a 1:5 ratio. Repetition of the procedure gave (162) (34 mg, 69\%) as a yellow solid.

If the reaction is uninterrupted for $48 \mathrm{~h}$ the yellow oil $(20 \mathrm{mg})$ so obtained contains only traces of (162) ( ${ }^{1} \mathrm{H}$ n.m.r.); a similar outcome follows from reaction with potassium hydroxide.

Attempted preparation of 1,1-bis(trimethylsilyl)-1H-cyclopropa[b]anthracene-3,8-dione (240).

To a stirred solution of quinone (162) $(47 \mathrm{mg}, 0.21 \mathrm{mmol})$ in dry thf $(20 \mathrm{ml})$, cooled to $-70^{\circ} \mathrm{C}$ an under an argon atmosphere was added butyllithium $(1.92 \mathrm{M}, 0.11 \mathrm{ml}$, $0.21 \mathrm{mmol}$ ). The mixture which developed a dark red/orange colouration was then allowed to warm to $-43^{\circ} \mathrm{C}$ where it was stirred for $30 \mathrm{~min}$ and then recooled to $-70^{\circ} \mathrm{C}$ whereupon trimethylsilyl chloride $(0.027 \mathrm{ml}, 0.21 \mathrm{mmol})$ was added. After stirring at $-43^{\circ} \mathrm{C}$ for $25 \mathrm{~min}$ the mixture was recooled to $-70^{\circ} \mathrm{C}$ whereupon a second portion of butyllithium (1.92 M, $0.055 \mathrm{ml}, 0.105 \mathrm{mmol}$ ) was added. The now bright orange mixture was stirred at $-43^{\circ} \mathrm{C}$ for $25 \mathrm{~min}$ and then recooled to $-70^{\circ} \mathrm{C}$ and treated with a second portion of trimethylsilyl chloride $(0.014 \mathrm{ml}, 0.105 \mathrm{mmol})$. The last two steps were repeated then the mixture was allowed to warm slowly to ambient temperature overnight. The orange mixture was poured into water/dichloromethane $(200 \mathrm{ml} ; 1: 1)$, the phases separated, and the aqueous phase extracted (dichloromethane, $2 \times 50 \mathrm{ml}$ ). The combined organic layers were washed (water, $100 \mathrm{ml}$ ), dried $\left(\mathrm{MgSO}_{4}\right)$, and concentrated under reduced pressure to a yellow oil that contained a complex mixture of products $\left({ }^{1} \mathrm{H}\right.$ n.m.r.) none of which corresponded to monosilane (239), disilane (240), or unchanged quinone (162). Although attempts were made to separate the components from the mixture their identity remains unresolved.

Upon repeating the above procedure with the sterically congested $t$ butyldiphenylsilyl chloride a similar outcome was obtained.

\section{1,4,4a,9a-Tetrahydroanthracene-9,10-dione (242).}

To a pre-calibrated Carius tube was placed 1,4-naphthoquinone (15.8 g, $0.1 \mathrm{~mol})$, benzene and a magnetic follower and the mixture cooled to $-50^{\circ} \mathrm{C}$ whereupon buta-1,3diene $(5.4,8.6 \mathrm{ml}, 0.1 \mathrm{~mol})$ was added by vapour-phase transfer. The tube was sealed, and its contents stirred at $75^{\circ} \mathrm{C}$ for $4-5$ days. After cooling to liquid nitrogen temperature the tube was opened and the excess butadiene and solvent were removed under reduced 
pressure to give a grey solid. Recrystallisation (dichloromethane, $-16^{\circ} \mathrm{C}$ ) gave the title compound (242) $\left(15.8 \mathrm{~g}, 75 \%\right.$ ) as a pale grey solid, m.p. $102-104^{\circ} \mathrm{C}$ (lit. $\left.{ }^{157} 102-103^{\circ} \mathrm{C}\right)$.

9,10-Dimethoxy-1,4-dihydroanthracene (243).

To a stirred solution of 1,4,4a,9a-tetrahydroanthracene-9,10-dione (242) and dimethyl sulfate $(6.25 \mathrm{ml}, 0.066 \mathrm{mmol})$ in dry acetone $(80 \mathrm{ml})$ was added anhydrous potassium carbonate $(17 \mathrm{~g}, 0.122 \mathrm{mmol})$ and the mixture refluxed for $16 \mathrm{~h}$. After cooling, the mixture was filtered through a pad of celite under vacuum and the filtrate concentrated under reduced pressure to an off-white oily solid. Recrystallisation (dichloromethane, $\left.-16^{\circ} \mathrm{C}\right)$ gave the known ${ }^{158}$ title compound $(4.89 \mathrm{~g}, 62 \%)$ as colourless crystals, m.p. 147$148^{\circ}$ C. ${ }^{1} \mathrm{H}$ n.m.r. $\delta 3.58$ (d, J $\left.1.5 \mathrm{~Hz}, 4 \mathrm{H}, \mathrm{H} 1 / 4\right), 3.92$ (s, 6H, 2×OMe), 6.04 (t, J $1.7 \mathrm{~Hz}$, H2/3), 7.46-7.50 (BB', 2H, H6/7), 8.06-8.09 (AA, 2H, H5/8).

Attempted preparation of 1,1-dichloro-3,8-dimethoxy-1a,2,9,9a-tetrahydro-1H-cyclopropa[b] anthracene (244) from the reaction of 9,10-dimethoxy-5,8-dihydroanthracene (243), with potassium hydroxide and chloroform.

To a mixture of 9,10-dimethoxy-5,8-dihydroanthracene (243) (1 g, $4.17 \mathrm{mmol})$, benzyltriethylammonium bromide $(71 \mathrm{mg}, 0.0003 \mathrm{mmol})$, and freshly powdered potassium hydroxide $(666 \mathrm{mg}, 16.68 \mathrm{mmol})$ in diethyl ether $(50 \mathrm{ml})$ cooled to $0^{\circ} \mathrm{C}$, was added chloroform $(50 \mathrm{ml}, 0.62 \mathrm{~mol})$ dropwise over $20 \mathrm{~min}$. The muddy-yellow mixture was then allowed to warm slowly to ambient temperature in the cold bath overnight. The crude product was filtered though a pad of celite under vacuum and the inorganic residue washed (diethyl ether, $100 \mathrm{ml}$ ). The filtrates were combined and concentrated under reduced pressure to give the title compound $(243)(1 \mathrm{~g}, 100 \%)$ as a pale brown solid. Spectral data were identical in all respects to an authentic sample.

\section{1,1-Dichloro-3,8-dimethoxy-1a,2,9,9a-tetrahydro-1H-cyclopropa[b]anthracene (244).}

To a stirred suspension of 9,10-dimethoxy-1,4-dihydroanthracene (243) (1 g, 4.17 $\mathrm{mmol})$ and sodium methoxide $(1.12 \mathrm{~g}, 4.17 \mathrm{mmol})$ in benzene $(35 \mathrm{ml})$, cooled to $0^{\circ} \mathrm{C}$ and under argon, was added ethyl trichloroacetate $(2.8 \mathrm{ml}, 4.17 \mathrm{mmol})$ dropwise over $1 \mathrm{~h}$. The mixture was then allowed to slowly warm to room temperature overnight and was then poured into water/benzene $(100 \mathrm{ml} ; 1: 1)$. After separation of the phases the aqueous layer was then extracted (benzene, $50 \mathrm{ml}$ ) and the combined organic layers washed (water, $100 \mathrm{ml})$, dried $\left(\mathrm{MgSO}_{4}\right)$, and concentrated under reduced pressure to give a red/brown solid 
which contained ( ${ }^{1} \mathrm{H}$ n.m.r.) unchanged alkene (243) and carbene adduct (244) in a 1:1 ratio. The crude residue was then treated with a further portion of sodium methoxide $(1.12 \mathrm{~g}, 4.17 \mathrm{~mol})$ and ethyl trichloroacetate $(2.8 \mathrm{ml}, 4.17 \mathrm{mmol})$ as described above. Conventional workup including radial chromatography (dichloromethane elution) and collection of the major and most mobile component gave 1,1-dichloro-3,8-dimethoxy1a,2,9,9a-tetrahydro-1H-cyclopropa[b]anthracene $(244)(910 \mathrm{mg}, 67 \%)$ as a pale yellow solid; an analytical sample was obtained as white crystals that become pink on standing from recrystallisation (dichloromethane) $\left(320 \mathrm{mg}, 24 \%\right.$ ), m.p. $122-124^{\circ} \mathrm{C}$ (Found C, 63.30; $\mathrm{H} \mathrm{5.03;} \mathrm{Cl}, 22.04 ;[\mathrm{M}+\mathrm{H}]^{+}$(electrospray) 323.0629. $\mathrm{C}_{17} \mathrm{H}_{17} \mathrm{O}_{2} \mathrm{Cl}_{2}$ requires $\mathrm{C}, 63.17 ; \mathrm{H}$ 4.99; Cl, 21.94\%; [M+H] 323.0606). IR $v_{\max } 2933,2895,2834,1588,1455,1361,1328$, 1105, 1091, 1065, 1039, 777, $697 \mathrm{~cm}^{-1}$. UV $\lambda_{\max }$ (acetonitrile) 236 (4.80), $293 \mathrm{~nm}(\log \varepsilon$ 3.69). ${ }^{1} \mathrm{H}$ n.m.r. $\delta 2.06-2.10$ ( $2 \times \mathrm{X}$ of AMX, $\left.2 \mathrm{H}, \mathrm{H1a} / 9 \mathrm{a}\right), 2.71-2.79$ (2×A of AMX, $2 \mathrm{H}$,

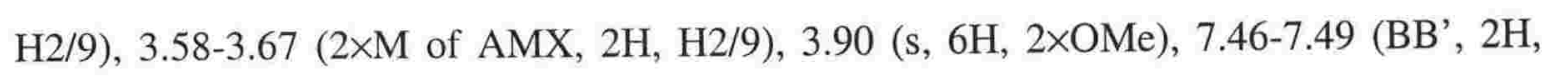
H5/6), 8.03-8.06 (AA', 2H, H4/7); ${ }^{13}$ C n.m.r. $\delta 19.3$ (C2/9), 27.4 (C1a/9a), 61.5 (2×OMe), $66.5(\mathrm{C} 1), 122.0$ (C4/7), 124.5 (C2a/8a), 125.6 (C5/6), 127.0 (C3a/7a), $149.1(\mathrm{C} 8)$.

Attempted preparation of 3,8-dimethoxy-1H-cyclopropa[b]anthracene (163) from treatment of 1,1-dichloro-3,8-dimethoxy-1a,2,9,9a-tetrahydro-1H-cyclopropa[b]anthracene (244) with potassium $\mathrm{t}-$ butoxide .

To a stirred suspension of freshly sublimed potassium $t$-butoxide ( $209 \mathrm{mg}$, $1.86 \mathrm{~mol})$ in dry thf $(10 \mathrm{ml})$, cooled to $0^{\circ} \mathrm{C}$ and under argon, was added 1,1-dichloro-3,8dimethoxy-1a,2,9,9a-tetrahydro- $1 H$-cyclopropa[ $b]$ anthracene $(244)$ in the same solvent $(5 \mathrm{ml})$ dropwise over $5 \mathrm{~min}$. The mixture was then allowed to warm slowly to ambient temperature overnight, poured into water/dichloromethane $(100 \mathrm{ml} ; 1: 1)$, the phases were separated, and the aqueous mixture extracted (dichloromethane, $100 \mathrm{ml}$ ). The combined organic layers were washed (water, $100 \mathrm{ml})$, dried $\left(\mathrm{MgSO}_{4}\right)$, and concentrated under reduced pressure to a bright yellow solid. Radial chromatography (dichloromethane elution) and collection of the major component gave 9,10-dimethoxy-2-( $t$-butoxymethyl)anthracene $(245)^{153}(22.3 \mathrm{mg}, 44 \%)$ as the only identifiable component in the crude mixture. All spectral data were in agreement with those reported by Kay. ${ }^{153}$

When the reaction is performed at $-25^{\circ} \mathrm{C}$ or $-80^{\circ} \mathrm{C}$ the $t$-butyl ether (245) is the only identifiable product of reaction $\left(49 \%\right.$ in each case). At $-80^{\circ} \mathrm{C}$ and with only two molar equivalents of potassium $t$-butoxide an 8:1 mixture of unchanged 1,1-dichloro-3,8- 
(22.2 $\mathrm{mg})$ was obtained.

\section{1,1-Dichloro-1a,2,9,9a-tetrahydro-1H-cyclopropa[b] anthracene-3,8-dione (249).}

To a stirred solution of 1,1-dichloro-3,8-dimethoxy-1a,2,9,9a-tetrahydro-1H-cyclopropa $[b]$ anthracene $(244)(620 \mathrm{mg}, 1.92 \mathrm{mmol})$ in acetonitrile/dichloromethane $(120 \mathrm{ml}$; $3: 2)$ was added cerium ammonium nitrate $(3.18 \mathrm{~g}, 5.8 \mathrm{mmol})$ in water $(50 \mathrm{ml})$ in $5 \mathrm{ml}$ portions over $10 \mathrm{~min}$. The mixture was then stirred for a further $30 \mathrm{~min}$ and was then poured into water/dichloromethane $(400 \mathrm{ml} ; 1: 1)$. The phases were separated and the aqueous extracted (dichloromethane, $200 \mathrm{ml}$ ), and the combined organic layers were washed (water, $400 \mathrm{ml}$ ), dried $\left(\mathrm{MgSO}_{4}\right)$, and concentrated under reduced pressure to a red/orange solid. Radial chromatography (dichloromethane/light petroleum elution; 1:1) and collection of the major component gave 1,1-dichloro-1a,2,9,9a-tetrahydro- $1 \mathrm{H}$-cyclopropa[b] anthracene-3,8-dione (249) (280 mg, 50\%) as a bright orange solid; an analytical sample was obtained as yellow crystals from recrystallisation (dichloromethane/light

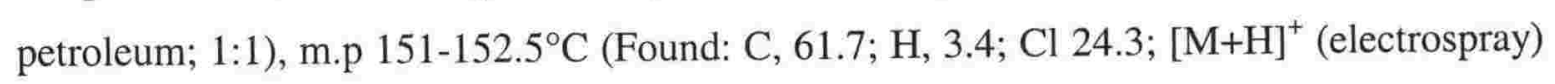
293.0135. $\mathrm{C}_{15} \mathrm{H}_{10} \mathrm{O}_{2} \mathrm{Cl}_{2}$ requires: $\mathrm{C}, 61.4(5) ; \mathrm{H}, 3.4 ; \mathrm{Cl} 24.2 \%$; $\left.[\mathrm{M}+\mathrm{H}]^{+} 293.0131\right)$. IR $v_{\max } 3016,2921,2907,1660,1639,1592,1334,1289,1167,933,803,774,693 \mathrm{~cm}^{-1}$. UV $\lambda_{\max }$ (acetonitrile) 245 (4.31), $266 \mathrm{sh}$ (4.17), 270 (4.18), $330 \mathrm{~nm}\left(\log\right.$ ع 3.50). ${ }^{1} \mathrm{H}$ n.m.r.

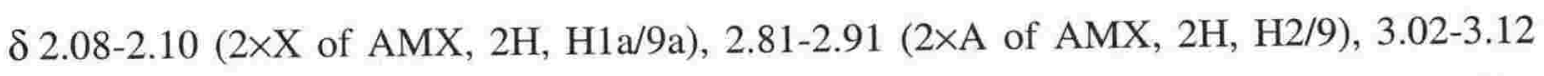
( $2 \times \mathrm{M}$ of $\mathrm{AMX}, 2 \mathrm{H}, \mathrm{H} 2 / 9), 7.67-7.73$ (AA', $2 \mathrm{H}, \mathrm{H} 5 / 6), 8.04-8.08\left(\mathrm{BB}^{\prime}, 2 \mathrm{H}, \mathrm{H} 4 / 7\right) ;{ }^{13} \mathrm{C}$ n.m.r. $\delta 19.0$ (C2/9), 24.0 (C1a/9a), 64.3 (C1), 126.3 (C4/7), 131.8 (C3a/7a), 133.6 (C5/6), $140.8(\mathrm{C} 2 \mathrm{a} / 8 \mathrm{a}), 183.8(\mathrm{C} 3 / 8)$.

Attempted preparation of $1 \mathrm{H}$-cyclopropa $[\mathrm{b}$ ] anthracene-3,8-dione from treatment of 1,1-dichloro-1 a,2,9,9a-tetrahydro-1H-cyclopropa[b] anthracene-3,8-dione (249) with potassium $\mathrm{t}$-butoxide.

To a stirred suspension of freshly sublimed potassium $t$-butoxide ( $245 \mathrm{mg}, 2.18$ $\mathrm{mol})$ in dry thf $(15 \mathrm{ml})$, cooled to $0^{\circ} \mathrm{C}$ and under argon was added 1,1-dichloro-1a,2,9,9atetrahydro- $1 H$-cyclopropa $[b]$ anthracene-3,8-dione $(249)$ in the same solvent $(2 \mathrm{ml})$ dropwise over $5 \mathrm{~min}$. The dark red/black mixture was then allowed to warm slowly to ambient temperature overnight and then was poured into water/dichloromethane $(100 \mathrm{ml}$; 1:1). The phases were separated and the aqueous mixture extracted with dichloromethane $(100 \mathrm{ml})$. The combined organic layers were washed (water, $100 \mathrm{ml})$, dried $\left(\mathrm{MgSO}_{4}\right)$, and 
concentrated under reduced pressure to a brown residue that contained a complex mixture of components ( ${ }^{1} \mathrm{H}$ n.m.r.). Radial chromatography and collection of the only mobile component on silica gel (dichloromethane elution) gave a brown oil ( $7.6 \mathrm{mg}, 20 \%$ mass return) the n.m.r. spectrum of which did not correspond to either the sought after quinone (162) or unchanged (249).

Attempted preparation of 1,1-dichloro-3,8-dimethoxy-1H-cyclopropa[b]anthracene (251) from 1,1-dichloro-3,8-dimethoxy-1a,2,9,9a-tetrahydro-1H-cyclopropa[b]anthracene (244) and $D D Q$.

A mixture of 1,1-dichloro-3,8-dimethoxy-1a,2,9,9a-tetrahydro-1H-cyclopropa[b]anthracene (244) (70 mg, $0.217 \mathrm{mmol})$ and DDQ (250 $\mathrm{mg}, 1.1 \mathrm{mmol}$ ) was suspended in dry benzene $(7 \mathrm{ml})$ and the mixture stirred under argon at ambient temperature for $24 \mathrm{~h}$ the mixture. The solvent was removed, the residue was suspended in dichloromethane $(5 \mathrm{ml})$ and then filtered through a sintered glass funnel. The filtrate was concentrated under reduced pressure to a yellow solid that contained a complex mixture of products $\left({ }^{1} \mathrm{H}\right.$ n.m.r. $)$ none of which corresponded to unchanged (244) or the sought-after product (251). Attempts to purify the mixture were not made

Attempted preparation of 1,1-dichloro-1H-cyclopropa[b]anthracene-3,8-dione (252) from 1,1-dichloro-1a,2,9,9a-tetrahydro-1H-cyclopropa[b]anthracene-3,8-dione (249) and $D D Q$.

A mixture of 1,1-dichloro-1a,2,9,9a-tetrahydro-1H-cyclopropa[b]anthracene-3,8dione (249) (25 mg, $0.09 \mathrm{mmol}$ ) and DDQ (97 mg, $0.43 \mathrm{mmol}$ ) was suspended in dry benzene $(5 \mathrm{ml})$ and the mixture stirred under argon at ambient temperature for $48 \mathrm{~h}$. The solvent was removed, the residue was suspended in dichloromethane $(5 \mathrm{ml})$ and then filtered through a sintered glass funnel. The filtrate was concentrated under reduced pressure to a yellow solid that contained a complex mixture of products $\left({ }^{1} \mathrm{H}\right.$ n.m.r.) none of which corresponded to unchanged (249) or the sought-after product (252).

Attempted preparation of 3,8-dimethoxy-1a,2,9,9a-tetrahydro-1H-cyclopropa[b]anthracene (253).

Zinc powder $(1.36 \mathrm{~g}, 20.8 \mathrm{mmol})$ and copper(I) chloride $(2.0620 .8 \mathrm{mmol})$ were suspended in anhydrous diethyl ether $(40 \mathrm{ml})$ and the mixture stirred at room temperature for $40 \mathrm{~min}$ after which point diiodomethane $(2.79 \mathrm{~g}, 0.84 \mathrm{ml}, 10.4 \mathrm{mmol})$ was added. The 
mixture was stirred for a further $5 \mathrm{~min}$ and then 3,8-dimethoxy-4,7-tetrahydroanthracene (243) $(500 \mathrm{mg}, 2.08 \mathrm{mmol})$ was added in dry thf $(20 \mathrm{ml})$. The mixture was stirred at room temperature for a further $16 \mathrm{~h}$ and then filtered though a pad of celite under reduced pressure and the solid residue washed (diethyl ether, $100 \mathrm{ml}$ ). Concentration of the combined filtrates under reduced pressure followed by recrystallisation (dichloromethane, $-16^{\circ} \mathrm{C}$ ) gave unchanged (243) $(425-500 \mathrm{mg}, 85-100 \%)$ as colourless crystals that were identical to an authentic sample from above.

When the above reaction is repeated at reflux for $16 \mathrm{~h}$ unchanged starting material (450 $\mathrm{mg}, 90 \%)$ is recovered.

Attempted preparation of 3,8-dimethoxy-1a,2,9,9a-tetrahydro-1H-cyclopropa[b]anthracene (253).

Diazomethane ${ }^{*}$ gas $(\sim 4.16 \mathrm{mmol})$ was generated in a diazomethane generation and distillation kit that was placed behind a plastic safety shield in a well vented fume cupboard, from the careful addition of a solution of diazald $\circledast(1.26 \mathrm{~g}, 5.86 \mathrm{mmol})$ in diethyl ether $(11.3 \mathrm{ml})$ to a stirred solution of potassium hydroxide $(1.26 \mathrm{~g}, 22.5 \mathrm{mmol})$ in water $(2.13 \mathrm{ml})$ and ethanol $(2.34 \mathrm{ml})$ at $60^{\circ} \mathrm{C}$. The diazomethane gas was distilled directly into a receiver flask containing 3,8-dimethoxy-4,7-tetrahydroanthracene (243) (500 mg, $2.08 \mathrm{mmol})$ in dichloromethane $(15 \mathrm{ml})$ and the resultant mixture was allowed to stir at room temperature for $22 \mathrm{~h}$ whereupon it was quenched (acetic acid, $5 \mathrm{ml}$ ) and stirred for a further $10 \mathrm{~min}$. The mixture was then poured into water/dichloromethane (200 $\mathrm{ml} ; 1: 1)$, the phases separated, and the aqueous phases extracted (dichloromethane, $50 \mathrm{ml}$ ). The combined organic layers were washed (water, $100 \mathrm{ml}$ ), dried $\left(\mathrm{MgSO}_{4}\right)$, and concentrated under reduced pressure to a yellow solid. Radial chromatography (dichloromethane/light petroleum elution; 1:1) and collection of the major and most mobile component returned unchanged 3,8-dimethoxy-4,7-tetrahydroanthracene (243) (348 mg, 70\%) as a colourless solid that was identical to the starting material. Further elution (dichloromethane/light petroleum elution; 1:1) gave anthraquinone $(35 \mathrm{mg}, 8 \%$ ) as pale yellow needles whose spectral data were in agreement with an authentic sample.

\section{Chapter 6.}

"Diazomethane is highly toxic and explosive and should be prepared with extreme caution in a well vented fume cupboard. 
A solution of vinylene trithiocarbonate $(269)(4 \mathrm{~g}, 29.8 \mathrm{mmol})$ in dimethyl sulfate $(28 \mathrm{ml})$, under an argon atmosphere, was heated to $95^{\circ} \mathrm{C}$ with stirring for $40 \mathrm{~min}$. The mixture was cooled to $0^{\circ} \mathrm{C}$, glacial acetic acid added $(5.6 \mathrm{ml}, 97.8 \mathrm{mmol})$ and the mixture was stirred for a further $10 \mathrm{~min}$ before being treated with tetrafluoroboric acid solution $(54 \% \mathrm{w} / \mathrm{v}$ in diethyl ether, $7.52 \mathrm{ml}, 29.8 \mathrm{mmol})$. After stirring the mixture for a further 10 min dry diethyl ether $(400 \mathrm{ml})$ was added at which point a precipitate formed. The solid was collected, washed thoroughly (diethyl ether, $4 \times 250 \mathrm{ml}$ ) and dried under high vacuum. The crude residue was recrystallised (ethanol, $-16^{\circ} \mathrm{C}$ ) to give the known ${ }^{176}$ salt $(270)$ $(6.36 \mathrm{~g}, 90 \%)$ as colourless needles which became green upon standing, m.p. $107-108^{\circ} \mathrm{C}$. ${ }^{1}$ H n.m.r. $\delta 3.37$ (s, 3H), 8.94 (s, 2H). Salt (270) is best stored under argon.

\section{2-Methyl-1,3-dithiole (271).}

To a stirred solution of 2-methylthio-1,3-dithiolium tetrafluoborate (270) (5 g, $21.2 \mathrm{mmol})$ in anhydrous ethanol $(175 \mathrm{ml})$ at $0^{\circ} \mathrm{C}$ and under argon was added sodium borohydride $(0.84 \mathrm{~g}, 22.2 \mathrm{mmol})$ portion wise over $\sim 15 \mathrm{~min}$. The suspension was then stirred at room temperature for $3 \mathrm{~h}$ then the solvent was removed. The residue was dissolved in water/dichloromethane $(200 \mathrm{ml} ; 1: 1)$, the phases separated, and the aqueous extracted (dichloromethane, $2 \times 50 \mathrm{ml}$ ). The combined organic layers were washed (water, $200 \mathrm{ml}$ ), dried $\left(\mathrm{MgSO}_{4}\right)$, and concentrated under reduced pressure to give a pure sample of the title compound (271) as a malodorous pale yellow oil $(3.0 \mathrm{~g}, 94 \%) .{ }^{1} \mathrm{H}$ n.m.r. data were in excellent agreement with the literature values. ${ }^{177}$

\section{1,3-Dithiolium tetrafluoborate (272).}

To a stirred solution of 2-methyl-1,3-dithiole (271) $(0.85 \mathrm{~g}, 5.7 \mathrm{mmol})$ in glacial acetic acid $(8 \mathrm{ml}, 139.8 \mathrm{mmol})$, cooled to $0^{\circ} \mathrm{C}$ and under argon, was added dropwise over $5 \mathrm{~min}$ tetrafluoroboric acid solution $(54 \% \mathrm{w} / \mathrm{v}$ in diethyl ether, $1.45 \mathrm{ml}, 5.7 \mathrm{mmol}$ ); by the end of the addition a precipitate had formed. The suspension was then stirred at room temperature for $20 \mathrm{~min}$, poured into dry diethyl ether $(50 \mathrm{ml})$, stirred for a further $5 \mathrm{~min}$ and then the solid was collected. The solid was washed thoroughly (diethyl ether, $4 \times 50 \mathrm{ml})$ and dried under high vacuum to give the known ${ }^{177}$ salt $(272)(0.95 \mathrm{~g}, 89 \%)$ as a white powder. The solid was used without further ado. 
Trimethyl phosphite $(2.4 \mathrm{ml}, 20.52 \mathrm{mmol})$ and sodium iodide $(3 \mathrm{~g}, 19.88 \mathrm{mmol})$ were added successively to a stirred solution of 1,3-dithiolium tetrafluoroborate (272) $(3.55 \mathrm{~g}, 18.70 \mathrm{mmol})$ in dry acetonitrile $(20 \mathrm{ml})$ under an argon atmosphere. The bright yellow mixture was stirred for $3 \mathrm{~h}$ and then poured into water $(50 \mathrm{ml})$. The mixture was extracted (dichloromethane, $3 \times 30 \mathrm{ml}$ ), and the combined organic extracts washed (water, $100 \mathrm{ml})$, dried $\left(\mathrm{MgSO}_{4}\right)$, and concentrated under reduced pressure to a pale yellow oil. The oil was purified by flash column chromatography (basic alumina, ethyl acetate elution) to give the title compound (268) (3.50 g, 88\%) (lit. ${ }^{164} 95 \%$ ) as a pale yellow hygroscopic oil that becomes red upon standing. N.m.r. data were in agreement with the literature values. ${ }^{164}$ It is best to store (268) under argon in a flask protected from light.

\section{2-(Diphenylmethylidene)-1,3-dithiole (273).}

To a stirred solution of phosphonate ester (264) (100 mg, $0.47 \mathrm{mmol})$ in dry thf $(20 \mathrm{ml})$, cooled to $-78^{\circ} \mathrm{C}$ and under an argon atmosphere, was added butyllithium $(2.5 \mathrm{M}$, $0.19 \mathrm{ml}, 0.47 \mathrm{mmol})$. The suspension was stirred for $30 \mathrm{~min}$ at this temperature and then benzophenone was added $(86 \mathrm{mg}, 0.47 \mathrm{mmol})$ in the same solvent $(1 \mathrm{ml})$ dropwise over 5 min. The mixture was then allowed to warm slowly to room temperature overnight and poured into water/dichloromethane $(100 \mathrm{ml} ; 1: 1)$. The phases were separated and the aqueous extracted (dichloromethane, $50 \mathrm{ml}$ ). The combined organic layers were washed (water, $100 \mathrm{ml}$ ), dried $\left(\mathrm{MgSO}_{4}\right)$, and concentrated under reduced pressure to a pale yellow oil. Radial chromatography (dichloromethane elution) and collection of major component followed by recrystallisation gave the title compound (273) as a cream coloured solid (93 mg, 74\%), m.p. $99-100^{\circ} \mathrm{C}$ (lit. ${ }^{164} 68 \%,>240^{\circ} \mathrm{C}$ ). All spectral data were in excellent agreement with the literature values. ${ }^{164}$

Attempted preparation of 3,6-bis(1,3-dithiol-2-ylidene)-3,6-dihydro-1H-cyclopropa[b]naphthalene (168).

To a stirred solution of phosphonate ester (264) $(250 \mathrm{mg}, 1.18 \mathrm{mmol})$ in dry thf $(15 \mathrm{ml})$, cooled to $-78^{\circ} \mathrm{C}$ and under an argon atmosphere, was added butyllithium $(2.5 \mathrm{M}$, $0.47 \mathrm{ml}, 1.18 \mathrm{mmol}$ ). The suspension was stirred for $30 \mathrm{~min}$ at this temperature and then $1 H$-cyclopropa[b]naphthalene-3,6-dione (154) was added $(100 \mathrm{mg}, 0.59 \mathrm{mmol})$ in the same solvent $(3 \mathrm{ml})$ dropwise over $5 \mathrm{~min}$. The mixture which developed a turquoise and then dark green colouration though out the addition was then allowed to warm slowly to room 
temperature overnight and then poured into water/dichloromethane $(200 \mathrm{ml} ; 1: 1)$. The phases were separated and the aqueous extracted (dichloromethane, $100 \mathrm{ml}$ ) and the combined organic layers were washed (water, $100 \mathrm{ml})$, dried $\left(\mathrm{MgSO}_{4}\right)$, and concentrated under reduced pressure to a thick red oil which contained a complex mixture of components ( ${ }^{1} \mathrm{H}$ n.m.r. and t.l.c.) none of which corresponded to quinone (154), phosphonate ester (264), or dithioles (168) or (266). Attempts to purify the mixture and identify its components were unsuccessful.

A similar outcome to that recorded above was obtained when phosphonate ester (264) was treated with one molar equivalent of cyclopropanaphthoquinone (154).

\section{3,8-Bis(1,3-dithiol-2-ylidene)-3,8-dihydro-1H-cyclopropa[b]anthracene (169).}

To a stirred solution of phosphonate ester (264) (145 mg, $0.68 \mathrm{mmol})$ in dry thf $(5 \mathrm{ml})$, cooled to $-78^{\circ} \mathrm{C}$ and under an argon atmosphere, was added butyllithium $(1.65 \mathrm{M}$, $0.41 \mathrm{ml}, 0.68 \mathrm{mmol}$ ). The suspension was stirred for $30 \mathrm{~min}$ at this temperature and then $1 H$-cyclopropa $[b]$ anthracene-3,8-dione (162) was added $(150 \mathrm{mg}, 0.68 \mathrm{mmol})$ in the same solvent $(4 \mathrm{ml})$ dropwise over $5 \mathrm{~min}$. The mixture developed a colouration thoughout the addition. The mixture was then allowed to warm slowly to room temperature overnight and then poured into water/dichloromethane $(200 \mathrm{ml} ; 1: 1)$. The phases were separated and the aqueous extracted (dichloromethane, $100 \mathrm{ml}$ ). The combined organic layers were washed (water, $100 \mathrm{ml}$ ), dried $\left(\mathrm{MgSO}_{4}\right)$, and concentrated under reduced pressure to a red/orange oil. Column chromatography (basic alumina, dichloromethane/light petroleum elution; $4: 1$ ) gave a yellow solid that contained predominately a product proposed as 3,8-bis(1,3-dithiol-2-ylidene)-3,8-dihydro-1 $H$-cyclopropa[b]anthracene (169) (49 mg, 23\%). (Found: $[\mathrm{M}+\mathrm{H}]^{+}$392.9890. $\quad \mathrm{C}_{21} \mathrm{H}_{12} \mathrm{~S}_{4}$ requires: $[\mathrm{M}+\mathrm{H}]^{+}$392.9895). N.m.r. data abstracted for (169): 3.14 (d, J 4.2 Hz, H1), 3.60 (d, J 4.2 Hz. H1), 6.27 (bs, 4H, H4'/5'/9'/10'), 7.297.32 (BB', 2H, H5/6), 7.65 (s, 2H, H2/9), 7.68-7.73 (AA', 2H, H4/7); 21.0 (C1), 113.1 (C2/9), 117.0(3) (C4'/9' or C5'/10'), 117.0(5) (C5'/10' or $\left.\mathrm{C}^{\prime}{ }^{\prime} 9^{\prime}\right), 124.8$ (C4/7), 125.9 $(\mathrm{C} 5 / 6)$. Further attempts to purify (169) by chromatography or recrystallisation resulted in its decomposition.

\section{8-(1,3-Dithiol-2-ylidene)-3,8-dihydro-1H-cyclopropa[b]anthracene-3(8H)-one (267).}

To a stirred solution of phosphonate ester (264) (193 mg, $0.90 \mathrm{mmol})$ in dry thf $(3 \mathrm{ml})$, cooled to $-78^{\circ} \mathrm{C}$ and under an argon atmosphere, was added butyllithium (1.65 M, $0.55 \mathrm{ml}, 0.90 \mathrm{mmol}$ ). The suspension was stirred for $30 \mathrm{~min}$ at this temperature and then $1 H$-cyclopropa[ $b]$ anthracene,3-8-dione (162) was added $(100 \mathrm{mg}, 0.45 \mathrm{mmol})$ in the same 
solvent $(3 \mathrm{ml})$ dropwise over $5 \mathrm{~min}$. The mixture developed a colouration thoughout the addition. The mixture was then allowed to warm slowly to room temperature overnight and then poured into water/dichloromethane $(200 \mathrm{ml} ; 1: 1)$. The phases were separated and the aqueous extracted (dichloromethane, $100 \mathrm{ml}$ ). The combined organic layers were washed (water, $100 \mathrm{ml}$ ), dried $\left(\mathrm{MgSO}_{4}\right)$, and concentrated under reduced pressure to a red/orange oil. Column chromatography (basic alumina, dichloromethane/light petroleum elution; 4:1) gave a red solid as a near analytical sample of 8-(1,3-dithiol-2-ylidene)-3,8-dihydro-1H-cyclopropa[b]anthracene-3(8H)-one $(267)(40 \mathrm{mg}, 23 \%)$ as a rust coloured powder, m.p. $>350^{\circ} \mathrm{C}$ (Found: $[\mathrm{M}+\mathrm{H}]^{+}$307.0253. $\mathrm{C}_{18} \mathrm{H}_{10} \mathrm{~S}_{2} \mathrm{O}$ requires $[\mathrm{M}+\mathrm{H}]^{+} 307.0246$ ). IR $v_{\max } 3093,3059,2926,2852,1636,1594,1573,1479,1443,1285,1231,1123,1099$, $759,688 \mathrm{~cm}^{-1}$. UV $\lambda_{\max }$ (hexane) 251 (4.00), 358 (3.34), 431 (3.56), $451 \mathrm{~nm}(\log \varepsilon 3.64)$; UV $\lambda_{\max }$ (acetonitrile) 252 (4.57), 365 (3.89), $467 \mathrm{~nm}(\log \varepsilon 4.14) .{ }^{1} \mathrm{H}$ n.m.r. $\delta 3.39$ (s, $2 \mathrm{H}$, H1), 6.42 (bs, 2H, H4'/5'), 7.38-7.43 (m, 1H, H6), 7.59-7.65 (m, 1H, H5), 7.81 (d, J $1.8 \mathrm{~Hz}, \mathrm{H} 9), 7.80-7.92$ (m, 1H, H7), 8.10 (d, J $1.8 \mathrm{~Hz}, \mathrm{H} 2), 8.24-8.27$ (m, $1 \mathrm{H}, \mathrm{H} 4) ;{ }^{13} \mathrm{C}$ n.m.r. $\delta 19.8(\mathrm{C} 1), 113.0$ (C9), 113.8 (C2), $117.8\left(4^{\circ}\right), 117.9$ (C4' or C5'), 118.0 (C5' or C4'), $125.3\left(4^{\circ}\right), 125.8$ (C7), 126.4 (C6), 126.9 (C4), $130.6\left(4^{\circ}\right), 130.9$ (4º), 131.5 (C5), $133.0\left(4^{\circ}\right), 139.9\left(4^{\circ}\right), 142.7\left(4^{\circ}\right), 145.7\left(4^{\circ}\right), 183.6(\mathrm{C} 3) . \quad$ Recrystallisation (dichloromethane/hexane; $1: 1,-16^{\circ} \mathrm{C}$ ) resulted in significant decomposition and $<1 \mathrm{mg}$ of truncated rust coloured cones were obtained.

Preparation of 1-\{[10-(6,6-diphenyl-2,5-dithiapentafulvenyl)phenyl]methylidene $\}-1 H$ cyclopropa[b]naphthalene-0.25 $\mathrm{C}_{6} \mathrm{H}_{14}$ (274).

To a stirred solution of phosphonate ester (264) $(88 \mathrm{mg}, 0.42 \mathrm{mmol})$ in dry thf $(2 \mathrm{ml})$, cooled to $-78^{\circ} \mathrm{C}$ and under an argon atmosphere, was added butyllithium $(1.65 \mathrm{M}$, $0.25 \mathrm{ml}, 0.42 \mathrm{mmol}$ ). The suspension was stirred for $30 \mathrm{~min}$ at this temperature and then 1-[(p-benzoylphenyl)phenylmethylidene $]-1 H$-cyclopropa[ $b]$ naphthalene- $0.9 \mathrm{CH}_{2} \mathrm{Cl}_{2}$ (200) was added $(170 \mathrm{mg}, 0.45 \mathrm{mmol})$ in the same solvent $(4 \mathrm{ml})$ dropwise over $5 \mathrm{~min}$. The dark red mixture was then allowed to warm slowly to room temperature overnight and then poured into water/dichloromethane $(200 \mathrm{ml} ; 1: 1)$. The phases were separated, the aqueous phase extracted (dichloromethane, $100 \mathrm{ml}$ ), and the combined organic layers washed (water, $100 \mathrm{ml}$ ), dried $\left(\mathrm{MgSO}_{4}\right)$, and concentrated under reduced pressure to a red/orange oil. Column chromatography (basic alumina, dichloromethane/hexane; 1:4 elution) gave an analytical sample of 1-\{[10-(6,6-diphenyl-2,5-dithiapentafulvenyl)phenyl]methylidene $\}$ $1 \mathrm{H}$-cyclopropa[b]-naphthalene-0.25 $C_{6} H_{4}(274)(134 \mathrm{mg}, 66 \%)$ as a bright red powder, 
m.p. $69-70^{\circ} \mathrm{C}$ (Found: $[\mathrm{M}+\mathrm{H}]^{+} 495.1249 . \mathrm{C}_{34} \mathrm{H}_{22} \mathrm{~S}_{2}$ requires $[\mathrm{M}+\mathrm{H}]^{+} 495.1236$ ). IR $v_{\max }$ $3048,2951,1773,1593,1487,1442,1419,1345,1135,1029,845,799,748,697,635$ $\mathrm{cm}^{-1}$. UV $\lambda_{\max }$ (hexane) 230 (4.77), 278 (4.47), $292 \mathrm{sh}$ (4.42), $396 \mathrm{sh}$ (4.35), $449 \mathrm{~nm}(\log \varepsilon$ 4.62); UV $\lambda_{\max }$ (acetonitrile) 230 (4.75), 275 (4.45), $294 \mathrm{sh}(4.41), 441 \mathrm{~nm}(\log \varepsilon 4.59) .{ }^{1} \mathrm{H}$ n.m.r. $\delta 6.24$ (d, $J 6.6 \mathrm{~Hz}, \mathrm{H}^{\prime}{ }^{\prime}$ or H5'), 6.28 (d, J 6.6 Hz, H4' or H5'), 7.28-7.50 (m, 10H), 7.54 (d, $J 1.8 \mathrm{~Hz}, 1 \mathrm{H}, \mathrm{H} 2$ or $\mathrm{H} 7), 7.58$ (d, $J 1.8 \mathrm{~Hz}, 1 \mathrm{H}, \mathrm{H} 7$ or $\mathrm{H} 2$ ), 7.70-7.78 (m, 4H), 7.86-7.90 (AA', 2H, H3/6); ${ }^{13}$ C n.m.r. $\delta$ 107.0(6) (C2 or C7), 107.1(6) (C7 or C2), 111.8 (C1), 117.4 (C4' or C5'), 117.6 (C5' or C4'), 119.5 (C8), $124.6\left(4^{\circ}\right), 126.7(\mathrm{C} 4 / 5), 127.2$ $(\mathrm{CH}), 127.4(\mathrm{CH}), 127.6\left(4^{\circ}\right), 127.9(\mathrm{CH}), 128.1(\mathrm{CH}), 128.3(5)(\mathrm{CH}), 128.4(3)(\mathrm{CH})$, 128.7(0) (C3 or C6), 128.7(2) (C6 or C3), $128.9(\mathrm{CH}), 136.5\left(4^{\circ}\right), 137.4\left(4^{\circ}\right), 138.7\left(4^{\circ}\right)$, $138.8\left(4^{\circ}\right), 139.3\left(4^{\circ}\right), 140.9\left(4^{\circ}\right), 142.8\left(4^{\circ}\right)$.

Attempted preparation of a charge-transfer salt from 1-\{[10-(6,6-diphenyl-2,5-dithiapentafulvenyl)phenyl]methylidene $-1 H$-cyclopropa[b] naphthalene-0.25 hexane (274) and DDQ.

To a stirred solution of 1-\{[10-(6,6-diphenyl-2,5-dithiapentafulvenyl)-phenyl]methylidene $\}-1 H$-cyclopropa[b]naphthalene- $0.25 \quad \mathrm{C}_{6} \mathrm{H}_{14} \quad$ (274) $(20 \mathrm{mg}, 0.04 \mathrm{mmol})$ in dry acetonitrile $(6 \mathrm{ml})$ was added a solution of DDQ $(18.4 \mathrm{mg}, 0.08 \mathrm{mmol})$ at which point the initially red coloured solution developed a dark green colouration; UV $\lambda_{\max }$ (acetonitrile) 252 (4.56), 382 (4.07), 294 (4.04), 312 (4.03), 347 (4.07), 436 (3.88) 540-620 nm (log $\varepsilon$ 3.34). Attempts to obtain crystals were unsuccessful and the mixture become brown (even upon standing in the freezer for $16 \mathrm{~h}$ ).

\section{0-(1,3-Dithiol-2-ylidene) anthracene-9(10H)-one (265)}

To a mixture of salt $(270)(1 \mathrm{~g}, 4.24 \mathrm{mmol})$ and anthrone $(0.82 \mathrm{mmol})$ under argon was added glacial acetic acid $(10 \mathrm{ml}, 174.7 \mathrm{mmol})$ followed by pyridine $(2 \mathrm{ml}, 24.7 \mathrm{mmol})$. The mixture was then refluxed with stirring for $30 \mathrm{~min}$, cooled, and the orange precipitate filtered. The solid was washed (water, $5 \times 40 \mathrm{ml}$ ), dried under high vacuum, and recrystallised from dichloromethane to give the title compound ( $940 \mathrm{mg}, 79 \%$ ) as bright orange needles, m.p. $220-221^{\circ} \mathrm{C}$ (lit. ${ }^{181} 85 \%, 219-221^{\circ} \mathrm{C}$ ), ${ }^{1} \mathrm{H}$ n.m.r. data were in agreement with those reported. ${ }^{164}{ }^{13} \mathrm{C}$ n.m.r. $\delta 116.1\left(4^{\circ}\right), 117.1,124.9,125.9,126.1,129.7\left(4^{\circ}\right)$, $130.9,138.5\left(4^{\circ}\right), 145.0\left(4^{\circ}\right), 182.7\left(4^{\circ}\right)$.

9-(1H-Cyclopropa[b]naphthalene-1-ylidene)-10-(1,3-dithiol-2-ylidene)-9H,10H-dihydroanthracene (275). 
To a stirred suspension of dithiole (265) (96 mg, $0.34 \mathrm{mmol}$ ) and disilylcyclopropanaphthalene (105) $(100 \mathrm{mg}, 0.34 \mathrm{mmol})$ in dry thf $(15 \mathrm{ml})$ cooled to $-78^{\circ} \mathrm{C}$ and under argon was added potassium $t$-butoxide $(40 \mathrm{mg}, 0.34 \mathrm{mmol})$ in the same solvent $(3 \mathrm{ml})$ dropwise over $5 \mathrm{~min}$. The dark red mixture was then allowed to warm to room temperature slowly overnight and then poured into water/dichloromethane $(200 \mathrm{ml} ; 1: 1)$. The phases were separated and the aqueous extracted (dichloromethane, $100 \mathrm{ml}$ ). The combined organic layers were washed (water, $100 \mathrm{ml})$, dried $\left(\mathrm{MgSO}_{4}\right)$, and concentrated under reduced pressure to a dark red oil. Column chromatography (basic alumina, dichloromethane elution) and trituation (same solvent) gave the 9-(1H-cyclopropa[b]naphthalene1-ylidene)-10-(1,3-dithiol-2-ylidene)-9H,10-H-dihydroanthracene (275) (4.5 mg, 3\%) as red/black needles, m.p. darkens $111-112{ }^{\circ} \mathrm{C}$, melts $253-254{ }^{\circ} \mathrm{C}$ (Found: $[\mathrm{M}+\mathrm{H}]^{+} 417.0760$. $\mathrm{C}_{28} \mathrm{H}_{16} \mathrm{~S}_{2}$ requires $[\mathrm{M}+\mathrm{H}]^{+}$417.0766). IR $v_{\max } 3057,1631,1497,1442,1416,1142,1115$, $752,742,659,622 \mathrm{~cm}^{-1}$. UV $\lambda_{\max }$ (acetonitrile) 236 (4.59), 268 (3.95), $295 \mathrm{sh}$ (3.89), 382 (3.98), 420 (4.07), 508 nm (log ع 4.18). ${ }^{1} \mathrm{H}$ n.m.r. $\delta 6.28$ (s, 2H, H20/21), 7.40-7.44 (m, 4H, H10/11/15/16), 7.47-7.50 (BB', 2H, H4/5), 7.65 (s, 2H, H2/7), 7.76-7.78 (m, 2H, H12/14), 7.89-7.92 (AA', 2H, H3/6); ${ }^{13} \mathrm{C}$ n.m.r. $\delta 107.9$ (C2/7), 109.7 (C1), 113.6 (C8), 117.1 (C20/21), 122.3 (C13), 123.5 (C9/17), 126.0 (C12/14), 126.4 (C10/16 or C11/15), 126.7(0) (C11/15 or $\mathrm{C} 10 / 16), 126.7(2)(\mathrm{C} 1 \mathrm{a} / 7 \mathrm{a}), 126.9(\mathrm{C} 4 / 5), 128.7(\mathrm{C} 3 / 6), 132.9$ (C8a/17a or C12a/13a), 135.0 (C12/13a or C8a/17a), 136.8 (C18), 139.1 (C2a/6a).

Further attempts to prepare (275) gave the compound in yields of 1-6\%.

\section{2[(Benzoyl)phenyl]methylidene-1,3-dithiole (276)}

To a mixture of salt (270) (500 mg, $2.11 \mathrm{mmol})$ and 1,2-diphenylethanone (416 mg, $2.11 \mathrm{mmol})$, under an argon atmosphere was added sequentially glacial acetic acid $(5 \mathrm{ml}$, $87.4 \mathrm{mmol})$ and pyridine $(1 \mathrm{ml}, 12.4 \mathrm{mmol})$. The mixture was then refluxed with stirring for $45 \mathrm{~min}$., cooled, and then poured into water/dichloromethane $(200 \mathrm{ml} ; 1: 1)$. The phases were separated, the aqueous extracted (dichloromethane, $3 \times 40 \mathrm{ml}$ ), and the combined organic layers were washed (water, $200 \mathrm{ml})$, dried $\left(\mathrm{MgSO}_{4}\right)$, and concentrated under reduced pressure to a brown oil that contained ( ${ }^{1} \mathrm{H}$ n.m.r.) a 1:1 mixture of unchanged ketone and product (276). The residue was then dissolved in acetic acid (5 ml), salt (270) (213 mg, $1.06 \mathrm{mmol})$ was added followed by pyridine $(0.5 \mathrm{ml})$ and the mixture refluxed with stirring for $45 \mathrm{~min}$. After cooling, workup was performed as above and the crude residue was purified by column chromatography (ethyl acetate elution). Recrystallisation of the yellow/brown residue obtained (thf/hexane; $\left.1: 1,-16^{\circ} \mathrm{C}\right)$, gave $(276)(575 \mathrm{mg}, 90 \%$; 
based on ketone used) as lustrous yellow crystals, m.p. $134-136^{\circ} \mathrm{C}$ (Found: C, 68.7; H, 4.0; $[\mathrm{M}+\mathrm{H}]^{+}$297.0395. $\mathrm{C}_{17} \mathrm{H}_{12} \mathrm{OS}_{2}$ requires: $\mathrm{C}, 68.9 ; \mathrm{H}, 4.1 \% ;[\mathrm{M}+\mathrm{H}]^{+}$297.0402). IR $v_{\max }$ $3095,3068,1597,1584,1564,1545,1443,1413,1386,1330,1305,1263,1072,989,741$, 698, $666 \mathrm{~cm}^{-1}$. UV $\lambda_{\max }$ (acetonitrile) 231 (4.13), 250 (3.88), $400 \mathrm{~nm}(\log \varepsilon 4.39) .{ }^{1} \mathrm{H}$ n.m.r. $\delta 6.80$ (d, $J 6.7 \mathrm{~Hz}, 1 \mathrm{H}, \mathrm{H} 4$ or H5), 7.00 (d, $J 6.7 \mathrm{~Hz}, 1 \mathrm{H}, \mathrm{H} 5$ or H4), 7.11-7.17 (m 2H), 7.22-7.39 (m, 8H); ${ }^{13} \mathrm{C}$ n.m.r. $\delta 118.9$ (C4 or C5), $121.3\left(4^{\circ}\right), 125.4$ (C5 or C4), 127.4 $(\mathrm{CH}), 127.9(\mathrm{CH}), 129.0(\mathrm{CH}), 129.3(\mathrm{CH}), 129.8(\mathrm{CH}), 130.3(\mathrm{CH}), 139.3\left(4^{\circ}\right), 140.0$ $\left(4^{\circ}\right), 167.8\left(4^{\circ}\right), 185.8(\mathrm{C} 8)$.

1-[1,2-Diphenyl-2-(1,3-dithiol-2-ylidene)] ethylidene-1H-cyclopropa[b]naphthalene (277).

To a stirred solution of dithiole (276) $(156 \mathrm{mg}, 0.52 \mathrm{mmol})$ and disilylcyclopropanaphthalene $(105)(150 \mathrm{mg}, 0.52 \mathrm{mmol})$ in dry thf $(3 \mathrm{ml})$ cooled to $-78^{\circ} \mathrm{C}$ and under argon was added potassium $t$-butoxide $(60 \mathrm{mg}, 0.52 \mathrm{mmol})$ in the same solvent $(3 \mathrm{ml})$ dropwise over $15 \mathrm{~min}$. The dark red mixture was then allowed to warm to room temperature slowly overnight and then poured into water/dichloromethane $(200 \mathrm{ml} ; 1: 1)$. The phases were separated and the aqueous extracted (dichloromethane, $100 \mathrm{ml}$ ). The combined organic layers were washed (water, $100 \mathrm{ml}$ ), dried $\left(\mathrm{MgSO}_{4}\right)$, and concentrated under reduced pressure to a dark red/orange oil. Column chromatography (basic alumina, dichloromethane/light petroleum elution; 4:1) gave 9- $(1 \mathrm{H}$-cyclopropa/b]naphthalene-1-ylidene $)$ $10-(1,3$-dithiol-2-ylidene)-9H,10H-dihydroanthracene $(277)(28.5 \mathrm{mg}, 13 \%)$ as a red oil (Found: $[\mathrm{M}+\mathrm{H}]^{+}$419.0925. $\mathrm{C}_{28} \mathrm{H}_{18} \mathrm{~S}_{2}$ requires $[\mathrm{M}+\mathrm{H}]^{+}$419.0923). IR $v_{\max } 3048,1765$, $1630,1592,1542,1486,1439,1413,1344,1144,905,845,760,744,727,692,643 \mathrm{~cm}^{-1}$. UV $\lambda_{\max }$ (hexane) 230 (4.69), 290 (4.33), 380 (4.24), 428 (3.99), 474 (4.18), $502 \mathrm{~nm}(\log \varepsilon$ 4.21); UV $\lambda_{\max }$ (acetonitrile) 232 (4.65), 288 (4.31), 378 (4.22), 422 (3.99), $476 \mathrm{~nm}$ (log $\varepsilon$ 4.02). ${ }^{1} \mathrm{H}$ n.m.r. $\delta 6.26$ (d, $J 6.7 \mathrm{~Hz}, 1 \mathrm{H}, \mathrm{H} 18$ or H19), 6.31 (d, $J 6.7 \mathrm{~Hz}, 1 \mathrm{H}, \mathrm{H} 19$ or H18), 7.16-7.45 (m, 9H), 7.57-7.60 (m, 2H, H10/14 or H22/26), 7.68 (d, $J 1.6 \mathrm{~Hz}, 1 \mathrm{H}, \mathrm{H} 2$ or H7), 7.82-7.93 (m, $4 \mathrm{H}, \mathrm{H} 3 / 6$ and $\mathrm{H} 10 / 14$ or $\mathrm{H} 22 / 26) ;{ }^{13} \mathrm{C}$ n.m.r. $\delta 107.7$ (C2 or C7), 109.0 (C7 or C2), 112.6 (C1), 116.9 (C18 or C19), 119.5 (C19 or C18), $120.6\left(4^{\circ}\right), 121.8\left(4^{\circ}\right), 126.5$ $(\mathrm{CH}), 126.6(8)(\mathrm{CH}), 126.7(1)(\mathrm{CH}), 127.1(\mathrm{CH}), 127.4\left(4^{\circ}\right), 127.7\left(4^{\circ}\right), 128.1(\mathrm{CH}), 128.5$ $(\mathrm{CH}), 128.5(\mathrm{CH}), 128.7(\mathrm{CH}), 128.8(\mathrm{CH}), 137.5\left(4^{\circ}\right), 138.7\left(4^{\circ}\right), 138.9\left(4^{\circ}\right), 141.2\left(4^{\circ}\right)$. 


\title{
Appendix A
}

\section{Re-prints of Published Papers}

\section{Studies in the Cycloproparene Series: Formation of a New Dimer of $1 H$-Cyclopropa $[b]$ naphthalene}

\author{
Brian Halton, ${ }^{\mathrm{A}, \mathrm{B}}$ Carissa S. Jones, ${ }^{\mathrm{A}}$ Peter T. Northcote ${ }^{\mathrm{A}}$ and \\ Roland Boese ${ }^{\mathrm{C}}$ \\ A School of Chemical and Physical Sciences, Victoria University of Wellington, \\ P.O. Box 600, Wellington, New Zealand. \\ B Author to whom correspondence may be addressed. \\ C Institute for Inorganic Chemistry, University of Essen, 45117 Essen, Germany, \\ and to whom inquiries about the crystallography should be addressed.
}

\begin{abstract}
1-(Trimethylsilyl)-1 $H$-cyclopropa[b]naphthalene (10) and its 1-methyl derivative (11) have been isolated as pure compounds from use of a lipophilic size exclusion gel. Acylation of the $1 H$-cyclopropa[ $b]$ naphthalenyl anion (2) is effected with $N, N$-dimethyl-benzamide and -acetamide to give (5) and (6), respectively. Analogous reactions with the 1-(trimethylsilyl)- $1 H$-cyclopropa[b]naphthalenyl anion (9) do not yield the 1-acyl-1-(trimethylsilyl)-1 $H$-cyclopropa[b]naphthalenes (12) and (13); instead the novel 6-methyl$7 \mathrm{H}$-dibenzo[ $b, g]$ fluorene (15) results from attempted acetylation. Compound (15), a formal dimer of $1 H$-cyclopropa $[b]$ naphthalene (1), is formed also from anion (9) both in toluene and from addition of hydrocarbon (1); its structure is assigned from spectroscopic data and confirmed by single-crystal $\mathrm{X}$-ray analysis.
\end{abstract}

Despite major advances in the chemistry of the cycloproparenes, ${ }^{1-5}$ many simple facets of their behaviour remain to be explored. In this context our previous studies directed at the interception of $1 H$-cyclopropa $[b]$ naphthalenyl anions with simple electrophiles met with only limited success ${ }^{6,7}$ when compared to use of the $\alpha$-silyl anion in Peterson olefination and direct formation of $\mathrm{C} 1$ exocyclic methylidenecycloproparenes. ${ }^{1,8,9}$ Capture of the C1 unsubstituted anion (2) has provided the monosubstituted derivatives (3) and (4) (Scheme 1) in only modest yield. ${ }^{6}$ The 1-trimethylsilyl analogue (9) gave the $\mathrm{C}_{1}$ silane (10) and its 1-methyl derivative (11) (Scheme 2) as easily identifiable components of hitherto inseparable product mixtures. ${ }^{7}$

The formation of anions (2) and (9) requires rigorous exclusion of moisture as even catalytic amounts of hydroxide (or t-butoxide) ion with water convert disilane (8) into hydrocarbon (1) via anions (9) and (2). ${ }^{7}$ Controlled protonation of anion (9) to give the monosilane (10) in reasonable yields has not proved easy and viable amounts were obtained previously only when trifluoroacetic acid was employed as proton source. ${ }^{8}$ Effective protonation of (9) requires the conjugate base thus generated to be too weak to remove the silyl group of (10) and in this context the effect of toluene was assessed. While addition of toluene to anion (9) does not provide silane (10) except in trace amounts, a new product was formed that was subsequently identified as the dibenzo $[b, g]$ fluorene (15) (see below). Despite this, we have now found that anion generation employing t-butoxide in the range from -70 to $-40^{\circ} \mathrm{C}$ followed by the addition of water, pouring into water, and workup, provides a product mixture from which monosilane (10) has been isolated successfully (9\%) with the aid of chromatography employing the size exclusion gel Sephadex LH-20. At<smiles></smiles>

(1)<smiles>CC1c2cc3ccccc3cc2C1C</smiles>

(2)<smiles>[R]C([2H])(O)[C@H]1c2cc3ccccc3cc2[C@H]1[3H]</smiles>

(3) $\mathrm{R}=\mathrm{Me}$ or $\mathrm{Ph}$<smiles>CC1Cc2cc3ccccc3cc21</smiles>

(4)<smiles>O=CC1CC2CC1C2</smiles>

(5) $\mathrm{R}=\mathrm{Ph}$

(6) $\mathrm{R}=\mathrm{Me}$<smiles>CC(=O)C1(C(C)=O)C2c3ccccc3C1c1ccccc12</smiles>

(7)
Scheme 1 
ambient temperature (10) is a colourless oil that slowly solidifies in the freezer (Experimental section) and rapidly turns yellow. The spectroscopic data recorded for (10) are fully compatible with those abstracted for it from the product mixture with (8) and (1), but they show some minor chemical shift changes as one would expect with an uncontaminated sample. Most notable are the observations of small long-range couplings between the $\mathrm{C} 2 / \mathrm{C} 7$ aromatic protons $(\delta$ $7 \cdot 33)$ and the benzylic $C 1$ proton $(\delta 3 \cdot 23, J<1 \mathrm{~Hz})$, and to the $\mathrm{H} 3 / \mathrm{H} 6$ sromatics $(J<0.5 \mathrm{~Hz})$. Spurred by the success in using size exclusion gel chromatography, the capture of anion (9) with iodomethane was repeated as described previously. ${ }^{7}$ Separation of the ensuing mixture of products was again brought about with use of the gel and 1-methyl-1-(trimethylsilyl)- $1 \mathrm{H}$ cyclopropa[b]naphthalene (11) has been obtained as a colourless oil in 47\% yield; (10) and (11) are now fully characterized (Experimental section).<smiles>CCC1(C)c2cc3ccccc3cc21</smiles>

(8)

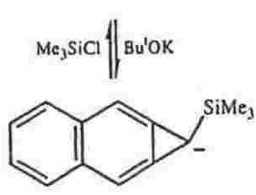

(9)<smiles>CCC1Cc2cc3ccccc3cc21</smiles>

(10)

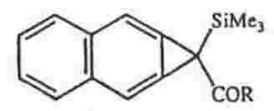

(12) $\mathrm{R}=\mathrm{Ph}$

(13) $\mathrm{R}=\mathrm{Me}$

(14) $\mathrm{R}=\mathrm{NMe}_{2}$

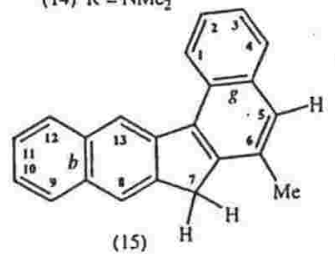

(15)

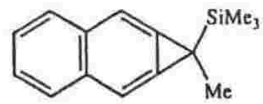

(11)
Scheme 2

the monoamide is deprotonated by unreacted anion (2) thereby allowing for disubstitution to take place. The structure of compound (7) is fully supported by the appearance of a singlet for $\mathrm{H} 2 / \mathrm{H} 7$ at $7.85 \mathrm{ppm}$ and an $\mathrm{AA}^{\prime} \mathrm{BB}^{\prime}$ pattern for $\mathrm{H} 3 / \mathrm{H} 6$ and $\mathrm{H} 4 / \mathrm{H} 5$ as well as shielded $\mathrm{C} 2 / \mathrm{C} 7$ resonances at $112.9 \mathrm{ppm}$ in the ${ }^{13} \mathrm{C}$ spectrum; the $N$-methyl groups appear with distinct six-proton $(\delta 2.94$ and 3.67$)$ and carbon $(\delta$ 35.9 and 38.2$)$ resonances.

The $\alpha$-silyl anion (9) is known to react with acid halides and cyanides to give a range of carbonylcontaining compounds, e.g. (12)-(14), in yields that range from 50 to $65 \% .{ }^{12}$ In contrast to this, and to the successful reactions with (2) described above, anion (9) gives only complex product mixtures from reactions with $N, N$-dimethyl-benzamide and -acetamide. While the product mixture from the former remains unresolved in our hands that from treatment of anion (9) with $N, N$-dimethylacetamide has been separated and provides, not the expected acetyl compound (13) but the novel 6-methyl-7 $H$-dibenzo $[b, g]$ fluorene (15), a formal dimer of $1 H$-eyclopropa[b]naphthalene (1). The compound is isolated as straw-coloured blocks in yields that range from 4 to $18 \%$ and its formation is accompanied by the regeneration of (1) in yields of $2-20 \%$. The formation of these products cannot involve the $N, N$-dimethylacetamide reagent as the same dimer is formed from attempted protonation of (9) by toluene but in lower $(6 \%)$ yield. Moreover, an increase in the yield of (15) to $28 \%$ is obtained when $1 H$-cyclopropa $[b]$ naphthalene ( 1 ) is added to anion (9). Clearly, a second desilylation occurs and proton transfers are required. In the presence of water, anion (9) is protonated to give (10) that reacts with base and affords (1) via anion (2). ${ }^{7}$ Thus it seems likely that traces of water are present and that hydrocarbon (1) and anion (9) are intimately involved in the formation of (15). However, it would be mere speculation to propose a pathway(s) from (9) to (15) at this time.

The assignment of structure to (15) is based upon

Other electrophilic captures by anions (2) and (9) have been assessed. Whereas attempts to effect benzoylation of (2) with either benzoyl chloride or methyl benzoate were unsuccessful, ${ }^{6}$ use of $N, N$ dimethylbenzamide and $N, N$-dimethylacetamide has provided the acyl derivatives (5) and (6) in yields of 46 and $26 \%$, respectively; spectroscopic data are fully compatible with the assigned structures. Comparable formation of anion (2) and reaction with dimethylcarbamoyl chloride did not provide a monoamide but instead gave disubstitution in the form of diamide (7) (34\%) (Scheme 1) together with unchanged (1) (20\%). Despite variations in the ratio of the carbamoyl chloride from 0.5 to $10 \mathrm{~mol}$. equiv. the same two products were obtained with little variation in the isolated yields. This is reminiscent of the silylation of cyclopropa $[b]$ naphthalene (1) which give disilane (8) instead of the expected monosilane $(10)^{9-11}$ and suggests that

the $\mathrm{C}_{22} \mathrm{H}_{16}$ hydrocarbon nature of the compound, the appearance of methyl and methylene signals $(\delta 2 \cdot 59$ and 4.06 , respectively) in addition to 11 aromatic protons in the ${ }^{1} \mathrm{H}$ n.m.r. spectrum, and the presence of 22 distinct ${ }^{13} \mathrm{C}$ resonances. Three of the eleven aromatic protons appear as broadened singlets, and there are nine quaternary and eleven aromatic methine carbon atoms. The methyl signal shows long-range proton-proton spin-spin coupling to an adjacent but otherwise isolated aromatic proton $(7.66 \mathrm{ppm}, J \mathrm{c}$. $1 \mathrm{~Hz}$ ) whereas the methylene group is an uncoupled singlet. While the symmetry expected of a cycloproparene framework clearly is absent, the presence of two other broadened aromatic singlets $\left(\begin{array}{ll}\delta & 8.03\end{array}\right.$ and 8.77) implies one tetrasubstituted arene ring with two para protons, and the presence of an adjacent methyl group and aromatic proton suggest comparable but angular fusion elsewhere. Plausible structures are the 
dibenzo $[b, g \mid$ fluorene (15) and its $[a, h]$ isomer (16). Differentiation between the two in favour of (15) has come only from detailed n.O.e., TOCSY, HSQC, and HMBC n.m.r. experiments with the proton resonance multiplicities derived from Hомо-2DJ measurements. Nuclear Overhauser enhancements recorded between the methyl and methylene groups, and between the former and the long-range coupled aromatic hydrogen atom at 7.66 ppm and vice versa (Fig. 1), are especially important as they require the dibenzo[ $[b, g]$ fluorene skeleton (15) with the $\mathrm{C} 6$ methyl group located spatially close to the $\mathrm{CH}_{2}$ moiety and adjacent to the isolated aromatic proton (H 5); the dibenzo $[a, h]$ isomer (16) contains the latter but not the former feature and thus is unlikely.

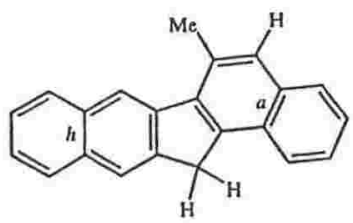

(16)

An n.O.e. from the $\mathrm{C} 7$ methylene group to the signal at $8.03 \mathrm{ppm}$ allows for identification of $\mathrm{H} 8$ which in turn provides for conventional assignment of the para analogue, H13 (8.77 ppm). Upon irradiation H13 gives a positive n.O.e. with two protons, one at 8.04 and the other at $8.90 \mathrm{ppm}$; irradiation of the latter confirms the effect with $\mathrm{H} 13$. One is the peri proton (H12) and the other the spatially proximal, though six bonds removed, H1. The presence of three contiguous protons from the proton that resonates at $8.04 \mathrm{ppm}$ ( $\delta 7.44,7.55$, and 7.87 as multiplets) as well as from that at $8.90 \mathrm{ppm}(\delta 7.66,7.57$, and 7.91 as multiplets) is confirmed by Tocsy experiments thereby providing appropriate evidence for the presence of the two benzo-fused rings. An HMBC experiment showed a distinct correlation of the well separated $\mathrm{C} 8$ resonance at $122.9 \mathrm{ppm}$ (assigned from HSQC to $\mathrm{H} 8$ at 8.03 ppm) with the proton at $7.87 \mathrm{ppm}$; this proton is H9. The rocsY experiments correlate $\mathrm{H} 12$ through $\mathrm{H} 11$ and $\mathrm{H} 10$ to $\mathrm{H} 9(\delta 7.87)$ and thus require the peri proton $\mathrm{H} 12$ to be the one that resonates at 8.04 ppm. In turn, this leads to the assignment of the resonance at $8.90 \mathrm{ppm}$ to $\mathrm{H} 1$. This is consistent with its location in the bay region of (15) and fits well with the data recently assigned to dibenzo $[a, i]$ fluorene by Wentrup and Régimbald. ${ }^{13}$ Despite all of the data available an unambiguous proof that structure (15) is the hydrocarbon product was sought and it has come from an X-ray structure analysis.

The results of a room-temperature single-crystal Xray study of 6 -methyl-7H-dibenzo $[b, g]$ fluorene $(15)$ as crystallized from dichloromethane/hexane (Tables 1-3 and Fig. 2) are fully consistent with the benzo ring fusion as depicted by (15) and shown in the ORTEP plot (Fig. 2); the compound crystallizes in the monoclinic crystal system. The intramolecular geometries (Tables 2 and 3 ) are largely as expected for a polycyclic fluorenyl system. However, while dibenzofluorenes have been listed in Chemical Abstracts, to the best of our knowledge 6methyl-7H-dibenzo[ $b, g]$ fluorene (15) is the first formally characterized $[b, g]$ hydrocarbon derivative. There has been a claim that $7 H$-dibenzo[ $b, g]$ fluorene-7-one is an environmental pollutant ${ }^{14}$ and $5 H$-dibenzo $[b, g]$ fluorene$5,8,13$-trione has been isolated and characterized. ${ }^{15}$

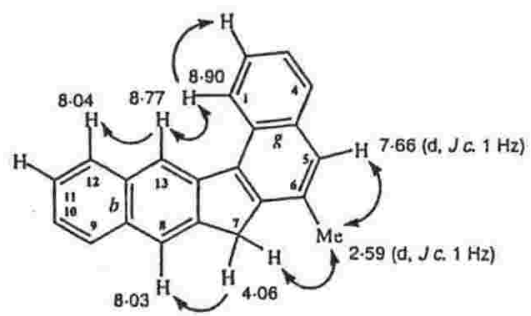

Fig. 1. ${ }^{1} \mathrm{H}$ n.m.r. chemical shifts (ppm) and nuclear Overhauser enhancements (arrows) recorded for 6-methyl-7Hdibenzo $[b, g]$ fluorene $(15)$.

Table 1. Non-hydrogen positional and isotropic displacement parameters for (15)

\begin{tabular}{lcrrr}
\hline Atom & $10^{4} x$ & \multicolumn{1}{c}{$10^{4} y$} & $10^{4} z$ & $10^{3} U_{\text {oq }}\left(\AA^{2}\right)$ \\
\hline$C(1)$ & $3457(2)$ & $9117(3)$ & $1754(1)$ & $55(1)$ \\
$C(2)$ & $2738(2)$ & $10544(3)$ & $1967(1)$ & $65(1)$ \\
$C(3)$ & $1621(2)$ & $10211(3)$ & $1752(1)$ & $66(1)$ \\
$C(4)$ & $1234(2)$ & $8469(3)$ & $1327(1)$ & $63(1)$ \\
$C(5)$ & $1545(2)$ & $5122(3)$ & $648(1)$ & $59(1)$ \\
$C(6)$ & $2222(2)$ & $3637(3)$ & $412(1)$ & $55(1)$ \\
$C(7)$ & $4230(1)$ & $2486(3)$ & $449(1)$ & $53(1)$ \\
$C(8)$ & $6312(1)$ & $2945(3)$ & $875(1)$ & $53(1)$ \\
$C(9)$ & $8289(2)$ & $3617(3)$ & $1336(1)$ & $59(1)$ \\
$C(10)$ & $9096(2)$ & $4915(3)$ & $1709(1)$ & $63(1)$ \\
$C(11)$ & $8844(2)$ & $6917(3)$ & $2025(1)$ & $61(1)$ \\
$C(12)$ & $7788(1)$ & $7578(3)$ & $1962(1)$ & $54(1)$ \\
$C(13)$ & $5820(1)$ & $6878(2)$ & $1543(1)$ & $49(1)$ \\
$C(14)$ & $3092(1)$ & $7280(3)$ & $1310(1)$ & $49(1)$ \\
$C(15)$ & $1954(1)$ & $6942(3)$ & $1095(1)$ & $54(1)$ \\
$C(16)$ & $3351(1)$ & $3958(3)$ & $627(1)$ & $50(1)$ \\
$C(17)$ & $5262(1)$ & $3580(3)$ & $835(1)$ & $49(1)$ \\
$C(18)$ & $7180(1)$ & $4248(3)$ & $1260(1)$ & $50(1)$ \\
$C(19)$ & $6921(1)$ & $6260(3)$ & $1585(1)$ & $48(1)$ \\
$C(20)$ & $4993(1)$ & $5558(2)$ & $1186(1)$ & $46(1)$ \\
$C(21)$ & $3795(1)$ & $5721(2)$ & $1061(1)$ & $47(1)$ \\
$C(22)$ & $1786(2)$ & $1713(3)$ & $-59(1)$ & $65(1)$ \\
\hline
\end{tabular}

Table 2. Molecular non-hydrogen distances $(\AA)$ for (15)

\begin{tabular}{llll}
\hline \multicolumn{1}{c}{ Atoms } & Distance & \multicolumn{1}{c}{ Atoms } & Distance \\
\hline $\mathrm{C}(1)-\mathrm{C}(2)$ & $1 \cdot 370(2)$ & $\mathrm{C}(9)-\mathrm{C}(10)$ & $1 \cdot 365(3)$ \\
$\mathrm{C}(1)-\mathrm{C}(14)$ & $1.419(2)$ & $\mathrm{C}(9)-\mathrm{C}(18)$ & $1.421(2)$ \\
$\mathrm{C}(2)-\mathrm{C}(3)$ & $1 \cdot 396(3)$ & $\mathrm{C}(10)-\mathrm{C}(11)$ & $1.408(3)$ \\
$\mathrm{C}(3)-\mathrm{C}(4)$ & $1 \cdot 361(3)$ & $\mathrm{C}(11)-\mathrm{C}(12)$ & $1.365(2)$ \\
$\mathrm{C}(4)-\mathrm{C}(15)$ & $1 \cdot 425(2)$ & $\mathrm{C}(12)-\mathrm{C}(19)$ & $1.422(2)$ \\
$\mathrm{C}(5)-\mathrm{C}(6)$ & $1 \cdot 376(3)$ & $\mathrm{C}(13)-\mathrm{C}(20)$ & $1.376(2)$ \\
$\mathrm{C}(5)-\mathrm{C}(15)$ & $1.426(2)$ & $\mathrm{C}(13)-\mathrm{C}(19)$ & $1.419(2)$ \\
$\mathrm{C}(6)-\mathrm{C}(16)$ & $1.409(2)$ & $\mathrm{C}(14)-\mathrm{C}(15)$ & $1.421(2)$ \\
$\mathrm{C}(6)-\mathrm{C}(22)$ & $1.507(2)$ & $\mathrm{C}(14)-\mathrm{C}(21)$ & $1.438(2)$ \\
$\mathrm{C}(7)-\mathrm{C}(16)$ & $1.509(2)$ & $\mathrm{C}(16)-\mathrm{C}(21)$ & $1.397(2)$ \\
$\mathrm{C}(7)-\mathrm{C}(17)$ & $1.512(2)$ & $\mathrm{C}(17)-\mathrm{C}(20)$ & $1.441(2)$ \\
$\mathrm{C}(8)-\mathrm{C}(17)$ & $1.360(2)$ & $\mathrm{C}(18)-\mathrm{C}(19)$ & $1.425(2)$ \\
$\mathrm{C}(8)-\mathrm{C}(18)$ & $1.424(2)$ & $\mathrm{C}(20)-\mathrm{C}(21)$ & $1.479(2)$ \\
\hline
\end{tabular}


Table 3. Molecular non-hydrogen angles (degrees) for (15)

\begin{tabular}{llll}
\multicolumn{1}{c}{ Atoms } & \multicolumn{1}{c}{ Angle } & \multicolumn{1}{c}{ Atoms } & \multicolumn{1}{c}{ Angle } \\
\hline$C(2)-C(1)-C(14)$ & $121 \cdot 3(2)$ & $\mathrm{C}(14)-C(15)-C(5)$ & $120 \cdot 2(2)$ \\
$C(1)-C(2)-C(3)$ & $120 \cdot 4(2)$ & $C(4)-C(15)-C(5)$ & $120 \cdot 9(2)$ \\
$C(4)-C(3)-C(2)$ & $120 \cdot 3(2)$ & $C(21)-C(16)-C(6)$ & $122 \cdot 5(2)$ \\
$C(3)-C(4)-C(15)$ & $121 \cdot 1(2)$ & $C(21)-C(16)-C(7)$ & $111 \cdot 2(2)$ \\
$C(6)-C(5)-C(15)$ & $122 \cdot 1(2)$ & $C(6)-C(16)-C(7)$ & $126 \cdot 4(2)$ \\
$C(5)-C(6)-C(16)$ & $117 \cdot 8(2)$ & $C(8)-C(17)-C(20)$ & $121 \cdot 3(2)$ \\
$C(5)-C(6)-C(22)$ & $121 \cdot 9(2)$ & $C(8)-C(17)-C(7)$ & $129 \cdot 2(2)$ \\
$C(16)-C(6)-C(22)$ & $120 \cdot 3(2)$ & $C(20)-C(17)-C(7)$ & $109 \cdot 44(14)$ \\
$C(16)-C(7)-C(17)$ & $103 \cdot 04(13)$ & $C(9)-C(18)-C(8)$ & $122 \cdot 8(2)$ \\
$C(17)-C(8)-C(18)$ & $120 \cdot 6(2)$ & $C(9)-C(18)-C(19)$ & $118 \cdot 8(2)$ \\
$C(10)-C(9)-C(18)$ & $121 \cdot 0(2)$ & $C(8)-C(18)-C(19)$ & $118 \cdot 4(2)$ \\
$C(9)-C(10)-C(11)$ & $120 \cdot 4(2)$ & $C(13)-C(19)-C(12)$ & $121 \cdot 50(14)$ \\
$C(12)-C(11)-C(10)$ & $12 C \cdot 3(2)$ & $C(13)-C(19)-C(18)$ & $120 \cdot 1(2)$ \\
$C(11)-C(12)-C(19)$ & $121 \cdot 2(2)$ & $C(12)-C(19)-C(18)$ & $118 \cdot 4(2)$ \\
$C(20)-C(13)-C(19)$ & $120 \cdot 60(14)$ & $C(13)-C(20)-C(17)$ & $118 \cdot 9(2)$ \\
$C(1)-C(14)-C(15)$ & $118 \cdot 0(2)$ & $C(13)-C(20)-C(21)$ & $133 \cdot 22(14)$ \\
$C(1)-C(14)-C(21)$ & $124 \cdot 6(2)$ & $C(17)-C(20)-C(21)$ & $107 \cdot 84(13)$ \\
$C(15)-C(14)-C(21)$ & $117 \cdot 4(2)$ & $C(16)-C(21)-C(14)$ & $120 \cdot 0(2)$ \\
$C(14)-C(15)-C(4)$ & $118 \cdot 8(2)$ & $C(16)-C(21)-C(20)$ & $108 \cdot 47(14)$ \\
\hline
\end{tabular}

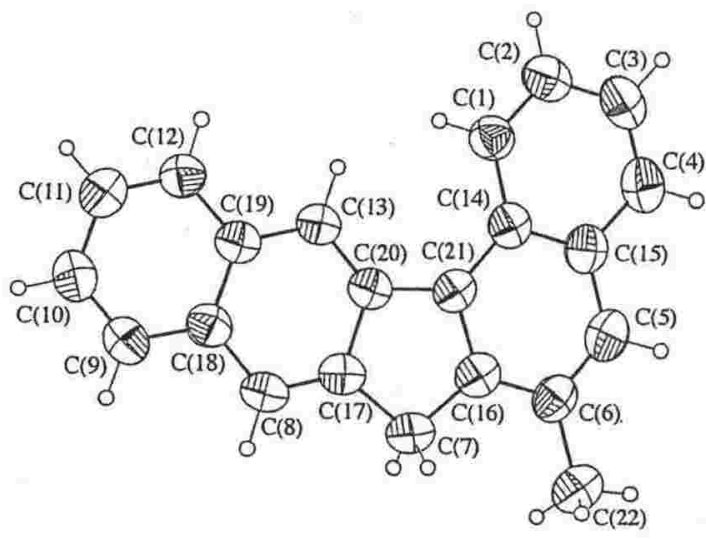

Fig. 2. Projection of (15) normal to the 'plane'; $50 \%$ thermal ellipsoids are shown for the non-hydrogen atoms, hydrogen atoms having an arbitrary radius of $0.1 \AA$.

Experiments aimed at providing other simple functionalized cycloproparenes and a better understanding of the reactivities of the cycloproparenyl anions continue to attract our attention and will form the basis of future publications.

\section{Experimental}

General conditions were as described previously. ${ }^{16}$ N.m.r. experiments were performed for $\left({ }^{2} \mathrm{H}\right)$ chloroform solutions with the chemical shifts $(\delta)$ referenced ${ }^{17}$ to the chloroform resonance ${ }^{1} \mathrm{H}, 7.26 \mathrm{ppm}$, and ${ }^{13} \mathrm{C}, 77.0 \mathrm{ppm}$, on a Varian UNITY Inova $300 \mathrm{MHz}$ instrument operating at ambient temperature. Short- and long-range ${ }^{1} \mathrm{H}-{ }^{13} \mathrm{C}$ correlations were determined with gradient-enhanced inverse-detected HSQC and iments. One-dimensional TocsY experiments were performed with shaped-pulse-selective excitation and spin lock mixing times of $15-80 \mathrm{~ms}$. Enhancements from n.O.e. experiments were detected with a GOSEY experiment with Gausian-selective excitation and a $0.5 \mathrm{~s}$ mixing time.

1-Benzoyl-1 H-cyclopropa/b/naphthalene (5)

To a stirred solution of cyclopropa[b]naphthalene (1) (141 mg, $1.0 \mathrm{mmol})$ in thf (tetrahydrofuran) $(15 \mathrm{ml})$ at $-70^{\circ} \mathrm{C}$ and under a dry nitrogen atmosphere was added butyllithium in hexane $(0.4 \mathrm{ml}, 2.5 \mathrm{M}, 1 \mathrm{mmol})$. The dark mixture was stirred for
$30 \mathrm{~min}$, and $N, N$-dimethylbenzamide ( $149 \mathrm{mg}, 1.0 \mathrm{mmol}$ ) was added under a stream of dry nitrogen. The mixture was warmed to room temperature overnight and then poured into dichloromethane/water $(60 \mathrm{ml} ; 1: 1)$. The phases were separated, the aqueous phase was extracted with dichloromethane $(3 \times 15 \mathrm{ml})$, and the combined organic solutions were dried $\left(\mathrm{MgSO}_{4}\right)$ and concentrated in vacuum to a yellow solid. Radial chromatography (dichloromethane/light petroleum elution; $1: 1)$ gave as the major component a yellow solid. Recrystallization (dichloromethane/light petroleum; $1: 1$ ) gave pale yellow plates (111 mg, 46\%) that are heat- and acidsensitive. An analytical sample of the title compound (5) (26 mg, 11\%) was obtained from further radial chromatography and recrystallization (dichloromethane, $-16^{\circ}$ ) as colourless plates that slowly turned yellow on standing, m.p. $101^{\circ}$ (dec.) (Found: $\mathrm{C}, 88 \cdot 3 ; \mathrm{H}, 4 \cdot 8 . \mathrm{C}_{18} \mathrm{H}_{12} \mathrm{O}$ requires $\mathrm{C}, 88 \cdot 5 ; \mathrm{H}, 4.9 \%$ ). $\nu_{\max } 3065,3040,3021,1684,1674,1591,1578,1522,1446$, $1323,1213,1179,1065,1009,868,816,754,723,686 \mathrm{~cm}^{-1}$. $\lambda_{\max }$ (hexane) 244 ( $\left.\log \epsilon 4 \cdot 13\right), 252 \mathrm{sh}(3 \cdot 97), 270(3 \cdot 72), 280 \mathrm{sh}$ (3.69), 290sh (3.51), 310 (3.18), $322 \mathrm{~nm}(3 \cdot 29)$. ${ }^{1} \mathrm{H}$ n.m.r. $\delta 5 \cdot 34, s, \mathrm{H} 1_{7} 7 \cdot 49-7 \cdot 53, \mathrm{BB}^{\prime}, \mathrm{H} 4 / 5 ; 7 \cdot 54-7 \cdot 59$, apparent t, $J_{\text {ortho }} 7 \cdot 3 \mathrm{~Hz}, \mathrm{H}^{\prime} / 5^{\prime} ; 7 \cdot 63-7.69$, apparent tt, $J_{\text {ortho }} 7 \cdot 3$, $1.7 \mathrm{~Hz}, \mathrm{H}_{4}^{\prime} ; 7 \cdot 78$ s, H2/7; $7 \cdot 94-7 \cdot 98, \mathrm{AA}^{\prime}, \mathrm{H} 3 / 6 ; 8 \cdot 23-$ $8.26, \mathrm{~m}, \mathrm{H} 22^{\prime} / 6^{\prime} .{ }^{13} \mathrm{C}$ n.m.r. $\delta 32.8, \mathrm{C} 1 ; 109 \cdot 0, \mathrm{C} 2 / 7 ; 118 \cdot 0$, $\mathrm{C} 1 \mathrm{a} / 7 \mathrm{a} ; 121 \cdot 5, \mathrm{C} 4 / 5 ; 124 \cdot 3, \mathrm{C}^{\prime} / 5^{\prime} ; 124.4, \mathrm{C} 3 / 6 ; 124.5$, $\mathrm{C} 2^{\prime} / 6^{\prime} ; 129 \cdot 1, \mathrm{C}^{\prime} ; 131 \cdot 5, \mathrm{Cl}^{\prime}, 132 \cdot 9, \mathrm{C} 2 \mathrm{a} / 6 \mathrm{a} ; 189 \cdot 6, \mathrm{CO}$. Mass spectrum $m / z 245$ (20\%), 244 (M, 100), 215 (33), 139 $(\mathrm{M}-\mathrm{PhCO}, 60), 105(\mathrm{PhCO}, 16), 77$ (12).

\section{1-Acetyl-1H-cyclopropa/b/naphthalene (6)}

The cycloproparenyl anion (2) was prepared from cyclopropal $b$ naphthalene (1) (500 mg, $3.6 \mathrm{mmol}$ ) and butyllithium $(2.02 \mathrm{ml}, 1.78 \mathrm{M}, 3.6 \mathrm{mmol})$ and allowed to react with $N, N$ dimethylacetamide $(0.33 \mathrm{ml}, 3.6 \mathrm{mmol})$ as described for the preparation of (5) above. Conventional workup afforded an oil which upon radial chromatography (light petroleum elution) gave unchanged cycloproparene (1) $(64 \mathrm{mg}, 13 \%)$ as the most mobile component followed by (dichloromethane/light petroleum elution; $1: 4$ ) an orange oil $(203 \mathrm{mg}$ ). Resubjection of the oil to radial chromatography and elution with the same mixture of solvents gave the acetyl compound (168 mg, 26\%) as a yellow oil. An analytical sample of compound (6) (110 mg, $17 \%$ ) was obtained as a yellow oil of limited stability from one further radial chromatography (Found: $\mathrm{C}, 84 \cdot 6 ; \mathrm{H}, 5.6$. $\mathrm{C}_{13} \mathrm{H}_{10} \mathrm{O}$ requires $\left.\mathrm{C}, 85 \cdot 7 ; \mathrm{H}, 5 \cdot 5 \%\right) . \nu_{\max } 3048,2973,1703$, $1524,1357,1253,1222,1180,1164,1140,863,748 \mathrm{~cm}^{-1} \cdot \lambda_{\max }$ (acetonitrile) 227sh (log $€ 4.57), 268(3.73), 278(3.77), 288 \mathrm{sh}$ (3.68), $308(3 \cdot 27), 322$ nm (3.31). ${ }^{1} \mathrm{H}$ n.m.r. $\delta 1.86, \mathrm{~s}, \mathrm{Me}$; $4 \cdot 52, \mathrm{~s}, \mathrm{H} 1 ; 7 \cdot 53-7 \cdot 58, \mathrm{BB}^{\prime}, \mathrm{H} 4 / 5 ; 7 \cdot 73, \mathrm{~s}, \mathrm{H} 2 / 7 ; 7 \cdot 96-7 \cdot 99$, $\mathrm{AA}^{\prime}, \mathrm{H} 3 / 6 .{ }^{13} \mathrm{C}$ n.m.r. $\delta 26 \cdot 0, \mathrm{Me} ; 40 \cdot 7, \mathrm{C} 1 ; 113 \cdot 3, \mathrm{C} 2 / 7$; $122 \cdot 3, \mathrm{C} 1 \mathrm{a} / 7 \mathrm{a} ; 126 \cdot 1, \mathrm{C} 4 / 5 ; 128 \cdot 7, \mathrm{C} 3 / 6 ; 137 \cdot 3, \mathrm{C} 2 \mathrm{a} / 6 \mathrm{a}$; $204 \cdot 0$, CO. Mass spectrum $m / z 183(9 \%), 182(\mathrm{M}, 63), 181$ (32), 153 (16), 152 (18), 140 (14), 139 (M-MeCO, 100), 63 (17), $43(\mathrm{MeCO}, 21)$.

$\mathrm{N}, \mathrm{N}, \mathrm{N}^{\prime}, \mathrm{N}^{\prime}$-Tetramethyl-1 H-cyclopropa/b/naphthalene-1,1dicarboxamide (7)

To a stirred solution of cyclopropanaphthalene (1) $(140 \mathrm{mg}$, $1.0 \mathrm{mmol})$ in the $(10 \mathrm{ml})$ at $-70^{\circ}$, and under an oxygen-free dry nitrogen atmosphere, was added butyllithium in hexane $(0.56 \mathrm{ml}, 1.77 \mathrm{M}, 1.0 \mathrm{mmol})$. The mixture was stirred for 30 $\mathrm{min}$ at $-70^{\circ}$ and then dimethylcarbamoyl chloride $(0.092 \mathrm{ml}$, $1 \mathrm{mmol}$ ) was added. The still dark mixture was allowed to warm slowly to ambient temperature whereupon conventional workup provided a pale yellow oil. Radial chromatography gave (light petroleum elution) unchanged (1) $(28 \mathrm{mg}, 20 \%)$ and the major component (ethyl acetate elution) as a pale brown solid. Recrystallization (dichloromethane, $-16^{\circ}$ ) provided light brown rectangular prisms of the title compound ( 7$)$ ( $97 \mathrm{mg}, 34 \%$ ), 
m.p. $126-130^{\circ}$ (Found: C, $72 \cdot 3 ; \mathrm{H}, 6 \cdot 4 ; \mathrm{N}, 9 \cdot 9, \mathrm{C}_{17} \mathrm{H}_{18} \mathrm{~N}_{2} \mathrm{O}_{2}$ requires $\mathrm{C}, 72 \cdot 3 ; \mathrm{H}, 6 \cdot 4 ; \mathrm{N}, 9 \cdot 9 \%) . \nu_{\max } 3004,2925,2855$, $1643,1498,1460,1390,1256,1235,1166,1136,1057,1020$ $891,839,747,696 \mathrm{~cm}^{-1} . \lambda_{\max }$ (acetonitrile) $239(\log \in 4.72)$, 244 sh (4.49), $271(3 \cdot 76), 279(3.77), 290$ sh $(3.67), 310(3.46)$, $314 \mathrm{sh}(3.42), 323 \mathrm{~nm}(3.55)$. ${ }^{1} \mathrm{H}$ n.m.r. $82.94, \mathrm{~s}, 2 \times \mathrm{Me} ; 3.67$, $\mathrm{s}, 2 \times \mathrm{Me} ; 7 \cdot 49-7 \cdot 53, \mathrm{BB}^{\prime}, \mathrm{H} 4 / 5 ; 7 \cdot 85, \mathrm{~s}, \mathrm{H} 2 / 7 ; 7 \cdot 93-7.97$, $\mathrm{AA}^{\prime}, \mathrm{H} 3 / 6 .{ }^{13} \mathrm{C}$ n.m.r. $\delta 35.9,2 \times \mathrm{Me} ; 38.2,2 \times \mathrm{Me} ; 46.6$ $\mathrm{C} 1 ; 112 \cdot 9, \mathrm{C} 2 / 7 ; 124 \cdot 3, \mathrm{Cla} / 7 \mathrm{a} ; 126 \cdot 3, \mathrm{C} 4 / 5 ; 128 \cdot 9, \mathrm{C} 3 / 6$; $137 \cdot 7, \mathrm{C} 2 \mathrm{a} / 6 \mathrm{a} ; 165 \cdot 7,2 \times \mathrm{CO}$. Mass spectrum $\mathrm{m} / z 283(16 \%)$, $282(\mathrm{M}, 82), 265(8), 239(20), 238\left(\mathrm{M}-\mathrm{NMe}_{2}, 100\right), 211(33)$, 210 (31), 196 (17), 195 (30), $182(13), 168(13), 167$ (12), 166 (13), $153(23), 152(26), 141(20), 140(20), 139(38), 127(13)$, $126(28), 72\left(\mathrm{Me}_{2} \mathrm{NCO}, 46\right), 58(60), 44(36), 43(25), 42(92)$.

Variations in the proportion of the carbamoyl chloride used (from 0.5 to 10 mol. equiv.) had little impact on the isolated yield of (7), and no evidence was obtained to support the presence of a monoamide.

\section{1-(Trimethylsilyl)- $1 \mathrm{H}$-cyclopropa/b/naphthalene (10)}

To a stirred solution of disilane (8) $(200 \mathrm{mg}, 0.7 \mathrm{mmol})$ in thf $(10 \mathrm{ml})$ at $-70^{\circ}$ under a dry nitrogen atmosphere was added potassium t-butoxide $(79 \mathrm{mg}, 0.7 \mathrm{mmol})$ in the same solvent $(10 \mathrm{ml})$. The mixture was warmed to $-43^{\circ}$ for $90 \mathrm{~min}$, and then cooled to $-70^{\circ}$ whereupon water $(1 \mathrm{ml}$, $55.6 \mathrm{mmol}$ ) was added to the dark yellow-black mixture. The now turbid and lighter coloured mixture was stirred for 2 min, poured into dichloromethane/water $(60 \mathrm{ml} ; 1: 1)$, and the phases were separated. The aqueous phase was extracted (dichloromethane, $3 \times 15 \mathrm{ml}$ ), and the combined organic phases were dried $\left(\mathrm{MgSO}_{4}\right)$ and concentrated in vacuum to a pale yellow oil. Radial chromatography (light petroleum elution) gave a yellow oil $(84 \mathrm{mg})$ as the major and most mobile component. ${ }^{1} \mathrm{H}$ n.m.r. analysis of the oil indicated a mixture of monosilane (10), cyclopropanaphthalene (1) and an unidentified component, thought to be ring-opened, in a ratio of $c .16: 5: 6$.

A sample (c. $40 \mathrm{mg}$ ) of the mixture was chromatographed over the LH-20 size exclusion gel (thf elution) to give an oil that contained two components. A second radial chromatography (hexane elution) afforded the $1 \mathrm{H}$-cycloproparene (10) as a colourless oil $(6 \cdot 2 \mathrm{mg}, 9 \%)$ that solidified at $-16^{\circ}$ and turned yellow on standing (Found: $\mathrm{M}^{\circ+} 212 \cdot 10263 . \mathrm{C}_{14} \mathrm{H}_{16} \mathrm{Si}$ requires $\left.\mathrm{M}^{*+} 212 \cdot 10213\right) . \quad \nu_{\max } 3069,3038,2955,2895,1248,1227$ $1157,1045,1020,924,916,891,860,839,743,698,671 \mathrm{~cm}^{-1}$ $\lambda_{\max }$ (hexane) $228 \mathrm{sh}(\log \epsilon 4.42), 240(4 \cdot 23), 246 \mathrm{sh}(4 \cdot 11), 262$ (3.72), $272(3 \cdot 69), 284(3 \cdot 52), 311(3 \cdot 35), 324 \mathrm{~nm}(3 \cdot 54) .{ }^{1} \mathrm{H}$ n.m.r. $\delta 0 \cdot 02, \mathrm{~s}, \mathrm{SiMe}_{3} ; 3 \cdot 23, \mathrm{t}, J<1 \mathrm{~Hz}, \mathrm{H} 1 ; 7 \cdot 33$, dd, $J<$ $1, J<0.5 \mathrm{~Hz}, \mathrm{H} 2 / 7 ; 7 \cdot 37-7 \cdot 40, \mathrm{BB}^{\prime}, \mathrm{H} 4 / 5 ; 7 \cdot 77-7.80, \mathrm{AA}^{\prime}$ H $3 / 6 .{ }^{13} \mathrm{C}$ n.m.r. $\delta-2 \cdot 4, \mathrm{SiMe}_{3} ; 24 \cdot 3, \mathrm{C} 1 ; 110 \cdot 0, \mathrm{C} 2 / 7$; $125 \cdot 0, \mathrm{C} 4 / 6 ; 127 \cdot 1, \mathrm{C} 1 \mathrm{a} / 7 \mathrm{a} ; 127 \cdot 9, \mathrm{C} 3 / 6 ; 136 \cdot 2, \mathrm{C} 2 \mathrm{a} / 6 \mathrm{a}$. Mass spectrum $m / z 213(6 \%), 212(\mathrm{M}, 26), 211(9), 198(20)$ 197 (M-Me, 100), 196 (20), 195 (78), 181 (12), 171 (20), 169 (17), $167(13), 165(10), 155(15), 154(13), 153(14), 152(12)$ $139\left(\mathrm{M}-\mathrm{SiMe}_{3}, 45\right), 89(15), 74(13), 73(77), 63(21), 59$ (12), 58 (18), $53(13), 45(62)$.

\section{1-Methyl-1-(trimethylsilyl)-1 H-cyclopropa/b/naphthalene (11)}

Anion (9) was prepared from disilane (8) $(300 \mathrm{mg}, 1.06 \mathrm{mmol})$ and potassium t-butoxide $(118.5 \mathrm{mg}, 1.06 \mathrm{mmol})$ in the same manner as described above for compound (10), and iodomethane $(0.066 \mathrm{ml}, 1.06 \mathrm{mmol})$ in thf $(0.934 \mathrm{ml})$ was added by syringe at $-70^{\circ}$. The dark-coloured mixture became turbid and orange-red in colour. The mixture was warmed to ambient temperature overnight, poured into dichloromethane/water $(60 \mathrm{ml} ; 1: 1)$, and the phases were separated. The aqueous phase was extracted with dichloromethane $(3 \times 15 \mathrm{ml})$, and the combined organic phases were washed (water, $50 \mathrm{ml}$ ), dried $\left(\mathrm{MgSO}_{4}\right)$ and concentrated in vacuum to a red oil. Radial chromatography (light petroleum elution) gave a pale yellow oil (204 mg) as the major and most mobile component that contained ( ${ }^{1} \mathrm{H}$ n.m.r.). silane (11) and cycloproparene (1) in a ratio of c. $9: 1$.

A sample of the oil $(59 \mathrm{mg})$ was subjected to chromatography over LH-20 (thf elution). Concentration of the productcontaining fraction in vaccum and resubjection productchromatography (hexane elution) gave an analytically pure sample of the title compound (11) $(32.6 \mathrm{mg}, 47 \%$ overall) as a colourless oil that solidified at $-20^{\circ}$, m.p. $46-50^{\circ}$ (Found: $\mathrm{C}, 79.7 ; \mathrm{H}, 8.3 . \mathrm{C}_{15} \mathrm{H}_{18} \mathrm{Si}$ requires $\left.\mathrm{C}, 79.6 ; \mathrm{H}, 8.0 \%\right)$. $\nu_{\max }$ $3073,3038,3030,2955,2917,2893,2859,1447,1248,870,830$, $743,692,648 \mathrm{~cm}^{-1} . \lambda_{\max }$ (hexane) $226(\log \epsilon 4.59), 264 \mathrm{sh}$ $(3.82), 272(3.75), 284(3.55), 312(3.42), 326 \mathrm{~nm}(3.55) .{ }^{1} \mathrm{H}$ n.m.r. $\delta 0.02, \mathrm{~s}, \mathrm{SiMe}_{3} ; 1.69$, s, Me; $7 \cdot 34, \mathrm{~s}, \mathrm{H} 2 / 7 ; 7 \cdot 39-7.42$, $\mathrm{BB}^{\prime}, \mathrm{H} 4 / 5 ; 7 \cdot 79-7 \cdot 82, \mathrm{AA}^{\prime}, \mathrm{H} 3 / 6 .{ }^{13} \mathrm{C}$ n.m.r. $\delta-3 \cdot 3, \mathrm{SiMe}_{3}$; $22.8, \mathrm{Me} ; 31.9, \mathrm{C}_{1} ; 109.9, \mathrm{C} 2 / 7 ; 124.9, \mathrm{C} 4 / 5 ; 128.0, \mathrm{C} 3 / 6$; $134 \cdot 2, \mathrm{C} 1 \mathrm{a} / 7 \mathrm{a} ; 135 \cdot 9, \mathrm{C} 2 \mathrm{a} / 6 \mathrm{a}$. Mass spectrum $\mathrm{m} / \mathrm{z} 227(4 \%)$, $226(\mathrm{M}, 20), 212(21), 211(\mathrm{M}-\mathrm{Me}, 100), 210$ (10), $209(34)$, 195 (41), $185(23), 183(21), 171(15), 167(13), 165(24), 155$ (11), 153 (M- $\left.\mathrm{SiMe}_{3}, 24\right), 152$ (34), 151 (13), 74 (10), 73 (64), $59(56), 45(42), 43(24)$.

\section{6-Methyl-7H-dibenzo/b,g/fluorene (15)}

Anion (9) was prepared from disilane (8) $(100 \mathrm{mg}, 0.35 \mathrm{mmol})$ and potassium t-butoxide $(40 \mathrm{mg}, 0.35 \mathrm{mmol}$ ) in the same manner as described above for compound (10). After return of the temperature to $-70^{\circ}$ one of the procedures (A)-(c) below was followed.

(A) From addition of toluene. Dry toluene $(0.04 \mathrm{ml}$, $0.35 \mathrm{mmol}$ ) was added by syringe and the dark mixture was allowed to attain room temperature over $16 \mathrm{~h}$. The now red mixture was decanted into dichloromethane/water $(60 \mathrm{ml}$; $1: 1$ ), the phases were separated and the aqueous phase was reextracted with dichloromethane $(3 \times 25 \mathrm{ml})$. The combined organic layers were washed (water, $50 \mathrm{ml}$ ), dried $\left(\mathrm{MgSO}_{4}\right)$ and concentrated in vacuum to a bright orange oil. Radial chromatography (light petroleum elution) gave as the most mobile fraction a yellow oily solid $(10.2 \mathrm{mg})$ shown (n.m.r.) to contain monosilane (10), cyclopropanaphthalene (1) and an unidentified component in a ratio of c. $53: 10: 5$. Continued elution over c. 20-30 min. provided a further component as a solid $(20.4 \mathrm{mg})$ that was markedly fluorescent on ultraviolet irradiation. Recrystallization (dichloromethane/light petroleum; $1: 1$ ) at $-20^{\circ}$ afforded the fluorene (15) $(5.9 \mathrm{mg}, 6 \%)$-see below.

(B) From addition of N,N-dimethylacetamide. $N, N$ Dimethylacetamide $(0.033 \mathrm{ml}, 0.35 \mathrm{mmol})$ in the $(1 \mathrm{ml})$ was added and the mixture allowed to attain room temperature slowly overnight. Workup as described in method (A) above gave a dark red-brown oil. Radial chromatography (light petroleum elution) gave as the most mobile component $1 \mathrm{H}$ cyclopropa $[b]$ naphthalene $(1)$ as a white solid (9.6 mg, 20\%). Further elution and recrystallization of the resultant yellow solid as in (A) above afforded compound (15) (17.7 mg, 18\%). Repetitions of the reaction with differing amounts of (8) provided (15) in yields that ranged from 4 to $18 \%$.

The product samples were combined, dissolved in dichloromethane/hexane $(1: 1)$, and recrystallized by solvent diffusion in a sealed container containing hexane to afford the title fluorene (15) as straw-coloured blocks suitable for analysis and crystal structure determination, m.p. $174^{\circ}$ (sublimes), 212-213 (sublimate melts) (Found: $\mathrm{C}, 94 \cdot 3 ; \mathrm{H}, 5 \cdot 7 . \mathrm{C}_{22} \mathrm{H}_{16}$ requires $\mathrm{C}$, $94 \cdot 3 ; \mathrm{H}, 5 \cdot 7 \%) . \nu_{\max } 3050,3019,2918,2878,1686,1516,1425$, $1414,1370,1213,1024,951,934,862,762,741,650 \mathrm{~cm}^{-1}$ $\lambda_{\max }$ (acetonitrile) $249(\log \epsilon 4.70), 254 \operatorname{sh}(4.63), 269(3.89)$ 280 (4.02), $292(4 \cdot 07), 316 \operatorname{sh}(4 \cdot 13), 328(4 \cdot 27), 335(4 \cdot 24)$, 
$344(4 \cdot 26), 350 \mathrm{~nm}(4 \cdot 38) .{ }^{1} \mathrm{H}$ n.m.r. $\dagger \delta 2 \cdot 59$, d, $J$ c. $1 \mathrm{~Hz}$, Me; $4.06, \mathrm{~s}, \mathrm{CH}_{2} ; 7 \cdot 44-7 \cdot 57, \mathrm{~m}, 3 \mathrm{H}, \mathrm{H} 3 / 10 / 11 ; 7 \cdot 66$; d, J c. $1 \mathrm{~Hz}, \mathrm{H} 5 ; 7.66$; apparent dt, J 7.5, $1.3 \mathrm{~Hz}, \mathrm{H} 2 ; 7.87$, , d, c. $9.2 \mathrm{~Hz}, \mathrm{H} 9 ; 7.91,{ }^{\circ}$ apparent dd, $J 8.2,2.8 \mathrm{~Hz}, \mathrm{H} 4 ; 8.03$," d, $J<1 \mathrm{~Hz}, \mathrm{H} 8$; 8.04, apparent dd, $J 6 \cdot 7,2.4 \mathrm{~Hz}, \mathrm{H} 12$; 8.77 , broadened $\mathrm{s}, \mathrm{H} 13 ; 8.90, \mathrm{~d}, \mathrm{~J} 8.1 \mathrm{~Hz}, \mathrm{H} 1 .{ }^{13} \mathrm{C}$ n.m.r. $\delta$ $19.4, \mathrm{Me} ; 36.6, \mathrm{CH}_{2} ; 121.1, \mathrm{C} 13 ; 122.9, \mathrm{C} 8 ; 123.6, \mathrm{C} 12$ or $\mathrm{C} 1 ; 125 \cdot 2, \mathrm{C} 10$ or $\mathrm{C} 3 ; 125 \cdot 3, \mathrm{C} 3$ or $\mathrm{C} 10 ; 125 \cdot 4, \mathrm{C} 2$ or C 11 ; $125.9, \mathrm{C} 11$ or $\mathrm{C} 2 ; 127 \cdot 4, \mathrm{C} 4$ or $\mathrm{C} 9 ; 127.9, \mathrm{C} 5 ; 128.5(2), \mathrm{C} 1$ or C $12 ; 128 \cdot 5(6), \mathrm{C} 9$ or C $4 ; 128 \cdot 4(6), 132 \cdot 0,132 \cdot 5,133 \cdot 2$, $134 \cdot 0,135 \cdot 0,141 \cdot 7(8), 141 \cdot 8(4), 143 \cdot 6$, all quaternary. Mass spectrum $\mathrm{m} / \mathrm{z} 281(21 \%), 280(\mathrm{M}, 100), 279(27), 278(12)$, $276(14), 266(14), 265(\mathrm{M}-\mathrm{Me}, 64), 263(10), 140(15), 139$ (17) 138 (17), 133 (29), $132(17), 126(19), 125$ (11).

(c) From addition of $1 \mathrm{H}$-cyclopropa/b/naphthalene (1). Cycloproparene (1) $(49 \mathrm{mg}, 0.35 \mathrm{mmol})$ in the $(4 \mathrm{ml})$ was added and the dark mixture warmed to room temperature overnight. Workup and radial chromatography as described above returned compound (1) $(1.1 \mathrm{mg}, 2 \%)$ as the most mobile component and the fluorene (15) $(36.4 \mathrm{mg})$ as the second component. Recrystallization (dichloromethane/light petroleum; $1: 1)$ at $-16^{\circ}$ gave the dibenzo $[b, g]$ fluorene $(15)(28 \mathrm{mg}, 28 \%)$ as pale straw-coloured needles, m.p. $178^{\circ}$ (sublimes), and identical to the samples described above.

\section{Crystal Structure Determination of (15)}

A room-temperature area detector diffractometer data set was measured on a Siemens SMART-CCD diffractometer (monochromatized Mo $\mathrm{K} \alpha$ radiation, $\lambda \quad 0.7107_{3} \AA$; temperature $298 \mathrm{~K}$ ). Data collection was of 12165 intensities, 3052 independent $\left(R_{\text {merg }}=0.0272,1.69^{\circ}<\theta<28.27^{\circ}\right), 2193$ observed $\left[F_{0}\right.$ $\left(R_{\text {marg }}=0.0272,1.69^{\circ}<\theta<\right.$,
$\geq 4 \sigma(F)]$ absorption correction with Siemens (Madison, Wis consin, U.S.A., 1998) SADABS program: $R_{\text {merg }}$ before/after $0.0315 / 0.0244$, maximum/minimum transmission $1.00 / 0.81$. Structure solution was with direct methods and refinement on $F^{2}$ (shelXTL 5.03, Siemens, Madison, Wisconsin, U.S.A.) (199 parameters). The hydrogen atom positions were calculated and refined as riding groups with the $1 \cdot 2$-fold $U_{\text {(iso) }}(1 \cdot 5$-fold for the methyl group) to that for the equivalent atomic displacement parameter of the corresponding carbon atom. Methyl hydrogen atoms were taken from a difference-Fourier map, idealized and also refined as a riding group. $R_{1}=0.0544, w R_{2}=0.1501$ (all data) $w^{-1}=\sigma^{2}\left(F_{\mathrm{o}}{ }^{2}\right)+(0.074 P)^{2}+0.221 P$, where $P=$ $\left[\max \left(F_{\mathrm{o}}{ }^{2}\right)+\left(2 F_{\mathrm{c}}{ }^{2}\right)\right] / 3$; the maximum residual electron density was 0.242 e $\AA^{-3}$. Pertinent data are given in Fig. 2 and Tables $1-3$, and the material deposited comprises hydrogen and displacement parameters and structure factor amplitudes.

Crystal data. $\mathrm{C}_{22} \mathrm{H}_{16}$. Monoclinic crystal system, space group $P 2_{1} / n, a 12.6005(2), b 5.8620(1), c 20.4002(3) \AA, \beta$ $102 \cdot 330(1)^{0}, V 1472 \cdot 09(4) \AA^{3} . D_{\mathrm{c}}(Z=4) 1 \cdot 265 \mathrm{~g} \mathrm{~cm}^{-3}, \mu$ $0.071 \mathrm{~mm}^{-1}$; crystal dimensions 0.26 by 0.21 by $0.16 \mathrm{~mm}$.

\section{Acknowledgments}

We are indebted to $\mathrm{Mr} \mathrm{D}$. Bläser for assistance with the X-ray structure analysis. Generous support of this work in New Zealand from the Victoria University Science Faculty Grants Committee, the NZ Lotteries Grants Board-Science Distribution Committee, and the Curtis-Gordon Scholarship Committee (to C.S.J.), and in Germany from Fonds der Chemischen Industrie is gratefully acknowledged.

\section{References}

${ }^{1}$ Halton, B., and Stang, P. J., Synlett, 1997, 145.

2 Müller, P., 'Cycloproparenes' in 'Carbocyclic Threemembered Ring Compounds' (Ed. A. de Meijere), HoubenWeyl Methoden Organischen Chemie, Vol. E/17d (Georg Thieme: Stuttgart 1997).

3 Halton, B., 'Cycloproparenes' in 'The Chemistry of the Cyclopropyl Group' (Ed. Z. Rappoport) Vol. 3, p. 707 (John Wiley: Chichester 1995).

4 Müller, P., 'Chemistry of Cycloproparenes' in 'Advances in Theoretically Interesting Molecules' (Ed. R. P. Thummel)

Vol. 3, p. 37 (JAI Press: Greenwich, Connecticut, 1995).

5 Halton, B., Chem. Rev., 1989, 89, 1161.

6 Cutler, C. A., and Halton, B., Aust. J. Chem., 1997, 50, 267.

${ }^{7}$ Halton, B., and Jones, C. S., J. Chem. Soc., Perkin Trans. 2, 1998, 2505.

8 Halton, B., Cooney, M. J., Boese, R., and Maulitz, A. H., J. Org. Chem., 1998, 63, 1583.

9 Halton, B., Cooney, M. J., Davey, T. W., Forman, G. S., Lu, Q., Boese, R., Bläser, D., and Maulitz, A. H., J. Chem. Soc., Perkin Trans. 1, 1995, 2819.

10 Halton, B., Kay, A. J., Zhi-mei, Z., Boese, R., and Haumann, T., J. Chem. Soc., Perkin Trans. 1, 1996, 1445.

11 Halton, B., Randall, C. J., Gainsford, G. J., and Stang, P. J., J. Am. Chem. Soc., 1986, 108, 5949.

12 McNichols, A. T., Stang, P. J., Addington, D. M., and Halton, B., Tetrahedron Lett., 1994, 35, 437.

13 Régimbald, M., and Wentrup, C., J. Org. Chem., 1998, 63, 8417. See also: Harvey, R. G., Pataki, J., Cortez, C., Di Raddo, P., and Yang, C.-X., J. Org. Chem., 1991, 56, 1210.

14 Thomas, R., Environ. Sci. Technol., 1983, 17, 666 (Chem. Abstr., 1983, 99, 145276g).

15 Dean, F. M., Houghton, L. E., Nayyir-Mashir, R., and Thektaranonth, C., J. Chem. Soc., Perkin Trans. 1, 1980, 1994.

${ }_{16}$ Cooney, M. J., and Halton, B., Aust. J. Chem., 1996, 49, 533.

17 Gottlieb, H. E., Kotlyar, V., and Nudelman, A., J. Org. Chem., 1997, 62, 7512 . † Asterisks $\left(^{*}\right)$ indicate that chemical shifts, apparent peak multiplicities and coupling constants were obtained from HoMO-2D.
experiments and not from expanded ${ }^{1} \mathrm{H}$ n.m.r. spectra. the Australian Journal of Chemistry, P.O. Box 1139, Collingwood, Vic. 3066 (until December 2004).

\section{hitp://www.publish.csiro.aujoumals/ajc}




\title{
Studies in the cycloproparene series: chemistry of 1-acyl- $1 H$-cyclopropa[b]naphthalenes and synthesis of cyclopropa $[b]$ naphthalenylidene enol ethers
}

\author{
Brian Halton, ${ }^{\mathrm{a}, *}$ Carissa S. Jones ${ }^{\mathrm{a}}$ and Davor Margetic ${ }^{\mathrm{b}}$ \\ "School of Chemical and Physical Sciences, Victoria University of Wellington, P.O. Box 600, Wellington, New Zealand \\ ${ }^{b}$ Centre for Molecular Architecture, Central Queensland University, Rockhampton, Queensland 4702, Australia
}

Received 12 September 2000; accepted 16 November 2000

\begin{abstract}
The $1 H$-cyclopropa $[b]$ naphthalenyl anion reacts with $N, N$-dimethylamides to provide $\mathrm{C}-1$ acyl derivatives in good yield in what are rare examples of mono-substitution in the cycloproparene series. The availability of these acylcycloproparenes provides for subsequent benzylic proton abstraction and enolate ion interception to give $O$-silyl and $O$-phosphinate derivatives. This reaction sequence lends to first cyclopropa $[b]$ naphthalenylidene enol ethers and provides the only example of exocyclic alkene formation taking place in one step from the benzylic centre. Protonation of the acyl derivatives by mineral acid triggers three-membered ring opening to 2,3-disubstituted naphthaa naphthofuran occurs. (c) 2001
\end{abstract}

Although the chemistry of the cycloproparenes, e.g. 1, has received significant attention over the past years, ${ }^{1-6}$ the number of examples of compounds carrying a single $\mathrm{C}-1$ substituent are remarkably few. Thus while Eaborn and co-workers $^{7,8}$ were able to isolate the monosilyl cyclopropabenzene derivative $3 \mathbf{a}$, all attempts to obtain the equivalent naphthalene analogue $3 \mathrm{~b}$ led to the disilane $5 \mathrm{~b}$ instead (Scheme 1). ${ }^{6,9,10}$ Base-induced proton abstraction of $3 \mathrm{a}$ and desilylation of $5 \mathrm{a}$ and $5 \mathrm{~b}$ gives the silicon-stabilized anions $4 \mathrm{a}$ and $4 \mathrm{~b}$ that have been used in a range of Peterson olefinations to provide $\mathrm{C}-1$ exocyclic alkenes 6 (alkylidenecycloproparenes) in what has become the major pathway to this class of compounds. ${ }^{6.11-13}$ An alternative route to such compounds ${ }^{14}$ involves trapping $4 \mathrm{~b}$ with a modified carbonyl

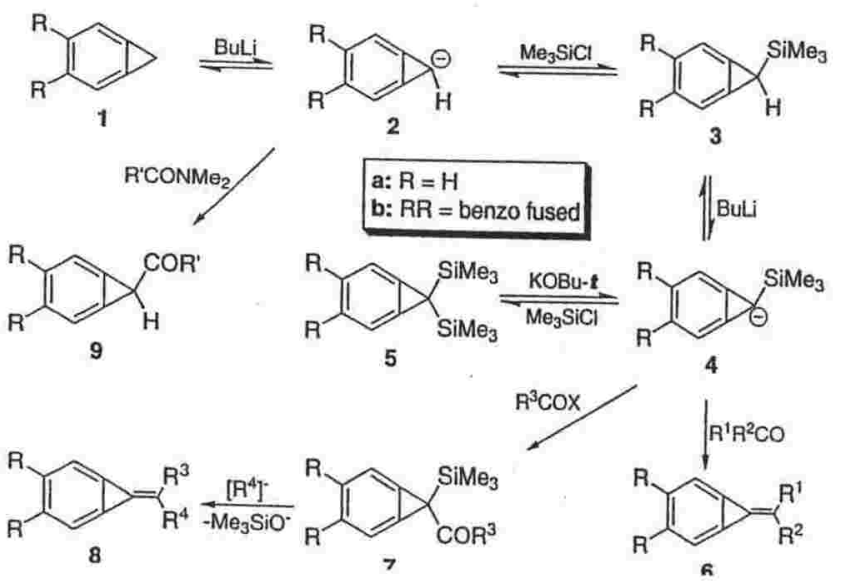

Scheme 1. Keywords: arenes; alkenes; bicyclic aromatics; carbonyl compounds; enol ethers; enolates; fulvenes; molecular modelling; naphthalenes; phosphonic acid
ester; rearrangements; silicon compounds; strained compounds. 


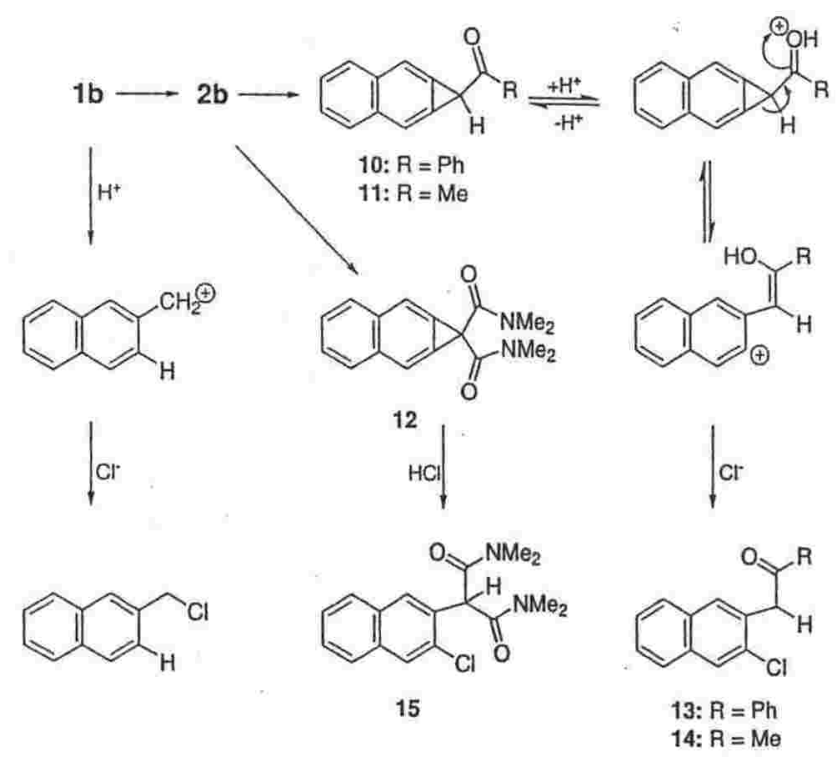

Scheme 2.

compound to give a 1-acyl-1-trimethylsilyl derivative $7 \mathbf{b}$ which, in turn, reacts with an appropriate nucleophile at the carbonyl carbon atom to induce deoxysilylation and formation of alkene $\mathbf{8 b}$.

The capture of $\mathbf{2}$ or $\mathbf{4}$ with electrophilic species other than those described above has proved more difficult. The studies have attracted less attention but they have shown that the chemistry of the C-1 cyclopropanaphthalenyl anion is frustrated by its fast protonation and by the formation of monosubstituted derivatives that are difficult to isolate. ${ }^{15-17}$ Nonetheless, monosilyl $\mathbf{3 b}$ has been isolated from the desilylation of $\mathbf{5 b}$ and protonation of $\mathbf{4 b}$, although particular care was needed. ${ }^{15} \mathrm{~A}$ modification of the reaction procedure from 1 b also provided ${ }^{15} 1$-acetyl and 1-benzoyl (and 1,1dicarboxamido) derivatives, cf. $9 \mathrm{~b}$, via the interception of $\mathbf{2 b}$ (Scheme 2). It is the chemistry of these last compounds that forms the basis of the present paper. The location of electron withdrawing functionality adjacent to the cycloproparenyl C-1 centre in 9 opens up the possibility of chemistry in which the electron demand is reversed from that normally observed in the electrophilic opening of the cycloproparene three-membered ring; this last path is illustrated for the formation of 2-(chloromethyl)naphthalene from $1 \mathrm{~b}$ in Scheme 2. ${ }^{1-4}$ Furthermore, the presence of a benzylic $\alpha$ hydrogen atom in 9 augurs well for enolate ion formation from C-1 with direct formation of an exocyclic olefin, while the location of the carbonyl group suggests that thermolysis and/or photolysis will provide naphtho[b]furans from cleavage of the three-membered ring. ${ }^{18-20}$ We report herein on the ring opening reactions of 1-acylcyclopropa $[b]$ naphthalenes and on the formation and characterization of cycloproparenylidene enol ethers.

J-Benzoyl- (10), 1-acetylcyclopropa[b]naphthalene (11), and the dicarboxamide derivative 12 were prepared from Ib in moderate yields, as described previously. ${ }^{15}$ On treat- ment with $\mathrm{HCl}$ these compounds undergo easy ring opening but do not provide the monosubstituted arenes expected on the basis of typical protonation of the cycloproparene $\pi$ framework (Scheme 2). ${ }^{21-23}$ Rather, it is the electrophilic carbonyl group that is protonated, thereby facilitating cleavage of the three-membered ring which, in turn, requires capture of the nucleophile at the arene site; the 2-chloro-3[(substituted)methyl]naphthalenes $13-15$ are formed in yields of $60-74 \%$. Although $2^{\prime}$-chlorophenylpropan-2one $^{24-26}$ and 2-(2'-chlorophenyl)-1-phenylethanone ${ }^{27,28}$ are known, the naphthalene analogues $13-15$ have not been recorded previously. Their structures are assigned with confidence from the spectroscopic data (Section 1) and especially the appearance of broadened singlets for the (now) non-equivalent para protons $\mathrm{H}-1$ ( $7.70-7.85 \mathrm{ppm})$ and $\mathrm{H}-4$ (7.88-7.93 ppm), with their corresponding carbon resonances in the ranges $130.2-130.6$ and 127.4$127.7 \mathrm{ppm}$, respectively. The benzylic protons for 13 and 14 appear at 4.59 and $4.00 \mathrm{ppm}$ whereas the tertiary $\mathrm{ArCH}(\mathrm{CONMe})_{2}$ of 15 is at $5.56 \mathrm{ppm}$. The methyl groups of the bis(dimethylamido) derivative 15 appear as two sixproton singlets at 2.94 and $3.02 \mathrm{ppm}$, respectively, as is expected on the basis of restricted rotation about such $\mathrm{CO}-\mathrm{N}$ bonds. ${ }^{29-32}$

In contrast to the behaviour described above, treatment of the cycloproparenes $\mathbf{1 0}$ and $\mathbf{1 1}$ with base (BuLi) is expected to effect the removal of the benzylic proton $\mathrm{H}-1$ and generate the enolate anions 16 and 17 (Scheme 3). As enolates can be captured by electrophiles at both carbon and oxygen, we sought to effect the latter since this would provide new and novel alkylidenecycloproparenes generated for the first time in one step from the cycloproparene simply by proton removal at C-1. As discussed above, the conventional method for preparation of methylidenecycloproparenes is by Peterson olefination from the silyl derivatives employing a carbonyl-containing 
<smiles>O=CCc1ccc2ccccc2c1</smiles>

10: $\mathrm{R}^{1}=\mathrm{Ph}$

11: $\mathrm{R}^{1}=\mathrm{Me}$<smiles>C[In]</smiles><smiles></smiles><smiles>CCC=C1C(=CO)c2cc3ccccc3cc21</smiles>

16: $\mathrm{R}^{1}=\mathrm{Ph}$

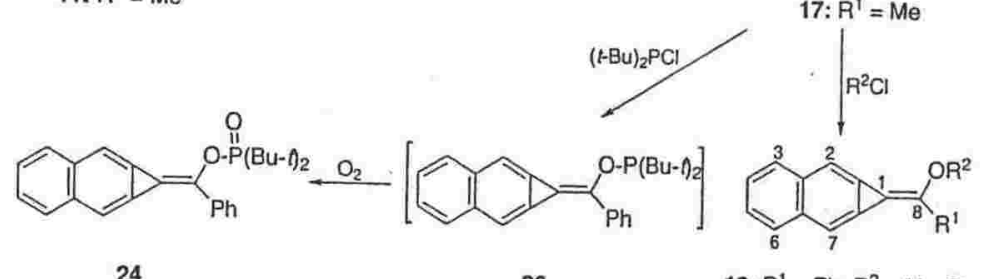

Scheme 3.

$$
\begin{aligned}
& \text { 18: } R^{1}=\mathrm{Ph} ; R^{2}=\mathrm{Me}_{3} \mathrm{Si} \\
& \text { 19: } R^{1}=\mathrm{Ph} ; R^{2}=t-\mathrm{BuMe}_{2} \mathrm{Si} \\
& \text { 20: } R^{1}=\mathrm{Ph} ; R^{2}=t-\mathrm{BuPh}_{2} \mathrm{Si} \\
& \text { 21: } R^{\prime}=\mathrm{Me} ; R^{2}=t-\mathrm{BuMe}_{2} \mathrm{Si} \\
& 22: R^{1}=\mathrm{Me} ; R^{2}=t-\mathrm{BuPh}_{2} \mathrm{Si}
\end{aligned}
$$

compound and it has yielded almost one hundred exocyclic alkene derivatives. ${ }^{6,10,33}$ In the event, use of the base with 10 followed by addition of chlorotrimethylsilane led to an unstable solid that provided spectroscopic data compatible with enol ether 18. In particular, the C-1 benzylic proton of $10(5.34 \mathrm{ppm})$ was absent, the formerly equivalent $\mathrm{H}-2 / 7$ $(7.78 \mathrm{ppm})$ were non-equivalent and shifted to 7.31 and $7.41 \mathrm{ppm}$ and the protons of the new trimethylsiloxy group appeared at $0.46 \mathrm{ppm}$. The ${ }^{13} \mathrm{C}$ NMR spectrum showed the absence of the carbonyl carbon of the substrate $10(189.6 \mathrm{ppm})$ but provided characteristically shielded and
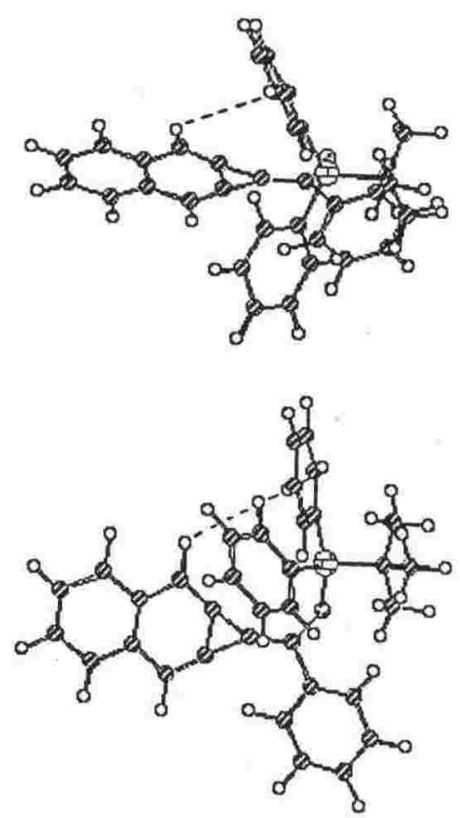

Figure 1. Two views of the favoured conformation of siloxyalkene 20 showing the shielding of $\mathrm{H}-2$ by an $\mathrm{Si}-\mathrm{Ph}$ group; the distunce $\mathrm{H}-2$ to ring centre is $411 \mathrm{ppm}$. distinct signals for $\mathrm{C}-2$ ( $Z$ with respect to the silyloxy group) and C-7 ( $E$ with respect to the silyloxy group) of 18 at 104.2 and $104.9 \mathrm{ppm}$, respectively, that provide adequate proof for retention of the cyclopropanaphthalene framework. ${ }^{1-6,11,12}$ In addition, a shielded C-1 olefinic resonance was recorded at $96.0 \mathrm{ppm}$ and the trimethylsiloxy carbons resonated at $0.90 \mathrm{ppm}$. Unfortunately all attempts to isolate the pure compound resulted in its decomposition, likely from traces of water, as it is well known that trimethylsilyl enol ethers are very sensitive to it and to acid..$^{34}$

By employing sterically more demanding $t$-butyldimethyland $t$-butyldiphenyl-chlorosilanes with $\mathbf{1 0}$ the siloxyalkenes 19 and 20 were isolated in yields of 57 and $60 \%$, respectively; the acetyl derivative $\mathbf{1 1}$ is likewise transformed into enol ethers 21 and 22 but they are markedly less stable (Section 1). The structures of these methylidenecyclopropanaphthalenes follow from their spectroscopic data. The bright yellow $t$-butyldimethyl derivative 19 displays methyl proton resonances at $\delta 0.48\left(\mathrm{OSiMe}_{2}\right)$ and $1.19(\mathrm{OSiBu}-t)$ while the C-8 silyl ether and phenyl substituents cause nonequivalence in $\mathrm{H}-2 / 7$ which appear at $\delta 7.29 / 7.40$; the corresponding carbon resonances are at $104.1 / 104.9$ while C-1 and C-8 appear at 95.9 and $135.8 \mathrm{ppm}$, respectively. The data recorded for the yellow $t$-butyldiphenyl derivative 20 have many similarities and show an OSiBu- $t(\delta$ 1.30) and shielded and deshielded olefinic carbons (C-1 and C-8: $\delta$ 97.5 and 136.9), but it is the non-equivalence of $\mathrm{H}-2$ and $\mathrm{H}-7$ that shows the most dramatic change; $\mathrm{H}-2$ ( $\mathrm{Z}$ to the $\mathrm{O}$ atom) resonates $1.5 \mathrm{ppm}$ to higher field than $\mathrm{H}-7$ ( $\delta 5.78 \mathrm{vs}$ 7.27). The $\mathrm{C}-2 / 7$ resonances differ in chemical shift by $1 \mathrm{ppm}(\delta$ 104.9/103.9), a value somewhat larger than normal. ${ }^{6}$ Because of the large chemical shift difference between $\mathrm{H}-2$ and $\mathrm{H}-7, \mathrm{HMBC}$ correlations for the assignment of H-3 and H-6 (and their respective carbon resonances) are straightforward (Section L). It is abundantly clear that the dramatic difference in the environment of $\mathrm{H}-2$ and $\mathrm{H}-7$ in $\mathbf{2 0}$ is a result of the sterically very demanding diphenyl( $($-butyl)silyl group. Semiempirical calculations at the restricted Hartree-Fock level using the AM1 


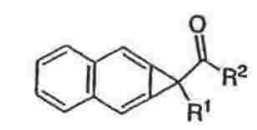

10: $R^{1}=H ; R^{2}=P h$

11: $R^{1}=H_{;} R^{2}=M e$

12: $R^{1}=\mathrm{CONMe} ; R^{2}=\mathrm{NMe}_{2}$
Scheme 4.

method ${ }^{35,36}$ place $\mathrm{H}-2$ in the shielding cone of one of the silicon phenyl substituents in the most favoured conformer $\left(546 \mathrm{~kJ} \mathrm{~mol}^{-1}\right)$, as shown in Fig. 1. The distance from the centre of this pendant phenyl ring to $\mathrm{H}-2$ is $411 \mathrm{ppm}$ and it is twisted $70^{\circ}$ with respect to the plane carrying the cycloproparenyl moiety. The range of isomers derived from rotations about the $\mathrm{C}-8-\mathrm{O}, \mathrm{O}-\mathrm{Si}$, and $\mathrm{Si}-\mathrm{C}_{i p s o}$ bonds of this flexible molecule all fall within ca. $12 \mathrm{~kJ} \mathrm{~mol}^{-1}$. The electronic absorption spectra of these brightly coloured compounds show little solvatochromy between hexane and acetonitrile but there seems little doubt that the molecules typify the polar alkylidenecycloproparenes and have charge separation. ${ }^{6}$ The assignment of structure to the analogous, though sterically somewhat less crowded, $t$-butyldimethylsilyl eno ether $\mathbf{2 1}$ from the acetyl-substituted $\mathbf{1 1}$ follows by analogy but the compound undergoes easy oxidation during attempted purification. In contrast, the more bulky diphenyl derivative $22\left(\delta_{\mathrm{H}} 5.99, \mathrm{H}-2 ; 7.04, \mathrm{H}-7\right)$ is formed in only low yield and could not be obtained pure prior to its decom position. It seems likely that the presence of the enolizable $\mathrm{MeCO}$-centre also provides for alternative, competing reaction pathways.

Despite the drawbacks associated with acetylcycloproparene 11, di-t-butylchlorophosphine intercepts phenyl substituted enolate 16. Work-up in a manner analogous to that employed for the siloxy ethers 19 and 20 provides product, but it is not the phosphinous acid ester 23 that is isolated. Rather the phosphonic acid ester 24 is the bright yellow solid obtained and its formation is presumed to result from aerial oxidation ${ }^{37}$ of initially formed 23 . The two $t$-butyl groups exhibit a phosphorus-coupled proton doublet $\left({ }^{3} J_{\mathrm{P}-\mathrm{H}}=14.7 \mathrm{~Hz}\right)$ in the ' $\mathrm{H}$ NMR spectrum and the $\mathrm{C}-\mathrm{H}(2 / 7)$ groups appear at $\delta 7.53 / 106.6$ and $7.65 / 108.6$; the phosphorus resonance is at $68.6 \mathrm{ppm}$.

Upon thermolysis and/or photolysis 1,1-diester-substituted cycloproparenes are known to provide benzofurans from ring expansion involving the carbonyl group. ${ }^{18-20}$ Comparable reactions of the acylcycloproparenes 10-12 described herein, therefore, should result in novel and new naphtho[b]furans (Scheme 4). This is borne out from refluxing a solution of the diamide 12 in benzene. Rearrangement occurs to give the aminofuran 28 quantitatively (Section 1). At ambient temperature rotation about the $\mathrm{CO}-\mathrm{N}$ bond ${ }^{29-32}$. in this compound is comparable with the time-scale of NMR measurement in $d$-chloroform solution as the CONMe protons appear as a broad singlet $(\delta 3.17)$ but the resonance of the methyl carbons is not discernible above the baseline. HSQC and HMBC experiments allow for the location chemical shift at $\delta \mathrm{ca}$. 35.2 . At $-20^{\circ} \mathrm{C}$ rotation is slowed and the signals are clearly evident in the expected $1: 1$ ratios
$\left(\delta_{\mathrm{H}} 3.18\right.$ and $3.19 ; \delta_{\mathrm{C}} 35.2$ and 39.3$)$. In the same way, the $\mathrm{C}-1$ acetyl derivative 11 provides the known ${ }^{38,39}$ analogous product 27 but the yield is only $20 \%$. With this and the phenyl substituted substrate 10, added scope likely exists for alternative reactions at least formally through diradical
25 dimerizations ${ }^{40,41}$ and involvement of the C-8 phenyl group of 10 through radical insertions ${ }^{42-44}$ become relevant. It is not surprisingly, therefore, that the 2-phenylnaphthofuran 26 is isolated in low (7\%) yield but it proved to be the only identifiable product from a multi-component mixture formed upon thermolysis of 10 at $80^{\circ} \mathrm{C}$. In contrast, reaction in the solid state at $101^{\circ} \mathrm{C}$ gives some $17 \%$ of 26 while the photo-rearrangement $(254 \mathrm{~nm})$ provides the same product in a meagre $5 \%$ yield.

In conclusion, we have demonstrated that the novel $\mathrm{C}-1$ acylcycloproparenes 10-12 undergo ring opening in an acid medium. The ortho-disubstituted products are formed in reactions triggered by enol formation. This contrasts with electrophilic addition to the aromatic $\pi$ framework and opening to a benzylic cation as observed in simpler cyclo proparenes. The formation observed in simpler cycloproparenes. The formation of enolate ion upon base removal of the C-1 hydrogen atom has been demonstrated from trapping with sterically congested chlorosilanes. This has provided the first example of cycloproparenylidene enol ethers whose ultraviolet absorptions are compatible with extended conjugation and 'push-pull' character within the molecules. The thermal behaviour of these acyl derivatives compares well with that of C-1 esters reported some 30 years ago and has provided new and novel naphthofurans. We continue to explore the chemistry of the cycloproparene framework and in particular the development of more extended conjugation through less common functionality
at C-1.

\section{Experimental}

\subsection{General}

Microanalyses were performed by the Analytical Facility of Otago University, Dunedin. Accurate mass measurements were recorded by $\mathrm{Mr} \mathrm{O}$. Zubkov on a PE Biosystems Mariner spectrometer operating in electrospray mode except where stated otherwise. ${ }^{1} \mathrm{H}$ and ${ }^{13} \mathrm{C} N \mathrm{NMR}$ spectra were recorded on a Varian Unity INOVA $300 \mathrm{MHz}$ instrument for $d$-chloroform solutions using the residual solvent peak as internal standard; the ${ }^{31}$ P NMR spectrum (same instrument) used the $P$ atom of $10 \%$ phosphoric acid in $D_{2} \mathrm{O}$ as $0.00 \mathrm{ppm}$. NMR multiplicities are defined by the usual notation and coupling constants are in hertz. The assignments of ${ }^{13} \mathrm{C}$ and ${ }^{1} \mathrm{H}$ NMR resonances were made with the aid of 
DEPT and ' $\mathrm{H}-{ }^{\prime} \mathrm{H}$ COSY and ${ }^{13} \mathrm{C}-{ }^{\prime} \mathrm{H}$ HSQC experiments, and heteronuclear multiple bond connectivity (HMBC) for $\mathrm{KBr}$ disks IR spectra of solid samples were recorded UV $\mathrm{KBr}$ disks using a Biorad FTS 7 spectrophotometer. 8452A direments were acquired from a Hewlett-Packard determinode array spectrophotometer. Melting points were and are uncorrected

Thin layer chromatographic (TLC) analyses were performed using Merck Kieselgel (Alufoilen) $60 \mathrm{~F}_{254}$ to a thickness of $0.2 \mathrm{~mm}$. Components were detected under an ultraviolet lamp at 254 or $350 \mathrm{~nm}$, or in an iodine chamber. Preparative TLC plates were coated with Merck Kieselge $\mathrm{GF}_{254}$ to a thickness of $0.75 \mathrm{~mm}$ and radial chromatography plates were coated with Merck Kieselgel $60 \mathrm{GF}_{254}$ to a thickness of 2.0 or $4.0 \mathrm{~mm}$. Column chromatography employed Riedel de Haën silica gel S (230-400 ASTM)
unless otherwise stated.

\subsubsection{Reaction of the 1-acyl-1H-cyclopropa[b]naphtha-} lenes with mineral acid. To the stirred 1 -acyl- $1 H$-cyclopropa[b]naphthalene $(57-100 \mathrm{mg}, 0.31-0.41 \mathrm{mmol})$ in THF $(5 \mathrm{ml})$ under nitrogen was added $\mathrm{HCl}(2 \mathrm{M}, 1 \mathrm{ml}$, $2 \mathrm{mmol}$ ) and the stirring continued at ambient temperature until the reaction was complete (TLC). The yellow product mixture was poured into $\mathrm{CH}_{2} \mathrm{Cl}_{2} / \mathrm{H}_{2} \mathrm{O}(1: 1 ; 100 \mathrm{ml})$, the ( $\left.\mathrm{CH}_{2} \mathrm{Cl}, 3 \times 15 \mathrm{mi}\right)$. $\left(\mathrm{CH}_{2} \mathrm{Cl}_{2}, 3 \times 15 \mathrm{ml}\right)$. The combined organic layers were washed $\left(\mathrm{H}_{2} \mathrm{O}, 50 \mathrm{ml}\right)$, dried $\left(\mathrm{MgSO}_{4}\right)$, and concentrated under reduced pressure to an oily yellow solid that was purified by radial chromatography.

(a) From $10^{15}$ (100 mg, $0.41 \mathrm{mmol}$ ) after $40 \mathrm{~h}$ was obtained 2-phenylnaphtho[2,3-b]furan (26) (3.8 mg, 4\%-see below) as a white solid from radial chromatography (light petroleum elution). Further elution (dichloromethes gave 2-(3-chloro-2-naphthalenyl)-1-phenylethanone (13) (90 mg, 79\%) as a white solid and the major component; an analytical sample was obtained (THF) as lustrous, fluffy white needles, mp 164-166 ${ }^{\circ} \mathrm{C}$ (Found: $\mathrm{C}, 76.8 ; \mathrm{H}, 4.6 ; \mathrm{Cl}$, 12.6; $[\mathrm{M}+\mathrm{H}]^{+} 281.0728$. $\mathrm{C}_{18} \mathrm{H}_{13} \mathrm{ClO}$ requires: $\mathrm{C}, 77.0 ; \mathrm{H}$ 4.7; $\mathrm{Cl}, 12.6 \% ;\left[\mathrm{M}+\mathrm{H}^{+}{ }^{+} 281.0739\right) . \nu_{\max } 3055,3034,3025$, $1694,1319,1209,976,764,750,687 \mathrm{~cm}^{-1}$. 229 (4.80), $250 \mathrm{sh}(4.26), 271(3.84), 280(3.82), 290 \mathrm{~nm}$ (log $\epsilon$ 3.62). 'H NMR $\delta 4.59$ (s, CH $\mathrm{CH}_{2}, 7.44-7.54(\mathrm{~m}, \mathrm{H} 6 / 7$ and H13/15), 7.59-7.64 (app th, J=7.3,1.7 Hz, H14), 7.74 (bs, H1), 7.75-7.79 (m, H5/8), 7.93 (bs, H4), 8.08-8.12 (m H12/16); ${ }^{13} \mathrm{C}$ NMR $\delta 43.5\left(\mathrm{CH}_{2}\right), 126.3 / 126.6(\mathrm{C} 6$ and $(\mathrm{m}$, 126.7/127.5 (C5 and C8) 127.6 (C4), 128.4 (C. (C13/15), 130.6 (C1), 131.6 (C4), 128.4 (C12/16), 128.7 $133.2(5)$ (q), 133.3 (2) $(\mathrm{C} 14), 136.5$ (C11), (q), 132.3 (q) (C11), 196.6 (CO).

(b) From $11^{15}(57 \mathrm{mg}, 0.31 \mathrm{mmol})$ after $36 \mathrm{~h}$ was obtained 1-(3-chloro-2-naphthalenyl)propan-2-one (14) (4I mg, $60 \%)$ as a pale yellow solid from radial chromatography (dichloromethane/light petroleum elution, 1:1). An analytiless plates, was obtained (same solvent mixture) as colour219.0571. $\mathrm{C}_{13} \mathrm{H}_{11} \mathrm{OCl}$ (Found: $\mathrm{C}, 71.5 ; \mathrm{H}, 5.1 ;[\mathrm{M}+\mathrm{H}]^{+}$ 219.0571), $\nu_{13} \mathrm{H}_{11} \mathrm{OCl}$ requires: $\mathrm{C}, 71.4 ; \mathrm{H} 5.1 \% ;[\mathrm{M}+\mathrm{H}]^{+}$ 1402 , 1361, $\nu_{\max } 3045,2922,2903,2853,1721,1588,1492$, $763 \mathrm{~cm}^{-1} .1334,1319,1165,1135,1006,966,888$,
(3.67), 281 (3.67), $290 \mathrm{~nm}(\log \in 3.49), 1 \mathrm{H} N 3.3$ (s, Me), $4.00\left(\mathrm{~s}, \mathrm{CH}_{2}\right), 7.47-7.50(\mathrm{~m}, \mathrm{H})$. H NMR $\delta 2.26$ $7.73-7.80$ (m, H5/8), 7.90 (bs, H4): ${ }^{13} \mathrm{H} 6 / 7$ ). 7.70 (bs, H1), $48.6\left(\mathrm{CH}_{2}\right), 126.4 / 126.7(2)$ (C6s, $\left.\mathrm{C} 4\right):{ }^{13} \mathrm{C}$ NMR $\delta 29.7(\mathrm{Me})$, and $\mathrm{C} 8$ ), 127.7 (C4), 130.6 (C1), 130.7 126.7(7)/127.4 (C5 (C8a or $\mathrm{C} 4 \mathrm{a}) ; 132.2$ (C3 or $\mathrm{C} 2), 133.7$ (C2 or $\mathrm{C} 3$ ), 131.9 (CO).

(c) From $12^{15}$ (100 mg, $\left.0.35 \mathrm{mmol}\right)$ after $168 \mathrm{~h}$ was obtained 2-chloro-3-[bis( $N, N$-dimethylcarboxamido)methyl]naphthalene (15) (84 mg, 74\%) as a pale yellow solid from radial chromatography (ethyl acetate elution); analytical sample was obtained from (ethl acetate elution); an petroleum (1:1) as obtained from dichloromethane/light (Found: C, 63.7; colourless blocks, mp $145-146^{\circ} \mathrm{C}$ 319.1223 $\mathrm{C}_{17} 3.7 \mathrm{H}, 6.0 ; \mathrm{N}, 8.8 ; \mathrm{Cl}, 10.9 ;[\mathrm{M}+\mathrm{H}]^{+}$ Cl, $10.0 \%$. $\mathrm{C}_{17} \mathrm{H}_{19} \mathrm{O}_{2} \mathrm{~N}_{2} \mathrm{Cl}$ requires: C, $64.0 ; \mathrm{H}, 6.0 ; \mathrm{N}, 8.8$. $1656,1495,1455,1439.1385) . \nu_{\max } 3059,2924,2854$ $1054,1017,959,880,851385,1352,1271,1205,1129$ (4.67), $276 \mathrm{~nm}, 880,851,755 \mathrm{~cm}^{-1} \cdot \lambda_{\max }(\mathrm{MeCN}) 231$ $3.02(\mathrm{~s}, 2 \times \mathrm{NMe}), 5.56(\mathrm{~s}, \mathrm{ArCH})$, $7.70-7.73(\mathrm{~m}, \mathrm{H} 5), 7.79-7.83$ ( $7.42-7.49$ (m, H6/7), 7.88 (bs, H4); ${ }^{13} \mathrm{C}$ NMR 836.0 (m, H8), 7.84 (bs, HI), 51.3 (ArCH), 126.3/126.9 (C6 (2XNMe), 37.2 (2XNMe), (C6 and C7), 126.4 (C5), 127.4 131.9/133.3 (C4), 129.9/131.0 (C2 and C3), 130.2 (C1). $(2 \times \mathrm{CO})$

1.1.2. Preparation of enol ethers from 1-acyl- $1 H$-cyclopropa $b]$ naphthalenes. To a stirred solution of the 1-acyl$1 H$-cyclopropa $b]$ naphthalene $(100-150 \mathrm{mg}, 0.41$ $0.61 \mathrm{mmol})$ in anhydrous THF (15 ml) at $-78^{\circ} \mathrm{C}$ under argon was added BuLi $(2.5 \mathrm{M}, 0.16-0.25 \mathrm{ml}, 1 \mathrm{~mol}$ equiv.). The dark mixture was stirred at this temperature for $30 \mathrm{~min}$ prior to the dropwise addition of alkyl balide (1 mol equiv.). The mixture was warmed to ambient temperature overnight, poured into $\mathrm{Et}_{3} \mathrm{O} / \mathrm{H}_{2} \mathrm{O}$ (1:1 $100 \mathrm{ml}$ ) and the phases separated. The aqueous phase was extracted (ether, $3 \times 15 \mathrm{ml}$ ) and the combined organic layers were washed $\left(\mathrm{H}_{2} \mathrm{O}, 50 \mathrm{ml}\right)$, dried $\left(\mathrm{MgSO}_{4}\right)$ and concentrated under reduced pressure to a thick yellow oil which was purified by radial chromatography.

(a) From 1-benzoyl-1H-cyclopropa[b]naphthalene $(\mathbf{1 0})^{15}$ $(120 / 150 \mathrm{mg}, \quad 0.49 / 0.61 \mathrm{mmol}$ ): (i) with trimethylsilyl chloride $(0.08 \mathrm{ml}, 0.61 \mathrm{mmol})$ was obtained a bright yellow solid (84 mg) after radial chromatography (dichloromethane/light petroleum, 1:1) that contained ('H NMR) 1[(trimethylsiloxy)phenylmethylidene]-1 $\mathrm{H}$-cyclopropa[b] naphthalene (18) and unchanged 10 in a 17:3 ratio. Attempts to obtain the pure compound resulted in its decomposition. Partial NMR data for 18 abstracted from spectra are: 'H NMR $\delta 0.55\left(\mathrm{~s}, \mathrm{Me}_{3} \mathrm{Si}\right), 7.30-7.31(\mathrm{~d}, J \sim 1 \mathrm{~Hz}, 1 \mathrm{H})$, $7.37-7.43$ (app tt, $J=7.3,1.7 \mathrm{~Hz}, 1 \mathrm{H}$ ), $7.41-7.42$ (d, $J \sim 1 \mathrm{~Hz}, 1 \mathrm{H}$ ), 7.47-7.50 (BB', 2H), 7.51-7.57 (app t, $J=7.6 \mathrm{~Hz}, 2 \mathrm{H}), 7.84-7.89\left(\mathrm{AA}^{\prime}, 2 \mathrm{H}\right), 8.05-8.09$ (app t, $J=8.5 \mathrm{~Hz}, 2 \mathrm{H}) ;{ }^{13} \mathrm{C}$ NMR $\delta 0.90\left(\mathrm{Me}_{3} \mathrm{Si}\right), 96.0$ (q), 104.2 $(\mathrm{CH}), 104.9(\mathrm{CH}), 124.7(2 \times \mathrm{CH}), 127.0(\mathrm{CH}), 126.2$ $(\mathrm{CH}), 127.8(\mathrm{CH}), 128.1(5)(\mathrm{CH}), 128.2(6)(\mathrm{CH}), 128.3$
$(2 \times \mathrm{CH})$ $(2 \times \mathrm{CH})$.

(ii) With $t$-butyldimethylsilyl chloride $(93 \mathrm{mg}, 0.61 \mathrm{mmol}$ ) in THF ( $2 \mathrm{ml})$ was obtained 1-[( $t$-butyldimethylsiloxy)phenylmethylidene $]-1 H$-cyclopropa[ $b]$ naphthalene (19) 
(125 mg, 57\%) as a bright yellow solid from radial chromatography (dichloromethane/light petroleum, 1:4). An analytical sample was obtained as bright yellow plates from dichloromethane/light petroleum (1:1), mp 104$106^{\circ} \mathrm{C}$ (Found: $\mathrm{C}, 80.5 ; \mathrm{H}, 7.2(5) ;[\mathrm{M}+\mathrm{H}]^{+}$(APCD 359.1826. $\mathrm{C}_{24} \mathrm{H}_{26} \mathrm{OSi}$ requires: C, $80.4 ; \mathrm{H}, 7.4 \%$; $[\mathrm{M}+\mathrm{H}]^{+}$ $359.1807) . \nu_{\max } 3041,2948,2925,2854,1785,1592,1549$ $1509,1487,1419,1311,1292,1251,1177,1160,1138$ $1074,841,783,763,747,689,608,553 \mathrm{~cm}^{-1} . \lambda_{\max }$ (hexane) 230 (4.58), $246 \mathrm{sh}(4.25), 254$ (4.15), 291 (4.28) 368 (4.07), 374 (4.14), 393 (4.56), $422 \mathrm{~nm}(\log \epsilon 4.82)$; UV $\lambda_{\max }(\mathrm{MeCN}) 230$ (4.56), $246 \mathrm{sh}(4.25), 254$ (4.14), 291 (4.26), 368 (4.03), 372 (4.07), 392 (4.49), $420 \mathrm{~nm}(\log \epsilon$ 4.72). ${ }^{1} \mathrm{H}$ NMR $\delta 0.48\left(\mathrm{~s}, \mathrm{Me}_{2} \mathrm{Si}\right), 1.19$ (s, Me $\left.\mathrm{CSSi}_{3}\right), 7.29$ (d, $J=1.5 \mathrm{~Hz}, \mathrm{H} 2$ or H7), 7.37-7.42 (app tt, $J=7.3,1.7 \mathrm{~Hz}$ H12), 7.40 (d, $J=1.5 \mathrm{~Hz}, \mathrm{H} 7$ or H2), 7.45-7.50 (BB', H4/5), $7.51-7.56(\mathrm{t}, J=7.6 \mathrm{~Hz}, \mathrm{H} 11 / 13), 7.84-7.88\left(\mathrm{AA}^{\prime}, \mathrm{H} 3 / 6\right)$ $8.07-8.10$ (app d, $J=8.6 \mathrm{~Hz}, \mathrm{H} 10 / 14) ;{ }^{13} \mathrm{C}$ NMR $\delta-3.7$ $\left(\mathrm{Me}_{2} \mathrm{Si}\right), 18.4\left(\mathrm{Me}_{3} \mathrm{C}\right), 25.9\left(\mathrm{Me}_{3} \mathrm{CSi}\right), 95.9(\mathrm{Cl}), 104.1$ 104.9 (C2 and C7), 114.6 (Cla or C7a), 124.7 (C10/14), $126.1(5) / 126.2$ (C4 and C5), 126.4 (C7a or Cla), 127.8 (C12), 128.1/128.2 (C3 and C6), 128.3 (C11/13), 135.8 (C8), 137.1 (C9), 137.7/137.8 (C2a and C6a).

(iii) With t-butyldiphenylsilyl chloride $(0.13 \mathrm{ml}, 0.49$ $\mathrm{mmol}$ ) was obtained from radial chromatography (dichloromethane/light petroleum, 1:4) an analytical sample of 1-[( $t$ butyldiphenylsiloxy)phenylmethylidene $]-1 H$-cyclopropa[b]naphthalene (20) $(150 \mathrm{mg}, 63 \%)$ as a viscous tar which solidified after scratching, mp $134-140^{\circ} \mathrm{C}$ (Found: C, 84.5(5); H, 6.5; $\left[\mathrm{M}+\mathrm{H}^{+}\right.$(APCI) 483.2139. $\mathrm{C}_{34} \mathrm{H}_{30} \mathrm{OSi}$ requires: C, 84.6; $\left.\mathrm{H}, 6.3 \% ;[\mathrm{M}+\mathrm{H}]^{+} 483.2140\right), \nu_{\max } 3071$, $3050,2927,2855,1589,1547,1506,1486,1471,1445$, $1418,1386,1287,1151,1132,1114,1073,1025,886$, $828,762,742,701,620,602,552,503,490,469 \mathrm{~cm}^{-1}$ $\lambda_{\max }$ (hexane) 227 (4.73), $254 \mathrm{sh}(4.33), 282$ (4.23), 292 (4.39), 369 (4.16), 374 (4.22), 394 (4.64), $422 \mathrm{~nm}$ (log 4.90); $\lambda_{\max }(\mathrm{MeCN}) 228(4.58), 254 \mathrm{sh}(4.19), 282(4.09)$, 292 (4.24), $368(4.01), 374(4.08), 393(4.47) 421 \mathrm{~nm}(\log \epsilon$ 4.71). ${ }^{1} \mathrm{H}$ NMR $\delta 1.30\left(\mathrm{~s}, \mathrm{Me}_{3} \mathrm{CSi}\right), 5.78(\mathrm{~d}, J=1.2 \mathrm{~Hz}, \mathrm{H} 2)$ 7.27 (d, $J=1.2 \mathrm{~Hz}, \mathrm{H} 7), 7.33-7.52(\mathrm{~m}, 10 \mathrm{H}), 7.61-7.66(\mathrm{bd}$ $\mathrm{t}, J=7.7 \mathrm{~Hz}, \mathrm{H} 11 / 13), 7.74$ (bd d, $J=8.1 \mathrm{~Hz}, \mathrm{H}$ ), $7.99-8.03$ (m, 4H), 8.27-8.31 (bd d, $J=7.3 \mathrm{~Hz}, \mathrm{H10} / 14$ ); ${ }^{13} \mathrm{C}$ NMR $\delta$ 19.5 ( $\left.\mathrm{Me}_{3} \mathrm{CSi}\right), 26.5\left(\mathrm{Me}_{3} \mathrm{CSi}\right), 97.5(\mathrm{Cl}), 103.9$ (C7), 104.9 (C2), 124.6 (C10/14), 125.5 (C1a or C7a), 125.7/125.8 (C4 and C5), $126.4(\mathrm{C} 7 \mathrm{a}$ or C1a), $127.7(2 \times \mathrm{CH}), 127.8(\mathrm{CH})$ $127.9(\mathrm{CH}), 128.2(\mathrm{CH}), 128.5(\mathrm{C} 11 / 13), 129.8(\mathrm{C} 18), 133.3$ (C15), 134.6 (C8), 135.1 (2×CH), 136.9 (C9), 137.5/137.7 (C2a and $\mathrm{C} 6 \mathrm{a})$

(iv) With di-t-butylchlorophosphine $(0.09 \mathrm{ml}, 0.49 \mathrm{mmol})$ was obtained after radial chromatography (ethyl acetate elution) a mixture of di-t-butylphosphinic acid 8-[1(phenylmethylidene)-1H-cyclopropa[b]naphthalene]- $O$ ether (24) together with an unidentified phosphorouscontaining compound as a yellow oil. Subsequent radial chromatography (ethyl acetate/dichloromethane elution, 6:1) gave an analytical sample of $24(32 \mathrm{mg}, 16 \%)$ as a bright yellow oil which solidified on standing, mp 158$161^{\circ} \mathrm{C}$ (Found: C, 76.7; H, 7.3; $[\mathrm{M}+\mathrm{H}]^{+}{ }^{+} 405.1978$. $\mathrm{C}_{26} \mathrm{H}_{29} \mathrm{O}_{2} \mathrm{P}$ requires: $\mathrm{C}, 77.2 ; \mathrm{H}, 7.2 \%$; $[\mathrm{M}+\mathrm{H}]^{+} 405.1990$ ). $\nu_{\max } 3053,2924,2854,1770,1742,1713,1632,1469,1448$, $1423,1384,1274,1231,1143,1115,1074,1011,939,889$,
$822,748,692,672,594,476 \mathrm{~cm}^{-1}, \lambda_{\max }$ (hexane) 230 (4.73), 252 sh (4.31), 290 (4.43), 3.71 sh (4.25), 392 (4.63) $420 \mathrm{~nm}(\log \epsilon 4.75)$; UV $\lambda_{\max }(\mathrm{MeCN}) 230$ (4.73), $252 \mathrm{sh}$ (4.30), 290 (4.41), $3.69 \mathrm{sh}(4.22), 391$ (4.57), $418 \mathrm{~nm}$, $(\log \epsilon 4.71) .{ }^{1} \mathrm{H}$ NMR $\delta 1.44\left(\mathrm{~d},{ }^{3} J_{\mathrm{P}-\mathrm{H}}=14.7 \mathrm{~Hz}, 2 \times \mathrm{Me}_{3} \mathrm{C}\right)$, 7.33-7.37 (app tt, $J=7.3,1.2 \mathrm{~Hz}, \mathrm{H} 12$ ), 7.42-7.47 (BB', H4/5), $7.48-7.54$ (app t, $J=7.7 \mathrm{~Hz}, \mathrm{H} 11 / 13$ ), 7.53 (d, $J=1.7 \mathrm{~Hz}, \mathrm{H} 2$ or $\mathrm{H} 7), 7.65(\mathrm{~d}, J=1.7 \mathrm{~Hz}, \mathrm{H} 7$ or $\mathrm{H} 2$ ), 7.85-7.89 (AA', H3/6), 7.95-7.99 (app d, $J=8.5 \mathrm{~Hz}, \mathrm{H} 10$ ) $14) ;{ }^{13} \mathrm{C}$ NMR $\delta 26.7\left(2 \times \mathrm{Me}_{3} \mathrm{C}\right), 37.2$ (d, ${ }^{1} J_{\mathrm{P}-\mathrm{C}}=80.6 \mathrm{~Hz}$, $\left.2 \times \mathrm{Me}_{3} \mathrm{C}\right), 99.9(\mathrm{Cl}), 106.6 / 108.6(\mathrm{C} 2$ and $\mathrm{C} 7), 124.0(\mathrm{C} 10$ l 14), $126.0\left(\mathrm{~d},{ }^{4} \mathrm{~J}_{\mathrm{P}-\mathrm{C}}=1.0 \mathrm{~Hz}, \mathrm{Cla}\right.$ or $\left.\mathrm{C} 7 \mathrm{a}\right), 126.3(3) / 126.3(8)$ (C4 and C5), $127.0\left(\mathrm{~d},{ }^{4} \mathrm{~J}_{\mathrm{p}-\mathrm{C}}=1.0 \mathrm{~Hz}, \mathrm{C} 7 \mathrm{a}\right.$ or $\left.\mathrm{Cla}\right), 127.7$ (C12), 128.4 (C3 or C6), 128.6 (C11/13), 128.9 (C6 or C3), $132.4\left(\mathrm{~d},{ }^{2} J_{\mathrm{P}-\mathrm{C}}=12.6 \mathrm{~Hz}, \mathrm{C} 8\right), 135.5\left(\mathrm{~d},{ }^{3} J_{\mathrm{P}-\mathrm{C}}=4.5 \mathrm{~Hz}, \mathrm{C} 9\right)$ $138.3 / 138.9$ (C2a and C6a); ${ }^{31} \mathrm{P}$ NMR $\delta$ 68.6.

(b) From 1-acetyl-1 $H$-cyclopropa[b]naphthalene (11) ${ }^{15}$ (100 mg, 0.55): (i) with $t$-butyldimethylsilyl chloride ( $84 \mathrm{mg}, 0.55 \mathrm{mmol})$ in THF $(2 \mathrm{ml}$ ) was obtained after radial chromatography (dichloromethane/light petroleum elution, $1: 4)$ a bright yellow oil $(60 \mathrm{mg})$ that contained predominantly 1-[(1-t-butyldimethylsiloxy)ethylidene]-1H-cyclopropa $[b]$ naphthalene $(21)$ (ca. $55 \mathrm{mg}$, ca. $35 \%$ ). Subsequent attempts to purify compound 21 resulted in its decomposition. NMR data abstracted from the spectra of the crude product are: ${ }^{1} \mathrm{H}$ NMR $\delta 0.37\left(\mathrm{~s}, \mathrm{Me}_{2} \mathrm{Si}\right), 1.05$ (s, $\left.\mathrm{Me}_{3} \mathrm{CSi}\right), 2.29(\mathrm{~s},=\mathrm{CMe}), 7.09 / 7.10(2 \times \mathrm{bs}, \mathrm{H} 2$ and $\mathrm{H} 7)$ 7.38-7.41 (BB' $\left.\mathrm{BB}^{\prime} \mathrm{H} / 5\right), 7.73-7.77$ (AA $\left.{ }^{\prime}, \mathrm{H} 3 / 6\right) ;{ }^{13} \mathrm{CNMR} \delta$ $-3.9\left(\mathrm{Me}_{2} \mathrm{Si}\right), 18.0\left(\mathrm{Me}_{3} \mathrm{CSi}\right), 22.3(=\mathrm{CMe}), 25.6$ $\left(\mathrm{Me}_{3} \mathrm{CSi}\right), 95.4(\mathrm{Cl}), 103.4 / 103.6(\mathrm{C} 2 / 7), 125.6 / 125.7$ (C4/ 5), 127.9/128.1 (C3/6), 126.2, 128.4, 136.7, 137.3, 137.5 (all q).

(ii) With $t$-butyldiphenylsilyl chloride $(0.143 \mathrm{ml}, 0.55$ $\mathrm{mmol}$ ) was obtained a thick yellow tar $(150 \mathrm{mg})$ as the crude product of reaction. ' $\mathrm{H}$ NMR analysis indicated the presence of olefin 22 from the presence of doublets (ca. $J=1 \mathrm{~Hz}$ ) at $\delta 5.99(\mathrm{H} 2)$ and $7.04(\mathrm{H} 7)$ with the shielding caused by a phenyl group in analogy with the lowest energy conformer of 20. Attempts to isolate this product resulted in its decomposition.

1.1.3. Preparation of naptho[2,3-b]furans 26-28. (a) A solution of the requisite 1 -acyl- $1 H$-cyclopropa[ $b]$ naphthalene $(100-340 \mathrm{mg}, 0.35-1.87 \mathrm{mmol})$ was refluxed in dry benzene $(5 \mathrm{ml})$ under argon for $16 \mathrm{~h}$. The residue obtained by concentration to dryness was purified as described

(i) 1-Benzoyl-1 $H$-cyclopropa $[b]$ naphthalene $(10)(120 \mathrm{mg}$, $0.49 \mathrm{mmol}$ ) gave 2-phenylnaphtho[2,3-b]furan (26) $(8 \mathrm{mg}, 7 \%)$ as cream plates from radial chromatography (light petroleum elution), mp $204^{\circ} \mathrm{C}$ (sub.), $225-233^{\circ} \mathrm{C}$ (sublimate vapourizes) (Found: C, $86.8 ; \mathrm{H}, 4.8 ;[\mathrm{M}+\mathrm{H}]^{+}$ 245.0953. $\mathrm{C}_{18} \mathrm{H}_{12} \mathrm{O}$ requires: C, $88.5 ; \mathrm{H}, 4.9$ (5)\%; $\left.[\mathrm{M}+\mathrm{H}]^{+} 245.0961\right) . \nu_{\max } 3059,3039,1564,1490,1448$, $1264,1018,902,867,761,742,719,689,478 \mathrm{~cm}^{-1} . \lambda_{\max }$ (MeCN) 222 (4.77), 272 (4.77) 279 (4.71), 330sh (4.59) 340 (4.64), 356sh nm (log $\epsilon 4.25)$. 'H NMR $\delta 7.15$ (d, $J \sim 1 \mathrm{~Hz}, 1 \mathrm{H}), 7.41-7.52(\mathrm{~m}, 5 \mathrm{H}), 7.92-7.97(\mathrm{~m}, 5 \mathrm{H})$, $8.04(\mathrm{bs}, 1 \mathrm{H}) ;{ }^{13} \mathrm{C}$ NMR $\delta 100.7(\mathrm{CH}), 106.6(\mathrm{CH}) 118.5$ (CH) $124.0(\mathrm{CH}), 124.7(\mathrm{CH}), 125.3(2 \times \mathrm{CH}), 127.8(\mathrm{CH})$, $127.9(\mathrm{CH}), 128.8(2 \times \mathrm{CH}), 129.1(\mathrm{CH}), 130.1(\mathrm{C}), 130.2$ (C), 130.7 (C), 131.5 (C), 153.9 (C), 158.1 (C). 
When 10 (50 mg, $0.20 \mathrm{mmol}$ ) was slowly heated to its melting point $\left(101^{\circ} \mathrm{C}\right)$ the sample decomposed violently. The cooled black residue was dissolved in acetone and filtered though silica gel in a micropipette. The eluent was purified by radial chromatography (light petroleum) to give naphthofuran $26(8.6 \mathrm{mg}, 17 \%)$ identical to the sample obtained above.

(ii) 1-Acetyl- $1 H$-cyclopropa[b]naphthalene (11) $(340 \mathrm{mg}$, $1.87 \mathrm{mmol}$ ) gave 2-methylnaphtho[2,3-b]furan (27) (67 mg, 20\%) from radial chromatography (dichloromethane/light petroleum elution, 1:1) as a white solid, $\mathrm{mp}$ $73-74^{\circ} \mathrm{C}$ (lit. ${ }^{39} 71-72^{\circ} \mathrm{C}$ ). ${ }^{~} \mathrm{H}$ NMR $\delta 2.54(\mathrm{~d}, J \sim 1 \mathrm{~Hz}, \mathrm{Me}$ ), 6.50 (t, J 1 Hz, H3), 7.43-7.47 (m, H6/7), 7.84 (bs, H9), 7.93-7.96 (m, H4 and H5/8); ${ }^{13} \mathrm{C}$ NMR $\delta 14.3(\mathrm{Me}), 102.2$ (C3), 106.0 (C9), 117.4 (C4), 123.7/124.3 (C6 and C7), 127.7 (6)/127.7 (9) (C5 and $\mathrm{C} 8$ ), 130.4 (C3a and $\mathrm{C} 4 \mathrm{a}$ or C8a), 130.8 (C8a or C4a), 154.0 (C9a), 158.1 (C2).

(iii) $\operatorname{Bis}(N, N$-dimethyl $)-1 H$-cyclopropa[b]naphthalene-1,1dicarboxamide (12) (100 mg, $0.35 \mathrm{mmol}$ ) gave $2-\mathrm{N}, \mathrm{N}$ dimethylamino-3- $N, N$-dimethylamidobenzo $[2,3-b]$ furan (28) (100 mg, 100\%) as a brown wax. Recrystallization (dichloromethane/light petroleum, 1:1) gave an analytical sample as brown plates, mp $135-136^{\circ} \mathrm{C}$ (Found: $\mathrm{C}, 71.5$; $\mathrm{H}, 6.6 ; \mathrm{N}, 9.9(5) ;[\mathrm{M}+\mathrm{H}]^{+}$283.1442. $\mathrm{C}_{17} \mathrm{H}_{18} \mathrm{O}_{2} \mathrm{~N}_{2}$ requires C, 72.3; H, 6.4; N 9.9\%; $\left.[\mathrm{M}+\mathrm{H}]^{+} 283.1441\right) . \nu_{\max } 3053$ $2924,1643,1633,1607,1579,1430,1416,1370,1042$, $857 \mathrm{~cm}^{-1} \cdot \lambda_{\max }(\mathrm{MeCN}) 222(4.56), 272(4.56), 292$ (3.83), $305(4.01), 318(4.15), 353 \mathrm{~nm}(\log \epsilon 4.11) .{ }^{1} \mathrm{H}$ NMR $\delta 3.15\left(\mathrm{~s},=\mathrm{CNMe}_{2}\right), 3.17$ (bs, $\left.\mathrm{CONMe}_{2}\right), 7.29-$ 7.40 (m, H6/7), 7.42 (bs, H4), 7.58 (bs, H9), 7.79-7.82 $(\mathrm{m}, \mathrm{H} 5 / 8) ;{ }^{13} \mathrm{C}$ NMR $\delta 35.2^{\dagger} \quad\left(\mathrm{CONMe}_{2}\right), 39.4$ $\left(=\mathrm{CNMe}_{2}\right), 85.7$ (C3), 104.9 (C9), 113.2 (C4), 123.2/ 124.1 (C6 and C7), 126.9 (C5), 127.6 (C8), 129.1/131.3 (C4a and C8a), 130.8 (C3a), 149.0 (C9a), 160.6 (C2), 166.9 (CO). The chemical shift data for the formally nonequivalent $\mathrm{CONMe} \mathrm{N}_{2}$ methyl groups were resolved at $-20^{\circ} \mathrm{C}$ giving $\delta_{\mathrm{H}} 3.18(\mathrm{~s}, 3 \mathrm{H})$ and $3.19(\mathrm{~s}, 3 \mathrm{H})$, and $\delta_{\mathrm{C}} 35.2$ and 39.3.

(b) A solution of benzoyl-1H-cyclopropa[b]naphthalene (10) $(80 \mathrm{mg}, 0.33 \mathrm{mmol})$ in $\mathrm{MeCN}(30 \mathrm{ml})$ was irradiated at $254 \mathrm{~nm}$ for $16 \mathrm{~h}$ in a Rayonette RP203 preparative photochemical reactor in a quartz tube fitted with a reflux condenser. Concentration to dryness and purification of the orange residue by radial chromatography (light petroleum) gave naphthofuran $26(3.6 \mathrm{mg}, 5 \%)$ as a cream solid identical to that obtained above.

\subsection{Computational methods}

Semiempirical calculations were performed at the restricted Hartree-Fock level using the AM1 method. ${ }^{35,36}$ Calculations were performed using SPARTAN ${ }^{45}$ on an R5000 Silicon Graphics workstation.

\footnotetext{
This chemical shift value was obtained from the HSQC and gHMBC spectra as no signal was observed in the ${ }^{13} \mathrm{C}$ NMR experiment.
}

\section{Acknowledgements}

The work has received generous financial support from the Victoria University Science Faculty Leave and Grants Committee (B. H., C. S. J.), and the Curtis-Gordon and VUW Alumni Scholarship Funds (C. S. J.).

\section{References}

1. Halton, B. Chem. Rev. 1989, 89, 1161-1185.

2. Halton, B. In Cycloproparenes. Chemistry of the Cyclopropyl Group; Rappoport, Z., Ed.; Wiley: Chichester, 1995; Vol. 2, pp. 707-772.

3. Müller, P. In Cycloproparenes. Carbocyclic Three-Membered Ring Compounds; de Meijere, A., Ed.; Thieme: Stuttgart, 1997; Vol. E17d, pp. 2866-2948.

4. Müller, P. In Chemistry of Cycloproparenes. Advances in Theoretically Interesting Molecules; Thummel, R. P., Ed.; JAI: Greenwich, CT, 1995; Vol. 3, pp. 37-107.

5. Halton, B.; Jones, C. S.; Kay, A. J.; Margetic, D.; Sretenovic, S. J. Chem. Soc., Perkin Trans. 1 2000, 2205-2210.

6. Halton, B.; Stang, P. J. Synlett 1997, 145-158.

7. Eaborn, C.; Eidenschink, R.; Harris, S. J.; Walton, D. R. M. J. Organomet. Chem. 1977, I24, C27-C29.

8. Eaborn, C.; Stamper, J. G. J. Organomet. Chem. 1980, 192, $155-161$.

9. Halton, B.; Buckland, S. J.; Mei, Q.; Stang, P. J. Tetrahedron Lett. 1986, 27, 5159-5160.

10. Halton, B.; Randall, C. J.; Stang, P. J. J. Am. Chem. Soc. 1984, 106, 6108-6110.

11. Halton, B.; Cooney, M. J.; Boese, R.; Maulitz, A. H. J. Org. Chem. 1998, 63, 1583-1590.

12. Halton, B.; Buckland, S. J.; Lu, Q.; Mei, Q.; Stang, P. J. J. Org. Chem. 1988, 53, 2418-2422.

13. Apeloig, Y.; Boese, R.; Bläser, D.; Halton, B.; Maulitz, A. H. J. Am. Chem. Soc. 1988, 120, 10,147-10,153.

14. McNichols, A. T.; Stang, P. J.; Addington, D. M.; Halton, B. Tetrahedron Lett. 1994, 35, 437-440.

15. Halton, B.; Jones, C. S.; Northcote, P. T.; Boese, R. Aust. J. Chem. 1999, 52, 285-290.

16. Halton, B.; Jones, C. S. J. Chem. Soc., Perkin Trans. 21998 , 2505-2508 (1999, p. 387).

17. Cutler, C. A.; Halton, B. Aust. J. Chem. 1997, 50, 267-270.

18. Dürr, H.; Schrader, L. Angew. Chem., Int. Ed. Engl. 1969, 8, $446-447$.

19. Dürr, H.; Schrader, L. Chem. Ber. 1970, 103, 1334-1346.

20. Lüddecke, E.; Rau, H.; Dürr, H.; Schmitz, H. Tetrahedron 1977, 33, 2677-2681.

21. Saracoglu, N.; Durucasu, I.; Balci, M. Tetrahedron 1995, 51, $10,979-10,986$.

22. Billups, W. E.; Rodin, W. A. J. Org. Chem. 1988, 53, $1312-$ 1314.

23. Bee, L. K.; Garratt, P. J.; Mansuri, M. M. J. Am. Chem. Soc. 1980, 102, 7076-7079.

24. Mayer, K. K.; Stoeber, G.; Knefeli, F.; Lee, D. U.; Striegel, H. G.; Weiss, I.; Wiegrebe, W. Acta Pharm. 1992, 42, 293 298.

25. Striegel, H. G.; Mayer, K. K.; Wiegrebe, W.; Schlunegger, U. P.; Siegrist, P.; Aebi, B. Arch. Pharm. 1992, 325, 751-760.

26. Zielinski, W. Pol. J. Chem. 1982, 56, 93-100.

27. Chen, G.-S.; Gibson, M. S. J. Chem. Soc., Perkin Trans. I $1975,1138-1139$. 
28. Moriarty, R. M.; Gill, U. S. Organometallics 1986, 5, 253256.

29. Wiberg, K. B. Origin of the amide rotational barrier. In Amide Linkage; Greenberg, A., Breneman, C. M., Liebman, J. L., Eds.; Wiley: New York, 2000; pp. 33-45.

30. Fong, C. W. Aust. J. Chem. 1980, 33, 1285-1290.

31. Spaargaren, K.; Korver, P. K.; Van der Haak, P. J; De Boer, Th. J. Org. Magn. Reson. 1971, 3, 605-614.

32. Jackman, L. M.; Kavanagh, T. E.; Haddon, R. C. Org. Magn. Reson. 1969, l, 109-123.

33. Some forty new derivatives have been prepared for physicochemical examination: Dixon, G. M.; Halton, B. Unpublished data.

34. House, H. O.; Czuba, L. J.; Gall, M.; Olmstead, H. D. J. Org. Chem. 1969, 34, 2324-2336.

35. Dewar, M. J. S.; Zoebisch, E. G.; Healy, E. F. J. Am. Chem. Soc. 1985, 107, 3902-3909.

36. Stewart, J. J. P. In Reviews in Computational Chemistry; Lipkowitz, K. B., Boyd, D. B., Eds.; VCH: New York, 1991; Vol. 2, pp. 45.

37. See for example: Davies, J. E.; Klunduk, M. C.; Mays, M. J.;
Raithby, P. R.; Shields, G. P.; Tompkin, P. K. J. Chem. Soc. Dalton Trans. 1997, 715-719. Mikolajczyk, M.; Kielbasinski, P.; Wieczorek, M. W.; Blaszczyk, J.; Kolbe, A. J. Org. Chem. 1990, 55, 1198-1203. Baudler, M.; Michels, M.; Pieroth, M. Hahn, J. Angew. Chem. 1990, 98, 465-467.

38. Cagniant, P.; Kirsch, G.; Legendre, P. C. R. Acad. Sci, Ser. C. $1973,22,1629-1631$.

39. Narasimhan, N. S.; Paradkar, M. V. Indian J. Chem. 1969, 7, 536-537.

40. Billups, W. E.; McCord, D. J.; Maughon, B. R. Tetrahedron Lett. 1994, 35, 4493-4496.

41. Vogel, E.; Grimme, W.; Korte, S. Tetrahedron Lett. 1965 , 3625-3631,

42. Burgert, W.; Grosse, M.; Rewicki, D. Chem. Ber. 1982, 115, 309-323.

43. Halton, B.; Woolhouse, A. D.; Milsom, P. J. J. Chem. Soc., Perkin Trans. 1 1977, 735-740.

44. Staab, H. A.; Ipaktschi, J. Chem. Ber. 1968, 101, 1457-1472.

45. SPARTAN v. 5.0, 1997: Wavefunction Inc., 18401 Von Karman Avenue, Suite 370, Irvine, CA 92612, USA. 


\title{
$1 H$-Cyclopropa $[b]$ anthracene-3,8-dione
}

\author{
Brian Halton* and Carissa S. Jones \\ School of Chemical and Physical Sciences, Victoria University, PO Box 600, Wellington, New Zealand
}

Received 16 September 1999; accepted 19 October 1999

\begin{abstract}
The first cyclopropa-fused anthraquinone, $1 H$-cyclopropa[ $b]$ anthracene-3,8-dione, is available in four steps from homologation of $1 H$-cyclopropa[b]naphthalene-3,6-dione. ( 1999 Elsevier Science Ltd. All rights reserved.

Keywords: anthraquinones; aromatics; dehydrogenation; Diels-Alder reactions; oxidation; strained molecules.

The number of oxygenated cycloproparenes recorded was small until recently. ${ }^{1,2}$ Rarer still are the cyclopropaquinones. ${ }^{3-5}$ The 1,4-benzo- and naphthoquinones 1 and 2 have been prepared by conventional methods (Scheme 1) but the location of the $\pi$ bond across the sites of three-membered ring fusion reduces the stability of the compounds to such an extent that the molecules have transient existence only; their structures have been adduced from Diels-Alder trapping experiments with dienes. ${ }^{3.5}$ In contrast, the naphthalene-3,6-dione 3 is a stable crystalline solid that has all the characteristics expected of a quinone and it represents the only stable member of the series. ${ }^{4}$ We report here the synthesis of the second stable cyclopropaquinone, $1 H$-cyclopropa[b]anthracene-3,8-dione (4), by what we believe to be the first homologation in the cycloproparene series.
\end{abstract}

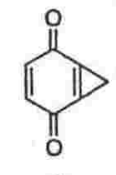

1<smiles>O=C1C2=C(C2)C(=O)c2ccccc21</smiles>

2<smiles>O=c1ccc(=O)c2cc3ccc2cc13</smiles>

3

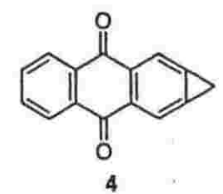

4

Scheme 1.

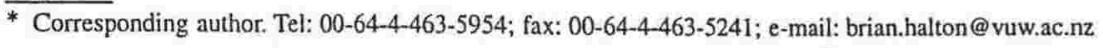
0040-4039/99/\$ - see front matter \& 1999 Elsevier Science Ltd. All rights reserved. PII: $50040-4039(99) 01985-1$
} 
The easily available ${ }^{4}$ dione 3 reacts with butadiene at $45^{\circ} \mathrm{C}$ to give the Diels-Alder adduct 5 with high regioselectivity (Scheme 2). ${ }^{\dagger \ddagger}$ As the reaction temperature is increased diadduct $6^{\S}$ is formed and becomes the sole product at $100^{\circ} \mathrm{C}$ in the presence of excess diene. Thus, addition across the $\mathrm{C} 4-\mathrm{C} 5$ $\pi$ bond of 3 precedes the $\left[\sigma^{2}+\pi^{2}\right]$ addition that opens ${ }^{1,6,7}$ the three-membered ring. Compounds 5 and 6 follow from their spectroscopic data which eliminate addition to the strained $\mathrm{Cla}-\mathrm{C} 7 \mathrm{a}$ bond ${ }^{8.9}$ of 3 . Tetrahydroanthracene 5 shows NMR shifts typical of the cycloproparenes ${ }^{1}$ with $\mathrm{H} 1$ as a singlet at $\delta$ 3.36 and the ortho carbons $\mathrm{C} 2 / 9$ at $\delta 113.2$. In diadduct $6 \mathrm{H} 1 / \mathrm{H} 2$ appear as an $\mathrm{ABX}$ system and the mono-substituted vinyl group at $\mathrm{C} 2$ is clearly evident.

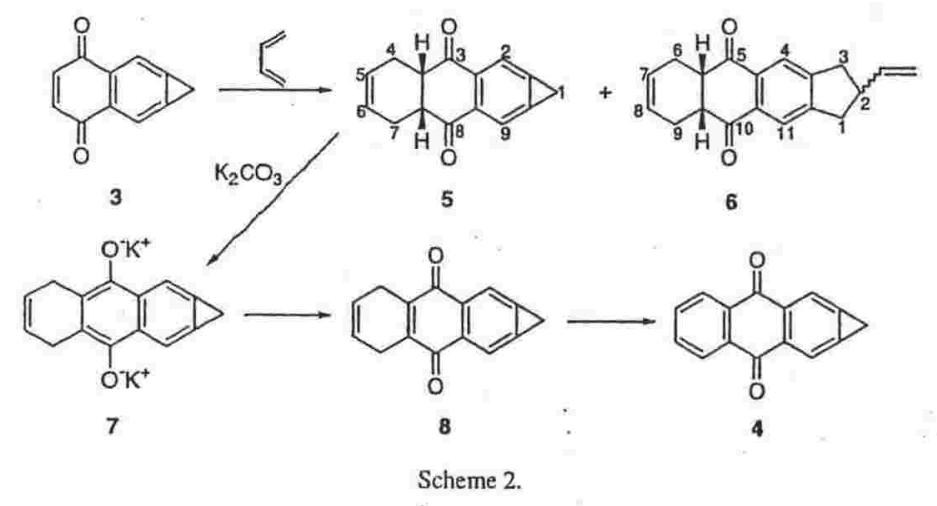

Reaction of dione 5 with $\mathrm{K}_{2} \mathrm{CO}_{3}$ results in proton abstraction from both $\mathrm{C} 3 \mathrm{a}$ and $\mathrm{C} 7 \mathrm{a}$ and the bisphenolate $7^{\text {I }}$ is formed. Exposure of 7 to air results in oxidation to quinone $8,{ }^{11}$ the dihydro-derivative of the desired anthraquinone 4 . Subsequent treatment of 8 with $\mathrm{K}_{2} \mathrm{CO}_{3}, \mathrm{DDQ}$ or air all effect oxidation to $1 \mathrm{H}$-cyclopropa[b]anthraquinone $(4)^{\dagger \dagger}$ but use of $\mathrm{K}_{2} \mathrm{CO}_{3}$ provides the most efficient procedure. Compound $\mathbf{4}$ is a yellow crystalline solid that is markedly more stable than the only other oxygenated cyclopropanthracene reported, namely 3,8-dioxacyclopropanthracene which dimerizes below $0^{\circ} \mathrm{C} .{ }^{10} \mathrm{The}$ characteristic shielding of the carbons adjacent to the strained ring fusion ${ }^{1}$ is evident in $4(\delta 113.5)$ and the methylene protons resonate as a singlet at $\delta 3.55$. Notable is the fact that the impact of the strained ring is detectable from NMR data only for the cyclopropa-fused benzenoid ring; the dione carbons and the remote $\mathrm{C} 3 \mathrm{a}-\mathrm{C} 7 \mathrm{a}$ ring have ${ }^{1} \mathrm{H}$ and ${ }^{13} \mathrm{C}$ resonances that match well those of anthraquinone.

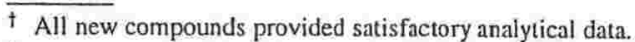

* Compound 5: 27\%, mp 96-97 ${ }^{\circ} \mathrm{C}$. NMR $\left(\mathrm{CDCl}_{3}\right) \delta_{\mathrm{H}} 2.19-2.29(2 \mathrm{H}), 2.47-2.57(2 \mathrm{H}), 3.36(\mathrm{~s}, 2 \mathrm{H}), 3.36-3.41(\mathrm{~m}, 2 \mathrm{H}), 5.73$ $(\mathrm{t}, J=1.5 \mathrm{~Hz}, 2 \mathrm{H}), 7.87(\mathrm{~s}, 2 \mathrm{H}) ; \delta_{\mathrm{c}} 19.5,24.4,46.5,113.2,124.6,133.0,137.7,198.2$. The low yield follows from easy aerial oxidation. The product from radial chromatography $(65-80 \%)$ can be used for transformation into 4.

5 Compound 6: $31 \%, \mathrm{mp} 128-129^{\circ} \mathrm{C}$. NMR $\left(\mathrm{CDCl}_{3}\right) \delta_{\mathrm{H}} 2.20(\mathrm{bd}, J=15.4 \mathrm{~Hz}, 2 \mathrm{H}), 2.50(\mathrm{bd}, J=15.4 \mathrm{~Hz}, 2 \mathrm{H}), 2.80-2.89(\mathrm{~m}$, 2H), 3.09-3.21 (m, 3H), 3.35 (bd t, $J=10.3 \mathrm{~Hz}, 2 \mathrm{H}), 5.01$ (bd, $J=10.3 \mathrm{~Hz}, 1 \mathrm{H}), 5.10$ (bd, $J=17 \mathrm{~Hz}, 1 \mathrm{H}), 5.73(\mathrm{~s}, 2 \mathrm{H}), 5.87-5.98$ $(\mathrm{m}, 1 \mathrm{H}), 7.84(\mathrm{~s}, 2 \mathrm{H}) ; \delta_{\mathrm{C}} 24.4,39.0,43.9,46.5,114.4,122.4(5), 124.6,133.1,140.7,150.5,198.3$.

1 Compound 7: Quantitative by NMR. NMR [( $\left.\left(\mathrm{CD}_{3}\right)_{2} \mathrm{CO}\right] \delta_{\mathrm{H}} 3.42\left(\mathrm{~s}, \mathrm{CH}_{2}\right), 3.50$ (d, $\left.J=\mathrm{ca} .1 \mathrm{~Hz}, 4 \mathrm{H}\right), 5.96$ (bt, $J=\mathrm{ca} .1 \mathrm{~Hz}$, $2 \mathrm{H}), 7.95(\mathrm{~s} 2 \mathrm{H}) ; \delta_{\mathrm{C}} 18.2,25.32,107.0,117.7,122.1,124.4,127.8,144.2$.

II Compound $8: 41 \%$, mp $130^{\circ} \mathrm{C}$ (dec.). NMR $\left(\mathrm{CDCl}_{3}\right) \delta_{\mathrm{H}} 3.24(\mathrm{~d}, J=1.2 \mathrm{~Hz}, 4 \mathrm{H}), 3.34\left(\mathrm{~s}, \mathrm{CH}_{2}\right), 5.87(\mathrm{t}, J=1.2 \mathrm{~Hz}, 2 \mathrm{H}), 7.94(\mathrm{~s}$, $2 \mathrm{H}) ; \delta_{\mathrm{C}} 19.1,24.4,112.7,122.7,131.9,135.4,141.1,184.8$. The crude product $(96 \%)$ can be used directly for transformation into 4.

${ }^{\dagger}+$ Compound 4: $80 \%$, mp $132^{\circ} \mathrm{C}$ (sub.). NMR $\left(\mathrm{CDCl}_{3}\right) \delta_{\mathrm{H}} 3.41\left(\mathrm{~s} \mathrm{CH}_{2}\right), 7.79-7.81(2 \mathrm{H}), 8.16(\mathrm{~s}, \mathrm{H} 2 / 9), 8.31-8.34(2 \mathrm{H}) ; \delta \mathrm{C}$ $19.2,113.5,127.2,132.8,133,1,134.0,137.0,183.3 . v_{\max } 1668 \mathrm{~cm}^{-1} ; \lambda_{\max }(\mathrm{MeCN}) 259(4.65), 280 \mathrm{sh}(4.04), 324 \mathrm{~nm}(10 \mathrm{~g}$ E3.61).
} 


\section{References}

1. Halton, B. In The Cyclopropy/ Group, Pt. 3; Rappoport, Z., Ed. The chemistry of the functional groups. Wiley: Chichester, 1995, pp. 707-772; Chem. Rev. 1989, 89, 1161-1185.

2. Halton, B.; Stang, P. J. Synlett 1997, 145-158.

3. Collis, G. E.; Jayatilaka, D.; Wege, D. Aust. J. Chem. 1997, 50, 505-513.

4. Halton, B.; Kay, A. J.; Zhi-mei, Z.; Boese, R.; Haumann, T. J. Chem. Soc.. Perkin Trans. 1 1996, 1445-1452, 1993. 2239-2240).

5. Watabe, T.; Okada, K.; Oda, M. J. Org. Chem. 1988, 53, 216-218

6. Ando, S.; Imamura, J.; Tajitsu, M.; Saito, K. Heterocycles 1998, 48, 1769-1776.

7. Saracoglu, N.; Durucasu, I.; Balci, M. Tetrahedron 1995, 51, 10979-10986.

8. Laue, J.; Seilz, G.; Wassmuth, H. Z. Naturforsch. Teil B. 1996, 51, 348-358.

8. Vogel, E.; Grimme, W.; Korte, S. Tetrahedron Lett. 1965, 3625-3631; Korte, S. PhD Thesis, University of Köln, 1968.

10. Cooney, M. J.; Halton, B. Aust. J. Chem. 1996, 49, 533-538. 
Studies in the cycloproparene series: chemistry of $1 \mathrm{H}$-cyclopropa $[b]$ naphthalene-3,6-dione and its transformation into $1 H$-cyclopropa[b]anthracene-3,8-dione ${ }^{1}$

\author{
Brian Halton, ${ }^{* a}$ Carissa S. Jones, ${ }^{a}$ Andrew J. Kay, ${ }^{\circ} \dagger$ Davor Margetic ${ }^{b}$ and Sanja Sretenovic ${ }^{a}$ \\ a School of Chemical \& Physical Sciences, Victoria University of Wellington, PO Box 600, \\ Wellington, New Zealand \\ ' Centre for Molecular Architecture, Central Queensland University, Rockhampton, \\ Queensland 4702, Australia
}

Received (in Cambridge, UK) 4th April 2000, Accepted 2nd June 2000

Published on the Web 3rd July 2000

$1 \mathrm{H}$-Cyclopropa[ $b]$ naphthalene-3,6-dione 3 adds bromine stoichiometrically across the enedione $\mathrm{C}=\mathrm{C}$ bond to give dibromodihydrocyclopropanaphthalenedione 10 while with an excess of the reagent (bromomethyl)tribromonaphthoquinone 12 is formed. Typical quinone character is exhibited by 3 in Diels-Alder cycloadditions and it gives the endo-methanocyclopropanthraquinone 14 with cyclopentadiene. With buta-1,3-diene the analogous tetrahydrocyclopropanthraquinone 15 is formed from a temperature dependent reaction. Above $45^{\circ} \mathrm{C}$ opening of the threemembered ring of 4 also occurs and the cyclopentanthracenedione 16 is obtained from $[\pi 2+\pi 4]$ and $[02+\pi 2]$ me sole product at $100^{\circ} \mathrm{C}$. Enolisation of the tetrahydroanthraquinone 15 provides diphenolate 18 and this can be diverted to diether 19 or readily oxidized to the dihydroanthraquinone 20 . In turn, 20 is dehydrogenated to the fully aromatic cyclopropa[ $b]$ anthracene-3,8-dione 4 , the first anthraquinone of the cycloproparene series.

Although the chemistry of the cycloproparenes has received significant attention over the past years, ${ }^{2}$ only three cyclopropaquinones $(1-3)$ have been reported. ${ }^{3-3}$ The first and<smiles>O=C1C=CC(=O)C2=C1C2</smiles><smiles>O=C1C2=CC2C2C=CC12</smiles><smiles></smiles><smiles>O=C1c2ccccc2C(=O)c2ccccc21</smiles>

simplest of these, $1 H$-cyclopropabenzene-2,5-dione 1 , was prepared by Oda and his colleagues, but it was too reactive for isolation under normal conditions and its formation was deduced from appropriate trapping experiments. ${ }^{3}$ The same was found to hold $\mathrm{d}^{4}$ for the cyclopropa[b]naphthalene analogue 2. However, incorporation of an enedione moiety into the ring remote from cyclopropa fusion led to cyclopropa[ $b]$ naphthalene-3,6-dione 3 as a stable, yellow crystalline compound whose structure has been confirmed by $\mathrm{X}$-ray analysis. ${ }^{5}$ This compound is intermediate to naphthoquinone and anthraquinone in its ease of reduction as established from cyclic voltammetry. ${ }^{5}$ We now report on the essential chemical reactivity of quinone 3 that provides for its use as a synthon to more complex polycyclic assemblies by way of $[4+2]$ DielsAlder methodology. As the cycloproparene framework is

† Present address: Industrial Research Ltd., Gracefield Research Centre, PO Box 31-310, Lower Hutt, New Zealand.

DOI: $10.1039 / \mathrm{b} 002683 \mathrm{~m}$ usually retained in the absence of electrophilic reagents, ${ }^{2}$ this allows for the elaboration of the series from cyclopropanaphthalene-3,6-dione 3 to cyclopropanthracene-3,8dione 4 . Quinone 4 has possible use as a new molecular dione 4. Quinone 4 has possible use as a new molecular conjugation through a C-1 exocyclic olefin.

It is known that cyclopropabenzene behaves as an electron rich dienophile in Diels-Alder cycloadditions and adds electron deficient dienes across the C-1a-C-5a bridge bond. ${ }^{2}$ Howtron deficient dienes across thembered ring can also occur. ${ }^{6}$ Thus, with butadiene at $80^{\circ} \mathrm{C}$ cyclopropabenzene gives 2-vinylindane 5 from $[3+2]$ addition across the $\sigma$ bond, small amounts of the analogous $[3+4]$ adduct 6 , and some of the dihydro[10]annulene 7 that arises from sequential $[2+4]$ addition across the $\mathrm{Cla}-\mathrm{C} 5 \mathrm{a}$ bond followed by norcaradiene valence isomerthe $\mathrm{Cla}-\mathrm{C} 5 \mathrm{a}$ bond followed $\mathrm{Ag}(\mathrm{l})$ catalysis the regioselectivity of addition to the $\sigma$ bond is changed and it is the regioisomeric 1-vinylindane 8 that is obtained. ${ }^{8}$ In similar vein, easy

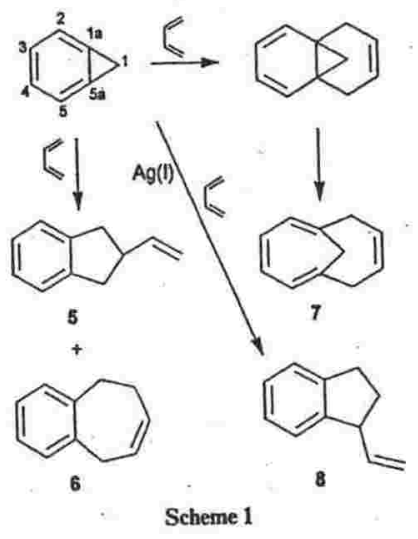

J. Chem. Soc., Perkin Trans. I, 2000, 2205-2210

This journal is $\odot$ The Royal Society of Chemistry 2000 
ring-opening with electrophilic reagents is illustrated by the formation of benzyl ethers from reaction with alcohols in a reaction that is catalysed by $\mathrm{Ag}(1)$ (Scheme 2). ${ }^{\mathrm{g}-17}$ In order to

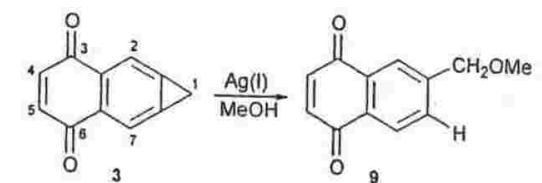<smiles>Cc1cc2c(cc1Br)C(=O)C(Br)C(Br)C2=O</smiles><smiles>[R]c1cc2c(cc1[R])C(C(=O)O)C(Br)C(Br)C2=O</smiles><smiles>O=C1C(Br)=C(Br)C(=O)c2cc3c(cc21)C3(Br)Br</smiles>

Scheme 2

compare the reactivity of the three-membered ring $\sigma$ bond with that of the enedione olefinic bond of quinone 3 , the behaviour with methanol, bromine, cyclopentadiene and butadiene has been examined.

As part of the formal characterization of quinone 3, we reported ${ }^{5}$ that $\mathrm{Ag}(1)$-catalysed methanolysis results in the known" 6-(methoxymethyl)naphthalene-1,4-dione 9 from electrophilic opening of the three-membered ring (Scheme 2). What is more relevant here, however, is the fact that in competition for molecular bromine ( 1 mol equiv.) it is the enedione $\pi$ bond that adds the reagent and the trans-dibromide 10 is formed almost quantitatively from reaction in tetrachloromethane. The structure of the product is fully compatible with the spectroscopic data. The two proton singlet at $\delta 4.99$ is expected for a trans-1,2-dibromocyclohexane ring ${ }^{12}$ and it replaces that at $\delta 6.95$ for $4-\mathrm{H} / 5-\mathrm{H}$ of 3 . The retention of the methylene moiety [10: $\delta 3.44 / 19.7 ; 3: \delta 3.36 / 19.1]$, the presence of only two methine signals, one of which corresponds to the shielded C-2(5) carbon atoms $(\delta 114.4)$, and the appearance of three quaternary carbons are fully consistent with the assigned structure. In a modification of the experimental procedure tetrachloromethane-methanol was employed as the solvent system whereupon a different outcome was recorded. In this case the product is precipitated as the reaction proceeds and addition of a further aliquot of bromine provides more of the same compound. The product is insoluble in common solvents and is neither dibromide 10 nor the diastereomeric tetrabromides 11 from dibromination. Rather, facile oxidation of 11 provides the aromatic tetrabromide 12 as the isolated compound (Scheme 2). While the path from 3 to 12 via 10 and 11 is entirely plausible. we cannot exclude the alternative route via 9 in which the oxidative dehydrogenation precedes opening of the three-membered ring (Scheme 2). Compound 12 displays the mree-membered ter of a tetrabromide $(m / 2484 / 486 / 488 / 490 / 492: 4 / 15 / 20 / 13 / 3)$ and the three ' $\mathrm{H}$ NMR signals $\left(\delta 4.65, \mathrm{CH}_{2} ; 8.22, \mathrm{CH} ; 8.36\right.$, $\mathrm{CH}$ ) closely resemble the corresponding peaks of 2-bromo-3(bromomethyl)naphthalene. ${ }^{13}$

Reaction of quinone 3 with excess cyclopentadiene at ambient temperature results in a $1: 1$ adduct that is assigned as the<smiles>O=C1C=CC(=O)c2cc3c(cc21)C1CCC3C1C1C(=O)c2cc3c(cc2C1=O)C3</smiles><smiles></smiles>

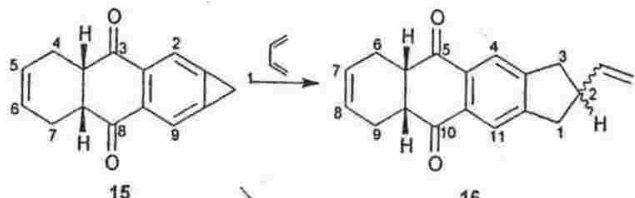

15

16<smiles>CC(C)(C)CCCC(C)(C)O</smiles><smiles>[R]c1c2c(c([R])c3cc4c(cc13)C4)CC=CC2</smiles>

$\mathrm{Me}_{2} \mathrm{SO}_{4}\left[\begin{array}{rl}18 \mathrm{R} & =\mathrm{OK}^{+} \\ 19 \mathrm{R} & =\mathrm{OMe}\end{array}\right.$<smiles>C[C@@H]1C2=CC3(C)CC=CCC13C=C1C(=O)CC=CC[C@H]1C2=O</smiles>

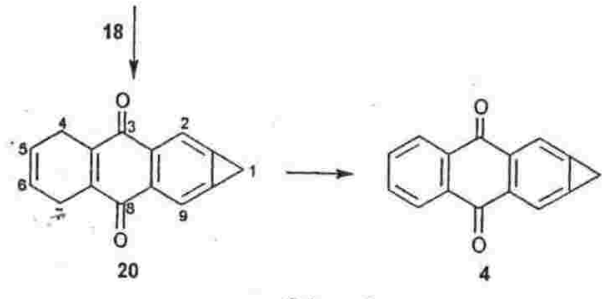

Scheme 3

endo-product 14 from Diels-Alder addition across the enedione olefinic double bond (Scheme 3). The presence of only nine signals in the ${ }^{13} \mathrm{C}$ NMR spectrum is nicely compatible with 14 and excludes the product from addition across the threemembered ring $\sigma$ bond. The highly stereoselective formation of the endo-adduct is deduced from the characteristic coupling between the bridge ( $3 \mathrm{a}-\mathrm{H} / 7 \mathrm{a}-\mathrm{H}: \delta 3.44$ ) and bridgehead protons (4-H/7-H: $\delta 3.65$ ) that requires the bridge protons to be oriented exo.

The ease with which quinone 3 adds not only cyclopentadiene but also isobenzofuran ' across the olefinic $\pi$ bond suggests its use as a synthon for the annelated cyclopropanthracene-3,8-dione 4 (Scheme 3). Indeed, the addition of buta-1,3diene occurs easily under sealed tube conditions but the excess of this reagent that is invariably present impacts upon the outcome. When the Carius tube reaction is effected at $100^{\circ} \mathrm{C}$ cycloaddition takes place at both the enedione $\pi$ bond and the cyclopropene $\sigma$ bond to give a symmetrical diadduct that is identified as [5a $\alpha, 9 \mathrm{a} \alpha$ ]-2-ethenyl-5a, 6,9,9a-tetrahydro- $1 \mathrm{H}$ cyclopenta[b]anthracene-5,10-dione 16 rather than the desired mono-addition product 15 (Scheme 3 ). The symmetry of 16 is evident from the ${ }^{13} \mathrm{C}$ NMR spectrum which displays only 11 lines for the centrosymmetric molecule (Experimental section). These observations exclude the unsymmetrical regioisomeric homologue of 8 with the vinyl substituent at $C-1$, as well as the benzocycloheptene equivalent to 6 from $[4+3]$ addition across the three-membered ring $\sigma$ bond (Scheme 1). The assignment of the product as indane 16 rather than the norcaradiene 17 (or its ring opened valence bond isomer) from addition across the C-1a-9a bridge also follows from the spectroscopic data. The ${ }^{1} \mathrm{H}$ NMR resonances at $\delta 5.01,5.10$, and 5.93 are compatible 
with the monosubstituted vinyl group, that at $\delta 5.73$ with the symmetrical disubstituted olefin $(7-\mathrm{H} / 8-\mathrm{H})$, and the two-proton aromatic singlet $(\delta 7.84)$ is required for 16 but not 17 (Scheme 3 ). Although the opening of the three-membered ring recorded for 3 matches the behaviour of cyclopropabenzene discussed above (Scheme 1), a more appropriate model for cyclopentanthracene 16 was sought. Hence cyclopropa[b]naphthalene 21 was reacted with buta-1,3-diene at $100^{\circ} \mathrm{C}$. The single product isolated in $57 \%$ yield is confidently assigned as 2-ethenyl-2,3-dihydrocyclopenta[b]naphthalene 22 (Scheme 4)

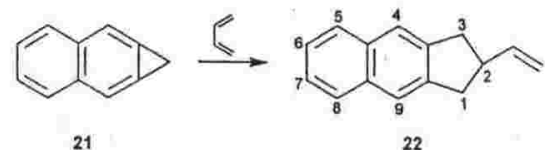

Scheme 4

and the NMR data for its 2-vinylindane moiety mirror those found for 16 (Experimental section).

On the scale at which this work was performed it was not possible to exclude excess butadiene from the cycloadditions, but by careful adjustment of the reaction conditions the outcome can be controlled. As the temperature of the reaction is decreased from $100^{\circ} \mathrm{C}$ the proportion of cyclopentanthracene 16 decreases and at $45^{\circ} \mathrm{C}$ the addition of butadiene is predominantly to the enedione $\mathrm{C}=\mathrm{C}$ bond of quinone 3 . After 4 days the crude reaction mixture contains mono-adduct 15 and substrate 3 with only traces of di-adduct 16. Crystallization of the crude product mixture (benzene, $5^{\circ} \mathrm{C}$ ) provides two distinct crystal types, green and pale yellow, that are easily separated by hand. The green material is a mixture of 3 and 15 ( $\mathrm{ca} .2: 1$; NMR) whereas the pale yellow crystals are almost pure 15 that can be used directly for subsequent transformations. An analytical sample of dione $\mathbf{1 5}$ was obtained, however, only after several recrystallizations because of easy oxidation (vide infra). ${ }^{14}$ Retention of the cycloproparene skeleton in 15 is evident from the NMR spectra as the $\mathrm{C}-1$ methylene protons appear as a broadened singlet $(\delta 3.36)$ and the ortho carbon atoms C-2/9 are characteristically ${ }^{2}$ shielded and appear at $\delta$ 113.2. The resonances recorded for the AMX spin system of $4-\mathrm{H} / 7-\mathrm{H}$ at $\delta 2.19-2.29$ and $2.47-2.57$, and $3 \mathrm{a}-\mathrm{H} / 7 \mathrm{a}-\mathrm{H}$ at $\delta 3.36-$ 3.41 , respectively, are complex. Dreiding molecular models of 15 show a distinct preference for the new ring that spans $C-4$ to $\mathrm{C}-7$ to adopt a half-chair conformation and molecular orbital calculations at the $6-31 \mathrm{G}^{*}$ level (without geometrical constraints) confirm this as the energy minimum (Fig. la) with dihedral angles for $3 \mathrm{a}-\mathrm{H}-\mathrm{C}-3 \mathrm{a}-\mathrm{C}-7 \mathrm{a}-7 \mathrm{a}-\mathrm{H}$ and $\mathrm{C}-4-\mathrm{C}-3 \mathrm{a}-\mathrm{C}$ $7 \mathrm{a}-\mathrm{C}-7$ of $58.2^{\circ}$ and $58.0^{\circ}$, respectively; calculated bond lengths and angles are shown in Fig. 2. By constraining the $3 \mathrm{a}-\mathrm{H}-\mathrm{C}-3 \mathrm{a}-$ $\mathrm{C}-7 \mathrm{a}-7 \mathrm{a}-\mathrm{H}$ dihedral angle to $0^{\circ}$ the calculations also detect four shallow energy wells $\mathrm{ca}$. $32-37 \mathrm{~kJ} \mathrm{~mol}^{-1}$ above the minimum and these correspond to the distinct conformers of 15 shown in Fig. 1b-e. Despite cooling to $-50^{\circ} \mathrm{C}$ the ' $\mathrm{H}$ NMR spectrum of 15 remains unchanged.

The ring-fused tetrahydroanthraquinone 15 contains a dihydroxynaphthalene valence isomer and aromatization of this moiety to 18 is achieved quantitatively with $\mathrm{K}_{2} \mathrm{CO}_{3}$. This salt has been characterized in solution from its NMR data and by conversion into the dimethoxy derivative 19. Attempts to protonate 18 have been frustrated because upon workup its oxidation product, dihydrocyclopropanthraquinone 20 is isolated (Experimental section). Apart from displaying comparable $1-\mathrm{H}$ and $2-\mathrm{H} / 9-\mathrm{H}$ proton singlets, and shielded $\mathrm{C}-2 / 9$ carbon resonances, 18 and 19 display oxygen-substituted ipso aromatic carbons at $\delta 144.2$ and 150.9 whereas diones 15 and 20 have the carbonyl resonances at $\delta 198.1$ and 184.8 , respectively; 1,4 dihydroxybenzene is likewise transformed to its dianion with $\mathrm{K}_{2} \mathrm{CO}_{3}$ under the same reaction conditions and it provides comparable NMR data. The 4,7-dihydroanthraquinone $\mathbf{2 0}$,
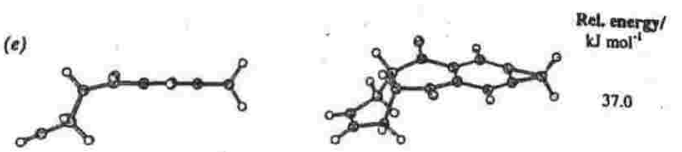

(d)
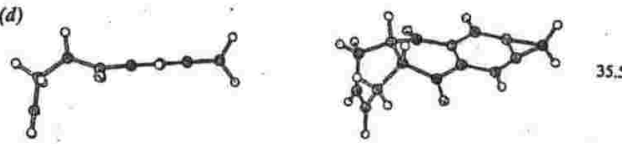

(c)
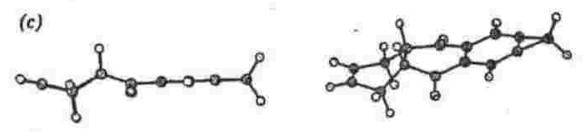

32.2

(b)
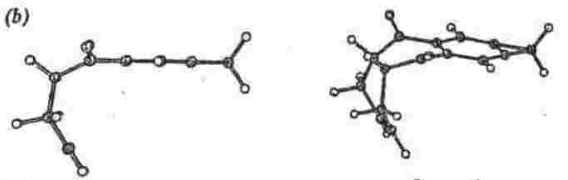

31.9

(a)
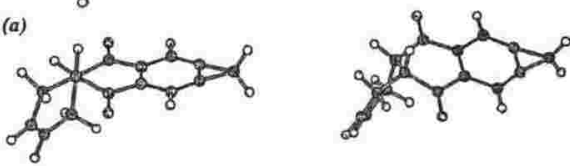

0.0

Fig. 1 Calculated $\left(6-31 \mathrm{G}^{*}\right)$ conformations of Diels-Alder adduct 15 in order of increasing energy $(a)$ without constraint and $(b)-(e)$ with the $3 \mathrm{a}-\mathrm{H}-\mathrm{C}-3 \mathrm{a}-\mathrm{C}-7 \mathrm{a}-7 \mathrm{a}-\mathrm{H}$ dihedral angle constrained to $0^{\circ}$.

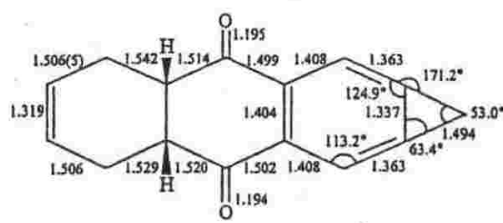

Fig. 2 Calculated geometry of tetrahydroanthracenedione 15; bond lengths in $\AA$ and angles in degrees.

obtained by oxidation of 18 , is a yellow solid isolated in $41 \%$ yield and it displays the spectral characteristics expected of a ring-annelated variant of cyclopropanaphthalenedione 3 . More notable, however, is the relative instability of the compound as standing for a period of days results in darkening and even at $-16^{\circ} \mathrm{C}$ some $9 \%$ of 20 is oxidized to cyclopropanthraquinone 4 over 17 days. This desired transformation can be brought about more efficiently by passing oxygen through a solution of $\mathbf{2 0}$ ( $80 \%$ yield), by treating the dihydro derivative with DDQ $(58 \%)$, or by standing 20 over $\mathrm{K}_{2} \mathrm{CO}_{3}(69 \%)$. The cyclopropanthraquinone 4 so obtained is a yellow crystalline compound that is markedly more stable than the only other oxygenated cyclopropanthracene recorded, namely the 3,8-dioxa derivative which dimerizes below $0{ }^{\circ} \mathrm{C}{ }^{15}$ It is the first quinone in the cyclopropanthracene series and, to the best of our knowledge, only the seventh cyclopropa[b]anthracene prepared. ${ }^{10.15,16}$ The compound provides a cyclic voltammogram ${ }^{5}$ almost identical to that of 9,10-anthraquinone recorded under the same conditions. This shows that in the anthracene series cyclopropa[b] fusion does not alter the quinone redox characteristics. Quinone 4 shows the conjugated carbonyl stretching frequency at $1668 \mathrm{~cm}^{-1}$ and in the NMR spectra the methylene group appears at $\delta 3.55 / 19.2, \mathrm{CH}(2 / 9)$ at $8.14 / 113.5$, and $4-\mathrm{H} / 7-\mathrm{H}$ as an $\mathrm{AA}^{\prime} \mathrm{BB}^{\prime}$ coupled system at $\delta 7.76-7.82$ and 8.28-8.32 with their respective carbons at $\delta 134.0$ and 127.2 . This AA' $^{\prime} B^{\prime}$ system and the carbonyl carbon resonance match well those of 9,10 -anthraquinone in the same solvent. In like manner the resonances of the cyclopropabenzenediyl moiety complement those of cyclopropabenzene and demonstrate that the impact 
of cyclopropa fusion on the NMR parameters cannot be detected beyond the ring in which it is locited.

The potential of naphthoquinone 3 as a synthon for other linear cycloproparene homologues forms a part of our continuing studies.

\section{Experimental \\ General}

Microanalyses were performed by the Analytical Facility of Otago University, Dunedin. Low-resolution mass spectra were recorded on a Hewlett Packard HP-5995C instrument and accurate mass measurements made either by $\mathrm{Mr} \mathrm{B}$. Clark of the Chemistry Department, University of Canterbury, Christchurch, on a Kratos MS80 RFA instrument or Mr O. Zubkov on Victoria University PE Biosystems Mariner operating in electrospray mode. ${ }^{1} \mathrm{H}$ and ${ }^{13} \mathrm{C}$ NMR spectra were recorded on a Varian Unity INOVA $300 \mathrm{MHz}$ instrument for (D)chloroform solutions, except where stated, using the residual solvent peak as internal standard. 'H NMR multiplicities are defined by the usual notation and coupling constants are in hertz. The assignment of ${ }^{13} \mathrm{C}$ and ${ }^{1} \mathrm{H}$ NMR resonances were made with the aid of DEPT and ${ }^{1} \mathrm{H}-{ }^{-} \mathrm{H}$ COSY and ${ }^{13} \mathrm{C}-{ }^{1} \mathrm{H}$ HSQC experiments, and heteronuclear multiple bond connectivity (HMBC) experiments. IR spectra of solid samples were recorded for $\mathrm{KBr}$ disks and all other samples between $\mathrm{NaCl}$ plates using a Biorad FTS 7 spectrophotometer. UV measurements were acquired from a Hewlett-Packard 8452A diode array spectrophotometer. Melting points were determined on a Reichert hot-stage melt ing point apparatus and are uncorrected. Geometry optimiz ations were performed using the SPARTAN programme ${ }^{17}$ employing the ab initio $6-31 \mathrm{G}^{\circ}$ basis set ${ }^{18}$ on a Silicon Graphics $\mathrm{O} 2 \mathrm{R} 5000$ workstation.

Thin layer chromatographic (TLC) analyses were performed using Merck Kieselgel (Alufoilen) $60 \mathrm{~F}_{254}$ to a thickness of 0.2 $\mathrm{mm}$. Components were detected under an ultraviolet lamp at 254 or $350 \mathrm{~nm}$, or in an iodine chamber. Preparative TLC plates were coated with Merck Kieselgel GF $_{254}$ to a thickness of 0.75 $\mathrm{mm}$ and radial chromatography plates were coated with Merck Kieselgel $60 \mathrm{GF}_{254}$ to a thickness of 2.0 or $4.0 \mathrm{~mm}$. Column chromatography employed Riedel-de Haën silica gel S (230-400 ASTM) unless otherwise stated.

\section{Reactions of $1 H$-cyclopropa[ $b]$ naphthalene-3,6-dione 3 with bromine}

A. In tetrachloromethane: trans-4,5-dibromo-4,5-dihydro- $1 \mathrm{H}$ cyclopropa $[b]$ naphthalene-3,6-dione 10 . To a stirred solution of dione $3(85 \mathrm{mg}, 0.5 \mathrm{mmol})$ in dry tetrachloromethane $(10 \mathrm{ml})$ under oxygen-free nitrogen was added dropwise bromine $(88$ $\mathrm{mg}, 28 \mathrm{~L}, 0.55 \mathrm{mmol})$ in the same solvent $(2 \mathrm{ml})$. The paleorange solution was stirred for $1 \mathrm{~h}$, concentrated under vacuum and light petroleum $(c a .15 \mathrm{ml})$ added. The mixture was then shaken v,gorously, the upper petroleum layer decanted from the dark insoluble residue, and the extraction repeated. The combined organic extracts were then concentrated under vacuum to afford 10 as a thick orange oil (150 mg, 91\%) (Found: $\mathrm{M}^{+}$ 329.8712 and 327.8736. $\mathrm{C}_{11} \mathrm{H}_{6}{ }^{81} \mathrm{Br}^{79} \mathrm{Br}$ and $\mathrm{C}_{11} \mathrm{H}_{6}{ }^{79} \mathrm{Br}_{2}$ require M. 329.8714 and 327.8735, respectively); $v_{\max } / \mathrm{cm}^{-1} 3001.2951$, $1703,1698,1694,1557,1410,1283,1223,1127,1063,978,880$, 735; NMR $\delta_{\mathrm{H}} 3.44\left(2 \mathrm{H}, \mathrm{s}, \mathrm{CH}_{2}\right), 4.99(2 \mathrm{H}, \mathrm{s} 4-\mathrm{H} / 5-\mathrm{H}), 7.95$ $(2 \mathrm{H}, \mathrm{s}, 2-\mathrm{H} / 7-\mathrm{H}) ; \delta_{\mathrm{c}} 19.7(\mathrm{C}-1), 45.9(\mathrm{C}-4 / 5), 114.4(\mathrm{C}-2 / 7)$, 133.8 (C-2a/6a), 134.5 (C-1a/7a), $186.6(\mathrm{C}-3 / 6) ; m i=332 / 330 / 328$ $\left(\mathrm{M}^{+}, 19 / 42 / 20 \%\right), 251 / 249(91 / 100, \mathrm{M}-\mathrm{Br}), 170(45), 114(47)$, 88 (29).

B. In tetrachloromethane-methanol: 2,3,6-tribromo-7-(bromomethyl)naphthalene-1,4-dione 12 . To a stirred solution of 3 (50 $\mathrm{mg}, 0.29 \mathrm{mmol})$ in tetrachloromethane $(10 \mathrm{ml})$ at $0{ }^{\circ} \mathrm{C}$ under oxygen-free nitrogen, was added dropwise a solution of brom- ine ( $47 \mathrm{mg}, 15 \mathrm{~L}, 0.29 \mathrm{mmol})$ in the same solvent $(1.5 \mathrm{ml})$. The solution was stirred for $10 \mathrm{~min}$ and methanol $(10 \mathrm{ml})$ was added rapidly. The solution stirred for $24 \mathrm{~h}$ and the pale-yellow precipitate collected by filtration. A further portion of bromine $(0.29 \mathrm{mmol})$ in tetrachloromethane $(1.5 \mathrm{ml})$ was added to the filtrate and stirring was continued for $36 \mathrm{~h}$. More of the paleyellow precipitate formed and was collected by filtration. Mass spectrometry and 'H NMR spectroscopy indicated the isolated precipitates to be identical. The compound, insoluble in common solvents, is assigned as $12(106.4 \mathrm{mg}, 75 \%)$, mp $242-$ $244^{\circ} \mathrm{C}$ (Found: $\mathrm{C}, 27.1 ; \mathrm{H}, 0.67 ; \mathrm{Br}, 65.0 . \mathrm{C}_{11} \mathrm{H}_{4} \mathrm{Br}_{4} \mathrm{O}_{2}$ requires C, $27.1 ; \mathrm{H}, 0.83 ; \mathrm{Br}, 65.5 \%) ; 1_{\max } / \mathrm{cm}^{-1} 3088,3028,1676,1588$, $1539,1283,1213,1190,1092,970,943,831,797,725,718$; NMR $\delta_{\mathrm{H}} 4.65\left(2 \mathrm{H}, \mathrm{s}, \mathrm{CH}_{2} \mathrm{Br}\right), 8.22(1 \mathrm{H}, \mathrm{s}, 4-\mathrm{H}) .8 .36(1 \mathrm{H}, \mathrm{s}$, $1-\mathrm{H}) ; m /=492 / 490 / 488 / 486 / 484(3.4 / 13.4 / 20.3 / 14.6 / 3.8 \%, M)$, $411 / 409 / 407 / 405(32 / 100 / 100 / 34, \mathrm{M}-\mathrm{Br}), 383 / 381 / 379 / 377$ $(9 / 27 / 27 / 10, \mathrm{M}-\mathrm{Br}-\mathrm{CO})$.

Reaction of $1 H$-cyclopropa[ $b$ ]naphthalene-3,6-dione 3 with cyclopenta-1,3-diene: [3aa,4a,7a,7a $\alpha]-4,7$-methano-3a,4,7,7atetrahydro-1 $H$-cyclopropa[ $b]$ anthracene-3,8-dione 14

To a stirred solution of $3(170 \mathrm{mg}, 1.0 \mathrm{mmol})$ in benzene $(20 \mathrm{ml})$ at RT and under oxygen-free nitrogen was added dropwise freshly distilled cyclopenta-1,3-diene $(225 \mathrm{mg}, 0.28 \mathrm{ml}, 3.4$ $\mathrm{mmol})$. The bright yellow solution was stirred until colourless $(4 \mathrm{~h})$ and then concentrated under vacuum to a white solid. TLC (dichloromethane) indicated two components $\left(R_{\mathrm{F}} 0.0,0.8\right)$ and the most mobile fraction was isolated by radial chromatography (dichloromethane elution). The resultant white solid $(216 \mathrm{mg}$ ) was recrystallized (light petroleum) to afford 14 as fine white platelets ( $190 \mathrm{mg}, 80 \%$ ), mp $90-91.5^{\circ} \mathrm{C}$ (Found: C, 81.5 ; $\mathrm{H}$ 4.95. $\mathrm{C}_{16} \mathrm{H}_{12} \mathrm{O}_{2}$ requires $\left.\mathrm{C}, 81.3 ; \mathrm{H}, 5.1 \%\right) ; v_{\max } / \mathrm{cm}^{-1} 3061$, $3005,2936,2874,1667,1564,1402,1335,1294,1265,1229$, $1084,1061,982,893,785,712$; NMR $\delta_{\mathrm{H}} 1.52-1.59(2 \mathrm{H}, \mathrm{m}, 10-$ $\mathrm{H}), 3.33(2 \mathrm{H}, \mathrm{s}, 1-\mathrm{H}), 3.44(2 \mathrm{H}, \mathrm{dd}, \mathrm{l} .5,2.3,3 \mathrm{a}-\mathrm{H} / 7 \mathrm{a}-\mathrm{H}), 3.63-$ $3.66(2 \mathrm{H}, \mathrm{m}, 4-\mathrm{H} / 7-\mathrm{H}), 5.96(2 \mathrm{H}, \mathrm{t}, 1.8,5-\mathrm{H} / 6-\mathrm{H}), 7.84(2 \mathrm{H}, \mathrm{s}$ $2-\mathrm{H} / 9-\mathrm{H}) ; \delta_{\mathrm{C}} 19.2(\mathrm{C}-1), 49.2(\mathrm{C}-10), 49.3 / 49.4(\mathrm{C}-3 \mathrm{a} / 7 \mathrm{a}$ and $\mathrm{C}-4 / 7), 113.0$ (C-2/9), 132.7 (C-1a/9a), 135.5 (C-5/6), 139.9

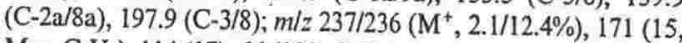
$\left.\mathrm{M}-\mathrm{C}_{5} \mathrm{H}_{5}\right), 114(17), 66\left(100, \mathrm{C}_{5} \mathrm{H}_{6}\right)$.

cis-3a,4,7,7a-Tetrahydro-1 $H$-cyclopropa[ $b]$ anthracene-3,8-dione 15

To a calibrated Carius tube $(300 \mathrm{ml})$ was added naphthoquinone $3(1.5 \mathrm{~g}, 8.8 \mathrm{mmol})$, a magnetic follower and benzene ( $30 \mathrm{ml}$ ). The mixture was cooled to $-50^{\circ} \mathrm{C}$ and buta-1,3-diene $(5 \mathrm{ml}, 56.8 \mathrm{mmol})$ was added by vapour transfer. The sealed tube was warmed to $45^{\circ} \mathrm{C}$ and the contents maintained at this temperature with stirring for 4 days. The cooled tube (liquid nitrogen) was opened, slowly warmed to RT and the mixture filtered though a plug of cotton wool. The filtrate was concentrated under reduced pressure to a yellow-brown oily solid $(1.90 \mathrm{~g})$ that contained ('H NMR) unchanged 3 and tetrahydrocycloproparene 15 ( $c a .3: 17$ ) and only traces of diadduct. The crude solid product was purified by:

Method A. Recrystallization from benzene at $5{ }^{\circ} \mathrm{C}$ predominantly gave pale yellow crystals $(565 \mathrm{mg}, 27 \%$ ) together with some green crystals $(50 \mathrm{mg}$ ) that were separated by hand. The pale yellow crystals contained ( ${ }^{1} \mathrm{H}$ NMR) 15 with traces $(<2 \%)$ of 3 while the green crystals contained these same compounds in a $2: 1$ ratio. The pale yellow crystals, suitable for subsequent use (see below), were recrystallized first from benzene and then from ether-tetrahydrofuran $(1: 1)$ to give an analytical sample (19.4 mg) of adduct 15 as pale yellow diamond-shaped crystals, mp $96-97^{\circ} \mathrm{C}$ (Found: $\mathrm{C}, 80.2 ; \mathrm{H}, 5.5 . \mathrm{C}_{15} \mathrm{H}_{12} \mathrm{O}_{2}$ requires $\mathrm{C}$, 80.3; H, 5.4\%); $\lambda_{\max }$ (acetonitrile)/nm 235 (log $\left.\varepsilon 4.42\right), 273$ (3.81); $v_{\max } / \mathrm{cm}^{-1} 3029,2974,2938,2900,2851,1678,1556,1431,1246$, $1178,1053,878,706$; NMR $\delta_{\mathrm{H}} 2.19-2.29\left(2 \mathrm{H}_{\mathrm{A}}, \mathrm{AMX}, 4-\mathrm{H} / 7-\right.$ 
H), 2.47-2.57 (2 $\left.\mathrm{H}_{\mathrm{M}}, \mathrm{AMX}, 4-\mathrm{H} / 7-\mathrm{H}\right), 3.36(2 \mathrm{H}, \mathrm{s}, \mathrm{I}-\mathrm{H}) ; 3.36-$ $3.41\left(2 \mathrm{H}_{\mathrm{x}}\right.$, AMX, 3a-H/7a-H), $5.73(2 \mathrm{H}, \mathrm{t}, 1.5, \mathrm{H} 5 / 6), 7.87$ $(2 \mathrm{H}, \mathrm{s}, \mathrm{H} 2 / 9) ; \delta_{\mathrm{C}} 19.5(\mathrm{C}-1), 24.4(\mathrm{C}-4 / 7), 46.5(\mathrm{C}-3 \mathrm{a} / 7 \mathrm{a}), 113.2$ (C2/9), 124.6 (C5/6), 133.0 (C-1a/9a), 137.7 (C-2a/8a), 198.2 $(\mathrm{C}-3 / 8) ; m /=225 / 224\left(\mathrm{M}^{+}, 10 / 60 \%, 196(69, \mathrm{M}-28), 178(52)\right.$, $152(31), 116(30), 105(38), 89(51), 88(82), 87(33), 77(52), 63$ (43), $62(100)$.

Method B. Radial chromatography (dichloromethane-ligh petroleum elution; $1: 1)$ to give a yellow solid $(1.3-1.6 \mathrm{~g}, \mathrm{ca} .65$ $80 \%$ ) that contained enedione 15, dihydroquinone 20 (see below), and naphthoquinone 3 in varying ratios from $15: 0: 5$ to $150: 1: 0.5$. The appearance of dihydroquinone 20 depends on the time that the product mixture resides on the column and this purification procedure is the method of choice for subsequent transformation into anthraquinone 4.

\section{[5aa,9aa]-2-Ethenyl-5a,6,9,9a-tetrahydro-1 $H$ -} cyclopenta[b]anthracene-5,10-dione 16

A freshly prepared solution of dione $3(400 \mathrm{mg}, 2.35 \mathrm{mmol})$ in benzene $(7 \mathrm{ml})$ was placed in a calibrated Carius tube $(30 \mathrm{ml})$. The solution was frozen (liquid nitrogen) and buta-1,3-diene (ca. $3 \mathrm{ml}$, ca. $35 \mathrm{mmol}$ ) added by vapour transfer. After warming to RT the sealed tube was heated at $100^{\circ} \mathrm{C}$ for $24 \mathrm{~h}$ (Carius furnace). The tube was cooled, refrozen to liquid air temperature, opened and the contents slowly warmed to RT. The liquid was concentrated under reduced pressure to a yellow oily solid $(1.49 \mathrm{~g})$ that was pumped under vacuum for $4 \mathrm{~h}$ to give a white solid $(1.07 \mathrm{~g})$. TLC (dichloromethane-light petroleum; $5: 1$ elution) indicated three components $\left(R_{\mathrm{F}} 0.0,0.5,0.7\right)$ and column chromatography (silica gel; dichloromethane-light petroleum, $5:$ I elution) provides a first fraction $(140 \mathrm{mg}$ ) thought to be (NMR) the dimer of butadiene. The second and major component provided a solid which gave $16\left(200 \mathrm{mg} .3 \mathrm{I}^{\mathrm{t}} / \mathrm{11}\right)$ as colourless crystals (dichloromethane-light petroleum, 1:2; $c a$. $-16^{\circ} \mathrm{C}$ ). mp $128-129^{\circ} \mathrm{C}$ (Found: $\mathrm{C}, 81.7 ; \mathrm{H}$, 6.6. $\mathrm{C}_{19} \mathrm{H}_{18} \mathrm{O}_{2}$ requires $\mathrm{C}, 82.0 ; \mathrm{H}, 6.5 \%) ; \lambda_{\max }$ (hexane)/nm $214 \operatorname{sh}(\log \varepsilon 3.58$ ), $235 \mathrm{sh}(4.63), 240(4.70), 268(4.00): v_{\max } / \mathrm{cm}^{-1} 3077,3025,2880$, $2828,1682,1603,1418,1364,1341,1288,1172,1130,920,868$ 835,$752 ; \mathrm{NMR} \delta_{\mathrm{H}} 2.20\left(2 \mathrm{H}_{\mathrm{A}}\right.$, br d, 15.2, 6- H/9-H), $2.50\left(2 \mathrm{H}_{\mathrm{B}}\right.$. br d. 15.2,6-H/9-H), 2.80-2.89 (2 H, m, I-H/3-H), 3.09-3.21

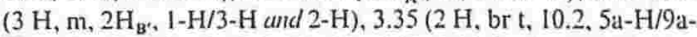
H), $5.01\left(1 \mathrm{H}\right.$, br d, 10.2, $\left.13_{\mathrm{A}}-\mathrm{H}\right), 5.10\left(1 \mathrm{H}\right.$, br d, 17.0, $\left.13_{\mathrm{B}}-\mathrm{H}\right)$, 5.73 (2 H, s, 7-H/8-H), 5.87-5.98 (1 H, m, 12-H), $7.84(2 \mathrm{H}, \mathrm{s}$, $4-\mathrm{H} / 1 \mathrm{l}-\mathrm{H}): \delta_{\mathrm{C}} 24.4(\mathrm{C}-6 / 9), 39.0(\mathrm{C}-1 / 3), 43.9(\mathrm{C}-2), 46.5(\mathrm{C}-5 \mathrm{a} /$ 9a), $114.4(\mathrm{C}-13), 122.5(\mathrm{C}-4 / 11), 124.6(\mathrm{C}-7 / 8), 133.1(\mathrm{C}-4 \mathrm{a}$ 10a), 140.7 (C-12), 150.5 (C-3a/11a), 198.3 (C-5/10); $m /=279$ $278\left(\mathrm{M}^{+}, 17 / 82 \%\right), 250(38, \mathrm{M}-28) .170(17), 115(100)$.

\section{3,8-Dihydroxy-4,7-dihydro- $1 H$-cyclopropa[ $b]$ anthracene dipotassium salt 18}

To an NMR tube flushed with argon and containing anhydrous potassium carbonate $(24 \mathrm{mg}, 0.17 \mathrm{mmol}$ ) was added butadien adduct 15 (24 mg, $0.17 \mathrm{mmol})$ in $d_{6}$-acetone $(0.45 \mathrm{ml})$. After I h cycloproparene 15 was unchanged ('H NMR). The tube was then sonicated for $30 \mathrm{~min}$ after which time substrate 15 and salt 18 were present in a ca. 10:1 ratio. Further sonication for 30 min showed complete conversion into the dipotassium salt 18; NMR (d $d_{6}$-acetone) $\delta_{\mathrm{H}} 3.42\left(2 \mathrm{H}, \mathrm{s}, \mathrm{CH}_{2}\right), 3.50(4 \mathrm{H}, \mathrm{d}, 1.0$, $\left.2 \times \mathrm{CH}_{2}, 4-\mathrm{H} / 7-\mathrm{H}\right), 5.96(2 \mathrm{H}, \mathrm{t}, 1.3,5-\mathrm{H} / 6-\mathrm{H}), 7.95(2 \mathrm{H}, \mathrm{s}$, $2-\mathrm{H} / 9-\mathrm{H}) ; \delta_{\mathrm{C}} 18.2(\mathrm{C}-1), 25.3(\mathrm{C}-4 / 7), 107.0(\mathrm{C}-2 / 9), 117.7$ (C-3a/7a), 122.1 (C-1a/9a), 124.4 (C-5/6), 127.8 (C-2a/8a), 144.2 (C-3/8).

1,4-Dihydroxybenzene displays a single $\mathrm{OH}$ resonance in $d_{6}$-acetone and this was likewise transformed into its dianion under the same conditions.

\section{3,8-Dimethoxy-4,7-dihydro-1 $H$-cyclopropa[b]anthracene 19}

To a stirred solution of 15 (100 $\mathrm{mg}, 0.45 \mathrm{mmol})$ and anhydrous potassium carbonate $(222 \mathrm{mg}, 1.62 \mathrm{mmol})$ in anhydrous acetone $(7 \mathrm{ml})$ under argon at $0{ }^{\circ} \mathrm{C}$, was added dimethyl sulfate $(0.106 \mathrm{ml}, 1.13 \mathrm{mmol})$ over $30 \mathrm{~s}$ by syringe. After stirring overnight (RT) the lemon coloured mixture was filtered under vacuum though Celite and the residue was washed with acetone $(4 \times 25 \mathrm{ml})$. The combined filtrates were concentrated (reduced pressure) to a dark yellow solid which gave a strongly fluorescent pure white solid $(72 \mathrm{mg}, 63 \%)$ as the major and most mobile component on radial chromatography (dichloromethane-light petroleum elution: 1:1). Recrystallization (dichloromethane-light petroleum, $1: 1,-16^{\circ} \mathrm{C}$ ) provided 19 (22 mg, 20\%) as colourless plates, mp $126-130^{\circ} \mathrm{C}$ (Found C. $80.7: \mathrm{H}$ 6.3. $\mathrm{C}_{15} \mathrm{H}_{12} \mathrm{O}_{2}$ requires $\mathrm{C}, 80.9 ; \mathrm{H}, 6.4 \%$ ); $\lambda_{\max }$ (acetonitrile)/nm $216(\log \& .4 .45), 246(4.62), 300$ (3.69); m $/ \mathrm{cm}^{-1} 3072,3029,2993,2932,2876,2827,1744,1671,1600$ $1451,1423,1330.1297,1063,1052,995,950,853 ;$ NMR $_{\mathrm{H}} 3.49$ $\left(2 \mathrm{H}, \mathrm{s}, \mathrm{CH}_{2}\right), 3.56\left(4 \mathrm{H}, \mathrm{d}, 1.2,2 \times \mathrm{CH}_{2}\right), 3.91(6 \mathrm{H}, \mathrm{s}$, $2 \times \mathrm{OMe}), 6.02(2 \mathrm{H}, \mathrm{t}, 1.4,5-\mathrm{H} / 6-\mathrm{H}), 7.82(2 \mathrm{H}, \mathrm{s}, 2-\mathrm{H} / 9-\mathrm{H})$ $\delta_{\mathrm{C}} 18.4(\mathrm{C}-1), 24.3(\mathrm{C}-4 / 7), 61.0(2 \times \mathrm{OMe}), 106.8(\mathrm{C}-2 / 9), 122.9$ (C-la/9a), 124.1 (C-5/6), 124.5 (C-3a/7a), $130.0(\mathrm{C}-2 \mathrm{a} / 8 \mathrm{a}), 150.0$ (C-3/8); $m /=253 / 252\left(\mathrm{M}^{+}, 19 / 100^{\circ} / 11\right), 237(62, \mathrm{M}-\mathrm{Me}), 206$ (70), 205 (38), $178(69), 177(34), 176(44), 165(77), 163(30), 89$ (36), 88 (47), 87 (41), 77 (65), 76 (35), 75 (33), 63 (58), 62 (48), $51(67), 50(37)$

Further elution of the chromatotron plate (same solvent mixture) gave an oily yellow solid (24 mg) that contained ( ${ }^{1} \mathrm{H}$ NMR) 19, cyclopropanthraquinone 4 (see below) and unreacted dimethyl sulfate in a $20: 6: 1$ ratio; purification was not attempted.

\section{4,7-Dihydro- $1 H$-cyclopropa[ $b]$ anthracene-3,8-dione 20}

To a stirred solution of adduct $15(100 \mathrm{mg}, 0.45 \mathrm{mmol})$ in anhydrous acetone $(10 \mathrm{ml})$ under argon, was added anhydrous potassium carbonate $(620 \mathrm{mg}, 4.49 \mathrm{mmol})$ under a stream of argon. The mixture instantly darkened and was stirred for a further $2.5 \mathrm{~h}$ then filtered though a pad of Celite under vacuum. The residue was washed with acetone $(4 \times 25 \mathrm{ml})$ and the combined filtrates concentrated under reduced pressure to a purpleblue sparingly soluble solid ( $97 \mathrm{mg}$ ) shown ('H NMR) to be a mixture of dihydroquinone $\mathbf{2 0}$ with varying amounts of cyclopropanthraquinone 4 (see below). The solid was either left in air until it became yellow or stirred as a suspension in dichloromethane until it dissolved; use of an oxygen atmosphere accelerates the oxidation. Radial chromatography (dichloromethane-light petroleum elution; 1:4) gave, as the major and most mobile component, dihydrodione $\mathbf{2 0}$ as a yellow solid (41 $\mathrm{mg}, 41 \%$ ) that rapidly darkened on standing. Recrystallization (diethyl ether, $-16^{\circ} \mathrm{C}$ ) provided an analytical sample of 20 (23 mg. $23 \%$ ) as yellow crystals, mp $122-124^{\circ} \mathrm{C}$ (decomp.) after sublimation at $111{ }^{\circ} \mathrm{C}$ (Found: $\mathrm{C}, 81.2 ; \mathrm{H} 4.5(5) . \mathrm{C}_{15} \mathrm{H}_{10} \mathrm{O}_{2}$ requires $\mathrm{C}, 81.1 ; \mathrm{H}, 4.5 \%) ; \lambda_{\max }$ (acetonitrile)/nm $260(\log$ \& 4.41), 326 (3.30); $v_{\max } / \mathrm{cm}^{-1} 3037,3010,2948,2923,2852,1655$, $1626,1557,1416,1294,1123,1058,980,933,875,856,723,715$ NMR $\delta_{\mathrm{H}} 3.25\left(4 \mathrm{H}\right.$, d, I.2, $\left.2 \times \mathrm{CH}_{2}\right), 3.34\left(2 \mathrm{H}, \mathrm{s}, \mathrm{CH}_{2}\right), 5.87$ $(2 \mathrm{H}, \mathrm{t}, 1.2,5-\mathrm{H} / 6-\mathrm{H}), 7.94(2 \mathrm{H}, \mathrm{s}, 2-\mathrm{H} / 9-\mathrm{H}) ; \delta_{\mathrm{C}} 19.1(\mathrm{C}-1), 24.4$ (C-4/7), 112.7 (C-2/9), 122.7 (C-5/6), 131.9 (C-1a/9a), 135.4 (C-2a/8a), 141.1 (C-3a/7a), 184.8 (C-3/8); $m /=$ (electrospray) 223 $(\mathrm{M}+\mathrm{H})^{+}$

Quinone 20 is readily oxidised to cycloproanthracene 4 and on storing in the freezer for 17 days $9 \%$ conversion to compound 4 was observed. It is best that 4 be stored under an inert atmosphere.

\section{H-Cyclopropa[ $b]$ anthracene-3,8-dione 4}

Method A. A slow stream of oxygen was bubbled through a mixture of quinones 20 and 4 (from above) $(103 \mathrm{mg}$, ca. 0.46 mmol) suspended in dichloromethane $(15 \mathrm{ml})$. After $2 \mathrm{~h}$ the solid had dissolved but the oxygen flow was continued for 12 days with constant volume being maintained by solvent 
addition as required. Solvent removal under reduced pressure gave a yellow solid that was purified by column chromatography (dichloromethane elution) (yellow powder; $82 \mathrm{mg}$, $80 \%$ ). Recrystallization (dichloromethane, $-16^{\circ} \mathrm{C}$ ) gave the anthracenedione $4(28 \mathrm{mg}, 27 \%)$ as yellow crystals, mp $132^{\circ} \mathrm{C}$ (subl., sublimate $\mathrm{mp}>350^{\circ} \mathrm{C}$ (Found: $\mathrm{C}, 81.7 ; \mathrm{H} 3.7 ; \mathrm{m} / \mathrm{z}$ $(\mathrm{M}+\mathrm{H})^{+}$221.0601. $\mathrm{C}_{15} \mathrm{H}_{8} \mathrm{O}_{2}$ requires $\mathrm{C}, 81.8 ; \mathrm{H}, 3.7 \%$; $\left.(M+H)^{+} 221.0602\right) ; \lambda_{\max }$ (acetonitrile)/nm $206(\log \& 4.27), 259$ (4.65), 280 sh (4.04), 324 (3.61); $v_{\max } / \mathrm{cm}^{-1} 2924,2853,1668$, $1592,1581,1557,1317,1292,1123$; NMR $\delta_{\mathrm{H}} 3.41\left(2 \mathrm{H}, \mathrm{s}, \mathrm{CH}_{2}\right)$, 7.79-7.81 (2 H, AA', 5-H/6-H), 8.16 (2 H, s, 2-H/9-H), 8.31$8.34\left(2 \mathrm{H}, \mathrm{BB}^{\prime}, 4-\mathrm{H} / 7-\mathrm{H}\right) ; \delta_{\mathrm{C}} 19.2$ (C-1), 113.5 (C-2/9), 127.2 (C-4/7), 132.8 (C-1a/9a), 133.1 (C3a/7a), 134.0 (C-5/6), 137.0 (C2a/8a), $183.3(\mathrm{C}-3 / 8)$.

Method B. The quinones 20 and $4(50 \mathrm{mg}, 0.22 \mathrm{mmol})$ and DDQ (128 $\mathrm{mg}, 0.56 \mathrm{mmol}$ ) were suspended in anhydrous benzene ( $5 \mathrm{ml})$ at RT for 6.5 days under argon. Workup as in A above gave a mixture of 4 and $D D Q$, and further chromatography provided $4(29 \mathrm{mg}, 58 \%)$ identical to that described in $\mathbf{A}$ above.

Method C. To the mixture of 20 and $4(50 \mathrm{mg}, 0.22 \mathrm{mmol})$ in dry acetone $(5 \mathrm{ml})$ under argon was added anhydrous potassium carbonate $(311 \mathrm{mg}, 2.26 \mathrm{mmol}$ ) and the suspension was stirred for $24 \mathrm{~h}$. Workup as in A above gave 20 and $\mathbf{4}$ in a 1:5 ratio ('H NMR). However, upon repeating the procedure 4 (34 $\mathrm{mg}, 69 \%$ ) was obtained as a yellow solid. If the reaction is left uninterrupted for $48 \mathrm{~h}$ a yellow oil $(20 \mathrm{mg})$ that contains only traces of $\mathbf{4}$ is obtained; a similar outcome follows from reaction with potassium hydroxide.

\section{2-Ethenyl-2,3-dihydro-1 $H$-cyclopenta[ $b$ ]naphthalene 22}

A solution of cyclopropanaphthalene $21(200 \mathrm{mg}, 1.4 \mathrm{mmol})$ in benzene $(5 \mathrm{ml})$ was placed in a calibrated Carius tube $(20 \mathrm{ml})$, cooled to liquid air temperature and buta-1,3-diene (ca. $1 \mathrm{ml}$, ca. $12 \mathrm{mmol}$ ) added by vapour transfer. The sealed tube was slowly warmed and then heated at $100^{\circ} \mathrm{C}$ for $18-19 \mathrm{~h}$ (Carius furnace). The cooled tube (liquid nitrogen) was opened, slowly warmed to room temperature and then excess diene and solvent were removed under vacuum. The product was pumped under vacuum $(5-6 \mathrm{~h})$ to give a white solid ( $300 \mathrm{mg})$ and column chromatography (silica gel; dichloromethane-light petroleum, $1: 4$ elution) afforded from the most mobile fraction a white solid $(180 \mathrm{mg}, 64 \%)$. Recrystallization (dichloromethane, ca. $-16^{\circ} \mathrm{C}$ ) gave 22 as colourless crystals $(160 \mathrm{mg}, 57 \%)$, mp 55$57^{\circ} \mathrm{C}$ (Found: $\mathrm{C}, 92.5 ; \mathrm{H}, 7.5 . \mathrm{C}_{13} \mathrm{H}_{14}$ requires $\mathrm{C}, 92.7 ; \mathrm{H}, 7.3 \%$ ); $\lambda_{\max }$ (hexane)/nm 248 (log $\varepsilon$ 3.46), 268 (3.72), 279 (3.76), 290 (3.60), 308 (3.09), $320(3.15) ; v_{\max } / \mathrm{cm}^{-1} 3079,3054,2980,2834$ $1640,1605,1429,1337,1267,1148,992,911,868,741$; NMR $\delta_{\mathrm{H}} 2.87-2.95\left(2 \mathrm{H}_{\mathrm{A}}, \mathrm{m}, 1-\mathrm{H} / 3-\mathrm{H}\right), 3.10-3.25\left(2 \mathrm{H}, 2 \mathrm{H}_{\mathrm{B}}, \mathrm{m}, 1-\mathrm{H} /\right.$ $3-\mathrm{H}$ and $2-\mathrm{H}), 5.09\left(1 \mathrm{H}\right.$, br d, $\left.10.3,11_{\mathrm{A}}-\mathrm{H}\right), 5.14(1 \mathrm{H}$, br d, $\left.17.3 ; 11_{\mathrm{B}}-\mathrm{H}\right), 5.95-6.06(1 \mathrm{H}, \mathrm{m}, 10-\mathrm{H}), 7.36-7.39\left(2 \mathrm{H}, \mathrm{AA}^{\prime}\right.$, 5-H/8-H), $7.62(2 \mathrm{H}, \mathrm{s}, 4-\mathrm{H} / 9-\mathrm{H}), 7.73-7.76\left(2 \mathrm{H}, \mathrm{BB}^{\prime}, 6-\mathrm{H} / 7\right.$ $\mathrm{H}) ; \delta_{\mathrm{C}} 38.8$ (C-1/3), 44.8 (C-2), 113.8 (C-11), 122.1 (C-5/8), 124.9 (C-4/9), 127.4 (C-6/7), 132.9 (C-4a/8a), 141.8 (C-10), 142.2 (C-3a/9a); m/z 195/194 (M+ $12 / 77 \%), 193(18, \mathrm{M}-\mathrm{H})$, $179\left(100, \mathrm{M}-\mathrm{CH}_{3}\right), 116\left(38, \mathrm{M}-\mathrm{C}_{2} \mathrm{H}_{4}\right), 165\left(83, \mathrm{M}-\mathrm{C}_{2} \mathrm{H}_{5}\right)$, $152\left(20, \mathrm{M}-\mathrm{C}_{3} \mathrm{H}_{6}\right), 139\left(15, \mathrm{M}-\mathrm{C}_{4} \mathrm{H}_{7}\right)$.

\section{Acknowledgements}

The work has received generous financial support from the former Victoria University Grants Committee (B. H.), the Victoria University Science Faculty Leave and Grants Committee (B. H., C. S. J.), and the Curtis-Gordon and VUW Alumni Scholarship Funds (C. S. J.).

\section{References}

1 Preceding paper in the series: B. Halton, D. A. C. Evans and R. N. Warrener, Aust. J. Chem., 1999, 52, 1123.

2 P. Müller, in Carbocyclic Three-Membered Ring Compounds, ed. A. de Meijere, Thieme, Stutgart, 1997, vol. E/17d; B. Halton and P. J. Stang, Synlett, 1997, 145; P. Müller, in Advances in Theoretically Interesting Molecules, ed. R P. Thummel, JAI Press, Greenwich, 1995, vol. 3, p. 37; B. Halton, in The Chemistry of the Cyclopropyl Group, ed. Z. Rappoport, Wiley, Chichester, 1995, vol. 2, p. 707 B. Halton, Chem. Rev., 1989, 89, 1161.

3 T. Watabe, K. Okada and M. Oda, J. Org. Chem, 1988, 53, 216.

4 G. E. Collis, D. Jayatilaka and D. Wege, Aust. J. Chem., 1997, 50 , 505.

5 B. Halton, A. J. Kay, Z. Zhi-mei, R. Boese and T. Haumann. J. Chem. Soc, Perkin Trans. 1, 1996, 1445; B. Halton, A. J. Kay and Z. Zhi-mei, J. Chem. Soc., Perkin Trans. 1, 1993, 2239.

6 S. Ando, J. Imamura, M. Tajitsu and K. Saito, Heterocycles, 1998 48, 1769; J. Laue, G. Seitz and H. Wassmuth, Z. Naturforsch., Tei B., 1996, 51, 348; N. Saracoglu, I. Durucasu and M. Balci, Tetrahedron, 1995, 51, 10979; B. Halton, R. Boese, D. Bläser and Q. Lu, Aust. J. Chem., 1991, 44, 265; U. H. Brinker and H. Wüster. Tetruhedron Lett., 1991, 32, 593; B. Halton and S. G. G. Russell, Aust. J. Chem., 1990, 43, 2099; S. Kagabu and T. Inoue, Chem. Leth. 1989. 2181; H. Kato, S. Toda, Y. Arikawa, M. Masuzawa, M. Hashimoto, K. Ikoma, S.-Z. Wang and A. Miyasaka, J. Chem. Soc., Perkin Trans. 1, 1990, 2035; K. Saito, H. Ishihara and S. Kagabu, Bull. Soc. Chem. Jpn., 1987, 60, 4141.

7 E. Vogel, W. Grimme and S. Korte, Tetrahedron Lett., 1965, 3625; S. Korte, PhD Thesis, University of Köln, 1968.

8 W. E. Billups, W. Y. Chow and C. V. Smith, J. Am. Chem. Soc. 1974, W. E. Billups, 1979.

9 W. E. Billups, W. Luo, D. Harmon, D.McCord and R. Wagner, Tetrahedron Lett., 1997, 38, 4533; W. E. Billups and W. A. Rodin, J. Org. Chem., 1988, 53, 1312; W. E. Billups and W. Y. Chow, J. Am. Chem. Soc. 1973, 95, 4099.

10 W. E. Billups, M. M. Haley, R. C. Claussen and W. A. Rodin, J. Am. Chem. Soc, 1991, 113, 4331.

11 J. Skarzewski, Tetrahedron, 1984, 62, 1995.

12 L. M. Jackman and S. Sternhell, Applications of Nuclear Magnetic Resonance Spectroscopy in Organic Chemistry, Pergamon Press, London, 1969, 2nd. edn., p. 288.

13 C. C. L. Chai, D. Christen, B. Halton, R. Neidlein and M. A. E. Starr, Aust. J. Chem., 1995, 48, 577.

14 Preliminary communication: B. Halton and C. S. Jones, Tetrahedron Lett., 1999, 40, 9367.

15 M. J. Cooney and B. Halton, Aust. J. Chem., 1996, 49, 533

16 J. Lecoultre, E. Heilbronner, P. Müller and D. Rodriguez, Collect. Czech. Chem. Commun., 1988, 53, 2385; W. E. Billups, L.-J. Lin, B. E. Arney, W. A. Rodin and E. W. Casserly, Tetrahedron Lett., 1984, 25, 3935; W. E. Billups, E. W. Casserly and B. E. Arney, J. Am. Chem. Soc., 1984, 106, 440; P. Müller and D. Rodriguez, Helv. Chin. Acta, 1983, 66, 2540; P. Müller and M. Rey, Helv. Chim. Acta, 1982. 65, 1157; P. Müller and M. Rey, Helv. Chim. Acta, 1981, 64, 354; D. Davalian and P. J. Gurratt, Tetrahedron Lett., 1976, 2815.

17 Spartan v. 5.0. Wavefunction. Inc. 18401 Von Karman Avenue, Suite 370, Irvine, CA, 92612, 1997.

18 W. J. Hehre, L. Radom, P. v R. Schleyer and J. A. Pople, Ab Initio Molecular Orbilal Theory, Wiley, New York, 1986. 


\section{References}

1. Greenberg, A., and Liebman, J. F., Strained Organic Molecules, Academic: New York, 1978; Perkin, W. H., J. Chem. Soc., 1888, 1.

2. Advances in Strained and Interesting Organic Molecules, Vol. 8, (Ed. Halton, B.), Jai Press Inc: Connecticut, 2000.

3. Ullman, E. F., and Buncel, E., J. Am. Chem. Soc., 1963, 85, 2106

4. Wiberg, K. B., and Fenoglio, R. A., J. Am. Chem. Soc., 1968, 90, 3395.

5. Dem'yanov, N. Y., and Doyarenko, M. N., Bull. Acad. Sci. Russie., 1926, 16, 297;

Dem'yanov, N. Y., and Doyarenko, M. N., Ber. 1923, 562200 (Chem. Abstr., 1923, 20, 2988); Schlatter, M. J., J. Am. Chem. Soc., 1941, 63, 1733; Wiberg, K. B., Bartley, W. J., J. Am. Chem. Soc., 1960, 82, 6375; Stigliani, W. M., Laurie, V. W., and Li, J. C., J. Chem. Phys, 1975, 62, 1890.

6. Maier, G., Angew. Chem., Int. Ed. Engl., 1988, 27, 309.

7. Breslow, R., Pure Appl. Chem., 1971, 28, 111.

8. Korth, A., Marcon. M. L., Mendis, D. A., Krueger, F. R., Richter, A, K., Lin, R. P., Mithell, D. L., Anderson, K. A., Carlson, C. W., Reme, H., Sauvand, J. A., and d'Uston, C., Nature, 1989, 52, 337.

9. Breslow, R., Groves, J. T., and Ryan, G., J. Am. Chem. Soc., 1967, 89, 5048; Breslow, R., and Groves, J., J. Am. Chem. Soc., 1970, 92, 984.

10. Glukhovtsev, M. N., Laiter, S., and Pross, A., J. Phys. Chem., 1996, 100, 17801.

11. McMurry J., Organic Chemistry, $3^{\text {rd }}$ Ed., Brooks/Cole: California, 1992.

12. Hofmann, R. W., Dehydrobenzene and Cycloalkynes, Academic: New York, 1967.

13. Chapman, O. L., Mattes, K., McIntosh, C. L., Pacansky, J., Calder, G. V., and Orr, G., J. Am. Chem. Soc., 1973, 95, 6134.

14. Cava, M. P., and Napier, D. R., J. Am. Chem. Soc., 1958, 80, 2255.

15. Ullman, E. F., and Buncel, E., J. Am. Chem. Soc., 1963, 85, 2106.

16. Vogel, E., Grimme, W., and Korte, S., Tetrahedron Lett., 1965, 3625.

17. Mills, W. H., and Nixon, I. G., J. Chem Soc., 1968, 90, 3395.

18. Longuet-Higgins, H. C., and Coulson, C. A., Trans. Faraday Soc., 1946, 42, 756.

19. Apeloig, Y., and Arad, D., J. Am. Chem. Soc., 1986, 108, 3241.

20. Baldridge. K. K., and Siegel, J. S., J. Am. Chem. Soc., 1992, 114, 9583.

21. Hiberty, P. C., Ohanessian, G., and Delbecq, F., J. Am. Chem. Soc., 1985, 107, 3095; Mó, O., Yáñez, M., Eckert-Maksic, M., and Maksic, Z. B., J. Org. Chem., 1995, 60, 1638, and references cited therein. 
22. Neidlen, R., Christen, D., Poignée, V., Boese, R., Bläser, D., Gieren, A., RuizPérez, C., Hüber, T., Angew. Chem., Int. Ed. Engl., 1988, 27, 294.

23. Billups, W. E., Chow, W. Y., Leavell, K. H., Lewis, E. S., Margrave, J. L., Sass, R. L., Shieh, J, J., Werness, P. G., and Wood, J. L., J. Am. Chem. Soc., 1973, 95, 7878.

24. Halton, B., Randall, C. J., Gainsford, G. J., and Stang, P. J., J. Am. Chem. Soc., 1986, 108, 5949 .

25. Bläser, D., Boese, R., Brett, W. A., Rademacher, P., Schwager, H., Stanger, A., and Vollhardt, K. P. C., Angew. Chem., Int. Ed. Engl., 1989, 28, 206.

26. Boese, R., Bläser, D., Billups, W. E., Haley, M. M., Maulitz, A. H., Mohler, D. L., and Vollhardt, K. P. C., Angew. Chem., Int. Ed. Engl., 1994, 33, 313.

27. Apeloig, Y., Boese, R., Halton, B., and Maulitz, A. H., J. Am. Chem. Soc., 1998, $120,10147$.

28. Halton, B., Cooney, M. J., Boese, R., and Maulitz, A. H., J. Org. Chem., 1998, 63, 1583.

29. Diercks, R., and Vollhardt, K. P. C., J. Am. Chem. Soc., 1986, 108, 3150; Stanger, A., and Vollhardt, K. P. C., J. Org. Chem., 1988, 53, 4889.

30. Bürgi, H. B., Baldridge, K. K., Hardcastle, K., Frank, N. L., Gantzel, P., Siegel, J. S., and Ziller, J., Angew. Chem., Int. Ed. Engl., 1995, 34, 1454.

31. De, S. C., and Dutt, D. N., J. Indian Chem. Soc., 1930, 86, 525.

32. Halton, B., Harrison, S. A. R., and Spangler, C. W., Aust. J. Chem. 1975, 28, 681.

33. Anet, R., and Anet, F. A. L., J. Am. Chem. Soc., 1964, 86, 525.

34. Dürr, H., and Schrader, L., Angew. Chem., Int. Ed. Engl., 1969, 8, 446; Chem. Ber., 1970, 103, 1334.

35. Halton, B., "Cycloproparenes." In The Chemistry of the Cyclopropyl Group (Ed. Rappoport, Z.), Wiley: Chichester, 1995.

36. Halton, B., Chem. Rev., 1989, 89, 1161.

37. Dürr, H., and Schmitz, H., Angew. Chem., Int. Ed. Engl., 1975, 14, 647.

38. Brogli, F., Giovannini, E., Heilbronner, E., and Schurter, R., Chem. Ber., 1973, 106, 961 .

39. Korte, S., Ph. D. Thesis, University of Köln, 1968.

40. Okazaki, R., O-oko, M., Tokitoh, N., and Inamoto, N., J. Org. Chem., 1985, 50, 180 .

41. Davalian, D., Garratt, P. J., and Mansuri, M. M., J. Am. Chem. Soc., 1978, 100, 980 .

42. Bee, L. K., Garratt, P. J., and Mansuri, M. M., J. Am. Chem. Soc., 1980, 102, 7076.

43. Billups, W. E., Rodin, W. A., and Haley, M. M., Tetrahedron, 1988, 44, 1305. 
44. Billups, W. E., Chow, W. Y., and Smith, C. V., J. Am. Chem. Soc., 1974, 96, 1979.

45. Brinker, U. D., and Wüster, H., Tetrahedron Lett., 1991, 32, 593.

46. Vogel, E., Ippen, J., and Buch, V., Angew. Chem., Int. Ed. Engl., 1975, 14, 566.

47. Neidlein, R., and Tadesse, L., Helv. Chim. Acta, 1988, 71, 249.

48. Neidlein, R., Kohl, M., and Kramer, W., Helv. Chim. Acta, 1989, 72, 1311.

49. Kagabu, S., and Saito, K., Tetrahedron Lett., 1988, 29, 675.

50. Wentrup, C., Wentrup-Byrne, E., and Müller, P., J. Chem. Soc., Chem. Commun., 1977, 210; Wentrup, C., Wentrup-Byrne, E., and Müller, P., Tetrahedron Lett., $1979,4249$.

51. Dürr, H., and Ahr, H. -J., Tetrahedron Lett., 1977, 1991.

52. Lüddecke, E., Rau, H., Dürr, H., and Schmitz, H., Tetrahedron, 1977, 33, 2677.

53. Billups, W. E., and Chow, W. Y., J. Am. Chem. Soc., 1973, 95, 4099.

54. Tanimoto, S., Schäfer, R., Ippen, J., and Vogel, E., Angew. Chem., Int. Ed. Engl., 1976, 15, 613.

55. Apeloig, Y., unpublished observations, 1989. Professor Apeloig is thanked for making these data available.

56. Billups, W. E., Casserly, E. W., and Arney, B. E. Jr., J. Am. Chem. Soc., 1984, 106, 440 .

57. Billups, W. E., Lin, L. -J., Arney, B. E. Jr., Rodin, W. A., and Casserly, E. W., Tetrahedron Lett., 1984, 25, 3935.

58. Halton, B., Dent, B. R., Böhm, S., Officer, D. L., Schmickler, H., Schophoff, F., and Vogel, E., J. Am. Chem. Soc., 1985, 107, 7176.

59. Billups, W. E., Blakeney, A. J., Chow, W. Y., J. Chem. Soc., Chem. Commun., 1971, 1461.

60. Billups, W. E., Blakeney, A. J., Chow, W. Y., Org. Synth., 1976, 55, 12.

61. Prestien, J., and Günther, H., Angew. Chem., Int. Ed. Engl., 1974, 13, 276.

62. Halton, B., Diggins, M. D., Zhi-Mei, Z., and Kay, A. J., J. Org. Chem., 1992, 57, 4080 .

63. Browne, A. R., Halton, B., and Spangler, C. W., Tetrahedron, 1974, 30, 3289.

64. Halton, B., Kay, A. J., Zhi-Mei, Z., Boese, R., and Haumann, T., J. Chem. Soc., Perkin Trans. 1, 1996, 1445.

65. Ippen, J., and Vogel, E., Angew. Chem., Int. Ed. Engl., 1974, 13, 736; Halton, B., Boese, R., Bläser, D., and Lu, Q., Aust. J. Chem., 1991, 44, 265.

66. Davalian, D., and Garratt, P. J., Tetrahedron Lett., 1976, 2815.

67. Casserly, E. W., Ph. D. Thesis, Rice University, 1983. 
68. Billups, W. E., Reed, L. E., Casserly, E. W., and Lin, L. -J., J. Org. Chem., 1981, 46, 1326.

69. Billups, W. E., Haley, M. M., Claussen, R., C., and Rodin, W. A., J. Am. Chem. Soc., 1991, 113, 4331.

70. Billups, W. E., McCord, D. J., and Maughon, B. R., J. Am. Chem. Soc., 1994, 116, 8831.

71. Halton, B., and Milsom, P. J., J. Chem. Soc., Chem. Commun., 1971, 814.

72. Halton, B., Milsom, P. J., and Woolhouse, A. D., J. Chem. Soc., Perkin Trans. 1, $1977,731$.

73. Browne, A. R., and Halton, B., J. Chem. Soc., Chem. Commun., 1972, 1341.

74. Browne, A. R., and Halton, B., J. Chem. Soc., Perkin Trans. 1, 1977, 1177.

75. Müller, P., Etienne, R., Pfyffer, J., Pineda, N., and Schipoff, M., Helv. Chim. Acta, 1978, 61, 2482.

76. Müller, P., and Schaller, J. -P., Helv. Chim. Acta, 1990, 73, 1228.

77. Billups, W. E., Arney, B. E., and Lin, L. -J., J. Org. Chem., 1984, 49, 3436.

78. Dent, B. R., and Halton, B., Aust. J. Chem., 1987, 40, 925.

79. Müller, P., Thi, H. C. N., and Pfyffer, J., Helv. Chim. Acta, 1986, 69, 855.

80. Dent, B. R., and Halton, B., Tetrahedron Lett., 1984, 25, 4279.

81. Dent, B. R., and Halton, B., Aust. J. Chem., 1986, 39, 1789.

82. Halton, B., Woolhouse, A. D., Hügel, H. M., and Kelly, D. P., J. Chem. Soc., Chem. Commun. 1974, 247.

83. Halton, B., Hügel, H. M., Kelly, D. P., Müller, P., and Burger, U., J. Chem. Soc., Perkin Trans. 2, 1976, 258.

84. Roberts, J. D., Streitweiser, A., and Regan, C. M., J. Am. Chem. Soc., 1952, 74, 4579 .

85. Müller, P., Helv. Chim. Acta, 1974, 57, 704.

86. Müller, P., J. Chem. Soc., Chem. Commun., 1973, 895.

87. Halton, B., Woolhouse, A. D., and Milsom, P. J., J. Chem. Soc., Perkin Trans. 1, $1977,735$.

88. Müller, P., Helv. Chim. Acta, 1973, 56, 500.

89. See citation 231 in ref. 31 .

90. Eaborn, C., and Stamper, J. G., J. Organomet. Chem., 1980, 192, 155; Eaborn, C., Eidenschink, R., Harris, S. J., and Walton, D. R. M., J. Organomet. Chem., 1977, 124, C27-C29.

91. Moore, L., Lubinski, R., Baschky, M. C., Dahlke, G. D., Hare, M., Arrowood, T., Glasovac, Z., Eckert-Maksic, M., and Kass, S. R., J. Org. Chem., 1997, 62, 7390. 
92. Szeimies, G., and Wimmer, P., unpublished observations, 1985; Wimmer, P., Diplomarbiet, University of Munich. Professor Szeimies is thanked for making these data available.

93. Cutler, C. A., and Halton, B., Aust. J. Chem., 1997, 50, 267.

94. Weber, W. P., Silicon Reagents for Organic Synthesis, Springer-Verlag: New York, 1983.

95. McNicholls, A. T., Stang, P. J., Addington, D. M., and Halton, B., Tetrahedron Lett., 1994, 35, 437.

96. Jones, C. S., B.Sc (Hons.) Project Report, Victoria University of Wellington, 1997.

97. Halton, B., and Jones, C. S., J. Chem. Soc., Perkin Trans. 2, 1998, 2505.

98. Halton, B., Randall, C. J., and Stang, P. J., J. Am. Chem. Soc., 1984, 106, 6108.

99. Halton, B., and Stang, P. J., Acc. Chem. Res., 1987, $20,443$.

100. Halton, B., and Stang, P. J., SYNLETT, 1997, 145.

101. Halton, B., Lu, Q., and Stang, P. J., J. Chem. Soc., Chem. Commun., 1988, 879.

102. Halton, B., Cooney, M. J., Davey, T. W., Forman, G. S., Lu, Q., Boese, R., Bläser, D., and Maulitz, A. H., J. Chem. Soc., Perkin Trans. 1, 1995, 2819.

103. Halton, B., Buckland, S. J., Lu, Q., Mei, Q., and Stang, P. J., J. Org. Chem., 1988, $\mathbf{5 3}, 2418$.

104. Dixon, G. M., and Halton, B., unpublished observations, 1997-2001.

105. Halton, B., Buckland, S. J., Mei, Q., and Stang, P. J., Tetrahedron Lett., 1986, 27, 5159.

106. Apeloig, Y., Karni, M., Arad, D., "Cycloprobenzene and Alkylidenecyclopropabenzenes. A Synergistic Relationship Between Theory and Experiment." In "Strain and Its Implications" (de Meijere, A., and Blechert, S., Eds.), Reidel, Dordrecht, 1989.

107. See citation 202 in ref. 35 .

108. Halton, B., Lu, Q., and Melhuish, W. H., J. Photochem. Photobiol., A: Chem., 1990, 52, 205.

109. Buckland, S. J., Halton, B., and Stang, P. J., Aust. J. Chem., 1988, 41, 845.

110. Buckland, S. J., Halton, B., Mei, Q., and Stang, P. J., Aust. J. Chem., 1987, 40, 1375.

111. Forman, G. S., and Halton, B., unpublished observations, 1994.

112. McNichols, A. T., Stang, P. J., Halton, B., and Kay, A. J., Tetrahedron Lett., 1993, 34, 3131.

113. Halton, B., Kay, A. J., McNichols, A. T., Stang, P. J., Apeloig, Y., Maulitz, A. H., Boese, R., and Haumann, T., Tetrahedron Lett., 1993, 34, 6151. 
114. Halton, B., Cooney, M. J., and Wong, H., J. Am. Chem. Soc., 1994, 116, 11574.

115. Bickers, P. Y., Halton, B., Kay, A. J., and Northcote, P. T., Aust. J. Chem., 1999, 52, 647 .

116. Halton, B., Kay, A. J., Zhi-Mei, Z., Boese, R., and Haumann, T., J. Chem. Soc., Perkin Trans. 1, 1996, 1445.

117. Fritzsche, G., Gleiter, R., Irngartinger, H., and Oeser, T., Eur. J. Org. Chem., 1999, 73.

118. Halton, B., Kay, A. J., and Zhi-Mei, Z., J. Chem. Soc., Perkin Trans. 1, 1993, 2239.

119. Halton, B., Jones, C. S., Kay, A. J., Margetic, D., and Stretenovic, S., J. Chem. Soc., Perkin Trans. 1, 2000, 2205.

120. Halton, B., Evans, D. A. C., and Warrener, R. N., Aust. J. Chem., 1999, 52, 1123.

121. Sretenovic, S., M.Sc. Thesis, Victoria University of Wellington, 1998.

122. Wudl, F., Smith, G. M., and Hufnage, E. J., J. Chem. Soc., Chem. Commun., 1970, 1453.

123. Moore, A. J., and Bryce, M. R., J. Chem. Soc., Perkin Trans. 1, 1991, 157.

124. For a review see: Bryce, M. R., Chem. Soc. Rev., 1991, 20, 355.

125. Kurata, H., Kawase, T., and Oda, M., Tetrahedron Lett., 34, 3445.

126. Cook, E. S., and Hill, A. J., J. Am. Chem. Soc., 1940, 62, 1995.

127. Halton, B., Jones, C. S., Northcote, P. T., and Boese, R., Aust. J. Chem., 1999, 52, 285 .

128. Gerkin, R, E., Lundstedt, A. P., and Reppart, W. J., Acta Cryst., 1984, C40, 1892.

129. Dean, F. M., Houghton, L. E., Nayyir-Mashir, R., and Thebtaranonth, C., J. Chem. Soc., Perkin Trans. 1, 1980, 1994.

130. Thomas, R., Environ. Sci. Technol., 1983, 17, 666 (Chem. Abst., 1983, 99, 145276y).

131. Olmsted, J. III, J. Phys, Chem, 1979, 83, 2581.

132. Cooney, M. J., and Halton, B., unpublished observations.

133. For an extensive review see: Zeller, K., -P In "Carbocyclische $\pi$-Electronen Systeme, Methoden der Organischen Chemie (Houben Weyl)", Band 5, Teil 2C, Georg Thieme Velag, Stuttgart, 1985.

134. Thiele, J., Ber., 1900, 33, 672.

135. The Chemistry of the Metal-Carbon Bond, Vol. 2 (Ed. Hartley, F. R., Patai, S.), Wiley: Chichester, 1985.

136. Peterson, M. L., Strnad, J. T., Markotan, T. P., Morales, C. A., Scaltrito, D. V., and Staley, S. W., J. Org. Chem., 1999, 64, 9067. 
137. Wiberg, K. B., "Origin of the Amide Rotational Barrier". In "Amide Linkage" (Eds. Greenberg, A., Breneman, C. M., Liebman, J. L.), Wiley: New York, 2000.

138. Narasimhan, N. S., and Paradkar, M. V., Indian. J. Chem., 1969, 7, 536.

139. Levy, G. C., Lichter, R. L., and Nelson, G. J., Carbon-13 Nuclear Magnetic Resonance Spectroscopy, 2nd Edition, Wiley, New York, 1980.

140. Reich, H. J., Holtan, R. C., and Bolm, C., J. Am. Chem. Soc., 1990, 112, 5609.

141. House, H. O., Czuba, L. J., Gall, M., Olmstead, H. D., J. Org. Chem., 1969, 34, 2324.

142. Halton, B., Jones, C. S., and Margetic, D., Tetrahedron, 57, 2001, 3529.

143. Walker, B. J., Organophosphorous Chemistry (Eds. Griffiths, V. S., Feather, N., Lee, W. H., Holliday, A. K., and Williams, G. H.), Penguin Books Ltd., Middlesex, 1972.

144. Davies, J. E., Klunduk, M. C., Mays, M. J., Raithby, P. R., Shields, G. P., Tompkin, P. K., J. Chem. Soc., Dalton Trans., 1997, 715; Mikolajczyk, M., Kielbasinski, P., Wieczorek, M. W., Blaszczyk, J., Kolbe, A., J. Org. Chem., 1990, 55. 1998; Baulder, M., Michels, M., Pieroth, M., Hahn, J., Angew. Chem., Int. Ed. Engl., 1990, 98, 465.

145. Arad, D., Ph.D. Thesis, Technion-Israel Institute of Technology, 1986.

146. Staab, H. A., and Ipaktschi, J., Chem. Ber., 1968, 101, 1457.

147. Cooney, M. J., and Halton, B., Aust. J. Chem., 1996, 49, 533.

148. Wege, D., Eur. J. Org. Chem., 2001, 849.

149. Collins, G. E., Jayatilaka, D., and Wege, D., Aust. J. Chem., 1997, 50, 505.

150. Watabe, T., Okada, K., and Oda, M., J. Org. Chem., 1988, 53, 216.

151. Diels, O., and Alder, K., Chem. Ber., 1929, 62, 2337.

152. Rao, A. V. R., Yadar, J. S., Reddy, K. B., and Mehendale, A. R., Tetrahedron, 1984, 4643; Alexander, J., and Mitcher, L. A., Tetrahedron Lett., 1978, 19, 3403.

153. Kay, A. J., Ph.D. Thesis, Victoria University of Wellington, 1996.

154. Daumas, M., Vo Quang, Y., Vo Quang, L., and Le Goffic, F., Synthesis, 1989, 64; Fischer, A., and Henderson, G. N., Synthesis, 1985, 7, 641.

155. Singh, I., Ogata, R. T., Moore, R. E., Chang, C. W. J., and Schewer, P. J., Tetrahedron, 1968, 24, 6053; St. Berger, and Rieker, A., "Identification and Determination of Quinones". In "The Chemistry of Quinonoid Compounds" (Ed. Patai, S.), Vol. 1, Part 2, Wiley, Chichester, 1974, pp. 195-204.

156. Halton, B., Jones, C. S., Tetrahedron Lett., 1999, 40, 9367.

157. Diels, O., and Alder, K., Liebigs, Ann, Chem., 1928, 98, 460. 
158. Joshi, B. S., Jiang, Q., Rho, T., and Pelletier, S. W., J. Org. Chem., 1994, 59, 8220;

Kato, M., Kasai, M., Shiraki, K., Furuichi, K., and Miwa, T., Chem. Lett., 1987, 4, 669.

159. Simmons, H. E., and Smith, R. D., J. Am. Chem. Soc., 1958, 80, 5323; Ibid, 1959, 81, 4256; Denis, J. M., Girard, J. M., and Coria, J. M., Synthesis, 1972, 549.

160. Neidlein, R., Poignee, V., Kramer, W., and Glueck, C., Angew. Chem., Int. Ed. Engl., 1986, 98, 735 .

161. For example see: Segura, J. L., and Martín, N., Angew. Chem., Int. Ed., 2001, 40, 1372; Bryce, M. R., J. Mater. Chem., 2000, 10, 589; Nielse, M. B., Lomholt, C., and Becher, J., Chem. Soc. Rev., 2000, 29, 153; Bryce, M. R., J. Mater. Chem., 1995, 5, 1481; Garín, J., Adv. Heterocycl. Chem., 1995, 62, 249.

162. For example see: Brisset, H., Moustarderle, S., Blanchard, P., Illien, B., Riou, A., Orduna, J., Garín, J., Roncali, J., J. Mater. Chem., 1997, 7, 2027; Bryce, M. R., J. Mater. Chem., 1995, 5, 1481; Brisset, H., Thobie-Gautier, C., Jubault, M., Gorgues, and Roncali, J., J. Chem. Soc., Chem. Commun., 1994, 1765.

163. Yamashita, Y., Kobayashi, Y., and Miyashi, T., Angew. Chem., Int. Ed. Engl., 1989, 28, 1052.

164. Moore, A. J., Bryce, M. R., J. Chem. Soc., Perkin Trans. 1, 1991, 157.

165. Bryce, M. R., Finn, T., Moore, A. J., Batsanov, A. S., and Howard, J. A. K., Eur. J. Org. Chem., 2000, 51.

166. Bryce, M. R., Moore, A. J., Hasan, M., Ashwell, G. J., Fraser, A. T., Clegg, W., Hursthouse, M. B., Karahlov, A. I., Angew. Chem. Int. Ed. Engl., 1990, 29, 1450.

167. Yamashita, Y., Tomura, M., Zaman, M. B., and Imaeda, K., J. Chem., Soc., Chem. Commun., 1998, 1657; Moore, A. J., Bryce, M. R., Batsanov, A. S., Green, A., Howard, J. A. K., McKervey, M. A., McGuigan, P., Ledoux, I., Ork, E., Viruela, R., Virulea, P. M., and Tabit, B., J. Mater. Chem., 1998, 8, 1173; Bryce, M. R., Moore, A. J., Tanner, B. K., Whitehead, R., Cleggs, W., Gerson, F., Lamprecht, A., and Pfenninger, S., Chem. Mater., 1996, 8, 1182.

168. Yoshida, Z., Kawase, T., Awaji, H., Sugimoto, T., and Yoneda, S., Tetrahedron Lett., 1983, 24, 3469; Awaji, H., Sugimoto, T., Misaka, Y., Kawase, T., Yoneda, S., and Yoshida, Z., Chem. Mater., 1989, 1, 535.

169. Ohta, A., and Yamashita, Y., J. Chem., Soc., Chem. Commun., 1995, 557; Elandaloussi, E. H., Benahmed-Gasmi, A., Riou, A., Gorgues, A., and Roncali, J., J. Mater. Chem., 1996, 6. 1859. 
170. Takahashi, K., and Tomitani, K., J. Chem. Soc., Chem. Commun., 1991, 821; Takahashi, K., Nirhira, T., and Tomitani, K., J. Chem., Soc., Chem., Commun., 1993, 1617.

171. Martín, N., Ortí, E., Sánchez, L., Viruela, P. M., and Viruela, R., Eur. J. Org. Chem., 1999, 1239.

172. Suzuki, T., Sakimura, T., Tanaka, S., Yamashita, Y., Shiohara, H., and Miyashi, T., J. Chem. Soc., Chem. Commun., 1994, 1431.

173. Finn, T., Bryce, M. R., Batsanov, A. S., and Howard, J. A. K., J. Chem. Soc., Chem. Commun., 1999, 1835.

174. Hansen, T. K., Jørgensen, T., Jensen, F., Thygesen, P. H., Christiansen, K., Hursthouse, M. B., Harman, M. E., Malik, M. A., Girmay, B., Underhill, A. E., Begtrup, M., Kilburn, J. D., Belmore, K., Roepstorff, P., and Becher, J., J. Org. Chem., 1993, 58, 1359.

175. Godbert, N., Batsanov, A. S., Bryce, M. R., and Howard., J. A. K., J. Org. Chem., $2001,66,713$.

176. Khodorkovskii, V. Y., Bite, D., Petrova, M. V., Neilands, O., Khim. Geterotsikl. Soedin., 1984, 5705 (Chem. Abstr., 1984, 101, 110783u).

177. Wudl, F., and Kaplan, M. L., J. Org. Chem., 1974, 39, 3608.

178. Boulle, C., Desmars, O., Gautier, N., Hudhomme, P., Cariou, M., and Gorgues, A., J. Chem. Soc., Chem. Commun., 1998, $20,2197$.

179. Boese, R., and Bläser, D., unpublished observations (2000).

180. Foster, R., Organic Charge-Transfer Complexes, Academic Press: London, 1969.

181. Bryce, M. R., Moore, A. J., Lorcy, D., Dhindsa, A. S., and Robert, A., J. Chem. Soc., Chem. Commun., 1990, 470.

182. Perrin, D. D., Armarego, W. L. F., and Perrin, D. R., Purification of Laboratory Chemicals, $2^{\text {nd }} E d$., Pergamon Press: London, 1980.

183. Winkle, M. R., Lansinger, J. M., and Ronald, R. C., J. Chem. Soc., Chem. Commun., 1980, 87.

184. CHEM 363 Laboratory Manual, Victoria University of Wellington, 1973, p. 87. 